\title{
Entwurf von modellbasierten Anti-Windup-Methoden für Systeme mit Stellbegrenzungen
}

\author{
Vom Fachbereich \\ Elektrotechnik und Informationstechnik \\ der Technischen Universität Darmstadt \\ zur Erlangung des akademischen Grades \\ eines Doktor-Ingenieurs (Dr.-Ing.) \\ genehmigte Dissertation
}

Von

\section{Dipl.-Ing. Andreas Ortseifen}

geboren am 8. Oktober 1983 in Karlsruhe

Referent: Prof. Dr.-Ing. J. Adamy

Korreferent: Prof. Dr.-Ing. S. Liu

Tag der Einreichung: 23. November 2012

Tag der mündlichen Prüfung: 27. Mai 2013

D17

Darmstadt 2012 



\section{Vorwort}

Die vorliegende Arbeit entstand im Rahmen meiner Tätigkeit als wissenschaftlicher Mitarbeiter in der Zeit von Mai 2009 bis August 2012 am Institut für Automatisierungstechnik und Mechatronik, Fachgebiet Regelungstheorie und Robotik, der TU Darmstadt.

Mein ganz besonderer Dank gilt Herrn Prof. Dr.-Ing. Jürgen Adamy, der mir die Möglichkeit gegeben hat, diese Arbeit unter seiner Betreuung anzufertigen. Seine vielfältige Unterstützung, der mir gewährte fachliche Freiraum und das hervorragende wissenschaftliche Arbeitsklima am Fachgebiet haben sehr zum Gelingen der Arbeit beigetragen.

Herrn Prof. Dr.-Ing. Steven Liu bin ich für die Übernahme des Korreferats und die kritische Durchsicht der Arbeit sehr verbunden.

Des Weiteren möchte ich allen Kollegen und Freunden vom Institut meinen Dank für die vielen fachlichen und nichtfachlichen Diskussionen und die herausragende Arbeitsatmosphäre aussprechen. Für die notwendige organisatorische und administrative Unterstützung danke ich Birgit Heid, Susanne Muntermann und Sylvia Gelman.

Schließlich gilt mein Dank Dieter Lens, Arne Wahrburg und Klaus Kefferpütz für die inhaltliche Durchsicht der Arbeit. Ihre Anmerkungen und Kommentare waren eine wertvolle Unterstützung bei der Fertigstellung. Valentina Ansel hat die grafisch anspruchsvollen Bilder der Beispielsysteme erstellt, wofür ich mich ebenfalls recht herzlich bedanke.

Nicht zuletzt möchte ich meiner Familie und Rianne Engberink für die großartige und vielfältige Unterstützung ganz herzlich danken. 



\section{Inhaltsverzeichnis}

Abkürzungen und Symbole

IX

Kurzfassung

XIV

1 Einleitung 1

1.1 Zielsetzung der Arbeit . . . . . . . . . . . . . . 2

1.2 Gliederung der Arbeit . . . . . . . . . . . . 3

2 Die Windup-Problematik $\mathbf{5}$

2.1 Definition des betrachteten Regelkreises . . . . . . . . 5

2.1.1 Güteforderungen . . . . . . . . . . . . . 6

2.1.2 Reale Aktoren . . . . . . . . . . . . . . . 10

2.2 Charakterisierung des Windup-Begriffs . . . . . . . . . 13

2.2.1 Windup im klassischen Sinne . . . . . . . . . . 13

2.2 .2 Windup heute . . . . . . . . . . . . 15

2.2.3 Beispiel: hydraulisches Positioniersystem . . . . . . . 17

2.3 Zusammenfassung und Fazit . . . . . . . . . . . . 18

3 Umgang mit Stellbegrenzungen $\quad 20$

3.1 Stabilisierung eingangsbeschränkter Systeme . . . . . . . 20

3.2 Grundlegende Vorgehensweisen . . . . . . . . . . . . . 22

3.2.1 Direkte Berücksichtigung der Stellbegrenzungen . . . 22

3.2.2 Modellbasierte prädiktive Regelung . . . . . . . . . . 24

3.2.3 Governor-Konzepte . . . . . . . . . . . . . 26

3.3 Anti-Windup-Methoden . . . . . . . . . . . . 27

3.3 .1 Grundidee . . . . . . . . . . . . . 27

3.3.2 Historische Entwicklung . . . . . . . . . . . . 28

3.3.3 Komplexität und Leistungsfähigkeit . . . . . . . . . 34

3.4 Zusammenfassung und Fazit . . . . . . . . . . . . . 36

4 Modernes Anti-Windup: Grundlagen $\quad 37$

4.1 Definition des Anti-Windup-Regelkreises . . . . . . . . . 37

4.2 Ziele von modernem Anti-Windup . . . . . . . . . . 40 
4.3 Allgemeines lineares Anti-Windup . . . . . . . . . . . 44

4.3.1 Problematik beim Entwurf . . . . . . . . . . 45

4.3.2 Entwurf mit virtuellen Ausgängen . . . . . . . . . . 47

4.4 Modellbasiertes Anti-Windup . . . . . . . . . . . . . 48

4.4.1 Entkopplung durch Zustandstransformation . . . . . 49

4.4.2 Entwurf der Zustandsrückführung . . . . . . . 53

4.5 Modulares Anti-Windup . . . . . . . . . . . . . . . . . 59

4.5.1 Vermeidung von Regler-Windup . . . . . . . . . 59

4.5.2 Vermeidung von Strecken-Windup . . . . . . . . . 64

4.5.3 Lösung des Direktionalitätsproblems . . . . . . . . . 67

4.6 Zusammenfassung und Ausblick . . . . . . . . . . . 68

5 Ein Entwurfsverfahren für die Beobachtertechnik $\quad \mathbf{7 0}$

5.1 Problembeschreibung . . . . . . . . . . . . . 70

5.2 Analyse der Stabilität . . . . . . . . . . . . . 73

5.2 .1 Vorüberlegungen . . . . . . . . . . . . . 73

5.2.2 Eigenschaften der Sättigungsfunktion . . . . . . . 74

5.2.3 Stabilitätsprüfung mit dem Satz von Lee . . . . . . . 76

5.3 Analyse der Regelgüte . . . . . . . . . . . . . . 78

5.3.1 Grundidee: Ein Ersatzsystem für die Analyse . . . . 79

5.3.2 Abschätzung der L2-Verstärkung . . . . . . . . . 87

5.3.3 Präzisere Abschätzung für kleine Signale . . . . . . . 89

5.3.4 Charakterisierung der Abweichung . . . . . . . . . 92

5.4 Eine systematische Auswahlmethode . . . . . . . . . . . 94

5.4.1 Zusammenfassung der Entwurfsschritte . . . . . . . 94

5.4.2 Beispiel: hydraulisches Positioniersystem . . . . . . . 96

5.5 Zusammenfassung und Fazit . . . . . . . . . . . . . . . 99

6 Stabilität von modellbasiertem Anti-Windup 100

6.1 Regelkreis und Struktur des Anti-Windup . . . . . . . . . 100

6.2 Transformation in Mismatch-Koordinaten . . . . . . . . . 102

6.3 Stabilität des Ursprungs . . . . . . . . . . . . . . . . . 105

6.3.1 Stabilitätseigenschaften des linearen Teilsystems . . 106

6.3.2 Eigenschaften des Anti-Windup-Ersatzsystems . . . 107

6.4 Stabilität anderer Ruhelagen . . . . . . . . . . . . 113

6.4.1 Ruhelagen des unbeschränkten Regelkreises . . . . . 113

6.4.2 Zulässige Ruhelagen . . . . . . . . . . . . . . . . . . 114

6.4.3 Eine zusätzliche Entwurfsbedingung . . . . . . . 115

6.5 Zusammenfassung und Fazit . . . . . . . . . . . 120 
7 Lineares Anti-Windup für stabile Strecken 121

7.1 Problembeschreibung . . . . . . . . . . . . . . 121

7.2 Entwurfsbedingungen . . . . . . . . . . . . . . 123

7.2.1 Bedingungen für globale Stabilität . . . . . . . . . . 124

7.2.2 Bedingungen für Regelgüte . . . . . . . . . . . 126

7.3 Entwurf mit Path-Following . . . . . . . . . . . . . 130

7.4 Beispiel: Längsdynamik eines Flugzeugs . . . . . . . . . 137

7.4.1 Parametrierung des Anti-Windup . . . . . . . . 139

7.4 .2 Führungsregelung . . . . . . . . . . . . . . . 142

7.5 Zusammenfassung und Fazit . . . . . . . . . . . . . 144

8 Anti-Windup mit variabler Struktur für stabile Strecken 145

8.1 Problembeschreibung . . . . . . . . . . . . . 145

8.2 Weich strukturvariable Regelung . . . . . . . . . . 146

8.2 .1 Grundlagen . . . . . . . . . . . . . . 147

8.2.2 Entwurf mittels konvexer Optimierung . . . . . . . 150

8.2.3 Erweiterung des Regelkonzeptes . . . . . . . . . 153

8.3 Weich strukturvariables Anti-Windup . . . . . . . . 156

8.4 Beispiel: elektrisches Netzwerk . . . . . . . . . . . . . 160

8.5 Zusammenfassung und Fazit . . . . . . . . . . . . 163

9 Lineares Anti-Windup für grenz- und instabile Strecken 164

9.1 Problembeschreibung . . . . . . . . . . . . . . . . . 164

9.2 Abschätzen der Störenergie . . . . . . . . . . . . . 168

9.2.1 Zustandstransformation . . . . . . . . . . 168

9.2.2 Ausgangsenergie eines linearen Systems . . . . . . 170

9.3 Anti-Windup-Entwurf . . . . . . . . . . . . . . . 173

9.3.1 Parametrierung der Anti-Windup-Matrix . . . . . 173

9.3.2 Zusammenfassung der Entwurfsschritte . . . . . 176

9.4 Beispiel: Tauchtiefe eines U-Bootes . . . . . . . . . . . . 178

9.5 Zusammenfassung und Fazit . . . . . . . . . . . . 181

10 Zusammenfassung 182

A Mathematischer Anhang 184

A.1 Eigenschaften der Spektralnorm . . . . . . . . . . . . . 184

A.2 Sätze über lipschitzstetige Funktionen . . . . . . . . . . . 184

A.3 Matrizen und Details zu den Beispielen . . . . . . . . 186

A.3.1 Matrizen des linearen Teilsystems . . . . . . . . 186

A.3.2 Matrizen des Anti-Windup-Regelkreises . . . . . . 186 
A.3.3 Reglermatrizen aus Abschnitt 7.4 . . . . . . . . 187

A.3.4 Gütefunktion aus Abschnitt 3.3.3 . . . . . . . . 187

$\begin{array}{ll}\text { B Konvexe Optimierung } & 188\end{array}$

B.1 Optimierungsprobleme . . . . . . . . . . . . . . 188

B.2 Lineare Matrixungleichungen und semidefinite Programme . 189

B.3 Rechenregeln für LMIs . . . . . . . . . . . . . . . . . . . . 191

C Herleitungen und Beweise 193

C.1 Herleitung des Ersatzsystems . . . . . . . . . . . . 193

C.1.1 Äquivalenz . . . . . . . . . . . . . . . . . . 193

C.1.2 Abschätzung . . . . . . . . . . . . . 195

C.2 Schrittweite beim Path-Following . . . . . . . . . . 199

C.3 Beweise . . . . . . . . . . . . . . . . . 200

C.3.1 Beweis von Lemma $5.2 \ldots \ldots . . \ldots 200$

C.3.2 Beweis von Lemma 5.3 . . . . . . . . . . . . . 203

C.3.3 Beweis von Satz 5.2 . . . . . . . . . . . . . . 203

C.3.4 Beweis von Satz $5.3 \ldots \ldots 206$

C.3.5 Beweis von Satz 6.1 . . . . . . . . . . . . 208

C.3.6 Beweis von Lemma 6.1 . . . . . . . . . . . . . 209

C.3.7 Beweis von Satz 6.2 . . . . . . . . . . . . . . 210

C.3.8 Beweis von Satz 6.3 . . . . . . . . . . . . . . 211

C.3.9 Beweis von Satz 6.5 . . . . . . . . . . . . . . 211

C.3.10 Beweis von Lemma 6.2 . . . . . . . . . . . . . . 212

C.3.11 Beweis von Lemma 7.3 . . . . . . . . . . . . . . 213

C.3.12 Beweis von Lemma 8.1 . . . . . . . . . . . 215

C.3.13 Beweis von Lemma 8.3 . . . . . . . . . . . . 217

C.3.14 Beweis von Lemma 8.4 . . . . . . . . . . . . . 218

C.3.15 Beweis von Lemma 9.1 . . . . . . . . . . . . . . 223

Literaturverzeichnis 


\section{Abkürzungen und Symbole}

\section{Abkürzungen}

GAW generalized anti-windup, generalisiertes Anti-Windup

ISOVAW implicit soft variable-structure anti-windup, implizites weich strukturvariables Anti-Windup

LMI linear matrix inequality, lineare Matrixungleichung

MIMO multiple-input multiple-output, System mit mehreren Eingängen und mehreren Ausgängen

MPC model predictive control, modellprädiktive Regelung

MPR modellprädiktive Regelung

SISO single-input single-output, System mit einem Eingang und einem Ausgang

SOS sum-of-squares

\section{Notation}

$x$

$\boldsymbol{x}$

$x_{\mathrm{s}, i}$

$\boldsymbol{x}^{\top}$

$\boldsymbol{X}$

0

II

$\boldsymbol{X}_{\langle i\rangle}$

$\boldsymbol{X}^{\top}$

$\boldsymbol{X}^{-1}$

He $\boldsymbol{X}$

$\operatorname{det}(\boldsymbol{X})$

$\operatorname{adj}(\boldsymbol{X})$
Skalar

Spaltenvektor

$i$-te Komponente des Vektors $\boldsymbol{x}_{\mathrm{s}}$

Zeilenvektor

Matrix

Nullmatrix

Einheitsmatrix

$i$-te Zeile der Matrix $\boldsymbol{X}$

transponierte Matrix

inverse Matrix

Summe aus $\boldsymbol{X}$ und $\boldsymbol{X}^{\top}$, d. h. He $\boldsymbol{X}=\boldsymbol{X}+\boldsymbol{X}^{\top}$

Determinante der Matrix $\boldsymbol{X}$

Adjunkte der Matrix $\boldsymbol{X}$ 
$\operatorname{rang}(\boldsymbol{X}) \quad$ Rang der Matrix $\boldsymbol{X}$

$\operatorname{diag}(\cdot) \quad$ Diagonalmatrix oder Blockdiagonalmatrix

$$
\begin{aligned}
\operatorname{diag}\left(x_{1}, \ldots, x_{n}\right) & =\left[\begin{array}{cccc}
x_{1} & 0 & \cdots & 0 \\
0 & \ddots & \ddots & \vdots \\
\vdots & \ddots & \ddots & 0 \\
0 & \cdots & 0 & x_{n}
\end{array}\right] \\
\operatorname{diag}\left(\boldsymbol{X}_{1}, \ldots, \boldsymbol{X}_{n}\right) & =\left[\begin{array}{cccc}
\boldsymbol{X}_{1} & \mathbf{0} & \cdots & \mathbf{0} \\
\mathbf{0} & \ddots & \ddots & \vdots \\
\vdots & \ddots & \ddots & \mathbf{0} \\
\mathbf{0} & \cdots & \mathbf{0} & \boldsymbol{X}_{n}
\end{array}\right]
\end{aligned}
$$

$\boldsymbol{X} \succ \mathbf{0} \quad \boldsymbol{X}$ ist symmetrisch und positiv definit

$\boldsymbol{X} \succeq \mathbf{0} \quad \boldsymbol{X}$ ist symmetrisch und positiv semidefinit

$\boldsymbol{X} \prec \mathbf{0} \quad \boldsymbol{X}$ ist symmetrisch und negativ definit

$\boldsymbol{X} \preceq \mathbf{0} \quad \boldsymbol{X}$ ist symmetrisch und negativ semidefinit

$\mathcal{X}$

Menge von Skalaren, Vektoren oder Matrizen

$\operatorname{co} \mathcal{X} \quad$ konvexe Hülle einer Menge, siehe (9.10) auf S. 172

$\partial \mathcal{X} \quad$ Rand einer kompakten Menge

$|\cdot| \quad$ Betrag eines Skalars

$\|\cdot\| \quad$ Norm eines Vektors oder einer Matrix

$\|\cdot\|_{2} \quad$ Euklidische Vektornorm, Spektralnorm einer Matrix

$\|\cdot\|_{L_{2}} \quad L_{2}$-Norm eines Signals, siehe Definition 4.1, S. 43

$\operatorname{Re}\{s\} \quad$ Realteil der komplexen Zahl $s$

$\operatorname{Im}\{s\} \quad$ Imaginärteil der komplexen Zahl $s$

$\dot{\boldsymbol{x}}$

Ableitung von $\boldsymbol{x}$ nach der Zeit

$\frac{\partial f(\boldsymbol{x})}{\partial \boldsymbol{x}}$

Ableitung von $f$ nach $\boldsymbol{x}: \frac{\partial f(\boldsymbol{x})}{\partial \boldsymbol{x}}=\left[\frac{\partial f(\boldsymbol{x})}{\partial x_{1}} \ldots \frac{\partial f(\boldsymbol{x})}{\partial x_{n}}\right]$

\section{Symbole}

\section{Lateinische Großbuchstaben}

$\boldsymbol{A} \quad$ Systemmatrix eines linearen Systems

A dynamisches System: Aktor

AW dynamisches System: Anti-Windup 


\begin{tabular}{|c|c|}
\hline$B$ & Eingangsmatrix eines linearen MIMO-Systems \\
\hline$C$ & Ausgangsmatrix eines linearen MIMO-Systems \\
\hline $\mathbb{C}$ & Menge der komplexen Zahlen \\
\hline$D$ & Durchgangsmatrix eines linearen MIMO-Systems \\
\hline ES & Ersatzsystem für den Anti-Windup-Entwurf \\
\hline $\mathcal{E}$ & offene Hyperellipse im $\mathbb{R}^{n}$ \\
\hline$\overline{\mathcal{E}}$ & kompakte Hyperellipse im $\mathbb{R}^{n}$, siehe (8.13) auf S. 154 \\
\hline $\boldsymbol{F}$ & statisches Vorfilter eines Kontrollbeobachters \\
\hline $\boldsymbol{G}(s)$ & Laplace-Übertragungsmatrix \\
\hline $\mathcal{G}$ & allgemeine Menge im $\mathbb{R}^{n}$ \\
\hline $\mathcal{G}\left(\boldsymbol{x}^{(\mathrm{R})}\right)$ & ein Einzugsgebiet der Ruhelage $\boldsymbol{x}^{(\mathrm{R})}$ \\
\hline $\boldsymbol{H}(v)$ & spezielle Diagonalmatrix, siehe (8.3) auf S. 147 \\
\hline II & Einheitsmatrix \\
\hline $\mathcal{J}$ & Menge spezieller Vektoren, siehe S. 84 \\
\hline KB & dynamisches System: Kontrollbeobachter \\
\hline $\mathcal{L}$ & Menge spezieller Vektoren, siehe Abschnitt 5.1, S. 72 \\
\hline $\mathbf{L}$ & dynamisches System: linearer Regelkreis \\
\hline$M$ & spezielle Diagonalmatrix, siehe (5.7) auf S. 76 \\
\hline $\mathcal{M}$ & Menge spezieller Diagonalmatrizen, siehe S. 76 \\
\hline$N$ & spezielle Diagonalmatrix, siehe Satz 8.1 auf S. 148 \\
\hline $\mathbb{N}$ & Menge der natürlichen Zahlen \\
\hline $\mathbf{P}$ & linearer Teil des nichtlinearen Standardregelkreises \\
\hline $\mathcal{P}$ & Menge der Eckpunkte eines Polytops \\
\hline$Q$ & symmetrische, positiv definite Matrix \\
\hline $\boldsymbol{R}$ & symmetrische, positiv definite Ljapunov-Matrix \\
\hline $\mathbf{R}$ & dynamisches System: Regler \\
\hline $\mathbb{R}$ & Menge der reellen Zahlen \\
\hline $\mathbb{R}_{>0}$ & Menge der positiven reellen Zahlen \\
\hline $\mathbb{R}_{\geq 0}$ & Menge der positiven reellen Zahlen einschließlich null \\
\hline $\mathbb{R}_{<0}^{-}$ & Menge der negativen reellen Zahlen \\
\hline $\mathbb{R}_{\leq 0}$ & Menge der negativen reellen Zahlen einschließlich null \\
\hline $\mathbf{S}$ & dynamisches System: Strecke \\
\hline $\mathbb{S}^{n}$ & $\begin{array}{l}\text { Menge der symmetrischen } n \times n \text {-Matrizen mit reellwer- } \\
\text { tigen Elementen }\end{array}$ \\
\hline $\mathcal{T}_{i}$ & kompaktes Intervall \\
\hline $\mathcal{T}$ & kompakte Menge: $\mathcal{T}_{1} \times \cdots \times \mathcal{T}_{m}$ \\
\hline $\mathcal{U}$ & Menge zulässiger Stellsignale, siehe (2.12) auf S. 12 \\
\hline
\end{tabular}


$\mathcal{W} \quad$ Menge bestimmter Signale mit einer maximalen Amplitude, siehe Abschnitt (5.3.3), S. 91

$\mathcal{X}^{(0)} \quad$ Menge der Anfangszustände eines Systems

$\boldsymbol{\mathcal { X }}^{(\mathrm{R})} \quad$ Menge der Ruhelagen stellgrößenbegrenzter Systeme, siehe S. 21

$\mathcal{Y} \quad$ Klasse bestimmter Signale, siehe S. 91

$\boldsymbol{Z} \quad$ Rosenbrock-Matrix eines linearen Systems

\section{Lateinische Kleinbuchstaben}

$a$

$b$

$c$

$d$

$e, e$

e

j

$\boldsymbol{k}_{\mathrm{r}}$

$l_{\mathrm{r}}$

$\boldsymbol{r}(t)$

$s$

$t$

$v$

$\boldsymbol{u}(t)$

$\overline{\mathbf{u}}$

$\boldsymbol{w}(t)$

$\boldsymbol{w}_{0}$

$\boldsymbol{x}(t)$

$\boldsymbol{x}^{(0)}$

$\boldsymbol{x}^{(\mathrm{R})}$

$\boldsymbol{y}(t)$

$\boldsymbol{y}^{(\mathrm{R})}$

$\boldsymbol{z}(t)$

$z_{0}$

Vektor, dessen Elemente die Koeffizienten des charakteristischen Polynoms eines linearen Systems sind, siehe (8.2) auf S. 147

Eingangsvektor eines linearen SISO-Systems

Ausgangsvektor eines linearen SISO-Systems

Durchgriff eines linearen SISO-Systems

Regelabweichung im MIMO-Fall bzw. SISO-Fall

Eulersche Zahl

imaginäre Einheit, $j=\sqrt{-1}$

Vektor eines linearen Zustandsreglers im SISO-Fall

Beobachtervektor im SISO-Fall

mehrdimensionales Eingangssignal

Laplace-Variable

Zeit

Selektionsparameter, siehe S. 147

mehrdimensionales Eingangssignal

vektorielle Sättigungsgrenze, siehe S. 11

Sollwert

konstanter Sollwert

Zustandsvektor eines dynamischen Systems

Zustandsvektor zum Zeitpunkt $t=0$, Anfangswert $\boldsymbol{x}(0)$

Ruhelage eines dynamischen Systems, siehe S. 7

Ausgang eines dynamischen Systems

stationäre Ausgangsgröße für $t \rightarrow \infty$

Störung, die auf ein dynamisches System wirkt

konstante Störung 


\section{Griechische Großbuchstaben}

$\Lambda \quad$ Menge der Eigenwerte des unbeschränkten Regelkreises

$\Theta \quad$ Matrixvariable, siehe Abschnitt 5.2.3, S. 76

$\Sigma^{i}[v] \quad$ Menge der SOS-Matrixpolynome der Dimension $i$, siehe Definition 8.2, S. 151

$\hat{\Psi} \quad$ Diagonalmatrix der Sektorbedingung, siehe Lemma 5.3

$\Psi \quad$ Diagonalmatrix der Sektorbedingung nach Substitution

$\Phi \quad$ obere Grenze eines mehrdimensionalen Sektors, siehe Definition 5.1, S. 74

\section{Griechische Kleinbuchstaben}

\begin{tabular}{|c|c|}
\hline$\alpha$ & $\begin{array}{l}\text { Skalierungsfaktor für die Stellgröße des Anti-Windup- } \\
\text { Ersatzsvstems, siehe Abschnitt 6.2. S. } 102 \mathrm{ff} \text {. }\end{array}$ \\
\hline$\gamma$ & $\begin{array}{l}\text { Obere Grenze für die } L_{2} \text {-Verstärkung eines Systems, sie- } \\
\text { he Definition } 4.2, \mathrm{~S} .47\end{array}$ \\
\hline$\gamma_{y}$ & $\begin{array}{l}\text { Obere Grenze für die } L_{2} \text {-Kleinsignalverstärkung eines } \\
\text { Systems, siehe Definition } 5.3 \text {, S. } 91\end{array}$ \\
\hline$\delta$ & Sättigungsgrad, siehe (4.2) auf S. 37 \\
\hline$\epsilon$ & beliebig kleiner, positiver Skalar \\
\hline$t)$ & $\begin{array}{l}\text { Störgröße des Anti-Windup-Ersatzsystems, siehe Ab- } \\
\text { schnitt 4.4.2, S. } 53 \text { ff. }\end{array}$ \\
\hline$(v)$ & Polynom zweiten Grades in $v$, siehe Abschnitt 8.2 .2 \\
\hline & Schrittweite beim Path-Following, siehe S. 134 \\
\hline$\lambda$ & Eigenwert einer Matrix \\
\hline & Sektornichtlinearität, siehe Abschnitt 5.2, S. 73 ff. \\
\hline & $\begin{array}{l}i \text {-te Komponente einer Sektornichtlinearität, siehe Ab- } \\
\text { schnitt } 5.2 \text {, S. } 73 \mathrm{ff} .\end{array}$ \\
\hline (1) & transformierter Zustandsvektor, siehe S. 48 ff. \\
\hline & Anfangswert $\boldsymbol{\xi}(0)$ \\
\hline & transformierte Ruhelage \\
\hline & Skalar, der $1-\alpha$ entspricht \\
\hline & $i$-te Komponente der Sektorgrenze $\boldsymbol{\Phi}$ \\
\hline & Hilfspolynom in $v$, siehe Abschnitt 8.2.2, S. $150 \mathrm{ff}$. \\
\hline & Kreisfrequenz \\
\hline
\end{tabular}




\section{Kurzfassung}

Durch die Nichtbeachtung der immer vorhandenen Stellbegrenzungen bei der Auslegung eines Reglers kann es während des Betriebs zu einem unerwünschten Fehlverhalten des geschlossenen Regelkreises kommen. Dieses Fehlverhalten wird auch als Windup bezeichnet und besitzt die unterschiedlichsten Ausprägungen: heftige Oszillationen, ein extrem verlangsamtes Ausregelverhalten oder sogar Instabilität.

Die vorliegende Arbeit beschäftigt sich mit dem systematischen Entwurf von Anti-Windup-Maßnahmen, die in Kombination mit dem zuvor ausgelegten Regler einen sicheren und effizienten Betrieb des Regelkreises trotz vorhandener Stellbegrenzungen gewährleisten. Neben einer Stabilitätsgarantie für den Regelkreis und einer hohen Regelgüte liegt der Fokus auf einem einfach durchführbaren Entwurf mit möglichst wenigen vom Anwender zu wählenden Parametern. Letzteres wird durch eine auf konvexer Optimierung basierende Parametrierung des Anti-Windup erreicht.

Die Hauptbeiträge dieser Arbeit bestehen in einem systematischen Entwurfsverfahren für die von Hippe vorgeschlagene Beobachtertechnik zur Vermeidung von Regler-Windup sowie in der Entwicklung von drei neuartigen Entwurfsmethoden für verschiedene lineare und nichtlineare modellbasierte Anti-Windup-Strukturen.

Die erste Methode basiert auf stückweise quadratischen LjapunovFunktionen und ermöglicht neben globaler Stabilität eine im Vergleich zum Stand der Technik hohe Regelgüte für lineares Anti-Windup. Diese wird durch die Optimierung eines lokalen Gütemaßes erreicht. Die zweite Methode formuliert die Parametrierung eines ebenfalls neu entwickelten weich strukturvariablen Anti-Windup als konvexes Optimierungsproblem. Durch die variable Struktur dieses Ansatzes kann die Regelgüte im Vergleich zu linearen Ansätzen beträchtlich gesteigert werden. Das dritte Entwurfsverfahren setzt auf lokale Stabilitätsergebnisse für lineares Anti-Windup und eignet sich somit für Regelkreise mit grenz- oder instabilen Strecken. Die präzise Charakterisierung des Stabilitätsgebietes in Kombination mit einem auf die Regelgüte fokussierten Entwurf ist für den praktischen Einsatz wichtig und stellt den Hauptunterschied zu existierenden Verfahren dar. 


\section{Abstract}

Neglecting actuator limitations when designing a feedback control may cause a severe malfunctioning of the control loop during operation. This faulty behavior is often termed windup and manifests itself in intense oscillations, a significantly prolonged settling time or even instability.

The main objective of this work is the development of systematic design methods for anti-windup strategies, guaranteeing - in cooperation with the pre-designed controller - a safe and efficient operation of the control loop. Concerning the design approaches, three objectives are targeted. Stability of the control loop as well as a high performance, i.e. a significant reduction of windup, are desired characteristics. Furthermore, the procedure to parameterize the anti-windup should be as user-friendly as possible. Therefore, all design methods in this work are based on convex optimization.

The primary contribution of this thesis consists in a systematic design method for Hippe's observer technique, preventing controller windup, and the development of three new design methods suited for different linear and nonlinear model-based anti-windup structures.

The first method uses piecewise quadratic Ljapunov functions to establish global stability of the closed-loop system with a linear anti-windup. A local performance criterion is exploited to achieve a drastic reduction of windup compared to existing approaches. The second design strategy parameterizes a newly developed soft variable-structure anti-windup by solving a convex optimization problem. Due to its variable structure, the anti-windup outperforms linear state-of-the-art approaches. The third design algorithm aims at local stability results for linear anti-windup structures. Therefore, it is suited for control loops with marginally stable or unstable plants. The main difference compared to existing approaches is the precise characterization of the stability region in combination with a performance-oriented design. Both aspects are necessary and important for a practical implementation of the proposed method. 



\section{Einleitung}

Die Aufgabe der Regelungstechnik besteht darin, ein technisches System oder einen Prozess von außen so zu beeinflussen, dass ein gewünschtes Systemverhalten resultiert bzw. der Prozess in gewünschter Weise abläuft.

Um dieses Ziel zu erreichen, ist das reibungslose Zusammenspiel verschiedener Komponenten erforderlich. Eine Grundvoraussetzung für jede Regelung sind in gewissen Zeitabständen aktualisierte Informationen über den Betriebszustand des zu regelnden Systems, das auch als Strecke bezeichnet wird. Aus diesen Informationen berechnet der Regler einen Zahlenwert, die sogenannte kommandierte Stellgröße. Diese Größe quantifiziert, wie die Strecke zu beeinflussen ist, damit sich das gewünschte Verhalten einstellt. Die eigentliche Beeinflussung der Strecke übernimmt dann der Aktor, der das Bindeglied zwischen Regler und Strecke darstellt. Aktoren, wie beispielsweise Netzteile oder Antriebe, setzen die kommandierte Stellgröße in eine physikalische Größe um, die im Weiteren als reale Stellgröße bezeichnet wird. Folglich wirken reale Stellgrößen, wie zum Beispiel Kräfte, Drehmomente oder elektrische Spannungen, auf die Strecke. Den Aufbau des so entstehenden Regelkreises zeigt Abbildung 1.1.

Aus physikalischen Gründen ist die reale Stellgröße immer begrenzt. Deshalb lässt sich nicht jede beliebige vom Regler kommandierte Stellgröße exakt auf die Strecke übertragen. Eine Vernachlässigung dieser Stellgrößenbegrenzung bei der Auslegung des Reglers kann zu einem unerwünschten Regelkreisverhalten führen und im schlimmsten Fall katastrophale Folgen nach sich ziehen [178]. Dieses Fehlverhalten des Regelkreises wird in der Literatur oft als Windup bezeichnet und motiviert die Entwicklung von Regelkonzepten für Strecken mit stellbegrenzten Aktoren.

Im industrienahen Umfeld hat sich ein zweistufiges Entwurfskonzept für Regler etabliert. Zuerst wird die Stellgrößenbeschränkung ignoriert und ein linearer Regler für ein lineares Modell der Strecke entworfen. Aufgrund der Linearität kann in diesem Entwurfsschritt auf ein breites Spektrum an einfach anwendbaren Entwurfsmethoden zurückgegriffen werden. Der zweite Schritt besteht in der Erweiterung des Regelkreises um eine strukturelle Maßnahme, die Stabilität und eine hohe Regelgüte garantiert, falls sich der nichtlineare Einfluss der Stellbegrenzung bemerkbar macht und der 


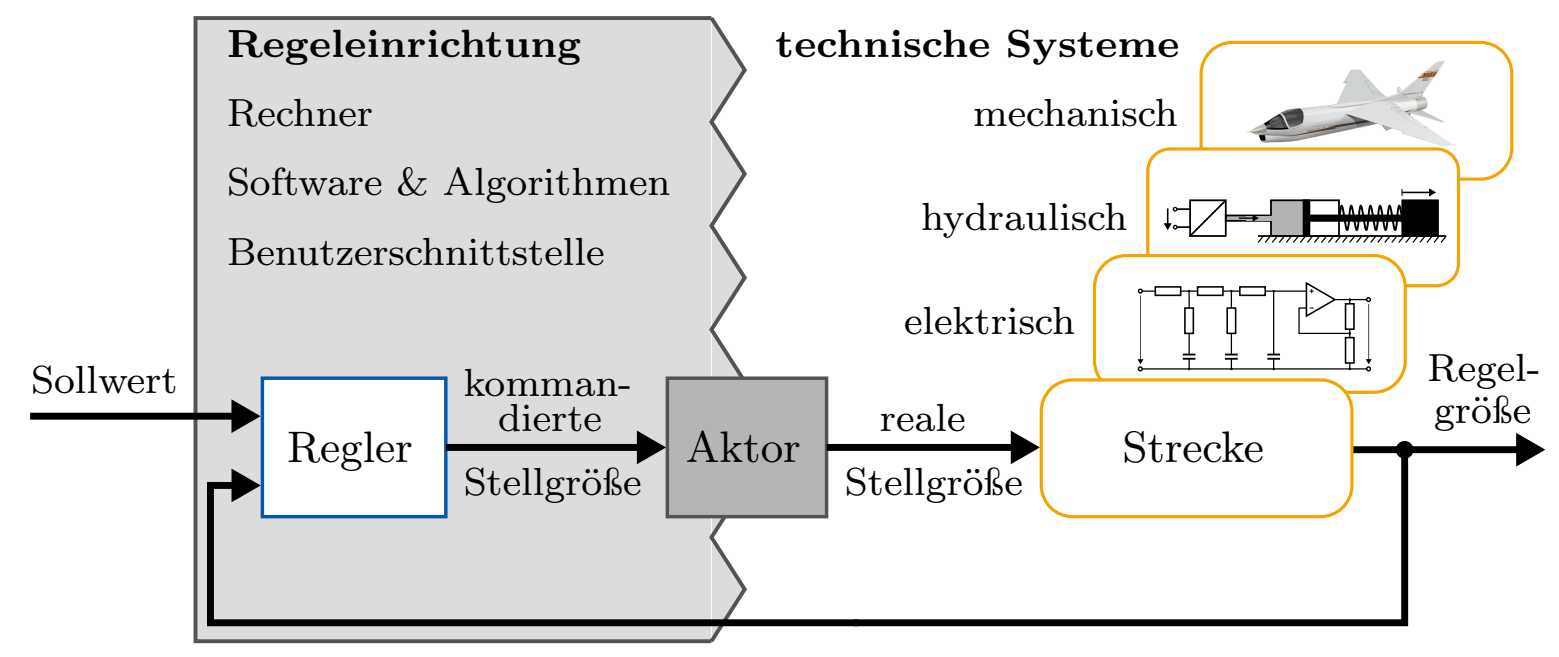

Abbildung 1.1: Aufbau eines Regelkreises. Der Aktor stellt das Bindeglied zwischen der Regeleinrichtung und dem zu regelnden System, der Strecke, dar.

Aktor an seinen Grenzen operiert. Diese Maßnahme wird aus historischen Gründen mit Anti-Windup bezeichnet und ist Hauptgegenstand der vorliegenden Arbeit. Der nächste Abschnitt beschreibt die konkrete Zielsetzung.

\subsection{Zielsetzung der Arbeit}

Ziel dieser Arbeit ist die Entwicklung von neuen systematischen Entwurfsverfahren für die Parametrierung existierender Anti-Windup-Maßnahmen sowie die Entwicklung neuer Anti-Windup-Strukturen. Dabei liegt der Fokus auf der Kombination folgender Anforderungen:

- eine Stabilitätsgarantie für die jeweils betrachtete Ruhelage des geschlossenen Regelkreises, wobei ein möglichst großes genau spezifiziertes Stabilitätsgebiet angestrebt wird,

- eine möglichst hohe garantierte Regelgüte,

- ein möglichst einfacher, automatisierbarer Entwurf mit möglichst wenigen vom Anwender zu wählenden Entwurfsparametern.

Viele existierende Ansätze berücksichtigen nur jeweils einen der drei genannten Punkte, d. h. zielen entweder auf ein großes Stabilitätsgebiet oder eine hohe Regelgüte ab. Einfach anwendbare Entwurfsverfahren basieren oft auf iterativen Entwürfen und geben keine Stabilitätsgarantie. Dann ist Ausprobieren und ausgiebiges Simulieren notwendig. 
Um den Entwurf auf eine systematische Weise in vertretbarer Rechenzeit durchführen zu können, wird der Anti-Windup-Entwurf als konvexes Optimierungsproblem formuliert. Dieses Vorgehen hat sich in vielen Bereichen der Regelungstechnik durchgesetzt, da zuverlässige Algorithmen für die numerische Lösung derartiger Probleme existieren. Anhang B bietet eine Einführung in die Thematik, die im Folgenden als bekannt vorausgesetzt wird. Den weiteren Aufbau der Arbeit beschreibt der nächste Abschnitt.

\subsection{Gliederung der Arbeit}

Die vorliegende Arbeit gliedert sich in zehn Kapitel. Dabei stellt das zweite Kapitel den Anknüpfungspunkt zu den einleitenden Worten dar und beschreibt detailliert den betrachteten Regelkreis sowie die im Zusammenhang mit Stellbegrenzungen auftretenden Windup-Probleme. Existierende Lösungen für die Windup-Problematik stehen im Mittelpunkt des dritten Kapitels. Dazu zählen eine direkte Berücksichtigung der Begrenzungen beim Reglerentwurf, modellbasierte prädiktive Regelungen sowie Anti-Windup-Maßnahmen. Letztere behandelt Kapitel 4 intensiver und stellt den Stand der Technik ausführlich dar.

Die Kapitel 5 bis 9 enthalten die neuen Beiträge der Arbeit, die in Auszügen bereits in den Publikationen [147-150] veröffentlicht wurden. Kapitel 5 befasst sich mit einer systematischen Entwurfsmethode für die auf Hippe zurückgehende Beobachtertechnik eines modularen Anti-WindupKonzeptes [85]. Wesentliche Neuerung ist die Bewertung der Regelgüte anhand der Abweichung von einem idealen Verhalten.

Kapitel 6 untersucht die Stabilität von Regelkreisen mit sogenanntem modellbasierten Anti-Windup. Der Fokus liegt auf der Herleitung von einfach zu handhabenden, universell einsetzbaren Stabilitätssätzen, die eine Erweiterung von $[52,185]$ darstellen. Diese Ergebnisse bilden die Grundlage für die neuen Entwurfsmethoden der Kapitel 7 bis 9. Die Besonderheit des Entwurfs in Kapitel 7 ist die Verwendung von stückweise quadratischen Ljapunov-Funktionen. Dadurch kann eine höhere Regelgüte im Vergleich zum Stand der Technik erzielt werden, wie ein Beispiel demonstriert.

Kapitel 8 stellt eine neues weich strukturvariables Anti-Windup vor, das auf der gleichnamigen Zustandsregelung [98] basiert. Es zeichnet sich durch eine hohe Regelgüte und einen, im Vergleich zum Stand der Technik, einfachen Entwurf sowie eine gute Implementierbarkeit aus.

Lokale Ergebnisse werden in Kapitel 9 verfolgt. Hauptaugenmerk liegt auf einem modellbasierten Anti-Windup mit hoher Regelgüte, das loka- 


\section{Kapitel 2}

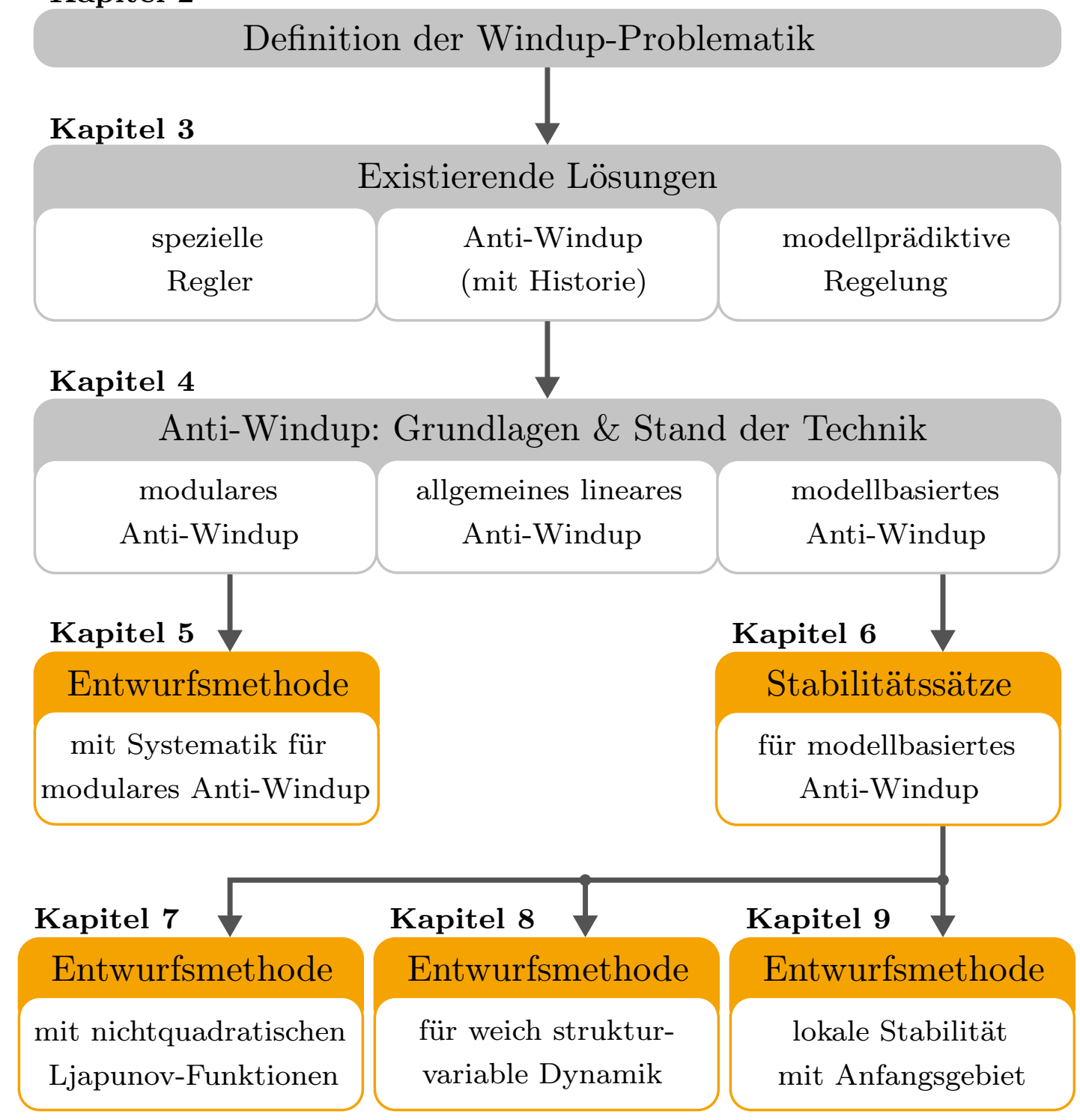

Abbildung 1.2: Gliederung der vorliegenden Arbeit. Die neuen Beiträge und Entwurfsmethoden sind orange hervorgehoben.

le Stabilität des Regelkreises für alle Anfangszustände aus einem genau charakterisierten Gebiet garantiert. Die präzise Charakterisierung des Anfangsgebietes ist für den praktischen Einsatz wichtig und stellt den Hauptunterschied zu existierenden Entwurfsmethoden dar.

Abbildung 1.2 visualisiert die Gliederung der Arbeit und zeigt die Verbindungen der einzelnen Kapitel auf. Die Arbeit schließt mit einer Zusammenfassung in Kapitel 10. 


\section{Die Windup-Problematik}

Dieses Kapitel charakterisiert das Windup-Problem. Nach der Definition des betrachteten Regelkreises wird auf die Entstehung des Begriffs Windup eingegangen und der klassischen Definition eine moderne Variante gegenübergestellt. Ein Beispiel demonstriert die Windup-Problematik.

\subsection{Definition des betrachteten Regelkreises}

Das zu regelnde dynamische System, die Strecke, wird durch ein lineares, zeitinvariantes, steuer- und beobachtbares Zustandsraummodell

$$
\mathbf{S}:\left\{\begin{array}{l}
\dot{\boldsymbol{x}}_{\mathrm{s}}(t)=\boldsymbol{A}_{\mathrm{s}} \boldsymbol{x}_{\mathrm{s}}(t)+\boldsymbol{B}_{\mathrm{s}, u} \boldsymbol{u}_{\mathrm{s}}(t)+\boldsymbol{B}_{\mathrm{s}, z} \boldsymbol{z}(t) \\
\boldsymbol{y}_{\mathrm{s}}(t)=\boldsymbol{C}_{\mathrm{s}} \boldsymbol{x}_{\mathrm{s}}(t)
\end{array}\right.
$$

beschrieben. Dabei bezeichnet $\boldsymbol{x}_{\mathrm{s}}(t) \in \mathbb{R}^{n_{\mathrm{s}}}$ den Zustand, $\boldsymbol{u}_{\mathrm{s}}(t) \in \mathbb{R}^{m}$ die Stellgröße und $\boldsymbol{z}(t) \in \mathbb{R}^{l}$ die auf das System wirkende Störgröße. Der messbare Streckenausgang ist $\boldsymbol{y}_{\mathrm{s}}(t) \in \mathbb{R}^{m}$. Die konstanten Matrizen $\boldsymbol{A}_{\mathrm{s}}$, $\boldsymbol{B}_{\mathrm{s}, u}, \boldsymbol{B}_{\mathrm{s}, z}, \boldsymbol{C}_{\mathrm{S}}$ besitzen passende Dimensionen.

Vor allem interessiert das Verhalten der Strecke im Zeitintervall $[0, \infty)$. Anfangsbedingungen lassen sich durch einen Anfangszustand $\boldsymbol{x}_{\mathrm{s}}(0)=\boldsymbol{x}_{\mathrm{s}}^{(0)}$ berücksichtigen. Die Zeitabhängigkeit des Zustands und der Ein- und Ausgangsgrößen wird im Weiteren nicht mehr ausgeschrieben.

Um der Strecke ein gewünschtes dynamisches Verhalten aufzuprägen, kommt ein linearer Regler zum Einsatz, der durch

$$
\mathbf{R}:\left\{\begin{array}{l}
\dot{\boldsymbol{x}}_{\mathrm{r}}=\boldsymbol{A}_{\mathrm{r}} \boldsymbol{x}_{\mathrm{r}}+\boldsymbol{B}_{\mathrm{r}, u} \boldsymbol{u}_{\mathrm{r}}+\boldsymbol{B}_{\mathrm{r}, w} \boldsymbol{w} \\
\boldsymbol{y}_{\mathrm{r}}=\boldsymbol{C}_{\mathrm{r}} \boldsymbol{x}_{\mathrm{r}}+\boldsymbol{D}_{\mathrm{r}, u} \boldsymbol{u}_{\mathrm{r}}+\boldsymbol{D}_{\mathrm{r}, w} \boldsymbol{w}
\end{array}\right.
$$

mit $\boldsymbol{x}_{\mathrm{r}}(0)=\boldsymbol{x}_{\mathrm{r}}^{(0)}$ beschrieben wird. Dabei ist $\boldsymbol{x}_{\mathrm{r}} \in \mathbb{R}^{n_{\mathrm{r}}}$ der Reglerzustand, $\boldsymbol{u}_{\mathrm{r}} \in \mathbb{R}^{m}$ der Messeingang für die Ausgangssignale $\boldsymbol{y}_{\mathrm{s}}$ der Strecke, $\boldsymbol{w} \in \mathbb{R}^{m}$ der Eingang zur Vorgabe von Sollwerten und $\boldsymbol{y}_{\mathrm{r}} \in \mathbb{R}^{m}$ die vom Regler berechnete Stellgröße, der Reglerausgang.

Die Stellgröße $\boldsymbol{y}_{\mathrm{r}}$ wird mittels eines Aktors auf die Strecke übertragen. Der Aktor kann zum Beispiel ein Netzteil sein, welches die vom Regler 


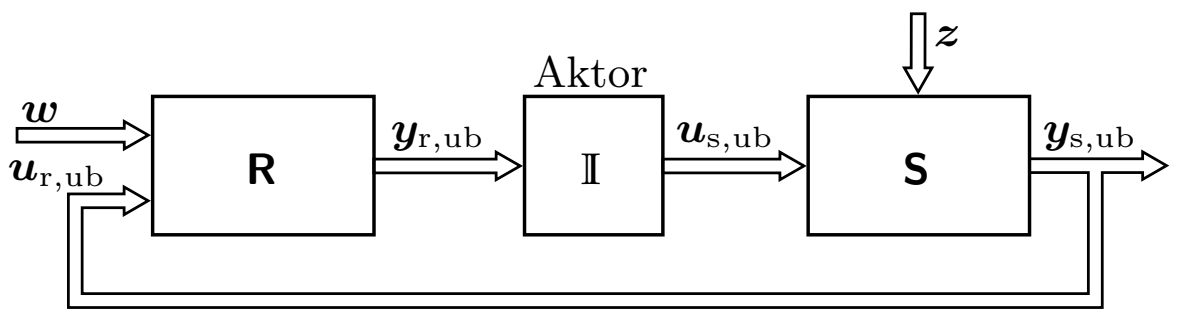

Abbildung 2.1: Unbeschränkter Regelkreis mit idealem Aktor. Die kommandierte Stellgröße wird immer exakt eingestellt, d.h. $\boldsymbol{u}_{\mathrm{s}, \mathrm{ub}}=\boldsymbol{y}_{\mathrm{r}, \mathrm{ub}}$.

gewünschte Spannung $\boldsymbol{y}_{\mathrm{r}}$ an den Eingang $\boldsymbol{u}_{\mathrm{s}}$ der Strecke (2.1) anlegt oder ein mechatronisches System, das durch Öffnen eines Ventils am Eingang der Strecke den vom Regler berechneten Durchfluss einstellt. Der Aktor setzt somit einen vom Regler berechneten Zahlenwert in eine physikalische Größe wie Weg, Kraft oder elektrische Spannung um.

Bei einer exakten Umsetzung durch einen idealen Aktor kann die vom Regler berechnete Stellgröße $\boldsymbol{y}_{\mathrm{r}}$ immer genau eingestellt werden, d. h., es gilt $\boldsymbol{u}_{\mathrm{s}}=\mathbb{I} \boldsymbol{y}_{\mathrm{r}}=\boldsymbol{y}_{\mathrm{r}}$. Der so entstehende Regelkreis in Abbildung 2.1 wird als unbeschränkter Regelkreis bezeichnet, weil die Stellgröße nicht durch den Aktor beschränkt ist. Der Index s,ub bzw. r,ub für Zustände, Ein- und Ausgänge der Strecke und des Reglers betonen dabei die Unbeschränktheit des Regelkreises. Mit dem Zustandsvektor $\boldsymbol{x}_{\mathrm{ub}}^{\top}=\left[\begin{array}{ll}\boldsymbol{x}_{\mathrm{s}, \mathrm{ub}}^{\top} & \boldsymbol{x}_{\mathrm{r}, \mathrm{ub}}^{\top}\end{array}\right]$ und dem Eingangsvektor $\boldsymbol{r}^{\top}=\left[\begin{array}{ll}\boldsymbol{w}^{\top} & \boldsymbol{z}^{\top}\end{array}\right]$ ergibt sich für die Zustandsraumdarstellung des unbeschränkten Regelkreises

$$
\dot{\boldsymbol{x}}_{\mathrm{ub}}=\boldsymbol{A}_{\mathrm{ub}} \boldsymbol{x}_{\mathrm{ub}}+\boldsymbol{B}_{\mathrm{ub}} \boldsymbol{r},
$$

wobei die Systemmatrizen folgende Gestalt besitzen

$$
\boldsymbol{A}_{\mathrm{ub}}=\left[\begin{array}{cc}
\boldsymbol{A}_{\mathrm{s}}+\boldsymbol{B}_{\mathrm{s}, u} \boldsymbol{D}_{\mathrm{r}, u} \boldsymbol{C}_{\mathrm{s}} & \boldsymbol{B}_{\mathrm{s}, u} \boldsymbol{C}_{\mathrm{r}} \\
\boldsymbol{B}_{\mathrm{r}, u} \boldsymbol{C}_{\mathrm{s}} & \boldsymbol{A}_{\mathrm{r}}
\end{array}\right], \quad \boldsymbol{B}_{\mathrm{ub}}=\left[\begin{array}{cc}
\boldsymbol{B}_{\mathrm{s}, u} \boldsymbol{D}_{\mathrm{r}, w} & \boldsymbol{B}_{\mathrm{s}, z} \\
\boldsymbol{B}_{\mathrm{r}, w} & \mathbf{0}
\end{array}\right] .
$$

Die Aufgabe beim Entwurf des Reglers besteht darin, die Matrizen des Regelgesetzes (2.2) so zu wählen, dass die Dynamik des unbeschränkten Regelkreises (2.3) gewisse Güteforderungen erfüllt, die nachfolgend kurz behandelt werden.

\subsubsection{Güteforderungen}

Die konkreten Forderungen an die Regelung hängen von der jeweiligen Anwendung ab. Im Weiteren sind typische Güteforderungen aus der Literatur aufgelistet [132, 133]. 


\section{Forderung 1: Stabilität}

Stabilität lässt sich im Kontext dynamischer Systeme allgemein beschreiben als die Eigenschaft, ,[...] auf eine beschränkte Erregung mit einer beschränkten Bewegung zu reagieren" [132]. Konkret kann die Erregung zum Beispiel die Auslenkung des Zustandes aus einer Ruhelage sein. Der Begriff Ruhelage ist dabei für allgemeine dynamische Systeme

$$
\dot{\boldsymbol{x}}=\boldsymbol{f}(\boldsymbol{x}, \boldsymbol{u})
$$

mit dem Zustand $\boldsymbol{x}$ und dem Eingang $\boldsymbol{u}$ wie folgt definiert.

Definition 2.1 (Ruhelage, nach [3]). Gegeben sei das System (2.5). Dann heißt ein Punkt $\boldsymbol{x}^{(\mathrm{R})}$ des Zustandsraums Ruhelage, wenn für einen konstanten Eingangsvektor $\boldsymbol{u}=\boldsymbol{u}^{(\mathrm{R})}$ gilt

$$
\dot{\boldsymbol{x}}=\boldsymbol{f}\left(\boldsymbol{x}^{(\mathrm{R})}, \boldsymbol{u}^{(\mathrm{R})}\right)=\mathbf{0} .
$$

Bezüglich der Stabilität dieser Ruhelage gilt

Definition 2.2 (nach $[11,107]$ ). Gegeben sei eine Ruhelage $\boldsymbol{x}^{(\mathrm{R})}$ des Systems (2.5). Dann heißt die Ruhelage stabil im Sinne von Ljapunov, wenn für jedes $\epsilon>0$ ein $\delta=\delta(\epsilon)>0$ existiert, so dass für die Trajektorien $\boldsymbol{x}(t)$ des Systems gilt

$$
\left\|\boldsymbol{x}(0)-\boldsymbol{x}^{(\mathrm{R})}\right\|<\delta \Rightarrow\left\|\boldsymbol{x}(t)-\boldsymbol{x}^{(\mathrm{R})}\right\|<\epsilon \quad \forall t \geq 0,
$$

wobei $\|\cdot\|$ eine beliebige Vektornorm ist. Die Ruhelage $\boldsymbol{x}^{(\mathrm{R})}$ heißt attraktiv, wenn für ein $\eta>0$ gilt

$$
\left\|\boldsymbol{x}(0)-\boldsymbol{x}^{(\mathrm{R})}\right\|<\eta \Rightarrow \lim _{t \rightarrow \infty} \boldsymbol{x}(t)=\boldsymbol{x}^{(\mathrm{R})} .
$$

Ist die Ruhelage $\boldsymbol{x}^{(\mathrm{R})}$ stabil im Sinne von Ljapunov und attraktiv, dann wird sie als asymptotisch stabil bezeichnet. Ist sie stabil im Sinne von Ljapunov und attraktiv für ein beliebig großes $\eta$, dann wird die Ruhelage als global asymptotisch stabil bezeichnet.

Aus der linearen Systemtheorie ist bekannt, dass der unbeschränkte Regelkreis (2.3) genau eine asymptotisch stabile Ruhelage

$$
\boldsymbol{x}_{\mathrm{ub}}^{(\mathrm{R})}=-\boldsymbol{A}_{\mathrm{ub}}^{-1} \boldsymbol{B}_{\mathrm{ub}} \boldsymbol{r}
$$

besitzt, wenn alle Eigenwerte der Systemmatrix $\boldsymbol{A}_{\mathrm{ub}}$ einen negativen Realteil aufweisen $[3,132]$. Des Weiteren ist eine asymptotisch stabile Ruhelage eines linearen Systems immer global asymptotisch stabil, d. h., die Trajektorien $\boldsymbol{x}_{\mathrm{ub}}(t)$ laufen von beliebigen Anfangswerten $\boldsymbol{x}_{\mathrm{ub}}(0)=\boldsymbol{x}_{\mathrm{ub}}^{(0)} \in \mathbb{R}^{n_{\mathrm{s}}+n_{\mathrm{r}}}$ nach $\boldsymbol{x}_{\mathrm{ub}}^{(\mathrm{R})}$. Garantiert der Regler negative Realteile der Eigenwerte von $\boldsymbol{A}_{\mathrm{ub}}$, wird der gesamte Regelkreis vereinfachend als stabil bezeichnet. 


\section{Forderung 2: Sollwertfolge und Störkompensation}

Die Stabilität des unbeschränkten Regelkreises ist eine notwendige Bedingung, die jeder Regler garantieren muss. Darüber hinaus ist es Aufgabe des Reglers, den Ausgang der Strecke $\boldsymbol{y}_{\mathrm{s}}$, die sogenannte Regelgröße, mit der Sollgröße $\boldsymbol{w}$ in Übereinstimmung zu bringen oder zumindest die asymptotische Annäherung

$$
\lim _{t \rightarrow \infty}\left(\boldsymbol{w}(t)-\boldsymbol{y}_{\mathrm{s}}(t)\right)=\mathbf{0}
$$

trotz auftretender Störungen $\boldsymbol{z}(t)$ zu garantieren. Dabei wird die Differenz aus Soll- und Regelgröße als Regelabweichung e bezeichnet. Es gilt

$$
e=w-y_{\mathrm{s}} .
$$

Die Sollgrößen $\boldsymbol{w}$ und Störgrößen $\boldsymbol{z}$ lassen sich in vielen Fällen mit linearen Zustandsraummodellen

$$
\begin{aligned}
{\left[\begin{array}{c}
\dot{\boldsymbol{x}}_{w} \\
\dot{\boldsymbol{x}}_{z}
\end{array}\right] } & =\left[\begin{array}{cc}
\boldsymbol{A}_{w} & \mathbf{0} \\
\mathbf{0} & \boldsymbol{A}_{z}
\end{array}\right]\left[\begin{array}{l}
\boldsymbol{x}_{w} \\
\boldsymbol{x}_{z}
\end{array}\right], \quad\left[\begin{array}{l}
\boldsymbol{x}_{w}(0) \\
\boldsymbol{x}_{z}(0)
\end{array}\right]=\left[\begin{array}{l}
\boldsymbol{x}_{w}^{(0)} \\
\boldsymbol{x}_{z}^{(0)}
\end{array}\right], \\
{\left[\begin{array}{c}
\boldsymbol{w} \\
\boldsymbol{z}
\end{array}\right] } & =\left[\begin{array}{cc}
\boldsymbol{C}_{w} & \mathbf{0} \\
\mathbf{0} & \boldsymbol{C}_{z}
\end{array}\right]\left[\begin{array}{l}
\boldsymbol{x}_{w} \\
\boldsymbol{x}_{z}
\end{array}\right]
\end{aligned}
$$

beschreiben. Sind die Eigenwerte $\lambda_{i}$ der Systemmatrix diag $\left(\boldsymbol{A}_{w}, \boldsymbol{A}_{z}\right)$ des Führungs- und Störgrößenmodells nicht identisch mit den invarianten Nullstellen der Strecke $\mathbf{S}$, d. h.

$$
\operatorname{rang}\left[\begin{array}{cc}
\lambda_{i} \mathbb{I}-\boldsymbol{A}_{\mathrm{s}} & -\boldsymbol{B}_{\mathrm{s}, u} \\
\boldsymbol{C}_{\mathrm{s}} & \mathbf{0}
\end{array}\right]=n_{\mathrm{s}}+m \quad \forall \lambda_{i},
$$

dann existiert ein Regler, der Sollwertfolge und Störkompensation garantiert [132, Abschnitt 4.4.5].

Für die weiteren Ausführungen gilt die vereinfachende

Annahme 2.1. Die Führungsgröße $\boldsymbol{w}$ und die auftretende Störgröße $\boldsymbol{z}$ sind konstant für alle $t \geq 0$, d. h.

$$
\boldsymbol{w}(t)=\boldsymbol{w}_{0} \forall t \geq 0, \quad \boldsymbol{z}(t)=\boldsymbol{z}_{0} \forall t \geq 0 .
$$

Somit sind $\boldsymbol{w}(t)$ und $\boldsymbol{z}(t)$ stetige Funktionen in $t$ für alle $t \geq 0$. 
Für den hier betrachteten Fall konstanter Führungs- und Störgrößen gilt

$$
\boldsymbol{A}_{w}=\mathbf{0}, \boldsymbol{A}_{z}=\mathbf{0}
$$

Die Strecke darf somit keine invarianten Nullstellen im Ursprung besitzen, wenn Sollwertfolge und Störkompensation angestrebt wird. Ist diese Bedingung erfüllt, lässt sich immer ein Regler $\mathbf{R}$ finden, der die Ruhelage

$$
\boldsymbol{x}_{\mathrm{ub}}^{(\mathrm{R})}=\left[\begin{array}{c}
\boldsymbol{x}_{\mathrm{s}, \mathrm{ub}}^{(\mathrm{R})} \\
\boldsymbol{x}_{\mathrm{r}, \mathrm{ub}}^{\mathrm{R})}
\end{array}\right] \quad \text { mit } \quad \boldsymbol{C}_{\mathrm{s}} \boldsymbol{x}_{\mathrm{s}, \mathrm{ub}}^{(\mathrm{R})}=\boldsymbol{w}_{0}
$$

des Regelkreises (2.3) global asymptotisch stabilisiert und somit

$$
\lim _{t \rightarrow \infty} \boldsymbol{y}_{\mathrm{s}}(t)=\lim _{t \rightarrow \infty} \boldsymbol{C}_{\mathrm{s}} \boldsymbol{x}_{\mathrm{s}}(t)=\boldsymbol{C}_{\mathrm{s}} \boldsymbol{x}_{\mathrm{s}, \mathrm{ub}}^{(\mathrm{R})}=\boldsymbol{w}_{0}
$$

garantiert.

\section{Forderung 3: Dynamisches Verhalten}

Die Güteforderung der Sollwertfolge und Störkompensation stellt nur sicher, dass sich die Regelgröße $\boldsymbol{y}_{\mathrm{s}}$ dem Sollwert $\boldsymbol{w}=\boldsymbol{w}_{0}$ asymptotisch annähert. Für die Güte der Regelung ist aber auch die Art und Weise der Annäherung entscheidend. Diese lässt sich anhand verschiedener Kenngrößen wie Ausregelzeit oder Überschwingweite quantifizieren. Die Ausregelzeit ist definiert als die Zeitspanne zwischen der Änderung des Sollwertes und dem Einschwingen der Regelgröße in einen Schlauch der Breite $\pm \epsilon$ um den neuen Sollwert. Die Überschwingweite gibt an, wie weit sich die Regelgröße nach dem ersten Erreichen des Sollwertes wieder von ihm entfernt, bevor sie in den $\epsilon$-Schlauch eintritt.

\section{Forderung 4: Schwache Kopplungen zwischen Regelgrößen}

Im Fall einer Mehrgrößenregelung, d. h. $m>1$, wird häufig eine schwache Kopplung zwischen den Regelgrößen $y_{\mathrm{s}, 1}, \ldots, y_{\mathrm{s}, m}$ angestrebt. Das bedeutet: Die Veränderung der Sollwertkomponente $w_{i}$ soll sich möglichst nur auf die ihm zugeordnete Regelgröße $y_{\mathrm{s}, i}$ auswirken.

\section{Zentrale Annahme}

Um ein Regelgesetz zu finden, für das der unbeschränkte Regelkreis die diskutierten Güteforderungen erfüllt, kann auf ein breites Spektrum an 
Entwurfsverfahren der linearen Regelungstheorie zurückgegriffen werden $[6,39,50,82,132,133]$. Ein linearer Regler $\mathbf{R}$ wird deshalb in dieser Arbeit als gegeben betrachtet und der Entwurf nicht behandelt. Des Weiteren gilt

Annahme 2.2 (Ideales Verhalten des unbeschränkten Regelkreises). Der gegebene Regler $\mathbf{R}$ gemäß (2.2) garantiert, dass der unbeschränkte Regelkreis (2.3) alle Güteforderungen erfüllt und somit ein ideales Verhalten aufweist.

Dieses ideale Verhalten des unbeschränkten Regelkreises entsteht durch das Zusammenspiel von Regler und idealem Aktor. Ein idealer Aktor existiert aber in der praktischen Anwendung nicht. Deshalb befasst sich der nächste Abschnitt mit realen Aktoren.

\subsubsection{Reale Aktoren}

Ein realer Aktor ist ein dynamisches System

$$
\mathbf{A}:\left\{\begin{array}{l}
\dot{\boldsymbol{x}}_{\mathrm{a}}=\boldsymbol{f}_{\mathrm{a}}\left(\boldsymbol{x}_{\mathrm{a}}, \boldsymbol{u}_{\mathrm{a}}\right) \\
\boldsymbol{y}_{\mathrm{a}}=\boldsymbol{h}_{\mathrm{a}}\left(\boldsymbol{x}_{\mathrm{a}}, \boldsymbol{u}_{\mathrm{a}}\right)
\end{array}\right.
$$

mit dem Eingang $\boldsymbol{u}_{\mathrm{a}} \in \mathbb{R}^{m}$, dem Ausgang $\boldsymbol{y}_{\mathrm{a}} \in \mathbb{R}^{m}$ sowie dem Zustand $\boldsymbol{x}_{\mathrm{a}} \in \mathbb{R}^{n_{\mathrm{a}}}$. Die Aufgabe des Aktors ist es, die am Eingang $\boldsymbol{u}_{\mathrm{a}}$ anliegende vom Regler kommandierte Stellgröße $\boldsymbol{y}_{\mathrm{r}}$ möglichst schnell und präzise am Ausgang $\boldsymbol{y}_{\mathrm{a}}$ einzustellen. Dabei gibt es zwei wesentliche Punkte, die einen realen von einem idealen Aktor unterscheiden:

1. Der Ausgang des realen Aktors ist in der Amplitude begrenzt, d. h., es sind nicht beliebig große Stellgrößen auf die Strecke übertragbar.

2. Aufgrund physikalischer Gesetzmäßigkeiten besitzt der reale Aktor eine Dynamik, d. h., die vom Regler angeforderte Stellgröße $\boldsymbol{y}_{\mathrm{r}}$ wird sich erst nach einer gewissen Zeit am Aktorausgang $\boldsymbol{y}_{\mathrm{a}}$ einstellen.

Der zweite Punkt kann bei der mathematischen Modellierung des Regelkreises vernachlässigt werden, wenn die Streckendynamik um ein Vielfaches langsamer ist als die Dynamik des Aktors. Davon wird im Weiteren ausgegangen $^{1}$. Die Aktorzustände zur Modellierung der Dynamik werden

\footnotetext{
${ }^{1}$ Ist das nicht der Fall, besteht die Möglichkeit, die Aktordynamik als Teil der Strecke aufzufassen und ein um die Aktorzustände erweitertes Streckenmodell zu betrachten. In [85, Abschnitt 7.1] wird die Aktordynamik beispielsweise als stabiles Verzögerungsglied erster Ordnung vor dem eigentlichen Streckeneingang modelliert.
} 
dann nicht benötigt und es gilt für den Aktorausgang

$$
\boldsymbol{y}_{\mathrm{a}}=\boldsymbol{h}_{\mathrm{a}}\left(\boldsymbol{u}_{\mathrm{a}}\right) .
$$

Der erste Punkt, die Beschränkung des Aktorausganges, ist im Fall eines Ventils sofort einsichtig: Mehr als 100\% lässt es sich nicht öffnen. Auch ein Netzteil besitzt eine maximale Ausgangsspannung. Mathematisch lässt sich eine derartige Begrenzung der $i$-ten Komponente $y_{\mathrm{a}, \mathrm{i}}$ des Aktorausganges $\boldsymbol{y}_{\mathrm{a}}$ durch die nichtlineare Sättigungskennlinie

$$
y_{\mathrm{a}, \mathrm{i}}=\operatorname{sat}_{\left[\underline{\mathrm{u}}_{i}, \overline{\mathrm{u}}_{i}\right]}\left(u_{\mathrm{a}, \mathrm{i}}\right)=\left\{\begin{array}{cr}
\underline{\mathrm{u}}_{i}, & \underline{\mathrm{u}}_{i}>u_{\mathrm{a}, \mathrm{i}}, \\
u_{\mathrm{a}, \mathrm{i}}, & \underline{\mathrm{u}}_{i} \leq u_{\mathrm{a}, \mathrm{i}} \leq \overline{\mathrm{u}}_{i}, \\
\overline{\mathrm{u}}_{i}, & u_{\mathrm{a}, \mathrm{i}}>\overline{\mathrm{u}}_{i}
\end{array}\right.
$$

beschreiben. In vektorieller Schreibweise ergibt sich folgende Beziehung

$$
\boldsymbol{y}_{\mathrm{a}}=\operatorname{sat}_{[\underline{\mathbf{u}}, \overline{\mathbf{u}}]}\left(\boldsymbol{u}_{\mathrm{a}}\right)=\left[\begin{array}{c}
\operatorname{sat}_{\left[\underline{\mathbf{u}}_{1}, \overline{\mathrm{u}}_{1}\right]}\left(u_{\mathrm{a}, 1}\right) \\
\vdots \\
\operatorname{sat}_{\left[\underline{\underline{u}}_{m}, \overline{\mathrm{u}}_{m}\right]}\left(u_{\mathrm{a}, \mathrm{m}}\right)
\end{array}\right]
$$

mit den vektoriellen Sättigungsgrenzen

$$
\begin{aligned}
\underline{\mathbf{u}} & =\left[\begin{array}{lll}
\underline{u}_{1} & \ldots & \underline{\mathrm{u}}_{m}
\end{array}\right]^{\top}, \\
\overline{\mathbf{u}} & =\left[\begin{array}{lll}
\overline{\mathrm{u}}_{1} & \ldots & \overline{\mathrm{u}}_{m}
\end{array}\right]^{\top} .
\end{aligned}
$$

In vielen Fällen liegen symmetrische Grenzen der Stellgröße vor, d. h. $\overline{\mathrm{u}}_{i}=-\underline{\mathrm{u}}_{i}$. Zwecks einer übersichtlicheren Notation wird die $i$-te Komponente der Sättigungsfunktion dann durch

$$
y_{\mathrm{a}, \mathrm{i}}=h_{\mathrm{a}, \mathrm{i}}\left(u_{\mathrm{a}, \mathrm{i}}\right)=\operatorname{sat}_{\overline{\mathrm{u}}_{i}}\left(u_{\mathrm{a}, \mathrm{i}}\right)=\operatorname{sgn}\left(u_{\mathrm{a}, \mathrm{i}}\right) \min \left\{\left|u_{\mathrm{a}, \mathrm{i}}\right|, \overline{\mathrm{u}}_{i}\right\}
$$

beschrieben. Der Index $\underline{u}_{i}$ entfällt. In vektorieller Schreibweise folgt

$$
\boldsymbol{y}_{\mathrm{a}}=\left[\begin{array}{c}
y_{\mathrm{a}, 1} \\
\vdots \\
y_{\mathrm{a}, \mathrm{m}}
\end{array}\right]=\left[\begin{array}{c}
\operatorname{sat}_{\overline{\mathrm{u}}_{1}}\left(u_{\mathrm{a}, 1}\right) \\
\vdots \\
\operatorname{sat}_{\overline{\mathrm{u}}_{m}}\left(u_{\mathrm{a}, \mathrm{m}}\right)
\end{array}\right]=\operatorname{sat}_{\overline{\mathbf{u}}}\left(\boldsymbol{u}_{\mathrm{a}}\right)
$$

mit der vektoriellen Sättigungsgrenze (2.8).

Nun kann der Regelkreis mit begrenzter Stellgröße definiert werden. Die Verschaltung von Regler, Aktor und Strecke gemäß

$$
\boldsymbol{u}_{\mathrm{r}}=\boldsymbol{y}_{\mathrm{s}}, \quad \boldsymbol{u}_{\mathrm{a}}=\boldsymbol{y}_{\mathrm{r}}, \quad \boldsymbol{u}_{\mathrm{s}}=\boldsymbol{y}_{\mathrm{a}}=\operatorname{sat}_{\overline{\mathbf{u}}}\left(\boldsymbol{u}_{\mathrm{a}}\right)
$$




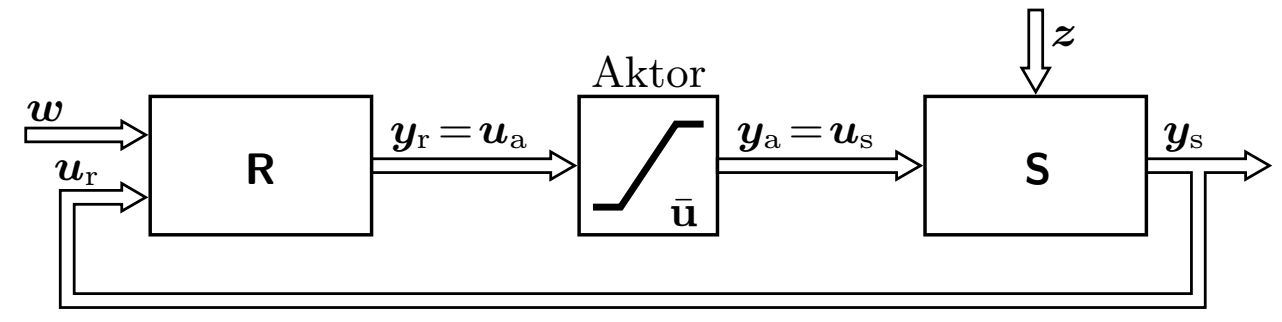

Abbildung 2.2: Beschränkter Regelkreis mit realem Aktor.

führt zu dem in Abbildung 2.2 dargestellten beschränkten Regelkreis.

Mit dem zusammengesetzten Zustandsvektor

$$
\boldsymbol{x}_{\mathrm{b}}^{\top}=\left[\begin{array}{ll}
\boldsymbol{x}_{\mathrm{s}}^{\top} & \boldsymbol{x}_{\mathrm{r}}^{\top}
\end{array}\right]
$$

und dem Eingangsvektor

$$
\boldsymbol{r}^{\top}=\left[\begin{array}{ll}
\boldsymbol{w}^{\top} & \boldsymbol{z}^{\top}
\end{array}\right]
$$

ergibt sich die Zustandsraumdarstellung

$$
\begin{aligned}
\dot{\boldsymbol{x}}_{\mathrm{b}} & =\boldsymbol{A}_{\mathrm{b}} \boldsymbol{x}_{\mathrm{b}}+\boldsymbol{B}_{\mathrm{b}, \mathrm{u}} \boldsymbol{u}_{\mathrm{s}}+\boldsymbol{B}_{\mathrm{b}, \mathrm{r}} \boldsymbol{r} \\
\boldsymbol{u}_{\mathrm{s}} & =\operatorname{sat}_{\overline{\mathbf{u}}}\left(\boldsymbol{C}_{\mathrm{b}} \boldsymbol{x}_{\mathrm{b}}+\boldsymbol{D}_{\mathrm{b}} \boldsymbol{r}\right)
\end{aligned}
$$

des beschränkten Regelkreises mit den Systemmatrizen

$$
\begin{aligned}
& \boldsymbol{A}_{\mathrm{b}}=\left[\begin{array}{cc}
\boldsymbol{A}_{\mathrm{s}} & \mathbf{0} \\
\boldsymbol{B}_{\mathrm{r}, u} \boldsymbol{C}_{\mathrm{s}} & \boldsymbol{A}_{\mathrm{r}}
\end{array}\right], \boldsymbol{B}_{\mathrm{b}, \mathrm{u}}=\left[\begin{array}{c}
\boldsymbol{B}_{\mathrm{s}, u} \\
\mathbf{0}
\end{array}\right], \boldsymbol{B}_{\mathrm{b}, \mathrm{r}}=\left[\begin{array}{cc}
\mathbf{0} & \boldsymbol{B}_{\mathrm{s}, z} \\
\boldsymbol{B}_{\mathrm{r}, w} & \mathbf{0}
\end{array}\right], \\
& \boldsymbol{C}_{\mathrm{b}}=\left[\begin{array}{ll}
\boldsymbol{D}_{\mathrm{r}, u} \boldsymbol{C}_{\mathrm{s}} & \boldsymbol{C}_{\mathrm{r}}
\end{array}\right], \boldsymbol{D}_{\mathrm{b}}=\boldsymbol{D}_{\mathrm{r}, w} .
\end{aligned}
$$

Für kleine Stellgrößen zeigen der beschränkte Regelkreis (2.11) und der unbeschränkte Regelkreis (2.3) das gleiche Verhalten, weil dann keine Sättigung auftritt. Der beschränkte Regelkreis erfüllt in diesem nichtsättigenden Betrieb auch alle Güteforderungen. Bei entsprechend großen Störungen bzw. großen Sollwertänderungen werden aber durch den linearen Regler große Stellgrößen angefordert, die aufgrund der Sättigung des Aktors nicht komplett auf die Strecke übertragen werden können. Die Eingangsgröße der Strecke $\boldsymbol{u}_{\mathrm{s}}$ bleibt somit begrenzt auf die Menge

$$
\mathcal{U}=\left\{\boldsymbol{u}_{\mathrm{s}} \in \mathbb{R}^{m}|| u_{\mathrm{s}, i} \mid \leq \overline{\mathrm{u}}_{i} \forall i=1, \ldots, m\right\} .
$$

Im sättigenden Betrieb kommt es deshalb häufig zu großen Abweichungen von dem erwünschten und nach Annahme 2.2 idealen dynamischen Verhalten des unbeschränkten Regelkreises. Die Ursache sind sogenannte Windup-Effekte, auf die im nächsten Abschnitt näher eingegangen wird. 


\subsection{Charakterisierung des Windup-Begriffs}

Der Begriff Windup ${ }^{2}$ steht heute im regelungstechnischen Kontext für ein durch Stellgrößenbegrenzungen ausgelöstes Fehlverhalten des Regelkreises.

Ursprünglich stammt der Begriff aus der Anfangszeit der industriellen Regelungstechnik in den ersten Dekaden des zwanzigsten Jahrhunderts. Damals beschrieb er ein die Regelgüte beeinträchtigendes Phänomen, das im Zusammenhang mit PID-Reglern und Stellgrößenbegrenzungen auftritt. Der nächste Abschnitt geht darauf detaillierter ein, bevor in Abschnitt 2.2.2 die aktuelle Bedeutung von Windup vorgestellt wird. Abschließend folgt ein illustrierendes Beispiel in Abschnitt 2.2.3.

\subsubsection{Windup im klassischen Sinne}

Um die klassische Definition des Begriffs Windup zu erläutern, wird der beschränkte Regelkreis in Abbildung 2.3 mit einem PID-Regler und einer zu regelnden linearen SISO-Strecke $\mathbf{S}$ gemäß (2.1) betrachtet. Die Güteforderungen an den PID-Regler bestehen darin, die Regelabweichung

$$
e=w-y_{\mathrm{s}}
$$

auszuregeln ${ }^{3}$ sowie eine kurze Ausregelzeit bei minimaler Überschwingweite zu garantieren.

Die Ausgangsgröße des PID-Reglers wird durch das Regelgesetz

$$
y_{\mathrm{r}}(t)=K_{\mathrm{p}} e(t)+K_{\mathrm{i}} \int_{0}^{t} e(\tau) d \tau+K_{\mathrm{d}} \frac{d}{d t} e(t)
$$

berechnet. Die Wahl der Reglerparameter $K_{\mathrm{p}}, K_{\mathrm{i}}$ und $K_{\mathrm{d}}$ erfolgt dabei häufig ohne Berücksichtigung des realen Aktors und der damit verbundenen beschränkten Stellgröße.

Solange eine ausreichend kleine Regelabweichung $e$ vorliegt, die sich nur langsam ändert, ist diese Vereinfachung gerechtfertigt, da dann aufgrund der Linearität des Reglers der Reglerausgang $y_{\mathrm{r}}$ ebenfalls klein bleibt und

$$
u_{\mathrm{s}}=y_{\mathrm{a}}=\operatorname{sat}_{\overline{\mathrm{u}}}\left(u_{\mathrm{a}}\right)=u_{\mathrm{a}}=y_{\mathrm{r}}
$$

${ }^{2}$ engl. to wind up: aufwickeln, aufspulen.

${ }^{3}$ Es wird hier vorausgesetzt, dass ausschließlich Sollgrößen $w$ auftreten, die sich mit einer begrenzten Stellgröße $u_{\mathrm{s}} \in \mathcal{U}$ am Ausgang der Strecke einstellen lassen. 


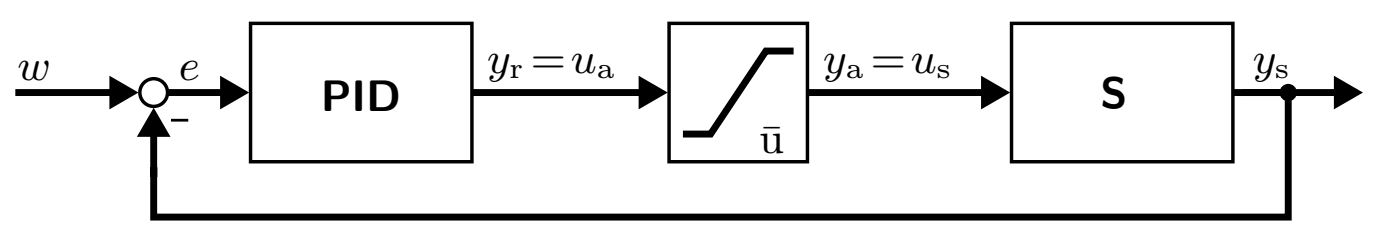

Abbildung 2.3: Regelkreis mit PID-Regler, linearer Regelstrecke $\mathbf{S}$ und einem realen Aktor mit Stellgrößenbegrenzung.

gilt. Der Regelkreis operiert somit ausschließlich im linearen Bereich der Sättigungskennlinie, was auch als nichtsättigender Betrieb bezeichnet wird. Die vom Regler kommandierte Stellgröße $y_{\mathrm{r}}$ wird dabei immer exakt auf die Strecke übertragen und es resultiert das gewünschte dynamische Verhalten des Regelkreises.

Eine große Regelabweichung $e$ führt zu einer großen kommandierten Stellgröße $y_{\mathrm{r}}$ des Reglers. Diese kann jedoch aufgrund der Ausgangsbegrenzung des realen Aktors nicht vollständig auf die Strecke übertragen werden. Offensichtlich wird eine kommandierte Stellgröße $y_{\mathrm{r}}>\overline{\mathrm{u}}$ durch die Sättigung auf den Wert $\overline{\mathrm{u}}$ begrenzt und folglich gilt $u_{\mathrm{s}}=\overline{\mathrm{u}}$.

Tritt der Sättigungsfall ein, macht es keinen Sinn, zur Beschleunigung des Ausregelvorganges die kommandierte Stellgröße $y_{\mathrm{r}}$ weiter zu erhöhen, da der Streckeneingang $u_{\mathrm{s}}$ seinen maximalen Wert $\overline{\mathrm{u}}$ schon erreicht hat. Genau das passiert aber, da das Regelgesetz (2.13) ein Integral enthält und dieser I-Anteil des Reglers die noch nicht vollständig abgebaute Regelabweichung $e$ weiter aufintegriert. Der Wert des Integrals wächst so lange weiter an, bis die Regelabweichung ihr Vorzeichen ändert. Das Anwachsen des I-Anteils wird als Windup bzw. als Integral-Windup bezeichnet.

Nach dem Vorzeichenwechsel der Regelabweichung muss der Integralanteil wieder abgebaut werden, was sich ungünstig auf das dynamische Verhalten des Regelkreises auswirkt. Häufig beobachtete Symptome von Integral-Windup sind ein erhöhtes Überschwingen, ein oszillatorisches Verhalten, eine lange Ausregelzeit und Instabilität.

Vergleichbare negative Auswirkungen auf die Regelkreisdynamik können allerdings auch dann auftreten, wenn der Regler keinen I-Anteil besitzt. Deshalb wurde im Laufe der Zeit und mit der zunehmenden systematischen Erforschung der durch Stellgrößenbegrenzungen ausgelösten negativen Effekte die Bedeutung des Windup-Begriffs erweitert und verallgemeinert. Darauf wird im nächsten Abschnitt eingegangen. 


\subsubsection{Windup heute}

\section{Eine allgemeine Definition}

In der modernen Literatur $[55,85,173,182,183,191,211]$ umfasst der Begriff Windup alle durch Stellgrößenbegrenzungen ausgelösten Effekte, die zu Abweichungen des Regelkreisverhaltens von einem erwünschten nominalen Verhalten führen. Das erwünschte nominale Verhalten entspricht dabei häufig dem idealen Führungs- und Störverhalten des unbeschränkten Regelkreises aus Abbildung 2.1.

Diese moderne Definition trägt der Tatsache Rechnung, dass es aufgrund des realen Aktors auch in Regelkreisen ohne PID-Regler zu einem unerwünschten Verhalten kommen kann. Das Ausmaß der störenden WindupEffekte lässt sich anhand der Abweichungen zwischen den Zuständen des beschränkten und des unbeschränkten Regelkreises quantifizieren.

\section{Eine tiefergehende Definition}

Die allgemeine Definition des Begriffs Windup wird von Hippe in [85, 87] detaillierter aufgeschlüsselt. Dabei wird zwischen drei grundsätzlichen Phänomenen unterschieden, aus denen sich alle Windup-Effekte zusammensetzen: Regler-Windup, Strecken-Windup und das Direktionalitätsproblem. Diese drei Phänomene können sich in einem Regelkreis überlagern und so die beobachtbaren Abweichungen des beschränkten Regelkreises von dem erwünschten unbeschränkten Verhalten verursachen. Nachfolgend sind die drei Phänomene kurz erläutert.

Regler-Windup. Nach Hippe umfasst der Begriff Regler-Windup alle Windup-Effekte, die eindeutig dem Regler zuzuordnen sind. ReglerWindup tritt auf, wenn die Reglerdynamik grenz- oder instabil ist [87], d. h. wenn $\boldsymbol{A}_{\mathrm{r}}$ nichtnegative Eigenwerte besitzt. Dies lässt sich wie folgt plausibilisieren: Im Sättigungsfall ist der beschränkte Regelkreis praktisch unterbrochen, weil Änderungen des Streckenausgangs $\boldsymbol{y}_{\mathrm{s}}$ nicht mehr über den Regler den Streckeneingang $\boldsymbol{u}_{\mathrm{s}}$ beeinflussen. Sind beispielsweise alle Stellgrößen in Sättigung, wirkt auf die Strecke die konstante Eingangsgröße $\boldsymbol{u}_{\mathrm{s}}=\overline{\mathbf{u}}$, wie Abbildung 2.4 zeigt. Eine grenz- oder instabile Reglerdynamik wird dann nicht mehr durch eine Rückkopplung stabilisiert. Das führt zu einem Anwachsen der Reglerzustände. Klassisches Integral-Windup bei PID-Reglern ist somit ein Spezialfall des Regler-Windup, weil der I-Anteil ein instabiles Element im offenen Kreis darstellt. Nach dem Ende der Sättigungsphase müssen die großen Reglerzustände wieder abgebaut werden, was zu den bekannten negativen Auswirkungen führt. 


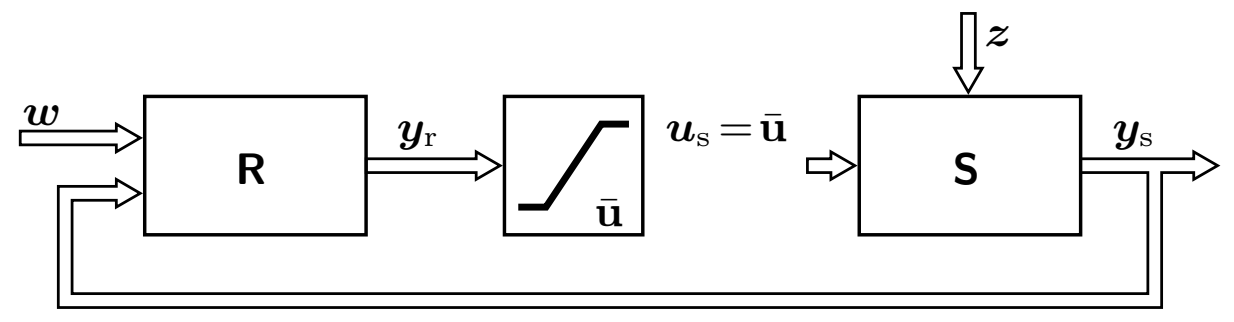

Abbildung 2.4: Unterbrochener Regelkreis im Sättigungsfall.

Strecken-Windup. Analog zum Regler-Windup definiert Hippe den Begriff Strecken-Windup in [89]. Damit sind alle Windup-Effekte gemeint, die sich der geregelten Strecke zuordnen lassen [87]. Strecken-Windup entsteht während des Ausregelvorganges durch stark anwachsende Streckenzustände, die sich durch das begrenzte Stellsignal nicht mehr schnell genug abbauen lassen, so dass der Sollwert über- bzw. unterschritten wird. Ein oszillierendes Verhalten ist die Folge.

Anschaulich lässt sich der Effekt an einem Fahrzeug demonstrieren, das ein festgelegtes Ziel möglichst schnell erreichen möchte. Zunächst wird stark beschleunigt, die Geschwindigkeit wächst an, der Abstand zum Ziel verringert sich. Kurz vor dem Ziel muss gebremst werden, damit das Fahrzeug punktgenau zum Stehen kommt. Ist allerdings die Geschwindigkeit zu hoch und die Bremskraft begrenzt, wird das Fahrzeug erst in einigem Abstand vom Ziel anhalten, wenden und wieder auf das Ziel zufahren.

Ob Strecken-Windup auftritt und in welchem Ausmaß, hängt von dem verwendeten Regler ab. Die Beispiele in [85] zeigen, dass die negativen Auswirkungen des Strecken-Windup umso stärker zu Tage treten, je schneller die Dynamik des unbeschränkten Regelkreises ist.

Direktionalitätsproblem. Im SISO-Fall lassen sich nach Hippe alle Windup-Effekte entweder dem Regler- oder dem Strecken-Windup zuordnen. Im MIMO-Fall kommt noch das nachfolgend beschriebene Direktionalitätsproblem hinzu, das bei Entkopplungsreglern auftritt. Eine entkoppelnde Regelung wird so entworfen, dass die Veränderung eines Sollwertes lediglich eine zugeordnete Ausgangsgröße der Strecke beeinflusst. Dazu erzeugt ein Entkopplungsregler Stellvektoren $\boldsymbol{y}_{\mathrm{r}}$ mit einer gewissen Länge und Richtung, die vom Aktor auf die Strecke übertragen werden müssen. Geht eine Komponente $u_{\mathrm{a}, \mathrm{i}}$ des Aktorausganges in Sättigung, so wird nicht nur die Länge, sondern auch die Richtung der entsprechenden Komponente $u_{\mathrm{s}, i}$ des Streckeneinganges verändert. Das ist beispielhaft für einen Stellvektor mit zwei Komponenten in Abbildung 2.5 dargestellt. Durch eine 
(a)

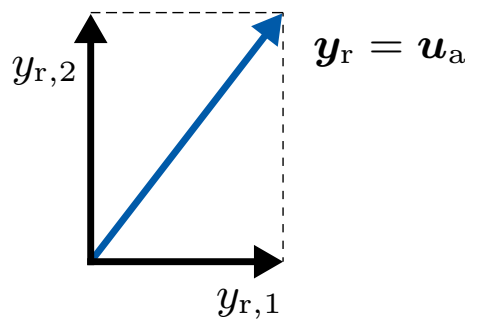

(b)

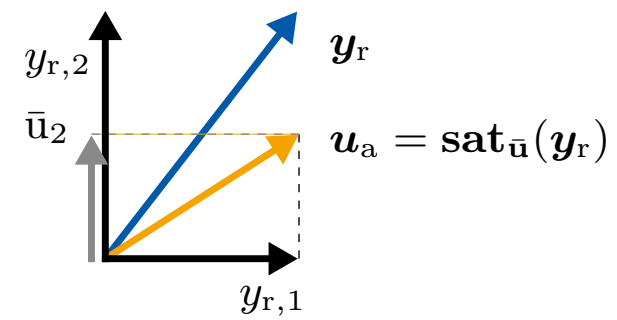

Abbildung 2.5: Das Direktionalitätsproblem. (a) Im unbeschränkten Regelkreis wird durch Vorgabe einer bestimmten Richtung des Stellgrößenvektors $\boldsymbol{y}_{\mathrm{r}}$ eine Entkopplung erreicht, da der ideale Aktor $\boldsymbol{u}_{\mathrm{a}}=\boldsymbol{y}_{\mathrm{r}}$ garantiert. (b) Im beschränkten Regelkreis kann der Aktor die Komponente $y_{\mathrm{r}, 2}$ nicht realisieren. Sättigung tritt auf und verändert Länge und Richtung des Aktorausganges, d. h. $\boldsymbol{u}_{\mathrm{a}}=\operatorname{sat}_{\overline{\mathbf{u}}}\left(\boldsymbol{y}_{\mathrm{r}}\right)$. Die entkoppelnde Eigenschaft des Reglers geht verloren.

derartige Änderung von Länge und Richtung des Stellvektors ist die entkoppelnde Wirkung nicht mehr gewährleistet, d. h., es können starke unerwünschte Kopplungen zwischen den Ein- und Ausgängen des Regelkreises auftreten. Diese Form von Windup wird als Direktionalitätsproblem bezeichnet.

\subsubsection{Beispiel: hydraulisches Positioniersystem}

Um das Auftreten von Windup zu demonstrieren, wird das vereinfachte Modell eines hydraulischen Positioniersystems betrachtet. Der schematische Aufbau ist in Abbildung 2.6 dargestellt. Die zu positionierende Lastmasse besitzt die Auslenkung $x_{\mathrm{s}, 1}$ in $\mathrm{cm}$ und die Geschwindigkeit $x_{\mathrm{s}, 2}=\dot{x}_{\mathrm{s}, 1}$ in $\mathrm{cm} / \mathrm{s}$. Die Eingangsspannung $u_{\mathrm{s}}$ des Systems in $\mathrm{V}$ wird über einen Spannungs-Druck-Wandler in den Druck $x_{\mathrm{s}, 3}$, angegeben in $\mathrm{N} / \mathrm{cm}^{2}$, gewandelt. Bei einer Differenz zwischen dem im Zylinder herrschenden Druck und $x_{\mathrm{s}, 3}$ entsteht ein Volumenstrom $\dot{q}$, der für Druckausgleich sorgt. Die entstehende Kraft auf den Zylinderkolben bewegt die Masse.

Mit dem Zustandsvektor $\boldsymbol{x}_{\mathrm{s}}=\left[\begin{array}{lll}x_{\mathrm{s}, 1} & x_{\mathrm{s}, 2} & x_{\mathrm{s}, 3}\end{array}\right]^{\top}$ lautet das in [121] hergeleitete lineare Zustandsraummodell der Strecke

$$
\begin{aligned}
& \dot{\boldsymbol{x}}_{\mathrm{s}}=\left[\begin{array}{ccc}
0 & 1 & 0 \\
-10 & -1,167 & 25 \\
0 & 0 & -0,8
\end{array}\right] \boldsymbol{x}_{\mathrm{s}}+\left[\begin{array}{c}
0 \\
0 \\
2,4
\end{array}\right] u_{\mathrm{s}}, \\
& y_{\mathrm{s}}=\left[\begin{array}{lll}
1 & 0 & 0
\end{array}\right] \boldsymbol{x}_{\mathrm{s}}
\end{aligned}
$$

mit $\boldsymbol{x}_{\mathrm{s}}(0)=\mathbf{0}$. Dabei besitzt die Matrix $\boldsymbol{A}_{\mathrm{s}}$ die Eigenwerte $\lambda_{1}=-0,8$ und 


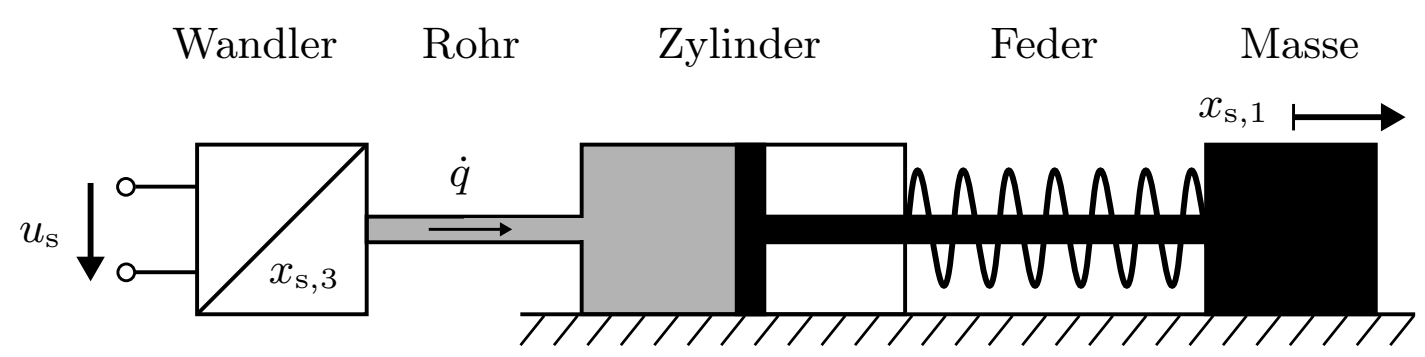

Abbildung 2.6: Hydraulisches Positioniersystem.

$\lambda_{2,3}=-0,584 \pm j 3,108$. Die Stellamplitude ist durch die Sättigungsfunktion gemäß $(2.9)$ mit $\overline{\mathrm{u}}=10,5 \mathrm{~V}$ begrenzt. Somit folgt $u_{\mathrm{s}}=\operatorname{sat}_{\overline{\mathrm{u}}}\left(y_{\mathrm{r}}\right)$.

Zur Regelung kommt ein linearer dynamischer Regler mit I-Anteil zum Einsatz, dessen Regelgesetz

$$
y_{\mathrm{r}}(s)=\frac{17,764(s+0,731)}{s} \frac{\left(s^{2}+1,167 s+10\right)}{(s+5)(s+100)}\left(w(s)-y_{\mathrm{s}}(s)\right)
$$

einen stabilen unbeschränkten Regelkreis garantiert und den Regelfehler $e(s)=w(s)-y_{\mathrm{s}}(s)$ stationär genau ausregelt.

Die Antwort des geschlossenen, unbeschränkten Regelkreises auf einen Sollwertsprung ist im oberen Teil der Abbildung 2.7 dargestellt. Deutlich ist die überschwingfreie Ausregelung auf den geforderten Sollwert von $w=20 \mathrm{~cm}$ zu sehen. Der dazu nötige Stellgrößenverlauf ist aber nur mit einem idealen Aktor realisierbar und verläuft anfangs kurz außerhalb des im unteren Teil von Abbildung 2.7 in weiß dargestellten physikalisch möglichen Stellgrößenbereiches von $\pm 10,5 \mathrm{~V}$.

Die Antwort des beschränkten Regelkreises aus Abbildung 2.2 auf den gleichen Sollwertsprung ist ebenfalls dargestellt. Aufgrund der vorhandenen Stellbegrenzung kommt es zu starken Abweichungen von dem erwünschten Verhalten des unbeschränkten Regelkreises. Das hohe Überschwingen begleitet von einem oszillatorischen Verhalten sind Auswirkungen des aufgetretenen Windup.

\subsection{Zusammenfassung und Fazit}

In diesem Kapitel wurde das Windup-Problem vorgestellt, das durch die Stellbegrenzungen eines realen Aktors entsteht. Der Begriff Windup umfasst alle durch sättigende Stellgrößen ausgelösten Effekte, die zu einer 

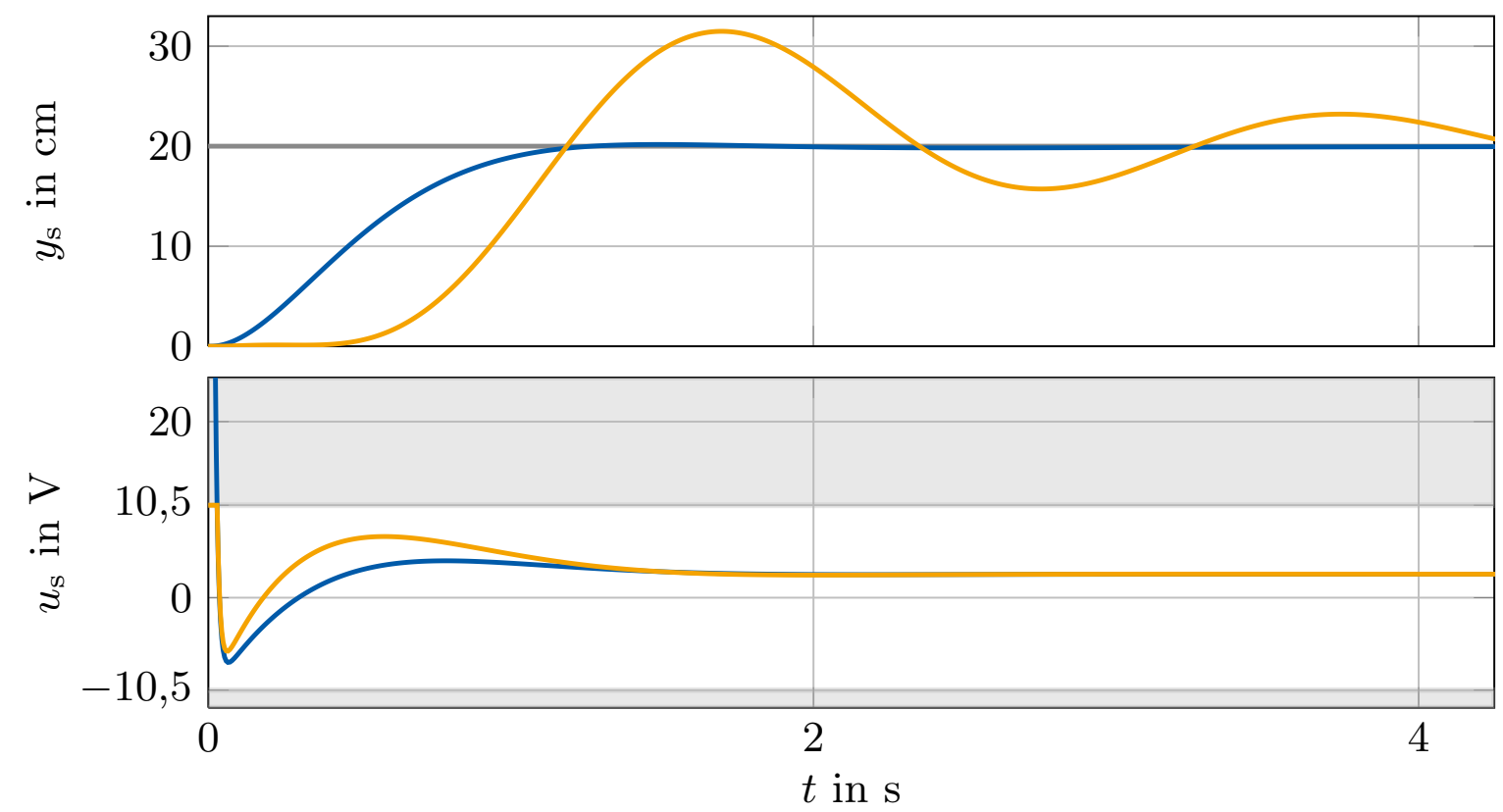

Abbildung 2.7: Sprungantworten des unbeschränkten (-) und des beschränkten (-) Regelkreises sowie zugehörige Stellgrößen. Der unbeschränkte Regelkreis erreicht den geforderten Sollwert $w=20 \mathrm{~cm}$ (-) praktisch ohne Überschwingen. Dazu ist für $t=0$ eine Stellgröße von $u_{\mathrm{s}}(0)=355 \mathrm{~V}$ erforderlich. Im beschränkten Regelkreis zeigen sich die Auswirkungen des Windup.

Abweichung des Regelkreisverhaltens von dem als ideal angesehenen unbeschränkten Verhalten führen. Typische Auswirkungen von Windup sind ein oszillierendes Verhalten, starkes Überschwingen oder eine sehr langsame Konvergenz des Streckenausganges zum gewünschten Sollwert. In MIMO-Regelkreisen kommt das Direktionalitätsproblem hinzu. Auch Instabilität des Regelkreises ist möglich. Zur Vermeidung dieser durch Stellbegrenzungen ausgelösten Probleme kommen unterschiedliche Lösungen in Frage, die im nächsten Kapitel diskutiert werden. 


\section{Umgang mit Stellbegrenzungen}

Wie im letzten Kapitel gezeigt, kann das Ignorieren von Stellbegrenzungen beim Reglerentwurf zu einer erheblichen Verschlechterung der Regelgüte führen. Im Extremfall ist sogar die Stabilität des Regelkreises gefährdet [178]. Die Entwicklung von Regelkonzepten für Strecken mit Stellbegrenzungen ist deshalb ein Forschungsgebiet mit hoher praktischer Relevanz. Grundsätzlich lassen sich vier Vorgehensweisen unterscheiden: direkte Berücksichtigung der Begrenzungen beim Reglerentwurf, modellbasierte prädiktive Regelung, Regelkreise mit Governor und die sogenannten AntiWindup-Methoden. Die ersten drei Konzepte werden in Abschnitt 3.2 behandelt. Anti-Windup-Maßnahmen folgen in Abschnitt 3.3. Konzeptunabhängig ist die Limitierung der erreichbaren Stabilitätseigenschaften des Regelkreises durch die Stellbegrenzung, worauf zunächst eingegangen wird.

\subsection{Stabilisierung eingangsbeschränkter Systeme: Grenzen des Möglichen}

Ausgangspunkt der Überlegungen ist ein lineares, steuer- und beobachtbares Mehrgrößensystem der Form

$$
\begin{aligned}
& \dot{\boldsymbol{x}}_{\mathrm{s}}=\boldsymbol{A}_{\mathrm{s}} \boldsymbol{x}_{\mathrm{s}}+\boldsymbol{B}_{\mathrm{s}, u} \boldsymbol{u}_{\mathrm{s}} \\
& \boldsymbol{y}_{\mathrm{s}}=\boldsymbol{C}_{\mathrm{s}} \boldsymbol{x}_{\mathrm{s}}
\end{aligned}
$$

das keine invarianten Nullstellen in Null besitzt (siehe Seite 8 f.). Im Fall eines unbeschränkten Eingangs $\boldsymbol{u}_{\mathrm{s}} \in \mathbb{R}^{m}$ lässt sich immer ein Regler finden, der Anfangszustände $\boldsymbol{x}_{\mathrm{s}}^{(0)} \in \mathbb{R}^{n_{\mathrm{s}}}$ in eine Ruhelage $\boldsymbol{x}_{\mathrm{s}}^{(\mathrm{R})}$ des Systems überführen kann. Diese Ruhelagen ergeben sich als Lösungen von

$$
\boldsymbol{A}_{\mathrm{s}} \boldsymbol{x}_{\mathrm{s}}^{(\mathrm{R})}+\boldsymbol{B}_{\mathrm{s}, u} \boldsymbol{u}_{\mathrm{s}}^{(\mathrm{R})}=\mathbf{0} \quad \Leftrightarrow \quad \boldsymbol{A}_{\mathrm{s}} \boldsymbol{x}_{\mathrm{s}}^{(\mathrm{R})}=-\boldsymbol{B}_{\mathrm{s}, u} \boldsymbol{u}_{\mathrm{s}}^{(\mathrm{R})} .
$$

Um den Systemzustand in der Ruhelage $\boldsymbol{x}_{\mathrm{s}}^{(\mathrm{R})}$ zu halten, muss der Aktor die stationäre Stellgröße $\boldsymbol{u}_{\mathrm{s}}^{(\mathrm{R})}$ aufbringen. Das gelingt im Fall einer beschränk- 
ten Eingangsgröße nur dann, wenn

$$
\boldsymbol{u}_{\mathrm{s}}^{(\mathrm{R})} \in \mathcal{U}=\left\{\boldsymbol{u}_{\mathrm{s}} \in \mathbb{R}^{m}|| u_{\mathrm{s}, i} \mid \leq \overline{\mathrm{u}}_{i} \forall i=1, \ldots, m\right\} \subset \mathbb{R}^{m} .
$$

Hier zeigt sich der erste Unterschied zum unbeschränkten Fall: Die Menge der Ruhelagen reduziert sich auf die Lösungen von (3.2) mit $\boldsymbol{u}_{\mathrm{s}}^{(\mathrm{R})} \in \mathcal{U}$, d. h. auf die Menge

$$
\mathcal{X}^{(\mathrm{R})}=\left\{\boldsymbol{x}_{\mathrm{s}}^{(\mathrm{R})} \in \mathbb{R}^{n_{\mathrm{s}}} \mid \boldsymbol{A}_{\mathrm{s}} \boldsymbol{x}_{\mathrm{s}}^{(\mathrm{R})}=-\boldsymbol{B}_{\mathrm{s}, u} \boldsymbol{u}_{\mathrm{s}}^{(\mathrm{R})}, \boldsymbol{u}_{\mathrm{s}}^{(\mathrm{R})} \in \mathcal{U}\right\} .
$$

Um die weiteren Betrachtungen zu vereinfachen, gilt ohne Beschränkung der Allgemeinheit $\boldsymbol{x}_{\mathrm{s}}^{(\mathrm{R})}=\mathbf{0}$. Denn das System (3.1) mit der Ruhelage $\boldsymbol{x}_{\mathrm{s}}^{(\mathrm{R})} \in \boldsymbol{\mathcal { X }}^{(\mathrm{R})}$ lässt sich mittels einer Zustands- und Eingangstransformation

$$
\begin{aligned}
& \boldsymbol{x}=\boldsymbol{x}_{\mathrm{s}}-\boldsymbol{x}_{\mathrm{s}}^{(\mathrm{R})}, \\
& \boldsymbol{u}=\boldsymbol{u}_{\mathrm{s}}-\boldsymbol{u}_{\mathrm{s}}^{(\mathrm{R})}
\end{aligned}
$$

in neuen Koordinaten darstellen $[3,6]$. Das transformierte System

$$
\dot{\boldsymbol{x}}=\boldsymbol{A}_{\mathrm{s}} \boldsymbol{x}+\boldsymbol{B}_{\mathrm{s}, u} \boldsymbol{u}
$$

besitzt die Ruhelage $\boldsymbol{x}^{(\mathrm{R})}=\mathbf{0}$ für $\boldsymbol{u}^{(\mathrm{R})}=\mathbf{0}$ und die Eingangsbeschränkung

$$
\boldsymbol{u}+\boldsymbol{u}_{\mathrm{s}}^{(\mathrm{R})} \in \mathcal{U} .
$$

Die Arbeiten [23, 165, 177] zeigen, dass sich der Zustand einer linearen, steuer- und beobachtbaren Strecke (3.1) nur dann von beliebigen Anfangswerten $\boldsymbol{x}_{\mathrm{s}}^{(0)} \in \mathbb{R}^{n_{\mathrm{s}}}$ mit einer beschränkten Stellgröße $\boldsymbol{u}_{\mathrm{s}} \in \mathcal{U}$ in die Ruhelage $\boldsymbol{x}_{\mathrm{s}}^{(\mathrm{R})}=\mathbf{0}$ überführen lässt, wenn alle Eigenwerte $\lambda_{i}$ der Systemmatrix $\boldsymbol{A}_{\mathrm{s}}$ einen nichtpositiven Realteil aufweisen, d. h. $\operatorname{Re}\left\{\lambda_{i}\right\} \leq 0 \forall i=1, \ldots, n_{\mathrm{s}}$. Dieses Ergebnis ist der zweite Unterschied im Vergleich zu dem Fall unbeschränkter Stellgröße und hat direkte Konsequenzen für die mit einer Regelung bestenfalls erreichbaren Stabilitätseigenschaften der Ruhelage. Offensichtlich sind diese durch die Eigenwerte $\lambda_{i}$ von $\boldsymbol{A}_{\mathrm{s}}$ bestimmt. Um die Aussage zu präzisieren, wird folgende Definition benötigt.

Definition 3.1 (Einzugsgebiet). Sei $\boldsymbol{x}^{(\mathrm{R})}=\mathbf{0} \in \mathbb{R}^{n}$ eine asymptotisch stabile Ruhelage des Systems

$$
\dot{\boldsymbol{x}}=\boldsymbol{f}\left(\boldsymbol{x}, \boldsymbol{u}^{(\mathrm{R})}=\mathbf{0}\right), \quad \boldsymbol{x}(0)=\boldsymbol{x}^{(0)} .
$$

Das Anfangswertproblem (3.4) besitze für alle $t \geq 0$ eine eindeutige Lösung. Eine Umgebung $\mathcal{G}(\mathbf{0}) \in \mathbb{R}^{n}$ der Ruhelage $\boldsymbol{x}^{(\mathrm{R})}$ heißt Einzugsgebiet der Ruhelage, wenn alle in $\mathcal{G}(\mathbf{0})$ startenden Trajektorien $\boldsymbol{x}(t)$ in $\mathcal{G}(\mathbf{0})$ verbleiben und in die Ruhelage streben. 
Ein unendlich großes Einzugsgebiet $\mathcal{G}(\mathbf{0})=\mathbb{R}^{n_{\mathrm{s}}}$, d. h. globale asymptotische Stabilität der Ruhelage $\boldsymbol{x}_{\mathrm{s}}^{(\mathrm{R})}=\mathbf{0}$ des Systems (3.3), lässt sich nur dann erreichen, wenn $\operatorname{Re}\left\{\lambda_{i}\right\} \leq 0 \forall i=1, \ldots, n_{\mathrm{s}}$ gilt. Im Allgemeinen wird dazu ein nichtlineares sättigendes Regelgesetz benötigt [48, 181].

Lineare, sättigende Regelgesetze führen zu weiteren Einschränkungen. Globale asymptotische Stabilität kann mit einem derartigen Regelgesetz nur für einige Spezialfälle, z. B. für alle Strecken erster und zweiter Ordnung mit nichtpositiven Eigenwerten oder Strecken beliebiger Ordnung mit ausschließlich negativen Eigenwerten, garantiert werden [181]. Semiglobale asymptotische Stabilität der Ruhelage, d.h. ein beliebig großes, aber begrenztes Einzugsgebiet, ist dagegen immer möglich [184].

Für instabile Strecken existiert unabhängig vom eingesetzten Regler nur ein begrenztes Einzugsgebiet $\mathcal{G}(\mathbf{0}) \subset \mathbb{R}^{n_{\mathrm{s}}}$. Die Ruhelage ist dann lokal asymptotisch stabil. Diese aufgezeigten Einschränkungen gelten für alle in den nächsten Abschnitten vorgestellten Regelkonzepte.

\subsection{Grundlegende Vorgehensweisen}

In den folgenden Abschnitten werden drei Regelkonzepte für Strecken mit Stellbegrenzungen vorgestellt, nämlich die direkte Berücksichtigung der Stellbegrenzungen beim Reglerentwurf, die modellbasierte prädiktive Regelung und das Governor-Konzept. Ebenso grundlegend ist das Konzept der Anti-Windup-Maßnahmen. Aufgrund ihrer besonderen Stellung in dieser Arbeit werden Anti-Windup-Methoden in Abschnitt 3.3 und detaillierter in Kapitel 4 beschrieben.

\subsubsection{Direkte Berücksichtigung der Stellbegrenzungen beim Reglerentwurf}

Ein naheliegender und systematischer Ansatz ist die direkte Berücksichtigung der Stellbegrenzungen beim Entwurf des Reglers R im beschränkten Regelkreis aus Abbildung 2.2. Nach der Wahl der Reglerstruktur wird diese dann so parametriert, dass Stabilität und eine hohe Regelgüte trotz der Stellbegrenzung garantiert sind. Das geschieht in vielen Fällen durch Lösen eines Optimierungsproblems mit passenden Nebenbedingungen.

Eine Zusammenstellung verschiedener Ansätze findet sich in [17, 91, 103, 163, 182]. Einen detaillierteren Überblick geben z. B. die Dissertationen [120, Abschnitt 2.2] und [38, Abschnitt 2.2] sowie [25]. Die existierenden Entwurfsverfahren lassen sich hinsichtlich der Reglerstruktur und der 
Stabilitätsgarantie voneinander unterscheiden. Grob kann eine Unterteilung in Zustandsregler und Ausgangsrückführungen mit jeweils globaler, semiglobaler oder lokaler Stabilität der Ruhelage erfolgen.

\section{Zustandsregelungen}

Viele Arbeiten widmen sich dem Entwurf von Zustandsreglern. Unter der Voraussetzung, dass alle Zustände der Strecke (2.1) messbar sind, d.h. $\boldsymbol{C}_{\mathrm{s}}=\mathbb{I}$ und somit $\boldsymbol{y}_{\mathrm{s}}=\boldsymbol{x}_{\mathrm{s}}$, lautet das Regelgesetz

$$
\boldsymbol{y}_{\mathrm{r}}=\boldsymbol{f}_{\mathrm{r}}\left(\boldsymbol{x}_{\mathrm{s}}\right) \text {. }
$$

Aufgrund der linearen Struktur der Strecke liegt ein lineares Regelgesetz der Form $\boldsymbol{y}_{\mathrm{r}}=\boldsymbol{K}_{\mathrm{r}} \boldsymbol{x}_{\mathrm{s}}$ oder ein sättigendes, lineares Regelgesetz der Form $\boldsymbol{y}_{\mathrm{r}}=\mathbf{s a t}_{\overline{\mathbf{u}}}\left(\boldsymbol{K}_{\mathrm{r}} \boldsymbol{x}_{\mathrm{s}}\right)$ nahe. Den Fall stabiler Strecken behandeln die Arbeiten $[124,161,170]$. Semiglobale Ergebnisse wurden in [125, 127, 163] publiziert. Lokale Stabilität des Ursprungs garantieren die Reglerentwürfe [31, 74, 91, 93]. Durch eine Modifikation des Regelgesetzes können auch andere Ruhelagen stabilisiert werden [19, 104, 106].

Der Einfachheit einer (sättigenden) linearen Zustandsrückführung steht eine mäßige Regelgüte in der Nähe des Ursprungs gegenüber. Durch strukturvariable Zustandsrückführungen lässt sich dieses Problem beheben. Die seit langem bekannte Grundidee ist, immer aggressivere Zustandsregler zu aktivieren, je näher die Systemzustände dem Ursprung kommen. Das führt zu strukturvariablen Zustandsreglern, die zwischen einer festen Anzahl an Zustandsreglern umschalten [109, 110, 169, 202]. Eine weitere Steigerung der Regelgüte ist durch unendlich viele Zustandsregler erreichbar. Die Reglerparameter ändern sich dann kontinuierlich und man spricht von weich strukturvariablen Regelungen [1, 4, 5, 38, 47, 97, 121] bzw. sättigenden weich strukturvariablen Regelungen [24, 98, 105, 122, 123]. Die theoretisch schnellste Regelung lässt sich mit zeitoptimalen Zustandsreglern erreichen $[3,9,49]$. Allerdings sind die dazu nötigen komplizierten nichtlinearen Regelgesetze nur für Strecken niedriger Ordnung bestimmbar.

Eine weitere Forschungsrichtung stellen sogenannte Low-and-High Gain Regelungen dar [124, 128, 129, 162]. Das Ziel einer solchen Regelung ist primär die Sicherstellung von semiglobaler Stabilität der Ruhelage mit einem linearen Regelgesetz (low gain). Dieser Teil der Regelung wird zuerst entworfen. Durch eine spezielle Variation der Reglerverstärkung in Abhängigkeit des Streckenzustands (scheduled low gain) lässt sich sogar globale Stabilität für alle Strecken erreichen, die nicht instabil sind. Um die Regelgüte zu erhöhen wird danach das Regelgesetz in der Nähe des Ursprungs 
modifiziert, so dass sich dort eine hohe Verstärkung ergibt (high gain). Eine Übersicht aktueller Entwicklungen findet sich in [126].

\section{Ausgangsrückführungen}

Da nicht immer alle Zustände der Strecke gemessen werden können, sind Zustandsregelungen nur bedingt einsetzbar. In der praktischen Anwendung stehen oftmals ausschließlich bestimmte Messgrößen $\boldsymbol{y}_{\mathrm{s}}=\boldsymbol{C}_{\mathrm{s}} \boldsymbol{x}_{\mathrm{s}}$ zur Verfügung. Deshalb liegt es nahe, einen Beobachter einzusetzen und die Messgrößen zur Schätzung der Streckenzustände zu verwenden. Die Zustandsregelung lässt sich dann mit den geschätzten Zuständen realisieren.

Viele Ausgangsrückführungen fußen auf diesem beobachterbasierten Prinzip. Die Methoden [124, 137] garantieren globale Stabilität für Strecken ohne instabile Eigenwerte. Semiglobale Ergebnisse für derartige Strecken werden in $[130,171,179]$ erreicht. Beobachterbasierte Ausgangsrückführungen mit lokaler Stabilitätsgarantie bieten [92, 120, 182].

Alternativ kann auch ein dynamischer Regler entworfen werden, der nicht auf einer Schätzung der Streckenzustände basiert. Die Arbeiten [120, 143, 203] verfolgen diesen Ansatz und beschreiben Ausgangsrückführungen mit garantierter lokaler Stabilität der Ruhelage für beliebige lineare Strecken. Globale Stabilität für stabile Strecken wird mit dem Ansatz in [140] erreicht. Die Ausgangsrückführungen in [37, 182] garantieren in Abhängigkeit der Strecke lokale, semiglobale oder globale Stabilität.

\subsubsection{Modellbasierte prädiktive Regelung}

Eine modellbasierte prädiktive Regelung (MPR) nutzt ein Modell der Regelstrecke, um deren zukünftiges Verhalten zu prädizieren [3, 159]. Insbesondere kann mit dem Modell eine Aussage über das Verhalten der Streckenzustände und -ausgänge bei gegebenen Eingangsverläufen getroffen werden. Diese Tatsache lässt sich ausnutzen, um durch Lösen eines Optimierungsproblems günstige Steuerfolgen für das Modell zu berechnen, die sich dann zur Regelung der Strecke nutzen lassen. Die beiden Hauptbestandteile einer modellbasierten prädiktiven Regelung sind somit das zeitdiskrete Streckenmodell für die Prädiktion und ein Optimierungsalgorithmus zur Online-Berechnung der optimalen Steuerfolge.

Der Vorteil dieser Vorgehensweise ist, dass Begrenzungen der Stellgröße explizit als Nebenbedingungen in das Optimierungsproblem integriert werden können $[27,136,158]$. Die Stellbegrenzungen werden somit immer eingehalten und die in Abschnitt 2.2 angesprochenen Probleme vermieden. 
Die Arbeitsweise einer MPR lässt sich wie folgt zusammenfassen:

1. Der zum Abtastzeitpunkt $\kappa$ aktuelle Zustand der Strecke $\boldsymbol{x}_{\mathrm{s}}(\kappa)$ wird gemessen oder mit einem Beobachter geschätzt.

2. Auf Basis eines zeitdiskreten Zustandsraummodells der Strecke

$$
\begin{aligned}
\boldsymbol{x}_{\mathrm{s}}(\kappa+1) & =\boldsymbol{A}_{\mathrm{s}} \boldsymbol{x}_{\mathrm{s}}(\kappa)+\boldsymbol{B}_{\mathrm{s}} \boldsymbol{u}_{\mathrm{s}}(\kappa), \\
\boldsymbol{y}_{\mathrm{s}}(\kappa) & =\boldsymbol{C}_{\mathrm{s}} \boldsymbol{x}_{\mathrm{s}}(\kappa)
\end{aligned}
$$

wird eine Steuerfolge $\boldsymbol{u}_{\mathrm{s}}(\kappa+1), \ldots, \boldsymbol{u}_{\mathrm{s}}(\kappa+N)$ für einen endlichen Zeithorizont $N \geq 1$ durch Lösen eines Optimalsteuerungsproblems bestimmt [136]. Als Gütemaß wird häufig

$$
J=\sum_{i=1}^{N}\left\|\boldsymbol{Q}\left(\boldsymbol{y}_{\mathrm{s}}(\kappa+i)-\boldsymbol{w}(\kappa+i)\right)\right\|^{2}+r \cdot \sum_{i=1}^{N}\left\|\boldsymbol{u}_{\mathrm{s}}(\kappa+i)\right\|^{2}
$$

mit $r>0$ und einer positiv definiten Matrix $\boldsymbol{Q}$ verwendet [3]. Typische Nebenbedingungen sind Beschränkungen der Stellamplitude.

3. Das erste Element $\boldsymbol{u}_{\mathrm{s}}(\kappa+1)$ der Steuerfolge wird zum Zeitpunkt $\kappa+1$ auf die Strecke geschaltet und der Algorithmus beginnt wieder bei Schritt 1 mit $\kappa:=\kappa+1$.

Ein großer Nachteil der MPR ist der hohe Rechenaufwand, der mit dem Lösen des Optimierungsproblems einhergeht und die damit verbundene Echtzeitproblematik. Die optimale Steuerfolge muss innerhalb eines Abtastschrittes berechnet worden sein. Das erfordert entweder schnelle Rechner oder lange Abtastzeiten. Folglich wurden modellbasierte prädiktive Regelungen zuerst bei langsamen Prozessen mit Abtastzeiten im Minutenbereich eingesetzt [118].

Die Lösung der Echtzeitproblematik ist noch immer Gegenstand der aktuellen Forschung. Eine Möglichkeit bietet die sogenannte explizite MPR $[14,44]$. Die Grundidee ist, eine explizite Form des Regelgesetzes als Funktion des Anfangszustandes offline zu berechnen [198]. Verschiedenen Gebieten des Zustandsraumes sind dann verschiedene Regelgesetze zugeordnet. Während des Ausregelvorganges reduziert sich der Rechenaufwand auf die Bestimmung des Gebietes, in dem sich der aktuelle Streckenzustand befindet. Die Anzahl der Gebiete und somit Regelgesetze kann jedoch exponentiell mit der Zahl der Streckenzustände, -eingänge und dem Zeithorizont $N$ steigen [198], was die Anwendbarkeit der expliziten MPC einschränkt. Alternative Ideen zur Verkürzung der Rechenzeit finden sich in $[152,198]$. 
(a)

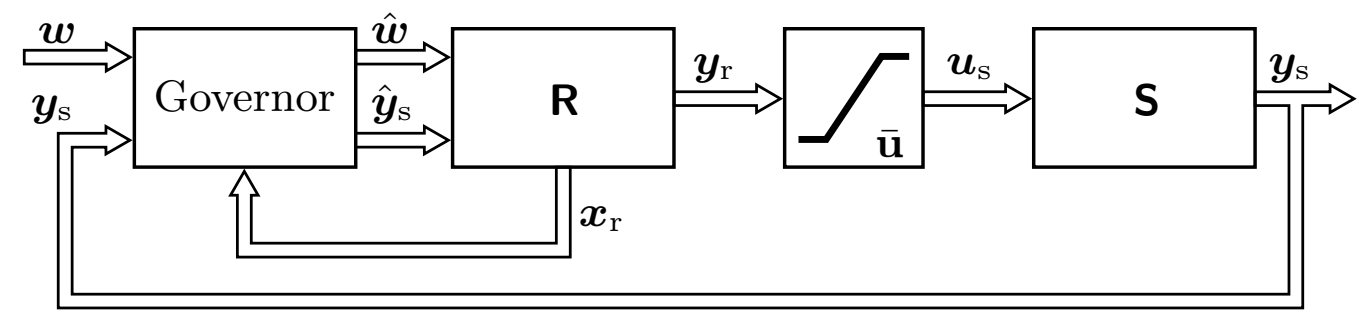

(b)

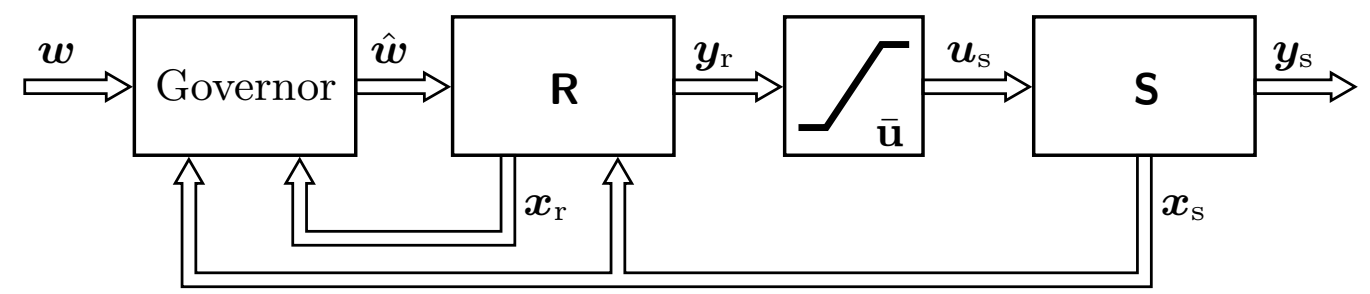

Abbildung 3.1: Governor-Ansätze. (a) Measurement Governor. (b) Reference oder Command Governor.

\subsubsection{Governor-Konzepte}

Governor-Konzepte basieren auf einem zweistufigen Ansatz. In einem ersten Schritt wird ein Regler ohne Berücksichtigung der Stellbegrenzungen entworfen. Danach wird unmittelbar vor dem Regler ein sogenannter Governor installiert. Dieses nichtlineare statische oder dynamische System modifiziert die Reglereingänge derart, dass ausschließlich Stellgrößen erzeugt werden, die vorhandene Stellbegrenzungen einhalten. Auch die Berücksichtigung von Zustandsbeschränkungen ist möglich. Die geeignete Modifikation der Reglereingänge erfordert allerdings ein nicht unerhebliches Maß an Rechenleistung.

Das Ziel von Governor-Ansätzen ist die konsequente Vermeidung der Sättigung. Wenn der Regelkreis ausschließlich im linearen Bereich der Sättigungskennlinie operiert, dann tritt nie Windup auf. Grob kann man zwei Varianten unterscheiden, den Measurement Governor in Abbildung 3.1 (a) und den Command Governor in Abbildung 3.1 (b).

Der Measurement Governor modifiziert das dem Regler zugeführte gemessene Ausgangssignal der Strecke $\boldsymbol{y}_{\mathrm{s}}$ und den Sollwert $\boldsymbol{w}$ derart, dass immer $\boldsymbol{y}_{\mathrm{r}}=\boldsymbol{u}_{\mathrm{s}}$ gilt. Erstmals wird dieses Konzept unter der Bezeichnung Error Governor in [102] vorgestellt, weil lediglich die Regelabweichung $\boldsymbol{e}=\boldsymbol{w}-\boldsymbol{y}_{\mathrm{s}}$ modifiziert wird. Eine weniger rechenaufwendige Variante für stabile zeitdiskrete Streckenmodelle findet sich in [62]. 
Der Command Governor [13, 30, 58-61, 168] modifiziert lediglich das Referenzsignal bzw. den Sollwert $\boldsymbol{w}$, benötigt aber eine Messung oder Schätzung des Streckenzustandes. Im Vergleich zum Measurement Governor können neben Stellgrößen- auch Zustandsbeschränkungen der Strecke berücksichtigt werden.

\subsection{Anti-Windup-Methoden}

Anti-Windup-Methoden basieren wie Governor ebenfalls auf einem zweiteiligen Ansatz. Sie werden in diesem Abschnitt kurz vorgestellt.

\subsubsection{Grundidee}

Steht der Begriff Windup für ein durch Stellgrößenbegrenzungen induziertes Fehlverhalten des Regelkreises, so bezeichnet man mit Anti-Windup spezielle strukturelle Erweiterungen des beschränkten Regelkreises aus Abbildung 2.2, die einem derartigen Fehlverhalten entgegenwirken. Charakteristisch ist, dass Anti-Windup-Maßnahmen erst aktiv werden, wenn Sättigung auftritt. Im nichtsättigenden Betrieb wird das Verhalten des Regelkreises allein durch den zuvor entworfenen Regler bestimmt.

Die Anti-Windup-Philosophie besteht demnach in einer Aufteilung des Regelkonzeptes: Zuerst wird unter Vernachlässigung der Sättigung ein Regler entworfen, der ein gutes Kleinsignalverhalten sicher stellt. Dann wird, wie in Abbildung 3.2 gezeigt, eine Anti-Windup-Strategie hinzugefügt, die im Sättigungsfall durch gezielte Beeinflussung des Reglers für Stabilität und eine zufriedenstellende Regelgüte sorgt. Allerdings gelten auch hier die in Abschnitt 3.1 beschriebenen Einschränkungen bezüglich der Stabilität des geschlossenen Regelkreises.

Der etwas aufwendigere zweistufige Entwurf eines solchen Regelkonzeptes wird von einigen vorteilhaften Eigenschaften begleitet, die ausschlaggebend für die Popularität von Anti-Windup im industriellen Umfeld sind. Besonders hervorzuheben ist die Tatsache, dass bereits entworfene Regler, die ein gutes Kleinsignalverhalten aufweisen, nachträglich um ein AntiWindup erweitert werden können. So ist ein sättigender Betrieb möglich, ohne einen komplett neuen Reglerentwurf durchführen zu müssen. Ein solches Retrofitting ${ }^{1}$ lohnt sich besonders dann, wenn viel Know-How bzw. Expertenwissen in den Reglerentwurf eingeflossen ist und Sättigungsprobleme nur gelegentlich auftreten.

\footnotetext{
${ }^{1}$ engl. Nachrüstung
} 


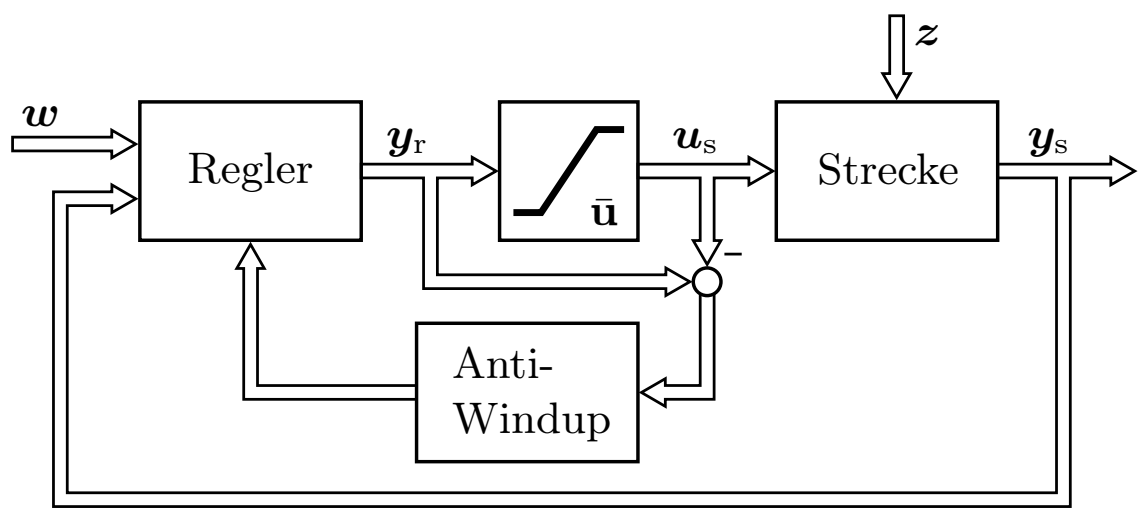

Abbildung 3.2: Allgemeine Anti-Windup-Architektur. Das Anti-Windup wird erstmalig aktiv, wenn Sättigung auftritt, d. h. für $\boldsymbol{y}_{\mathrm{r}} \neq \boldsymbol{u}_{\mathrm{s}}$. Im nichtsättigenden Betrieb bestimmt allein der Regler das Verhalten des Regelkreises.

\subsubsection{Historische Entwicklung}

Die Entwicklung von Anti-Windup lässt sich in vier Phasen einteilen, die in Abbildung 3.3 illustriert sind und nachfolgend kurz charakterisiert werden. Die Darstellung orientiert sich grob an [211], ergänzt diese aber um wesentliche Aspekte, wie z. B. das modulare Anti-Windup.

\section{Erste Phase: klassisches Anti-Windup}

Die erste Phase beginnt in den 1930er Jahren und ist vor allem durch die Entdeckung des Windup-Problems in der Industrie geprägt [183]. Die elektrisch, mechanisch, pneumatisch oder hydraulisch realisierten PI-Regler führen in Regelkreisen mit Stellbegrenzungen zu dem in Abschnitt 2.2.1 beschriebenen Integral-Windup und der damit verbundenen Abnahme der Regelgüte. Neben dem Problembewusstsein entstehen auch erste Ad-hocLösungen, die aber unveröffentlicht bleiben [183, 211].

Frühe Publikationen. Um 1950 kommt es zu ersten Veröffentlichungen. Anti-Windup-Maßnahmen sind zunächst eng mit der Realisierung der Regler verknüpft und folglich nur dann realisierbar, wenn sie konstruktiv in den gerätetechnischen Aufbau des Reglers integrierbar sind. Die Hardware limitiert die Möglichkeiten und führt zu Speziallösungen.

So beschreibt Geest in [57] einen pneumatischen PI-Regler, der durch Überdruckventile ein Hochlaufen des I-Anteils verhindert. Ähnliche Ideen erläutert Toop in [189]. Eine Anti-Windup-Methode von Clarridge [35] basiert auf einer speziellen Realisierung von PID-Reglern. Das Prinzip ist in Abbildung 3.4 illustriert und wird auch von Oppelt in [146] erläutert. 


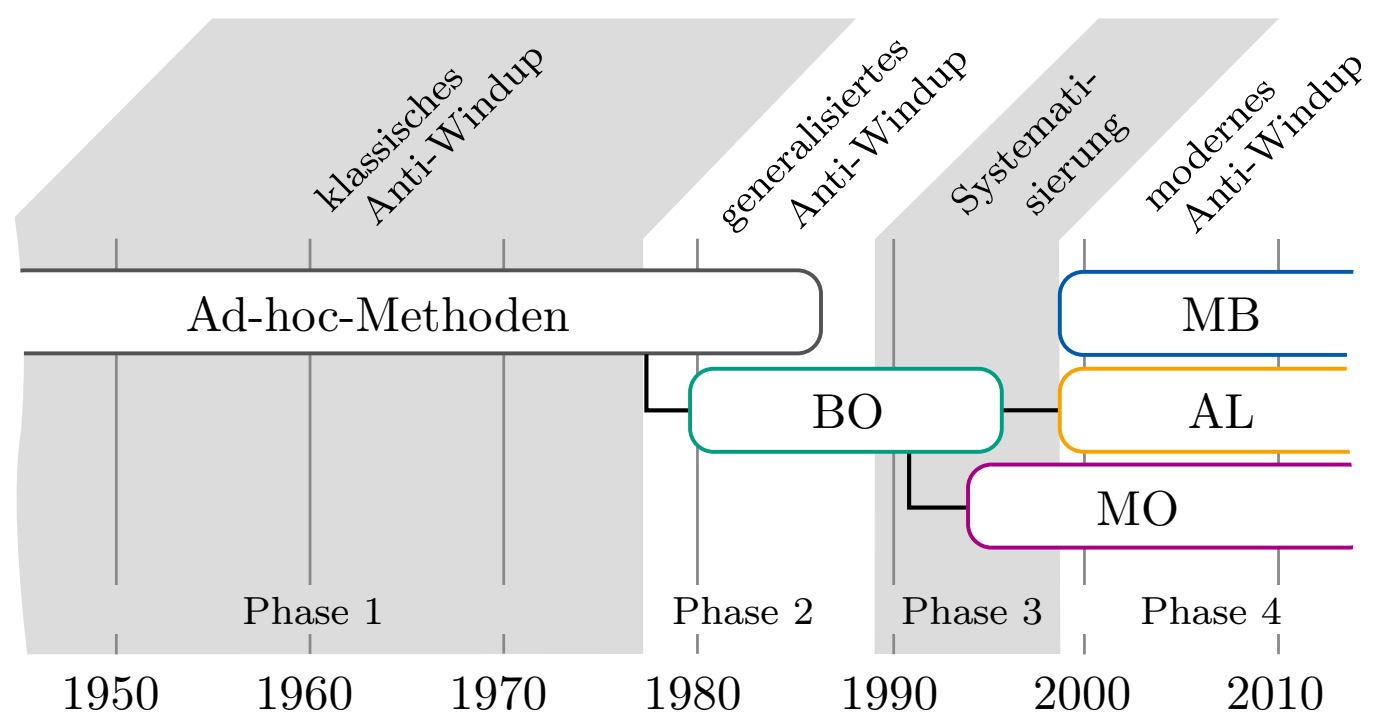

Abbildung 3.3: Historische Entwicklung von Anti-Windup. Nach den Ad-hocMethoden entwickelt sich Anfang der 1980er Jahre das beobachterbasierte (BO) Anti-Windup. Später entstehen modulares (MO), modellbasiertes (MB) und allgemeines lineares (AL) Anti-Windup.

Piwinger publiziert im Jahr 1962 eine Anti-Windup-Modifikation eines pneumatischen Reglers, die den problematischen I-Anteil bei großen Regelabweichungen deaktiviert und später wieder zuschaltet [157]. Ähnliche Ansätze verfolgen die Arbeiten [145, 172] für analog realisierte PID-Regler. Zusätzlich lässt sich der Integriererzustand vor dem Zuschalten manuell auf einen bestimmten Wert setzen. Abbildung 3.5 zeigt die unter der Bezeichnung Conditional Integration bekannte Anti-Windup-Strategie. Eine vergleichende Untersuchung ausgewählter Anti-Windup-Verfahren veröffentlicht Kollmann in einem Übersichtsartikel [111]. Auch [66] enthält verschiedene Ad-hoc-Methoden aus dieser Phase.

Theoretische Untersuchungen der Windup-Phänomene am Beispiel eines geregelten Servomotors werden schon 1956 von Lozier beschrieben [131]. Bezüglich der Reglerauslegung kommt Lozier zu dem Schluss, dass es nicht sinnvoll oder oft nicht möglich ist, ein gutes Kleinsignalverhalten aufzugeben, um Sättigung bei größeren Sollwertsprüngen oder Störungen zu vermeiden. Vielmehr sind spezielle Gegenmaßnahmen zu entwickeln, um die Windup-Problematik zu beheben. Das ist der zentrale Punkt der AntiWindup-Philosophie.

Neue Möglichkeiten durch digitale Regler. Die Arbeit von Fertik und Ross [45] legt 1967 mit der in Abbildung 3.6 gezeigten Back Calculation Methode den Grundstein für Anti-Windup bei digital reali- 


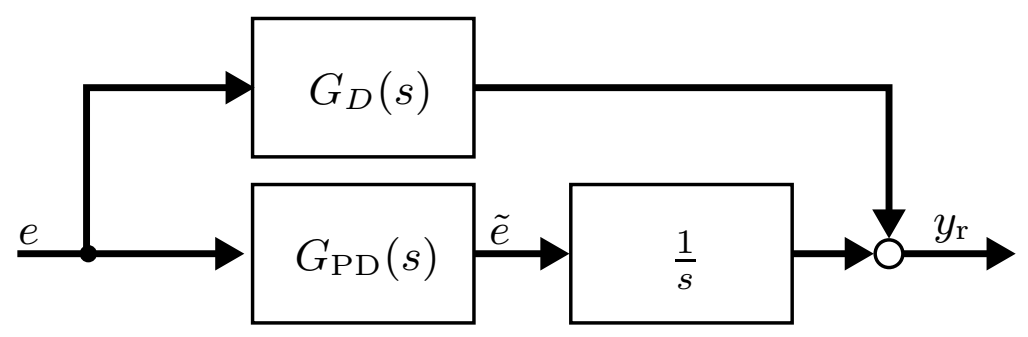

Abbildung 3.4: Spezielle Realisierung des PID-Reglers mit PD-Glied vor dem I-Anteil. Im Sättigungsfall integriert der I-Anteil dann nicht die Regelabweichung $e$ auf, sondern den Ausgang des PD-Gliedes $\tilde{e}=K_{i} e+K_{p} \dot{e}$. Das Aufwickeln des Integrierers endet, wenn $\tilde{e}$ das Vorzeichen wechselt, was aufgrund des Terms $K_{p} \dot{e}$ früher passiert als der Vorzeichenwechsel von $e$ [146, S. 523].

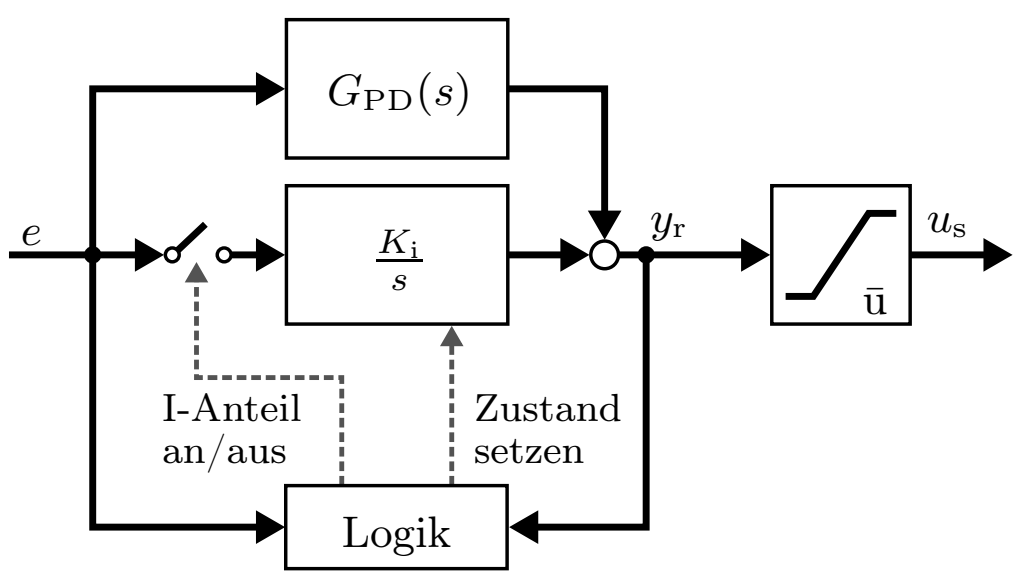

Abbildung 3.5: Conditional Integration. Der Integrierer wird nur dann zugeschaltet, wenn bestimmte Bedingungen erfüllt sind, z. B. wenn ein kleiner Regelfehler $e$ vorliegt und der Reglerausgang $y_{\mathrm{r}}$ innerhalb der Sättigungsgrenzen liegt. Zur Verbesserung der Regelgüte kann der Integriererzustand vor dem Zuschalten auf einen bestimmten Wert vorinitialisiert werden. 


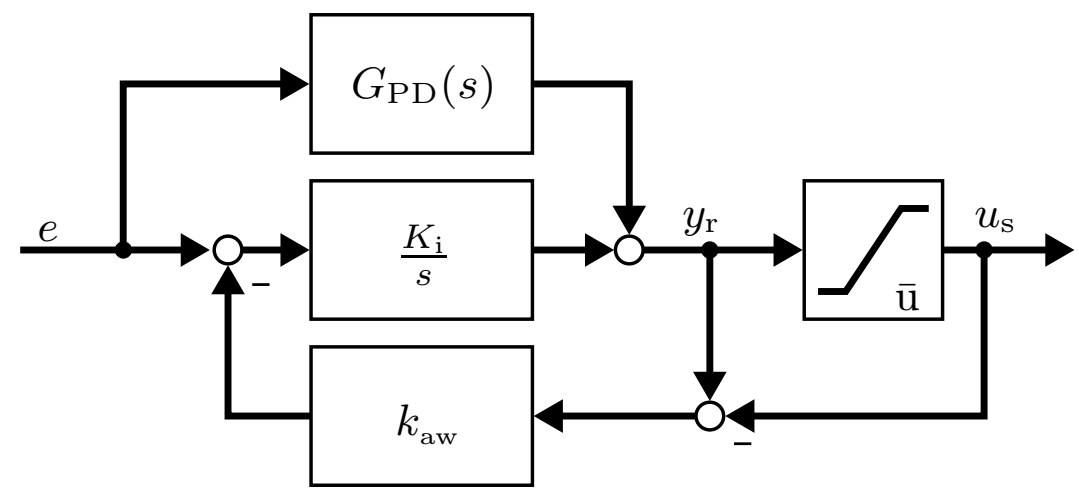

\begin{abstract}
Abbildung 3.6: Back Calculation. Die Grundidee ist, ein Aufwickeln des IAnteils durch die Rückkopplung der Differenz aus gesättigter und ungesättigter Stellgröße zu vermeiden. Liegt keine Sättigung vor, so ist die Rückführung inaktiv. Im Sättigungsfall wird der Integriererzustand durch die negative Rückkopplung über die Verstärkung $k_{\text {aw }}$ begrenzt und kann sich nicht aufwickeln.
\end{abstract}

sierten PID-Reglern. In [108] wird dieses digitale Anti-Windup zusammen mit anderen Ad-hoc-Methoden experimentell an einem chemischen Prozess evaluiert. Die Experimente zeigen, dass viele Ad-hoc-Techniken nur unter bestimmten Bedingungen gut funktionieren. Komplexere AntiWindup-Maßnahmen sind nötig, um unter möglichst vielen Bedingungen eine zufriedenstellende Regelgüte zu erreichen.

Kramer beschreibt in [115] ein Anti-Windup-Verfahren für digitale PIDRegler, bei dem der Integratorzustand optimal im Sinne eines Gütemaßes initialisiert wird. Letzteres ist nur deshalb möglich, weil das Regelgesetz im Speicher eines Rechners hinterlegt ist. Dieser flexibel beschreibbare Speicher und die vorhandene Rechenkapazität eröffnen neue Möglichkeiten für das Design von komplexeren Anti-Windup-Maßnahmen.

\title{
Zweite Phase: generalisiertes Anti-Windup
}

Kennzeichnend für die zweite Phase ist die Ausweitung von Anti-Windup auf eine breitere Klasse von Reglern. Zum einen werden bestehende Adhoc-Lösungen für PID-Regler auf PI-Zustandsregler [116, 117] und MIMORegler mit I-Anteil [101] übertragen, zum anderen entstehen die beobachterbasierten (BO) Anti-Windup-Methoden [8, 76-78, 82, 144, 207] für allgemeine lineare Regler. Auch erste Stabilitätsanalysen für Regelkreise mit Anti-Windup auf Basis grafischer Stabilitätskriterien, wie dem Kreis- oder Popov-Kriterium [3, 164, 212], finden sich in der Literatur [63-65, 67]. 
Die Grundlage für beobachterbasiertes Anti-Windup legt Hanus bereits 1980 mit der sogenannten Conditioning Technique [76]. Er interpretiert Windup als eine Inkonsistenz zwischen den Reglerzuständen $\boldsymbol{x}_{\mathrm{r}}$ und dem Aktorausgang bzw. Streckeneingang $\boldsymbol{u}_{\mathrm{s}}=\mathbf{s a t}_{\overline{\mathbf{u}}}\left(\boldsymbol{y}_{\mathrm{r}}\right)$ aufgrund der Stellbegrenzung. Die Lösung des Problems besteht darin, die Reglerzustände im Sättigungsfall in Übereinstimmung mit dem Streckeneingang zu bringen. Damit überträgt Hanus das Prinzip der Ad-hoc-Methoden für PID-Regler auf allgemeinere Reglerstrukturen.

Ähnliche aber weitreichendere Ergebnisse veröffentlichen Åström und Wittenmark in [8]. Der Kerngedanke ist, allgemeine lineare Regler als (reduzierte) Kontrollbeobachter aufzufassen und Windup als durch Stellbegrenzungen induzierte Beobachterfehler zu interpretieren. Wie auch von Wurmthaler [205] und Hippe [82] erläutert, lassen sich Beobachterfehler einfach vermeiden und das Windup-Problem lösen, indem

,[...] man dem Beobachter die Information über das tatsächliche

- nämlich begrenzte - Streckeneingangssignal zuführt."

Der Beobachter erhält somit $\operatorname{sat}_{\overline{\mathbf{u}}}\left(\boldsymbol{y}_{\mathrm{r}}\right)$ anstelle von $\boldsymbol{y}_{\mathrm{r}}$. Es zeigt sich später, dass die Conditioning Technique von Hanus und viele andere Verfahren [77, 78, 82, 144, 207] Spezialfälle der Beobachtermethode von Åström sind, die sich durch die Beobachterdynamik voneinander unterscheiden $[7,196]$. Als wichtigstes Ergebnis dieser Phase bleibt festzuhalten, dass sich die durch den Regler verursachten Windup-Probleme vollständig mit der Beobachtermethode, die auch als generalized anti-windup (GAW) bezeichnet wird, beheben lassen. Abschnitt 4.5.1 behandelt Details dieses Ansatzes.

\section{Dritte Phase: Systematisierung}

Die dritte Phase ist geprägt von zwei Entwicklungen. Zum einen erfolgt eine systematische Aufarbeitung der bisher veröffentlichten Verfahren zur Vermeidung von Regler-Windup. Dabei entstehen die Übersichtsartikel [7, 20, 41, 155, 201]. Die Arbeiten [28, 112, 196] geben ebenfalls einen Überblick und verdeutlichen, dass vielen bisher veröffentlichten Anti-WindupMethoden eine gemeinsame Struktur innewohnt. Diese Erkenntnis lässt sich beispielsweise zur Stabilitätsanalyse nutzen [114].

Zum anderen zeigt sich, dass eine Vermeidung des Regler-Windup nicht immer ausreicht, um eine akzeptable Regelgüte im Sättigungsfall zu erzielen. Die häufig durch das Regler-Windup überdeckten Effekte, das sogenannte Strecken-Windup [89] und die Direktionalitätsproblematik in MIMO-Regelkreisen [40], rücken in den Fokus der Forschung. 
Strecken-Windup. Erste Methoden zur Eliminierung von StreckenWindup finden sich in $[88,90,160,195,206]$. Die Grundidee besteht in der temporären Reduzierung des Sollwertes bzw. des Reglereinganges im Sättigungsfall, was durch ein zusätzliches dynamisches Element im Regelkreis realisiert und in [160, 195] als filtered setpoint bezeichnet wird. Die temporäre Sollwertreduzierung führt zu kleineren Stellgrößen, das Anwachsen der Streckenzustände wird limitiert und Strecken-Windup vermieden.

Eine charakteristische Eigenschaft der oben angeführten Methoden ist die separate Behandlung von Regler- und Strecken-Windup durch unterschiedliche Modifikationen des Regelkreises. Dieses Prinzip wird später von Hippe systematisiert und um Lösungen für das Direktionalitätsproblem ergänzt [85]. Deshalb wird dieser Ansatz im Weiteren als modulares Anti-Windup bezeichnet. Details finden sich in Abschnitt 4.5.

Direktionalitätsproblematik. Auf das Problem der Direktionalität in MIMO-Regelkreisen mit Stellbegrenzung macht Doyle bereits 1987 in [40] aufmerksam:

,[...] the saturation may cause a change in the direction of the plant input resulting in disastrous consequences."

Wie in Abschnitt 2.2.2 erläutert, kann es zu unerwünschten Effekten in MIMO-Regelkreisen kommen, wenn eine Komponente der Stellgröße in Sättigung geht und somit die Richtung des Stellgrößenvektors ändert. Erste systematische Lösungsansätze bieten [32, 135, 156, 197, 213]. Die gemeinsame Grundidee besteht darin, die Anti-Windup-Maßnahme optimal im Sinne eines Gütekriteriums zu entwerfen. Durch Lösen eines Optimierungsproblems kann so die Richtungsabweichung des Stellgrößenvektors im Vergleich zum unbeschränkten Regelkreis minimiert werden. Die Formulierung des Entwurfs als Optimierungsproblem stellt einen wichtigen Schritt auf dem Weg zu modernen Anti-Windup-Methoden dar.

\section{Vierte Phase: modernes Anti-Windup}

Kennzeichnend für die vierte Phase ist die Entwicklung von systematischen Anti-Windup-Maßnahmen, die Stabilität und eine optimale Regelgüte garantieren. Dieser Trend wird durch die Verfügbarkeit von effizienten Lösungsalgorithmen für konvexe Optimierungsprobleme mit Nebenbedingungen in Form linearer Matrixungleichungen begünstigt [180, 188]. Aufgrund der fundamentalen Bedeutung der konvexen Optimierung für moderne Anti-Windup-Entwurfsverfahren findet sich eine kurze Einführung in die Thematik im Anhang B dieser Arbeit. 
Erste Arbeiten, die beim Anti-Windup-Entwurf die Norm einer Übertragungsmatrix minimieren, sind [36, 41, 42, 113, 138]. Richtungsweisend ist die Arbeit von Teel und Kapoor [185] aus dem Jahr 1997, der folgendes Konzept zugrunde liegt: Die Verhaltensabweichung des Regelkreises mit Anti-Windup von dem unbeschränkten Regelkreis aus Abbildung 2.1 wird über die $L_{2}$-Norm, die ein Maß für die Energie eines Signals ist, formal quantifiziert. Das Anti-Windup lässt sich dann durch Lösen eines Optimierungsproblems so entwerfen, dass diese Verhaltensabweichung im energetischen Sinne minimiert wird. Ähnliche Ideen finden sich in der Arbeit von Weston und Postlethwaite [199].

Die Anti-Windup-Ansätze dieser Phase lassen sich in drei Kategorien einteilen: modellbasiertes Anti-Windup (MB), allgemeines lineares Anti-Windup (AL) und modulares Anti-Windup (MO). Die Arbeiten $[55,182,183,211]$ geben einen Überblick über die ersten beiden Methoden. Eine Gemeinsamkeit dieser Ansätze ist, dass im Gegensatz zum modularen Anti-Windup keine Unterteilung der Windup-Effekte in Reglerund Strecken-Windup sowie das Direktionalitätsproblem erfolgt. Das AntiWindup wird durch Lösen eines Optimierungsproblems so parametriert, dass eine optimale Regelgüte erreicht wird. Details zu den drei modernen Anti-Windup-Konzepten finden sich in Kapitel 4.

\subsubsection{Komplexität und Leistungsfähigkeit}

Wie in den letzten Abschnitten erläutert, haben sich Anti-Windup-Strategien von simplen Ad-hoc-Methoden über generalisierte Anti-WindupVerfahren hin zu modernen systematischen Erweiterungen des beschränkten Regelkreises entwickelt. Der zu zahlende Preis für die steigende Leistungsfähigkeit der Anti-Windup-Methoden ist eine komplexere Struktur. Tabelle 3.1 grenzt die Entwicklungsstufen voneinander ab.

Nach [108, 111] können die klassischen Anti-Windup-Verfahren nur in bestimmten Situationen das Windup verhindern. In vielen Fällen lässt sich keine zufriedenstellende Regelgüte erreichen, was die Entwicklung von komplexeren Anti-Windup-Methoden notwendig machte. Das wird am Beispiel des hydraulischen Systems aus Abschnitt 2.2.3 demonstriert.

Das hydraulische Positioniersystem ist durch die Zustandsraumdarstellung (2.14) gegeben. Der Regler (2.15) garantiert ein gutes Ausregelverhalten im unbeschränkten Fall. Wie in Abschnitt 2.2.3 gezeigt, führt eine Begrenzung der Stellamplitude auf $\overline{\mathrm{u}}=10,5 \mathrm{~V}$ zu Windup. Ein starkes Überschwingen gepaart mit einem oszillierenden Verhalten ist die Folge. 
Tabelle 3.1: Entwicklungsstufen von Anti-Windup.

\begin{tabular}{lccc}
\hline & klassisch & generalisiert & modern \\
\hline Reglertyp & PID & linear & (nicht)linear \\
Stabilitätsgarantie & - & $(\checkmark)$ & $\checkmark$ \\
Regelgütegarantie & - & - & $(\checkmark)$ \\
Komplexität & niedrig & mittel & mittel - hoch \\
\hline
\end{tabular}

Zur Untersuchung der mit klassischen Anti-Windup-Methoden erzielbaren Regelgüte wird der Regler (2.15) in zwei Teile zerlegt. Es ergibt sich

$$
\begin{aligned}
y_{\mathrm{r}}(s) & =\frac{17,764(s+0,731)}{s} \cdot \frac{\left(s^{2}+1,167 s+10\right)}{(s+5)(s+100)}\left(w(s)-y_{\mathrm{s}}(s)\right) \\
& =\left(17,764+12,985 \frac{1}{s}\right) \cdot \frac{\left(s^{2}+1,167 s+10\right)}{(s+5)(s+100)} e(s) .
\end{aligned}
$$

Dabei ist der erste Teil der Übertragungsfunktion ein PI-Regler mit $K_{\mathrm{p}}=17,764$ sowie $K_{\mathrm{i}}=12,985$ und der zweite ein lineares Filter mit zwei Polen und Nullstellen. Die Ad-hoc-Methoden zur Vermeidung von Integral-Windup aus der ersten Entwicklungsphase lassen sich nun auf den PI-Regler anwenden. Untersucht werden zwei typische und häufig eingesetzte Anti-Windup-Strategien:

- Die Conditional Integration Methode aus Abbildung 3.5. Dabei wird der Integrierer des PI-Reglers bei $\pm 10,5$ angehalten.

- Die Back Calculation Methode aus Abbildung 3.6. Der Anti-WindupParameter $k_{\text {aw }}$ wird durch iteratives Ausprobieren und Simulieren zu $k_{\text {aw }}=7,26$ bestimmt. Als Gütemaß kommt dabei das Integral der quadrierten Regelabweichung $\int_{0}^{\infty} e^{2}(t) d t$ zum Einsatz. Dieses Gütemaß ist in Abschnitt A.3.4 für verschiedene $k_{\text {aw }}$ dargestellt.

Abbildung 3.7 zeigt die Simulationsergebnisse für einen Sollwert von $w=20 \mathrm{~cm}$. Zum Vergleich sind auch die Systemantworten des unbeschränkten und des beschränkten Regelkreises ohne Anti-Windup dargestellt. Als Ergebnis bleibt festzuhalten: Die beiden untersuchten AntiWindup-Methoden können das initiale Überschwingen reduzieren, eine zufriedenstellende Regelgüte wird aber von keinem Verfahren erreicht. Um 

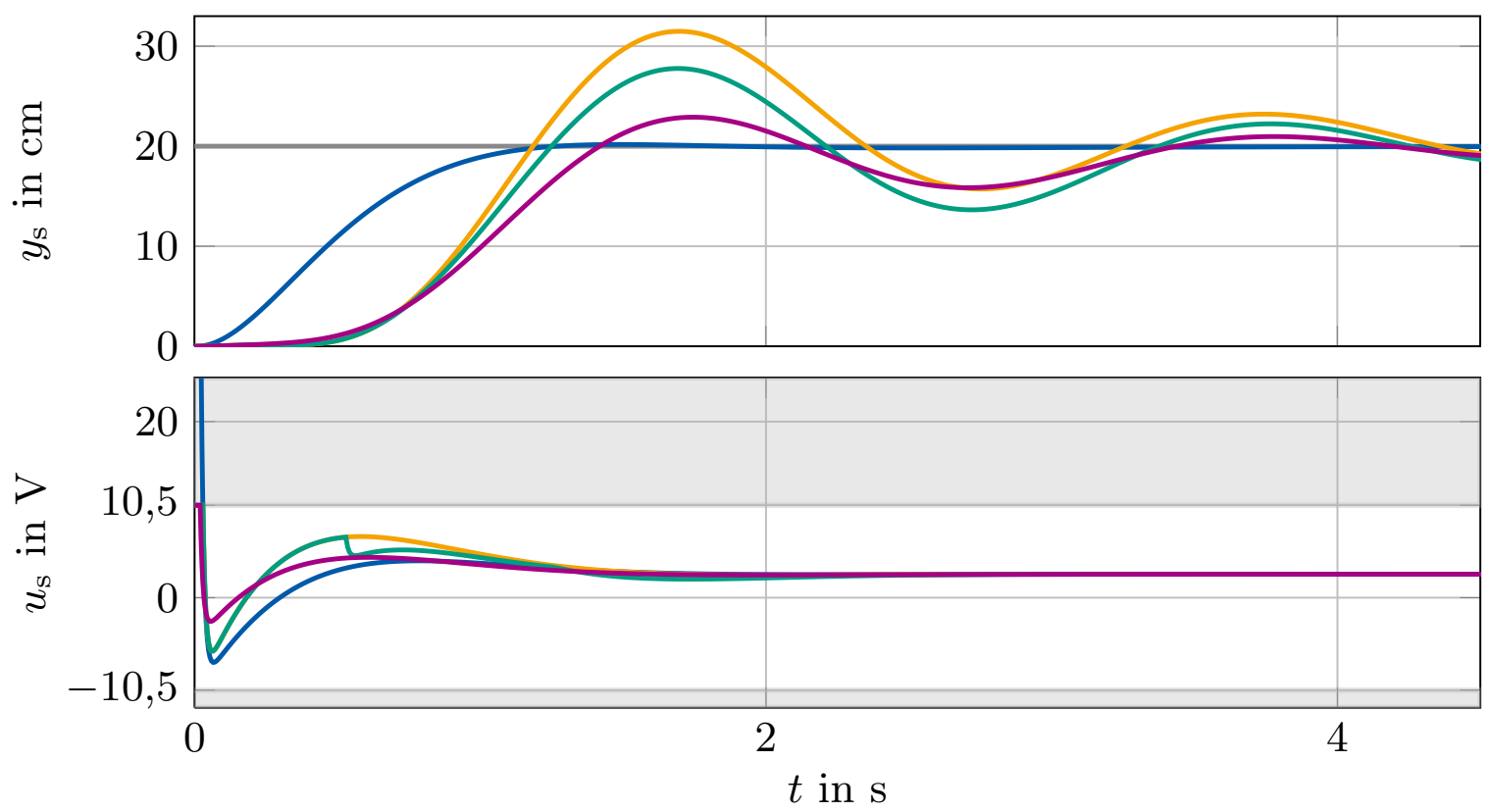

Abbildung 3.7: Simulation des hydraulischen Positioniersystems. Dargestellt sind die Systemantworten sowie Stellgrößen des unbeschränkten (-) und des beschränkten Regelkreises (-). Der Sollwert (-) ist $w=20 \mathrm{~cm}$. Bekannte klassische Anti-Windup-Methoden wie Conditional Integration (-) und Back Calculation (-) reduzieren das initiale Überschwingen, erreichen aber keine zufriedenstellende Regelgüte.

ein akzeptables Ergebnis zu erzielen ist eine systematische Herangehensweise an das Windup-Problem nötig, womit sich Kapitel 4 beschäftigt. Dass sich der Aufwand lohnt, wird in Kapitel 5 bei der erneuten Betrachtung des Beispiels deutlich werden.

\subsection{Zusammenfassung und Fazit}

In diesem Kapitel wurde gezeigt, welche Schwierigkeiten sich bei der Stabilisierung von Systemen mit Eingangsbeschränkung ergeben. Des Weiteren wurden verschiedene Konzepte zur Regelung von Strecken mit Stellbegrenzungen vorgestellt. Der Fokus lag dabei auf Anti-Windup-Verfahren und deren historischer Entwicklung von simplen Ad-hoc-Maßnahmen zu komplexeren Methoden. Diese Entwicklung war vor allem dadurch motiviert, dass die klassischen Anti-Windup-Verfahren bei komplexeren Reglern keine zufriedenstellende Regelgüte erreichen, was sich am Beispiel des schon zuvor betrachteten Positioniersystems demonstrieren ließ. 


\section{Modernes Anti-Windup: Grundlagen}

In diesem Kapitel werden zunächst der Anti-Windup-Regelkreis und die Anti-Windup-Ziele definiert. Danach folgt eine kurze Vorstellung der drei wichtigsten modernen Anti-Windup-Konzepte: allgemeines lineares AntiWindup, modellbasiertes Anti-Windup und modulares Anti-Windup.

\subsection{Definition des Anti-Windup-Regelkreises}

Um die durch Stellbegrenzungen ausgelösten Windup-Effekte zu minimieren, wird der beschränkte Regelkreis gemäß Abbildung 4.1 um die AntiWindup-Struktur AW erweitert [55, 182, 183, 211]. Diese ist durch

$$
\text { AW : }\left\{\begin{array}{c}
\dot{\boldsymbol{x}}_{\mathrm{aw}}=\boldsymbol{f}_{\mathrm{aw}}\left(\boldsymbol{x}_{\mathrm{aw}}, \boldsymbol{\delta}\right), \\
\boldsymbol{y}_{\mathrm{aw}, \mathrm{x}}=\boldsymbol{h}_{\mathrm{aw}, \mathrm{x}}\left(\boldsymbol{x}_{\mathrm{aw}}, \boldsymbol{\delta}\right), \\
\boldsymbol{y}_{\mathrm{aw}, \mathrm{y}}=\boldsymbol{h}_{\mathrm{aw}, \mathrm{y}}\left(\boldsymbol{x}_{\mathrm{aw}}, \boldsymbol{\delta}\right)
\end{array}\right.
$$

gegeben, mit dem Zustand $\boldsymbol{x}_{\text {aw }} \in \mathbb{R}^{n_{\text {aw }}}$ und dem Eingang $\boldsymbol{\delta} \in \mathbb{R}^{m}$. Letzterer beschreibt das Ausmaß der Abweichung zwischen Aktorein- und Aktorausgang. Im Weiteren wird dieses Ausmaß auch als Sättigungsgrad bezeichnet und wie folgt definiert

$$
\boldsymbol{\delta}=\boldsymbol{u}_{\mathrm{a}}-\boldsymbol{y}_{\mathrm{a}}=\boldsymbol{u}_{\mathrm{a}}-\operatorname{sat}_{\overline{\mathbf{u}}}\left(\boldsymbol{u}_{\mathrm{a}}\right) .
$$

Die Ausgänge $\boldsymbol{y}_{\mathrm{aw}, \mathrm{x}} \in \mathbb{R}^{n_{\mathrm{r}}}$ und $\boldsymbol{y}_{\mathrm{aw}, \mathrm{y}} \in \mathbb{R}^{m}$ beeinflussen die Reglerzustände bzw. den Reglerausgang. Der Aktoreingang $\boldsymbol{u}_{\mathrm{a}}$ ist dann durch folgende Dynamik bestimmt

$$
\begin{aligned}
\dot{\boldsymbol{x}}_{\mathrm{r}} & =\boldsymbol{A}_{\mathrm{r}} \boldsymbol{x}_{\mathrm{r}}+\boldsymbol{B}_{\mathrm{r}, u} \boldsymbol{u}_{\mathrm{r}}+\boldsymbol{B}_{\mathrm{r}, w} \boldsymbol{w}+\boldsymbol{y}_{\mathrm{aw}, \mathrm{x}} \\
\boldsymbol{y}_{\mathrm{r}} & =\boldsymbol{C}_{\mathrm{r}} \boldsymbol{x}_{\mathrm{r}}+\boldsymbol{D}_{\mathrm{r}, u} \boldsymbol{u}_{\mathrm{r}}+\boldsymbol{D}_{\mathrm{r}, w} \boldsymbol{w} \\
\boldsymbol{u}_{\mathrm{a}} & =\boldsymbol{y}_{\mathrm{r}}+\boldsymbol{y}_{\mathrm{aw}, \mathrm{y}}
\end{aligned}
$$

Beim Betrachten der Differentialgleichung wird deutlich, dass der Ausgang $\boldsymbol{y}_{\mathrm{aw}, \mathrm{x}}$ des Anti-Windup die Änderung der Reglerzustände direkt und 


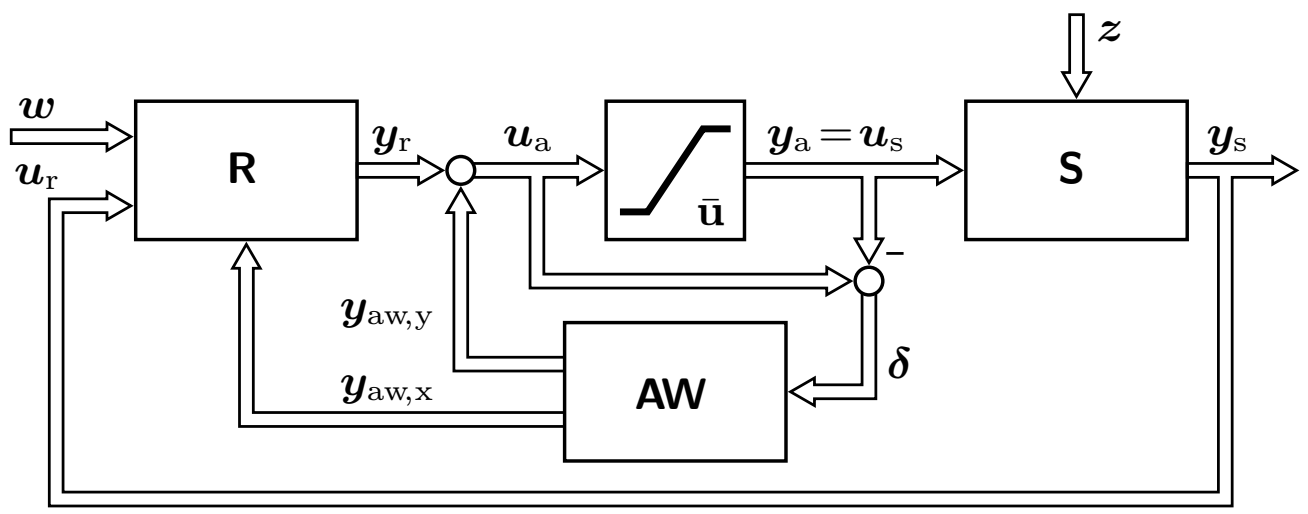

Abbildung 4.1: Allgemeine Anti-Windup-Struktur. Die Differenz aus Eingang und Ausgang des Aktors bildet den Eingang $\boldsymbol{\delta}$ des Anti-Windup. Die Ausgänge $\boldsymbol{y}_{\mathrm{aw}, \mathrm{x}}$ und $\boldsymbol{y}_{\mathrm{aw}, \mathrm{y}}$ beeinflussen die Reglerzustände bzw. den Reglerausgang.

unabhängig voneinander beeinflussen kann. Aufgrund dieser sehr engen Einbettung der Anti-Windup-Strategie in die Reglerdynamik wird im weiteren Verlauf dieser Arbeit auch der Begriff integriertes Anti-Windup verwendet $^{1}$. Die Dynamik des in Abbildung 4.1 dargestellten Anti-WindupRegelkreises ergibt sich mit $\boldsymbol{\delta}=\boldsymbol{u}_{\mathrm{a}}-\mathbf{s a t}_{\overline{\mathbf{u}}}\left(\boldsymbol{u}_{\mathrm{a}}\right)$ zu

$$
\begin{aligned}
\dot{\boldsymbol{x}}_{\mathrm{s}} & =\boldsymbol{A}_{\mathrm{s}} \boldsymbol{x}_{\mathrm{s}}+\boldsymbol{B}_{\mathrm{s}, u} \operatorname{sat}_{\overline{\mathbf{u}}}\left(\boldsymbol{u}_{\mathrm{a}}\right)+\boldsymbol{B}_{\mathrm{s}, z} \boldsymbol{z}, \\
\dot{\boldsymbol{x}}_{\mathrm{r}} & =\boldsymbol{A}_{\mathrm{r}} \boldsymbol{x}_{\mathrm{r}}+\boldsymbol{B}_{\mathrm{r}, u} \boldsymbol{y}_{\mathrm{s}}+\boldsymbol{B}_{\mathrm{r}, w} \boldsymbol{w}+\boldsymbol{h}_{\mathrm{aw}, \mathrm{x}}\left(\boldsymbol{x}_{\mathrm{aw}}, \boldsymbol{\delta}\right), \\
\dot{\boldsymbol{x}}_{\mathrm{aw}} & =\boldsymbol{f}_{\mathrm{aw}}\left(\boldsymbol{x}_{\mathrm{aw}}, \boldsymbol{\delta}\right), \\
\boldsymbol{y}_{\mathrm{s}} & =\boldsymbol{C}_{\mathrm{s}} \boldsymbol{x}_{\mathrm{s}} \\
\boldsymbol{u}_{\mathrm{a}} & =\boldsymbol{C}_{\mathrm{r}} \boldsymbol{x}_{\mathrm{r}}+\boldsymbol{D}_{\mathrm{r}, u} \boldsymbol{y}_{\mathrm{s}}+\boldsymbol{D}_{\mathrm{r}, w} \boldsymbol{w}+\boldsymbol{h}_{\mathrm{aw}, \mathrm{y}}\left(\boldsymbol{x}_{\mathrm{aw}}, \boldsymbol{\delta}\right) .
\end{aligned}
$$

\section{Spezialfall: externes Anti-Windup}

Falls die Reglerzustände nicht direkt für das Anti-Windup zugänglich sind, kann eine integrierte Anti-Windup-Maßnahme nicht verwendet werden. Die Beeinflussung der Reglerzustände muss dann über den Reglereingang $\boldsymbol{u}_{\mathrm{r}}$ erfolgen. Dazu wird eine externe Anti-Windup-Strategie ${ }^{2}$

$$
\mathbf{A W}:\left\{\begin{array}{c}
\dot{\boldsymbol{x}}_{\mathrm{aw}}=\boldsymbol{f}_{\mathrm{aw}}\left(\boldsymbol{x}_{\mathrm{aw}}, \boldsymbol{\delta}\right), \\
\boldsymbol{y}_{\mathrm{aw}, \mathrm{u}}=-\boldsymbol{h}_{\mathrm{aw}, \mathrm{u}}\left(\boldsymbol{x}_{\mathrm{aw}}, \boldsymbol{\delta}\right), \\
\boldsymbol{y}_{\mathrm{aw}, \mathrm{y}}=\boldsymbol{h}_{\mathrm{aw}, \mathrm{y}}\left(\boldsymbol{x}_{\mathrm{aw}}, \boldsymbol{\delta}\right)
\end{array}\right.
$$

\footnotetext{
${ }^{1}$ In der englischsprachigen Literatur: full-authority anti-windup.

${ }^{2}$ Das negative Vorzeichen von $\boldsymbol{h}_{\mathrm{aw}, \mathrm{u}}$ vereinfacht im Weiteren die Notation.
} 


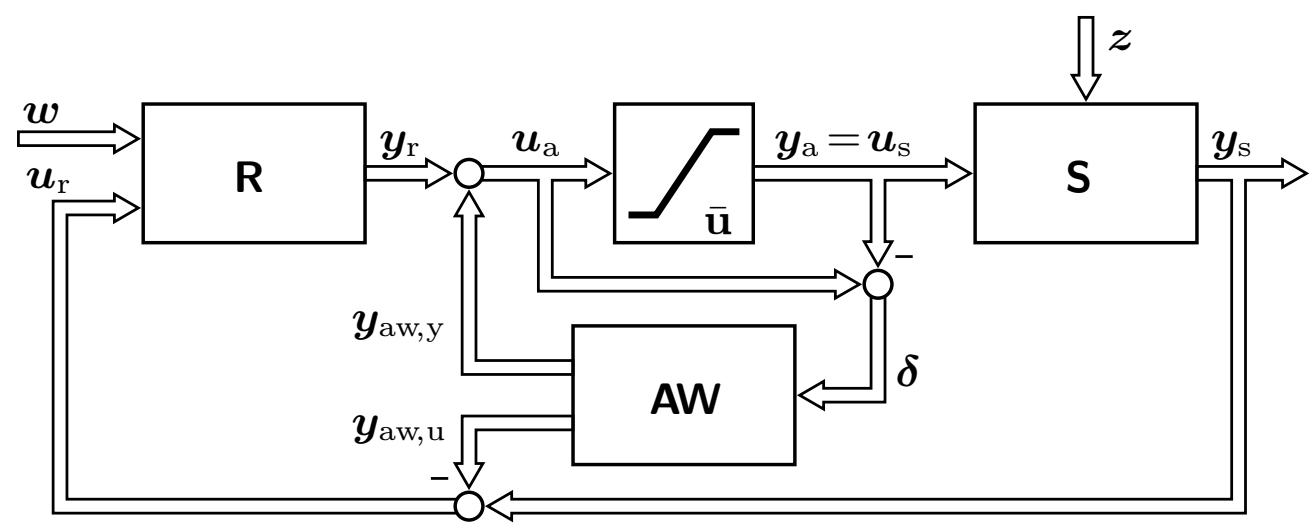

Abbildung 4.2: Externe Anti-Windup-Struktur. Die Ausgänge $\boldsymbol{y}_{\text {aw, u }}$ und $\boldsymbol{y}_{\text {aw,y }}$ beeinflussen den Reglereingang und den Reglerausgang.

mit dem Ausgang $\boldsymbol{y}_{\mathrm{aw}, \mathrm{u}} \in \mathbb{R}^{m}$ zur Beeinflussung des Reglereinganges eingesetzt. Die Verschaltung

$$
\boldsymbol{u}_{\mathrm{r}}=\boldsymbol{y}_{\mathrm{s}}-\boldsymbol{y}_{\mathrm{aw}, \mathrm{u}}
$$

führt zu dem in Abbildung 4.2 dargestellten Anti-Windup-Regelkreis. Die Reglerzustände können dann nur eingeschränkt über die Eingangsmatrix $\boldsymbol{B}_{\mathrm{r}, u}$ beeinflusst werden, d. h., es gilt

$$
\dot{\boldsymbol{x}}_{\mathrm{r}}=\boldsymbol{A}_{\mathrm{r}} \boldsymbol{x}_{\mathrm{r}}+\boldsymbol{B}_{\mathrm{r}, u} \boldsymbol{y}_{\mathrm{s}}+\boldsymbol{B}_{\mathrm{r}, w} \boldsymbol{w}-\boldsymbol{B}_{\mathrm{r}, u} \boldsymbol{y}_{\mathrm{aw}, \mathrm{u}} .
$$

Jedes externe Anti-Windup kann als Spezialfall eines integrierten AntiWindup aufgefasst werden. Dazu substituiert man in obiger Differentialgleichung $\boldsymbol{y}_{\mathrm{aw}, \mathrm{x}}=-\boldsymbol{B}_{\mathrm{r}, u} \boldsymbol{y}_{\mathrm{aw}, \mathrm{u}}$ und legt den Ausgang $\boldsymbol{y}_{\mathrm{aw}, \mathrm{x}}$ der AntiWindup-Dynamik (4.1) wie folgt fest

$$
\boldsymbol{y}_{\mathrm{aw}, \mathrm{x}}=\boldsymbol{h}_{\mathrm{aw}, \mathrm{x}}\left(\boldsymbol{x}_{\mathrm{aw}}, \boldsymbol{\delta}\right)=\boldsymbol{B}_{\mathrm{r}, u} \boldsymbol{h}_{\mathrm{aw}, \mathrm{u}}\left(\boldsymbol{x}_{\mathrm{aw}}, \boldsymbol{\delta}\right) .
$$

Nachdem der Anti-Windup-Regelkreis definiert ist, können die AntiWindup-Ziele formuliert werden.

\section{Anti-Windup-Ziele: Eine kurze Übersicht}

Unabhängig von der integrierten oder externen Anti-Windup-Variante wird die Anti-Windup-Dynamik (4.1) durch die Funktionen

$$
\begin{aligned}
\boldsymbol{f}_{\text {aw }}: & \mathbb{R}^{n_{\text {aw }}} \times \mathbb{R}^{m} \mapsto \mathbb{R}^{n_{\text {aw }}}, \\
\boldsymbol{h}_{\text {aw }, \mathrm{x}} & : \mathbb{R}^{n_{\text {aw }}} \times \mathbb{R}^{m} \mapsto \mathbb{R}^{n_{\mathrm{r}}}, \\
\boldsymbol{h}_{\text {aw }, \mathrm{y}} & : \mathbb{R}^{n_{\text {aw }}} \times \mathbb{R}^{m} \mapsto \mathbb{R}^{m}
\end{aligned}
$$


festgelegt. Eine grundlegende Forderung theoretischer Natur an $\boldsymbol{f}_{\text {aw }}$ ist, dass die Differentialgleichung

$$
\dot{\boldsymbol{x}}_{\mathrm{aw}}=\boldsymbol{f}_{\mathrm{aw}}\left(\boldsymbol{x}_{\mathrm{aw}}, \boldsymbol{\delta}\right)
$$

eine eindeutige Lösung $\boldsymbol{x}_{\text {aw }}(t)$ für alle $t \geq 0$, mögliche Anfangszustände $\boldsymbol{x}_{\text {aw }}(0)$ und Eingangssignale $\boldsymbol{\delta}(t)$ besitzt. Um weitere Forderungen formulieren zu können, ist es sinnvoll, die Ziele einer Anti-Windup-Maßnahme zu definieren. In der modernen Anti-Windup-Literatur [55, 85, 173, 182, 183, 191, 211] werden diese wie folgt beschrieben:

1. Erhalt des Kleinsignalverhaltens. Im nichtsättigenden Betrieb bleibt die Anti-Windup-Methode inaktiv. Das dynamische Verhalten des Regelkreises wird allein durch den Regler $\mathbf{R}$ bestimmt.

2. Stabilität im Sättigungsfall. Das Anti-Windup ermöglicht einen stabilen sättigenden Betrieb des Anti-Windup-Regelkreises. Die Stabilitätseigenschaften entsprechen dabei denen des unbeschränkten Regelkreises, wann immer dies mit begrenzter Stellgröße möglich ist.

3. Rückgewinnung des unbeschränkten Systemverhaltens. Während und nach dem Auftreten von Sättigung liegt das dynamische Verhalten des Anti-Windup-Regelkreises (4.3) so nahe am Verhalten des unbeschränkten Regelkreises (2.3) wie mit begrenzter Stellgröße möglich.

Die zunächst in Worten formulierten Ziele werden in den nächsten Abschnitten mathematisch formalisiert und entsprechende Forderungen an $\boldsymbol{f}_{\mathrm{aw}}, \boldsymbol{h}_{\mathrm{aw}, \mathrm{x}}$ und $\boldsymbol{h}_{\mathrm{aw}, \mathrm{y}}$ abgeleitet.

\subsection{Ziele von modernem Anti-Windup}

Der Begriff Windup steht für ein durch Stellgrößenbegrenzungen ausgelöstes Fehlverhalten des Regelkreises. Die Aufgabe eines Anti-Windup besteht konsequenterweise darin, dieses Fehlverhalten zu beseitigen und auch im Sättigungsfall ein wünschenswertes dynamisches Verhalten zu ermöglichen, so gut dies mit einer begrenzten Stellgröße umsetzbar ist. Diese Aufgabe lässt sich untergliedern in den Erhalt des Kleinsignalverhaltens, eine Stabilitätsgarantie und die Rückgewinnung des unbeschränkten Verhaltens im Sättigungsfall. Nachfolgend werden diese Teilaufgaben charakterisiert. 


\section{Erhalt des Kleinsignalverhaltens}

Der Ausgangspunkt von Anti-Windup ist der unbeschränkte Regelkreis aus Abbildung 2.1 mit der linearen Dynamik

$$
\dot{\boldsymbol{x}}_{\mathrm{ub}}=\boldsymbol{A} \boldsymbol{x}_{\mathrm{ub}}+\boldsymbol{B}_{w} \boldsymbol{w}+\boldsymbol{B}_{z} \boldsymbol{z}
$$

gemäß (2.3). Aufgrund der Annahme 2.2 besitzt dieser ein ideales dynamisches Verhalten. Der beschränkte Regelkreis weist ein identisches Verhalten auf, solange die Stellgröße nicht sättigt. Das ist für ausreichend kleine Sollwerte, Anfangszustände und Störungen der Fall.

Dieses ideale Kleinsignalverhalten des beschränkten Regelkreises soll durch eine Anti-Windup-Maßnahme nicht gestört werden. Deshalb wird die Anti-Windup-Strategie erst beim Auftreten von Sättigung aktiv, d. h., wenn für den Sättigungsgrad (4.2) erstmalig $\boldsymbol{\delta} \neq \mathbf{0}$ gilt. Bezüglich der Dynamik des Anti-Windup (4.1) erwachsen daraus die Anforderungen

$$
\begin{aligned}
\boldsymbol{f}_{\mathrm{aw}}(\mathbf{0}, \mathbf{0}) & =\mathbf{0}, \\
\boldsymbol{h}_{\mathrm{aw}, \mathrm{x}}(\mathbf{0}, \mathbf{0}) & =\mathbf{0}, \\
\boldsymbol{h}_{\mathrm{aw}, \mathrm{y}}(\mathbf{0}, \mathbf{0}) & =\mathbf{0}, \\
\boldsymbol{x}_{\mathrm{aw}}(0) & =\mathbf{0} .
\end{aligned}
$$

Besonders hervorzuheben ist der Anfangszustand $\boldsymbol{x}_{\text {aw }}(0)=\mathbf{0}$ des AntiWindup. Diese Wahl ist keine Einschränkung der Allgemeinheit, sondern zwingend notwendig für den Erhalt des Kleinsignalverhaltens, was ein charakteristisches Merkmal von Anti-Windup-Methoden ist.

\section{Stabilität im Sättigungsfall}

Eine grundlegende Aufgabe von Anti-Windup-Maßnahmen ist es, die Stabilität der Ruhelage des Anti-Windup-Regelkreises (4.3) im Sättigungsfall zu garantieren. Die daraus folgenden Forderungen an die Anti-WindupDynamik, d. h. an die Funktionen $\boldsymbol{f}_{\mathrm{aw}}, \boldsymbol{h}_{\mathrm{aw}, \mathrm{x}}$ und $\boldsymbol{h}_{\mathrm{aw}, \mathrm{y}}$, lassen sich formal mithilfe der Stabilitätstheorie von Ljapunov ausdrücken. Zwecks einer einfacheren Darstellung wird wieder von einer Ruhelage im Ursprung des Zustandsraumes ausgegangen. Es gilt dann folgendes zentrales Ergebnis für die Stabilitätsanalyse dynamischer Systeme.

Satz 4.1 (Direkte Methode von Ljapunov, nach [3, 107]). Gegeben ist das durch die Differentialgleichung $\dot{\boldsymbol{x}}=\boldsymbol{f}(\boldsymbol{x})$ beschriebene System. Die Funktion $\boldsymbol{f}: \mathbb{D} \mapsto \mathbb{R}^{n}$ sei derart, dass die Differentialgleichung für alle 
$\boldsymbol{x}(0) \in \mathbb{D} \subset \mathbb{R}^{n}$ eine eindeutige Lösung besitzt. Des Weiteren sei $\mathbb{D}$ eine Umgebung der Ruhelage $\boldsymbol{x}^{(\mathrm{R})}=\mathbf{0}$ des Systems. Wenn eine stetig differenzierbare Funktion $V: \mathbb{D} \mapsto \mathbb{R}$ existiert, so dass

$$
\begin{aligned}
& V(\mathbf{0})=0, \\
& V(\boldsymbol{x})>0 \forall \boldsymbol{x} \in \mathbb{D} \backslash\{\mathbf{0}\}, \\
& \dot{V}(\boldsymbol{x})<0 \forall \boldsymbol{x} \in \mathbb{D} \backslash\{\mathbf{0}\},
\end{aligned}
$$

dann ist die Ruhelage $\boldsymbol{x}^{(\mathrm{R})}=\mathbf{0}$ asymptotisch stabil.

Die Funktion $V(\boldsymbol{x})$ wird dabei als Ljapunov-Funktion bezeichnet. Gilt $\mathbb{D}=\mathbb{R}^{n}$ und die Ljapunov-Funktion ist radial unbeschränkt, d. h.

$$
\|\boldsymbol{x}\| \rightarrow \infty \Rightarrow V(\boldsymbol{x}) \rightarrow \infty
$$

dann ist die Ruhelage $\boldsymbol{x}^{(\mathrm{R})}=\mathbf{0}$ des Systems global asymptotisch stabil. Mit einer Ljapunov-Funktion lässt sich jedoch nicht nur die Stabilität einer Ruhelage nachweisen, sondern auch, im Fall lokaler asymptotischer Stabilität, ein Einzugsgebiet abschätzen, wie der folgende Satz zeigt.

Satz 4.2 (nach [3]). Ist $V(\boldsymbol{x})$ eine Ljapunov-Funktion für das System $\dot{\boldsymbol{x}}=\boldsymbol{f}(\boldsymbol{x})$ mit der Ruhelage $\boldsymbol{x}^{(\mathrm{R})}=\mathbf{0}$, dann ist das Gebiet

$$
\mathcal{G}(\mathbf{0})=\left\{\boldsymbol{x} \in \mathbb{R}^{n} \mid V(\boldsymbol{x})<c\right\} \quad \text { mit } c \in \mathbb{R}_{>0},
$$

falls es beschränkt ist, ein Einzugsgebiet der Ruhelage $\boldsymbol{x}^{(\mathrm{R})}=\mathbf{0}$, wenn

$$
\dot{V}(\boldsymbol{x})<0 \quad \forall \boldsymbol{x} \in \mathcal{G}(\mathbf{0})
$$

gilt.

Die Forderung nach asymptotischer Stabilität der Ruhelage des nichtlinearen Anti-Windup-Regelkreises lässt sich demnach mit der Stabilitätstheorie von Ljapunov formalisieren. Die Funktionen $\boldsymbol{f}_{\mathrm{aw}}, \boldsymbol{h}_{\mathrm{aw}, \mathrm{x}}$ und $\boldsymbol{h}_{\text {aw,y }}$ der Anti-Windup-Strategie sind folglich so zu wählen, dass die Existenz einer Ljapunov-Funktion $V(\boldsymbol{x})$ und ein ausreichend großes Einzugsgebiet $\mathcal{G}(\mathbf{0})$, wenn möglich sogar globale Stabilität, für den Anti-WindupRegelkreis garantiert sind.

\section{Rückgewinnung des unbeschränkten Verhaltens}

Neben der Sicherstellung eines stabilen Systemverhaltens besteht die Aufgabe einer Anti-Windup-Maßnahme darin, eine möglichst hohe Regelgüte 
des Anti-Windup-Regelkreises zu garantieren. Im nichtsättigenden Betrieb wird dies durch den kleinsignalerhaltenden Charakter des Anti-Windup erreicht. Dann gilt

$$
\boldsymbol{y}_{\mathrm{s}} \equiv \boldsymbol{y}_{\mathrm{s}, \mathrm{ub}} \quad \Leftrightarrow \quad \boldsymbol{\sigma}=\boldsymbol{y}_{\mathrm{s}}-\boldsymbol{y}_{\mathrm{s}, \mathrm{ub}} \equiv \mathbf{0}
$$

und der Ausgang des Anti-Windup-Regelkreises entspricht dem des unbeschränkten Regelkreises. Somit weist er gemäß Annahme 2.2 ein ideales Verhalten auf. Das ändert sich während und nach dem Auftreten von Sättigung. Es kommt zu Abweichungen zwischen den Ausgängen, so dass gilt

$$
\boldsymbol{y}_{\mathrm{s}} \neq \boldsymbol{y}_{\mathrm{s}, \mathrm{ub}} \quad \Leftrightarrow \quad \boldsymbol{\sigma} \neq \mathbf{0} .
$$

Das Ziel einer Anti-Windup-Maßnahme ist es, derartige Abweichungen zu minimieren und die Ausgänge bzw. Zustände möglichst schnell wieder in Übereinstimmung zu bringen, $\mathrm{d}$. $\mathrm{h}$.

$$
\boldsymbol{y}_{\mathrm{s}} \rightarrow \boldsymbol{y}_{\mathrm{s}, \mathrm{ub}} \quad \Leftrightarrow \quad \boldsymbol{\sigma} \rightarrow \mathbf{0} .
$$

Wenn das Anti-Windup die Stabilität der Ruhelage des Anti-WindupRegelkreises garantiert, dann wird eine Übereinstimmung in jedem Fall erreicht. Spätestens in der Ruhelage verschwindet die Abweichung. Die Frage ist, wie sich die Abweichung bis zu diesem Zeitpunkt entwickelt.

Ein sinnvolles Maß um die Größe der Abweichung $\boldsymbol{\sigma}(t)$ zwischen den Signalen $\boldsymbol{y}_{\mathrm{s}}(t)$ und $\boldsymbol{y}_{\mathrm{s}, \mathrm{ub}}(t)$ zu quantifizieren, ergibt sich durch Integrieren des quadrierten Betrags der Abweichung über die Zeit. Die Quadratwurzel dieses Integrals wird als $L_{2}$-Norm bezeichnet und ist wie folgt definiert.

Definition $4.1\left(L_{2}\right.$-Norm). Die $L_{2}$-Norm eines Signals $\boldsymbol{\sigma}(t)$ ist

$$
\|\boldsymbol{\sigma}\|_{L_{2}}=\left(\lim _{t \rightarrow \infty} \int_{0}^{t} \boldsymbol{\sigma}(\tau)^{\top} \boldsymbol{\sigma}(\tau) d \tau\right)^{\frac{1}{2}} .
$$

Die Forderung nach Rückgewinnung des unbeschränkten Systemverhaltens lässt sich demnach mit der $L_{2}$-Norm formalisieren. Die Funktionen $\boldsymbol{f}_{\mathrm{aw}}, \boldsymbol{h}_{\mathrm{aw}, \mathrm{x}}$ und $\boldsymbol{h}_{\mathrm{aw}, \mathrm{y}}$ der Anti-Windup-Strategie sind so zu wählen, dass die $L_{2}$-Norm der Abweichung $\boldsymbol{\sigma}=\boldsymbol{y}_{\mathrm{s}}-\boldsymbol{y}_{\mathrm{s}, \text { ub }}$ möglichst klein ist.

\section{Drei Wege zum Ziel}

Wie in Abschnitt 3.3.2 erläutert, lassen sich drei Konzepte für die Wahl der Anti-Windup-Dynamik (4.1) unterscheiden. Abbildung 4.3 zeigt die Unterteilung. Allgemeine lineare Ansätze werden in Abschnitt 4.3 behandelt. 


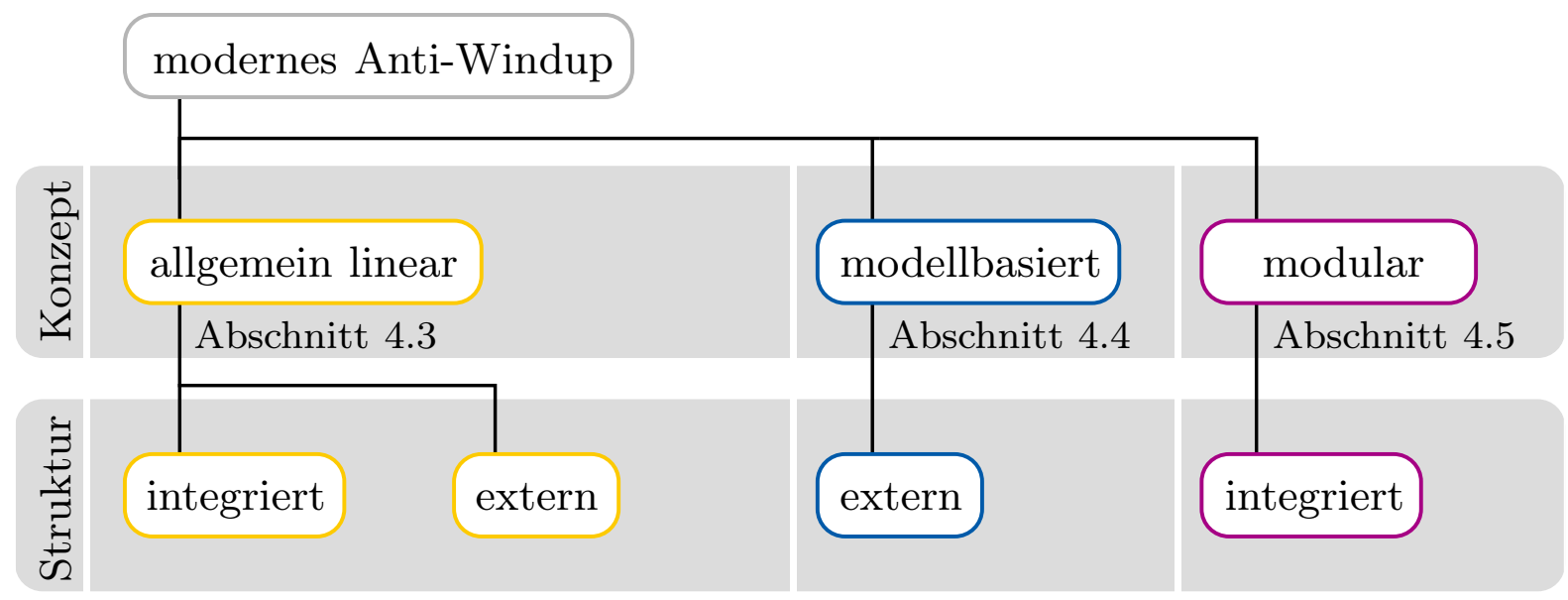

Abbildung 4.3: Klassifizierung von modernen Anti-Windup-Methoden.

Modellbasierte Methoden fasst der Abschnitt 4.4 zusammen und modulare Strategien erläutert Abschnitt 4.5.

\subsection{Allgemeines lineares Anti-Windup}

Die Grundidee des allgemeinen linearen Anti-Windup besteht darin, den Sättigungsgrad $\boldsymbol{\delta}=\boldsymbol{u}_{\mathrm{a}}-\mathbf{s a t}_{\overline{\mathbf{u}}}\left(\boldsymbol{u}_{\mathrm{a}}\right)$ über ein lineares System auf die Reglerzustände und den Reglerausgang zurückzuführen. Die Anti-WindupDynamik ergibt sich demnach zu

$$
\begin{aligned}
\dot{\boldsymbol{x}}_{\mathrm{aw}} & =\boldsymbol{A}_{\mathrm{aw}} \boldsymbol{x}_{\mathrm{aw}}+\boldsymbol{B}_{\mathrm{aw}} \boldsymbol{\delta}, \\
\boldsymbol{y}_{\mathrm{aw}, \mathrm{x}} & =\boldsymbol{C}_{\mathrm{aw}, \mathrm{x}} \boldsymbol{x}_{\mathrm{aw}}+\boldsymbol{D}_{\mathrm{aw}, \mathrm{x}} \boldsymbol{\delta}, \\
\boldsymbol{y}_{\mathrm{aw}, \mathrm{y}} & =\boldsymbol{C}_{\mathrm{aw}, \mathrm{y}} \boldsymbol{x}_{\mathrm{aw}}+\boldsymbol{D}_{\mathrm{aw}, \mathrm{y}} \boldsymbol{\delta},
\end{aligned}
$$

wobei die Dimension $n_{\text {aw }}$ des Zustands $\boldsymbol{x}_{\text {aw }}$ frei wählbar ist. Diese sehr allgemeine Struktur enthält praktisch alle linearen Anti-WindupMaßnahmen als Spezialfall. Bezüglich der Systemordnung werden in der Literatur [55, 182, 183, 211] drei Varianten unterschieden:

- Statisches Anti-Windup. Das Anti-Windup besitzt keine Dynamik und somit keine Zustände, d.h. $n_{\text {aw }}=0$. Nur die Matrizen $\boldsymbol{D}_{\text {aw, }}$ und $\boldsymbol{D}_{\text {aw,y }}$ müssen beim Entwurf gewählt werden.

- Anti-Windup voller Ordnung (engl. full-order anti-windup). Der Anti-Windup-Zustand besitzt eine mindestens so hohe Dimension wie der Streckenzustand. Somit gilt $n_{\text {aw }} \geq n_{\mathrm{s}}$. 
- Anti-Windup niedriger Ordnung (engl. low-order anti-windup). Die Ordnung des Anti-Windup ist kleiner als die Systemordnung der Strecke, d. h. $0<n_{\text {aw }}<n_{\text {s }}$.

Die klassische Anti-Windup-Maßnahme Back-Calculation für PID-Regler aus Abbildung 3.6 besitzt beispielsweise keine Dynamik. Es ist ein statisches Anti-Windup. Der Sättigungsgrad $\delta \in \mathbb{R}$ wird über eine konstante Verstärkung $-k_{\text {aw }}$ auf den Integriererzustand zurückgeführt. Das resultiert in der Parametrierung

$$
\begin{aligned}
\boldsymbol{A}_{\mathrm{aw}} & =\mathbf{0}, \quad \boldsymbol{B}_{\mathrm{aw}}=\mathbf{0}, \\
\boldsymbol{C}_{\mathrm{aw}, \mathrm{x}} & =\mathbf{0}, \quad \boldsymbol{D}_{\mathrm{aw}, \mathrm{x}}=-k_{\mathrm{aw}}, \\
\boldsymbol{C}_{\mathrm{aw}, \mathrm{y}} & =\mathbf{0}, \quad \boldsymbol{D}_{\mathrm{aw}, \mathrm{y}}=0 .
\end{aligned}
$$

Der Entwurf eines allgemeinen linearen Anti-Windup besteht in der Wahl der Systemordnung $n_{\text {aw }}$ und der Parametrierung der Matrizen in (4.5) gemäß den Entwurfszielen aus Abschnitt 4.2. Darauf wird im Folgenden detaillierter eingegangen.

\subsubsection{Problematik beim Entwurf}

In der Literatur findet sich eine Vielzahl von Entwurfsverfahren für lineare Anti-Windup-Maßnahmen gemäß (4.5), wie z.B. die Arbeiten $[51,55,70,95,182,187,211]$ und die darin angeführten Referenzen. Auffällig ist, dass die existierenden Entwurfsmethoden entweder Spezialfälle abdecken oder nicht auf konvexer Optimierung basieren. Bei einigen Verfahren ist beispielsweise die Systemordnung des Anti-Windup festgelegt und bestimmte Matrizen bleiben unberücksichtigt.

Eine zu hohe Systemordnung kann bei der Implementierung zu Schwierigkeiten führen, wenn Rechnerkapazitäten knapp bemessen sind. Nichtkonvexe Optimierungsprobleme lassen sich - wenn überhaupt - nur mit sehr hohem Aufwand lösen. Deshalb ist bei der Wahl des Entwurfsverfahrens ein Kompromiss zwischen der Systemordnung $n_{\text {aw }}$ des Anti-Windup, dem verwendeten Gütekriterium und der Art und Weise wie die AntiWindup-Matrizen parametriert werden, zu finden.

Kompromisse, die zu konvexen Optimierungsproblemen führen, werden nachfolgend beschrieben. Dabei garantieren alle Verfahren den Erhalt des Kleinsignalverhaltens und Stabilität im Sättigungsfall. Die Hauptschwierigkeit liegt darin, die konvexe Gütefunktion des Optimierungsproblems so zu gestalten, dass sie ein Maß für die Abweichung vom unbeschränkten Systemverhalten darstellt. Die in [18] vorgestellte Lösung führt zu 
Tabelle 4.1: Entwurfsverfahren für allgemeines lineares Anti-Windup. Nicht konvexe Optimierungsprobleme sind blau hervorgehoben.

\begin{tabular}{llll}
\hline & \multicolumn{3}{c}{ Systemordnung } \\
& $n_{\mathrm{aw}}=0$ & $n_{\mathrm{aw}} \geq n_{\mathrm{s}}$ & $n_{\mathrm{aw}}<n_{\mathrm{s}}$ \\
\hline Kompromiss 1 & {$[176]$} & {$[18]$} & - \\
\hline Kompromiss 2 & {$[29][68][69][182]$} & {$[182]$} & {$[182]$} \\
\hline \multirow{2}{*}{ Kompromiss 3 } & {$[70-72][95][139][182]$} & {$[70-72][95]$} & {$[51][182]$} \\
& {$[187][204][211]$} & {$[182][211]$} & \\
\hline
\end{tabular}

Kompromiss 1. Für den Entwurf wird der Anti-Windup-Regelkreis um ein Modell des unbeschränkten Regelkreises erweitert, so dass die Abweichung vom unbeschränkten Systemverhalten minimiert werden kann. Das resultierende Optimierungsproblem ist allerdings nur dann konvex, wenn für die Systemordnung des Anti-Windup $n_{\mathrm{aw}}=2\left(n_{\mathrm{s}}+n_{\mathrm{r}}\right)+m$ gilt.

Eine derart hohe Systemordnung ist in vielen Fällen nicht praktikabel, weshalb alternativ die folgenden Gütekriterien verwendet werden.

Kompromiss 2. Eine hohe Regelgüte des Anti-Windup spielt keine Rolle $[29,68,69]$. Das Ziel ist ein möglichst großes Einzugsgebiet der Ruhelage des Anti-Windup-Regelkreises zu erreichen. Für $n_{\text {aw }}=0$ ergibt sich ein konvexes Optimierungsproblem.

Kompromiss 3. Der Anti-Windup-Regelkreis wird um einen virtuellen Ausgang $\boldsymbol{y}_{\mathrm{p}}$ so erweitert, dass eine kleine $L_{2}$-Norm dieses Ausganges eine hohe Regelgüte impliziert. Als Gütekriterium beim Entwurf wird dann die $L_{2}$-Norm minimiert. Die Fälle $n_{\text {aw }}=0$ sowie $n_{\text {aw }}=n_{\mathrm{s}}$ führen zu konvexen Optimierungsproblemen [51, 70-72, 95, 139].

Tabelle 4.1 zeigt eine Klassifizierung der existierenden Entwurfsverfahren. Der Kompromiss 2 dürfte aufgrund der vernachlässigten Regelgüte nur für die wenigsten Anwendungen interessant sein. Mittels Kompromiss 3 lässt sich bei geeigneter Wahl des virtuellen Ausgangs eine hohe Regelgüte erreichen. Details sind im nächsten Abschnitt beschrieben. 


\subsubsection{Entwurf mit virtuellen Ausgängen}

Zur Definition des Entwurfsziels wird der Anti-Windup-Regelkreis (4.3) um einen virtuellen Ausgang $\boldsymbol{y}_{\mathrm{p}}$ erweitert, der sich wie folgt zusammensetzt

$$
\boldsymbol{y}_{\mathrm{p}}=\boldsymbol{C}_{\mathrm{p}} \boldsymbol{x}_{\mathrm{s}}+\boldsymbol{D}_{\mathrm{p}, \mathrm{u}} \boldsymbol{u}_{\mathrm{s}}+\boldsymbol{D}_{\mathrm{p}, \mathrm{z}} \boldsymbol{z}+\boldsymbol{D}_{\mathrm{p}, \mathrm{w}} \boldsymbol{w} .
$$

Über die Matrizen $\boldsymbol{C}_{\mathrm{p}}, \boldsymbol{D}_{\mathrm{p}, \mathrm{u}}, \boldsymbol{D}_{\mathrm{p}, \mathrm{z}}$ und $\boldsymbol{D}_{\mathrm{p}, \mathrm{w}}$ lassen sich die Komponenten des Streckenzustandes $\boldsymbol{x}_{\mathrm{s}}$, des Streckeneingangs $\boldsymbol{u}_{\mathrm{s}}$, der Störung $\boldsymbol{z}$ und des Sollwertes $\boldsymbol{w}$ gewichten. So können mithilfe dieses Ausgangs, je nach Festlegung der Matrizen, verschiedene für die Regelgüte relevante Größen dargestellt werden. Die Wahl

$$
C_{\mathrm{p}}=-C_{\mathrm{s}}, \quad D_{\mathrm{p}, \mathrm{u}}=\mathbf{0}, \quad D_{\mathrm{p}, \mathrm{z}}=\mathbf{0}, \quad D_{\mathrm{p}, \mathrm{w}}=\mathbb{I}
$$

führt beispielsweise zur Regelabweichung (2.6), denn es gilt

$$
\boldsymbol{y}_{\mathrm{p}}=-C_{\mathrm{s}} \boldsymbol{x}_{\mathrm{s}}+\boldsymbol{w}=\boldsymbol{w}-\boldsymbol{y}_{\mathrm{s}}=\boldsymbol{e} .
$$

Der virtuelle Ausgang $\boldsymbol{y}_{\mathrm{p}}$, genauer dessen $L_{2}$-Norm $\left\|\boldsymbol{y}_{\mathrm{p}}\right\|_{L_{2}}$, stellt somit ein Maß für die Regelgüte dar und bildet die Basis für die Parametrierung des Anti-Windup. Diese ist so zu gestalten, dass die $L_{2}$-Norm ${ }^{3}$ des Ausgangs $\boldsymbol{y}_{\mathrm{p}}$ für auftretende Sollwerte $\boldsymbol{w}$ und Störungen $\boldsymbol{z}$ möglichst klein wird.

Um diese Forderung als Gütemaßs in das Optimierungsproblem zu integrieren, wird das Ein-/Ausgangsverhalten des Anti-Windup-Regelkreises gemäß Abbildung 4.4 betrachtet. Die Eingangsgrößen $\boldsymbol{w}$ und $\boldsymbol{z}$ des Regelkreises sind in dem Vektor $\boldsymbol{r}$ zusammengefasst. Ein Maß dafür, wie stark sich Eingangssignale $\boldsymbol{r}$ auf den Ausgang $\boldsymbol{y}_{\mathrm{p}}$ im Sinne der $L_{2}$-Norm auswirken, ist die $L_{2}$-Verstärkung des Anti-Windup-Regelkreises gemäß

Definition $4.2\left(L_{2}\right.$-Verstärkung). Gegeben sei ein System

$$
\begin{aligned}
& \dot{\boldsymbol{x}}=\boldsymbol{f}(\boldsymbol{x}, \boldsymbol{r}), \\
& \boldsymbol{y}=\boldsymbol{h}(\boldsymbol{x}, \boldsymbol{r})
\end{aligned}
$$

mit dem Eingang $\boldsymbol{r}$ und dem Ausgang $\boldsymbol{y}$. Das System besitzt für $\boldsymbol{x}(0)=\mathbf{0}$ die $L_{2}$-Verstärkung $\gamma$, wenn

$$
\sup _{0<\|\boldsymbol{r}\|_{L_{2}}<\infty} \frac{\|\boldsymbol{y}\|_{L_{2}}}{\|\boldsymbol{r}\|_{L_{2}}} \leq \gamma
$$

${ }^{3}$ Andere Normen sind möglich. Für die $L_{2}$-Norm spricht, dass sie als Energie eines Signals interpretiert werden kann und einfach mathematisch handhabbar ist. 


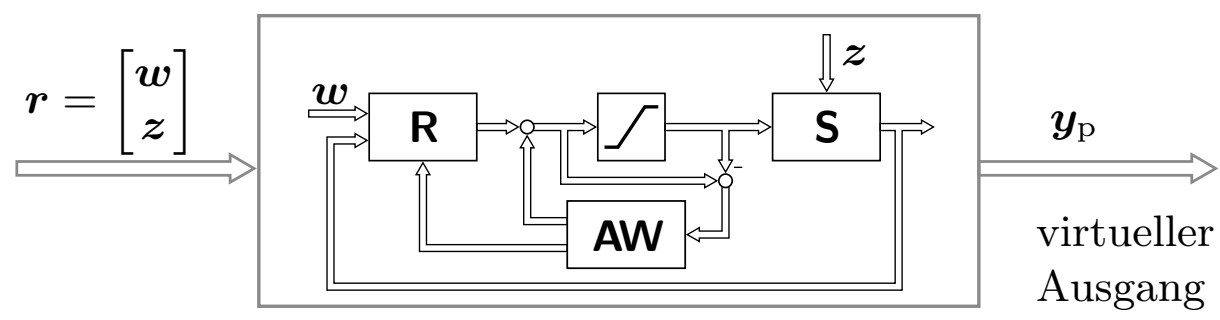

Abbildung 4.4: Das Ziel vieler Entwurfsverfahren für das allgemeine lineare Anti-Windup ist die Minimierung der $L_{2}$-Verstärkung des Anti-Windup-Regelkreises von dem Eingang $\boldsymbol{r}$ zu dem virtuellen Ausgang $\boldsymbol{y}_{\mathrm{p}}$.

Eine kleine $L_{2}$-Verstärkung $\gamma$ des Anti-Windup-Regelkreises vom Eingang $\boldsymbol{r}$ zu dem virtuellen Ausgang $\boldsymbol{y}_{\mathrm{p}}$ führt folglich dazu, dass die $L_{2}$-Norm $\left\|\boldsymbol{y}_{\mathrm{p}}\right\|_{L_{2}}$ klein bleibt und damit zu einer hohen Regelgüte. Als konvexe Gütefunktion des beim Entwurf zu lösenden Optimierungsproblems wird deshalb die $L_{2}$-Verstärkung $\gamma$ verwendet. Die Nebenbedingungen finden sich beispielsweise in [70, 95, 182, 211].

Nachteilig an der Methode der virtuellen Ausgänge ist der sehr starke Einfluss des virtuellen Ausgangs auf das Ergebnis. Diese Problematik wird anhand eines Beispiels in [70, Abschnitt 4] demonstriert. Letztlich bleibt die Wahl des Ausgangs (4.6) dem Anwender überlassen.

\subsection{Modellbasiertes Anti-Windup}

Modellbasierte Anti-Windup-Methoden ${ }^{4}$ besitzen die in Abbildung 4.2 dargestellte externe Struktur und nutzen ein Modell der Strecke um Windup zu verhindern $[55,182,183,211]$. Die Dynamik (4.4) ergibt sich zu

$$
\begin{aligned}
\dot{\boldsymbol{x}}_{\mathrm{aw}} & =\boldsymbol{A}_{\mathrm{s}} \boldsymbol{x}_{\mathrm{aw}}+\boldsymbol{B}_{\mathrm{s}, u} \boldsymbol{k}_{\mathrm{aw}}\left(\boldsymbol{x}_{\mathrm{aw}}\right)+\boldsymbol{B}_{\mathrm{s}, u} \boldsymbol{\delta}, \\
\boldsymbol{y}_{\mathrm{aw}, \mathrm{u}} & =-\boldsymbol{C}_{\mathrm{s}} \boldsymbol{x}_{\mathrm{aw}} \\
\boldsymbol{y}_{\mathrm{aw}, \mathrm{y}} & =-\boldsymbol{k}_{\mathrm{aw}}\left(\boldsymbol{x}_{\mathrm{aw}}\right)
\end{aligned}
$$

mit $\boldsymbol{x}_{\mathrm{aw}} \in \mathbb{R}^{n_{\mathrm{s}}}$. Im Vergleich zu dem allgemeinen linearen Anti-Windup fällt auf, dass große Teile der Anti-Windup-Dynamik und die Systemordnung $n_{\text {aw }}=n_{\mathrm{s}}$ durch das Modell der Strecke vorgegeben sind. Lediglich die Funktion $\boldsymbol{k}_{\text {aw }}: \mathbb{R}^{n_{\mathrm{s}}} \mapsto \mathbb{R}^{m}$ ist beim Entwurf wählbar. Diese einschränkende Festlegung hat mehrere Vorteile:

\footnotetext{
${ }^{4}$ In der englischsprachigen Literatur: model recovery anti-windup.
} 
- Nichtlineares Anti-Windup. Die Funktion $\boldsymbol{k}_{\text {aw }}$ ermöglicht nichtlineare Anti-Windup-Maßnahmen mit einer hohen Regelgüte.

- Einfacher Entwurf. Der Anti-Windup-Entwurf reduziert sich auf den Entwurf eines Zustandsreglers für das Modell der Strecke.

- Anschauliches Gütekriterium. Die Abweichung vom unbeschränkten Systemverhalten kann beim Entwurf direkt minimiert werden.

Um die letzten beiden Punkte zu zeigen, sind zwei Umformungen des AntiWindup-Regelkreises nötig, die in den nächsten Abschnitten detailliert erläutert werden. Ausgangspunkt ist der Anti-Windup-Regelkreis in Abbildung 4.5, dessen Dynamik sich aus der Strecke (2.1), dem Regler (2.2), dem Aktor (2.10), dem modellbasierten Anti-Windup (4.7) und dem Sättigungsgrad $\boldsymbol{\delta}$ gemäß (4.2) sowie den Verschaltungen

$$
\boldsymbol{u}_{\mathrm{s}}=\operatorname{sat}_{\overline{\mathbf{u}}}\left(\boldsymbol{y}_{\mathrm{r}}+\boldsymbol{y}_{\mathrm{aw}, \mathrm{y}}\right), \quad \boldsymbol{u}_{\mathrm{r}}=\boldsymbol{y}_{\mathrm{s}}-\boldsymbol{y}_{\mathrm{aw}, \mathrm{u}}
$$

zusammensetzt. Das Zustandsraummodell des Anti-Windup-Regelkreises mit modellbasierter Anti-Windup-Strategie lautet wie folgt

$$
\begin{aligned}
& \dot{\boldsymbol{x}}_{\mathrm{s}}=\boldsymbol{A}_{\mathrm{s}} \boldsymbol{x}_{\mathrm{s}}+\boldsymbol{B}_{\mathrm{s}, u} \mathbf{s a t}_{\overline{\mathbf{u}}}\left(\boldsymbol{y}_{\mathrm{r}}+\boldsymbol{y}_{\mathrm{aw}, \mathrm{y}}\right)+\boldsymbol{B}_{\mathrm{s}, z} \boldsymbol{z}, \\
& \dot{\boldsymbol{x}}_{\mathrm{r}}=\boldsymbol{A}_{\mathrm{r}} \boldsymbol{x}_{\mathrm{r}}+\boldsymbol{B}_{\mathrm{r}, u} \boldsymbol{C}_{\mathrm{s}}\left(\boldsymbol{x}_{\mathrm{s}}+\boldsymbol{x}_{\mathrm{aw}}\right)+\boldsymbol{B}_{\mathrm{r}, u} \boldsymbol{w}, \\
& \dot{\boldsymbol{x}}_{\mathrm{aw}}=\boldsymbol{A}_{\mathrm{s}} \boldsymbol{x}_{\mathrm{aw}}+\boldsymbol{B}_{\mathrm{s}, u} \boldsymbol{y}_{\mathrm{r}}-\boldsymbol{B}_{\mathrm{s}, u} \mathbf{s a t}_{\overline{\mathbf{u}}}\left(\boldsymbol{y}_{\mathrm{r}}+\boldsymbol{y}_{\mathrm{aw}, \mathrm{y}}\right), \\
& \boldsymbol{y}_{\mathrm{r}}=\boldsymbol{C}_{\mathrm{r}} \boldsymbol{x}_{\mathrm{r}}+\boldsymbol{D}_{\mathrm{r}, u} \boldsymbol{C}_{\mathrm{s}}\left(\boldsymbol{x}_{\mathrm{s}}+\boldsymbol{x}_{\mathrm{aw}}\right)+\boldsymbol{D}_{\mathrm{r}, w} \boldsymbol{w}, \\
& \boldsymbol{y}_{\mathrm{aw}, \mathrm{y}}=-\boldsymbol{k}_{\mathrm{aw}}\left(\boldsymbol{x}_{\mathrm{aw}}\right), \\
& \boldsymbol{y}_{\mathrm{s}}=\boldsymbol{C}_{\mathrm{s}} \boldsymbol{x}_{\mathrm{s}} .
\end{aligned}
$$

Diese nicht sonderlich übersichtliche Systembeschreibung basiert auf den Zuständen $\boldsymbol{x}_{\mathrm{s}}, \boldsymbol{x}_{\mathrm{r}}$ und $\boldsymbol{x}_{\mathrm{aw}}$ von Strecke, Regler und Anti-Windup, die für eine kompaktere Notation in dem Zustandsvektor

$$
\boldsymbol{x}_{\mathrm{awrk}}^{\top}=\left[\begin{array}{lll}
\boldsymbol{x}_{\mathrm{s}}^{\top} & \boldsymbol{x}_{\mathrm{r}}^{\top} & \boldsymbol{x}_{\mathrm{aw}}^{\top}
\end{array}\right]
$$

zusammengefasst werden. Mittels einer Koordinatentransformation lässt sich eine für den Entwurf besonders günstige Systemdarstellung erzielen.

\subsubsection{Entkopplung durch Zustandstransformation}

Bekanntlich ist die Wahl der Zustandsvariablen bei der Aufstellung eines Zustandsraummodells nicht eindeutig [132]. Oftmals lässt sich ein System 
besonders einfach analysieren, wenn die Zustandsvariablen geschickt gewählt sind oder durch eine Transformation in ein geeignetes Koordinatensystem überführt werden können. Bei der Auslegung des modellbasierten Anti-Windup, d. h. bei der Wahl der Funktion

$$
\boldsymbol{k}_{\mathrm{aw}}: \mathbb{R}^{n_{\mathrm{s}}} \mapsto \mathbb{R}^{m}
$$

ist man vor allem daran interessiert, wie stark das Verhalten des AntiWindup-Regelkreises (4.8) im Sättigungsfall von dem Verhalten des unbeschränkten Regelkreises (2.3) abweicht. Diese Diskrepanz (engl. mismatch) kann durch eine lineare Transformation der Zustandsvariablen

$$
\boldsymbol{\xi}_{\mathrm{ms}}=\boldsymbol{T} \boldsymbol{x}_{\mathrm{awrk}}
$$

mit einer regulären Matrix $\boldsymbol{T} \in \mathbb{R}^{\left(2 n_{\mathrm{s}}+n_{\mathrm{r}}\right) \times\left(2 n_{\mathrm{s}}+n_{\mathrm{r}}\right)}$ in die sogenannten Mismatch-Zustände $\boldsymbol{\xi}_{\mathrm{ms}}$ veranschaulicht werden [199, 209, 211]. Mittels der eineindeutigen Abbildung

$$
\boldsymbol{\xi}_{\mathrm{ms}}=\left[\begin{array}{l}
\boldsymbol{\xi}_{\mathrm{s}} \\
\boldsymbol{\xi}_{\mathrm{r}} \\
\boldsymbol{\xi}_{\mathrm{aw}}
\end{array}\right]=\left[\begin{array}{rrr}
\mathbb{I} & \mathbf{0} & \mathbb{I} \\
\mathbf{0} & \mathbb{I} & \mathbf{0} \\
\mathbf{0} & \mathbf{0} & -\mathbb{I}
\end{array}\right]\left[\begin{array}{l}
\boldsymbol{x}_{\mathrm{s}} \\
\boldsymbol{x}_{\mathrm{r}} \\
\boldsymbol{x}_{\mathrm{aw}}
\end{array}\right]=\left[\begin{array}{c}
\boldsymbol{x}_{\mathrm{s}}+\boldsymbol{x}_{\mathrm{aw}} \\
\boldsymbol{x}_{\mathrm{r}} \\
-\boldsymbol{x}_{\mathrm{aw}}
\end{array}\right]
$$

wird der Anti-Windup-Regelkreis (4.8) in die äquivalente MismatchDarstellung überführt. Es ergibt sich die Systemdynamik

$$
\begin{aligned}
& \dot{\boldsymbol{\xi}}_{\mathrm{s}}=\boldsymbol{A}_{\mathrm{s}} \boldsymbol{\xi}_{\mathrm{s}}+\boldsymbol{B}_{\mathrm{s}, u} \boldsymbol{y}_{\mathrm{r}}+\boldsymbol{B}_{\mathrm{s}, z} \boldsymbol{z}, \\
& \dot{\boldsymbol{\xi}}_{\mathrm{r}}=\boldsymbol{A}_{\mathrm{r}} \boldsymbol{\xi}_{\mathrm{r}}+\boldsymbol{B}_{\mathrm{r}, u} \boldsymbol{C}_{\mathrm{s}} \boldsymbol{\xi}_{\mathrm{s}}+\boldsymbol{B}_{\mathrm{r}, w} \boldsymbol{w}, \\
& \dot{\boldsymbol{\xi}}_{\mathrm{aw}}=\boldsymbol{A}_{\mathrm{s}} \boldsymbol{\xi}_{\mathrm{aw}}+\boldsymbol{B}_{\mathrm{s}, u}\left(\operatorname{sat}_{\overline{\mathbf{u}}}\left(\boldsymbol{y}_{\mathrm{r}}+\boldsymbol{y}_{\mathrm{aw}, \mathrm{y}}\right)-\boldsymbol{y}_{\mathrm{r}}\right), \\
& \boldsymbol{y}_{\mathrm{r}}=\boldsymbol{C}_{\mathrm{r}} \boldsymbol{\xi}_{\mathrm{r}}+\boldsymbol{D}_{\mathrm{r}, u} \boldsymbol{C}_{\mathrm{s}} \boldsymbol{\xi}_{\mathrm{s}}+\boldsymbol{D}_{\mathrm{r}, w} \boldsymbol{w}, \\
& \boldsymbol{y}_{\mathrm{aw}, \mathrm{y}}=-\boldsymbol{k}_{\mathrm{aw}}\left(-\boldsymbol{\xi}_{\mathrm{aw}}\right), \\
& \boldsymbol{y}_{\mathrm{s}}=\boldsymbol{C}_{\mathrm{s}}\left(\boldsymbol{\xi}_{\mathrm{s}}+\boldsymbol{\xi}_{\mathrm{aw}}\right)=\boldsymbol{y}_{\mathrm{s}, \mathrm{ub}}+\boldsymbol{y}_{\mathrm{aw}, \mathrm{u}} .
\end{aligned}
$$

Der Vorteil der Mismatch-Darstellung besteht in der Zerlegung des AntiWindup-Regelkreises in zwei übersichtliche Teilsysteme. Durch die Beziehungen (4.10a), (4.10b), (4.10d) wird ein lineares Teilsystem beschrieben, das dem unbeschränkten Regelkreis

$$
\mathbf{L}:\left\{\begin{aligned}
\dot{\boldsymbol{\xi}}_{\mathrm{s}} & =\boldsymbol{A}_{\mathrm{s}} \boldsymbol{\xi}_{\mathrm{s}}+\boldsymbol{B}_{\mathrm{s}, u} \boldsymbol{y}_{\mathrm{r}}+\boldsymbol{B}_{\mathrm{s}, z} \boldsymbol{z} \\
\dot{\boldsymbol{\xi}}_{\mathrm{r}} & =\boldsymbol{A}_{\mathrm{r}} \boldsymbol{\xi}_{\mathrm{r}}+\boldsymbol{B}_{\mathrm{r}, u} \boldsymbol{C}_{\mathrm{s}} \boldsymbol{\xi}_{\mathrm{s}}+\boldsymbol{B}_{\mathrm{r}, w} \boldsymbol{w} \\
\boldsymbol{y}_{\mathrm{r}} & =\boldsymbol{C}_{\mathrm{r}} \boldsymbol{\xi}_{\mathrm{r}}+D_{\mathrm{r}, u} C_{\mathrm{s}} \boldsymbol{\xi}_{\mathrm{s}}+D_{\mathrm{r}, w} \boldsymbol{w} \\
\boldsymbol{y}_{\mathrm{s}, \mathrm{ub}} & =\boldsymbol{C}_{\mathrm{s}} \boldsymbol{\xi}_{\mathrm{s}}
\end{aligned}\right.
$$


mit dem Ausgang $\boldsymbol{y}_{\mathrm{s}, \mathrm{ub}}$ entspricht. Das zweite Teilsystem (4.10c), (4.10e) wird auch als Anti-Windup-System bezeichnet, weil es die Sättigungsnichtlinearität und die Anti-Windup-Maßnahme beinhaltet. Es besitzt die Zustandsraumdarstellung

$$
\begin{aligned}
& \dot{\boldsymbol{\xi}}_{\mathrm{aw}}=\boldsymbol{A}_{\mathrm{s}} \boldsymbol{\xi}_{\mathrm{aw}}+\boldsymbol{B}_{\mathrm{s}, u}\left(\operatorname{sat}_{\overline{\mathbf{u}}}\left(\boldsymbol{y}_{\mathrm{r}}+\boldsymbol{y}_{\mathrm{aw}, \mathrm{y}}\right)-\boldsymbol{y}_{\mathrm{r}}\right), \\
& \boldsymbol{y}_{\mathrm{aw}, \mathrm{y}}=-\boldsymbol{k}_{\mathrm{aw}}\left(-\boldsymbol{\xi}_{\mathrm{aw}}\right) .
\end{aligned}
$$

Für punktsymmetrische Funktionen $\boldsymbol{k}_{\text {aw }}\left(\boldsymbol{\xi}_{\text {aw }}\right)$ lässt sich die Zustandsrückführung durch $\boldsymbol{y}_{\text {aw,y }}=\boldsymbol{k}_{\text {aw }}\left(\boldsymbol{\xi}_{\text {aw }}\right)$ beschreiben, so dass nach dem Hinzufügen eines zusätzlichen Ausganges $\boldsymbol{y}_{\mathrm{aw}, \mathrm{u}}$ die Systemdarstellung

$$
\begin{aligned}
& \dot{\boldsymbol{\xi}}_{\mathrm{aw}}=\boldsymbol{A}_{\mathrm{s}} \boldsymbol{\xi}_{\mathrm{aw}}+\boldsymbol{B}_{\mathrm{s}, u}\left(\boldsymbol{s a t}_{\overline{\mathbf{u}}}\left(\boldsymbol{y}_{\mathrm{r}}+\boldsymbol{y}_{\mathrm{aw}, \mathrm{y}}\right)-\boldsymbol{y}_{\mathrm{r}}\right) \\
& \boldsymbol{y}_{\mathrm{aw}, \mathrm{y}}=\boldsymbol{k}_{\mathrm{aw}}\left(\boldsymbol{\xi}_{\mathrm{aw}}\right) \\
& \boldsymbol{y}_{\mathrm{aw}, \mathrm{u}}=\boldsymbol{C}_{\mathrm{s}} \boldsymbol{\xi}_{\mathrm{aw}}
\end{aligned}
$$

des Anti-Windup-Teilsystems entsteht. Offensichtlich beinhaltet es ein Modell der Strecke

$$
\mathbf{S}^{*}:\left\{\begin{aligned}
\dot{\boldsymbol{\xi}}_{\mathrm{aw}} & =\boldsymbol{A}_{\mathrm{s}} \boldsymbol{\xi}_{\mathrm{aw}}+\boldsymbol{B}_{\mathrm{s}, u} \tilde{\boldsymbol{u}} \\
\boldsymbol{y}_{\mathrm{aw}, \mathrm{u}} & =\boldsymbol{C}_{\mathrm{s}} \boldsymbol{\xi}_{\mathrm{aw}}
\end{aligned}\right.
$$

dessen Zustände über $\boldsymbol{y}_{\text {aw,y }}=\boldsymbol{k}_{\text {aw }}\left(\boldsymbol{\xi}_{\text {aw }}\right)$ auf den Eingang

$$
\tilde{\boldsymbol{u}}=\operatorname{sat}_{\overline{\mathbf{u}}}\left(\boldsymbol{y}_{\mathrm{r}}+\boldsymbol{y}_{\mathrm{aw}, \mathrm{y}}\right)-\boldsymbol{y}_{\mathrm{r}}
$$

zurückgeführt werden. Abbildung 4.5 zeigt das aus den zwei Teilsystemen bestehende Gesamtsystem in Mismatch-Koordinaten mit dem Ausgang (4.10f). Dieser Ausgang besitzt die Struktur

$$
\boldsymbol{y}_{\mathrm{s}}=\boldsymbol{y}_{\mathrm{s}, \mathrm{ub}}+\boldsymbol{y}_{\mathrm{aw}, \mathrm{u}} \text {. }
$$

Auch der Anfangszustand des Anti-Windup-Regelkreises muss transformiert werden. Mittels (4.9) ergibt sich zunächst allgemein

$$
\boldsymbol{\xi}_{\mathrm{ms}}^{(0)}=\left[\begin{array}{c}
\boldsymbol{\xi}_{\mathrm{s}}^{(0)} \\
\boldsymbol{\xi}_{\mathrm{r}}^{(0)} \\
\boldsymbol{\xi}_{\mathrm{aw}}^{(0)}
\end{array}\right]=\left[\begin{array}{rrr}
\mathbb{I} & \mathbf{0} & \mathbb{I} \\
\mathbf{0} & \mathbb{I} & \mathbf{0} \\
\mathbf{0} & \mathbf{0} & -\mathbb{I}
\end{array}\right]\left[\begin{array}{l}
\boldsymbol{x}_{\mathrm{s}}^{(0)} \\
\boldsymbol{x}_{\mathrm{r}}^{(0)} \\
\boldsymbol{x}_{\mathrm{aw}}^{(0)}
\end{array}\right]=\left[\begin{array}{c}
\boldsymbol{x}_{\mathrm{s}}^{(0)}+\boldsymbol{x}_{\mathrm{aw}}^{(0)} \\
\boldsymbol{x}_{\mathrm{r}}^{(0)} \\
-\boldsymbol{x}_{\mathrm{aw}}^{(0)}
\end{array}\right] .
$$

Da der Anfangszustand $\boldsymbol{x}_{\mathrm{aw}}^{(0)}$ des Anti-Windup, wie in Abschnitt 4.2 diskutiert, immer null ist, folgt

$$
\boldsymbol{\xi}_{\mathrm{s}}^{(0)}=\boldsymbol{x}_{\mathrm{s}}^{(0)}, \quad \boldsymbol{\xi}_{\mathrm{r}}^{(0)}=\boldsymbol{x}_{\mathrm{r}}^{(0)}, \quad \boldsymbol{\xi}_{\mathrm{aw}}^{(0)}=\boldsymbol{x}_{\mathrm{aW}}^{(0)}=\mathbf{0} .
$$




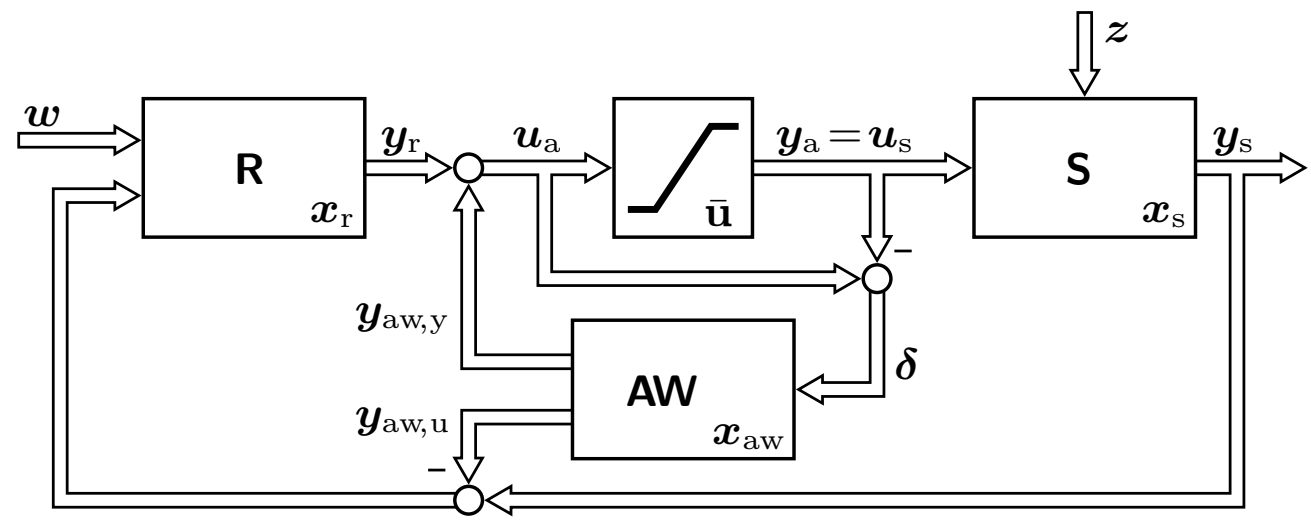

Anti-Windup-Regelkreis

Zustandstransformation

Mismatch-System

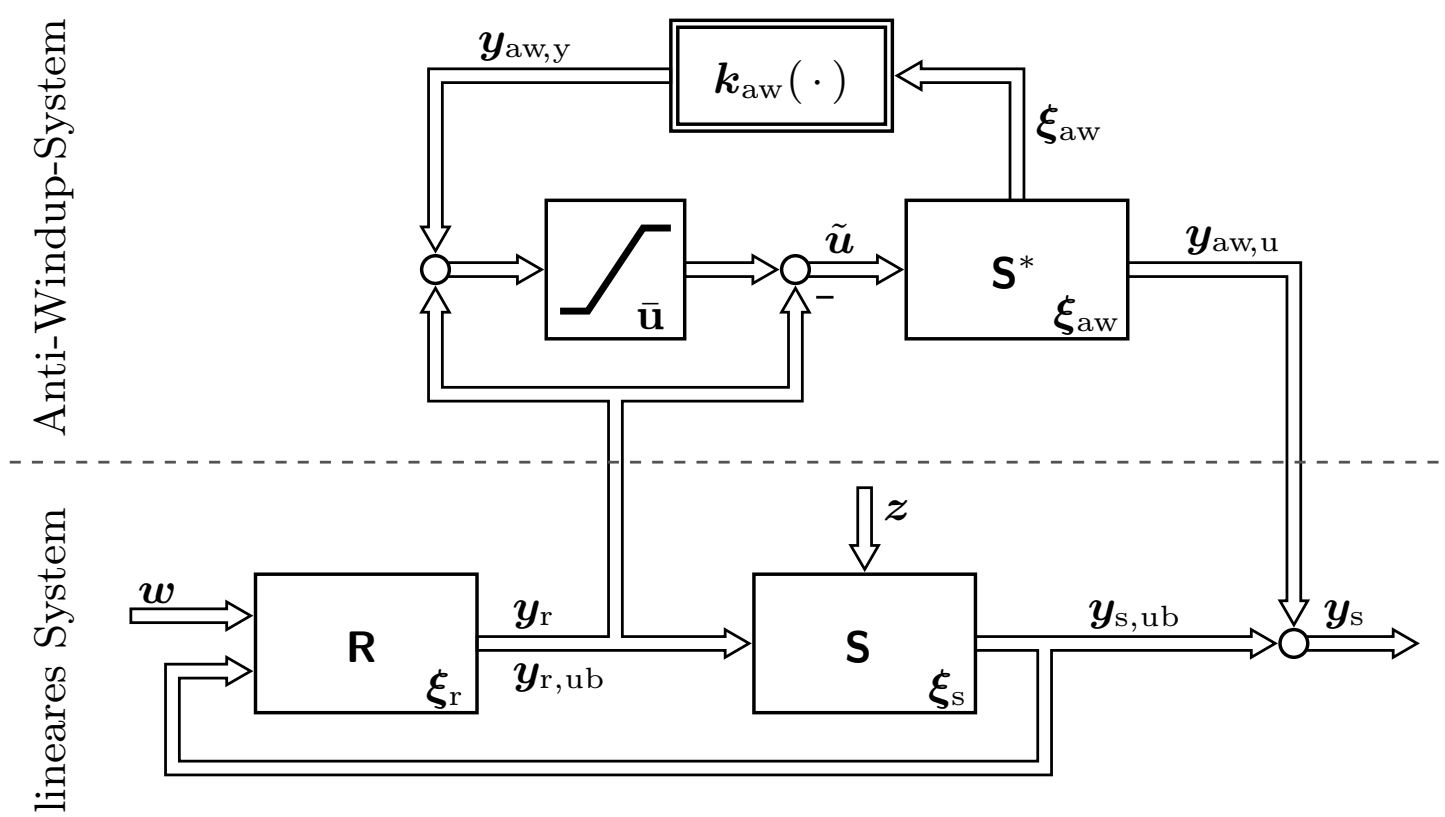

Abbildung 4.5: Umformung des Anti-Windup-Regelkreises in das äquivalente Mismatch-System, das aus dem unbeschränkten Regelkreis (lineares System) und dem Anti-Windup-System besteht. Der Vorteil der Umformung liegt in der Entkopplung der Dynamik des unbeschränkten Regelkreises von der Sättigung und dem Anti-Windup. 
Zusammenfassend lässt sich Folgendes festhalten:

- Die Mismatch-Darstellung entkoppelt das unbeschränkte Systemverhalten von der Sättigungskennlinie und dem Anti-Windup.

- Der Entwurf des Anti-Windup besteht in der Wahl einer nichtlinearen Zustandsrückführung $\boldsymbol{k}_{\text {aw }}$ für das Streckenmodell $\mathbf{S}^{*}$.

- Die Abweichung des Anti-Windup-Regelkreises von dem idealen Systemverhalten $\boldsymbol{y}_{\mathrm{s}, \mathrm{ub}}$ des unbeschränkten Regelkreises ist durch den Ausgang $\boldsymbol{y}_{\text {aw,u }}$ des Anti-Windup-Teilsystems (4.12) gegeben.

- Die Anfangszustände von Anti-Windup-Regelkreis und MismatchSystem sind für den hier betrachteten Fall $\boldsymbol{x}_{\mathrm{aw}}^{(0)}=\mathbf{0}$ identisch.

Problematisch für den Entwurf der Zustandsrückführung $\boldsymbol{k}_{\text {aw }}$ des AntiWindup-Systems ist der Einfluss des linearen Systems durch $\boldsymbol{y}_{\mathrm{r}}$. Eine Lösung beschreibt der nächste Abschnitt.

\subsubsection{Entwurf der Zustandsrückführung}

Betrachtet wird das Anti-Windup-System (4.12) mit der Dynamik

$$
\begin{aligned}
& \dot{\boldsymbol{\xi}}_{\mathrm{aw}}=\boldsymbol{A}_{\mathrm{s}} \boldsymbol{\xi}_{\mathrm{aw}}+\boldsymbol{B}_{\mathrm{s}, u}\left(\operatorname{sat}_{\overline{\mathbf{u}}}\left(\boldsymbol{y}_{\mathrm{r}}+\boldsymbol{y}_{\mathrm{aw}, \mathrm{y}}\right)-\boldsymbol{y}_{\mathrm{r}}\right), \\
& \boldsymbol{y}_{\mathrm{aw}, \mathrm{y}}=\boldsymbol{k}_{\mathrm{aw}}\left(\boldsymbol{\xi}_{\mathrm{aw}}\right), \\
& \boldsymbol{y}_{\mathrm{aw}, \mathrm{u}}=\boldsymbol{C}_{\mathrm{s}} \boldsymbol{\xi}_{\mathrm{aw}} .
\end{aligned}
$$

Die Hauptschwierigkeit bei dem Entwurf der Zustandsrückführung für das Anti-Windup-System stellt die zeitvariante Störgröße $\boldsymbol{y}_{\mathrm{r}}(t)$ dar. Problematisch ist nicht das Auftreten einer Störgröße an sich, sondern dass sie an zwei verschiedenen Stellen der Differentialgleichung auftaucht. In der AntiWindup-Literatur finden sich zwei Lösungen für dieses Problem. Die erste beruht auf einer Umformung des Systems, wobei eine Beziehung zwischen Sättigung und Totzone ausgenutzt wird.

\section{Lösung 1: Zustandsreglerentwurf für ein System mit Totzone}

In $[173,174,191,199]$ wird vorgeschlagen, die Sättigungsfunktion in (4.12) mittels einer Totzone auszudrücken. Zwischen einer Sättigung sat $(\cdot)$ und einer Totzone $\mathrm{dz}(\cdot)$ gilt bekanntlich der Zusammenhang

$$
\mathrm{dz}_{\overline{\mathrm{u}}_{i}}\left(u_{i}\right)=u_{i}-\operatorname{sat}_{\overline{\mathrm{u}}_{\mathrm{i}}}\left(u_{i}\right) .
$$




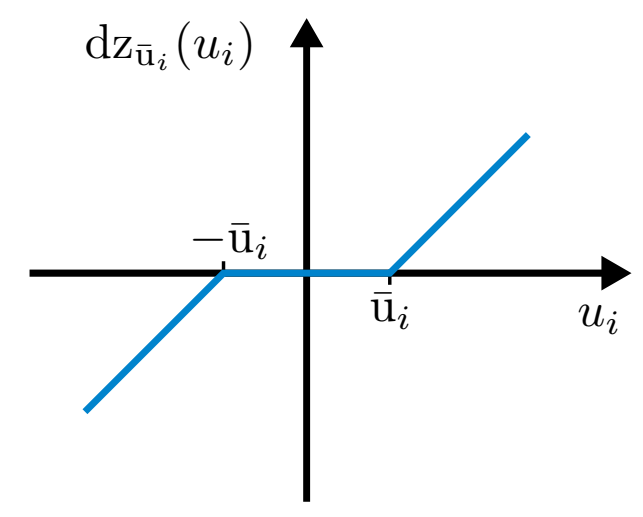

Abbildung 4.6: Totzone.

Eine derartige Totzone ist in Abbildung 4.6 dargestellt und lässt sich abschnittsweise wie folgt schreiben

$$
\mathrm{dz}_{\overline{\mathrm{u}}_{i}}\left(u_{i}\right)=\left\{\begin{array}{lr}
u_{i}+\overline{\mathrm{u}}_{i}, & -\overline{\mathrm{u}}_{i}>u_{i}, \\
0, & -\overline{\mathrm{u}}_{i} \leq u_{i} \leq \overline{\mathrm{u}}_{i}, \\
u_{i}-\overline{\mathrm{u}}_{i}, & u_{i}>\overline{\mathrm{u}}_{i} .
\end{array}\right.
$$

Überträgt man (4.13) auf den mehrdimensionalen Fall, so ergibt sich

$$
\mathbf{d z}_{\overline{\mathbf{u}}}(\boldsymbol{u})=\left[\begin{array}{lll}
\mathrm{dz}_{\overline{\mathbf{u}}_{1}}\left(u_{1}\right) & \ldots & \mathrm{dz}_{\overline{\mathbf{u}}_{m}}\left(u_{m}\right)
\end{array}\right]^{\top}=\boldsymbol{u}-\operatorname{sat}_{\overline{\mathbf{u}}}(\boldsymbol{u}) .
$$

Die Substitution der Sättigung in (4.12) durch eine Totzone führt zu der Systemdarstellung

$$
\dot{\boldsymbol{\xi}}_{\mathrm{aw}}=\boldsymbol{A}_{\mathrm{s}} \boldsymbol{\xi}_{\mathrm{aw}}+\boldsymbol{B}_{\mathrm{s}, u} \boldsymbol{y}_{\mathrm{aw}, \mathrm{y}}-\boldsymbol{B}_{\mathrm{s}, u} \mathbf{d z}_{\overline{\mathbf{u}}}\left(\boldsymbol{y}_{\mathrm{r}}+\boldsymbol{y}_{\mathrm{aw}, \mathrm{y}}\right) .
$$

Besonders für lineare Zustandsregler

$$
\boldsymbol{y}_{\mathrm{aw}, \mathrm{y}}=\boldsymbol{k}_{\mathrm{aw}}\left(\boldsymbol{\xi}_{\mathrm{aw}}\right)=-\underline{\boldsymbol{K}} \boldsymbol{\xi}_{\mathrm{aw}}
$$

ist dieser Ansatz geeignet, da sich das Differentialgleichungssystem

$$
\dot{\boldsymbol{\xi}}_{\mathrm{aw}}=\left(\boldsymbol{A}_{\mathrm{s}}-\boldsymbol{B}_{\mathrm{s}, u} \underline{\boldsymbol{K}}\right) \boldsymbol{\xi}_{\mathrm{aw}}+\boldsymbol{B}_{\mathrm{s}, u} \mathbf{d} \mathbf{z}_{\overline{\mathbf{u}}}\left(\underline{\boldsymbol{K}} \boldsymbol{\xi}_{\mathrm{aw}}-\boldsymbol{y}_{\mathrm{r}}\right)
$$

ergibt. Abbildung 4.7 zeigt das zugehörige Blockschaltbild. In [173, 174, 191] wird beschrieben, wie sich die Matrix $\underline{\boldsymbol{K}}$ einfach bestimmen lässt.

Das umgeformte System der Lösung 1 eignet sich aufgrund der zeitinvarianten Totzone sehr gut für die Analyse der Regelgüte des modellbasierten Anti-Windup, wie in Kapitel 5 gezeigt wird. Nachteile bestehen 


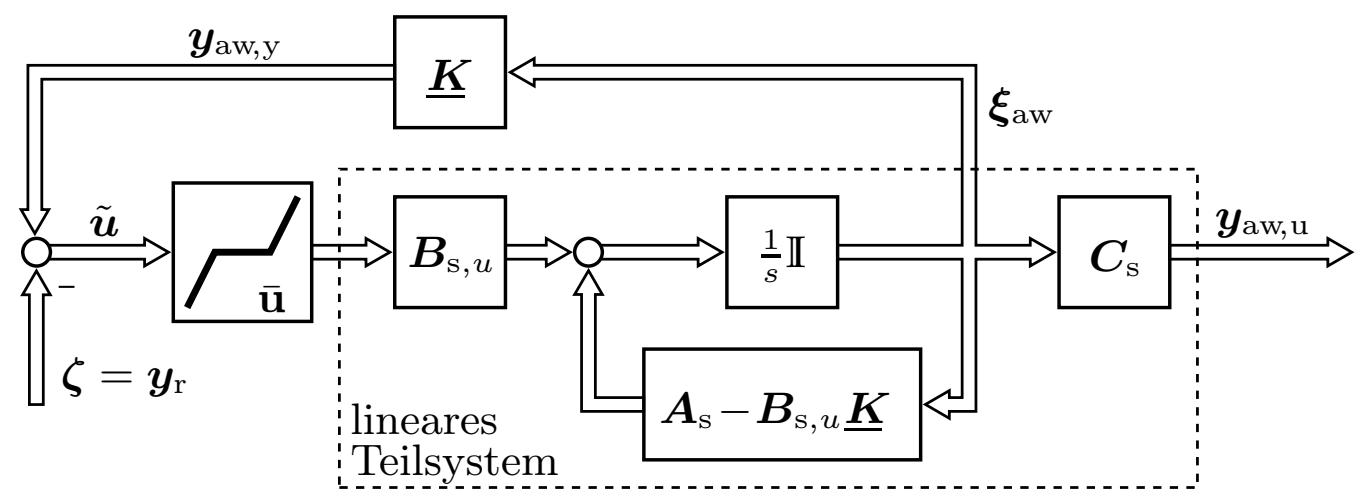

Abbildung 4.7: Blockschaltbild des umgeformten Anti-Windup-Systems mit Totzone und linearem Zustandsregler $\underline{\boldsymbol{K}}$ (Lösung 1).

darin, dass Entwurfsverfahren, die eine Sättigungsnichtlinearität voraussetzen, aufgrund der Totzone nicht angewendet werden können. Außerdem ist der Entwurf von nichtlinearen Zustandsreglern schwierig. Deshalb hat sich eine zweite Lösung etabliert, die nachfolgend beschrieben wird.

\section{Lösung 2: Ein System mit zeitvarianter Sättigung}

Eine alternative Vorgehensweise, die zu einer Systemstruktur ohne Totzone führt und für den Entwurf nichtlinearer Anti-Windup-Maßnahmen besser geeignet ist, wird in $[53,185]$ skizziert. Sie basiert auf einem Ersatzsystem, das zwar wesentliche Eigenschaften von (4.12) besitzt, aber nicht vollständig äquivalent ist. Die Äquivalenz geht durch Abschätzungen verloren, die zwecks einer Vereinfachung der Systemstruktur notwendig sind.

Dieses im Weiteren betrachtete Ersatzsystem besitzt die Dynamik

$$
\begin{aligned}
\dot{\boldsymbol{\xi}}_{\mathrm{aw}} & =\boldsymbol{A}_{\mathrm{s}} \boldsymbol{\xi}_{\mathrm{aw}}+\boldsymbol{B}_{\mathrm{s}, u} \mathbf{s a t}_{[-\overline{\mathbf{u}}+\boldsymbol{p}(t), \overline{\mathbf{u}}+\boldsymbol{p}(t)]}\left(\boldsymbol{y}_{\mathrm{aw}, \mathrm{y}}\right)+\boldsymbol{B}_{\mathrm{s}, u} \boldsymbol{\zeta}, \\
\boldsymbol{y}_{\mathrm{aw}, \mathrm{y}} & =\boldsymbol{k}_{\mathrm{aw}}\left(\boldsymbol{\xi}_{\mathrm{aw}}\right) \\
\boldsymbol{y}_{\mathrm{aw}, \mathrm{u}} & =\boldsymbol{C}_{\mathrm{s}} \boldsymbol{\xi}_{\mathrm{aw}} .
\end{aligned}
$$

Eine ausführliche, verständliche Herleitung ist in der Literatur noch nicht zu finden und deshalb in Abschnitt C.1 ab Seite 193 angegeben.

Abbildung 4.8 zeigt das Blockschaltbild des Systems. Dabei fallen zwei wesentliche Veränderungen gegenüber (4.12) auf. Erstens ist die Sättigungsfunktion sat ${ }_{[-\overline{\mathbf{u}}+\boldsymbol{p}(t), \overline{\mathbf{u}}+\boldsymbol{p}(t)]}$ zeitvariant, d. h., die Beschränkungen der Stellamplitude hängen von einer Größe $\boldsymbol{p}(t)$ ab. Zweitens bezeichnet $\boldsymbol{\zeta}(t)$ die neue Störgröße, die auf den Eingang des Streckenmodells wirkt. 


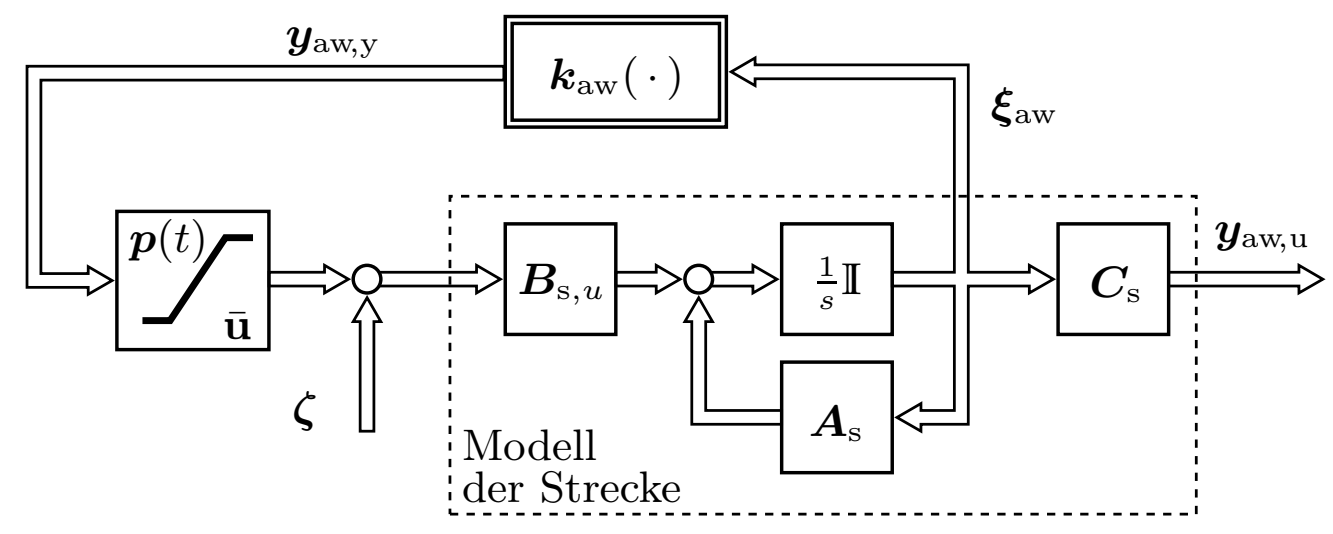

Abbildung 4.8: Blockschaltbild des Ersatzsystems für (4.12) mit nichtlinearem Zustandsregler $\boldsymbol{k}_{\text {aw }}(\cdot)$ und zeitvarianter Sättigung (Lösung 2).

Die beiden neu eingeführten zeitvarianten vektoriellen Größen

$$
\boldsymbol{p}^{\top}=\left[\begin{array}{lll}
p_{1} & \ldots & p_{m}
\end{array}\right], \quad \boldsymbol{\zeta}^{\top}=\left[\begin{array}{lll}
\zeta_{1} & \ldots & \zeta_{m}
\end{array}\right]
$$

hängen über einen beim Entwurf zu wählenden Parameter $\varrho \in(0,1)$ zusammen. Insbesondere gilt

$$
-\varrho \overline{\mathrm{u}}_{i} \leq p_{i} \leq \varrho \overline{\mathrm{u}}_{i} \quad \forall i=1, \ldots, m
$$

sowie die für jede Komponente von $\zeta$ gültige Abschätzung

$$
\left|\zeta_{i}\right| \leq 2\left|\operatorname{sat}_{\varrho \overline{\mathrm{u}}_{i}}\left(y_{\mathrm{r}, i}\right)-y_{\mathrm{r}, i}\right|,
$$

wobei $y_{\mathrm{r}, i}$ die $i$-te Komponente der Stellgröße des unbeschränkten Regelkreises (4.11) ist.

Somit steht beim Entwurf nicht nur ein Ersatzsystem, sondern ein ganzes Kontinuum an Ersatzsystemen zur Verfügung, die sich durch $\varrho \in(0,1)$ unterscheiden. Bei der Wahl des Ersatzsystems ist eine Abwägung zwischen der zur Verfügung stehenden Stellgröße und dem Betrag der Störgröße zu treffen. Je größer $\varrho$ ist, desto stärker können die Sättigungsgrenzen variieren, wie Abbildung 4.9 zeigt. Andererseits wird der Betrag in (4.17) für ein größeres $\varrho$ kleiner und damit die Abschätzung der auf das System wirkenden Störgröße.

Zusammenfassend lässt sich festhalten, dass (4.15) mit den Abschätzungen (4.16), (4.17) und $\varrho \in(0,1)$ ein Ersatzsystem für (4.12) darstellt, das für den Entwurf des Zustandsreglers verwendet werden kann. Erfüllt das geregelte Ersatzsystem bestimmte Entwurfsanforderungen, dann werden diese ebenfalls von dem System (4.12) erfüllt. Diese Aussage präzisiert 


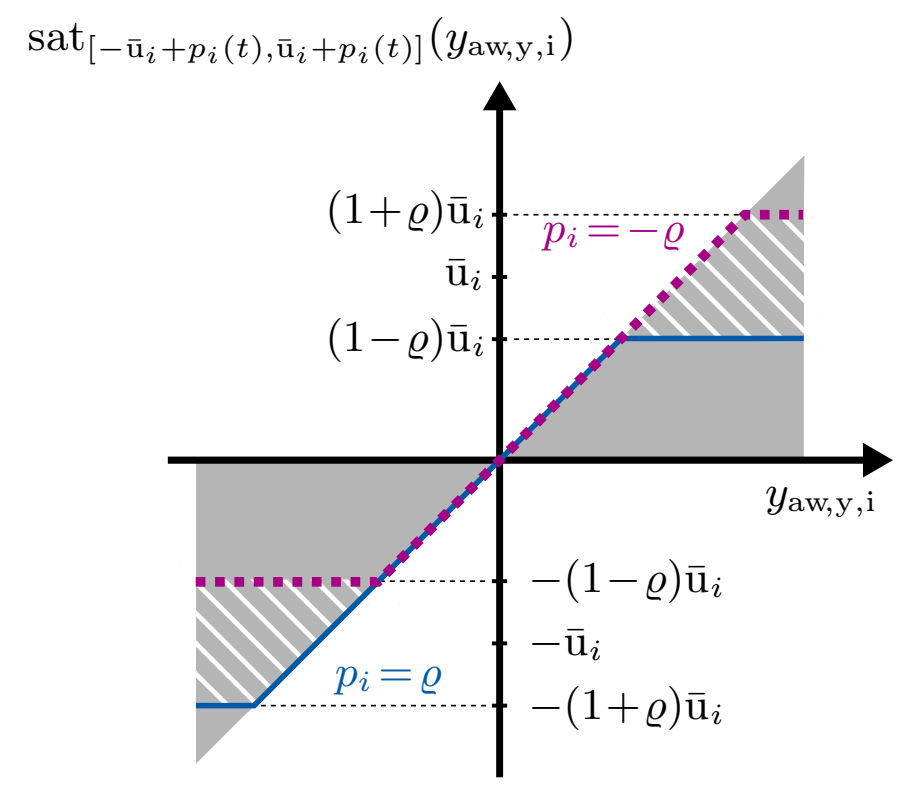

Abbildung 4.9: Entlang der ersten Winkelhalbierenden verschobene Sättigungsfunktion sat ${ }_{\left[-\overline{\mathrm{u}}_{i}+p_{i}(t), \overline{\mathrm{u}}_{i}+p_{i}(t)\right]}\left(y_{\mathrm{aw}, \mathrm{y}, \mathrm{i}}\right)$. Gezeigt sind die Sonderfälle für $p_{i}=$ $\varrho \overline{\mathrm{u}}_{i}$ in $(-)$ und für $p_{i}=-\varrho \overline{\mathrm{u}}_{i}$ in $(\cdots)$. Für $p_{i}(t) \in\left[-\varrho \overline{\mathrm{u}}_{i}, \varrho \overline{\mathrm{u}}_{i}\right]$ liegt die Sättigungsfunktion in dem weiß schraffierten Bereich.

Satz 4.3 (nach einer Idee aus [53]). Gegeben sei ein Zustandsregler $\boldsymbol{y}_{\text {aw,y }}=\boldsymbol{k}_{\text {aw }}\left(\boldsymbol{\xi}_{\text {aw }}\right)$ und ein beliebiges $\varrho \in(0,1)$. Für beliebige Anfangswerte $\boldsymbol{\xi}_{\text {aw }}(0)$ und Eingangssignale $\boldsymbol{y}_{\mathrm{r}}$ ist jede Trajektorie $\boldsymbol{\xi}_{\text {aw }}(t)$ des Systems (4.12) ebenfalls eine Trajektorie des Ersatzsystems (4.15) mit (4.16), (4.17), wenn die Verläufe von $\boldsymbol{p}(t)$ und $\boldsymbol{\zeta}(t)$ geeignet gewählt werden.

Beweis. In Abschnitt C.1 des Anhangs wird gezeigt, dass die Systeme (4.12) und (4.15) äquivalent sind für die spezielle Wahl

$$
\begin{aligned}
& \boldsymbol{p}=\operatorname{sat}_{\varrho \overline{\mathbf{u}}}\left(\boldsymbol{y}_{\mathrm{r}}\right), \\
& \boldsymbol{\zeta}=\boldsymbol{p}+\operatorname{sat}_{\overline{\mathbf{u}}}\left(\boldsymbol{y}_{\mathrm{r}}+\boldsymbol{y}_{\mathrm{aw}, \mathrm{y}}\right)-\mathbf{s a t}_{\overline{\mathbf{u}}}\left(\boldsymbol{p}+\boldsymbol{y}_{\mathrm{aw}, \mathrm{y}}\right)-\boldsymbol{y}_{\mathrm{r}} .
\end{aligned}
$$

Des Weiteren gilt nach Lemma C.2 in Abschnitt C.1, dass $\boldsymbol{\zeta}(t)$ der Abschätzung (4.17) genügt. Die Abschätzung (4.16) gilt offensichtlich für die angegebene spezielle Wahl $\boldsymbol{p}=\operatorname{sat}_{\varrho \overline{\mathbf{u}}}\left(\boldsymbol{y}_{\mathrm{r}}\right)$.

\section{Existierende Entwurfsverfahren}

Die in den letzten beiden Abschnitten vorgestellten Lösungen weisen jeweils bestimmte Vor- und Nachteile auf. Tabelle 4.2 stellt beide Varianten gegenüber. In der Literatur kommen beide Lösungen für den Entwurf von modellbasierten Anti-Windup-Maßnahmen zum Einsatz. 
Tabelle 4.2: Gegenüberstellung der zwei existierenden Lösungen für den Entwurf modellbasierter Anti-Windup-Maßnahmen.

\begin{tabular}{lll}
\hline & $\begin{array}{l}\text { Lösung 1: } \\
\text { Umformung }\end{array}$ & $\begin{array}{l}\text { Lösung 2: } \\
\text { Ersatzsystem }\end{array}$ \\
\hline Ursprung & {$[199]$} & {$[185]$} \\
Äquivalenz mit (4.12) & ja & nein \\
Struktur von $\boldsymbol{k}_{\text {aw }}$ & linear & nichtlinear \\
Nichtlinearität & Totzone & zeitvariante Sättigung \\
\hline
\end{tabular}

Tabelle 4.3: Entwurfsverfahren für modellbasiertes Anti-Windup. Verfahren, die eine asymptotisch stabile Strecke voraussetzen, sind blau hervorgehoben. Eine ausführlichere Übersicht findet sich in den Arbeiten [55, 182, 183, 211].

\begin{tabular}{lll}
\hline Gütekriterium & Lösung $\mathbf{1}$ & Lösung $\mathbf{2}$ \\
\hline$L_{2}$-Verstärkung & {$[80][173][174]$} & {$[53][210][211]$} \\
\hline quadratisches Gütemaß & - & \\
\hline \multirow{2}{*}{ anderes/kein Gütemaß } & {$[191]$} & {$[209][211]$} \\
\hline
\end{tabular}

Eine weitere Unterteilung der Entwurfsverfahren lässt sich hinsichtlich der Entwurfsziele und Gütekriterien vornehmen, wie Tabelle 4.3 zeigt. Das am meisten genutzte Gütekriterium für die Bewertung der Zustandsrückführung ist die $L_{2}$-Verstärkung (vgl. Definition 4.2) des Anti-WindupSystems bzw. die $L_{2}$-Verstärkung des für den Entwurf verwendeten Ersatzsystems vom Störeingang $\boldsymbol{\zeta}$ zum Ausgang $\boldsymbol{y}_{\text {aw, }}$. Eine kleine $L_{2}$-Verstärkung $\gamma$ impliziert eine kleine $L_{2}$-Norm von $\boldsymbol{y}_{\text {aw, }}$. Die vorhandene Abweichung zwischen dem Anti-Windup-Regelkreis und dem unbeschränkten Regelkreis ist somit klein im Sinne der $L_{2}$-Norm. 
Andere Verfahren [209, 211] nutzen ein quadratisches Gütemaß der Form

$$
\int_{0}^{\infty} \boldsymbol{\xi}_{\mathrm{aw}}^{\mathrm{T}}(t) \boldsymbol{Q} \boldsymbol{\xi}_{\mathrm{aw}}(t)+\boldsymbol{y}_{\mathrm{aw}, \mathrm{y}}^{\top}(t) \boldsymbol{R} \boldsymbol{y}_{\mathrm{aw}, \mathrm{y}}(t) d t
$$

für den Entwurf einer linearen Zustandsrückführung. Die Arbeiten [185, 208] geben kein Gütekriterium für den Entwurf an. Der Fokus liegt auf dem Entwurf einer global stabilisierenden Zustandsrückführung.

\subsection{Modulares Anti-Windup}

Modulares Anti-Windup [26, 83-89] setzt auf eine separate Behandlung der Windup-Effekte, d. h., Regler- und Strecken-Windup sowie das Direktionalitätsproblem werden getrennt voneinander durch verschiedene strukturelle Maßnahmen beseitigt.

\subsubsection{Vermeidung von Regler-Windup}

Regler-Windup wird durch die sogenannte Beobachtertechnik vermieden [82, 85, 87]. Der Grundgedanke ist, Windup als eine durch Stellbegrenzungen ausgelöste Inkonsistenz zwischen dem Reglerzustand $\boldsymbol{x}_{\mathrm{r}}$ und dem Streckeneingang $\boldsymbol{u}_{\mathrm{s}}$ zu interpretieren. Die Aufgabe des Anti-Windup besteht dann darin, beide Größen wieder in Übereinstimmung zu bringen.

Beim klassischen Integral-Windup beispielsweise wächst der Zustand des Integrierers an, die Eingangsgröße der Strecke bleibt allerdings aufgrund der Sättigung konstant. Die Lösung besteht im Anhalten des Integrierers. Die Beobachtermethode überträgt diesen Ansatz auf allgemeine Regler.

\section{Grundprinzip: Beobachterfehler vermeiden}

Es stellt sich die Frage, wie eine Anpassung der Reglerzustände an die Eingangsgröße der Strecke zu bewerkstelligen ist. Für Kontrollbeobachter, d. h. beobachterbasierte Zustandsregler, ist diese Frage einfach zu beantworten. Der Reglerzustand stellt nämlich in diesem Fall eine Schätzung des Streckenzustands dar und muss deshalb auch im sättigenden Betrieb möglichst nahe am Zustand der Strecke liegen.

Um zu demonstrieren, dass dies im Allgemeinen nicht der Fall ist, wird der in Abbildung 4.10 dargestellte Kontrollbeobachter mit dem Zustand $\boldsymbol{x}_{\mathrm{r}}$ betrachtet $[3,8,82,88]$. In Schalterstellung I benutzt der Beobachter 


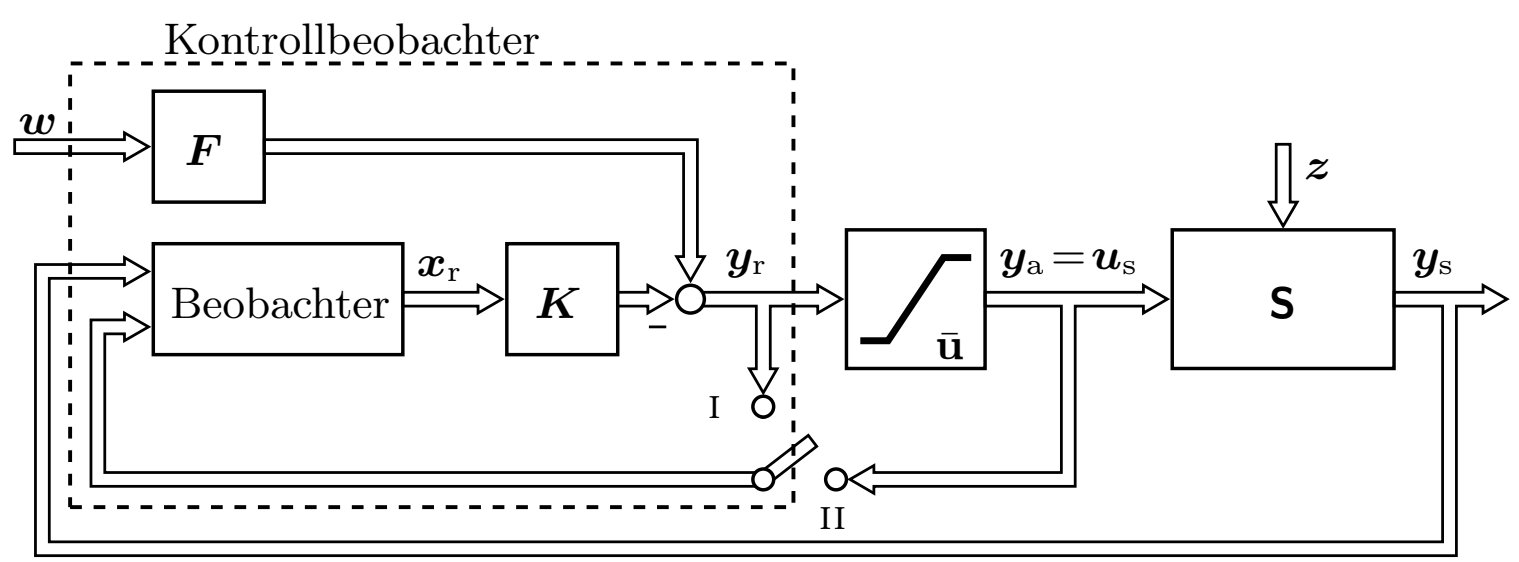

Abbildung 4.10: Vermeidung von Regler-Windup bei Kontrollbeobachtern. Dem Beobachter wird nicht der Reglerausgang $\boldsymbol{y}_{\mathrm{r}}$ (Schalterstellung I), sondern der Aktorausgang bzw. Streckeneingang $\boldsymbol{u}_{\mathrm{s}}$ (Schalterstellung II) zugeführt.

zur Rekonstruktion der Streckenzustände $\boldsymbol{x}_{\mathrm{s}}$ die Ausgangsgröße $\boldsymbol{y}_{\mathrm{r}}$ des Reglers. Mit einer geeignet gewählten Beobachtermatrix $\boldsymbol{L}_{\mathrm{bm}}$ ergibt sich für die Dynamik des Beobachters

$$
\dot{\boldsymbol{x}}_{\mathrm{r}}=\boldsymbol{A}_{\mathrm{s}} \boldsymbol{x}_{\mathrm{r}}+\boldsymbol{B}_{\mathrm{s}, u} \boldsymbol{y}_{\mathrm{r}}+\boldsymbol{L}_{\mathrm{bm}}\left(\boldsymbol{y}_{\mathrm{s}}-\boldsymbol{C}_{\mathrm{s}} \boldsymbol{x}_{\mathrm{r}}\right) .
$$

Solange die Stellgröße innerhalb der Sättigungsgrenzen bleibt, verhält sich der Aktor wie ein ideales Stellglied, d. h. $\boldsymbol{y}_{\mathrm{r}}=\boldsymbol{u}_{\mathrm{a}}=\boldsymbol{y}_{\mathrm{a}}=\boldsymbol{u}_{\mathrm{s}}$. Der Reglerausgang entspricht folglich dem Streckeneingang und es lässt sich für $\boldsymbol{z}=\mathbf{0}$ leicht zeigen, dass eine stabile Schätzfehlerdynamik

$$
\dot{\boldsymbol{x}}_{\mathrm{s}}-\dot{\boldsymbol{x}}_{\mathrm{r}}=\left(\boldsymbol{A}_{\mathrm{s}}-\boldsymbol{L}_{\mathrm{bm}} \boldsymbol{C}_{\mathrm{s}}\right)\left(\boldsymbol{x}_{\mathrm{s}}-\boldsymbol{x}_{\mathrm{r}}\right)
$$

vorliegt. Initiale Beobachterfehler $\boldsymbol{x}_{\mathrm{s}}^{(0)}-\boldsymbol{x}_{\mathrm{r}}^{(0)}$ klingen asymptotisch ab und der Zustand $\boldsymbol{x}_{\mathrm{r}}$ stellt nach einiger Zeit eine gute Schätzung von $\boldsymbol{x}_{\mathrm{s}}$ dar.

Im Sättigungsfall kann der Aktor die vom Regler angeforderte Stellgröße $\boldsymbol{y}_{\mathrm{r}}$ nicht mehr auf die Strecke übertragen und es gilt $\boldsymbol{u}_{\mathrm{s}}=\boldsymbol{y}_{\mathrm{a}} \neq \boldsymbol{u}_{\mathrm{a}}=\boldsymbol{y}_{\mathrm{r}}$. Dann weicht auch die Eingangsgröße der Strecke $\boldsymbol{u}_{\mathrm{s}}$ von der des Beobachters ab und die Dynamik des Schätzfehlers ergibt sich zu

$$
\dot{\boldsymbol{x}}_{\mathrm{s}}-\dot{\boldsymbol{x}}_{\mathrm{r}}=\left(\boldsymbol{A}_{\mathrm{s}}-\boldsymbol{L}_{\mathrm{bm}} \boldsymbol{C}_{\mathrm{s}}\right)\left(\boldsymbol{x}_{\mathrm{s}}-\boldsymbol{x}_{\mathrm{r}}\right)+\boldsymbol{B}_{\mathrm{s}, u}\left(\boldsymbol{u}_{\mathrm{s}}-\boldsymbol{y}_{\mathrm{r}}\right) .
$$

Durch die Differenz $\boldsymbol{u}_{\mathrm{s}}-\boldsymbol{y}_{\mathrm{r}}$ werden Beobachterfehler ausgelöst und die Stellgröße $\boldsymbol{y}_{\mathrm{r}}$ wird auf Basis einer fehlerhaften Schätzung der Streckenzustände bestimmt. Das führt zu einer Verschlechterung der Regelgüte, die als Windup sichtbar wird. 
Diese Inkonsistenz zwischen Reglerzuständen und Streckeneingang lässt sich durch Umlegen des Schalters in Position II vermeiden. Die Eingangsgröße des Beobachters ist dann immer identisch mit der Eingangsgröße der Strecke. Der Beobachterfehler weist folglich im Sättigungsfall die Dynamik (4.18) auf und ein Hochlaufen der Beobachterzustände wird vermieden.

\section{Alternative Sichtweise: Reglerdynamik stabilisieren}

Eine alternative Erklärung der Windup-Phänomene bei Kontrollbeobachtern liefert eine Betrachtung der Reglereigenwerte. Der Kontrollbeobachter in Schalterstellung I ist ein linearer, dynamischer Regler und wird durch

$$
\mathrm{KB}:\left\{\begin{array}{l}
\dot{\boldsymbol{x}}_{\mathrm{r}}=\boldsymbol{A}_{\mathrm{s}} \boldsymbol{x}_{\mathrm{r}}+\boldsymbol{B}_{\mathrm{s}, u} \boldsymbol{y}_{\mathrm{r}}+\boldsymbol{L}_{\mathrm{bm}}\left(\boldsymbol{y}_{\mathrm{s}}-\boldsymbol{C}_{\mathrm{s}} \boldsymbol{x}_{\mathrm{r}}\right) \\
\boldsymbol{y}_{\mathrm{r}}=-\boldsymbol{K} \boldsymbol{x}_{\mathrm{r}}+\boldsymbol{F} \boldsymbol{w}
\end{array}\right.
$$

beschrieben. Einsetzen des Ausgangs in die Differentialgleichung liefert

$$
\dot{\boldsymbol{x}}_{\mathrm{r}}=\boldsymbol{A}_{\mathrm{r}} \boldsymbol{x}_{\mathrm{r}}+\boldsymbol{L}_{\mathrm{bm}} \boldsymbol{y}_{\mathrm{s}}+\boldsymbol{B}_{\mathrm{s}, u} \boldsymbol{F} \boldsymbol{w}
$$

mit der Systemmatrix des Kontrollbeobachters

$$
\boldsymbol{A}_{\mathrm{r}}=\boldsymbol{A}_{\mathrm{s}}-\boldsymbol{L}_{\mathrm{bm}} \boldsymbol{C}_{\mathrm{s}}-\boldsymbol{B}_{\mathrm{s}, u} \boldsymbol{K} .
$$

Die Eigenwerte von $\boldsymbol{A}_{\mathrm{r}}$ werden beim Entwurf nicht explizit festgelegt und können deshalb rein imaginär sein oder sogar positive Realteile aufweisen. Der lineare Regelkreis ist natürlich trotzdem stabil, da das Separationstheorem gilt und somit die Eigenwerte des geschlossenen Regelkreises den Eigenwerten der geregelten Strecke und den Eigenwerten des Beobachters entsprechen [133]. Im Sättigungsfall ist der Regelkreis allerdings unterbrochen (vgl. Abbildung 2.4) und die grenz- oder instabile Reglerdynamik (4.19) wird nicht mehr durch eine Rückkopplung stabilisiert. Das führt zu einem Anwachsen des Reglerzustands und großen Beobachterfehlern.

Dieses Regler-Windup lässt sich durch Schalterstellung II verhindern. Anstatt $\boldsymbol{y}_{\mathrm{r}}$ wird dem Regler jetzt $\boldsymbol{y}_{\mathrm{a}}=\mathbf{s a t}_{\overline{\mathbf{u}}}\left(\boldsymbol{y}_{\mathrm{r}}\right)$ zugeführt. Es gilt

$$
\begin{aligned}
\dot{\boldsymbol{x}}_{\mathrm{r}} & =\boldsymbol{A}_{\mathrm{s}} \boldsymbol{x}_{\mathrm{r}}+\boldsymbol{B}_{\mathrm{s}, u} \boldsymbol{s a t}_{\overline{\mathbf{u}}}\left(\boldsymbol{y}_{\mathrm{r}}\right)+\boldsymbol{L}_{\mathrm{bm}}\left(\boldsymbol{y}_{\mathrm{s}}-\boldsymbol{C}_{\mathrm{s}} \boldsymbol{x}_{\mathrm{r}}\right) \\
& =\left(\boldsymbol{A}_{\mathrm{s}}-\boldsymbol{L}_{\mathrm{bm}} \boldsymbol{C}_{\mathrm{s}}\right) \boldsymbol{x}_{\mathrm{r}}+\boldsymbol{B}_{\mathrm{s}, u} \boldsymbol{s a t}_{\overline{\mathbf{u}}}\left(\boldsymbol{y}_{\mathrm{r}}\right)+\boldsymbol{L}_{\mathrm{bm}} \boldsymbol{y}_{\mathrm{s}} .
\end{aligned}
$$

Die Systemmatrix des Reglers entspricht dann der des Beobachters, d. h. $\boldsymbol{A}_{\mathrm{r}}=\boldsymbol{A}_{\mathrm{s}}-\boldsymbol{L}_{\mathrm{bm}} \boldsymbol{C}_{\mathrm{s}}$, und weist somit Eigenwerte mit ausschließlich negativem Realteil auf. 


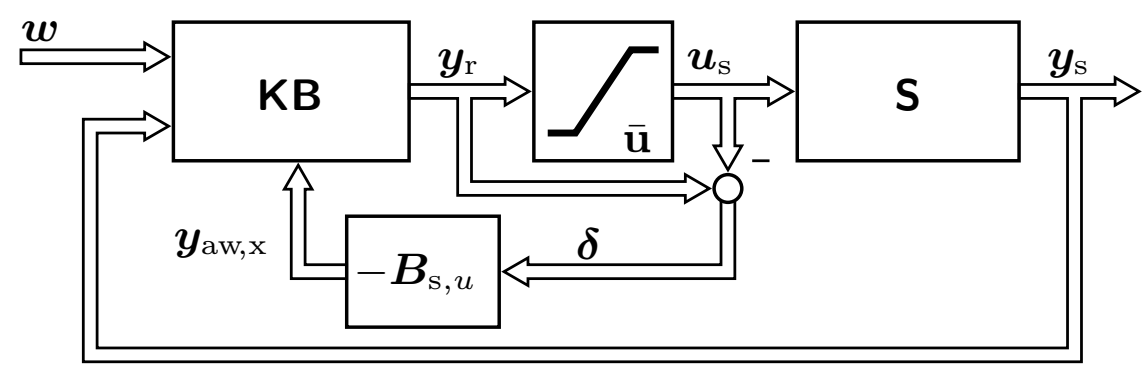

Abbildung 4.11: Regelkreis mit Kontrollbeobachter gemäß Abbildung 4.10. Die Schalterstellung II wird durch ein statisches Anti-Windup realisiert.

Die Beobachtertechnik für Kontrollbeobachter lässt sich demnach wie folgt zusammenfassen: Im Sättigungsfall werden dem Kontrollbeobachter die stabilen Eigenwerte des Beobachters zugewiesen. Dadurch wird ein starkes Anwachsen der Reglerzustände vermieden und Windup verhindert.

\section{Beziehung zu statischen Anti-Windup-Maßnahmen}

Die Beobachtertechnik lässt sich auch als statisches Anti-Windup realisieren. Dazu wird die Systemdynamik des Kontrollbeobachters (4.19) in Schalterstellung I betrachtet. Die Addition von $\boldsymbol{B}_{\mathrm{s}, u}\left(\mathbf{s a t}_{\overline{\mathbf{u}}}\left(\boldsymbol{y}_{\mathrm{r}}\right)-\boldsymbol{y}_{\mathrm{r}}\right)$ ändert die Dynamik des Reglers wie folgt

$$
\begin{aligned}
\dot{\boldsymbol{x}}_{\mathrm{r}} & =\boldsymbol{A}_{\mathrm{r}} \boldsymbol{x}_{\mathrm{r}}+\boldsymbol{L}_{\mathrm{bm}} \boldsymbol{y}_{\mathrm{s}}+\boldsymbol{B}_{\mathrm{s}, u} \boldsymbol{F} \boldsymbol{w}+\boldsymbol{B}_{\mathrm{s}, u}\left(\mathbf{s a t}_{\overline{\mathbf{u}}}\left(\boldsymbol{y}_{\mathrm{r}}\right)-\boldsymbol{y}_{\mathrm{r}}\right) \\
& =\left(\boldsymbol{A}_{\mathrm{s}}-\boldsymbol{L}_{\mathrm{bm}} \boldsymbol{C}_{\mathrm{s}}\right) \boldsymbol{x}_{\mathrm{r}}+\boldsymbol{B}_{\mathrm{s}, u} \mathbf{s a t}_{\overline{\mathbf{u}}}\left(\boldsymbol{y}_{\mathrm{r}}\right)+\boldsymbol{L}_{\mathrm{bm}} \boldsymbol{y}_{\mathrm{s}} .
\end{aligned}
$$

Das entspricht (4.20). Das Umlegen des Schalters in Position II ist demnach im Fall eines Kontrollbeobachters äquivalent zu einer Erweiterung des Regelkreises um ein lineares statisches Anti-Windup aus Abschnitt 4.3 gemäß (4.5) mit $\boldsymbol{D}_{\mathrm{aw}, \mathrm{x}}=-\boldsymbol{B}_{\mathrm{s}, u}$. Abbildung 4.11 illustriert dies.

Es liegt nahe, diese Vorgehensweise auf allgemeine Regler $\mathbf{R}$ zu übertragen $[7,8,196]$. Die Anti-Windup-Matrix $\boldsymbol{D}_{\mathrm{aw}, \mathrm{x}}$ ist dabei so zu wählen, dass im Sättigungsfall eine stabile Reglerdynamik

$$
\begin{aligned}
\dot{\boldsymbol{x}}_{\mathrm{r}} & =\boldsymbol{A}_{\mathrm{r}} \boldsymbol{x}_{\mathrm{r}}+\boldsymbol{B}_{\mathrm{r}, u} \boldsymbol{u}_{\mathrm{r}}+\boldsymbol{B}_{\mathrm{r}, w} \boldsymbol{w}+\boldsymbol{D}_{\mathrm{aw}, \mathrm{x}} \boldsymbol{\delta} \\
& =\boldsymbol{A}_{\mathrm{r}} \boldsymbol{x}_{\mathrm{r}}+\boldsymbol{B}_{\mathrm{r}, u} \boldsymbol{u}_{\mathrm{r}}+\boldsymbol{B}_{\mathrm{r}, w} \boldsymbol{w}+\boldsymbol{D}_{\mathrm{aw}, \mathrm{x}}\left(\boldsymbol{y}_{\mathrm{r}}-\operatorname{sat}_{\overline{\mathbf{u}}}\left(\boldsymbol{y}_{\mathrm{r}}\right)\right)
\end{aligned}
$$

resultiert. Einsetzen des Reglerausganges

$$
\boldsymbol{y}_{\mathrm{r}}=\boldsymbol{C}_{\mathrm{r}} \boldsymbol{x}_{\mathrm{r}}+\boldsymbol{D}_{\mathrm{r}, u} \boldsymbol{u}_{\mathrm{r}}+\boldsymbol{D}_{\mathrm{r}, w} \boldsymbol{w}
$$


führt schließlich zu der Differentialgleichung

$$
\begin{aligned}
\dot{\boldsymbol{x}}_{\mathrm{r}}=\left(\boldsymbol{A}_{\mathrm{r}}+\boldsymbol{D}_{\mathrm{aw}, \mathrm{x}} \boldsymbol{C}_{\mathrm{r}}\right) \boldsymbol{x}_{\mathrm{r}}+\left(\boldsymbol{B}_{\mathrm{r}, u}+\boldsymbol{D}_{\mathrm{aw}, \mathrm{x}} \boldsymbol{D}_{\mathrm{r}, u}\right) \boldsymbol{u}_{\mathrm{r}}+ \\
\quad\left(\boldsymbol{B}_{\mathrm{r}, w}+\boldsymbol{D}_{\mathrm{aw}, \mathrm{x}} \boldsymbol{D}_{\mathrm{r}, w}\right) \boldsymbol{w}-\boldsymbol{D}_{\mathrm{aw}, \mathrm{x}} \mathbf{s a t}_{\overline{\mathbf{u}}}\left(\boldsymbol{y}_{\mathrm{r}}\right) .
\end{aligned}
$$

Offensichtlich garantiert die Beobachtbarkeit des Matrixpaares $\left(\boldsymbol{A}_{\mathrm{r}}, \boldsymbol{C}_{\mathrm{r}}\right)$, dass sich die Eigenwerte der Systemmatrix beliebig über $\boldsymbol{D}_{\mathrm{aw}, \mathrm{x}}$ festlegen lassen [133]. Im Sättigungsfall ist die Dynamik des Reglers dann nicht durch die Matrix $\boldsymbol{A}_{\mathrm{r}}$, sondern durch die Eigenwerte von $\boldsymbol{A}_{\mathrm{r}}+\boldsymbol{D}_{\mathrm{aw}, \mathrm{x}} \boldsymbol{C}_{\mathrm{r}}$ bestimmt. Dieser Ansatz wird auch als generalized Anti-Windup (GAW) bezeichnet und ist beispielsweise in $[7,196]$ detailliert beschrieben.

\section{Beobachtertechnik als Spezialfall des GAW}

Die Beobachtertechnik ergibt sich als Spezialfall des GAW durch eine weitere Anforderung an die Eigenwerte von $\boldsymbol{A}_{\mathrm{r}}+\boldsymbol{D}_{\mathrm{aw}, \mathrm{x}} \boldsymbol{C}_{\mathrm{r}}$. Diese müssen mit $n_{\mathrm{r}}$ der insgesamt $n_{\mathrm{r}}+n_{\mathrm{s}}$ Eigenwerte des unbeschränkten Regelkreises übereinstimmen $^{5}$. Das führt unmittelbar zu der Frage, welche Eigenwerte zu wählen sind. Schon im SISO-Fall existieren bis zu

$$
\left(\begin{array}{c}
n_{\mathrm{s}}+n_{\mathrm{r}} \\
n_{\mathrm{r}}
\end{array}\right)=\frac{\left(n_{\mathrm{s}}+n_{\mathrm{r}}\right) !}{n_{\mathrm{s}} ! n_{\mathrm{r}} !}
$$

Möglichkeiten. Im Prinzip wird durch die Wahl der Eigenwerte eine (virtuelle) Beobachterdynamik festgelegt. Diese Festlegung ist in einem Regelkreis mit Strecke und Kontrollbeobachter eindeutig gegeben, in einem allgemeinen Regelkreis aber nicht, da unklar ist, welche Eigenwerte der geregelten Strecke und welche dem Beobachter zuzuordnen sind.

Eine Auswahlregel existiert in den bisherigen Arbeiten [26, 85, 87-90] nicht. Die naheliegende Wahl, die schnellsten $n_{\mathrm{r}}$ der $n_{\mathrm{r}}+n_{\mathrm{s}}$ Eigenwerte dem Beobachter und damit dem Regler zuzuordnen, kann zu einer extrem unbefriedigenden Regelgüte führen, wie in Abschnitt 5.4.2 an einem Beispiel demonstriert wird. Man ist deshalb auf Ausprobieren und Bewertung der Regelgüte durch Simulationen angewiesen. Eine systematischere Lösung wird in Kapitel 5 vorgestellt.

Sind die $n_{\mathrm{r}}$ Eigenwerte festgelegt, ist das statische Anti-Windup im SISO-Fall eindeutig bestimmt, da der Vektor $\boldsymbol{D}_{\mathrm{aw}, \mathrm{x}}=\boldsymbol{d}_{\mathrm{aw}, \mathrm{x}} \in \mathbb{R}^{n_{\mathrm{r}}}$ die

${ }^{5}$ Das ist dadurch begründet, dass die Beobachtertechnik dem Kontrollbeobachter im Sättigungsfall die Eigenwerte des Beobachters zuweist. Bekanntlich bilden die $n_{\mathrm{r}}$ Beobachtereigenwerte zusammen mit den Eigenwerten der geregelten Strecke die $n_{\mathrm{r}}+n_{\mathrm{s}}$ der Eigenwerte des unbeschränkten Regelkreises. 


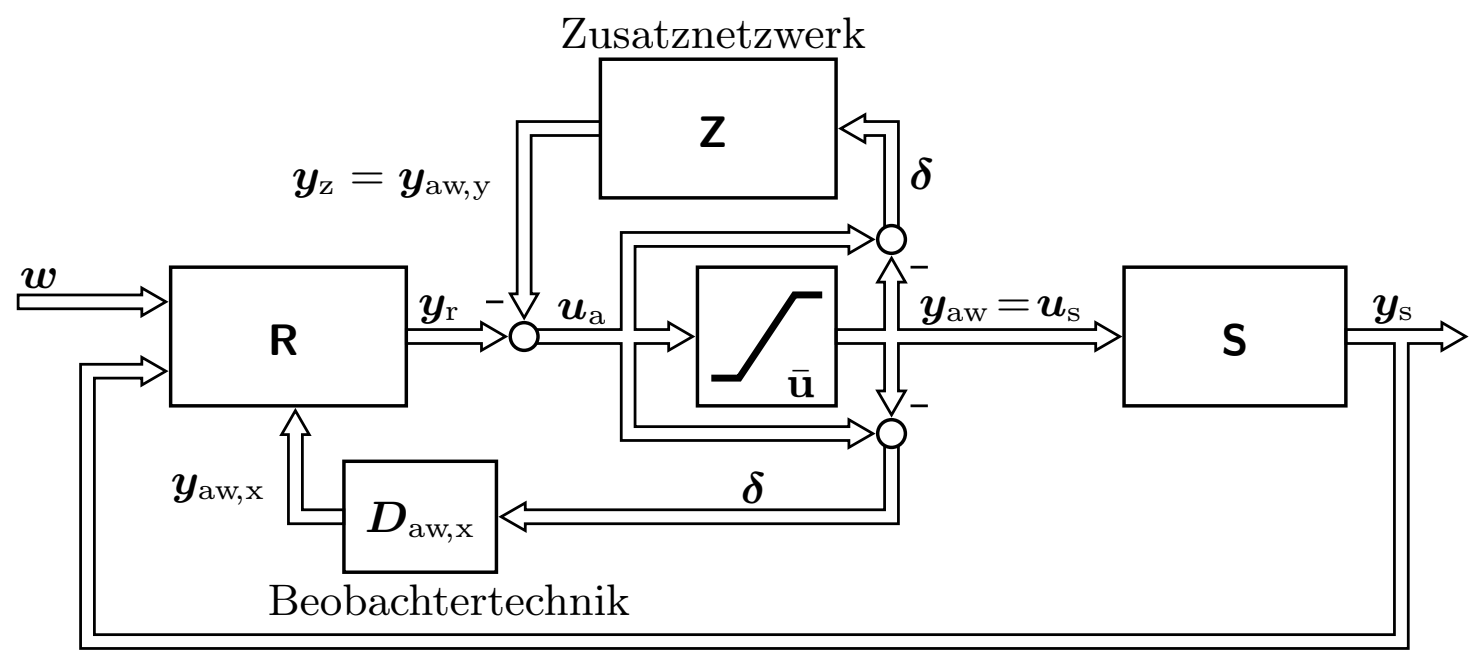

Abbildung 4.12: Modulares Anti-Windup. Regler-Windup wird durch die Beobachtertechnik vermieden, Strecken-Windup durch ein Zusatznetzwerk Z.

Dimension $n_{\mathrm{r}}$ besitzt. Im MIMO-Fall, d. h. $\boldsymbol{D}_{\mathrm{aw}, \mathrm{x}} \in \mathbb{R}^{n_{\mathrm{r}} \times m}$, können noch weitere $n_{\mathrm{r}}(m-1)$ Freiheitsgrade beim Entwurf ausgenutzt werden. Das verkompliziert den Entwurf und nimmt der Beobachtertechnik ihren Hauptvorteil, die einfache Anwendbarkeit.

\subsubsection{Vermeidung von Strecken-Windup}

Durch die Vermeidung des Regler-Windup mittels der Beobachtertechnik wird ein Hochlaufen der Reglerzustände im Sättigungsfall verhindert. Trotzdem kann es zu unerwünschten Verschlechterungen der Regelgüte im Sättigungsfall kommen. Diese negativen Auswirkungen der Sättigung sind auf das Strecken-Windup (vgl. Abschnitt 2.2.2) zurückzuführen und müssen durch eine zusätzliche Maßnahme verhindert werden [85, 87].

\section{Maßnahme für stabile Strecken: Zusatznetzwerk}

Für Strecken, die ausschließlich Eigenwerte mit negativem Realteil besitzen, schlägt Hippe zur Vermeidung des Strecken-Windup ein Zusatznetzwerk vor [85]. Der Regelkreis wird, wie in Abbildung 4.12 gezeigt, um ein lineares System $\mathbf{Z}$ mit dem Zustand $\boldsymbol{x}_{\mathrm{z}} \in \mathbb{R}^{n_{\mathrm{z}}}$ und der Dynamik

$$
\mathrm{Z}:\left\{\begin{array}{l}
\dot{x}_{\mathrm{z}}=A_{\mathrm{z}} \boldsymbol{x}_{\mathrm{z}}+\boldsymbol{B}_{\mathrm{z}} \boldsymbol{\delta} \\
\boldsymbol{y}_{\mathrm{z}}=C_{\mathrm{z}} \boldsymbol{x}_{\mathrm{z}}
\end{array}\right.
$$




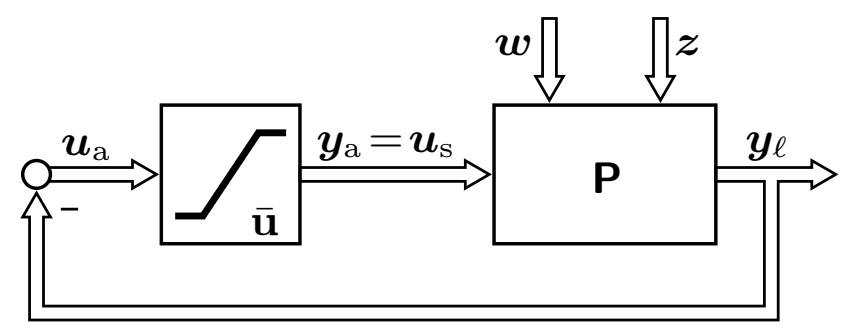

Abbildung 4.13: Nichtlinearer Standardregelkreis zur Stabilitätsanalyse und Untersuchung der Regelgüte.

sowie dem Eingang $\boldsymbol{\delta} \in \mathbb{R}^{m}$ und dem Ausgang $\boldsymbol{y}_{\mathrm{z}} \in \mathbb{R}^{m}$ erweitert. Die Matrizen $\boldsymbol{A}_{\mathrm{z}}, \boldsymbol{B}_{\mathrm{z}}$ und $\boldsymbol{C}_{\mathrm{z}}$ besitzen passende Dimensionen und sind beim Entwurf festzulegen. Dazu wird der Regelkreis in den nichtlinearen Standardregelkreis aus Abbildung 4.13 umgeformt. Mit dem Zustandsvektor

$$
\boldsymbol{x}_{\ell}^{\top}=\left[\begin{array}{lll}
\boldsymbol{x}_{\mathrm{s}}^{\top} & \boldsymbol{x}_{\mathrm{r}}^{\top} & \boldsymbol{x}_{\mathrm{z}}^{\top}
\end{array}\right]
$$

ergibt sich für den linearen Teil des nichtlinearen Standardregelkreises

$$
\mathbf{P}:\left\{\begin{array}{l}
\dot{\boldsymbol{x}}_{\ell}=\boldsymbol{A}_{\ell} \boldsymbol{x}_{\ell}+\boldsymbol{B}_{\ell, \mathrm{w}} \boldsymbol{w}+\boldsymbol{B}_{\ell, \mathrm{u}} \boldsymbol{y}_{\mathrm{a}}+\boldsymbol{B}_{\ell, \mathrm{z}} \boldsymbol{z} \\
\boldsymbol{y}_{\ell}=\boldsymbol{C}_{\ell} \boldsymbol{x}_{\ell}+\boldsymbol{D}_{\ell, \mathrm{w}} \boldsymbol{w}
\end{array}\right.
$$

Die Systemmatrizen sind in Abschnitt A.3.1 des Anhangs angegeben und hängen von der Parametrierung des Zusatznetzwerkes ab. Für den Entwurf ist die Übertragungsmatrix $\boldsymbol{P}(s)$ vom Eingang $\boldsymbol{y}_{\mathrm{a}}$ des linearen Teils zum Ausgang $\boldsymbol{y}_{\ell}$ von Interesse. Sie ergibt sich zu

$$
\boldsymbol{P}_{\ell}(s)=\boldsymbol{C}_{\ell}\left(s \mathbb{I}-\boldsymbol{A}_{\ell}\right)^{-1} \boldsymbol{B}_{\ell, \mathrm{u}} .
$$

Für SISO-Systeme lässt sich der Entwurf dann im Frequenzbereich mit dem folgenden empirischen Phasenkriterium ${ }^{6}$ durchführen.

Empirisches Phasenkriterium [26, 87]. Die lineare Regelung $\mathbf{R}$ sei gut gedämpft ausgelegt. Dann regt ein Ansprechen der Stellbegrenzung keine erhöhte Schwingneigung an, wenn die Phase des Frequenzganges $P_{\ell}(j \omega)+1$ für alle $\omega$ innerhalb des Intervalls $\left[-130^{\circ}, 130^{\circ}\right]$ verbleibt.

Abbildung 4.14 stellt das Kriterium in der komplexen Ebene grafisch dar. Bleibt die Ortskurve $P_{\ell}(j \omega)+1$ für alle $\omega$ außerhalb des grauen Sektors, so liegt vermutlich ein stabiler Regelkreis vor. Damit ist das empirische

\footnotetext{
${ }^{6}$ Ein formaler Stabilitätsbeweis existiert nicht.
} 


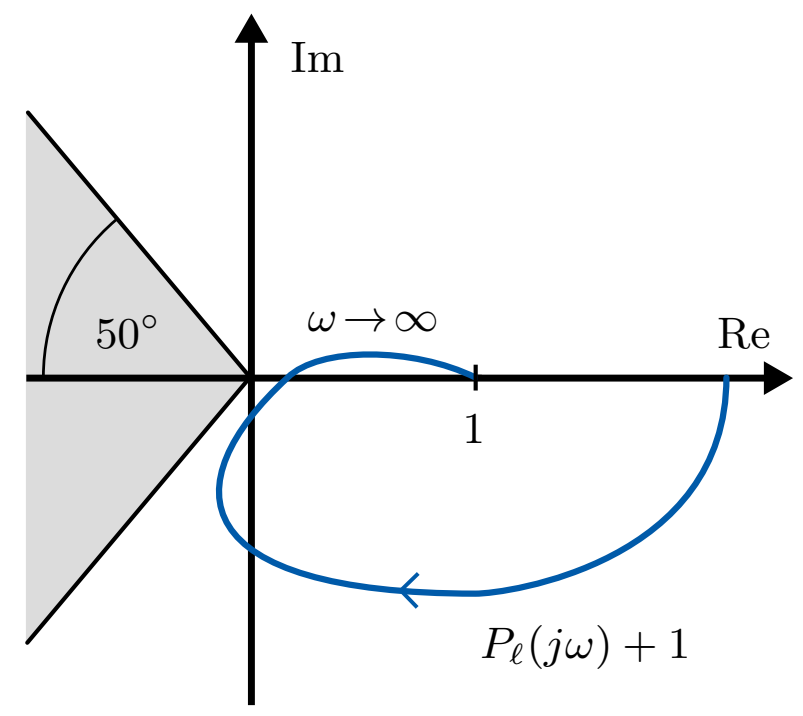

Abbildung 4.14: Visualisierung des Phasenkriteriums. Die in blau dargestellte Ortskurve von $P_{\ell}(j \omega)+1$ muss außerhalb des grauen Sektors verlaufen.

Phasenkriterium weniger konservativ als z. B. das Kreis- oder das PopovKriterium [3] und eröffnet mehr Freiheiten beim Entwurf. Allerdings ist die Stabilität nicht garantiert, was Simulationen nötig macht.

In [85, Abschnitt 3.3] wird gezeigt, wie der Entwurf für SISO-Systeme im Frequenzbereich durchgeführt werden kann. Er reduziert sich auf den Entwurf eines phasenabsenkenden oder -anhebenden Korrekturgliedes. Für den Mehrgrößenfall lässt sich ein dem Phasenkriterium äquivalentes Kriterium angeben, das ebenfalls weniger konservativ ist als die gängigen Stabilitätskriterien [166, 167]. Der MIMO-Entwurf im Frequenzbereich ist jedoch deutlich schwieriger. Deshalb wird in [85, 87] eine alternative Entwurfsmethode im Zeitbereich angegeben.

\section{Maßnahme für grenz- und instabile Strecken: Vorsteuerung}

Zur Vermeidung des Strecken-Windup bei grenz- und instabilen Strecken schlägt Hippe ein Regelungskonzept vor, das auf einem nichtlinearen Führungsfilter zur Vorsteuerung sowie einem Regler zur Stabilisierung und Störkompensation basiert [83, 85, 87]. Diese Regelung mit zwei Freiheitsgraden ist allerdings kein Anti-Windup im Sinne der in Abschnitt 3.3 skizzierten Grundidee. Anstatt im Sättigungsfall korrigierend einzugreifen wird die Regelung so ausgelegt, dass die Stellbegrenzung nie erreicht wird. Das macht auch die Vermeidung des Regler-Windup überflüssig.

Das Führungsfilter ist in Abbildung 4.15 dargestellt. Es besitzt den Zu- 


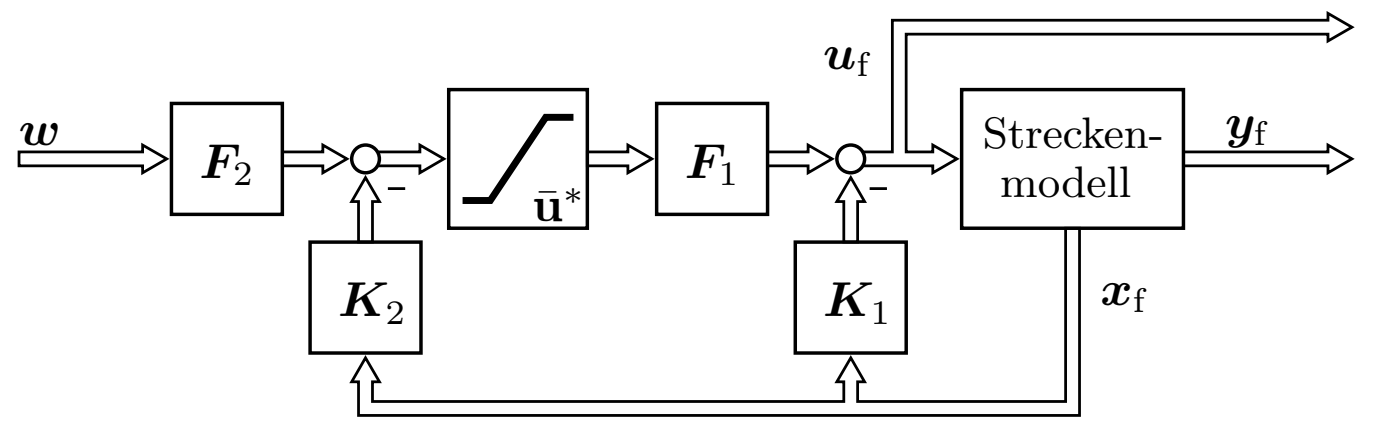

Abbildung 4.15: Nichtlineares Führungsfilter F.

stand $\boldsymbol{x}_{\mathrm{f}} \in \mathbb{R}^{n_{\mathrm{s}}}$, den Anfangswert $\boldsymbol{x}_{\mathrm{f}}(0)=\mathbf{0}$ und die Dynamik

$$
\mathbf{F}:\left\{\begin{array}{l}
\dot{\boldsymbol{x}}_{\mathrm{f}}=\boldsymbol{A}_{\mathrm{s}} \boldsymbol{x}_{\mathrm{f}}+\boldsymbol{B}_{\mathrm{s}, u} \boldsymbol{u}_{\mathrm{f}}, \\
\boldsymbol{u}_{\mathrm{f}}=\boldsymbol{F}_{1} \boldsymbol{s a t}_{\overline{\mathbf{u}}^{*}}\left(\boldsymbol{F}_{2} \boldsymbol{w}-\boldsymbol{K}_{2} \boldsymbol{x}_{\mathrm{f}}\right)-\boldsymbol{K}_{1} \boldsymbol{x}_{\mathrm{f}}, \\
\boldsymbol{y}_{\mathrm{f}}=\boldsymbol{C}_{\mathrm{s}} \boldsymbol{x}_{\mathrm{f}}
\end{array}\right.
$$

Der Ausgang $\boldsymbol{u}_{\mathrm{f}}$ ist dabei der durch das Führungsfilter berechnete Stellgrößenanteil, der vom Aktor realisiert werden muss. Wirken Störungen auf die Strecke kommt ein durch den Regler erzeugter Stellgrößenanteil $\boldsymbol{y}_{\mathrm{r}}$ hinzu.

Um eine Sättigung der Stellgröße zu vermeiden, wird die zur Verfügung stehende Stellamplitude $\overline{\mathbf{u}}$ aufgeteilt. Ein Teil wird für die Vorsteuerung reserviert und der verbleibende Teil steht dem Regler zur Verfügung. Die Aufteilung erfolgt komponentenweise und führt mit den Faktoren $\beta_{i} \in[0,1]$ zu den Forderungen

$$
\begin{aligned}
\left|y_{\mathrm{r}, i}\right| & <\beta_{i} \overline{\mathrm{u}}_{i}, \\
\left|u_{\mathrm{f}, \mathrm{i}}\right| & <\left(1-\beta_{i}\right) \overline{\mathrm{u}}_{i} \quad \forall i=1, \ldots, m .
\end{aligned}
$$

Beim Entwurf des Filters sind die Matrizen $\boldsymbol{F}_{1}, \boldsymbol{K}_{1}, \boldsymbol{F}_{2}, \boldsymbol{K}_{2}$ sowie die Stellbegrenzung $\overline{\mathbf{u}}^{*}$ so zu wählen, dass (4.21b) gilt. Für Details wird auf [85] verwiesen.

\subsubsection{Lösung des Direktionalitätsproblems}

Das Direktionalitätsproblem wird ebenfalls mit dem im letzten Abschnitt beschriebenen Führungsfilter gelöst. Dazu muss ein entkoppelnder $\mathrm{Zu}-$ standsregler $\boldsymbol{K}_{1}$ mit passendem Vorfilter $\boldsymbol{F}_{1}$ entworfen werden. 
Tabelle 4.4: Gegenüberstellung der drei modernen Anti-Windup-Maßnahmen.

\begin{tabular}{lccc}
\hline & allgemein linear & modellbasiert & modular \\
\hline $\begin{array}{l}\text { Struktur } \\
\text { Dynamik }\end{array}$ & $\begin{array}{c}\text { linear } \\
\text { eingeschränkt } \\
\text { variabel }\end{array}$ & $\begin{array}{c}\text { (nicht)linear } \\
\text { fest }\left(n_{\mathrm{s}}\right)\end{array}$ & linear \\
\hline Entwurf & mittel & niedriabel - mittel & mittel - hoch \\
Aufwand & konvexe & konvexe & iteratives \\
Vorgehen & Optimierung & Optimierung & Ausprobieren \\
\hline Garantien & ja & ja & nein \\
Stabilität & ja & ja & nein \\
Regelgüte & & &
\end{tabular}

Für instabile Strecken ist, wie im letzten Abschnitt bereits erwähnt, das Einhalten der Bedingungen (4.21) unbedingt erforderlich. Für stabile Strecken dagegen muss der Regler die Stellbegrenzung (4.21a) nicht unbedingt einhalten. Hippe schlägt vor den Regler so auszulegen, dass der Großteil der Störungen mit der zur Verfügung stehenden Stellgröße schnell ausgeregelt werden kann [85]. Für seltener vorkommende starke Störungen wird die Sättigung der Stellgröße in Kauf genommen. Die eventuell auftretenden Windup-Effekte lassen sich dann mit der Beobachtertechnik und dem Zusatznetzwerk verhindern. Details zum Entwurf des Führungsfilters und die Verschaltung finden sich in [85, Abschnitt 6.6].

\subsection{Zusammenfassung und Ausblick}

Dieses Kapitel erläuterte die Anti-Windup-Ziele und stellte drei moderne Anti-Windup-Maßnahmen vor: allgemeines lineares Anti-Windup, modellbasiertes Anti-Windup und modulares Anti-Windup. Die Gemeinsamkeit der Methoden besteht darin, dass sie erst im Sättigungsfall eingreifen und Windup durch geeignete Beeinflussung der Eingänge, Ausgänge und Zustände des Reglers verhindern. Eine Ausnahme stellt lediglich die Vorsteuerung des modularen Anti-Windup zur Lösung des Direktionalitätsproblems dar. Tabelle 4.4 vergleicht die drei Methoden. 
Die Vorteile des allgemeinen linearen Anti-Windup bestehen darin, dass die Ordnung des Anti-Windup prinzipiell frei wählbar ist. Auch statische Anti-Windup-Maßnahmen sind möglich. Der Entwurf kann in vielen Fällen auf das Lösen eines konvexen Optimierungsproblems zurückgeführt werden. Dabei muss jedoch zunächst ein sinnvolles Gütekriterium gewählt werden, was zeitaufwendig sein kann und iteratives Ausprobieren erfordert.

Diesen Nachteil weist das modellbasierte Anti-Windup nicht auf. Der ebenfalls auf konvexer Optimierung basierende Entwurf ist sehr intuitiv. Die Abweichung vom idealen unbeschränkten Systemverhalten kann direkt minimiert werden. Auch nichtlineare Anti-Windup-Strukturen, die eine höhere Regelgüte ermöglichen, lassen sich realisieren. Nachteilig ist die festgelegte Ordnung. Modellbasierte Anti-Windup-Maßnahmen besitzen immer genauso viele Zustände wie die Strecke.

Modulares Anti-Windup unterscheidet sich von den anderen Methoden durch eine separate Behandlung der Windup-Effekte. Regler- und Strecken-Windup sowie das Direktionalitätsproblem werden getrennt voneinander durch verschiedene strukturelle Maßnahmen beseitigt, was einen modularen Aufbau der Anti-Windup-Maßnahme ermöglicht. ReglerWindup lässt sich z. B. durch eine statische Rückführung vollständig beseitigen. Die Ordnung des Anti-Windup-Regelkreises wird somit nicht unnötig erhöht. Nachteilig ist, dass die Entwurfsverfahren auf Ausprobieren und Simulieren beruhen. Eine Stabilitätsgarantie für den geschlossenen Anti-Windup-Regelkreis fehlt. Gleiches gilt für die Regelgüte.

Die nächsten Kapitel dieser Arbeit bauen auf den beschriebenen Methoden auf und stellen Erweiterungen sowie neue Entwurfsverfahren vor. Der Fokus liegt dabei auf dem intuitiven modellbasierten Anti-Windup. In Kapitel 5 wird gezeigt, wie sich der Entwurf des modularen AntiWindup systematisieren lässt, wenn Ideen aus dem modellbasierten Ansatz zur Anwendung kommen. Kapitel 6 beschäftigt sich ausschließlich mit modellbasiertem Anti-Windup und untersucht die Stabilität des AntiWindup-Regelkreises. Dabei werden stabile, grenz- und instabile Strecken betrachtet und die Stabilisierung von verschiedenen Ruhelagen untersucht. Die resultierenden Stabilitätssätze lassen sich in den Kapiteln 7 bis 9 für die Entwicklung neuartiger Entwurfsmethoden für lineares und nichtlineares modellbasiertes Anti-Windup nutzen. Ziel der neuen Entwurfsverfahren ist eine sehr hohe Regelgüte, die entweder durch nichtquadratische Ljapunov-Funktionen (Kapitel 7), ein strukturvariables modellbasiertes Anti-Windup (Kapitel 8) oder die Beschränkung auf lokale Stabilität (Kapitel 9) erreicht wird. 


\section{Ein Entwurfsverfahren für die Beobachtertechnik}

In Abschnitt 4.5.1 wurde gezeigt, dass sich die Beobachtertechnik für allgemeine SISO-Regler als statisches Anti-Windup realisieren lässt, wie Abbildung 5.1 illustriert. Jedoch ist die Wahl des Anti-Windup-Vektors $\boldsymbol{l}_{\mathrm{aw}, \mathrm{x}}$ nicht eindeutig. Dieses Kapitel stellt ein neues, systematisches Entwurfsverfahren für die Beobachtertechnik des modularen Anti-Windup vor. Die Grundidee des Verfahrens wurde bereits in [147] veröffentlicht.

\subsection{Problembeschreibung}

Die vollständig steuer- und beobachtbare Strecke sei durch

$$
\mathbf{S}:\left\{\begin{array}{l}
\dot{\boldsymbol{x}}_{\mathrm{s}}=\boldsymbol{A}_{\mathrm{s}} \boldsymbol{x}_{\mathrm{s}}+\boldsymbol{b}_{\mathrm{s}, u} u_{\mathrm{s}}+\boldsymbol{b}_{\mathrm{s}, z} z \\
y_{\mathrm{s}}=\boldsymbol{c}_{\mathrm{s}}^{\top} \boldsymbol{x}_{\mathrm{s}}
\end{array}\right.
$$

gegeben, mit dem Zustand $\boldsymbol{x}_{\mathrm{s}} \in \mathbb{R}^{n_{\mathrm{s}}}$, dem Eingang $u_{\mathrm{s}} \in \mathbb{R}$, der Störgröße $z \in \mathbb{R}$ und dem Ausgang $y_{\mathrm{s}} \in \mathbb{R}$. Die Strecke sei stabil, d. h., die Matrix $\boldsymbol{A}_{\mathrm{s}}$ besitzt ausschließlich Eigenwerte mit negativem Realteil.

Um der Strecke ein gew ünschtes dynamisches Verhalten aufzuprägen

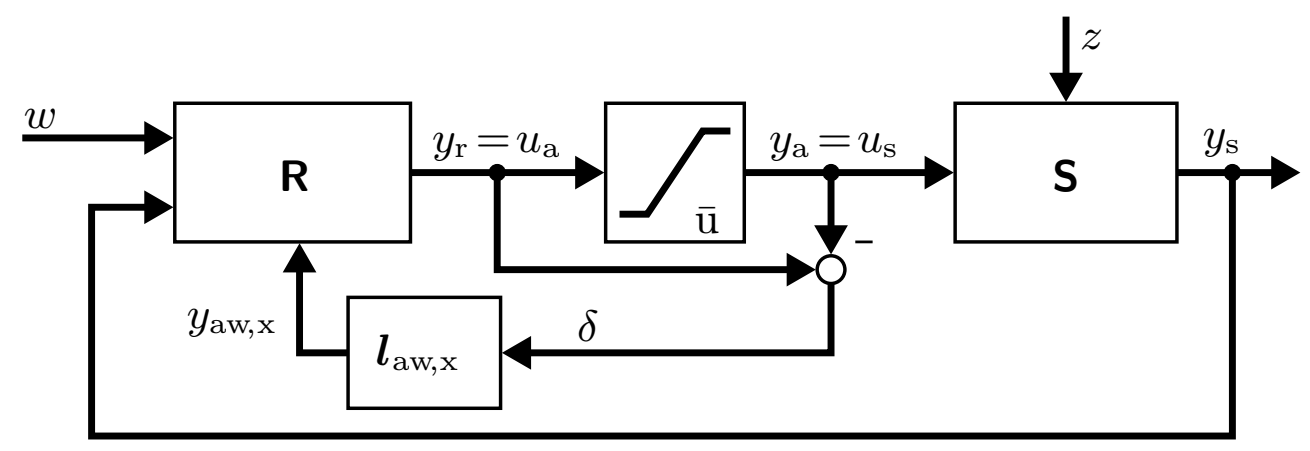

Abbildung 5.1: Anti-Windup-Regelkreis mit statischem Anti-Windup. 
und auftretende Störungen zu kompensieren, wird der Regler

$$
\mathbf{R}:\left\{\begin{array}{l}
\dot{\boldsymbol{x}}_{\mathrm{r}}=\boldsymbol{A}_{\mathrm{r}} \boldsymbol{x}_{\mathrm{r}}+\boldsymbol{b}_{\mathrm{r}, u} u_{\mathrm{r}}+\boldsymbol{b}_{\mathrm{r}, w} w \\
y_{\mathrm{r}}=\boldsymbol{c}_{\mathrm{r}}^{\top} \boldsymbol{x}_{\mathrm{r}}+d_{\mathrm{r}, u} u_{\mathrm{r}}+d_{\mathrm{r}, w} w
\end{array}\right.
$$

eingesetzt. Dabei bezeichnet $\boldsymbol{x}_{\mathrm{r}} \in \mathbb{R}^{n_{\mathrm{r}}}$ den Reglerzustand, $w \in \mathbb{R}$ den Sollwert, $u_{\mathrm{r}} \in \mathbb{R}$ den Messeingang und $y_{\mathrm{r}} \in \mathbb{R}$ den Ausgang. Des Weiteren sei das Paar $\left(\boldsymbol{A}_{\mathrm{r}}, \boldsymbol{c}_{\mathrm{r}}\right)$ beobachtbar und für die Parametrierung des Reglers gelte die Annahme 2.2 aus Abschnitt 2.1.1.

Durch die Stellbegrenzung des Aktors ausgelöste Windup-Probleme werden durch die Beobachtertechnik vermieden. Dazu wird der Regler um ein statisches Anti-Windup wie folgt erweitert

$$
\begin{aligned}
\dot{\boldsymbol{x}}_{\mathrm{r}} & =\boldsymbol{A}_{\mathrm{r}} \boldsymbol{x}_{\mathrm{r}}+\boldsymbol{b}_{\mathrm{r}, u} u_{\mathrm{r}}+\boldsymbol{b}_{\mathrm{r}, w} w+\boldsymbol{l}_{\mathrm{aw}, \mathrm{x}} \delta, \\
y_{\mathrm{r}} & =\boldsymbol{c}_{\mathrm{r}}^{\top} \boldsymbol{x}_{\mathrm{r}}+d_{\mathrm{r}, u} u_{\mathrm{r}}+d_{\mathrm{r}, w} w,
\end{aligned}
$$

wobei $\delta \in \mathbb{R}$ der Sättigungsgrad ist. Durch den realen Aktor mit dem Eingang $u_{\mathrm{a}}$ und dem Ausgang $y_{\mathrm{a}}$ ergeben sich die Verschaltungen

$$
u_{\mathrm{s}}=y_{\mathrm{a}}=\operatorname{sat}_{\overline{\mathrm{u}}}\left(y_{\mathrm{r}}\right), \quad u_{\mathrm{r}}=y_{\mathrm{s}}, \quad \delta=u_{\mathrm{a}}-y_{\mathrm{a}}=y_{\mathrm{r}}-\operatorname{sat}_{\overline{\mathrm{u}}}\left(y_{\mathrm{r}}\right) .
$$

Abbildung 5.1 zeigt das Blockschaltbild des Regelkreises. Solange keine Sättigung auftritt, gilt für den Sättigungsgrad $\delta=0$ und der Erhalt des Kleinsignalverhaltens ist sichergestellt.

Im Sättigungsfall ist der Regelkreis unterbrochen und eine instabile Reglerdynamik kann zu einem Anwachsen der Reglerzustände führen. Deshalb muss die Reglerdynamik durch das Anti-Windup stabilisiert werden. Nach dem Einsetzen von (5.2b) in (5.2a) folgt für die Reglerdynamik

$$
\begin{aligned}
\dot{\boldsymbol{x}}_{\mathrm{r}}=\left(\boldsymbol{A}_{\mathrm{r}}+\boldsymbol{l}_{\mathrm{aw}, \mathrm{x}} \boldsymbol{c}_{\mathrm{r}}^{\top}\right) \boldsymbol{x}_{\mathrm{r}}+\left(\boldsymbol{b}_{\mathrm{r}, u}+\boldsymbol{l}_{\mathrm{aw}, \mathrm{x}} d_{\mathrm{r}, u}\right) u_{\mathrm{r}}+ \\
\quad\left(\boldsymbol{b}_{\mathrm{r}, w}+\boldsymbol{l}_{\mathrm{aw}, \mathrm{x}} d_{\mathrm{r}, w}\right) w-\boldsymbol{l}_{\mathrm{aw}, \mathrm{x}} \operatorname{sat}_{\overline{\mathrm{u}}}\left(y_{\mathrm{r}}\right) .
\end{aligned}
$$

Die Beobachtbarkeit des Matrixpaares $\left(\boldsymbol{A}_{\mathrm{r}}, \boldsymbol{c}_{\mathrm{r}}\right)$ garantiert, dass sich die Eigenwerte $\lambda_{\mathrm{r}, i}, i=1, \ldots, n_{\mathrm{r}}$ des Reglers im Sättigungsfall beliebig über $\boldsymbol{l}_{\mathrm{aw}, \mathrm{x}}$ festlegen lassen. Diese Eigenwerte werden in der Menge

$$
\boldsymbol{\Lambda}_{\mathrm{r}}\left(\boldsymbol{l}_{\mathrm{aw}, \mathrm{x}}\right):=\left\{\lambda_{\mathrm{r}} \in \mathbb{C} \mid \operatorname{det}\left(\lambda_{\mathrm{r}} \mathbb{I}-\boldsymbol{A}_{\mathrm{r}}-\boldsymbol{l}_{\mathrm{aw}, \mathrm{x}} \boldsymbol{c}_{\mathrm{r}}^{\top}\right)=0\right\}
$$

zusammengefasst. Gemäß der Beobachtertechnik werden die $n_{\mathrm{r}}$ Eigenwerte so gewählt, dass sie mit $n_{\mathrm{r}}$ Eigenwerten der insgesamt $n_{\mathrm{r}}+n_{\mathrm{s}}$ Eigenwerte der Systemmatrix des unbeschränkten Regelkreises

$$
\boldsymbol{A}_{\mathrm{ub}}=\left[\begin{array}{cc}
\boldsymbol{A}_{\mathrm{s}}+\boldsymbol{b}_{\mathrm{s}, u} d_{\mathrm{r}, u} \boldsymbol{c}_{\mathrm{s}}^{\top} & \boldsymbol{b}_{\mathrm{s}, u} \boldsymbol{c}_{\mathrm{r}}^{\top} \\
\boldsymbol{b}_{\mathrm{r}, u} \boldsymbol{c}_{\mathrm{s}}^{\top} & \boldsymbol{A}_{\mathrm{r}}
\end{array}\right]
$$


übereinstimmen (vgl. (2.4) in Abschnitt 2.1). Für eine kompaktere Notation werden diese Eigenwerte in der Menge

$$
\boldsymbol{\Lambda}_{\mathrm{ub}}:=\left\{\lambda_{\mathrm{ub}} \in \mathbb{C} \mid \operatorname{det}\left(\lambda_{\mathrm{ub}} \mathbb{I}-\boldsymbol{A}_{\mathrm{ub}}\right)=0\right\}
$$

zusammengefasst. Die Menge an zulässigen Anti-Windup-Vektoren $\boldsymbol{l}_{\mathrm{aw}, \mathrm{x}}$ wird dann wie folgt definiert

$$
\mathcal{L}:=\left\{\boldsymbol{l}_{\mathrm{aw}, \mathrm{x}} \in \mathbb{R}^{n_{\mathrm{r}}} \mid \boldsymbol{\Lambda}_{\mathrm{r}}\left(\boldsymbol{l}_{\mathrm{aw}, \mathrm{x}}\right) \subset \boldsymbol{\Lambda}_{\mathrm{ub}}\right\} .
$$

In seltenen Fällen kann es vorkommen, dass $\mathcal{L}$ leer ist. Dazu müssen zwei Bedingungen gleichzeitig erfüllt sein. Erstens: Die Systemordnung des Regelkreises $n_{\mathrm{s}}+n_{\mathrm{r}}$ ist gerade und die Eigenwerte des unbeschränkten Regelkreises sind alle konjugiert komplex, d. h., $\boldsymbol{\Lambda}_{\mathrm{ub}}$ besitzt ausschließlich komplexe Elemente. Zweitens: die Dimension des Reglers $n_{\mathrm{r}}$ und damit auch die des Vektors $\boldsymbol{l}_{\mathrm{aw}, \mathrm{x}}$ ist ungerade. Dann muss die Matrix $\boldsymbol{A}_{\mathrm{r}}+\boldsymbol{l}_{\mathrm{aw}, \mathrm{x}} \boldsymbol{c}_{\mathrm{r}}^{\top}$ einen reellen Eigenwert besitzen, da komplexe Eigenwerte immer als konjugiert komplexes Paar auftreten. Folglich kann keine Menge $\boldsymbol{\Lambda}_{\mathrm{r}}\left(\boldsymbol{l}_{\mathrm{aw}, \mathrm{x}}\right)$ existieren, die eine Teilmenge von $\boldsymbol{\Lambda}_{\mathrm{ub}}$ ist. Liegt dieser Sonderfall vor, so lässt sich die in [3, Abschnitt 3.1.5] beschriebene Vorgehensweise für die Parametrierung des Anti-Windup anwenden.

Im Weiteren wird allerdings davon ausgegangen, dass $\mathcal{L}$ nicht leer ist. Welche Eigenwerte bzw. welche Vektoren $\boldsymbol{l}_{\mathrm{aw}, \mathrm{x}} \in \mathcal{L}$ eine hohe Regelgüte garantieren ist nicht bekannt. Bisher war man auf Ausprobieren und Simulationen angewiesen. In den folgenden Abschnitten wird eine systematische Entwurfsmethodik vorgestellt, die folgende Frage beantwortet. Wie ist der Anti-Windup-Vektor $\boldsymbol{l}_{\text {aw, }} \in \mathcal{L} \neq\{\}$ zu wählen, so dass der in Abbildung 5.1 dargestellte Anti-Windup-Regelkreis

1. eine global asymptotisch stabile Ruhelage besitzt sowie

2. eine Regelgüte aufweist, die möglichst nahe an die des unbeschränkten Regelkreises heranreicht?

Das Problem wird Stück für Stück gelöst. Der nächste Abschnitt beschäftigt sich mit der Stabilität der Ruhelage, d. h. der ersten Forderung. Die zweite Forderung nach einer möglichst kleinen Abweichung des AntiWindup-Regelkreises von dem dynamischen Verhalten des unbeschränkten Regelkreises wird in Abschnitt 5.3 untersucht. Der Abschnitt 5.4 fasst schließlich das Entwurfsverfahren zusammen und stellt die Lösung für das beschriebene Problem vor. Am Beispiel des hydraulischen Positioniersystems wird die neue Entwurfsmethodik demonstriert. 


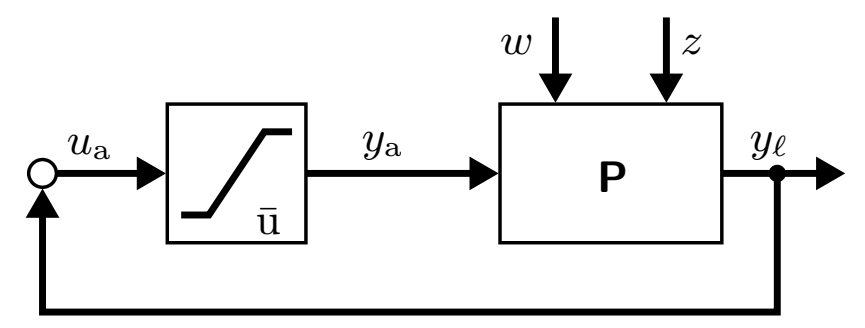

Abbildung 5.2: Der nichtlineare Standardregelkreis besteht aus einer statischen Nichtlinearität und einem linearen dynamischen System.

\subsection{Analyse der Stabilität}

Dieser Abschnitt beschäftigt sich mit der Stabilitätsanalyse des AntiWindup-Regelkreises aus Abbildung 5.1. Hierbei wird auf bekannte Ergebnisse aus [119] zurückgegriffen.

\subsubsection{Vorüberlegungen}

Zur Analyse der Stabilität wird der Regelkreis aus Abbildung 5.1 in den nichtlinearen Standardregelkreis umgeformt. Dieser ist in Abbildung 5.2 gezeigt. Mit dem Zustandsvektor

$$
\boldsymbol{x}_{\ell}^{\top}=\left[\begin{array}{ll}
\boldsymbol{x}_{\mathrm{s}}^{\top} & \boldsymbol{x}_{\mathrm{r}}^{\top}
\end{array}\right] \in \mathbb{R}^{n_{\mathrm{s}}+n_{\mathrm{r}}}
$$

ergibt sich das lineare dynamische Teilsystem

$$
\mathbf{P}:\left\{\begin{array}{l}
\dot{\boldsymbol{x}}_{\ell}=\boldsymbol{A}_{\ell} \boldsymbol{x}_{\ell}+\boldsymbol{b}_{\ell, \mathrm{w}} w+\boldsymbol{b}_{\ell, \mathrm{u}} y_{\mathrm{a}}+\boldsymbol{b}_{\ell, \mathrm{z}} z \\
y_{\ell}=\boldsymbol{c}_{\ell}^{\top} \boldsymbol{x}_{\ell}+d_{\ell, \mathrm{w}} w
\end{array}\right.
$$

wobei die Systemmatrizen folgende Gestalt besitzen

$$
\begin{aligned}
\boldsymbol{A}_{\ell} & =\left[\begin{array}{cc}
\boldsymbol{A}_{\mathrm{s}} & \mathbf{0} \\
\boldsymbol{b}_{\mathrm{s}, u} \boldsymbol{c}_{\mathrm{s}}^{\top}+\boldsymbol{l}_{\mathrm{aw}, \mathrm{x}} d_{\mathrm{r}, u} \boldsymbol{c}_{\mathrm{s}}^{\top} & \boldsymbol{A}_{\mathrm{r}}+\boldsymbol{l}_{\mathrm{aw}, \mathrm{x}} \boldsymbol{c}_{\mathrm{r}}^{\top}
\end{array}\right], \boldsymbol{b}_{\ell, \mathrm{w}}=\left[\begin{array}{c}
\mathbf{0} \\
\boldsymbol{b}_{\mathrm{r}, w}+\boldsymbol{l}_{\mathrm{aw}, \mathrm{x}} d_{\mathrm{r}, w}
\end{array}\right], \\
\boldsymbol{b}_{\ell, \mathrm{u}} & =\left[\begin{array}{c}
\boldsymbol{b}_{\mathrm{s}, u} \\
-\boldsymbol{l}_{\mathrm{aw}, \mathrm{x}}
\end{array}\right], \boldsymbol{b}_{\ell, \mathrm{z}}=\left[\begin{array}{c}
\boldsymbol{b}_{\mathrm{s}, z} \\
\mathbf{0}
\end{array}\right], \boldsymbol{c}_{\ell}^{\top}=\left[\begin{array}{ll}
d_{\mathrm{r}, u} \boldsymbol{c}_{\mathrm{s}}^{\top} & \boldsymbol{c}_{\mathrm{r}}^{\top}
\end{array}\right], d_{\ell, \mathrm{w}}=d_{\mathrm{r}, w} .
\end{aligned}
$$

Für den Nachweis globaler Stabilität der Ruhelage $\boldsymbol{x}_{\ell}^{(\mathrm{R})}=\mathbf{0}$ werden die Eingänge $w$ und $z$ zu null gesetzt. Es ist dann nur noch das lineare System

$$
\mathbf{P}_{0}:\left\{\begin{array}{l}
\dot{\boldsymbol{x}}_{\ell}=\boldsymbol{A}_{\ell} \boldsymbol{x}_{\ell}+\boldsymbol{b}_{\ell, \mathrm{u}} y_{\mathrm{a}} \\
y_{\ell}=\boldsymbol{c}_{\ell}^{\top} \boldsymbol{x}_{\ell}
\end{array}\right.
$$


in Kombination mit dem stellbegrenzten Aktor

$$
y_{\mathrm{a}}=\operatorname{sat}_{\overline{\mathrm{u}}}\left(y_{\ell}\right)
$$

zu betrachten, der durch eine Sättigungsfunktion beschrieben wird. Viele Stabilitätskriterien basieren auf bestimmten Eigenschaften dieser Sättigungsfunktion, wie der nächste Abschnitt beschreibt.

\subsubsection{Eigenschaften der Sättigungsfunktion}

Die Sättigungsfunktion gehört zur Klasse der Sektornichtlinearitäten. Das sind nichtlineare Funktionen, die in dem von der Abszisse und einer Ursprungsgeraden aufgespannten Sektor liegen. Im Fall der Sättigung besitzt die Ursprungsgerade die Steigung eins. Formal ist diese Eigenschaft für allgemeine Nichtlinearitäten wie folgt definiert.

Definition 5.1 (Globale Sektorzugehörigkeit). Eine nichtlineare, stückweise stetige Funktion der Gestalt

$$
\boldsymbol{y}=\boldsymbol{\mu}(\boldsymbol{u})=\left[\begin{array}{lll}
\mu_{1}\left(u_{1}\right) & \ldots & \mu_{m}\left(u_{m}\right)
\end{array}\right]^{\top}
$$

mit $\boldsymbol{y}, \boldsymbol{u} \in \mathbb{R}^{m}$ liegt global im Sektor $\mathcal{S}(\mathbf{0}, \boldsymbol{\Phi})$ mit

$$
\boldsymbol{\Phi}=\operatorname{diag}\left(\phi_{1}, \ldots, \phi_{m}\right) \succ \mathbf{0},
$$

wenn für alle Komponenten $\mu_{i}\left(u_{i}\right), i=1, \ldots, m$ gilt

$$
\begin{aligned}
0 & \leq \mu_{i}\left(u_{i}\right)=y_{i} \leq \phi_{i} u_{i} & & \forall u_{i} \in \mathbb{R}_{\geq 0}, \\
\phi_{i} u_{i} & \leq \mu_{i}\left(u_{i}\right)=y_{i} \leq 0 & & \forall u_{i} \in \mathbb{R}_{<0} .
\end{aligned}
$$

Anschaulich bedeuten die Ungleichungen (5.6a) und (5.6b), dass jede Komponente $\mu_{i}\left(u_{i}\right)$ der nichtlinearen Funktion von der Abszisse und einer Ursprungsgeraden mit der Steigung $\phi_{i}$ eingeschlossen wird. Abbildung 5.3 stellt dies grafisch dar.

Die Sättigungskennlinie liegt im Sektor $\mathcal{S}(0,1)$. Für die Überprüfung der Stabilität des nichtlinearen Standardregelkreises (5.5a), (5.5b) können folglich alle Kriterien angewendet werden, die für derartige Sektornichtlinearitäten geeignet sind; z. B. das in [3, 107] beschriebene Kreis-Kriterium oder das Popov-Kriterium [3, 21].

Ein Nachteil dieser Kriterien ist, dass sie die Sättigung nicht besonders genau charakterisieren. Die in beiden Kriterien zu überprüfenden Bedingungen sind strenger als nötig, da sie Stabilität für alle Sektornichtlinearitäten garantieren müssen. $\mathrm{Zu}$ einem weniger konservativen Stabilitätskriterium gelangt man durch Ausnutzen zusätzlicher Eigenschaften der 


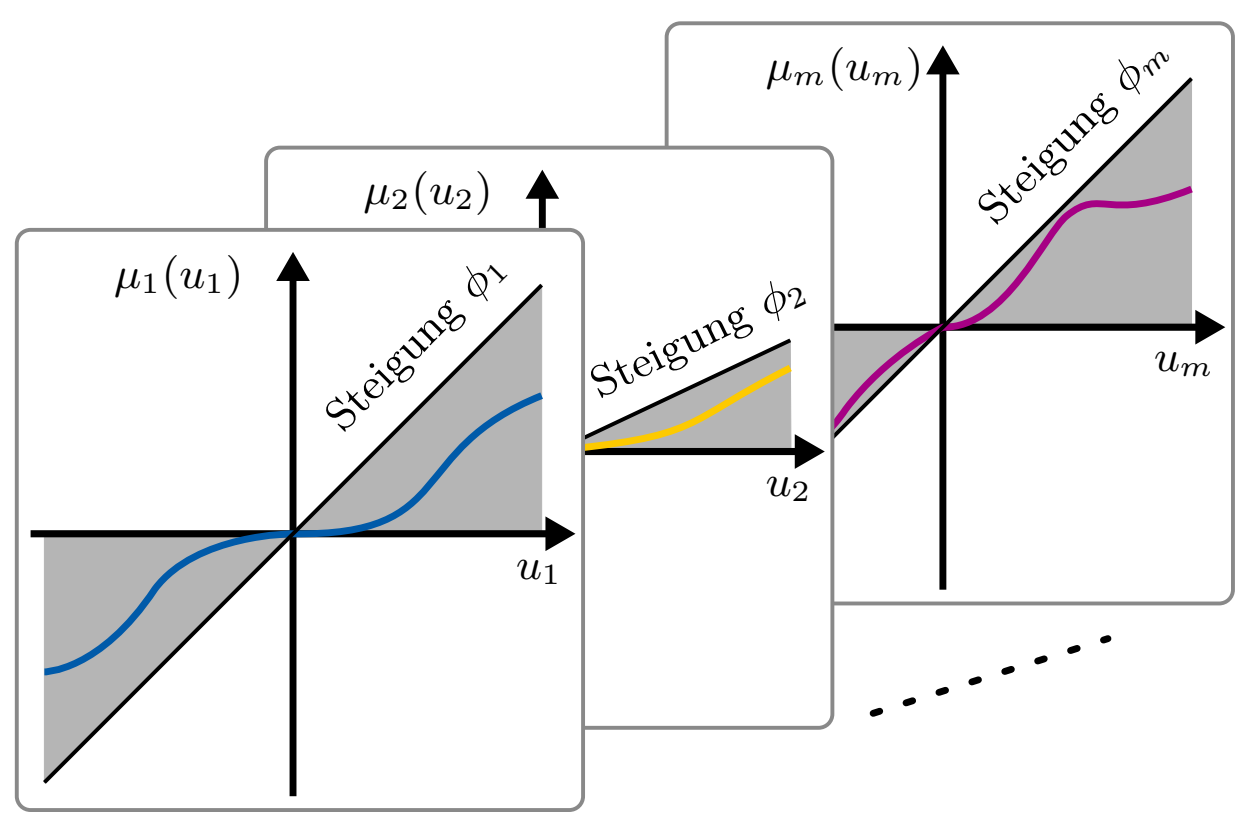

Abbildung 5.3: Veranschaulichung der Ungleichungen (5.6). Jede Komponente $\mu_{i}\left(u_{i}\right)$ liegt in einem Sektor $\mathcal{S}\left(0, \phi_{i}\right)$.

Sättigungsfunktion. Beispielsweise ist die Steigung der Sättigungskennlinie begrenzt. Das Kriterium der nicht-axialen Kreise [141, 166, 167] berücksichtigt diese Steigungsbegrenzung und führt zu weniger konservativen Ergebnissen bezüglich der Stabilität des Regelkreises (5.5).

In [147] wurde aus dem Kriterium der nicht-axialen Kreise ein einfach überprüfbares Stabilitätskriterium entwickelt, das Bedingungen an den Phasenverlauf der Übertragungsfunktion

$$
P(s)=\boldsymbol{c}_{\ell}^{\top}\left(s \mathbb{I}-\boldsymbol{A}_{\ell}\right)^{-1} \boldsymbol{b}_{\ell, \mathrm{u}}
$$

des linearen Teils $\mathbf{P}_{0}$ stellt. In dieser Arbeit wird ein alternativer Weg beschritten, der auf dem weniger bekannten Stabilitätssatz von Lee basiert [119]. Der Hauptvorteil ist, dass die zu überprüfenden Bedingungen als lineare Matrixungleichungen vorliegen. So kann direkt mit den Systemmatrizen $\boldsymbol{A}_{\ell}, \boldsymbol{b}_{\ell, \mathrm{u}}$ und $\boldsymbol{c}_{\ell}$ gearbeitet werden und die Stabilitätsprüfung reduziert sich auf ein konvexes Validierungsproblem. Eine Bestimmung der Übertragungsfunktion bzw. des Phasenganges entfällt. Details erläutert der nächste Abschnitt. 


\subsubsection{Stabilitätsprüfung mit dem Satz von Lee}

Um den Stabilitätssatz von Lee angeben zu können, wird die Menge $\mathcal{M}(m)$ benötigt. Diese besteht aus $4^{m}$ Diagonalmatrizen, deren Diagonalelemente entweder 0 oder 1 sind. Kompakt lässt sie sich wie folgt angeben

$$
\mathcal{M}(m):=\left\{\operatorname{diag}\left(\nu_{1}, \ldots, \nu_{2 m}\right) \in \mathbb{S}^{2 m} \mid \nu_{i} \in\{0,1\}\right\} .
$$

Die Diagonalmatrizen der Menge werden im Weiteren mit

$$
M_{i} \in \mathrm{S}^{2 m}, i=1, \ldots, 4^{m}
$$

bezeichnet und mit dem Index $i$ durchnummeriert. Für den Fall $m=1$ ergibt sich beispielsweise die Menge

$$
\begin{aligned}
\mathcal{M}(1) & =\left\{\boldsymbol{M}_{1}, \boldsymbol{M}_{2}, \boldsymbol{M}_{3}, \boldsymbol{M}_{4}\right\} \\
& =\left\{\left[\begin{array}{ll}
0 & 0 \\
0 & 0
\end{array}\right],\left[\begin{array}{ll}
1 & 0 \\
0 & 0
\end{array}\right],\left[\begin{array}{ll}
0 & 0 \\
0 & 1
\end{array}\right],\left[\begin{array}{ll}
1 & 0 \\
0 & 1
\end{array}\right]\right\} .
\end{aligned}
$$

Mit dem Satz von Lee aus [119] lässt sich die globale asymptotische Stabilität der Ruhelage $\boldsymbol{x}_{\ell}^{(\mathrm{R})}=\mathbf{0}$ eines nichtlinearen MIMO-Standardregelkreises überprüfen. Hier wird ein auf den vorliegenden Fall angepasstes Lemma angegeben, das den Operator $\mathrm{He} \boldsymbol{X}=\boldsymbol{X}+\boldsymbol{X}^{\top}$ verwendet.

Lemma 5.1 (Globale Stabilität). Gegeben sei das System

$$
\begin{aligned}
\dot{\boldsymbol{x}} & =\boldsymbol{A} \boldsymbol{x}+\boldsymbol{B} \boldsymbol{s a t}_{\overline{\boldsymbol{u}}}(\boldsymbol{u}), \\
\boldsymbol{u} & =\boldsymbol{C} \boldsymbol{x}
\end{aligned}
$$

mit dem Zustand $\boldsymbol{x} \in \mathbb{R}^{n}$ und der Rückführung $\boldsymbol{u} \in \mathbb{R}^{m}$. Wenn zwei symmetrische, positiv definite Matrizen $\boldsymbol{R} \in \mathbb{S}^{n+m}$ und $\boldsymbol{\Theta} \in \mathbb{S}^{4 m}$ existieren, so dass die Matrixungleichungen

$$
\begin{aligned}
\mathrm{He}\left[\begin{array}{cc}
\boldsymbol{A}_{*}^{\top} \boldsymbol{R}+\frac{1}{2} \boldsymbol{C}_{*}^{\top} \boldsymbol{\Theta} \boldsymbol{C}_{*} & \mathbf{0} \\
\boldsymbol{D}_{*}^{\top} \boldsymbol{\Theta} \boldsymbol{C}_{*}+\boldsymbol{B}_{*}^{\top} \boldsymbol{R} & \frac{1}{2} \boldsymbol{D}_{*}^{\top} \boldsymbol{\Theta} \boldsymbol{D}_{*}
\end{array}\right] & \prec \mathbf{0}, \\
{\left[\begin{array}{ll}
\mathbb{I} & \boldsymbol{M}_{i}
\end{array}\right] \boldsymbol{\Theta}\left[\begin{array}{c}
\mathbb{I} \\
\boldsymbol{M}_{i}
\end{array}\right] } & \succeq \mathbf{0} \forall i=1, \ldots, 4^{m}, \quad \boldsymbol{M}_{i} \in \mathcal{M}(m)
\end{aligned}
$$

mit der Menge $\mathcal{M}(m)$ gemäß (5.7) und den Abkürzungen

$$
\begin{array}{rlrl}
\boldsymbol{A}_{*} & =\left[\begin{array}{cc}
\boldsymbol{A} & \boldsymbol{B} \\
\mathbf{0} & \mathbf{0}
\end{array}\right] \in \mathbb{R}^{(n+m) \times(n+m)}, & \boldsymbol{B}_{*}=\left[\begin{array}{l}
\mathbf{0} \\
\mathbb{I}
\end{array}\right] \in \mathbb{R}^{(n+m) \times m}, \\
\boldsymbol{C}_{*}=\left[\begin{array}{cc}
\boldsymbol{C} & \mathbf{0} \\
\boldsymbol{C} \boldsymbol{A} & \boldsymbol{C} \boldsymbol{B} \\
\mathbf{0} & \mathbb{I} \\
\mathbf{0} & \mathbf{0}
\end{array}\right] \in \mathbb{R}^{4 m \times(n+m)}, & \boldsymbol{D}_{*}=\left[\begin{array}{l}
\mathbf{0} \\
\mathbf{0} \\
\mathbf{0} \\
\mathbb{I}
\end{array}\right] \in \mathbb{R}^{4 m \times m}
\end{array}
$$


erfüllt sind, dann ist die Ruhelage $\boldsymbol{x}^{(\mathrm{R})}=\mathbf{0}$ des Systems (5.9) global asymptotisch stabil.

Beweis. Das Lemma ist ein Spezialfall des Satzes von Lee. Der Beweis findet sich in [119]. Globale Stabilität wird mit einer Ljapunov-Funktion

$$
V(\boldsymbol{x})=\tilde{\boldsymbol{x}}^{\top} \boldsymbol{R} \tilde{\boldsymbol{x}}, \quad \boldsymbol{R} \succ \mathbf{0},
$$

und dem erweiterten Zustandsvektor $\tilde{\boldsymbol{x}}^{\top}=\left[\boldsymbol{x}^{\top} \operatorname{sat}_{\overline{\mathbf{u}}}(\boldsymbol{C} \boldsymbol{x})^{\top}\right]$ sichergestellt. Dabei ist zu beachten, dass die zeitliche Ableitung dieser nichtquadratischen Ljapunov-Funktion aufgrund der Sättigungsfunktion nur für fast alle $\boldsymbol{x} \in \mathbb{R}^{n}$ existiert. Mithilfe der verallgemeinerten Ableitung von Clark [186] lässt sich aber auch in diesem Fall globale asymptotische Stabilität nachweisen, wie beispielsweise [37] zeigt. Alternativ kann die Sättigungsfunktion durch eine stetig differenzierbare Funktion beliebig genau approximiert werden.

Das Lemma 5.1 kann für $m=1$ zur Stabilitätsprüfung der Ruhelage $\boldsymbol{x}_{\ell}^{(\mathrm{R})}=\mathbf{0}$ des Regelkreises (5.5) verwendet werden. Da die zu überprüfenden Nebenbedingungen lineare Matrixungleichungen sind, reduziert sich die Stabilitätsprüfung auf die Lösung eines konvexen Validierungsproblems, das nachfolgend angegeben ist.

Validierungsproblem 5.1. Gegeben seien die Matrizen

$$
\begin{aligned}
\boldsymbol{A}_{\ell} & =\left[\begin{array}{cc}
\boldsymbol{A}_{\mathrm{s}} & \mathbf{0} \\
\boldsymbol{b}_{\mathrm{s}, u} \boldsymbol{c}_{\mathrm{s}}^{\top}+\boldsymbol{l}_{\mathrm{aw}, \mathrm{x}} d_{\mathrm{r}, u} \boldsymbol{c}_{\mathrm{s}}^{\top} & \boldsymbol{A}_{\mathrm{r}}+\boldsymbol{l}_{\mathrm{aw}, \mathrm{x}} \boldsymbol{c}_{\mathrm{r}}^{\top}
\end{array}\right], \quad \boldsymbol{b}_{\ell, \mathrm{u}}=\left[\begin{array}{c}
\boldsymbol{b}_{\mathrm{s}, u} \\
-\boldsymbol{l}_{\mathrm{aw}, \mathrm{x}}
\end{array}\right], \\
\boldsymbol{c}_{\ell}^{\top} & =\left[\begin{array}{ll}
d_{\mathrm{r}, u} \boldsymbol{c}_{\mathrm{s}}^{\top} & \boldsymbol{c}_{\mathrm{r}}^{\top}
\end{array}\right]
\end{aligned}
$$

des nichtlinearen Standardregelkreises (5.5), die Menge $\mathcal{M}(1)$ gemäß (5.8) sowie die Abkürzungen $n=n_{\mathrm{s}}+n_{\mathrm{r}}$ und

$$
\begin{array}{rlrl}
\boldsymbol{A}_{*}= & {\left[\begin{array}{cc}
\boldsymbol{A}_{\ell} & \boldsymbol{b}_{\ell, \mathrm{u}} \\
\mathbf{0} & 0
\end{array}\right] \in \mathbb{R}^{(n+1) \times(n+1)},} & \boldsymbol{B}_{*}=\left[\begin{array}{l}
\mathbf{0} \\
1
\end{array}\right] \in \mathbb{R}^{(n+1) \times 1}, \\
\boldsymbol{C}_{*}=\left[\begin{array}{cc}
\boldsymbol{c}_{\ell}^{\top} & 0 \\
\boldsymbol{c}_{\ell}^{\top} \boldsymbol{A}_{\ell} & \boldsymbol{c}_{\ell}^{\top} \boldsymbol{b}_{\ell, \mathrm{u}} \\
\mathbf{0} & 1 \\
\mathbf{0} & 0
\end{array}\right] \in \mathbb{R}^{4 \times(n+1),} & \boldsymbol{D}_{*}=\left[\begin{array}{l}
0 \\
0 \\
0 \\
1
\end{array}\right] \in \mathbb{R}^{4 \times 1} .
\end{array}
$$




$$
\begin{aligned}
& \underset{\boldsymbol{R}, \boldsymbol{\Theta}}{\text { Finde }} \boldsymbol{R} \in \mathbb{S}^{n+1}, \boldsymbol{\Theta} \in \mathbb{S}^{4} \quad \text { unter den Nebenbedingungen } \\
& \boldsymbol{R} \succ \mathbf{0}, \boldsymbol{\Theta} \succ \mathbf{0}, \\
& \operatorname{He}\left[\begin{array}{cc}
\boldsymbol{A}_{*}^{\top} \boldsymbol{R}+\frac{1}{2} \boldsymbol{C}_{*}^{\top} \boldsymbol{\Theta} \boldsymbol{C}_{*} & \mathbf{0} \\
\boldsymbol{D}_{*}^{\top} \boldsymbol{\Theta} \boldsymbol{C}_{*}^{+}+\boldsymbol{B}_{*}^{\top} \boldsymbol{R} & \frac{1}{2} \boldsymbol{D}_{*}^{\top} \boldsymbol{\Theta} \boldsymbol{D}_{*}
\end{array}\right] \prec \mathbf{0}, \\
& {\left[\begin{array}{ll}
\mathbb{I} & \boldsymbol{M}
\end{array}\right] \boldsymbol{\Theta}\left[\begin{array}{c}
\mathbb{I} \\
\boldsymbol{M}
\end{array}\right] \succeq \mathbf{0} \quad \forall \boldsymbol{M} \in \mathcal{M}(1) .}
\end{aligned}
$$

Falls die positiv definiten, symmetrischen Matrizen $\boldsymbol{R}$ und $\boldsymbol{\Theta}$ unter den angeführten Nebenbedingungen existieren, ist die globale asymptotische Stabilität der Ruhelage $\boldsymbol{x}_{\ell}^{(\mathrm{R})}=\mathbf{0}$ des Regelkreises (5.5) nachgewiesen. $\mathrm{Zu}$ beachten ist dabei, dass die Nebenbedingungen nur hinreichend für die Stabilität sind. Jedoch zeigt der Vergleich in [119], dass globale Stabilität in vielen Fällen nachgewiesen werden kann, in denen andere Verfahren wie z. B. das Kreis- oder Popov-Kriterium sowie [75, 154] nur lokale Stabilität der Ruhelage garantieren.

Die globale Stabilität der Ruhelage $\boldsymbol{x}_{\ell}^{(\mathrm{R})}=\mathbf{0}$ des Anti-Windup-Regelkreises lässt sich somit anhand eines konvexen Validierungsproblems überprüfen. Die Lösbarkeit des Problems garantiert auch für andere Ruhelagen des Anti-Windup-Regelkreises, die sich für $w \neq 0, z \neq 0$ ergeben und mittels einer Transformation in den Ursprung verschoben werden können, globale asymptotische Stabilität. Folglich ist die erste Forderung des eingangs vorgestellten Problems gelöst. Der nächste Abschnitt beschäftigt sich mit der zweiten Forderung, d. h. mit der Regelgüte des Anti-WindupRegelkreises. Dabei ist die Frage zu beantworten, wie die Abweichung des Regelkreises vom unbeschränkten Verhalten charakterisiert werden kann.

\subsection{Analyse der Regelgüte}

Zur Analyse der Regelgüte ist ein Maß für die Abweichung des Regelkreisverhaltens von dem idealen Verhalten des unbeschränkten Regelkreises zu finden. Bei einem statischen Anti-Windup ist dies schwierig, wie in Abschnitt 4.3 erläutert wurde. Für ein modellbasiertes Anti-Windup dagegen relativ einfach, da der Regelkreis in Mismatch-Koordinaten transformiert werden kann (Abschnitt 4.4). Die hier verfolgte Grundidee ist deshalb für den Anti-Windup-Regelkreis mit Beobachtertechnik ein Ersatzsystem herzuleiten, das einem Regelkreis mit modellbasierten Anti-Windup entspricht und für die Analyse verwendet wird. 


\subsubsection{Grundidee: Ein Ersatzsystem für die Analyse}

Betrachtet wird der Anti-Windup-Regelkreis mit Beobachtertechnik aus Abbildung 5.1. Das Regelgesetz setzt sich aus dem Regler $\mathbf{R}$ und dem statischen Anti-Windup zusammen, das durch den Vektor $\boldsymbol{l}_{\mathrm{aw}, \mathrm{x}} \in \mathbb{R}^{n_{\mathrm{r}}}$ parametriert ist. Die resultierende Reglerdynamik ist

$$
\begin{aligned}
\dot{\boldsymbol{x}}_{\mathrm{r}}=\left(\boldsymbol{A}_{\mathrm{r}}+\right. & \left.\boldsymbol{l}_{\mathrm{aw}, \mathrm{x}} \boldsymbol{c}_{\mathrm{r}}^{\top}\right) \boldsymbol{x}_{\mathrm{r}} \\
& +\left(\boldsymbol{b}_{\mathrm{r}, u}+\boldsymbol{l}_{\mathrm{aw}, \mathrm{x}} d_{\mathrm{r}, u}\right) y_{\mathrm{s}}+\left(\boldsymbol{b}_{\mathrm{r}, w}+\boldsymbol{l}_{\mathrm{aw}, \mathrm{x}} d_{\mathrm{r}, w}\right) w-\boldsymbol{l}_{\mathrm{aw}, \mathrm{x}} y_{\mathrm{a}}
\end{aligned}
$$

und der den Aktor beeinflussende Ausgang ergibt sich zu

$$
u_{\mathrm{a}}=\boldsymbol{c}_{\mathrm{r}}^{\top} \boldsymbol{x}_{\mathrm{r}}+d_{\mathrm{r}, u} y_{\mathrm{s}}+d_{\mathrm{r}, w} w .
$$

Auf die Ableitung des Reglerzustandes (5.10a) wirken die drei Größen $w$, $y_{\mathrm{s}}$ und $y_{\mathrm{a}}$. Bezüglich des Übertragungsverhaltens lässt sich das Regelgesetz (5.10) folglich durch drei Übertragungsfunktionen von den Eingängen zu dem Ausgang (5.10b) beschreiben. Es gilt

$$
u_{\mathrm{a}}(s)=G_{1}(s) w(s)+G_{2}(s) y_{\mathrm{s}}(s)+G_{3}(s) y_{\mathrm{a}}(s)
$$

mit den Übertragungsfunktionen

$$
\begin{aligned}
& G_{1}(s)=\boldsymbol{c}_{\mathrm{r}}^{\top}\left(s \mathbb{I}-\boldsymbol{A}_{\mathrm{r}}-\boldsymbol{l}_{\mathrm{aw}, \mathrm{x}} \boldsymbol{c}_{\mathrm{r}}^{\top}\right)^{-1}\left(\boldsymbol{b}_{\mathrm{r}, w}+\boldsymbol{l}_{\mathrm{aw}, \mathrm{x}} d_{\mathrm{r}, w}\right)+d_{\mathrm{r}, w}, \\
& G_{2}(s)=\boldsymbol{c}_{\mathrm{r}}^{\top}\left(s \mathbb{I}-\boldsymbol{A}_{\mathrm{r}}-\boldsymbol{l}_{\mathrm{aw}, \mathrm{x}} \boldsymbol{c}_{\mathrm{r}}^{\top}\right)^{-1}\left(\boldsymbol{b}_{\mathrm{r}, u}+\boldsymbol{l}_{\mathrm{aw}, \mathrm{x}} d_{\mathrm{r}, u}\right)+d_{\mathrm{r}, u}, \\
& G_{3}(s)=-\boldsymbol{c}_{\mathrm{r}}^{\top}\left(s \mathbb{I}-\boldsymbol{A}_{\mathrm{r}}-\boldsymbol{l}_{\mathrm{aw}, \mathrm{x}} \boldsymbol{c}_{\mathrm{r}}^{\top}\right)^{-1} \boldsymbol{l}_{\mathrm{aw}, \mathrm{x}} .
\end{aligned}
$$

Die Grundidee zur Bewertung der Regelgüte ist, das Übertragungsverhalten (5.11) durch ein modellbasiertes lineares Anti-Windup nachzubilden. Dann lässt sich die Regelgüte anhand der Mismatch-Darstellung des Regelkreises analysieren. Abbildung 5.4 skizziert die Idee.

Ein modellbasiertes Anti-Windup besitzt die Systemstruktur (4.7). Hier wird der lineare Spezialfall mit $\boldsymbol{k}_{\mathrm{aw}}\left(\boldsymbol{x}_{\mathrm{aw}}\right)=-\underline{\boldsymbol{k}}^{\top} \boldsymbol{x}_{\mathrm{aw}}$ betrachtet, d. h.

$$
\mathbf{A W}:\left\{\begin{array}{l}
\dot{\boldsymbol{x}}_{\mathrm{aw}}=\left(\boldsymbol{A}_{\mathrm{s}}-\boldsymbol{b}_{\mathrm{s}, u} \underline{\boldsymbol{k}}^{\top}\right) \boldsymbol{x}_{\mathrm{aw}}+\boldsymbol{b}_{\mathrm{s}, u} \delta, \\
y_{\mathrm{aw}, \mathrm{y}}=\underline{\boldsymbol{k}}^{\top} \boldsymbol{x}_{\mathrm{aw}}, \\
y_{\mathrm{aw}, \mathrm{u}}=-\boldsymbol{c}_{\mathrm{s}}^{\top} \boldsymbol{x}_{\mathrm{aw}} .
\end{array}\right.
$$

Wie in Abschnitt (4.4) erläutert, ist die Systemdynamik durch die Strecke geprägt. Einzig der Vektor $\underline{\boldsymbol{k}} \in \mathbb{R}^{n_{\mathrm{s}}}$ muss beim Entwurf gewählt werden. Für den Eingang gilt

$$
\delta=u_{\mathrm{a}}-y_{\mathrm{a}}=u_{\mathrm{a}}-\operatorname{sat}_{\overline{\mathrm{u}}}\left(u_{\mathrm{a}}\right) .
$$


Anti-Windup-Regelkreis mit Beobachtertechnik

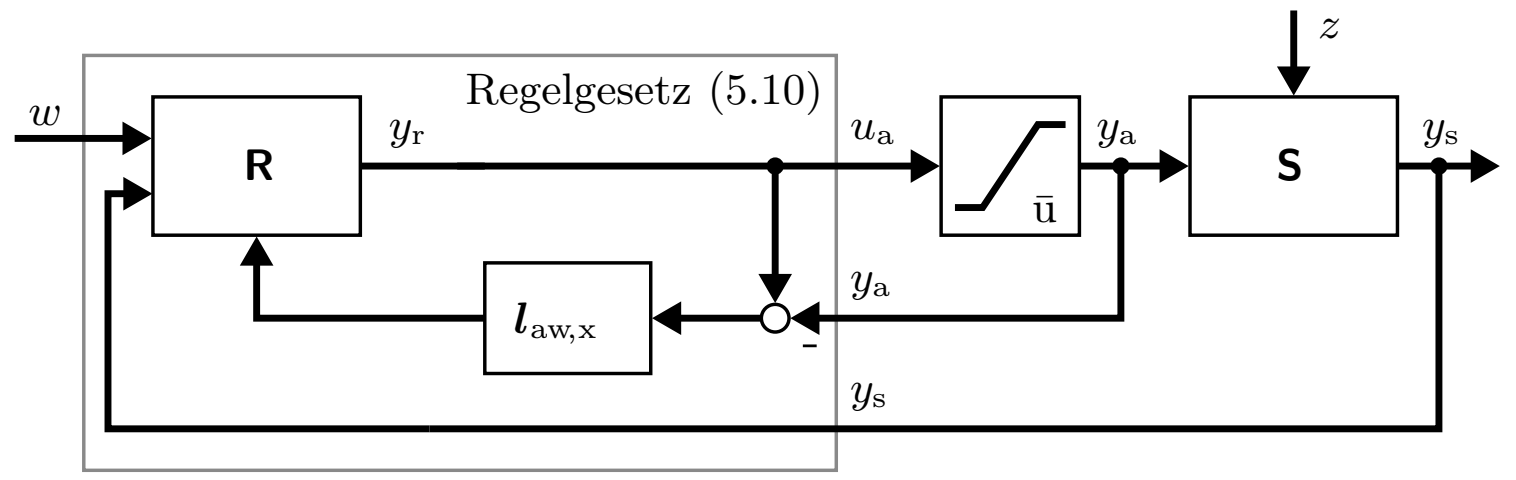

identisches Übertragungsverhalten
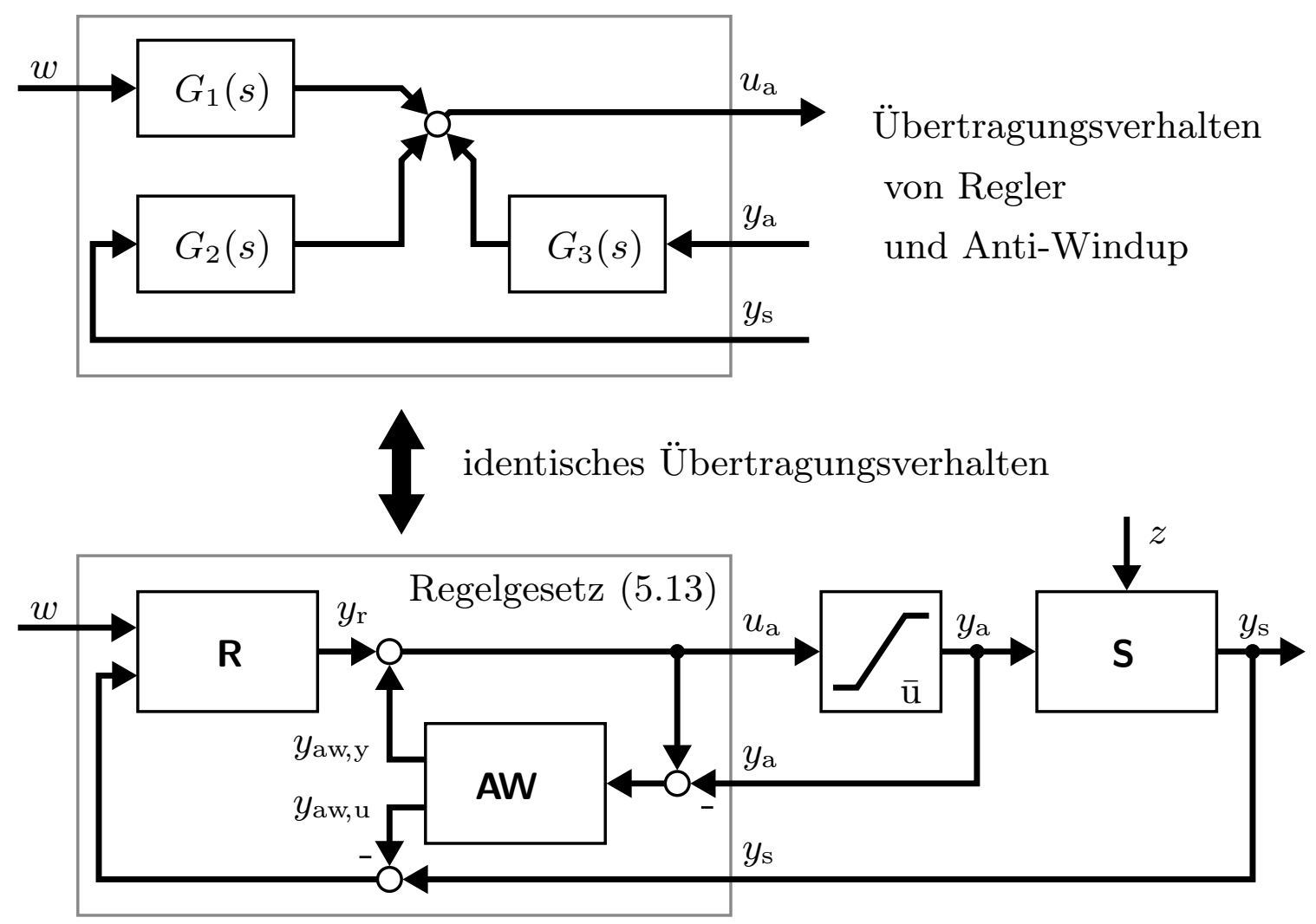

Ersatzsystem mit modellbasiertem Anti-Windup

Abbildung 5.4: Der Anti-Windup-Regelkreis mit Beobachtertechnik lässt sich in ein Ersatzsystem mit modellbasiertem Anti-Windup umformen, das ein identisches Übertragungsverhalten aufweist. Die Regelgüte des Ersatzsystems kann mithilfe der Mismatch-Darstellung einfach bewertet werden. 
Mit dem linearen Regler R gemäß (5.1) und der Verschaltung

$$
u_{\mathrm{a}}=y_{\mathrm{r}}+y_{\mathrm{aw}, \mathrm{y}}, \quad u_{\mathrm{r}}=y_{\mathrm{s}}-y_{\mathrm{aw}, \mathrm{u}}
$$

ergibt sich der im unteren Teil von Abbildung 5.4 veranschaulichte AntiWindup-Regelkreis. Nach dem Zusammenfassen der Zustände von Regler und Anti-Windup in dem Vektor

$$
\boldsymbol{x}^{\top}=\left[\begin{array}{ll}
\boldsymbol{x}_{\mathrm{aw}}^{\top} & \boldsymbol{x}_{\mathrm{r}}^{\top}
\end{array}\right] \in \mathbb{R}^{n_{\mathrm{s}}+n_{\mathrm{r}}}
$$

kann das Regelgesetz mit Anti-Windup wie folgt angegeben werden

$$
\begin{aligned}
\dot{\boldsymbol{x}} & =\boldsymbol{A}_{\mathrm{ub}} \boldsymbol{x}+\boldsymbol{b}_{1} w+\boldsymbol{b}_{2} y_{\mathrm{s}}+\boldsymbol{b}_{3} y_{\mathrm{a}}, \\
u_{\mathrm{a}} & =\boldsymbol{c}^{\top} \boldsymbol{x}+d_{\mathrm{r}, w} w+d_{\mathrm{r}, u} y_{\mathrm{s}} .
\end{aligned}
$$

Dabei gilt für die Systemmatrizen

$$
\begin{aligned}
\boldsymbol{A}_{\mathrm{ub}} & =\left[\begin{array}{cc}
\boldsymbol{A}_{\mathrm{s}}+\boldsymbol{b}_{\mathrm{s}, u} d_{\mathrm{r}, u} \boldsymbol{c}_{\mathrm{s}}^{\top} & \boldsymbol{b}_{\mathrm{s}, u} \boldsymbol{c}_{\mathrm{r}}^{\top} \\
\boldsymbol{b}_{\mathrm{r}, u} \boldsymbol{c}_{\mathrm{s}}^{\top} & \boldsymbol{A}_{\mathrm{r}}
\end{array}\right], \\
\boldsymbol{b}_{1} & =\left[\begin{array}{c}
\boldsymbol{b}_{\mathrm{s}, u} d_{\mathrm{r}, w} \\
\boldsymbol{b}_{\mathrm{r}, w}
\end{array}\right], \quad \boldsymbol{b}_{2}=\left[\begin{array}{c}
\boldsymbol{b}_{\mathrm{s}, u} d_{\mathrm{r}, u} \\
\boldsymbol{b}_{\mathrm{r}, u}
\end{array}\right], \quad \boldsymbol{b}_{3}=\left[\begin{array}{c}
-\boldsymbol{b}_{\mathrm{s}, u} \\
0
\end{array}\right], \\
\boldsymbol{c}^{\top} & =\left[\begin{array}{ll}
d_{\mathrm{r}, u} \boldsymbol{c}_{\mathrm{s}}^{\top}+\underline{\boldsymbol{k}}^{\top} & \boldsymbol{c}_{\mathrm{r}}^{\top}
\end{array}\right] .
\end{aligned}
$$

Die Matrix $\boldsymbol{A}_{\mathrm{ub}}$ entspricht der Systemmatrix des unbeschränkten Regelkreises (5.3). Das Übertragungsverhalten des Regelgesetzes (5.13) von den Eingängen $w, y_{\mathrm{s}}, y_{\mathrm{a}}$ zu dem Ausgang $u_{\mathrm{a}}$ ist gegeben durch

$$
u_{\mathrm{a}}(s)=H_{1}(s) w(s)+H_{2}(s) y_{\mathrm{s}}(s)+H_{3}(s) y_{\mathrm{a}}(s)
$$

mit den Übertragungsfunktionen

$$
\begin{aligned}
& H_{1}(s)=\boldsymbol{c}^{\top}\left(s \mathbb{I}-\boldsymbol{A}_{\mathrm{ub}}\right)^{-1} \boldsymbol{b}_{1}+d_{\mathrm{r}, w}, \\
& H_{2}(s)=\boldsymbol{c}^{\top}\left(s \mathbb{I}-\boldsymbol{A}_{\mathrm{ub}}\right)^{-1} \boldsymbol{b}_{2}+d_{\mathrm{r}, u}, \\
& H_{3}(s)=\boldsymbol{c}^{\top}\left(s \mathbb{I}-\boldsymbol{A}_{\mathrm{ub}}\right)^{-1} \boldsymbol{b}_{3} .
\end{aligned}
$$

Um das Übertragungsverhalten des Regelgesetzes (5.10) zu erreichen, muss der Vektor $\underline{\boldsymbol{k}}$ so gewählt werden, dass die Funktionen (5.14a), (5.14b), $(5.14 \mathrm{c})$ mit $(5.11 \mathrm{a}),(5.11 \mathrm{~b}),(5.11 \mathrm{c})$ übereinstimmen. Somit muss

$$
\begin{aligned}
& H_{1}(s)=G_{1}(s), \\
& H_{2}(s)=G_{2}(s), \\
& H_{3}(s)=G_{3}(s)
\end{aligned}
$$


für alle $s \in \mathbb{C}$ gelten. Aus diesen Forderungen lassen sich anhand der Darstellungen (5.11), (5.14) der Übertragungsfunktionen nur schwer Bedingungen an $\underline{\boldsymbol{k}}$ ableiten. Deshalb wird zunächst eine alternative Darstellung für SISO-Übertragungsfunktionen benötigt.

\section{Alternative Darstellung der Übertragungsfunktionen}

Ausgangspunkt der Überlegungen ist die Übertragungsfunktion

$$
G(s)=\boldsymbol{c}^{\top}(s \mathbb{I}-\boldsymbol{A})^{-1} \boldsymbol{b}+d .
$$

Mit dem in [133, Anhang A2.1] angegebenen Zusammenhang

$$
(s \mathbb{I}-\boldsymbol{A})^{-1}=\frac{1}{\operatorname{det}(s \mathbb{I}-\boldsymbol{A})} \operatorname{adj}(s \mathbb{I}-\boldsymbol{A})
$$

lässt sich die Übertragungsfunktion auch darstellen als

$$
G(s)=\frac{\boldsymbol{c}^{\top} \operatorname{adj}(s \mathbb{I}-\boldsymbol{A}) \boldsymbol{b}}{\operatorname{det}(s \mathbb{I}-\boldsymbol{A})}+d=\frac{\boldsymbol{c}^{\top} \operatorname{adj}(s \mathbb{I}-\boldsymbol{A}) \boldsymbol{b}+d \operatorname{det}(s \mathbb{I}-\boldsymbol{A})}{\operatorname{det}(s \mathbb{I}-\boldsymbol{A})} .
$$

Der Zähler kann dann mithilfe der Rosenbrock-Matrix

$$
\boldsymbol{Z}(s)=\left[\begin{array}{cc}
s \mathbb{I}-\boldsymbol{A} & \boldsymbol{b} \\
\boldsymbol{c}^{\mathrm{T}} & -d
\end{array}\right]
$$

nach [16, Gl. (12.10.12)] wie folgt geschrieben werden

$$
\boldsymbol{c}^{\top} \operatorname{adj}(s \mathbb{I}-\boldsymbol{A}) \boldsymbol{b}+d \operatorname{det}(s \mathbb{I}-\boldsymbol{A})=-\operatorname{det}(\boldsymbol{Z}(s)) .
$$

Daraus ergibt sich der folgende nützliche Zusammenhang

$$
G(s)=\frac{-\operatorname{det}(\boldsymbol{Z}(s))}{\operatorname{det}(s \mathbb{I}-\boldsymbol{A})}=\boldsymbol{c}^{\top}(s \mathbb{I}-\boldsymbol{A})^{-1} \boldsymbol{b}+d
$$

für SISO-Übertragungsfunktionen. Vorteilhaft an der Bruchdarstellung ist, dass die Matrixinversion entfällt. Des Weiteren lässt sich das Zählerpolynom durch die Determinante der Rosenbrock-Matrix darstellen und das Nennerpolynom mithilfe der Systemmatrix $\boldsymbol{A}$. Bezüglich der Übertragungsfunktionen (5.11) und (5.14) gilt

Lemma 5.2. Mit den Abkürzungen

$$
\boldsymbol{Z}_{\mathrm{r}, w}(s)=\left[\begin{array}{cc}
s \boldsymbol{I}-\boldsymbol{A}_{\mathrm{r}} & \boldsymbol{b}_{\mathrm{r}, w} \\
\boldsymbol{c}_{\mathrm{r}}^{\top} & -d_{\mathrm{r}, w}
\end{array}\right], \quad \boldsymbol{Z}_{\mathrm{r}, u}(s)=\left[\begin{array}{cc}
s \boldsymbol{I}-\boldsymbol{A}_{\mathrm{r}} & \boldsymbol{b}_{\mathrm{r}, u} \\
\boldsymbol{c}_{\mathrm{r}}^{\top} & -d_{\mathrm{r}, u}
\end{array}\right] .
$$


lassen sich die Übertragungsfunktionen (5.11) des Regelgesetzes mit Beobachtertechnik wie folgt schreiben

$$
\begin{aligned}
G_{1}(s) & =\frac{-\operatorname{det}\left(\boldsymbol{Z}_{\mathrm{r}, w}(s)\right)}{\operatorname{det}\left(s \mathbb{I}-\boldsymbol{A}_{\mathrm{r}}-\boldsymbol{l}_{\mathrm{aw}, \mathrm{x}} \boldsymbol{c}_{\mathrm{r}}^{\top}\right)}, \\
G_{2}(s) & =\frac{-\operatorname{det}\left(\boldsymbol{Z}_{\mathrm{r}, u}(s)\right)}{\operatorname{det}\left(s \mathbb{I}-\boldsymbol{A}_{\mathrm{r}}-\boldsymbol{l}_{\mathrm{aw}, \mathrm{x}} \boldsymbol{c}_{\mathrm{r}}^{\top}\right)}, \\
G_{3}(s) & =\frac{-\operatorname{det}\left(s \mathbb{I}-\boldsymbol{A}_{\mathrm{r}}\right)}{\operatorname{det}\left(s \mathbb{I}-\boldsymbol{A}_{\mathrm{r}}-\boldsymbol{l}_{\mathrm{aw}, \mathrm{x}} \boldsymbol{c}_{\mathrm{r}}^{\top}\right)}+1 .
\end{aligned}
$$

Für die Übertragungsfunktionen (5.14) gilt

$$
\begin{aligned}
& H_{1}(s)=\frac{-\operatorname{det}\left(s \mathbb{I}-\boldsymbol{A}_{\mathrm{s}}+\boldsymbol{b}_{\mathrm{s}, u} \underline{\boldsymbol{k}}^{\top}\right) \operatorname{det}\left(\boldsymbol{Z}_{\mathrm{r}, w}(s)\right)}{\operatorname{det}\left(s \mathbb{I}-\boldsymbol{A}_{\mathrm{ub}}\right)}, \\
& H_{2}(s)=\frac{-\operatorname{det}\left(s \mathbb{I}-\boldsymbol{A}_{\mathrm{s}}+\boldsymbol{b}_{\mathrm{s}, u} \underline{\boldsymbol{k}}^{\top}\right) \operatorname{det}\left(\boldsymbol{Z}_{\mathrm{r}, u}(s)\right)}{\operatorname{det}\left(s \mathbb{I}-\boldsymbol{A}_{\mathrm{ub}}\right)}, \\
& H_{3}(s)=\frac{-\operatorname{det}\left(s \mathbb{I}-\boldsymbol{A}_{\mathrm{s}}+\boldsymbol{b}_{\mathrm{s}, u} \underline{\boldsymbol{k}}^{\top}\right) \operatorname{det}\left(s \mathbb{I}-\boldsymbol{A}_{\mathrm{r}}\right)}{\operatorname{det}\left(s \mathbb{I}-\boldsymbol{A}_{\mathrm{ub}}\right)}+1 .
\end{aligned}
$$

Beweis. Der Beweis basiert auf (5.16) und elementaren Zeilenumformungen der beteiligten Matrizen. Er ist im Anhang C.3.1 angegeben.

Mithilfe dieses Lemmas lassen sich einfache Bedingungen an den Vektor $\underline{\boldsymbol{k}}$ formulieren, die Forderung (5.15) sicherstellen. Darauf geht der nächste Abschnitt detaillierter ein.

\section{Forderungen für äquivalentes Übertragungsverhalten}

Um (5.15) sicherzustellen, werden zunächst die Übertragungsfunktionen $H_{i}(s)$ betrachtet. Es fällt auf, dass sich deren Zählerpolynome aus zwei Faktoren zusammensetzen. Der erste Faktor ist durch

$$
\operatorname{det}\left(s \mathbb{I}-\boldsymbol{A}_{\mathrm{s}}+\boldsymbol{b}_{\mathrm{s}, u} \underline{\boldsymbol{k}}^{\top}\right)
$$

gegeben. Hierbei handelt es sich um das charakteristische Polynom der Matrix $\boldsymbol{A}_{\mathrm{s}}-\boldsymbol{b}_{\mathrm{s}, u} \underline{\boldsymbol{k}}^{\top}$. Der zweite Faktor entspricht nach (5.18) jeweils dem Zählerpolynom von $G_{i}(s)$. Damit Forderung (5.15) erfüllt werden kann, müssen sich die Nullstellen von (5.19) mit Nullstellen des Nennerpolynoms

$$
\operatorname{det}\left(s \mathbb{I}-\boldsymbol{A}_{\mathrm{ub}}\right)
$$


kürzen. In anderen Worten: alle $n_{\mathrm{s}}$ Eigenwerte der Matrix $\boldsymbol{A}_{\mathrm{s}}-\boldsymbol{b}_{\mathrm{s}, u} \underline{\boldsymbol{k}}^{\top}$ müssen mit $n_{\mathrm{s}}$ der $n_{\mathrm{s}}+n_{\mathrm{r}}$ Eigenwerte der Matrix $\boldsymbol{A}_{\mathrm{ub}}$ übereinstimmen.

Da das Matrixpaar $\left(\boldsymbol{A}_{\mathrm{s}}, \boldsymbol{b}_{\mathrm{s}, u}\right)$ steuerbar ist, können die Eigenwerte von $\boldsymbol{A}_{\mathrm{s}}-\boldsymbol{b}_{\mathrm{s}, u} \underline{\boldsymbol{k}}^{\top}$ durch $\underline{\boldsymbol{k}}$ beliebig platziert werden. Für eine kompaktere Notation werden diese Eigenwerte in der Menge

$$
\boldsymbol{\Lambda}_{\mathrm{aw}}(\underline{\boldsymbol{k}}):=\left\{\lambda \in \mathbb{C} \mid \operatorname{det}\left(\lambda \mathbb{I}-\boldsymbol{A}_{\mathrm{s}}+\boldsymbol{b}_{\mathrm{s}, u} \underline{\boldsymbol{k}}^{\top}\right)=0\right\}
$$

zusammengefasst. Die Eigenwerte von $\boldsymbol{A}_{\mathrm{ub}}$ sind in der Menge $\boldsymbol{\Lambda}_{\mathrm{ub}}$ nach (5.4) enthalten. Diese besitzt die Gestalt

$$
\boldsymbol{\Lambda}_{\mathrm{ub}}:=\left\{\lambda_{\mathrm{ub}} \in \mathbb{C} \mid \operatorname{det}\left(\lambda_{\mathrm{ub}} \mathbb{I}-\boldsymbol{A}_{\mathrm{ub}}\right)=0\right\} .
$$

Damit die Eigenwerte übereinstimmen, muss $\boldsymbol{\Lambda}_{\text {aw }}(\underline{\boldsymbol{k}})$ offensichtlich eine Teilmenge von $\boldsymbol{\Lambda}_{\mathrm{ub}}$ sein, d. h., der Vektor $\underline{\boldsymbol{k}}$ muss aus der Menge

$$
\mathcal{K}:=\left\{\underline{\boldsymbol{k}} \in \mathbb{R}^{n_{\mathrm{s}}} \mid \boldsymbol{\Lambda}_{\mathrm{aw}}(\underline{\boldsymbol{k}}) \subset \boldsymbol{\Lambda}_{\mathrm{ub}}\right\}
$$

gewählt werden. Diese Menge ist aufgrund der in Abschnitt 5.1 definierten Problemstellung nicht leer ${ }^{1}$.

Wie in Abschnitt 5.1 beschrieben, bilden die $n_{\mathrm{r}}$ Eigenwerte der Matrix $\boldsymbol{A}_{\mathrm{r}}+\boldsymbol{l}_{\mathrm{aw}, \mathrm{x}} \boldsymbol{c}_{\mathrm{r}}^{\top}$ für $\boldsymbol{l}_{\mathrm{aw}, \mathrm{x}} \in \mathcal{L}$ ebenfalls eine Untermenge der Eigenwerte von $\boldsymbol{A}_{\mathrm{ub}}$. Folglich lässt sich zu jedem Vektor $\boldsymbol{l}_{\mathrm{aw}, \mathrm{x}}$ aus der Menge $\mathcal{L}$ ein Vektor $\underline{\boldsymbol{k}}$ aus der Menge $\mathcal{K}$ finden, so dass gilt

$$
\boldsymbol{\Lambda}_{\mathrm{aw}}(\underline{\boldsymbol{k}}) \cup \boldsymbol{\Lambda}_{\mathrm{r}}\left(\boldsymbol{l}_{\mathrm{aw}, \mathrm{x}}\right)=\boldsymbol{\Lambda}_{\mathrm{ub}} .
$$

Diese speziellen Paare $\left(\boldsymbol{l}_{\mathrm{aw}, \mathrm{x}}, \underline{\boldsymbol{k}}\right) \in \mathcal{L} \times \mathcal{K}$ werden in der Menge

$$
\mathcal{J}:=\left\{\left(\boldsymbol{l}_{\mathrm{aw}, \mathrm{x}}, \underline{\boldsymbol{k}}\right) \in \mathcal{L} \times \mathcal{K} \mid \boldsymbol{\Lambda}_{\mathrm{aw}}(\underline{\boldsymbol{k}})=\boldsymbol{\Lambda}_{\mathrm{ub}} \backslash \boldsymbol{\Lambda}_{\mathrm{r}}\left(\boldsymbol{l}_{\mathrm{aw}, \mathrm{x}}\right)\right\}
$$

zusammengefasst. Für alle Paare $\left(\boldsymbol{l}_{\mathrm{aw}, \mathrm{x}}, \underline{\boldsymbol{k}}\right) \in \mathcal{L} \times \mathcal{K}$ lässt sich das Nennerpolynom (5.20) wie folgt faktorisieren

$$
\operatorname{det}\left(s \mathbb{I}-\boldsymbol{A}_{\mathrm{ub}}\right)=\operatorname{det}\left(s \mathbb{I}-\boldsymbol{A}_{\mathrm{s}}+\boldsymbol{b}_{\mathrm{s}, u} \underline{\boldsymbol{k}}^{\top}\right) \operatorname{det}\left(s \mathbb{I}-\boldsymbol{A}_{\mathrm{r}}-\boldsymbol{l}_{\mathrm{aw}, \mathrm{x}} \boldsymbol{c}_{\mathrm{r}}^{\top}\right) .
$$

Einsetzen dieser Beziehung in (5.18a), (5.18b), (5.18c) und kürzen der

\footnotetext{
${ }^{1}$ Der Fall eines unbeschränkten Regelkreises mit ausschließlich konjugiert komplexen Eigenwerten und einer ungeraden Systemordnung des Reglers und der Strecke wurde durch die Forderung $\mathcal{L} \neq\{\}$ ausgeschlossen.
} 
in Zähler und Nenner auftretenden Polynome liefert

$$
\begin{aligned}
& H_{1}(s)=\frac{-\operatorname{det}\left(\boldsymbol{Z}_{\mathrm{r}, w}(s)\right)}{\operatorname{det}\left(s \mathbb{I}-\boldsymbol{A}_{\mathrm{r}}-\boldsymbol{l}_{\mathrm{aw}, \mathrm{x}} \boldsymbol{c}_{\mathrm{r}}^{\top}\right)}=G_{1}(s), \\
& H_{2}(s)=\frac{-\operatorname{det}\left(\boldsymbol{Z}_{\mathrm{r}, u}(s)\right)}{\operatorname{det}\left(s \mathbb{I}-\boldsymbol{A}_{\mathrm{r}}-\boldsymbol{l}_{\mathrm{aw}, \mathrm{x}} \boldsymbol{c}_{\mathrm{r}}^{\top}\right)}=G_{2}(s), \\
& H_{3}(s)=\frac{-\operatorname{det}\left(s \mathbb{I}-\boldsymbol{A}_{\mathrm{r}}\right)}{\operatorname{det}\left(s \mathbb{I}-\boldsymbol{A}_{\mathrm{r}}-\boldsymbol{l}_{\mathrm{aw}, \mathrm{x}} \boldsymbol{c}_{\mathrm{r}}^{\top}\right)}+1=G_{3}(s) .
\end{aligned}
$$

Somit ist die Forderung (5.15) für alle geordneten Paare $\left(\boldsymbol{l}_{\mathrm{aw}, \mathrm{x}}, \underline{\boldsymbol{k}}\right) \in \mathcal{J}$ erfüllt. Um die Äquivalenz des Übertragungsverhaltens der Regelgesetze (5.10) und (5.13) sicher zu stellen, ist $\underline{\boldsymbol{k}}$ für ein gegebenes $\boldsymbol{l}_{\mathrm{aw}, \mathrm{x}} \in \mathcal{L}$ so zu wählen, dass $\left(\boldsymbol{l}_{\mathrm{aw}, \mathrm{x}}, \underline{\boldsymbol{k}}\right) \in \mathcal{J}$ gilt. Dieses Ergebnis präzisiert

Satz 5.1. Die Regelgesetze (5.10) und (5.13) sind für alle geordneten Paare $\left(\boldsymbol{l}_{\mathrm{aw}, \mathrm{x}}, \underline{\boldsymbol{k}}\right) \in \mathcal{J}$ äquivalent bezüglich des Übertragungsverhaltens von den Eingängen $w, y_{\mathrm{s}}, y_{\mathrm{a}} z u$ dem Ausgang $u_{\mathrm{a}}$.

Der Satz ermöglicht es, das auf der Beobachtertechnik basierende Regelgesetz (5.10) durch das Regelgesetz (5.13) mit einem linearen modellbasierten Anti-Windup zu ersetzen. Es ergibt sich das im unteren Teil von Abbildung 5.4 dargestellte Ersatzsystem, bestehend aus dem Regler R, dem modellbasierten AW gemäß (5.12) und der Strecke S. Eine Zustandsraumdarstellung dieses Ersatzsystems lautet wie folgt

$$
\begin{aligned}
& \dot{\boldsymbol{x}}_{\mathrm{s}}=\boldsymbol{A}_{\mathrm{s}} \boldsymbol{x}_{\mathrm{s}}+\boldsymbol{b}_{\mathrm{s}, u} \operatorname{sat}_{\overline{\mathrm{u}}}\left(y_{\mathrm{r}}+\underline{\boldsymbol{k}}^{\top} \boldsymbol{x}_{\mathrm{aw}}\right)+\boldsymbol{b}_{\mathrm{s}, z} z, \\
& \dot{\boldsymbol{x}}_{\mathrm{r}}=\boldsymbol{A}_{\mathrm{r}} \boldsymbol{x}_{\mathrm{r}}+\boldsymbol{b}_{\mathrm{r}, u} \boldsymbol{c}_{\mathrm{s}}^{\top}\left(\boldsymbol{x}_{\mathrm{s}}+\boldsymbol{x}_{\mathrm{aw}}\right)+\boldsymbol{b}_{\mathrm{r}, w} w, \\
& \dot{\boldsymbol{x}}_{\mathrm{aw}}=\boldsymbol{A}_{\mathrm{s}} \boldsymbol{x}_{\mathrm{aw}}+\boldsymbol{b}_{\mathrm{s}, u} y_{\mathrm{r}}-\boldsymbol{b}_{\mathrm{s}, u} \operatorname{sat}_{\overline{\mathrm{u}}}\left(y_{\mathrm{r}}+\underline{\boldsymbol{k}}^{\top} \boldsymbol{x}_{\mathrm{aw}}\right), \\
& y_{\mathrm{r}}=\boldsymbol{c}_{\mathrm{r}}^{\top} \boldsymbol{x}_{\mathrm{r}}+d_{\mathrm{r}, u} \boldsymbol{c}_{\mathrm{s}}^{\top}\left(\boldsymbol{x}_{\mathrm{s}}+\boldsymbol{x}_{\mathrm{aw}}\right)+d_{\mathrm{r}, w} w, \\
& y_{\mathrm{s}}=\boldsymbol{c}_{\mathrm{s}}^{\top} \boldsymbol{x}_{\mathrm{s}} .
\end{aligned}
$$

Der Nutzen des Ersatzsystems wird im nächsten Abschnitt deutlich.

\section{Vorteil des Ersatzsystems: Mismatch-Darstellung}

Mittels der Transformation (4.9) lässt sich das Ersatzsystem (5.21) in Mismatch-Koordinaten überführen. Dabei zerfällt das transformierte System, wie in Abschnitt 4.4.1 und 4.4.2 (Lösung 1) erläutert, in zwei Teil- 


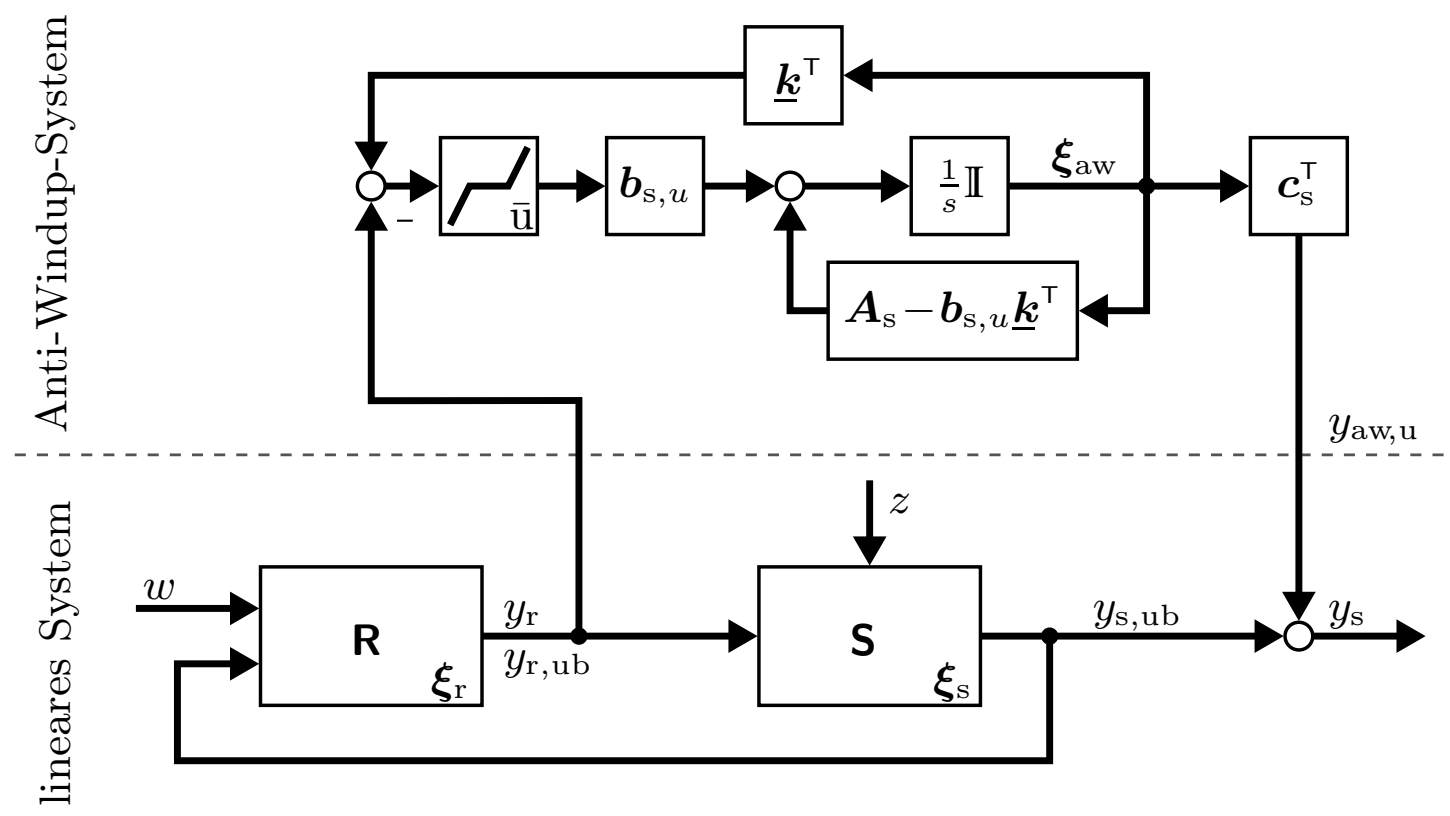

Abbildung 5.5: Das Ersatzsystem (5.21) kann mittels der Zustandstransformation (4.9) in das hier gezeigte Mismatch-System umgeformt werden. Die Abweichung des Anti-Windup-Regelkreises von dem unbeschränkten Verhalten lässt sich dann anhand der $L_{2}$-Verstärkung des Anti-Windup-Systems bestimmen.

systeme: den unbeschränkten Regelkreis und das Anti-Windup-System

$$
\begin{aligned}
\dot{\boldsymbol{\xi}}_{\mathrm{aw}} & =\left(\boldsymbol{A}_{\mathrm{s}}-\boldsymbol{b}_{\mathrm{s}, u} \underline{\boldsymbol{k}}^{\top}\right) \boldsymbol{\xi}_{\mathrm{aw}}+\boldsymbol{b}_{\mathrm{s}, u} \mathrm{dz} \mathrm{z}_{\overline{\mathrm{u}}}\left(\underline{\boldsymbol{k}}^{\top} \boldsymbol{\xi}_{\mathrm{aw}}-y_{\mathrm{r}}\right), \\
y_{\mathrm{aw}, \mathrm{u}} & =\boldsymbol{c}_{\mathrm{s}}^{\top} \boldsymbol{\xi}_{\mathrm{aw}},
\end{aligned}
$$

wobei $\boldsymbol{\xi}_{\mathrm{aw}}^{(0)}=\mathbf{0}$ gilt. Letzteres entspricht Lösung 1 aus Abbildung 4.7. Abbildung 5.5 zeigt das Mismatch-System und verdeutlicht, dass der Ausgang $y_{\mathrm{aw}, \mathrm{u}}$ des Anti-Windup-Systems die Abweichung des Anti-WindupRegelkreises von dem Verhalten des unbeschränkten Regelkreises darstellt.

Eine Möglichkeit, die Abweichung des Mismatch-Systems und damit auch die Abweichung des Anti-Windup-Regelkreises von dem unbeschränkten Verhalten zu charakterisieren, führt über die $L_{2}$-Verstärkung des AntiWindup-Systems (5.22) vom Eingang $y_{\mathrm{r}}$ zu dem Ausgang $y_{\mathrm{aw}, \mathrm{u}}$ (vgl. Abschnitt 4.2). Je kleiner die $L_{2}$-Verstärkung ist, desto kleiner wird auch die $L_{2}$-Norm des Ausgangs sein. Der nächste Abschnitt zeigt, wie sich die $L_{2}$-Verstärkung des Mismatch-Systems abschätzen lässt. 


\subsubsection{Abschätzung der L2-Verstärkung}

Die im Folgenden vorgestellten Methoden zur Abschätzung der $L_{2}$-Verstärkung sind nicht nur für SISO-Systeme der Form (5.22) geeignet. Um dies zu unterstreichen erfolgt die Darstellung anhand des MIMO-Systems

$$
\begin{aligned}
\dot{\boldsymbol{\xi}}_{\mathrm{aw}} & =\left(\boldsymbol{A}_{\mathrm{s}}-\boldsymbol{B}_{\mathrm{s}, u} \underline{\boldsymbol{K}}\right) \boldsymbol{\xi}_{\mathrm{aw}}+\boldsymbol{B}_{\mathrm{s}, u} \mathbf{d z}_{\overline{\mathbf{u}}}\left(\underline{\boldsymbol{K}} \boldsymbol{\xi}_{\mathrm{aw}}-\boldsymbol{y}_{\mathrm{r}}\right), \\
\boldsymbol{y}_{\mathrm{aw}, u} & =\boldsymbol{C}_{\mathrm{s}} \boldsymbol{\xi}_{\mathrm{aw}}
\end{aligned}
$$

mit $\boldsymbol{\xi}_{\text {aw }} \in \mathbb{R}^{n_{\mathrm{s}}}$ sowie $\boldsymbol{y}_{\mathrm{r}}, \boldsymbol{y}_{\mathrm{aw}, \mathrm{u}} \in \mathbb{R}^{m}$ und dem Anfangszustand $\boldsymbol{\xi}_{\mathrm{aw}}^{(0)}=\mathbf{0}$. Für $m=1$ ergibt sich der Spezialfall (5.22). Ähnlich wie bei dem Stabilitätssatz von Lee aus Abschnitt 5.2 spielen Eigenschaften der Sektornichtlinearität bei der Abschätzung der $L_{2}$-Verstärkung eine wichtige Rolle. Besonders wichtig ist die sogenannte klassische Sektorbedingung, die in dem folgenden Lemma für multivariable Nichtlinearitäten angegeben ist.

Lemma 5.3 (Klassische Sektorbedingung, [182]). Liegt eine Nichtlinearität $\boldsymbol{y}=\boldsymbol{\mu}(\boldsymbol{u})$ global im Sektor $\mathcal{S}(\mathbf{0}, \boldsymbol{\Phi})$, so gilt für alle $\boldsymbol{u} \in \mathbb{R}^{m}$ die folgende Ungleichung

$$
\boldsymbol{y}^{\top} \hat{\boldsymbol{\Psi}}(\boldsymbol{\Phi} \boldsymbol{u}-\boldsymbol{y}) \geq 0
$$

mit einer beliebigen positiv definiten Diagonalmatrix

$$
\hat{\boldsymbol{\Psi}}=\operatorname{diag}\left(\hat{\psi}_{1}, \ldots, \hat{\psi}_{m}\right) \text {. }
$$

Beweis. Siehe Abschnitt C.3.2 im Anhang.

Die Totzone des Anti-Windup-Systems (5.23) liegt beispielsweise global im Sektor $\mathcal{S}(\mathbf{0}, \mathbb{I})$. Folglich erfüllt sie die klassische Sektorbedingung mit $\boldsymbol{\Phi}=\mathbb{I}$. In [191] wurde die Sektorbedingung genutzt, um einen Entwurfssatz für lineare modellbasierte Anti-Windup-Maßnahmen zu beweisen. In modifizierter Form lässt sich dieser Satz auch für die Abschätzung der $L_{2}$-Verstärkung nutzen. Er ist nachfolgend angegeben.

Satz 5.2 (Abschätzung der $L_{2}$-Verstärkung). Gegeben sei das System

$$
\begin{aligned}
& \dot{\boldsymbol{\xi}}_{\mathrm{aw}}=\left(\boldsymbol{A}_{\mathrm{s}}-\boldsymbol{B}_{\mathrm{s}, u} \underline{\boldsymbol{K}}\right) \boldsymbol{\xi}_{\mathrm{aw}}+\boldsymbol{B}_{\mathrm{s}, u} \boldsymbol{d} z_{\overline{\boldsymbol{u}}}\left(\underline{\boldsymbol{K}} \boldsymbol{\xi}_{\mathrm{aw}}-\boldsymbol{y}_{\mathrm{r}}\right), \\
& \boldsymbol{y}_{\mathrm{aw}, \mathrm{u}}=\boldsymbol{C}_{\mathrm{s}} \boldsymbol{\xi}_{\mathrm{aw}}
\end{aligned}
$$

mit $\boldsymbol{\xi}_{\text {aw }} \in \mathbb{R}^{n}, \boldsymbol{y}_{\mathrm{r}}, \boldsymbol{y}_{\text {aw }, \mathrm{u}} \in \mathbb{R}^{m}, \underline{\boldsymbol{K}} \in \mathbb{R}^{m \times n}$. Wenn eine symmetrische Matrix $\boldsymbol{Q} \in \mathbb{S}^{n}$ und eine symmetrische Diagonalmatrix $\boldsymbol{\Psi} \in \mathbb{S}^{m}$ mit

$$
\boldsymbol{Q} \succ \mathbf{0}, \quad \boldsymbol{\Psi}=\operatorname{diag}\left(\psi_{1}, \ldots, \psi_{m}\right) \succ \mathbf{0}
$$


sowie ein positiver Skalar $\gamma$ existieren, so dass die Matrixungleichung

$$
\operatorname{He}\left[\begin{array}{crcc}
\left(\boldsymbol{A}_{\mathrm{s}}-\boldsymbol{B}_{\mathrm{s}, u} \underline{\boldsymbol{K}}\right) \boldsymbol{Q} & \mathbf{0} & \mathbf{0} & \mathbf{0} \\
\boldsymbol{\Psi} \boldsymbol{B}_{\mathrm{s}, u}^{\top}+\underline{\boldsymbol{K}} \boldsymbol{Q} & -\boldsymbol{\Psi} & \mathbf{0} & \mathbf{0} \\
\mathbf{0} & \mathbb{I} & -\frac{1}{2} \gamma \mathrm{II} & \mathbf{0} \\
\boldsymbol{C}_{\mathrm{s}} \boldsymbol{Q} & \mathbf{0} & \mathbf{0} & -\frac{1}{2} \gamma \mathrm{II}
\end{array}\right] \prec \mathbf{0}
$$

erfüllt ist, dann ist das System (5.25) für $\boldsymbol{y}_{\mathrm{r}} \equiv \mathbf{0}$ global asymptotisch stabil. Des Weiteren ist die $L_{2}$-Verstärkung des Systems vom Eingang $\boldsymbol{y}_{\mathrm{r}}$ zum Ausgang $\boldsymbol{y}_{\text {aw, }}$ kleiner gleich $\gamma$.

Beweis. In [191] ist kein vollständiger Beweis angegeben. Eine ausführliche Darstellung findet sich deshalb im Abschnitt C.3.3 des Anhangs.

Satz 5.2 lässt sich verwenden, um die $L_{2}$-Verstärkung von System (5.23) abzuschätzen. Dazu müssen eine symmetrische, positiv definite Matrix $\boldsymbol{Q} \in \mathbb{S}^{n_{\mathrm{s}}}$ und eine positiv definite Diagonalmatrix $\boldsymbol{\Psi} \in \mathbb{S}^{m}$ sowie ein positiver Skalar $\gamma$ gefunden werden, welche die Matrixungleichung (5.26) erfüllen. Für eine genaue Abschätzung sollte die obere Schranke $\gamma$ für die $L_{2}$-Verstärkung möglichst klein sein. Somit ergibt sich das konvexe

\section{Optimierungsproblem 5.1.}

$\underset{\boldsymbol{Q}, \boldsymbol{\Psi}, \gamma}{\operatorname{Minimiere}} \gamma \quad$ unter den Nebenbedingungen

$\boldsymbol{Q} \succ \mathbf{0}, \quad \boldsymbol{\Psi}=\operatorname{diag}\left(\psi_{1}, \ldots, \psi_{m}\right) \succ \mathbf{0}, \quad \gamma>0$,

$\operatorname{He}\left[\begin{array}{cccc}\left(\boldsymbol{A}_{\mathrm{s}}-\boldsymbol{B}_{\mathrm{s}, u} \underline{\boldsymbol{K}}\right) \boldsymbol{Q} & \mathbf{0} & \mathbf{0} & \mathbf{0} \\ \boldsymbol{\Psi} \boldsymbol{B}_{\mathrm{s}, u}^{\top}+\underline{\boldsymbol{K}} \boldsymbol{Q} & -\boldsymbol{\Psi} & \mathbf{0} & \mathbf{0} \\ \mathbf{0} & \mathbb{I} & -\frac{1}{2} \gamma \mathbb{I} & \mathbf{0} \\ \boldsymbol{C}_{\mathrm{s}} \boldsymbol{Q} & \mathbf{0} & \mathbf{0} & -\frac{1}{2} \gamma \mathbb{I}\end{array}\right] \prec \mathbf{0}$.

Mit Optimierungsproblem 5.1 steht eine Möglichkeit zur Verfügung, ein Maß für die Regelgüte des Mismatch-Systems abzuschätzen. Allerdings stellt sich die Frage, ob die Genauigkeit der Abschätzung verbessert werden kann, wenn die vorhandenen Informationen über die Nichtlinearität des Systems besser genutzt werden. Des Weiteren ist fraglich, ob eine Kenngröße zur Beurteilung der Regelgüte ausreichend ist. Den ersten Punkt behandelt der nächste Abschnitt ausführlich. Die Darstellung orientiert sich dabei an [191], ist aber an die Problemstellung angepasst. 


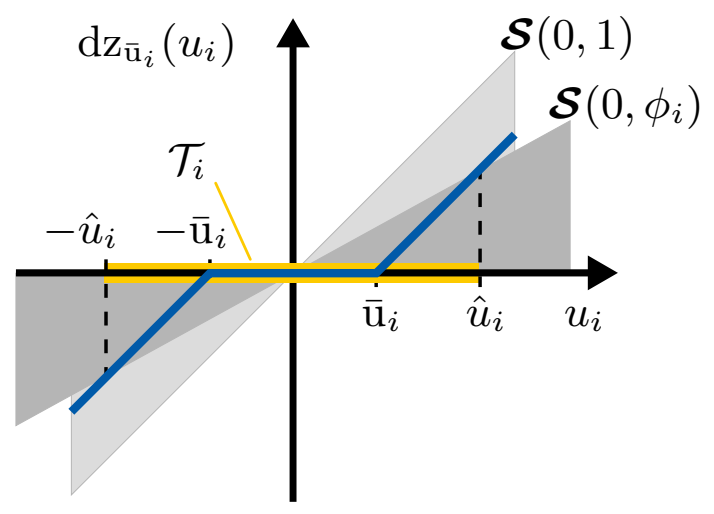

Abbildung 5.6: Die Totzone (-) liegt global im Sektor $\mathcal{S}(0,1)$ und lokal, d. h. für $u_{i} \in \mathcal{T}_{i}=\left[-\hat{u}_{i}, \hat{u}_{i}\right]$, in einem schmaleren Sektor $\mathcal{S}\left(0, \phi_{i}\right)$ mit $0<\phi_{i}<1$.

\subsubsection{Präzisere Abschätzung für kleine Signale}

Im letzten Abschnitt konnte mittels Optimierungsproblem 5.1 eine obere Schranke für die $L_{2}$-Verstärkung des Anti-Windup-Systems (5.23) vom Eingang $\boldsymbol{y}_{\mathrm{r}}$ zum Ausgang $\boldsymbol{y}_{\mathrm{aw}, \mathrm{u}}$ abgeschätzt werden. Diese Abschätzung gilt allerdings für beliebige Sektornichtlinearitäten im Sektor $\mathcal{S}(\mathbf{0}, \mathbb{I})$ und berücksichtigt nicht die speziellen Eigenschaften der Totzone. Konservative Ergebnisse sind die Folge. Eine Erhöhung der Genauigkeit lässt sich erreichen, wenn berücksichtigt wird, dass die Totzone lokal, d. h. für kleine Argumente, in einem viel schmaleren Sektor $\mathcal{S}(\mathbf{0}, \boldsymbol{\Phi})$ liegt.

Betrachtet wird zuerst die $i$-te Komponente der Totzone des AntiWindup-Systems (5.23). Gemäß (4.14) gilt

$$
\mathrm{dz}_{\overline{\mathrm{u}}_{i}}\left(u_{i}\right)=\left\{\begin{array}{lr}
u_{i}+\overline{\mathrm{u}}_{i}, & -\overline{\mathrm{u}}_{i}>u_{i}, \\
0, & -\overline{\mathrm{u}}_{i} \leq u_{i} \leq \overline{\mathrm{u}}_{i}, \\
u_{i}-\overline{\mathrm{u}}_{i}, & u_{i}>\overline{\mathrm{u}}_{i} .
\end{array}\right.
$$

Diese Funktion ist in Abbildung 5.6 dargestellt. Sie besitzt die Eigenschaft der lokalen Sektorzugehörigkeit, die für multivariable Funktionen wie folgt definiert ist.

Definition 5.2 (Lokale Sektorzugehörigkeit). Gegeben sei die Menge

$$
\begin{aligned}
& \mathcal{T}=\mathcal{T}_{1} \times \cdots \times \mathcal{T}_{m}, \\
& \mathcal{T}_{i}=\left[-\hat{u}_{i}, \hat{u}_{i}\right] \subset \mathbb{R}, \quad \hat{u}_{i} \in \mathbb{R}_{>0} .
\end{aligned}
$$

Eine nichtlineare stückweise stetige Funktion der Gestalt

$$
\boldsymbol{y}=\boldsymbol{\mu}(\boldsymbol{u})=\left[\begin{array}{lll}
\mu_{1}\left(u_{1}\right) & \ldots & \mu_{m}\left(u_{m}\right)
\end{array}\right]^{\top}
$$


mit $\boldsymbol{y}, \boldsymbol{u} \in \mathbb{R}^{m}$ liegt lokal, d. h. für alle $\boldsymbol{u} \in \mathcal{T}$, im Sektor $\mathcal{S}(\mathbf{0}, \boldsymbol{\Phi})$ mit

$$
\boldsymbol{\Phi}=\operatorname{diag}\left(\phi_{1}, \ldots, \phi_{m}\right) \succ \mathbf{0}
$$

wenn für alle Komponenten $\mu_{i}\left(u_{i}\right), i=1, \ldots, m$ gilt

$$
\begin{aligned}
& 0 \leq \mu_{i}\left(u_{i}\right)=y_{i} \leq \phi_{i, o} u_{i} \\
& \forall u_{i} \in \mathcal{T}_{i} \cap \mathbb{R}_{\geq 0}, \\
& \phi_{i, o} u_{i} \leq \mu_{i}\left(u_{i}\right)=y_{i} \leq \phi_{i, u} u_{i} \\
& \forall u_{i} \in \mathcal{T}_{i} \cap \mathbb{R}_{<0} .
\end{aligned}
$$

Anschaulich bedeuten die Ungleichungen (5.27a) und (5.27b), dass jede Komponente $\mu_{i}\left(u_{i}\right)$ der Nichtlinearität lokal, d. h. für alle $u_{i} \in \mathcal{T}_{i}$, von der Abszisse und der Ursprungsgeraden mit der Steigung $\phi_{i}$ eingeschlossen wird. Somit liegt die Totzone $\mathrm{dz}_{\bar{u}_{i}}\left(u_{i}\right)$ aus Abbildung 5.6 lokal, d. h. für

$$
u_{i} \in \mathcal{T}_{i}\left(\phi_{i}\right)=\left\{u_{i} \in \mathbb{R}|| u_{i} \mid \leq \hat{u}_{i}=\frac{\overline{\mathrm{u}}_{i}}{1-\phi_{i}}\right\}
$$

im Sektor $\mathcal{S}\left(0, \phi_{i}\right)$. Dieser Sektor schließst die Totzone lokal präziser ein als der Sektor $\mathcal{S}(0,1)$. Das gilt auch im Mehrgrößenfall und kann für eine präzisere Abschätzung der $L_{2}$-Verstärkung ausgenutzt werden. Zuvor wird noch ein Lemma benötigt, das die klassische Sektorbedingung aus Lemma 5.3 auf den lokalen Fall überträgt.

Lemma 5.4 (Lokale Sektorbedingung). Liegt die stückweise stetige Nichtlinearität $\boldsymbol{y}=\boldsymbol{\mu}(\boldsymbol{u})$ lokal, d. h. für alle $\boldsymbol{u} \in \mathcal{T}$, im Sektor $\mathcal{S}(\mathbf{0}, \boldsymbol{\Phi})$, so gilt die Sektorbedingung

$$
\boldsymbol{y}^{\top} \hat{\boldsymbol{\Psi}}(\boldsymbol{\Phi} \boldsymbol{u}-\boldsymbol{y}) \geq 0
$$

mit einer beliebigen positiv definiten Diagonalmatrix $\hat{\boldsymbol{\Psi}}$ für alle $\boldsymbol{u} \in \mathcal{T}$.

Beweis. Der Beweis erfolgt analog zu dem Beweis von Lemma 5.3.

Die lokale Sektorbedingung ist nur für betragsmäßig kleine Argumente der Totzone gültig. Eine auf dieser lokalen Sektorbedingung basierende Abschätzung der $L_{2}$-Verstärkung des Anti-Windup-Systems

$$
\begin{aligned}
\dot{\boldsymbol{\xi}}_{\mathrm{aw}} & =\left(\boldsymbol{A}_{\mathrm{s}}-\boldsymbol{B}_{\mathrm{s}, u} \underline{\boldsymbol{K}}\right) \boldsymbol{\xi}_{\mathrm{aw}}+\boldsymbol{B}_{\mathrm{s}, u} \mathbf{d} \mathbf{z}_{\overline{\mathbf{u}}}\left(\underline{\boldsymbol{K}} \boldsymbol{\xi}_{\mathrm{aw}}-\boldsymbol{y}_{\mathrm{r}}\right), \\
\boldsymbol{y}_{\mathrm{aw}, \mathrm{u}} & =\boldsymbol{C}_{\mathrm{s}} \boldsymbol{\xi}_{\mathrm{aw}}
\end{aligned}
$$

mit $\boldsymbol{\xi}_{\text {aw }}^{(0)}=\mathbf{0}$ gilt deshalb nur für bestimmte Eingangssignale $\boldsymbol{y}_{\mathrm{r}}(t)$. Um diese Eingangssignale genauer zu charakterisieren, wird das Argument

$$
\tilde{\boldsymbol{u}}=\underline{\boldsymbol{K}} \boldsymbol{\xi}_{\mathrm{aw}}-\boldsymbol{y}_{\mathrm{r}}
$$


der Totzone genauer betrachtet. Offensichtlich gilt die lokale Sektorbedingung für einen gegebenen Sektor $\mathcal{S}(\mathbf{0}, \boldsymbol{\Phi})$ mit $\boldsymbol{\Phi}=\operatorname{diag}\left(\phi_{1}, \ldots, \phi_{m}\right)$ nur für amplitudenbegrenzte Signale $\tilde{\boldsymbol{u}}(t)$, die in der Signalklasse

$$
\mathcal{W}(\boldsymbol{\Phi})=\left\{\tilde{\boldsymbol{u}}: \mathbb{R}_{\geq 0} \mapsto \mathbb{R}^{m}|| \tilde{u}_{i} \mid \leq \hat{u}_{i}=\frac{\overline{\mathrm{u}}_{i}}{1-\phi_{i}} \forall i=1, \ldots, m, \forall t \geq 0\right\}
$$

enthalten sind. Folglich ist die $L_{2}$-Verstärkung nur für die Eingangssignale $\boldsymbol{y}_{\mathrm{r}}$ gültig, die zu Signalen $\tilde{\boldsymbol{u}} \in \mathcal{W}(\boldsymbol{\Phi})$ führen. Kompakt lassen sich diese Eingangssignale in der Signalklasse

$$
\mathcal{Y}(\boldsymbol{\Phi})=\left\{\boldsymbol{y}_{\mathrm{r}}: \mathbb{R}_{\geq 0} \mapsto \mathbb{R}^{m} \mid \tilde{\boldsymbol{u}}(t) \in \mathcal{W}(\boldsymbol{\Phi}) \quad \forall t \leq 0\right\}
$$

zusammenfassen. Nun kann eine passende $L_{2}$-Verstärkung für alle Eingangssignale $\boldsymbol{y}_{\mathrm{r}} \in \mathcal{Y}(\boldsymbol{\Phi})$ definiert werden. In Anlehnung an [191] wird diese als $L_{2}$-Kleinsignalverstärkung bezeichnet.

Definition 5.3 ( $L_{2}$-Kleinsignalverstärkung). Gegeben sei das System (5.28) mit $\boldsymbol{\xi}_{\mathrm{aw}}^{(0)}=\mathbf{0}$. Das System besitzt die $L_{2}$-Verstärkung $\gamma_{\boldsymbol{y}}$ für Eingangssignale $\boldsymbol{y}_{\mathrm{r}} \in \mathcal{Y}(\boldsymbol{\Phi})$, wenn gilt

$$
\sup _{0<\left\|\boldsymbol{y}_{\mathrm{r}}\right\|_{L_{2}}<\infty, \boldsymbol{y}_{\mathrm{r}} \in \mathcal{Y}(\boldsymbol{\Phi})} \frac{\left\|\boldsymbol{y}_{\mathrm{aw}, \mathrm{u}}\right\|_{L_{2}}}{\left\|\boldsymbol{y}_{\mathrm{r}}\right\|_{L_{2}}} \leq \gamma_{\mathcal{Y}}
$$

Um für Systeme der Form (5.28) zu einer präziseren Abschätzung der $L_{2}$-Verstärkung zu gelangen, kann der folgende Satz verwendet werden.

Satz 5.3 (Abschätzung der $L_{2}$-Kleinsignalverstärkung). Gegeben sei eine positiv definite Diagonalmatrix $\mathbf{\Phi}=\operatorname{diag}\left(\phi_{1}, \ldots, \phi_{m}\right) \succ \mathbf{0}$ und das System

$$
\begin{aligned}
& \dot{\boldsymbol{\xi}}_{\mathrm{aw}}=\left(\boldsymbol{A}_{\mathrm{s}}-\boldsymbol{B}_{\mathrm{s}, u} \underline{\boldsymbol{K}}\right) \boldsymbol{\xi}_{\mathrm{aw}}+\boldsymbol{B}_{\mathrm{s}, u} \boldsymbol{d} z_{\overline{\boldsymbol{u}}}\left(\underline{\boldsymbol{K}} \boldsymbol{\xi}_{\mathrm{aw}}-\boldsymbol{y}_{\mathrm{r}}\right), \\
& \boldsymbol{y}_{\mathrm{aw}, \mathrm{u}}=\boldsymbol{C}_{\mathrm{s}} \boldsymbol{\xi}_{\mathrm{aw}}
\end{aligned}
$$

mit $\boldsymbol{\xi}_{\text {aw }} \in \mathbb{R}^{n}, \boldsymbol{y}_{\mathrm{r}}, \boldsymbol{y}_{\text {aw }, \mathrm{u}} \in \mathbb{R}^{m}, \underline{\boldsymbol{K}} \in \mathbb{R}^{m \times n}$ sowie einer Totzone

$$
\boldsymbol{d} z_{\overline{\boldsymbol{u}}}(\boldsymbol{u})=\left[\begin{array}{lll}
\mathrm{dz}_{\bar{u}_{1}}\left(u_{1}\right) & \ldots & \mathrm{dz}_{\bar{u}_{m}}\left(u_{m}\right)
\end{array}\right]^{\top} .
$$

Wenn eine symmetrische Matrix $\boldsymbol{Q} \in \mathbb{S}^{n}$ und eine Diagonalmatrix $\boldsymbol{\Psi} \in \mathbb{S}^{m}$ mit den Eigenschaften

$$
\boldsymbol{Q} \succ \mathbf{0}, \quad \boldsymbol{\Psi}=\operatorname{diag}\left(\psi_{1}, \ldots, \psi_{m}\right) \succ \mathbf{0}
$$


sowie ein positiver Skalar $\gamma_{\mathcal{y}}$ existieren, so dass die Matrixungleichung

$$
\mathrm{He}\left[\begin{array}{crcc}
\boldsymbol{A}_{\mathrm{s}} \boldsymbol{Q}-\boldsymbol{B}_{\mathrm{s}, u} \underline{\boldsymbol{K}} \boldsymbol{Q} & \mathbf{0} & \mathbf{0} & \mathbf{0} \\
\boldsymbol{\Psi} \boldsymbol{B}_{\mathrm{s}, u}^{\top}+\boldsymbol{\Phi} \underline{\boldsymbol{K}} \boldsymbol{Q} & -\boldsymbol{\Psi} & \mathbf{0} & \mathbf{0} \\
\mathbf{0} & \mathbf{\Phi} & -\frac{1}{2} \gamma_{\mathcal{Y}} \mathbb{I} & \mathbf{0} \\
\boldsymbol{C}_{\mathrm{s}} \boldsymbol{Q} & \mathbf{0} & \mathbf{0} & -\frac{1}{2} \gamma_{\mathcal{Y}} \mathbb{I}
\end{array}\right] \prec \mathbf{0}
$$

erfüllt ist, dann ist die Ruhelage $\boldsymbol{\xi}_{\mathrm{aw}}^{(0)}=\mathbf{0}$ des Systems (5.29) für $\boldsymbol{y}_{\mathrm{r}} \equiv \mathbf{0}$ lokal asymptotisch stabil. Des Weiteren ist die $L_{2}$-Kleinsignalverstärkung des Systems vom Eingang $\boldsymbol{y}_{\mathrm{r}}$ zum Ausgang $\boldsymbol{y}_{\text {aw, }}$ für Signale mit endlicher $L_{2}$-Norm aus der Signalklasse $\mathcal{Y}(\boldsymbol{\Phi})$ kleiner gleich $\gamma_{\mathcal{\nu}}$.

Beweis. Der Beweis basiert auf der Argumentation aus [175, 191] und findet sich im Anhang C.3.4.

Satz 5.3 lässt sich verwenden, um die $L_{2}$-Kleinsignalverstärkung von System (5.29) abzuschätzen. Für eine genaue Abschätzung sollte die obere Schranke $\gamma_{\mathcal{y}}$ für die $L_{2}$-Kleinsignalverstärkung möglichst klein sein. Somit ergibt sich das konvexe

Optimierungsproblem 5.2. Für eine gegebene Matrix

$$
\begin{aligned}
& \boldsymbol{\Phi}=\operatorname{diag}\left(\phi_{1}, \ldots, \phi_{m}\right) \succ \mathbf{0}, \\
& \underset{\boldsymbol{Q}, \boldsymbol{\Psi}, \gamma_{\boldsymbol{\nu}}}{\operatorname{minimier}} \gamma_{\boldsymbol{\nu}} \text { unter den Nebenbedingungen } \\
& \boldsymbol{Q} \succ \mathbf{0}, \quad \boldsymbol{\Psi}=\operatorname{diag}\left(\psi_{1}, \ldots, \psi_{m}\right) \succ \mathbf{0}, \quad \gamma_{\mathcal{\nu}}>0, \\
& \mathrm{He}\left[\begin{array}{crcc}
\left(\boldsymbol{A}_{\mathrm{s}}-\boldsymbol{B}_{\mathrm{s}, u} \underline{\boldsymbol{K}}\right) \boldsymbol{Q} & \mathbf{0} & \mathbf{0} & \mathbf{0} \\
\boldsymbol{\Psi} \boldsymbol{B}_{\mathrm{s}, u}^{\top}+\boldsymbol{\Phi} \underline{\boldsymbol{K}} \boldsymbol{Q} & -\boldsymbol{\Psi} & \mathbf{0} & \mathbf{0} \\
\mathbf{0} & \boldsymbol{\Phi} & -\frac{1}{2} \gamma_{\mathcal{Y}} \mathbb{I} & \mathbf{0} \\
\boldsymbol{C}_{\mathrm{S}} \boldsymbol{Q} & \mathbf{0} & \mathbf{0} & -\frac{1}{2} \gamma_{\mathcal{Y}} \mathbb{I}
\end{array}\right] \prec \mathbf{0 .}
\end{aligned}
$$

Für $\mathbf{\Phi}=\mathbb{I}$ ist dieses Optimierungsproblem äquivalent mit Optimierungsproblem 5.1. Dann schließt der Sektor $\mathcal{S}(\mathbf{0}, \mathbb{I})$ die Totzone komplett ein und die $L_{2}$-Verstärkung $\gamma_{\mathcal{y}}=\gamma$ gilt für alle Eingangssignale $\boldsymbol{y}_{\mathrm{r}}(t)$ mit endlicher $L_{2}$-Norm. Der Vorteil von Optimierungsproblem 5.2 ist die präzisere Abschätzung der $L_{2}$-Verstärkung für kleine Eingangssignale.

\subsubsection{Charakterisierung der Abweichung}

In den letzten Abschnitten wurden Methoden aufgezeigt, mit denen sich die $L_{2}$-Verstärkung bzw. die $L_{2}$-Kleinsignalverstärkung des Anti-WindupSystems abschätzen lassen. Anhand dieser Abschätzungen wird nun die 


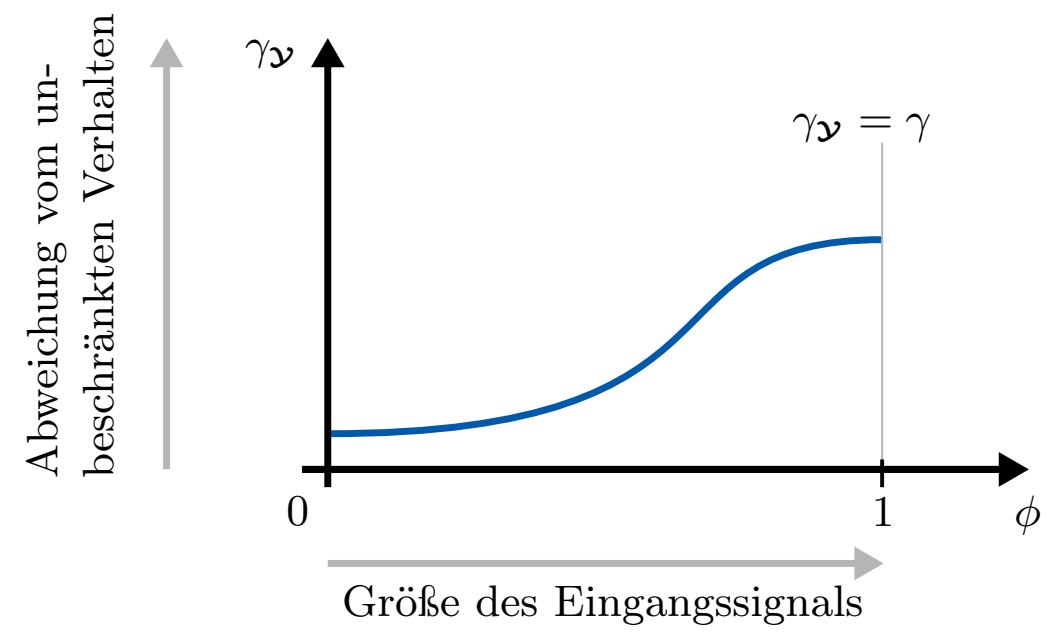

Abbildung 5.7: Visualisierung der Abweichung des Mismatch-Systems vom unbeschränkten Verhalten. Als Maß für die Abweichung ist eine obere Schranke der $L_{2}$-Kleinsignalverstärkung $\gamma_{\mathcal{y}}$ des Anti-Windup-Systems über der Sektorbreite $\phi$ aufgetragen, welche die Größe der Eingangssignale charakterisiert.

Abweichung des Anti-Windup-Regelkreises von dem Verhalten des unbeschränkten Regelkreises charakterisiert.

Offensichtlich ist die Verhaltensabweichung von der Eingangssignalklasse $\mathcal{Y}(\boldsymbol{\Phi})$ bzw. dem betrachteten Sektor $\mathcal{S}(\mathbf{0}, \boldsymbol{\Phi})$ abhängig. Deshalb wird die Abschätzung der $L_{2}$-Kleinsignalverstärkung $\gamma_{\mathcal{y}}$ des Anti-Windup-Systems für mehrere Eingangssignalklassen $\mathcal{Y}(\boldsymbol{\Phi})$, d. h. unterschiedlich breite Sektoren $\mathcal{S}(\mathbf{0}, \boldsymbol{\Phi})$, berechnet. Die Ergebnisse lassen sich in einem Diagramm visualisieren, das $\gamma_{\mathcal{y}}$ in Abhängigkeit der oberen Sektorgrenze $\boldsymbol{\Phi}=\phi \mathbb{I}$ mit $\phi \in(0,1]$ darstellt.

Abbildung 5.7 zeigt ein solches Verstärkungsdiagramm und beispielhaft einen Verlauf der abgeschätzten $L_{2}$-Kleinsignalverstärkung. Typischerweise ändert sich das Ausmaß der Abweichung vom unbeschränkten Verhalten mit der Größe des Eingangssignals. Große Eingangssignale führen im Allgemeinen zu größeren Abweichungen vom unbeschränkten Verhalten. Das ist plausibel, da die Stellgröße des Anti-Windup-Regelkreises begrenzt ist und somit die Ausregelung großer Anfangsauslenkungen mehr Zeit benötigt als die Ausregelung kleiner Anfangsauslenkungen. Der unbeschränkte Regelkreis dagegen ist ein lineares System mit konstanter Ausregelzeit.

Im Folgenden werden derartige Verstärkungsdiagramme genutzt, um die Abweichung des Anti-Windup-Regelkreises mit Beobachtertechnik von dem unbeschränkten Verhalten zu bewerten. Anhand des Verlaufs von $\gamma_{\mathcal{y}}$ kann entschieden werden, welcher Vektor $\boldsymbol{l}_{\mathrm{aw}, \mathrm{x}} \in \mathcal{L}$ die beste Regelgüte garantiert. Je dichter die Kurve der $L_{2}$-Kleinsignalverstärkung an der Ab- 


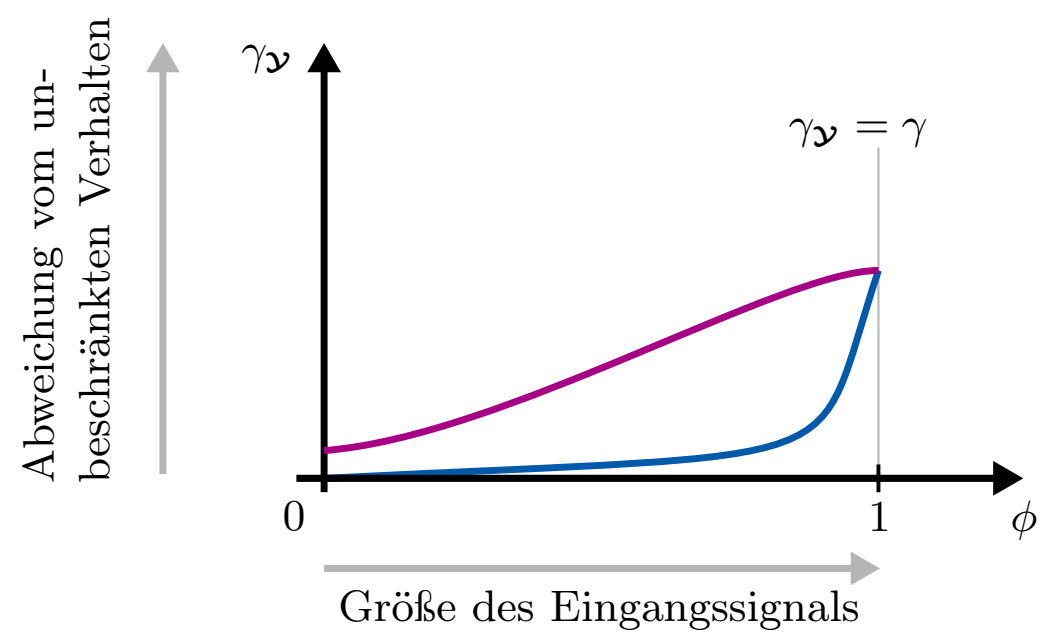

Abbildung 5.8: Vergleich der Regelgüte von zwei Regelkreisen anhand der Verstärkungsdiagramme der zugehörigen Anti-Windup-Systeme. Der Regelkreis (—) weist die kleinere Abweichung vom unbeschränkten Verhalten auf.

szisse liegt, desto kleiner die Abweichung vom unbeschränkten Verhalten im Sinne der $L_{2}$-Norm. Abbildung 5.8 illustriert dies beispielhaft.

\subsection{Eine systematische Auswahlmethode}

Ausgangspunkt dieses Kapitels war die Frage, wie der Vektor $\boldsymbol{l}_{\mathrm{aw}, \mathrm{x}} \in \mathcal{L}$ zu wählen ist, damit der Anti-Windup-Regelkreis eine global asymptotisch stabile Ruhelage besitzt und das dynamische Verhalten des Regelkreises möglichst wenig von dem Verhalten des unbeschränkten Regelkreises abweicht. Nach den Stabilitätsbetrachtungen in Abschnitt 5.2 und der Analyse der Regelgüte in Abschnitt 5.3 kann in diesem Abschnitt eine systematische Auswahlmethode für die Beobachtertechnik angegeben werden. $\mathrm{Zu}-$ nächst werden die nötigen Schritte kompakt zusammengefasst. Abschließend wird die Methode am Beispiel des hydraulischen Positioniersystems demonstriert.

\subsubsection{Zusammenfassung der Entwurfsschritte}

Dieser Abschnitt fasst die nötigen Entwurfsschritte zusammen. Die dabei auftretenden Validierungs- und Optimierungsprobleme lassen sich sehr schnell und komfortabel mit der Matlab-Toolbox [134] und dem Algorithmus [188] lösen. 


\section{Schritt 1: Stabilitätsanalyse}

In diesem Schritt werden alle Vektoren $\boldsymbol{l}_{\mathrm{aw}, \mathrm{x}} \in \mathcal{L}$ aussortiert, die keine globale asymptotisch stabile Ruhelage des Anti-Windup-Regelkreises garantieren und somit die erste Forderung der zu Beginn des Kapitels erläuterten Problemstellung nicht erfüllen. Die Überprüfung erfolgt für jedes $\boldsymbol{l}_{\mathrm{aw}, \mathrm{x}} \in \mathcal{L}$ mit Validierungsproblem 5.1. Die Vektoren, die zu einem global asymptotisch stabilen Regelkreis führen, werden für die weiteren Schritte zusammengefasst in der Menge

$$
\mathcal{L}^{*}=\left\{\boldsymbol{l}_{\mathrm{aw}, \mathrm{x}} \in \mathcal{L} \mid \text { Validierungsproblem } 5.1 \text { ist lösbar. }\right\} .
$$

\section{Schritt 2: Bestimmen der Ersatzsysteme}

In Abschnitt 5.3.1 wurde gezeigt, dass zur Abschätzung der Regelgüte des Anti-Windup-Regelkreises ein Ersatzsystem verwendet werden kann. Dieses besteht aus dem Regler $\mathbf{R}$, der Strecke $\mathbf{S}$ und einem linearen modellbasierten Anti-Windup, das durch den Vektor $\underline{\boldsymbol{k}}$ parametriert wird. Für jeden Vektor $\boldsymbol{l}_{\mathrm{aw}, \mathrm{x}} \in \mathcal{L}^{*}$ muss im zweiten Schritt das Ersatzsystem, d. h. der passende Vektor $\underline{\boldsymbol{k}}$, bestimmt werden.

Für die eindeutige Bestimmung des Vektors $\underline{\boldsymbol{k}}$ wird die Beziehung

$$
\operatorname{det}\left(s \mathbb{I}-\boldsymbol{A}_{\mathrm{s}}+\boldsymbol{b}_{\mathrm{s}, u} \underline{\boldsymbol{k}}^{\top}\right) \operatorname{det}\left(s \mathbb{I}-\boldsymbol{A}_{\mathrm{r}}-\boldsymbol{l}_{\mathrm{aw}, \mathrm{x}} \boldsymbol{c}_{\mathrm{r}}^{\top}\right)=\operatorname{det}\left(s \mathbb{I}-\boldsymbol{A}_{\mathrm{ub}}\right)
$$

ausgenutzt, wobei $\boldsymbol{A}_{\mathrm{ub}}$ die in (5.3) definierte Systemmatrix des unbeschränkten Regelkreises bezeichnet. Die Eigenwerte von $\boldsymbol{A}_{\mathrm{ub}}$ müssen sich folglich aus den Eigenwerten der Matrix $\boldsymbol{A}_{\mathrm{s}}-\boldsymbol{b}_{\mathrm{s}, u} \underline{\boldsymbol{k}}^{\top}$ und den Eigenwerten der Matrix $\boldsymbol{A}_{\mathrm{r}}+\boldsymbol{l}_{\mathrm{aw}, \mathrm{x}} \boldsymbol{c}_{\mathrm{r}}^{\top}$ zusammensetzen.

So ist jedem Vektor $\boldsymbol{l}_{\mathrm{aw}, \mathrm{x}} \in \mathcal{L}^{*}$ über (5.31) eindeutig ein Vektor $\underline{\boldsymbol{k}}$ zugeordnet bzw. für jeden Anti-Windup-Regelkreis mit der durch $\boldsymbol{l}_{\mathrm{aw}, \mathrm{x}}$ definierten Beobachtertechnik existiert ein Ersatzsystem mit modellbasiertem Anti-Windup, das durch $\underline{\boldsymbol{k}}$ parametriert ist. Diese Paare $\left(\boldsymbol{l}_{\mathrm{aw}, \mathrm{x}}, \underline{\boldsymbol{k}}\right)$ werden in der Menge $\mathcal{J}^{*}$ zusammengefasst.

\section{Schritt 3: Abschätzung der L2-Verstärkung des Ersatzsystems}

Im dritten Schritt wird für jedes Ersatzsystem ein Verstärkungsdiagramm erstellt. Dazu muss die $L_{2}$-Kleinsignalverstärkung des zu $\boldsymbol{l}_{\mathrm{aw}, \mathrm{x}}$ gehörenden und durch $\underline{\boldsymbol{k}}$ parametrierten Anti-Windup-Systems für unterschiedlich breite Sektoren $\mathcal{S}(0, \phi)$, wobei $\phi \in(0,1]$, abgeschätzt werden. Dies geschieht mithilfe des konvexen Optimierungsproblems 5.2. Für den hier betrachteten SISO-Fall vereinfacht sich dieses zu 
Optimierungsproblem 5.3. Für einen gegebenen positiven Skalar $\phi$,

$$
\begin{aligned}
& \underset{\boldsymbol{Q}, \psi, \gamma_{\boldsymbol{\nu}}}{\operatorname{minimiere}} \gamma_{\boldsymbol{\nu}} \quad \text { unter den Nebenbedingungen } \\
& \boldsymbol{Q} \succ \mathbf{0}, \quad \psi>0, \gamma_{\boldsymbol{\nu}}>0
\end{aligned}
$$$$
\mathrm{He}\left[\begin{array}{crcc}
\left(\boldsymbol{A}_{\mathrm{s}}-\boldsymbol{b}_{\mathrm{s}, u} \underline{\boldsymbol{k}}^{\top}\right) \boldsymbol{Q} & \mathbf{0} & \mathbf{0} & \mathbf{0} \\
\psi \boldsymbol{b}_{\mathrm{s}, u}^{\top}+\phi \underline{\boldsymbol{k}}^{\top} \boldsymbol{Q} & -\psi & \mathbf{0} & \mathbf{0} \\
\mathbf{0} & \phi & -\frac{1}{2} \gamma_{\mathcal{\nu}} & \mathbf{0} \\
\boldsymbol{c}_{\mathrm{S}}^{\top} \boldsymbol{Q} & 0 & 0 & -\frac{1}{2} \gamma_{\mathcal{\nu}}
\end{array}\right] \prec \mathbf{0} .
$$

\section{Schritt 4: Bewertung der Abweichung}

Im letzten Schritt wird die Abweichung des Anti-Windup-Regelkreises vom unbeschränkten Verhalten anhand der Verstärkungsdiagramme der Ersatzsysteme bewertet. Die Ersatzsysteme, deren Verstärkungskurven am dichtesten an der Abszisse liegen, gehören zu den Anti-Windup-Regelkreisen mit den kleinsten Abweichungen vom unbeschränkten Verhalten.

\subsubsection{Beispiel: hydraulisches Positioniersystem}

Um die vorgestellte Auswahlmethode an einem Beispiel zu demonstrieren, wird erneut das hydraulische Positioniersystem aus Abschnitt 2.2.3 betrachtet. Ein Modell dritter Ordnung der stabilen Strecke definiert (2.14).

Zur Regelung kommt der lineare dynamische Regler mit I-Anteil zum Einsatz, dessen Regelgesetz (2.15) einen stabilen unbeschränkten Regelkreis garantiert und den Regelfehler $e(s)=w(s)-y_{\mathrm{s}}(s)$ stationär genau ausregelt. Eine Zustandsraumdarstellung des Reglers ist durch

$$
\begin{aligned}
& \dot{\boldsymbol{x}}_{\mathrm{r}}=\left[\begin{array}{ccc}
0 & 2,4 & -8,55 \\
0 & -5,0 & 1 \\
0 & 0 & -100
\end{array}\right] \boldsymbol{x}_{\mathrm{r}}+\left[\begin{array}{c}
3,42 \\
0 \\
41,54
\end{array}\right] w+\left[\begin{array}{c}
-3,42 \\
0 \\
-41,54
\end{array}\right] y_{\mathrm{s}}, \\
& y_{\mathrm{r}}=\left[\begin{array}{lll}
3,80 & 12,47 & -44,40
\end{array}\right] \boldsymbol{x}_{\mathrm{r}}+17,76 w-17,76 y_{\mathrm{s}}
\end{aligned}
$$

gegeben. Die sechs Eigenwerte der Systemmatrix $\boldsymbol{A}_{\mathrm{ub}}$ des unbeschränkten Regelkreises lauten

$$
\begin{array}{ll}
\lambda_{\mathrm{ub}, 1}=-0,70, & \lambda_{\mathrm{ub}, 2,3}=-2,49 \pm j 2,20, \\
\lambda_{\mathrm{ub}, 4}=-100,11, & \lambda_{\mathrm{ub}, 5,6}=-0,58 \pm j 3,11
\end{array}
$$


Tabelle 5.1: Es existieren vier Möglichkeiten, den Vektor $\boldsymbol{l}_{\mathrm{aw}, \mathrm{x}} \mathrm{zu}$ wählen.

\begin{tabular}{|c|c|c|c|}
\hline Variante & Eigenwerte & Vektor $\boldsymbol{l}_{\mathrm{aw}}^{\top}$ & \\
\hline 1 & $\lambda_{\mathrm{ub}, 1}, \lambda_{\mathrm{ub}, 2,3}$ & {$[-0,192$} & $0,014 \quad-2,249]$ \\
\hline 2 & $\lambda_{\mathrm{ub}, 1}, \lambda_{\mathrm{ub}, 5,6}$ & {$[-0,192$} & $-0,00015 \quad-2,339]$ \\
\hline 3 & $\lambda_{\mathrm{ub}, 4}, \lambda_{\mathrm{ub}, 2,3}$ & {$[-0,684$} & $0,209 \quad 0,0025]$ \\
\hline 4 & $\lambda_{\mathrm{ub}, 4}, \lambda_{\mathrm{ub}, 5,6}$ & {$[-0,791$} & $0,548 \quad 0,0026]$ \\
\hline
\end{tabular}

und werden zusammengefasst in der Menge

$$
\boldsymbol{\Lambda}_{\mathrm{ub}}=\left\{\lambda_{\mathrm{ub}, 1}, \lambda_{\mathrm{ub}, 2}, \lambda_{\mathrm{ub}, 3}, \lambda_{\mathrm{ub}, 4}, \lambda_{\mathrm{ub}, 5}, \lambda_{\mathrm{ub}, 6}\right\} .
$$

Die symmetrische Stellbegrenzung des Aktors liegt bei $\overline{\mathrm{u}}=10,5 \mathrm{~V}$ und führt im Sättigungsfall zu Windup (vgl. Abschnitt 2.2.3). Als AntiWindup-Maßnahme kommt deshalb die Beobachtertechnik zum Einsatz, die der Systemmatrix des Reglers $\boldsymbol{A}_{\mathrm{r}}+\boldsymbol{l}_{\mathrm{aw}, \mathrm{x}} \boldsymbol{c}_{\mathrm{r}}^{\top}$ im Sättigungsfall drei Eigenwerte aus der Menge $\boldsymbol{\Lambda}_{\mathrm{ub}}$ zuweist. Wie Tabelle 5.1 zeigt, existieren vier Varianten die Eigenwerte bzw. den passenden Vektor $\boldsymbol{l}_{\mathrm{aw}, \mathrm{x}}$ zu wählen ${ }^{2}$. Die Vektoren werden in der Menge $\mathcal{L}$ zusammengefasst. Die Auswahl erfolgt mit der neuen Methode in vier Schritten.

Schritt 1. Die Stabilitätsprüfung im ersten Schritt verläuft für alle Vektoren positiv. Somit ist die Menge $\mathcal{L}^{*}$ der Vektoren, die globale asymptotische Stabilität des Ursprungs garantieren, identisch mit $\mathcal{L}$.

Schritt 2. Für jedes $\boldsymbol{l}_{\mathrm{aw}, \mathrm{x}} \in \mathcal{L}^{*}$ wird der passende Vektor $\underline{\boldsymbol{k}}$ bestimmt. Tabelle 5.2 präsentiert die Ergebnisse.

Schritt 3. Abbildung 5.9 zeigt die mit Optimierungsproblem 5.3 abgeschätzten $L_{2}$-Kleinsignalverstärkungen des Anti-Windup-Systems für jede der vier möglichen Varianten.

Schritt 4. Ausgewählt wird Variante 2, da die Kurve der $L_{2}$-Kleinsignalverstärkung für fast alle ausgewählten Werte $\phi$ am dichtesten an der Abszisse liegt. Nur für $\phi \rightarrow 1$, d. h. für unendlich große Signale, kommt es zu einem starken Anstieg der Verstärkung. Derartige Signale werden hier vernachlässigt, da sie während des normalen Betriebs nicht auftreten.

\footnotetext{
${ }^{2}$ Die Vektoren $\boldsymbol{l}_{\mathrm{aw}, \mathrm{x}}$ können z. B. mit der Ackermann-Formel berechnet werden [133].
} 
Tabelle 5.2: Für jede der vier Varianten existiert ein Vektor $\underline{\boldsymbol{k}}$, der das zugehörige Anti-Windup-System parametriert. Die drei Eigenwerte der Systemmatrix $\boldsymbol{A}_{\mathrm{s}}-\boldsymbol{b}_{\mathrm{s}, u} \underline{\boldsymbol{k}}^{\top}$ des Anti-Windup-Systems sind ebenfalls aufgelistet.

\begin{tabular}{cllll}
\hline Variante & Eigenwerte & Vektor $\underline{\boldsymbol{k}}^{\top}$ & & \\
\hline 1 & $\lambda_{\mathrm{ub}, 4}, \lambda_{\mathrm{ub}, 5,6}$ & {$\left[\begin{array}{llll}-1,66 \cdot 10^{-4} & 3,02 \cdot 10^{-4} & 41,380\end{array}\right]$} \\
2 & $\lambda_{\mathrm{ub}, 4}, \lambda_{\mathrm{ub}, 2,3}$ & {$\left[\begin{array}{llll}1,129 & 6,313 & 42,971\end{array}\right]$} & \\
3 & $\lambda_{\mathrm{ub}, 1}, \lambda_{\mathrm{ub}, 5,6}$ & {$\left[\begin{array}{llll}-3,16 \cdot 10^{-5} & -2,78 \cdot 10^{-6} & -0,040\end{array}\right]$} \\
4 & $\lambda_{\mathrm{ub}, 1}, \lambda_{\mathrm{ub}, 2,3}$ & {$\left[\begin{array}{llll}-0,624 & -0,012 & 1,550\end{array}\right]$} & \\
\hline
\end{tabular}

Abbildung 5.10 zeigt das Ausregelverhalten des unbeschränkten Regelkreises, des beschränkten Regelkreises und der vier Varianten des AntiWindup-Regelkreises. Der Anfangszustand von Strecke sowie Regler ist dabei jeweils null und der Sollwert beträgt $w=20 \mathrm{~cm}$. Die Simulation bestätigt die Aussage des Verstärkungsdiagramms; die Abweichung von Variante 2 des Anti-Windup-Regelkreises liegt am dichtesten an dem unbeschränkten Systemverhalten. Dann folgt Variante 1. Die Varianten 3 und 4 zeigen deutliche Abweichungen. Ein ähnliches Verhalten ergibt sich für kleinere und größere Sollwerte.

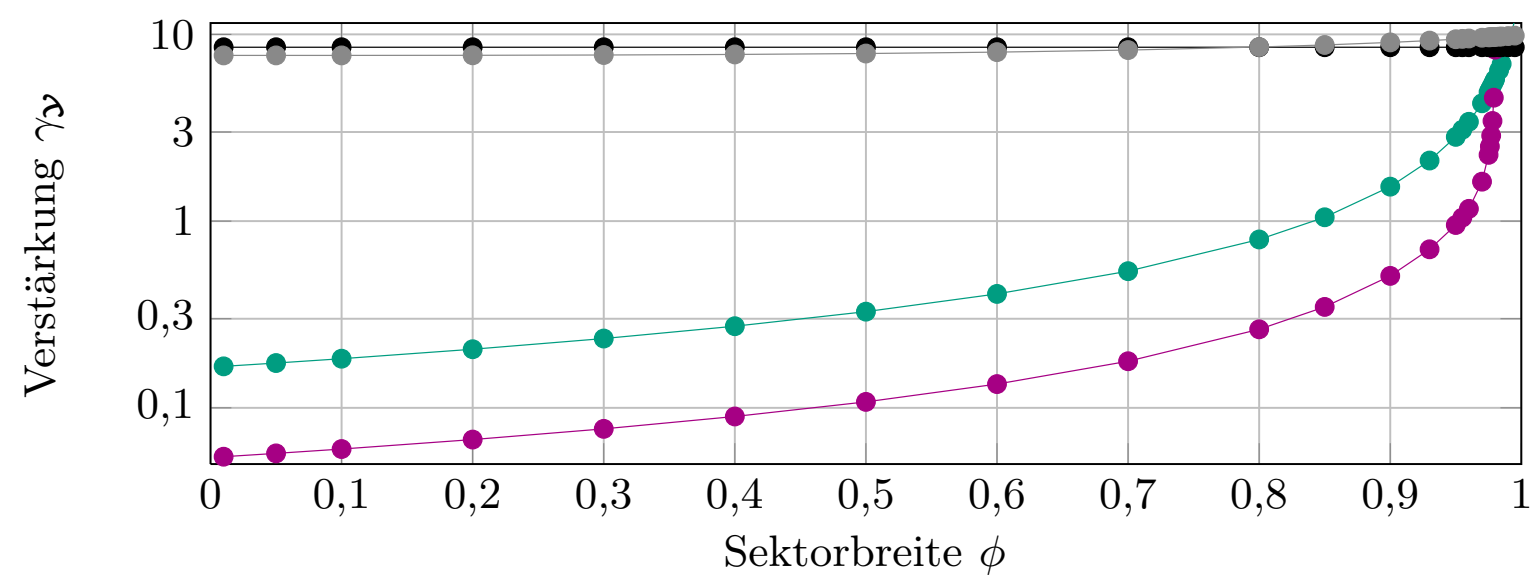

Abbildung 5.9: Das Verstärkungsdiagramm mit logarithmischer Ordinate zeigt die abgeschätzten $L_{2}$-Kleinsignalverstärkungen des Anti-Windup-Systems für Variante $1(\bullet-)$, Variante $2(\bullet-)$, Variante $3(\bullet-)$ und Variante $4(-\bullet)$. 

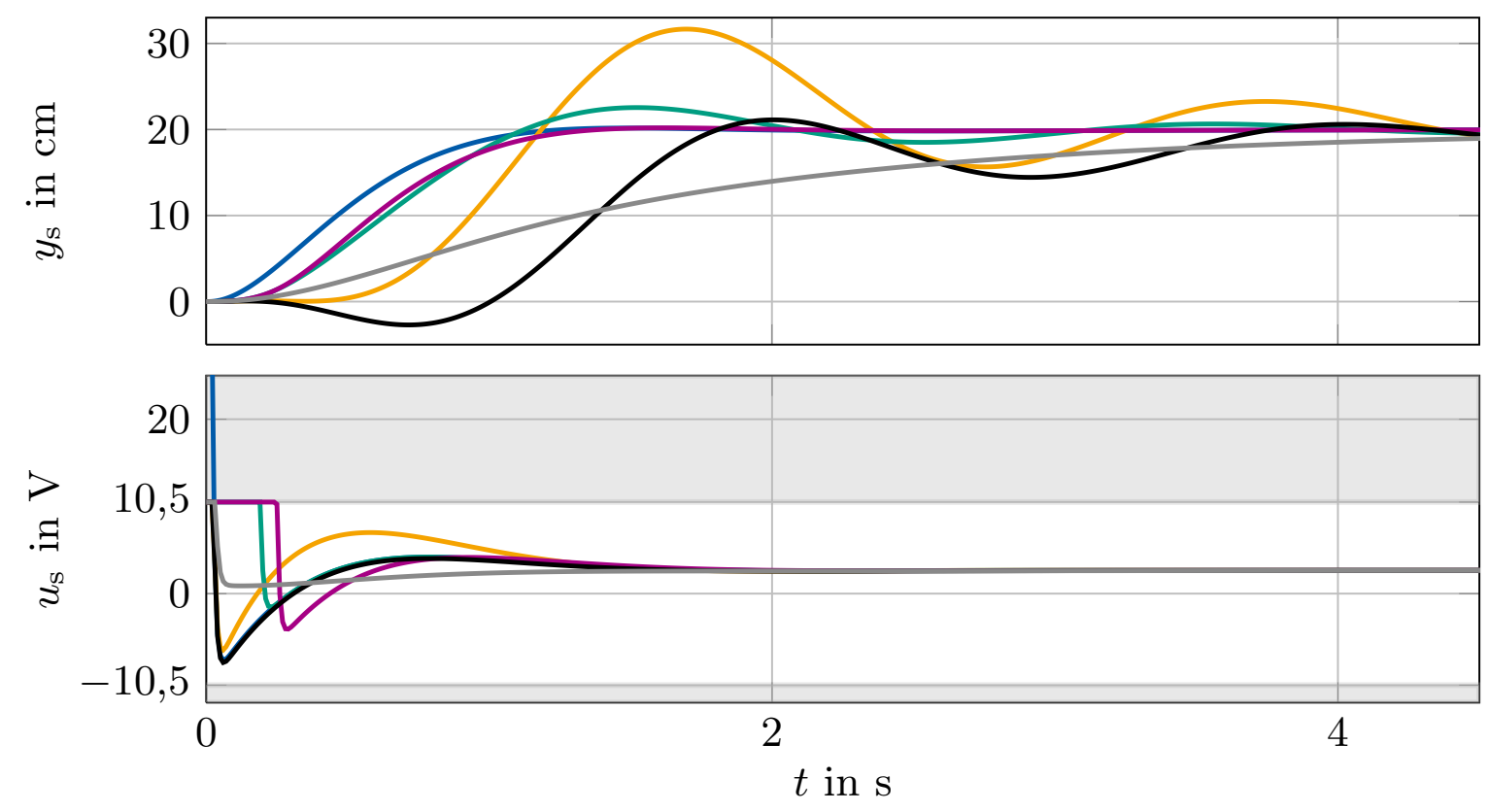

Abbildung 5.10: Ausregel- und Stellgrößenverläufe des unbeschränkten Regelkreises (—), des beschränkten Regelkreises (—) und der vier möglichen Varianten des Anti-Windup-Regelkreises für einen Sollwert von $w=20 \mathrm{~cm}$. Die Variante $2(-)$ liegt am dichtesten an dem Verhalten des unbeschränkten Regelkreises. Dann folgt Variante $1(-)$. Die Varianten $3(-)$ und $4(-)$ zeigen eine starke Abweichung.

\subsection{Zusammenfassung und Fazit}

In diesem Kapitel wurde eine systematische Entwurfsmethode für die Beobachtertechnik vorgestellt. Die Beobachtertechnik ist eine statische AntiWindup-Maßnahme, deren Entwurf sich auf die Wahl eines Vektors $\boldsymbol{l}_{\mathrm{aw}, \mathrm{x}}$ aus einer Menge $\mathcal{L}$ reduziert. Bisher war nicht klar, welche Vektoren dieser Menge eine hohe Regelgüte garantieren.

Die neue Entwurfsmethode trifft die Wahl anhand der abgeschätzten Abweichung zwischen zwei Regelkreisen. Verglichen wird das Verhalten des idealen unbeschränkten Regelkreises mit dem Verhalten des Anti-WindupRegelkreises, der sich durch den gewählten Vektor $\boldsymbol{l}_{\mathrm{aw}, \mathrm{x}}$ ergibt. Die Abschätzung der Abweichung basiert, wie bei modellbasierten Anti-WindupMaßnahmen, auf der $L_{2}$-Kleinsignalverstärkung eines Ersatzsystems. Das betrachtete Beispiel illustriert die Effektivität der Methode. 


\section{Stabilität von Regelkreisen mit modellbasiertem Anti-Windup}

Dieses Kapitel untersucht die Stabilität des Anti-Windup-Regelkreises mit einer modellbasierten Anti-Windup-Maßnahme. Dabei wird zwischen globalen und lokalen Stabilitätsaussagen für verschiedene Ruhelagen des Regelkreises, die von der Soll- und Störgröße abhängen, unterschieden. Das Hauptziel des Kapitels ist, einfach zu handhabende, möglichst universell einsetzbare Stabilitätssätze für den Entwurf modellbasierter Anti-WindupMaßnahmen zur Verfügung zu stellen. Eine derart allgemeine ausführliche Betrachtung fehlt bislang in der Literatur. Das Kapitel kann deshalb als Erweiterung der Ergebnisse in $[52,185]$ angesehen werden.

\subsection{Regelkreis und Struktur des Anti-Windup}

Betrachtet wird eine zu regelnde lineare Strecke

$$
\mathbf{S}:\left\{\begin{array}{l}
\dot{x}_{\mathrm{s}}=A_{\mathrm{s}} \boldsymbol{x}_{\mathrm{s}}+\boldsymbol{B}_{\mathrm{s}, u} \boldsymbol{u}_{\mathrm{s}}+\boldsymbol{B}_{\mathrm{s}, z} z \\
y_{\mathrm{s}}=C_{\mathrm{s}} \boldsymbol{x}_{\mathrm{s}}
\end{array}\right.
$$

mit dem Zustand $\boldsymbol{x}_{\mathrm{s}} \in \mathbb{R}^{n_{\mathrm{s}}}$, dem Stelleingang $\boldsymbol{u}_{\mathrm{s}} \in \mathbb{R}^{m}$, der Störgröße $\boldsymbol{z} \in \mathbb{R}^{l}$ und dem messbaren Ausgang $\boldsymbol{y}_{\mathrm{s}} \in \mathbb{R}^{m}$. Zur Regelung wird ein linearer Regler gemäß (2.2) mit der Dynamik

$$
\mathbf{R}:\left\{\begin{array}{l}
\dot{\boldsymbol{x}}_{\mathrm{r}}=\boldsymbol{A}_{\mathrm{r}} \boldsymbol{x}_{\mathrm{r}}+\boldsymbol{B}_{\mathrm{r}, u} \boldsymbol{u}_{\mathrm{r}}+\boldsymbol{B}_{\mathrm{r}, w} \boldsymbol{w} \\
\boldsymbol{y}_{\mathrm{r}}=\boldsymbol{C}_{\mathrm{r}} \boldsymbol{x}_{\mathrm{r}}+\boldsymbol{D}_{\mathrm{r}, u} \boldsymbol{u}_{\mathrm{r}}+\boldsymbol{D}_{\mathrm{r}, w} \boldsymbol{w}
\end{array}\right.
$$

verwendet. Dabei ist $\boldsymbol{x}_{\mathrm{r}} \in \mathbb{R}^{n_{\mathrm{r}}}$ der Zustand, $\boldsymbol{u}_{\mathrm{r}} \in \mathbb{R}^{m}$ der Messeingang für die Ausgangssignale der Strecke, $\boldsymbol{w} \in \mathbb{R}^{m}$ der Eingang zur Vorgabe von Sollwerten und $\boldsymbol{y}_{\mathrm{r}} \in \mathbb{R}^{m}$ die vom Regler berechnete Stellgröße. Bezüglich der Parametrierung des Reglers gilt Annahme 2.2, d. h., der unbeschränkte Regelkreis weist ein ideales Verhalten auf. 


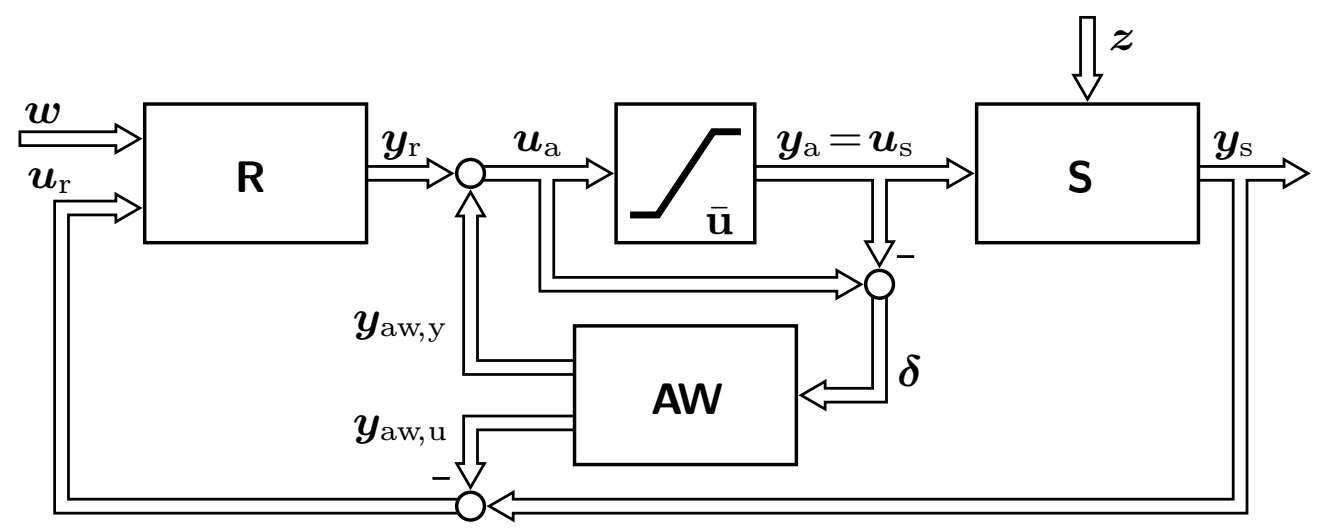

Abbildung 6.1: Anti-Windup-Regelkreis.

Um die durch den Aktor begrenzte Stellgröße zu berücksichtigen, kommt ein modellbasiertes Anti-Windup gemäß (4.7) mit der Dynamik

$$
\mathbf{A W}:\left\{\begin{aligned}
\dot{\boldsymbol{x}}_{\mathrm{aw}} & =\boldsymbol{A}_{\mathrm{s}} \boldsymbol{x}_{\mathrm{aw}}+\boldsymbol{B}_{\mathrm{s}, u} \boldsymbol{k}_{\mathrm{aw}}\left(\boldsymbol{x}_{\mathrm{aw}}\right)+\boldsymbol{B}_{\mathrm{s}, u} \boldsymbol{\delta} \\
\boldsymbol{y}_{\mathrm{aw}, \mathrm{u}} & =-\boldsymbol{C}_{\mathrm{s}} \boldsymbol{x}_{\mathrm{aw}} \\
\boldsymbol{y}_{\mathrm{aw}, \mathrm{y}} & =-\boldsymbol{k}_{\mathrm{aw}}\left(\boldsymbol{x}_{\mathrm{aw}}\right)
\end{aligned}\right.
$$

zum Einsatz. Der Anti-Windup-Regelkreis mit dem Zustand

$$
\boldsymbol{x}_{\mathrm{awrk}}^{\top}=\left[\begin{array}{lll}
\boldsymbol{x}_{\mathrm{s}}^{\top} & \boldsymbol{x}_{\mathrm{r}}^{\top} & \boldsymbol{x}_{\mathrm{aw}}^{\top}
\end{array}\right]
$$

entsteht durch die in Abbildung 6.1 gezeigte Verschaltung

$$
\begin{aligned}
\boldsymbol{\delta} & =\boldsymbol{u}_{\mathrm{a}}-\operatorname{sat}_{\overline{\mathbf{u}}}\left(\boldsymbol{u}_{\mathrm{a}}\right), & & \boldsymbol{u}_{\mathrm{a}}=\boldsymbol{y}_{\mathrm{r}}+\boldsymbol{y}_{\mathrm{aw}, \mathrm{y}}, \\
\boldsymbol{u}_{\mathrm{r}} & =\boldsymbol{y}_{\mathrm{s}}-\boldsymbol{y}_{\mathrm{aw}, \mathrm{u}}, & & \boldsymbol{u}_{\mathrm{s}}=\operatorname{sat}_{\overline{\mathbf{u}}}\left(\boldsymbol{u}_{\mathrm{a}}\right)
\end{aligned}
$$

der drei Systeme und besitzt die Dynamik

$$
\begin{aligned}
& \dot{\boldsymbol{x}}_{\mathrm{awrk}}=\tilde{\boldsymbol{A}} \boldsymbol{x}_{\mathrm{awrk}}+\tilde{\boldsymbol{B}}_{\mathrm{u}} \boldsymbol{s a t}_{\overline{\mathbf{u}}}\left(\boldsymbol{u}_{\mathrm{awrk}}\right)+\tilde{\boldsymbol{B}}_{\mathrm{w}} \boldsymbol{z}+\tilde{\boldsymbol{B}}_{\mathrm{z}} \boldsymbol{w}, \\
& \boldsymbol{u}_{\mathrm{awrk}}=\boldsymbol{C}_{\mathrm{r}} \boldsymbol{x}_{\mathrm{r}}+\boldsymbol{D}_{\mathrm{r}, u} \boldsymbol{C}_{\mathrm{s}}\left(\boldsymbol{x}_{\mathrm{s}}+\boldsymbol{x}_{\mathrm{aw}}\right)-\boldsymbol{k}_{\mathrm{aw}}\left(\boldsymbol{x}_{\mathrm{aw}}\right)+\boldsymbol{D}_{\mathrm{r}, w} \boldsymbol{w} .
\end{aligned}
$$

Die Systemmatrizen $\tilde{\boldsymbol{A}}, \tilde{\boldsymbol{B}}_{\mathrm{u}}, \tilde{\boldsymbol{B}}_{\mathrm{w}}$, und $\tilde{\boldsymbol{B}}_{\mathrm{z}}$ sind in Abschnitt A.3.2 des Anhangs gegeben. Der Anti-Windup-Entwurf besteht in der Wahl der Funktion $\boldsymbol{k}_{\text {aw }}: \mathbb{R}^{n_{\mathrm{s}}} \mapsto \mathbb{R}^{m}$ im Sinne der Entwurfsziele aus Abschnitt 4.2. Dabei sind einige Anforderungen zu berücksichtigen. Eine Grundvoraussetzung für die weiteren Überlegungen ist, dass das Differentialgleichungssystem (6.3) eine eindeutige Lösung für alle $t \geq 0$ besitzt. In diesem Zusammenhang spielt der Begriff der Lipschitz-Stetigkeit eine große Rolle. 
Definition 6.1. Gegeben sei eine Funktion $\boldsymbol{f}: \mathbb{D} \subseteq \mathbb{R}^{n} \mapsto \mathbb{R}^{m}$. Gibt es eine Konstante $L \geq 0$, so dass gilt

$$
\|\boldsymbol{f}(\boldsymbol{x})-\boldsymbol{f}(\tilde{\boldsymbol{x}})\|_{2} \leq L\|\boldsymbol{x}-\tilde{\boldsymbol{x}}\|_{2} \quad \forall \boldsymbol{x}, \tilde{\boldsymbol{x}} \in \mathbb{D},
$$

so genügt die Funktion $\boldsymbol{f}(\boldsymbol{x})$ auf ganz $\mathbb{D}$ einer globalen Lipschitz-Bedingung. Die Funktion wird dann auch als global lipschitzstetig bezeichnet. Wenn es zu jedem Punkt in $\mathbb{D}$ eine Umgebung $\mathcal{G} \subset \mathbb{D}$ gibt, so dass die Einschränkung von $\boldsymbol{f}(\boldsymbol{x})$ auf $\mathcal{G}$ dort einer Lipschitz-Bedingung genügt, so heißt $\boldsymbol{f}(\boldsymbol{x})$ lokal lipschitzstetig in $\mathbb{D}$.

Im Weiteren soll folgende Annahme bezüglich $\boldsymbol{k}_{\text {aw }}\left(\boldsymbol{x}_{\text {aw }}\right)$ gelten.

Annahme 6.1. Die Funktion $\boldsymbol{k}_{\mathrm{aw}}: \mathbb{R}^{n_{\mathrm{s}}} \mapsto \mathbb{R}^{m}$ ist lokal lipschitzstetig, punktsymmetrisch, d. h. $\boldsymbol{k}_{\text {aw }}\left(\boldsymbol{x}_{\text {aw }}\right)=-\boldsymbol{k}_{\text {aw }}\left(-\boldsymbol{x}_{\text {aw }}\right)$, und $\boldsymbol{k}_{\text {aw }}(\mathbf{0})=\mathbf{0}$.

Die erste Eigenschaft garantiert die globale Existenz einer eindeutigen Lösung des Differentialgleichungssystems (6.3). Es gilt

Satz 6.1 (Existenz und Eindeutigkeit der Lösung). Das durch (6.3) und den Anfangszustand $\boldsymbol{x}_{\mathrm{awrk}}(0)=\boldsymbol{x}_{\mathrm{awrk}}^{(0)} \in \mathbb{R}^{2 n_{\mathrm{s}}+n_{\mathrm{r}}}$ definierte Anfangswertproblem besitzt eine eindeutige Lösung $\boldsymbol{x}_{\text {awrk }}(t)$ für alle $t \geq 0$.

Beweis. Der Beweis findet sich in Abschnitt C.3.5.

Die zweite Eigenschaft der Funktion $\boldsymbol{k}_{\text {aw }}$, die Punktsymmetrie, vereinfacht die Systemdarstellung des Regelkreises in Mismatch-Koordinaten. Wie schon aus Abschnitt 4.4.1 bekannt, ist diese Systemdarstellung sehr hilfreich für den Entwurf des modellbasierten Anti-Windup. Details erläutert der nächste Abschnitt.

\subsection{Transformation in Mismatch-Koordinaten}

Transformiert man den Anti-Windup-Regelkreis (6.3) mittels der Transformation (4.9) in die Mismatch-Darstellung (4.10) mit dem Zustandsvektor

$$
\boldsymbol{\xi}_{\mathrm{ms}}^{\top}=\left[\begin{array}{lll}
\boldsymbol{\xi}_{\mathrm{s}}^{\top} & \boldsymbol{\xi}_{\mathrm{r}}^{\top} & \boldsymbol{\xi}_{\mathrm{aw}}^{\top}
\end{array}\right]
$$

ergibt sich eine Kaskade aus zwei Teilsystemen (vgl. Abbildung 4.5). Das erste Teilsystem

$$
\mathbf{L}:\left\{\begin{array}{l}
\dot{\boldsymbol{\xi}}_{\mathrm{s}}=\boldsymbol{A}_{\mathrm{s}} \boldsymbol{\xi}_{\mathrm{s}}+\boldsymbol{B}_{\mathrm{s}, u} \boldsymbol{y}_{\mathrm{r}}+\boldsymbol{B}_{\mathrm{s}, z} \boldsymbol{z} \\
\dot{\boldsymbol{\xi}}_{\mathrm{r}}=\boldsymbol{A}_{\mathrm{r}} \boldsymbol{\xi}_{\mathrm{r}}+\boldsymbol{B}_{\mathrm{r}, u} C_{\mathrm{s}} \boldsymbol{\xi}_{\mathrm{s}}+\boldsymbol{B}_{\mathrm{r}, w} \boldsymbol{w} \\
\boldsymbol{y}_{\mathrm{r}}=C_{\mathrm{r}} \boldsymbol{\xi}_{\mathrm{r}}+\boldsymbol{D}_{\mathrm{r}, u} C_{\mathrm{s}} \boldsymbol{\xi}_{\mathrm{s}}+\boldsymbol{D}_{\mathrm{r}, w} \boldsymbol{w}
\end{array}\right.
$$


ist linear und entspricht dem unbeschränkten Regelkreis. Der Ausgang $\boldsymbol{y}_{\mathrm{r}}$ wirkt als Störgröße auf das zweite Teilsystem

$$
\begin{aligned}
& \dot{\boldsymbol{\xi}}_{\mathrm{aw}}=\boldsymbol{A}_{\mathrm{s}} \boldsymbol{\xi}_{\mathrm{aw}}+\boldsymbol{B}_{\mathrm{s}, u}\left(\operatorname{sat}_{\overline{\mathbf{u}}}\left(\boldsymbol{y}_{\mathrm{r}}+\boldsymbol{y}_{\mathrm{aw}, \mathrm{y}}\right)-\boldsymbol{y}_{\mathrm{r}}\right), \\
& \boldsymbol{y}_{\mathrm{aw}, \mathrm{y}}=\boldsymbol{k}_{\mathrm{aw}}\left(\boldsymbol{\xi}_{\mathrm{aw}}\right), \\
& \boldsymbol{y}_{\mathrm{aw}, \mathrm{u}}=\boldsymbol{C}_{\mathrm{s}} \boldsymbol{\xi}_{\mathrm{aw}},
\end{aligned}
$$

das auch als Anti-Windup-System bezeichnet wird. Der Vorteil dieser Umformung besteht darin, dass sich der Anti-Windup-Entwurf auf den Entwurf eines Zustandsreglers für das System (6.5) reduziert. Schwierigkeiten bereitet die Tatsache, dass die Störgröße an zwei Stellen der Differentialgleichung auftaucht.

Lösungen für dieses Problem wurden in Abschnitt 4.4.2 diskutiert. Hier wird die zweite Lösungsmöglichkeit verfolgt und das Ersatzsystem

$$
\text { ES }^{*}:\left\{\begin{aligned}
\dot{\boldsymbol{\xi}}_{\mathrm{aw}} & =\boldsymbol{A}_{\mathrm{s}} \boldsymbol{\xi}_{\mathrm{aw}}+\boldsymbol{B}_{\mathrm{s}, u} \mathbf{s a t}_{[-\overline{\mathbf{u}}+\boldsymbol{p}(t), \overline{\mathbf{u}}+\boldsymbol{p}(t)]}\left(\boldsymbol{y}_{\mathrm{aw}, \mathrm{y}}\right)+\boldsymbol{B}_{\mathrm{s}, u} \boldsymbol{\zeta} \\
\boldsymbol{y}_{\mathrm{aw}, \mathrm{y}} & =\boldsymbol{k}_{\mathrm{aw}}\left(\boldsymbol{\xi}_{\mathrm{aw}}\right), \\
\boldsymbol{y}_{\mathrm{aw}, \mathrm{u}} & =\boldsymbol{C}_{\mathrm{s}} \boldsymbol{\xi}_{\mathrm{aw}}
\end{aligned}\right.
$$

mit zeitvarianter Sättigung und den stetigen Funktionen $\boldsymbol{p}(t), \boldsymbol{\zeta}(t)$ verwendet, die den komponentenweisen Abschätzungen

$$
\begin{aligned}
-\varrho \overline{\mathrm{u}}_{i} & \leq p_{i} \leq \varrho \overline{\mathrm{u}}_{i}, \\
\left|\zeta_{i}\right| & \leq 2\left|\operatorname{sat}_{\varrho \overline{\mathrm{u}}_{i}}\left(y_{\mathrm{r}, i}\right)-y_{\mathrm{r}, i}\right|
\end{aligned}
$$

genügen. Satz 4.3 garantiert die Äquivalenz dieses Ersatzsystems mit dem Anti-Windup-System (6.5) für ein beliebiges $\varrho \in(0,1)$ und eine spezielle - aber komplizierte - Wahl der Funktionen $\boldsymbol{p}(t), \boldsymbol{\zeta}(t)$.

Damit sich ein möglichst einfacher Entwurf ergibt, ist folgende Vorgehensweise ratsam. Die Funktion $\boldsymbol{k}_{\mathrm{aw}}$ wird so gewählt, dass sie die Entwurfsanforderungen für das Ersatzsystem ES* und alle stetigen Funktionen $\boldsymbol{p}(t), \boldsymbol{\zeta}(t)$, die den Abschätzungen (6.6) genügen, garantiert. Damit ist sichergestellt, dass die Funktion $\boldsymbol{k}_{\text {aw }}$ diese Eigenschaften auch für das Anti-Windup-System (6.5) garantiert. Anschaulich kann man sich das in Abbildung 6.2 gezeigte Blockschaltbild vorstellen. Die Systemdynamik der schwarzen Blöcke ist unbekannt, die stetigen Ausgänge $\boldsymbol{\zeta}(t)$ bzw. $\boldsymbol{p}(t)$ genügen aber den Abschätzungen (6.6).

Durch eine geschickte Wahl der Funktion $\boldsymbol{k}_{\text {aw }}$ lässt sich der Entwurf weiter vereinfachen. Die sättigende Zustandsrückführung

$$
\boldsymbol{y}_{\mathrm{aw}, \mathrm{y}}=\boldsymbol{k}_{\mathrm{aw}}\left(\boldsymbol{\xi}_{\mathrm{aw}}\right)=\mathbf{s a t}_{\alpha \overline{\mathbf{u}}}\left(-\boldsymbol{k}\left(\boldsymbol{\xi}_{\mathrm{aw}}\right)\right)
$$




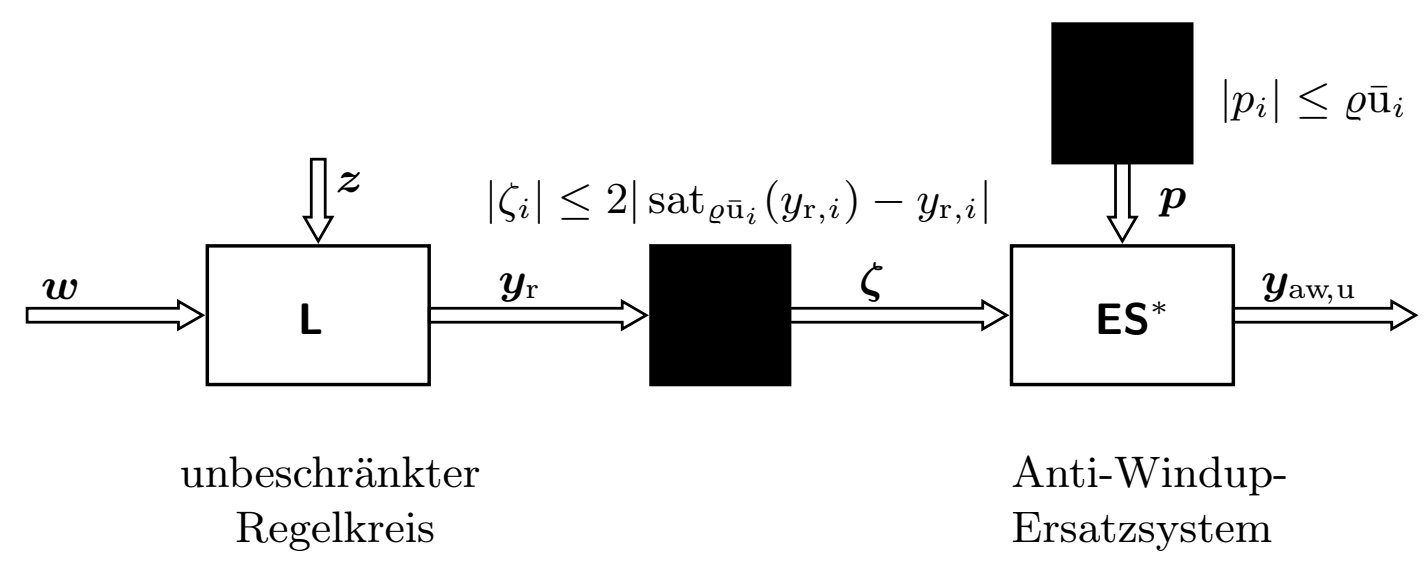

Abbildung 6.2: Für den Entwurf des Anti-Windup verwendete Systemdarstellung, wobei die Dynamik der schwarzen Blöcke unbekannt ist. Die Ausgänge $\boldsymbol{\zeta}(t)$ und $\boldsymbol{p}(t)$ sind stetige Funktionen, die den Abschätzungen (6.6) genügen.

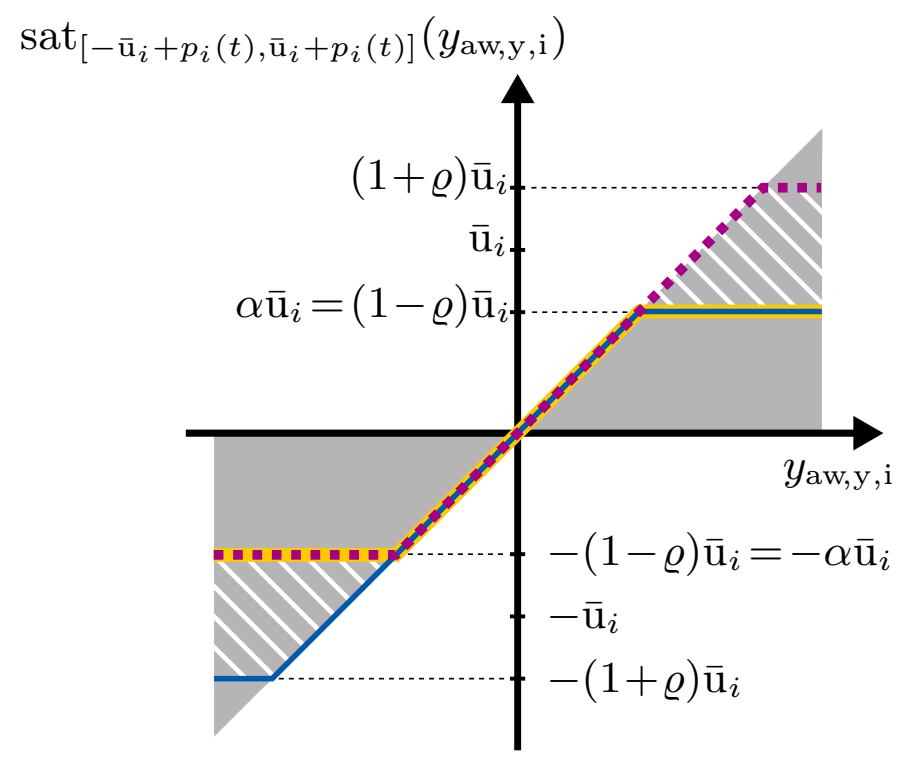

Abbildung 6.3: Zeitvariante Sättigungsfunktion $\operatorname{sat}_{\left[-\overline{\mathrm{u}}_{i}+p_{i}(t), \overline{\mathrm{u}}_{i}+p_{i}(t)\right]}\left(y_{\mathrm{aw}, \mathrm{y}, \mathrm{i}}\right)$. Gezeigt sind die Fälle $p_{i}=\varrho \overline{\mathrm{u}}_{i}$ in $(-)$ und $p_{i}=-\varrho \overline{\mathrm{u}}_{i}$ in $\left(\because \cdot{ }^{\prime}\right.$. Für jedes $p_{i}$ aus dem Intervall $\left[-\varrho \overline{\mathrm{u}}_{i}, \varrho \overline{\mathrm{u}}_{i}\right]$ liegt die Sättigungsfunktion in dem weiß schraffierten Bereich. Deshalb lässt sich die zur Verfügung stehende Stellgröße zu jedem Zeitpunkt durch die Funktion (-) abschätzen, d. h. durch $\operatorname{sat}_{\alpha \overline{\mathrm{u}}_{\mathrm{i}}}\left(y_{\mathrm{aw}, \mathrm{y}, \mathrm{i}}\right)$. 


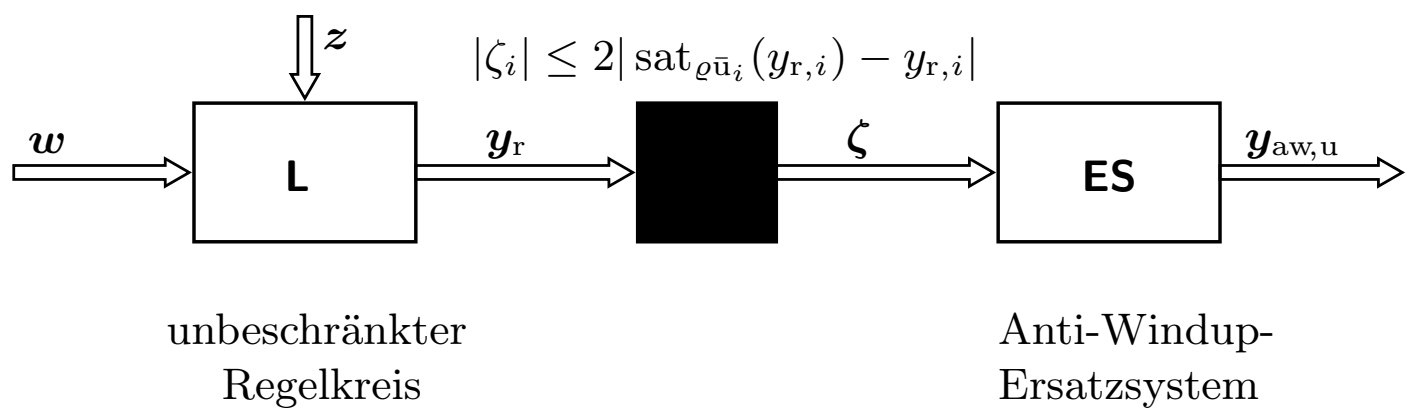

Abbildung 6.4: Eine sättigende Zustandsrückführung vereinfacht die Systemstruktur. Der Einfluss von $\boldsymbol{p}(t)$ wird eliminiert.

eliminiert den Einfluss von $\boldsymbol{p}(t)$ für $\alpha=1-\varrho$. Das folgt aus

$$
\begin{aligned}
\operatorname{sat}_{[-\overline{\mathbf{u}}+\boldsymbol{p}(t), \overline{\mathbf{u}}+\boldsymbol{p}(t)]}\left(\boldsymbol{y}_{\mathrm{aw}, \mathrm{y}}\right) & =\operatorname{sat}_{[-\overline{\mathbf{u}}+\boldsymbol{p}(t), \overline{\mathbf{u}}+\boldsymbol{p}(t)]}\left(\operatorname{sat}_{\alpha \overline{\mathbf{u}}}\left(-\boldsymbol{k}\left(\boldsymbol{\xi}_{\mathrm{aw}}\right)\right)\right) \\
& =\operatorname{sat}_{\alpha \overline{\mathbf{u}}}\left(-\boldsymbol{k}\left(\boldsymbol{\xi}_{\mathrm{aw}}\right)\right) .
\end{aligned}
$$

Für $\alpha=1-\varrho$ werden die Begrenzungen der zeitvarianten Sättigungsfunktion von $\boldsymbol{y}_{\text {aw,y }}$ nie überschritten, wie Abbildung 6.3 für eine Komponente der Stellgröße zeigt. Folglich kann die zeitvariante Sättigung in der Systembeschreibung entfallen. Es ergibt sich das vereinfachte Ersatzsystem

$$
\text { ES : }\left\{\begin{aligned}
\dot{\boldsymbol{\xi}}_{\mathrm{aw}} & =\boldsymbol{A}_{\mathrm{s}} \boldsymbol{\xi}_{\mathrm{aw}}+\boldsymbol{B}_{\mathrm{s}, u} \mathbf{s a t}_{\alpha \overline{\mathbf{u}}}\left(-\boldsymbol{k}\left(\boldsymbol{\xi}_{\mathrm{aw}}\right)\right)+\boldsymbol{B}_{\mathrm{s}, u} \boldsymbol{\zeta}, \\
\boldsymbol{y}_{\mathrm{aw}, \mathrm{u}} & =\boldsymbol{C}_{\mathrm{s}} \boldsymbol{\xi}_{\mathrm{aw}}
\end{aligned}\right.
$$

mit einer zeitinvarianten Sättigungsfunkion. Abbildung 6.4 zeigt das für den Entwurf des Anti-Windup relevante Blockschaltbild. Der große Vorteil dieser Reihenschaltung ist, dass Eigenschaften des Gesamtsystems und damit auch des Anti-Windup-Regelkreises auf Eigenschaften der Teilsysteme L und ES zurückgeführt werden können. Zunächst wird die Stabilität der Ruhelage $\boldsymbol{x}_{\text {awrk }}^{(\mathrm{R})}=\mathbf{0}$ des Anti-Windup-Regelkreises untersucht.

\subsection{Stabilität des Ursprungs}

Eine grundlegende Entwurfsanforderung an modellbasierte Anti-WindupMaßnahmen ist eine Stabilitätsgarantie für den Regelkreis (6.3). In diesem Abschnitt wird die globale und lokale Stabilität des Ursprungs, d. h. der Ruhelage $\boldsymbol{x}_{\mathrm{awrk}}^{(\mathrm{R})}=\mathbf{0}$, untersucht. Auf die Stabilität von Ruhelagen, die nicht im Ursprung liegen, d. h. $\boldsymbol{x}_{\text {awrk }}^{(\mathrm{R})} \neq \mathbf{0}$, geht Abschnitt 6.4 ein. 
Es ist offensichtlich, dass die Stabilitätsuntersuchungen auch in Mismatch-Koordinaten $\boldsymbol{\xi}_{\mathrm{ms}}$ durchgeführt werden können, da beide Koordinatensysteme über die lineare Abbildung (4.9) in Beziehung stehen. Im Weiteren wird deshalb die Stabilität der Ruhelage

$$
\boldsymbol{\xi}_{\mathrm{ms}}^{(\mathrm{R})}=\left[\begin{array}{l}
\boldsymbol{\xi}_{\mathrm{s}}^{(\mathrm{R})} \\
\boldsymbol{\xi}_{\mathrm{r}}^{\mathrm{R})} \\
\boldsymbol{\xi}_{\mathrm{aw}}^{(\mathrm{R})}
\end{array}\right]=\mathbf{0}
$$

des Mismatch-Systems untersucht. Aufgrund der besonderen Struktur des Mismatch-Systems lässt sich diese Aufgabe vereinfachen und auf eine Stabilitätsuntersuchung der beiden Teilsysteme $\mathbf{L}$ und ES mit den Ruhelagen

$$
\boldsymbol{\xi}_{\mathrm{L}}^{(\mathrm{R})}=\left[\begin{array}{c}
\boldsymbol{\xi}_{\mathrm{s}}^{(\mathrm{R})} \\
\boldsymbol{\xi}_{\mathrm{r}}^{(\mathrm{R})}
\end{array}\right]=\mathbf{0}, \quad \boldsymbol{\xi}_{\mathrm{aw}}^{(\mathrm{R})}=\mathbf{0}
$$

zurückführen. Dabei liegt auf der Hand, dass gilt

$$
\boldsymbol{\xi}_{\mathrm{L}}(t) \rightarrow \mathbf{0} \text { und } \boldsymbol{\xi}_{\mathrm{aw}}(t) \rightarrow \mathbf{0} \quad \Leftrightarrow \quad \boldsymbol{\xi}_{\mathrm{ms}}(t) \rightarrow \mathbf{0} \quad \Leftrightarrow \quad \boldsymbol{x}_{\mathrm{awrk}}(t) \rightarrow \mathbf{0} .
$$

Ebenso impliziert eine Beschränkung der Trajektorien

$$
\left\|\boldsymbol{\xi}_{\mathrm{L}}(t)\right\|<\mathrm{c}_{\mathrm{L}} \text { und }\left\|\boldsymbol{\xi}_{\text {aw }}(t)\right\|<\mathrm{c}_{\text {aw }}
$$

für alle $t \geq 0$ mit Konstanten $c_{L}>0, c_{\text {aw }}>0$ eine Beschränkung der Form

$$
\left\|\boldsymbol{\xi}_{\mathrm{ms}}(t)\right\|<\mathrm{c}_{\mathrm{ms}} \quad \Leftrightarrow \quad\left\|\boldsymbol{x}_{\mathrm{awrk}}\right\|<\mathrm{c}_{\mathrm{awrk}}
$$

für alle $t \geq 0$ mit positiven Konstanten $\mathrm{c}_{\mathrm{ms}}, \mathrm{c}_{\mathrm{awrk}}$.

Wie bereits angedeutet erfolgt in den nächsten Abschnitten die Stabilitätsuntersuchung der beiden Teilsysteme. Zunächst wird das lineare Teilsystem $\mathbf{L}$ mit der Ruhelage $\boldsymbol{\xi}_{\mathrm{L}}^{(\mathrm{R})}=\mathbf{0}$ betrachtet.

\subsubsection{Stabilitätseigenschaften des linearen Teilsystems}

Das Teilsystem L mit der Systemdynamik (6.4) entspricht dem unbeschränkten Regelkreis (2.3) und ist somit nach Annahme 2.2 ein stabiles, lineares System. Präziser charakterisiert dies

Lemma 6.1 (Exponentielle Stabilität des unbeschränkten Regelkreises). Es gelte $\boldsymbol{w}=\mathbf{0}, \boldsymbol{z}=\mathbf{0}$ und Annahme 2.2 sei erfüllt. Für den unbeschränkten Regelkreis (6.4) mit der Ruhelage $\boldsymbol{\xi}_{\mathrm{L}}^{(\mathrm{R})}=\mathbf{0}$ gilt dann Folgendes: 
(I) Es existieren positive Konstanten $\mathrm{c}_{\mathrm{L}}, \lambda$, so dass die Ungleichung

$$
\left\|\boldsymbol{\xi}_{\mathrm{L}}(t)\right\|_{2} \leq\left\|\boldsymbol{\xi}_{\mathrm{L}}(0)\right\|_{2} \mathrm{C}_{\mathrm{L}} \mathrm{e}^{-\lambda t}
$$

für beliebige Anfangswerte $\boldsymbol{\xi}_{\mathrm{L}}(0)$ und die euklidische Norm $\|\cdot\|_{2}$ gilt.

(II) Es existieren positive Konstanten $\mathrm{c}_{\mathrm{r}}, \lambda$, so dass die Ungleichung

$$
\left\|\boldsymbol{y}_{\mathrm{r}}(t)\right\|_{2} \leq\left\|\boldsymbol{\xi}_{\mathrm{L}}(0)\right\|_{2} \mathrm{c}_{\mathrm{r}} \mathrm{e}^{-\lambda t}
$$

für den Reglerausgang $\boldsymbol{y}_{\mathrm{r}}$ gilt.

Beweis. Der Beweis findet sich in Abschnitt C.3.6.

Offensichtlich folgt aus Aussage (I), dass die Ruhelage $\boldsymbol{\xi}_{\mathrm{L}}^{(\mathrm{R})}=\mathbf{0}$ des linearen Teilsystems $\mathbf{L}$ global asymptotisch stabil ist und die Norm des Zustandsvektors exponentiell mit der Zeit abnimmt. Eine Ruhelage mit diesen Eigenschaften wird auch als global exponentiell stabil bezeichnet [107]. Es bleibt festzuhalten: Das lineare Teilsystem ist, unabhängig von dem Anti-Windup, immer exponentiell stabil. Für die Stabilität der Ruhelage $\boldsymbol{\xi}_{\mathrm{ms}}^{(\mathrm{R})}=\mathbf{0}$ des Mismatch-Systems ist somit ausschlaggebend, wie es um die Stabilität des Anti-Windup-Ersatzsystems ES bzw. dessen Ruhelage $\boldsymbol{\xi}_{\mathrm{aw}}^{(\mathrm{R})}=\mathbf{0}$ bestellt ist. Dies wird nachfolgend diskutiert.

\subsubsection{Eigenschaften des Anti-Windup-Ersatzsystems}

Das in diesem Abschnitt betrachtete Anti-Windup-Ersatzsystem

$$
\mathbf{E S}:\left\{\begin{aligned}
\dot{\boldsymbol{\xi}}_{\mathrm{aw}} & =\boldsymbol{A}_{\mathrm{s}} \boldsymbol{\xi}_{\mathrm{aw}}+\boldsymbol{B}_{\mathrm{s}, u} \mathbf{s a t}_{\alpha \overline{\mathbf{u}}}\left(-\boldsymbol{k}\left(\boldsymbol{\xi}_{\mathrm{aw}}\right)\right)+\boldsymbol{B}_{\mathrm{s}, u} \boldsymbol{\zeta} \\
\boldsymbol{y}_{\mathrm{aw}, \mathrm{u}} & =\boldsymbol{C}_{\mathrm{s}} \boldsymbol{\xi}_{\mathrm{aw}}
\end{aligned}\right.
$$

ist kein autonomes System, da es durch die Störgröße $\boldsymbol{\zeta}(t) \neq \mathbf{0}$ beeinflusst wird. Deshalb ist der Ursprung $\boldsymbol{\xi}_{\text {aw }}=\mathbf{0}$ zunächst auch keine Ruhelage von ES. Das ändert sich jedoch ab einem Zeitpunkt $0<t^{*}<\infty$. Dann gilt $\boldsymbol{\zeta}(t)=\mathbf{0}$ für alle $t \geq t^{*}$.

Diese Tatsache ist durch die in Abbildung 6.4 gezeigte Reihenschaltung der Systeme $\mathbf{L}$ und $\mathbf{E S}$ bedingt und lässt sich wie folgt plausibilisieren. Die euklidische Norm der Stellgröße $\boldsymbol{y}_{\mathrm{r}}$ des Systems $\mathbf{L}$ nimmt nach Aussage (II) des Lemmas 6.1 exponentiell mit der Zeit ab. Daraus folgt, dass für jedes $\varrho \in(0,1)$ ein Zeitpunkt $0<t^{*}<\infty$ existieren muss, so dass

$$
\operatorname{sat}_{\varrho \overline{\mathbf{u}}}\left(\boldsymbol{y}_{\mathrm{r}}(t)\right)-\boldsymbol{y}_{\mathrm{r}}(t)=\mathbf{0}
$$


für alle $t \geq t^{*}$ gilt. Aus Abschätzung (6.6b) folgt dann $\zeta=\mathbf{0}$ für alle $t \geq t^{*}$ und ES wird nicht mehr durch die Störgröße beeinflusst.

Das dynamische Verhalten von ES lässt sich somit in zwei Phasen einteilen. In der ersten Phase, d. h. für $t \in\left[0, t^{*}\right)$, wird das System durch $\boldsymbol{\zeta}$ gestört und besitzt die Dynamik

$$
\dot{\boldsymbol{\xi}}_{\mathrm{aw}}=\boldsymbol{A}_{\mathrm{s}} \boldsymbol{\xi}_{\mathrm{aw}}+\boldsymbol{B}_{\mathrm{s}, u} \mathbf{s a t}_{\alpha \overline{\mathbf{u}}}\left(-\boldsymbol{k}\left(\boldsymbol{\xi}_{\mathrm{aw}}\right)\right)+\boldsymbol{B}_{\mathrm{s}, u} \boldsymbol{\zeta}(t)
$$

mit dem Anfangszustand $\boldsymbol{\xi}_{\text {aw }}(0)$. In der zweiten Phase, d. h. ab dem Zeitpunkt $t=t^{*}<\infty$, ist das System ES autonom und wird durch

$$
\dot{\boldsymbol{\xi}}_{\mathrm{aw}}=\boldsymbol{A}_{\mathrm{s}} \boldsymbol{\xi}_{\mathrm{aw}}+\boldsymbol{B}_{\mathrm{s}, u} \mathbf{s a t}_{\alpha \overline{\mathbf{u}}}\left(-\boldsymbol{k}\left(\boldsymbol{\xi}_{\mathrm{aw}}\right)\right)
$$

sowie den Anfangszustand $\boldsymbol{\xi}_{\text {aw }}\left(t^{*}\right)$ beschrieben. Es besitzt die zu stabilisierende Ruhelage $\boldsymbol{\xi}_{\mathrm{aw}}^{(\mathrm{R})}=\mathbf{0}$.

In der ersten Phase kann die Störgröße $\boldsymbol{\zeta}(t)$ die Systemdynamik (6.7) beliebig dominieren, weil der Einfluss des Anti-Windup bzw. des Zustandsreglers durch die Sättigung begrenzt ist. Durch Satz 6.1 ist jedoch sichergestellt, dass sich der Systemzustand $\boldsymbol{\xi}_{\text {aw }}(t)$ in dem begrenzten Zeitintervall $\left[0, t^{*}\right]$ nicht unendlich weit von dem Anfangszustand $\boldsymbol{\xi}_{\text {aw }}(0)$ entfernen kann. Eine endliche Entweichzeit der Lösung ist ausgeschlossen und für ein ausreichend großes $\tilde{c}>0$ gilt

$$
\left\|\boldsymbol{\xi}_{\text {aw }}(t)\right\|<\tilde{\mathrm{c}} \quad \forall t \in\left[0, t^{*}\right] .
$$

Die Trajektorien sind demnach in der ersten Phase beschränkt. Zu Beginn der zweiten Phase stellt sich der Zustand $\boldsymbol{\xi}_{\text {aw }}\left(t^{*}\right)$ ein. Liegt dieser in dem Einzugsgebiet $\mathcal{G}(\mathbf{0})$ der Ruhelage $\boldsymbol{\xi}_{\text {aw }}^{(\mathrm{R})}=\mathbf{0}$, so ist sichergestellt, dass

$$
\boldsymbol{\xi}_{\text {aw }}(t) \in \mathcal{G}(\mathbf{0}) \forall t \geq t^{*}, \quad \lim _{t \rightarrow \infty} \boldsymbol{\xi}(t)=\mathbf{0}
$$

gilt. Für $\boldsymbol{\xi}_{\text {aw }}\left(t^{*}\right) \in \mathcal{G}(\mathbf{0})$ sind die Trajektorien in der zweiten Phase ebenfalls beschränkt und streben in die Ruhelage. Über beide Phasen betrachtet liegt demnach ein stabiles Systemverhalten vor. Befindet sich $\boldsymbol{\xi}_{\text {aw }}\left(t^{*}\right)$ außerhalb des Einzugsgebietes der Ruhelage, werden die Trajektorien nicht in die Ruhelage konvergieren. Die entscheidende Bedingung für ein stabiles Systemverhalten ist folglich

$$
\boldsymbol{\xi}_{\text {aw }}\left(t^{*}\right) \in \mathcal{G}(\mathbf{0}) \quad \forall \boldsymbol{\zeta}(t) \text { gemäß }(6.6 \mathrm{~b}) .
$$




\section{Globale Stabilität}

Für ein unendlich großes Einzugsgebiet $\mathcal{G}(\mathbf{0})=\mathbb{R}^{n_{\mathrm{s}}}$ der Ruhelage ist die Bedingung (6.9) immer erfüllt. Der einfachste Anti-Windup-Entwurf besteht deshalb darin, die Funktion $\boldsymbol{k}$ so zu wählen, dass die Ruhelage $\boldsymbol{\xi}_{\text {aw }}^{(\mathrm{R})}=\mathbf{0}$ des autonomen Systems (6.8) global asymptotisch stabil ist. Wie aus Abschnitt 3.1 bekannt, ist dies prinzipiell nur dann möglich, wenn die Systemmatrix $\boldsymbol{A}_{\mathrm{s}}$ des autonomen Systems ES bzw. der Strecke $\mathbf{S}$ Eigenwerte mit nichtpositiven Realteilen besitzt. In diesen Fällen gilt

Satz 6.2. Gegeben sei das System

$$
\dot{\boldsymbol{\xi}}_{\mathrm{aw}}=\boldsymbol{A}_{\mathrm{s}} \boldsymbol{\xi}_{\mathrm{aw}}+\boldsymbol{B}_{\mathrm{s}, u} \boldsymbol{s} \boldsymbol{a} \boldsymbol{t}_{\alpha \overline{\boldsymbol{u}}}\left(-\boldsymbol{k}\left(\boldsymbol{\xi}_{\mathrm{aw}}\right)\right)+\boldsymbol{B}_{\mathrm{s}, u} \boldsymbol{\zeta}(t)
$$

mit dem Zustand $\boldsymbol{\xi}_{\text {aw }} \in \mathbb{R}^{n_{\mathrm{s}}}$ und der Störgröße $\boldsymbol{\zeta}(t)$. Für die Störgröße gelte $\boldsymbol{\zeta}(t)=0 \forall t \geq t^{*}$ mit $t^{*}<\infty$. Der Anfangszustand sei $\boldsymbol{\xi}_{\mathrm{aw}}^{(0)} \in \mathbb{R}^{n_{\mathrm{s}}}$.

Wenn die Ruhelage $\boldsymbol{\xi}_{\mathrm{aw}}^{(\mathrm{R})}=\mathbf{0}$ des autonomen Systems

$$
\dot{\boldsymbol{\xi}}_{\mathrm{aw}}=\boldsymbol{A}_{\mathrm{s}} \boldsymbol{\xi}_{\mathrm{aw}}+\boldsymbol{B}_{\mathrm{s}, u} \boldsymbol{s a t} \boldsymbol{t}_{\alpha \overline{\boldsymbol{u}}}\left(-\boldsymbol{k}\left(\boldsymbol{\xi}_{\mathrm{aw}}\right)\right)
$$

global asymptotisch stabil ist, dann existiert ein $\mathrm{c}>0$, so dass für die Trajektorien von (6.10) gilt

$$
\left\|\boldsymbol{\xi}_{\text {aw }}(t)\right\|<c \forall t \geq 0, \lim _{t \rightarrow \infty} \boldsymbol{\xi}_{\text {aw }}(t)=\mathbf{0} .
$$

Beweis. Der Beweis findet sich in Abschnitt C.3.7.

Aus der Beschränktheit der Trajektorien $\boldsymbol{\xi}_{\text {aw }}(t)$ und der Konvergenz in den Ursprung folgt zusammen mit der exponentiellen Stabilität des Teilsystems $\mathbf{L}$ die Stabilität der Reihenschaltung aus Abbildung 6.4. Somit impliziert die globale Stabilität der Ruhelage $\boldsymbol{\xi}_{\mathrm{aw}}^{(\mathrm{R})}=\mathbf{0}$ des autonomen Systems ES die Stabilität des Anti-Windup-Regelkreises. Diese erstaunliche Tatsache präzisiert

Satz 6.3. Gegeben sei der Anti-Windup-Regelkreis aus Abbildung 6.1 mit der Dynamik (6.3) und dem Anfangszustand $\boldsymbol{x}_{\text {awrk }}(0)=\boldsymbol{x}_{\text {awrk }}^{(0)} \in \mathbb{R}^{2 n_{\mathrm{s}}+n_{\mathrm{r}}}$. Wenn die Ruhelage $\boldsymbol{\xi}_{\mathrm{aw}}^{(\mathrm{R})}=\mathbf{0}$ des autonomen Anti-Windup-Ersatzsystems

$$
\dot{\boldsymbol{\xi}}_{\mathrm{aw}}=\boldsymbol{A}_{\mathrm{s}} \boldsymbol{\xi}_{\mathrm{aw}}+\boldsymbol{B}_{\mathrm{s}, u} \boldsymbol{s a t} \boldsymbol{t}_{\alpha \overline{\boldsymbol{u}}}\left(-\boldsymbol{k}\left(\boldsymbol{\xi}_{\mathrm{aw}}\right)\right)
$$

global asymptotisch stabil ist, dann existiert ein $\mathrm{c}>0$, so dass für die Trajektorien des Anti-Windup-Regelkreises gilt

$$
\left\|\boldsymbol{x}_{\mathrm{awrk}}(t)\right\|<\mathrm{c} \forall t \geq 0, \lim _{t \rightarrow \infty} \boldsymbol{x}_{\mathrm{awrk}}(t)=\mathbf{0} .
$$


Beweis. Der Beweis findet sich in Abschnitt C.3.8.

Für die globale Stabilisierung des autonomen Systems (6.8) durch den Zustandsregler $\boldsymbol{k}$ existiert immer eine einfache Lösung, falls die Strecke $\mathbf{S}$ stabil ist. Dann weist die Systemmatrix $\boldsymbol{A}_{\mathrm{s}}$ ausschließlich Eigenwerte mit negativem Realteil auf. Deshalb führt die Wahl $\boldsymbol{k}\left(\boldsymbol{\xi}_{\text {aw }}\right) \equiv \mathbf{0}$ zu dem linearen Anti-Windup-Ersatzsystem

$$
\mathbf{E S}_{0}:\left\{\begin{aligned}
\dot{\boldsymbol{\xi}}_{\mathrm{aw}} & =\boldsymbol{A}_{\mathrm{s}} \boldsymbol{\xi}_{\mathrm{aw}}+\boldsymbol{B}_{\mathrm{s}, u} \boldsymbol{\zeta} \\
\boldsymbol{y}_{\mathrm{aw}, \mathrm{u}} & =C_{\mathrm{s}} \boldsymbol{\xi}_{\mathrm{aw}}
\end{aligned}\right.
$$

Für $\boldsymbol{\zeta}=\mathbf{0}$ besitzt es eine global asymptotisch stabile Ruhelage im Ursprung. In der Literatur wird dieser Ansatz als Internal Model Control Anti-Windup bezeichnet [191]. Der Nachteil besteht in einer niedrigen Regelgüte, da die Systemdynamik der der ungeregelten Strecke entspricht. Ein stark schwingungsfähiges Verhalten oder hohe Ausregelzeiten können die Folge sein. Es empfiehlt sich deshalb, die Funktion $\boldsymbol{k}$ nicht identisch null zu wählen. Die Kapitel 7 und 8 beschreiben zwei attraktive Möglichkeiten.

\section{Lokale Stabilität}

Wenn die Systemmatrix $\boldsymbol{A}_{\mathrm{s}}$ der Strecke Eigenwerte mit positiven Realteilen besitzt und die Stellgröße beschränkt ist, kann globale Stabilität nicht erreicht werden [23, 165, 177]. Für die Ruhelage $\boldsymbol{\xi}_{\text {aw }}^{(\mathrm{R})}=\mathbf{0}$ des autonomen Systems ES lässt sich deshalb nur ein begrenztes Einzugsgebiet $\mathcal{G}(\mathbf{0}) \subset \mathbb{R}^{n_{\mathrm{s}}}$ realisieren.

Offensichtlich ist die Stabilitätsbedingung (6.9) mit einem begrenzten Einzugsgebiet $\mathcal{G}(\mathbf{0})$ nicht für beliebig große Störgrößen $\boldsymbol{\zeta}(t)$ erfüllbar. Nur für ausreichend kleine Störungen und kleine Anfangszustände $\boldsymbol{\xi}_{\text {aw }}(0)$ verbleibt der Zustand $\boldsymbol{\xi}_{\text {aw }}(t)$ des Systems ES für $t \in\left[0, t^{*}\right]$ in $\mathcal{G}(\mathbf{0})$. Die weiteren Überlegungen beschränken sich deshalb auf begrenzte Störungen. Denkbar ist zum Beispiel eine Amplitudenbegrenzung oder eine Begrenzung der Energie des Störsignals. Um die folgenden Stabilitätssätze möglichst allgemein formulieren zu können, wird die Art der Begrenzung aber zunächst nicht festgelegt. Ein allgemeines Stabilitätsresultat liefert

Satz 6.4. Gegeben sei das System

$$
\dot{\boldsymbol{\xi}}_{\mathrm{aw}}=\boldsymbol{A}_{\mathrm{s}} \boldsymbol{\xi}_{\text {aw }}+\boldsymbol{B}_{\mathrm{s}, u} \boldsymbol{s} \boldsymbol{a} \boldsymbol{t}_{\alpha \overline{\boldsymbol{u}}}\left(-\boldsymbol{k}\left(\boldsymbol{\xi}_{\mathrm{aw}}\right)\right)+\boldsymbol{B}_{\mathrm{s}, u} \boldsymbol{\zeta}(t)
$$

mit dem Zustand $\boldsymbol{\xi}_{\text {aw }} \in \mathbb{R}^{n_{\mathrm{s}}}$ und einem Anfangszustand $\boldsymbol{\xi}_{\mathrm{aw}}^{(0)} \in \mathcal{X}_{\mathrm{aw}}^{(0)} \subset \mathbb{R}^{n_{\mathrm{s}}}$. Die Störgröße $\boldsymbol{\zeta}(t)$ sei begrenzt, so dass der Zustand $\boldsymbol{\xi}_{\text {aw }}$ von (6.11) für alle $t \geq 0$ in einer begrenzten Umgebung $\mathcal{G}_{u} \supset \mathcal{X}_{\mathrm{aw}}^{(0)}$ des Ursprungs verbleibt. 
Wenn die Ruhelage $\boldsymbol{\xi}_{\mathrm{aw}}^{(\mathrm{R})}=\mathbf{0}$ des autonomen Systems

$$
\dot{\boldsymbol{\xi}}_{\mathrm{aw}}=\boldsymbol{A}_{\mathrm{s}} \boldsymbol{\xi}_{\mathrm{aw}}+\boldsymbol{B}_{\mathrm{s}, u} \boldsymbol{s} \boldsymbol{a} \boldsymbol{t}_{\alpha \overline{\boldsymbol{u}}}\left(-\boldsymbol{k}\left(\boldsymbol{\xi}_{\mathrm{aw}}\right)\right)
$$

asymptotisch stabil ist mit dem Einzugsgebiet $\mathcal{G}(\mathbf{0}) \supseteq \mathcal{G}_{u}$, dann existiert ein $\mathrm{c}>0$, so dass für die Trajektorien von (6.11) gilt

$$
\left\|\boldsymbol{\xi}_{\text {aw }}(t)\right\|<c \forall t \geq 0, \quad \lim _{t \rightarrow \infty} \boldsymbol{\xi}_{\text {aw }}(t)=\mathbf{0} .
$$

Beweis. Wenn der Zustand $\boldsymbol{\xi}_{\text {aw }}(t)$ für alle $t \geq 0$ in einer begrenzten Umgebung $\mathcal{G}_{u}$ des Ursprungs verbleibt, ist die Normbeschränkung des $\mathrm{Zu}$ standsvektors $\left\|\boldsymbol{\xi}_{\text {aw }}(t)\right\|<c$ mit $\mathrm{c}>0$ sichergestellt. Aus der Forderung $\mathcal{G}(\mathbf{0}) \supseteq \mathcal{G}_{u}$ und $\zeta(t)=0$ für alle $t \geq t^{*}$ folgt $\lim _{t \rightarrow \infty} \boldsymbol{\xi}_{\text {aw }}(t)=\mathbf{0}$.

Der Satz macht deutlich, dass die lokale Stabilität der Ruhelage $\boldsymbol{\xi}_{\mathrm{aw}}^{(\mathrm{R})}=\mathbf{0}$ des autonomen Anti-Windup-Ersatzsystems nicht ausreicht, um Stabilität des gesamten Anti-Windup-Regelkreises für beliebige Anfangszustände zu gewährleisten. Entscheidend für ein stabiles Systemverhalten sind

1. der Anfangszustand $\boldsymbol{\xi}_{\mathrm{aw}}^{(0)}$ bzw. die Größe des Gebietes $\boldsymbol{\mathcal { X }}_{\mathrm{aw}}^{(0)}$,

2. das Ausmaß der Störung $\boldsymbol{\zeta}$ und

3. die Größe des Einzugsgebietes $\mathcal{G}(\mathbf{0})$ der Ruhelage $\boldsymbol{\xi}_{\mathrm{aw}}^{(\mathrm{R})}=\mathbf{0}$.

Letzteres hängt entscheidend von der Struktur und Parametrierung des Zustandsreglers $\boldsymbol{k}\left(\boldsymbol{\xi}_{\text {aw }}\right)$ ab, so dass allgemeine Aussagen schwierig sind. Die Frage nach der Gebietsgröße $\mathcal{X}_{\text {aw }}^{(0)}$ lässt sich einfach beantworten. Um das Kleinsignalverhalten nicht zu stören, gilt für den Anfangszustand $\boldsymbol{\xi}_{\mathrm{aw}}^{(0)}$ nach Abschnitt 4.2 die Festlegung $\boldsymbol{x}_{\text {aw }}(0)=-\boldsymbol{\xi}_{\text {aw }}(0)=\mathbf{0}$. Das Anfangsgebiet $\mathcal{X}_{\mathrm{aw}}^{(0)}$ entartet somit zu einem Punkt.

Bezüglich der Störgröße lässt sich eine qualitative Aussage machen, wie nachfolgend gezeigt wird. Das Ausmaß der Störung $\zeta$ ist durch den Ausgang $\boldsymbol{y}_{\mathrm{r}}$ des Teilsystems $\mathbf{L}$, der dem Ausgang des Reglers $\mathbf{R}$ entspricht, gegeben. Bekanntlich gilt

$$
\left|\zeta_{i}\right| \leq 2\left|\operatorname{sat}_{\varrho \overline{\mathrm{u}}_{i}}\left(y_{\mathrm{r}, i}\right)-y_{\mathrm{r}, i}\right|=2\left|\mathrm{dz}_{\varrho \overline{\mathrm{u}}_{i}}\left(y_{\mathrm{r}, i}\right)\right| \leq 2\left|y_{\mathrm{r}, i}\right| .
$$

Daraus folgt sofort

$$
\|\boldsymbol{\zeta}\|_{2} \leq 2\left\|\boldsymbol{y}_{\mathrm{r}}\right\|_{2}
$$


Die euklidische Norm der Störung ist demnach zu jedem Zeitpunkt durch die euklidische Norm des Reglerausganges $\boldsymbol{y}_{\mathrm{r}}$ beschränkt, die wiederum von der Parametrierung des Reglers R und den Anfangszuständen

$$
\boldsymbol{\xi}_{\mathrm{L}}^{(0)}=\left[\begin{array}{l}
\boldsymbol{\xi}_{\mathrm{s}}^{(0)} \\
\boldsymbol{\xi}_{\mathrm{r}}^{(0)}
\end{array}\right]=\left[\begin{array}{l}
\boldsymbol{x}_{\mathrm{s}}^{(0)} \\
\boldsymbol{x}_{\mathrm{r}}^{(0)}
\end{array}\right]
$$

abhängt. Zusammen mit der Aussage (II) des Lemmas 6.1 folgt für geeignet gewählte positive Konstanten $\mathrm{c}_{\mathrm{r}}, \lambda$ die Abschätzung

$$
\|\boldsymbol{\zeta}(t)\|_{2} \leq 2\left\|\boldsymbol{y}_{\mathrm{r}}(t)\right\|_{2} \leq 2\left\|\boldsymbol{\xi}_{\mathrm{L}}(0)\right\|_{2} \mathrm{c}_{\mathrm{r}} \mathrm{e}^{-\lambda t}=2\left\|\left[\begin{array}{c}
\boldsymbol{x}_{\mathrm{s}}^{(0)} \\
\boldsymbol{x}_{\mathrm{r}}^{(0)}
\end{array}\right]\right\|_{2} \mathrm{c}_{\mathrm{r}} \mathrm{e}^{-\lambda t} .
$$

Der entscheidende Punkt ist, dass die Norm der Störung direkt von den Anfangszuständen des Regelkreises abhängt. Stabilität wird deshalb nur für ein begrenztes Gebiet von Anfangszuständen

$$
\mathcal{X}^{(0)}=\left\{\left[\begin{array}{l}
\boldsymbol{x}_{\mathrm{s}}^{(0)} \\
\boldsymbol{x}_{\mathrm{r}}^{(0)}
\end{array}\right] \in \mathbb{R}^{n_{\mathrm{s}}+n_{\mathrm{r}}} \mid\left\|\left[\begin{array}{c}
\boldsymbol{x}_{\mathrm{s}}^{(0)} \\
\boldsymbol{x}_{\mathrm{r}}^{(0)}
\end{array}\right]\right\|_{2}<\mathrm{c}_{0}\right\}
$$

mit $c_{0}>0$ nachgewiesen werden. Für den Anti-Windup-Regelkreis gilt

Satz 6.5. Gegeben sei der Anti-Windup-Regelkreis aus Abbildung 6.1 mit der Dynamik (6.3) und dem Anfangszustand $\boldsymbol{x}_{\mathrm{awrk}}(0)=\boldsymbol{x}_{\mathrm{awrk}}^{(0)}$ aus dem begrenzten Anfangsgebiet $\boldsymbol{\mathcal { X }}^{(0)} \times \mathbf{0}$ mit $\boldsymbol{\mathcal { X }}^{(0)}$ gemäß (6.12).

Wenn die Trajektorien $\boldsymbol{\xi}_{\text {aw }}(t)$ des Anti-Windup-Ersatzsystems

$$
\dot{\boldsymbol{\xi}}_{\mathrm{aw}}=\boldsymbol{A}_{\mathrm{s}} \boldsymbol{\xi}_{\text {aw }}+\boldsymbol{B}_{\mathrm{s}, u} \boldsymbol{s} \boldsymbol{a} \boldsymbol{t}_{\alpha \overline{\boldsymbol{u}}}\left(-\boldsymbol{k}\left(\boldsymbol{\xi}_{\mathrm{aw}}\right)\right)+\boldsymbol{B}_{\mathrm{s}, u} \boldsymbol{\zeta}
$$

für alle $t \geq 0$ in einer Umgebung $\mathcal{G}_{u}$ des Ursprungs verbleiben und die Ruhelage $\boldsymbol{\xi}_{\mathrm{aw}}^{(\mathrm{R})}=\mathbf{0}$ des autonomen Systems

$$
\dot{\boldsymbol{\xi}}_{\text {aw }}=\boldsymbol{A}_{\mathrm{s}} \boldsymbol{\xi}_{\text {aw }}+\boldsymbol{B}_{\mathrm{s}, u} \boldsymbol{s a t} \boldsymbol{t}_{\alpha \overline{\boldsymbol{u}}}\left(-\boldsymbol{k}\left(\boldsymbol{\xi}_{\mathrm{aw}}\right)\right)
$$

asymptotisch stabil ist mit dem Einzugsgebiet $\mathcal{G}(\mathbf{0}) \supseteq \mathcal{G}_{u}$, dann existiert ein $\mathrm{c}>0$, so dass für die Trajektorien des Anti-Windup-Regelkreises gilt

$$
\left\|\boldsymbol{x}_{\text {awrk }}(t)\right\|<\mathrm{c} \forall t \geq 0, \lim _{t \rightarrow \infty} \boldsymbol{x}_{\text {awrk }}(t)=\mathbf{0} .
$$

Beweis. Der Beweis findet sich in Abschnitt C.3.9.

Der Stabilitätssatz ist bewusst allgemein formuliert, so dass sich unterschiedliche Entwurfsverfahren entwickeln lassen. In Kapitel 9 werden beispielsweise lineare modellbasierte Anti-Windup-Maßnahmen der Form $\boldsymbol{k}\left(\boldsymbol{\xi}_{\text {aw }}\right)=\underline{\boldsymbol{K}} \boldsymbol{\xi}_{\text {aw }}$ entworfen. Das Gebiet $\mathcal{G}_{u}$ lässt sich dann für energiebegrenzte Störungen $\|\boldsymbol{\zeta}\|_{L_{2}}^{2} \leq \theta, \theta>0$ durch eine Höhenlinie der verwendeten quadratischen Ljapunov-Funktion charakterisieren. 


\subsection{Stabilität anderer Ruhelagen}

Der Regler $\mathbf{R}$ ist nach Annahme 2.2 so entworfen, dass Sollwertfolge und Störunterdrückung im Anti-Windup-Regelkreis garantiert sind, solange die Stellgröße nicht sättigt. In diesem Abschnitt wird untersucht, inwieweit ein modellbasiertes Anti-Windup der Form

$$
\mathbf{A W}:\left\{\begin{array}{l}
\dot{\boldsymbol{x}}_{\mathrm{aw}}=\boldsymbol{A}_{\mathrm{s}} \boldsymbol{x}_{\mathrm{aw}}+\boldsymbol{B}_{\mathrm{s}, u} \mathbf{s a t}_{\alpha \overline{\mathbf{u}}}\left(-\boldsymbol{k}\left(\boldsymbol{x}_{\mathrm{aw}}\right)\right)+\boldsymbol{B}_{\mathrm{s}, u} \boldsymbol{\delta}, \\
\boldsymbol{y}_{\mathrm{aw}, \mathrm{u}}=-\boldsymbol{C}_{\mathrm{s}} \boldsymbol{x}_{\mathrm{aw}} \\
\boldsymbol{y}_{\mathrm{aw}, \mathrm{y}}=-\mathbf{s a t}_{\alpha \overline{\mathbf{u}}}\left(-\boldsymbol{k}\left(\boldsymbol{x}_{\mathrm{aw}}\right)\right)
\end{array}\right.
$$

mit $\alpha=1-\varrho$ diese Eigenschaften auch im Sättigungsfall garantieren kann. Dabei spielt der bisher wenig beachtete Parameter $\varrho \in(0,1)$ des AntiWindup eine wichtige Rolle. Zunächst werden die von dem geforderten Sollwert $\boldsymbol{w}$ und auftretenden Störgrößen $\boldsymbol{z}$ abhängenden Ruhelagen des unbeschränkten Regelkreises betrachtet.

\subsubsection{Ruhelagen des unbeschränkten Regelkreises}

Ausgangspunkt der Überlegungen ist der unbeschränkte Regelkreis aus Abbildung 2.1. Möchte man den Zustand $\boldsymbol{x}_{\mathrm{s}}$ einer Strecke $\mathbf{S}$ in eine durch die Führungsgröße $\boldsymbol{w}$ vorgegebene Ruhelage $\boldsymbol{x}_{\mathrm{s}}^{(\mathrm{R})}$ überführen und dort halten, so ist dazu eine stationäre Stellgröße $\boldsymbol{y}_{\mathrm{r}}^{(\mathrm{R})}$ nötig. Gleiches gilt für die Stabilisierung einer beliebigen Ruhelage unter auftretenden konstanten Störungen $\boldsymbol{z} \neq \mathbf{0}$. Das lässt sich anhand der Systemdynamik (2.3) des unbeschränkten Regelkreises

$$
\dot{\boldsymbol{x}}_{\mathrm{ub}}=\boldsymbol{A}_{\mathrm{ub}} \boldsymbol{x}_{\mathrm{ub}}+\boldsymbol{B}_{\mathrm{ub}} \boldsymbol{r}
$$

mit den zusammengesetzten Zustands- und Eingangsvektoren

$$
\boldsymbol{x}_{\mathrm{ub}}^{\top}=\left[\begin{array}{ll}
\boldsymbol{x}_{\mathrm{s}, \mathrm{ub}}^{\top} & \boldsymbol{x}_{\mathrm{r}, \mathrm{ub}}^{\top}
\end{array}\right], \boldsymbol{r}^{\top}=\left[\begin{array}{ll}
\boldsymbol{w}^{\top} & \boldsymbol{z}^{\top}
\end{array}\right]
$$

leicht zeigen. Für konstante Eingangsgrößen

$$
\boldsymbol{r}_{0}^{\top}=\left[\begin{array}{ll}
\boldsymbol{w}_{0}^{\top} & \boldsymbol{z}_{0}^{\top}
\end{array}\right]
$$

ergeben sich die Ruhelagen $\boldsymbol{x}_{\mathrm{ub}}^{(\mathrm{R})}$ des unbeschränkten Regelkreises aus der bekannten Gleichung

$$
\dot{\boldsymbol{x}}_{\mathrm{ub}}=\mathbf{0}=\boldsymbol{A}_{\mathrm{ub}} \boldsymbol{x}_{\mathrm{ub}}^{(\mathrm{R})}+\boldsymbol{B}_{\mathrm{ub}} \boldsymbol{r}_{0}
$$


Auflösen nach $\operatorname{dem}$ Zustand ist wegen $\operatorname{det}\left(\boldsymbol{A}_{\mathrm{ub}}\right) \neq 0$ möglich und führt zu

$$
\boldsymbol{x}_{\mathrm{ub}}^{(\mathrm{R})}=-\boldsymbol{A}_{\mathrm{ub}}^{-1} \boldsymbol{B}_{\mathrm{ub}} \boldsymbol{r}_{0} .
$$

Befindet sich der Systemzustand in dieser Ruhelage, gilt für den Streckenausgang $\boldsymbol{y}_{\mathrm{s}}=\boldsymbol{w}_{0}$, da der Regler nach Annahme 2.2 Sollwertfolge und Störunterdrückung garantiert. Um den Zustand des Regelkreises in der Ruhelage zu halten, muss eine konstante Stellgröße auf die Strecke wirken. Diese Stellgröße entspricht im unbeschränkten Regelkreis dem Reglerausgang $\boldsymbol{y}_{\mathrm{r}}$. Gemäß (6.2) ergibt sich mit den Matrizen

$$
\boldsymbol{C}_{\mathrm{ub}}=\left[\begin{array}{ll}
\boldsymbol{D}_{\mathrm{r}, u} \boldsymbol{C}_{\mathrm{s}} & \boldsymbol{C}_{\mathrm{r}}
\end{array}\right], \quad \boldsymbol{D}_{\mathrm{ub}}=\left[\begin{array}{ll}
\boldsymbol{D}_{\mathrm{r}, w} & \mathbf{0}
\end{array}\right]
$$

der Reglerausgang zu

$$
\boldsymbol{y}_{\mathrm{r}, \mathrm{ub}}=\boldsymbol{C}_{\mathrm{ub}} \boldsymbol{x}_{\mathrm{ub}}+\boldsymbol{D}_{\mathrm{ub}} \boldsymbol{r} .
$$

Die benötigte stationäre Stellgröße hängt von dem geforderten Sollwert $\boldsymbol{w}_{0}$ und der auftretenden Störgröße $\boldsymbol{z}_{0}$ ab. Einsetzen von (6.13) liefert

$$
\boldsymbol{y}_{\mathrm{r}, \mathrm{ub}}^{(\mathrm{R})}=-\left(\boldsymbol{C}_{\mathrm{ub}} \boldsymbol{A}_{\mathrm{ub}}^{-1} \boldsymbol{B}_{\mathrm{ub}}-\boldsymbol{D}_{\mathrm{ub}}\right) \boldsymbol{r}_{0} .
$$

Diese stationäre Stellgröße kann in unbeschränkten Regelkreisen immer realisiert werden. Für die im Weiteren betrachteten Anti-WindupRegelkreise mit realem Aktor schränkt (6.15) die Menge der stabilisierbaren Ruhelagen ein. Details erläutert der nächste Abschnitt.

\subsubsection{Zulässige Ruhelagen}

Der Anti-Windup-Regelkreis weist durch die zusätzlichen Zustände des Anti-Windup eine höhere Systemordnung auf als der unbeschränkte Regelkreis. Folglich ist der Zustandsvektor

$$
\boldsymbol{x}_{\mathrm{awrk}}^{\top}=\left[\begin{array}{lll}
\boldsymbol{x}_{\mathrm{s}}^{\top} & \boldsymbol{x}_{\mathrm{r}}^{\top} & \boldsymbol{x}_{\mathrm{aw}}^{\top}
\end{array}\right]
$$

zu betrachten. Wie in Abschnitt 4.2 erläutert, ist das Ziel der Anti-Windup-Maßnahmen die Rückgewinnung des unbeschränkten Verhaltens. Die Zustände von Strecke und Regler des Anti-Windup-Regelkreises sollen deshalb möglichst dicht an den Zuständen von Strecke und Regler des unbeschränkten Regelkreises liegen. In einer Ruhelage dürfen keine Abweichungen vorhanden sein. Eine Ruhelage des unbeschränkten Regelkreises entspricht deshalb im Anti-Windup-Regelkreis der Ruhelage

$$
\boldsymbol{x}_{\mathrm{s}}^{(\mathrm{R})}=\boldsymbol{x}_{\mathrm{s}, \mathrm{ub}}^{(\mathrm{R})}, \quad \boldsymbol{x}_{\mathrm{r}}^{(\mathrm{R})}=\boldsymbol{x}_{\mathrm{r}, \mathrm{ub}}^{(\mathrm{R})}, \quad \boldsymbol{x}_{\mathrm{aw}}^{(\mathrm{R})}=\mathbf{0} .
$$


Für $\boldsymbol{x}_{\mathrm{aw}}^{(\mathrm{R})}=\mathbf{0}$ sind die Ausgänge des Anti-Windup ebenfalls null und der Regelkreis wird nicht mehr durch die Anti-Windup-Maßnahme beeinflusst.

Die auf die Strecke $\mathbf{S}$ wirkende Stellgröße des Anti-Windup-Regelkreises ist durch den Aktorausgang

$$
\boldsymbol{y}_{\mathrm{a}}=\operatorname{sat}_{\overline{\mathbf{u}}}\left(\boldsymbol{u}_{\mathrm{a}}\right)=\operatorname{sat}_{\overline{\mathbf{u}}}\left(\boldsymbol{y}_{\mathrm{r}}+\boldsymbol{y}_{\mathrm{aw}, \mathrm{y}}\right)
$$

gegeben. Aus $\boldsymbol{x}_{\mathrm{aw}}^{(\mathrm{R})}=\mathbf{0}$ folgt $\boldsymbol{y}_{\mathrm{aw}, \mathrm{y}}^{(\mathrm{R})}=\mathbf{0}$ und somit

$$
\boldsymbol{y}_{\mathrm{a}}^{(\mathrm{R})}=\operatorname{sat}_{\overline{\mathbf{u}}}\left(\boldsymbol{y}_{\mathrm{r}}^{(\mathrm{R})}\right) .
$$

Offensichtlich wird im Anti-Windup-Regelkreis die gleiche stationäre Stellgröße $\boldsymbol{y}_{\mathrm{r}}^{(\mathrm{R})}$ wie im unbeschränkten Regelkreis benötigt, um den Systemzustand in der Ruhelage (6.16) zu halten. Wenn die stationär benötigte Stellgröße die maximal vom Aktor realisierbare Stellgröße überschreitet, ist dies jedoch physikalisch unmöglich. Die weiteren Betrachtungen beschränken sich deshalb auf Ruhelagen, die eine realisierbare stationäre Stellgröße aufweisen und somit gemäß folgender Definition zulässig sind.

Definition 6.2 (Zulässige Ruhelage). Eine Ruhelage (6.16) des AntiWindup-Regelkreises mit den Komponenten

$$
\left[\begin{array}{c}
\boldsymbol{x}_{\mathrm{s}}^{(\mathrm{R})} \\
\boldsymbol{x}_{\mathrm{r}}^{(\mathrm{R})}
\end{array}\right]=\boldsymbol{x}_{\mathrm{ub}}^{(\mathrm{R})}=-\boldsymbol{A}_{\mathrm{ub}}^{-1} \boldsymbol{B}_{\mathrm{ub}} \boldsymbol{r}_{0}, \quad \boldsymbol{x}_{\mathrm{aw}}^{(\mathrm{R})}=\mathbf{0}
$$

heißt zulässig, wenn die stationär benötigte Stellgröße

$$
\boldsymbol{y}_{\mathrm{r}}^{(\mathrm{R})}=\boldsymbol{y}_{\mathrm{r}, \mathrm{ub}}^{(\mathrm{R})}=\boldsymbol{C}_{\mathrm{ub}} \boldsymbol{x}_{\mathrm{ub}}^{(\mathrm{R})}+\boldsymbol{D}_{\mathrm{ub}} \boldsymbol{r}_{0}
$$

folgende Bedingung erfüllt

$$
\left|y_{\mathrm{r}, i}^{(\mathrm{R})}\right|<\overline{\mathrm{u}}_{i} \forall i=1, \ldots, m .
$$

Im nächsten Abschnitt wird gezeigt, dass die Stabilisierung bestimmter zulässiger Ruhelagen durch das Anti-Windup möglich ist, wenn der AntiWindup-Parameter $\alpha \in(0,1)$ einer einfachen Bedingung genügt.

\subsubsection{Eine zusätzliche Entwurfsbedingung}

Um die Entwurfsbedingung für den Anti-Windup-Parameter $\alpha$ herzuleiten, ist erneut die Transformation (4.9) des Anti-Windup-Regelkreises in die Mismatch-Darstellung hilfreich. Abbildung 6.5 zeigt das für den AntiWindup-Entwurf maßgebliche Blockschaltbild. Der lineare Regelkreis L 
weist die gleiche Dynamik wie (2.3) auf. Somit ist nach Annahme 2.2 Stabilität, Sollwertfolge und Störkompensation gesichert und die global exponentiell stabile Ruhelage

$$
\boldsymbol{\xi}_{\mathrm{L}}^{(\mathrm{R})}=\left[\begin{array}{c}
\boldsymbol{\xi}_{\mathrm{s}}^{(\mathrm{R})} \\
\boldsymbol{\xi}_{\mathrm{r}}^{(\mathrm{R})}
\end{array}\right]
$$

stellt sich für $t \rightarrow \infty$ ein.

\section{Problem: Störung verschwindet nicht}

Der Unterschied zu den Betrachtungen in Abschnitt 6.3 liegt darin, dass $\boldsymbol{y}_{\mathrm{r}}$ nicht mehr in jedem Fall gegen null läuft, sondern gegen die stationäre Stellgröße (6.15). Es gilt

Lemma 6.2. Gegeben sei der stabile, unbeschränkte, lineare Regelkreis

$$
\begin{aligned}
& \dot{\boldsymbol{\xi}}_{\mathrm{L}}=\boldsymbol{A}_{\mathrm{ub}} \boldsymbol{\xi}_{\mathrm{L}}+\boldsymbol{B}_{\mathrm{ub}} \boldsymbol{r}, \quad \boldsymbol{\xi}_{\mathrm{L}}(0)=\boldsymbol{\xi}_{\mathrm{L}}^{(0)} \in \mathbb{R}^{n_{\mathrm{s}}+n_{\mathrm{r}}}, \\
& \boldsymbol{y}_{\mathrm{r}}=\boldsymbol{C}_{\mathrm{ub}} \boldsymbol{\xi}_{\mathrm{L}}+\boldsymbol{D}_{\mathrm{ub}} \boldsymbol{r} .
\end{aligned}
$$

Bezüglich der Parametrierung gelte Annahme 2.2. Der Eingangsvektor $\boldsymbol{r}(t)$ sei konstant, d. $h . \boldsymbol{r}(t)=\boldsymbol{r}_{0}$. Dann beträgt die stationäre Stellgröße

$$
\boldsymbol{y}_{\mathrm{r}}^{(\mathrm{R})}=\lim _{t \rightarrow \infty} \boldsymbol{y}_{\mathrm{r}}(t)=-\left(\boldsymbol{C}_{\mathrm{ub}} \boldsymbol{A}_{\mathrm{ub}}^{-1} \boldsymbol{B}_{\mathrm{ub}}-\boldsymbol{D}_{\mathrm{ub}}\right) \boldsymbol{r}_{0}
$$

und es existieren positive Konstanten c, $\lambda$, so dass gilt

$$
\left\|\boldsymbol{y}_{\mathrm{r}}(t)-\boldsymbol{y}_{\mathrm{r}}^{(\mathrm{R})}\right\|_{2} \leq \mathrm{ce}^{-\lambda t}
$$

Beweis. Der Beweis findet sich in Abschnitt C.3.10.

Die Konvergenz von $\boldsymbol{y}_{\mathrm{r}}$ gegen eine stationäre Stellgröße $\boldsymbol{y}_{\mathrm{r}}^{(\mathrm{R})} \neq \mathbf{0}$ hat Konsequenzen für die auf das zweite Teilsystem ES wirkende Störgröße $\boldsymbol{\zeta}$. Insbesondere ist nicht mehr garantiert, dass die Störung $\zeta$ verschwindet. Das folgt aus dem obigen Lemma sowie der Abschätzung

$$
\left|\zeta_{i}(t)\right| \leq\left|\operatorname{sat}_{\varrho \bar{u}_{i}}\left(y_{\mathrm{r}, i}(t)\right)-y_{\mathrm{r}, i}(t)\right|
$$

und lässt sich wie folgt plausibilisieren. Wenn beispielsweise die stationäre Stellgrößenkomponente $y_{\mathrm{r}, i}^{(\mathrm{R})}$ größer als der Sättigungslevel $\varrho \overline{\mathrm{u}}_{i}$ ist, dann gilt für die $i$-te Komponente der Störung die Abschätzung

$$
\lim _{t \rightarrow \infty}\left|\zeta_{i}(t)\right| \leq\left|\varrho \overline{\mathrm{u}}_{i}-y_{\mathrm{r}, i}^{(\mathrm{R})}\right|>0
$$

Die $i$-te Komponente von $\zeta$ kann demnach auch für $t \rightarrow \infty$ von null verschiedene Werte annehmen. Somit wirkt eine Störung $\boldsymbol{\zeta} \neq \mathbf{0}$ auf das Ersatzsystem und verschiebt die Ruhelage aus dem Ursprung. 


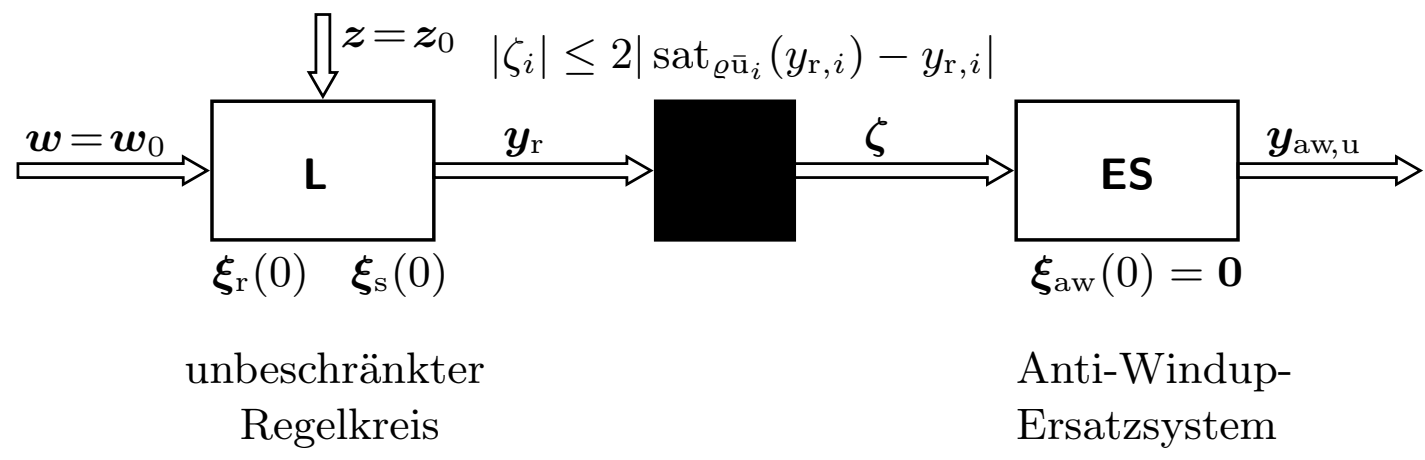

Abbildung 6.5: Mismatch-Darstellung des Anti-Windup-Regelkreises bestehend aus dem unbeschränkten Regelkreis und dem Anti-Windup-Ersatzsystem.

\section{Lösung: Anpassen des Anti-Windup-Parameters}

Der Ausweg besteht darin, den Parameter $\alpha=1-\varrho$ des Systems ES so zu wählen, dass für ein $0<t^{*}<\infty$ gilt

$$
\boldsymbol{\zeta}(t)=\mathbf{0} \quad \forall t \geq t^{*} .
$$

Das lässt sich durch eine geeignete Wahl von $\varrho$ erfüllen, wie im Folgenden gezeigt wird. Aus (6.21) und der Abschätzung (6.20) entsteht die Forderung nach der Existenz eines $\varrho \in(0,1)$, so dass gilt

$$
\operatorname{sat}_{\varrho \overline{\mathrm{u}}_{i}}\left(y_{\mathrm{r}, i}(t)\right)-y_{\mathrm{r}, i}(t)=0 \quad \forall t \geq t^{*}>0, \quad \forall i=1, \ldots, m .
$$

Das ist gleichbedeutend mit der Forderung

$$
-\varrho \overline{\mathrm{u}}_{i} \leq y_{\mathrm{r}, i}(t) \leq \varrho \overline{\mathrm{u}}_{i} \quad \forall t \geq t^{*}>0, \quad \forall i=1, \ldots, m .
$$

Im Weiteren wird diese Bedingung geschickt umgeformt. Zunächst führt eine Addition von null zu

$$
-\varrho \overline{\mathrm{u}}_{i} \leq y_{\mathrm{r}, i}(t)-y_{\mathrm{r}, i}^{(\mathrm{R})}+y_{\mathrm{r}, i}^{(\mathrm{R})} \leq \varrho \overline{\mathrm{u}}_{i} \quad \forall t \geq t^{*}>0, \quad \forall i=1, \ldots, m
$$

und nach einer Betragsbildung ergibt sich

$$
\left|y_{\mathrm{r}, i}(t)-y_{\mathrm{r}, i}^{(\mathrm{R})}+y_{\mathrm{r}, i}^{(\mathrm{R})}\right| \leq \varrho \overline{\mathrm{u}}_{i} \quad \forall t \geq t^{*}>0, \quad \forall i=1, \ldots, m .
$$

Unter Inkaufnahme von Konservativität ist diese Bedingung erfüllt, wenn der nach oben abgeschätzte Betrag kleiner als der Sättigungslevel ist, d. h.

$$
\left|y_{\mathrm{r}, i}(t)-y_{\mathrm{r}, i}^{(\mathrm{R})}+y_{\mathrm{r}, i}^{(\mathrm{R})}\right| \leq\left|y_{\mathrm{r}, i}(t)-y_{\mathrm{r}, i}^{(\mathrm{R})}\right|+\left|y_{\mathrm{r}, i}^{(\mathrm{R})}\right| \leq \varrho \overline{\mathrm{u}}_{i} .
$$


Da jede Komponente $y_{\mathrm{r}, i}(t)$ nach Lemma 6.2 für $t \rightarrow \infty$ exponentiell gegen die Komponente $y_{\mathrm{r}, i}^{\mathrm{R})}$ der stationären Stellgröße konvergiert, existiert für jedes beliebig kleine $\tilde{\epsilon}>0$ ein Zeitpunkt $0<t^{*}<\infty$, so dass

$$
\left|y_{\mathrm{r}, i}(t)-y_{\mathrm{r}, i}^{(\mathrm{R})}\right| \leq \tilde{\epsilon} \quad \forall t \geq t^{*}, \forall i=1, \ldots, m
$$

gilt. Die Kombination mit (6.22) führt zu der Bedingung

$$
\left|y_{\mathrm{r}, i}^{(\mathrm{R})}\right|+\tilde{\epsilon} \leq \varrho \overline{\mathrm{u}}_{i} \quad \forall i=1, \ldots, m,
$$

die für ein beliebig kleines $\tilde{\epsilon}>0$, ein $\varrho \in(0,1)$ und die zu den interessierenden zulässigen Ruhelagen gehörenden stationären Stellgrößen $\boldsymbol{y}_{\mathrm{r}}^{(\mathrm{R})}$ gelten muss. Auflösen nach $\varrho$ liefert schließlich

$$
1>\varrho>\max _{i \in\{1, \ldots, m\}}\left\{\frac{\left|y_{\mathrm{r}, i}^{(\mathrm{R})}\right|}{\overline{\mathrm{u}}_{i}}\right\},
$$

was mit der Beziehung $\alpha=1-\varrho$ zu der folgenden Vorschrift für die Wahl von $\alpha$ führt. Der Anti-Windup-Parameter $\alpha$ wird aus dem Intervall $(0, \hat{\alpha})$ gewählt, mit der oberen Intervallgrenze

$$
\hat{\alpha}=1-\max _{i \in\{1, \ldots, m\}}\left\{\left|y_{\mathrm{r}, i}^{(\mathrm{R})}\right| \overline{\mathrm{u}}_{i}^{-1}\right\} .
$$

Diese Wahl garantiert, dass $\zeta$ ab einem Zeitpunkt $0<t^{*}<\infty$ identisch null ist und somit die Ruhelage des Systems ES im Ursprung liegt. Das folgende Lemma fasst das Ergebnis dieses Abschnitts zusammen.

Lemma 6.3. Gegeben sei das Anti-Windup-Ersatzsystem

$$
\text { ES : }\left\{\begin{aligned}
\dot{\boldsymbol{\xi}}_{\mathrm{aw}} & =\boldsymbol{A}_{\mathrm{s}} \boldsymbol{\xi}_{\mathrm{aw}}+\boldsymbol{B}_{\mathrm{s}, u} \boldsymbol{s} \boldsymbol{a} \boldsymbol{t}_{\alpha \overline{\boldsymbol{u}}}\left(-\boldsymbol{k}\left(\boldsymbol{\xi}_{\mathrm{aw}}\right)\right)+\boldsymbol{B}_{\mathrm{s}, u} \boldsymbol{\zeta}, \\
\boldsymbol{y}_{\mathrm{aw}, \mathrm{u}} & =\boldsymbol{C}_{\mathrm{s}} \boldsymbol{\xi}_{\mathrm{aw}}
\end{aligned}\right.
$$

mit der Störgröße $\boldsymbol{\zeta}$, die der komponentenweisen Abschätzung (6.6b) genügt. Des Weiteren sei $\boldsymbol{x}_{\mathrm{awrk}}^{(\mathrm{R})}$ eine gemäß Definition 6.2 zulässige Ruhelage des Anti-Windup-Regelkreises (6.3) mit einer zugehörigen stationären Stellgröße $\boldsymbol{y}_{\mathrm{r}}^{(\mathrm{R})}$. Wenn der Anti-Windup-Parameter $\alpha$ aus dem offenen Intervall $(0, \hat{\alpha})$ mit

$$
\hat{\alpha}=1-\max _{i \in\{1, \ldots, m\}}\left\{\left|y_{\mathrm{r}, i}^{(\mathrm{R})}\right| \bar{u}_{i}^{-1}\right\}
$$

gewählt wird, dann existiert ein Zeitpunkt $0<t^{*}<\infty$, so dass $\boldsymbol{\zeta}(t)=\mathbf{0}$ für alle $t \geq t^{*}$. Ab diesem Zeitpunkt ist $\boldsymbol{\xi}_{\mathrm{aw}}^{(\mathrm{R})}=\mathbf{0}$ eine Ruhelage von $\mathbf{E S}$. 
Anmerkung 6.1. Um die Einflussmöglichkeit der Zustandsrückführung sat $_{\alpha \overline{\mathbf{u}}}\left(-\boldsymbol{k}\left(\boldsymbol{\xi}_{\text {aw }}\right)\right)$ auf die Systemdynamik von ES nicht zu stark einzuschränken, empfiehlt es sich, $\alpha$ möglichst groß zu wählen.

Wie der Anti-Windup-Parameter $\alpha$ zu wählen ist, damit die Störung $\boldsymbol{\zeta}$ verschwindet, hat dieser Abschnitt geklärt. Das Ergebnis kann nun genutzt werden, um zwei Stabilitätssätze zu beweisen.

\section{Stabilitätssätze}

Satz 6.3 gibt Bedingungen für die globale Stabilität der im Ursprung gelegenen Ruhelage des Anti-Windup-Regelkreises an. Mit Lemma 6.3 lässt sich dieses Ergebnis auf zulässige Ruhelagen $\boldsymbol{x}_{\text {awrk }}^{(\mathrm{R})} \neq \mathbf{0}$ erweitern.

Satz 6.6. Gegeben sei der Anti-Windup-Regelkreis (6.3) aus Abbildung 6.1 mit der Stellbegrenzung $\overline{\boldsymbol{u}}=\left[\bar{u}_{1} \ldots \bar{u}_{m}\right]^{\top}$ und dem Anfangszustand $\boldsymbol{x}_{\mathrm{awrk}}(0)=\boldsymbol{x}_{\mathrm{awrk}}^{(0)} \in \mathbb{R}^{2 n_{\mathrm{s}}+n_{\mathrm{r}}}$. Des Weiteren sei $\boldsymbol{x}_{\mathrm{awrk}}^{(\mathrm{R})}$ eine gemäß Definition 6.2 zulässige Ruhelage des Anti-Windup-Regelkreises mit einer zugehörigen stationären Stellgröße $\boldsymbol{y}_{\mathrm{r}}^{(\mathrm{R})}$. Wenn der Anti-Windup-Parameter $\alpha$ aus dem offenen Intervall $(0, \hat{\alpha})$ mit

$$
\hat{\alpha}=1-\max _{i \in\{1, \ldots, m\}}\left\{\left|y_{\mathrm{r}, i}^{(\mathrm{R})}\right| \bar{u}_{i}^{-1}\right\}
$$

gewählt wird und die Ruhelage $\boldsymbol{\xi}_{\mathrm{aw}}^{(\mathrm{R})}=\mathbf{0}$ des autonomen Anti-WindupErsatzsystems

$$
\dot{\boldsymbol{\xi}}_{\mathrm{aw}}=\boldsymbol{A}_{\mathrm{s}} \boldsymbol{\xi}_{\mathrm{aw}}+\boldsymbol{B}_{\mathrm{s}, u} \boldsymbol{s} \boldsymbol{a t} \boldsymbol{t}_{\alpha \overline{\boldsymbol{u}}}\left(-\boldsymbol{k}\left(\boldsymbol{\xi}_{\mathrm{aw}}\right)\right)
$$

global asymptotisch stabil ist, dann existiert ein $\mathrm{c}>0$, so dass für die Trajektorien des Anti-Windup-Regelkreises gilt

$$
\left\|\boldsymbol{x}_{\mathrm{awrk}}(t)-\boldsymbol{x}_{\mathrm{awrk}}^{(\mathrm{R})}\right\|<\mathrm{c} \forall t \geq 0, \lim _{t \rightarrow \infty} \boldsymbol{x}_{\mathrm{awrk}}(t)=\boldsymbol{x}_{\mathrm{awrk}}^{(\mathrm{R})} .
$$

Ist globale Stabilität des Anti-Windup-Regelkreises außer Reichweite, werden lokale Ergebnisse benötigt. Hinreichende Stabilitätsbedingungen für zulässige Ruhelagen $\boldsymbol{x}_{\text {awrk }}^{(\mathrm{R})} \neq \mathbf{0}$ liefert

Satz 6.7. Gegeben sei der Anti-Windup-Regelkreis (6.3) aus Abbildung 6.1 mit der Stellbegrenzung $\overline{\boldsymbol{u}}=\left[\bar{u}_{1} \ldots \bar{u}_{m}\right]^{\top}$ und dem Anfangszustand $\boldsymbol{x}_{\mathrm{awrk}}(0)=\boldsymbol{x}_{\mathrm{awrk}}^{(0)} \in \boldsymbol{\mathcal { X }}^{(0)} \times \mathbf{0}$. Des Weiteren sei $\boldsymbol{x}_{\mathrm{awrk}}^{(\mathrm{R})}$ eine gemäß Definition 6.2 zulässige Ruhelage des Anti-Windup-Regelkreises mit einer zugehörigen stationären Stellgröße $\boldsymbol{y}_{\mathrm{r}}^{(\mathrm{R})}$. Wenn der Anti-Windup-Parameter 
$\alpha$ aus dem offenen Intervall $(0, \hat{\alpha})$ mit

$$
\hat{\alpha}=1-\max _{i \in\{1, \ldots, m\}}\left\{\left|y_{\mathrm{r}, i}^{(\mathrm{R})}\right| \bar{u}_{i}^{-1}\right\}
$$

gewählt wird, die Trajektorien $\boldsymbol{\xi}_{\text {aw }}(t)$ des Anti-Windup-Ersatzsystems

$$
\dot{\boldsymbol{\xi}}_{\mathrm{aw}}=\boldsymbol{A}_{\mathrm{s}} \boldsymbol{\xi}_{\mathrm{aw}}+\boldsymbol{B}_{\mathrm{s}, u} \boldsymbol{s a t} \boldsymbol{t}_{\alpha \overline{\boldsymbol{u}}}\left(-\boldsymbol{k}\left(\boldsymbol{\xi}_{\mathrm{aw}}\right)\right)+\boldsymbol{B}_{\mathrm{s}, u} \boldsymbol{\zeta}
$$

für alle $t \geq 0$ in einer Umgebung $\mathcal{G}_{u}$ des Ursprungs verbleiben und die Ruhelage $\boldsymbol{\xi}_{\mathrm{aw}}^{(\mathrm{R})}=\mathbf{0}$ des autonomen Systems

$$
\dot{\boldsymbol{\xi}}_{\mathrm{aw}}=\boldsymbol{A}_{\mathrm{s}} \boldsymbol{\xi}_{\mathrm{aw}}+\boldsymbol{B}_{\mathrm{s}, u} \boldsymbol{s a t} \boldsymbol{t}_{\alpha \overline{\boldsymbol{u}}}\left(-\boldsymbol{k}\left(\boldsymbol{\xi}_{\mathrm{aw}}\right)\right)
$$

asymptotisch stabil ist mit dem Einzugsgebiet $\mathcal{G}(\mathbf{0}) \supseteq \mathcal{G}_{u}$, dann existiert ein $\mathrm{c}>0$, so dass für die Trajektorien des Anti-Windup-Regelkreises

$$
\left\|\boldsymbol{x}_{\mathrm{awrk}}(t)-\boldsymbol{x}_{\mathrm{awrk}}^{(\mathrm{R})}\right\|<\mathrm{c} \forall t \geq 0, \quad \lim _{t \rightarrow \infty} \boldsymbol{x}_{\mathrm{awrk}}(t)=\boldsymbol{x}_{\mathrm{awrk}}^{(\mathrm{R})}
$$

gilt.

\subsection{Zusammenfassung und Fazit}

In diesem Kapitel wurden grundlegende Stabilitätsaussagen für verschiedene Ruhelagen des Anti-Windup-Regelkreises hergeleitet. Dabei erfolgte eine Unterscheidung zwischen globaler und lokaler Stabilität. Als besonders wichtiges Ergebnis bleibt festzuhalten, dass sich im Fall stabiler Strecken immer globale Stabilität der Ruhelage des Anti-Windup-Regelkreises erreichen lässt. Dafür ist die Ruhelage eines linearen Modells der Strecke mit einem sättigenden Zustandsregler global asymptotisch zu stabilisieren.

Die angegebenen Stabilitätssätze stellen ein wichtiges Werkzeug für den Entwurf modellbasierter Anti-Windup-Maßnahmen dar und werden in den nächsten Kapiteln verwendet. In Kapitel 7 und 8 kommen die globalen Stabilitätssätze für ein lineares und ein nichtlineares modellbasiertes AntiWindup zum Einsatz. Kapitel 9 nutzt die lokalen Stabilitätsergebnisse für den Entwurf eines linearen modellbasierten Anti-Windup. 


\section{Lineares Anti-Windup für stabile Strecken}

In diesem Kapitel wird ein neues Entwurfsverfahren für lineares, modellbasiertes Anti-Windup vorgestellt. Der Fokus liegt dabei auf einer hohen lokalen Regelgüte und globaler Stabilität. Das Entwurfsverfahren erweitert die bereits in [148] veröffentlichte Grundidee und basiert auf den in Kapitel 6 vorgestellten Stabilitätssätzen.

\subsection{Problembeschreibung}

Im Folgenden wird ein Spezialfall des in Abschnitt 6.1 beschriebenen AntiWindup betrachtet, das eine besonders einfache Struktur aufweist. Die Zustandsrückführung $\boldsymbol{k}_{\text {aw }}$ des modellbasierten Anti-Windup

$$
\mathbf{A W}:\left\{\begin{aligned}
\dot{\boldsymbol{x}}_{\mathrm{aw}} & =\boldsymbol{A}_{\mathrm{s}} \boldsymbol{x}_{\mathrm{aw}}+\boldsymbol{B}_{\mathrm{s}, u} \boldsymbol{k}_{\mathrm{aw}}\left(\boldsymbol{x}_{\mathrm{aw}}\right)+\boldsymbol{B}_{\mathrm{s}, u} \boldsymbol{\delta}, \\
\boldsymbol{y}_{\mathrm{aw}, \mathrm{u}} & =-\boldsymbol{C}_{\mathrm{s}} \boldsymbol{x}_{\mathrm{aw}} \\
\boldsymbol{y}_{\mathrm{aw}, \mathrm{y}} & =-\boldsymbol{k}_{\mathrm{aw}}\left(\boldsymbol{x}_{\mathrm{aw}}\right)
\end{aligned}\right.
$$

besitzt die Gestalt eines linearen Sättigungsreglers, d. h.

$$
\boldsymbol{k}_{\mathrm{aw}}\left(\boldsymbol{x}_{\mathrm{aw}}\right)=\operatorname{sat}_{\alpha \overline{\mathbf{u}}}\left(-\underline{\boldsymbol{K}} \boldsymbol{x}_{\mathrm{aw}}\right) \text {. }
$$

Das so entstehende Anti-Windup wird als lineares modellbasiertes AntiWindup bezeichnet. Offensichtlich weist (7.1) die in Annahme 6.1 vorausgesetzten Eigenschaften auf, so dass alle Sätze aus Kapitel 6 Gültigkeit besitzen. Der Anti-Windup-Entwurf reduziert sich auf die Parametrierung der Matrix $\underline{\boldsymbol{K}} \in \mathbb{R}^{m \times n_{\mathrm{s}}}$ und die Wahl des Skalars $\alpha \in(0,1)$.

Eine vergleichbare Aufgabenstellung liegt den Arbeiten [55, 80, 173, 174, 182, 191, 209, 211] zugrunde, die den Entwurf der linearen Zustandsrückführung als Optimierungsproblem formulieren. Dabei tritt ein Zielkonflikt zwischen einer hohen Regelgüte, einem großen Stabilitätsgebiet und der Konvexität des Optimierungsproblems auf, wie Abbildung 7.1 illustriert. 


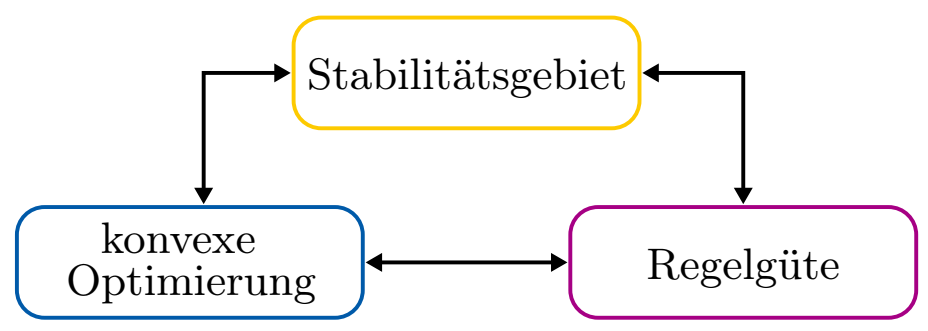

Abbildung 7.1: Zielkonflikt zwischen einem möglichst großen Stabilitätsgebiet, einer hohen Regelgüte und der Konvexität des Optimierungsproblems beim Entwurf von linearen Anti-Windup-Maßnahmen.

Der Grund für den Zielkonflikt ist, dass konvexe Optimierungsprobleme für lineares modellbasiertes Anti-Windup auf quadratischen LjapunovFunktionen basieren. Vor allem wenn eine stabile Strecke $\mathbf{S}$ vorliegt und globale Stabilität des Anti-Windup-Regelkreises beim Entwurf garantiert werden soll, ist dieser Ansatz konservativ. Das bedeutet, viele Matrizen $\underline{\boldsymbol{K}}$, die globale Stabilität und eine hohe Regelgüte garantieren, sind nicht Teil der zulässigen Menge ${ }^{1}$ des Optimierungsproblems.

Für viele dieser Anti-Windup-Matrizen lässt sich trotzdem die globale Stabilität der Ruhelage des Regelkreises beweisen. So wurden Verfahren zur Analyse der Stabilität von Regelkreisen entwickelt, die auf komplexeren Ljapunov-Funktionen basieren, wie das Popov-Kriterium, das Kriterium der nichtaxialen Kreise [141, 166, 167] oder zahlreiche andere Verfahren [75, 94, 99, 100, 119, 154, 192, 193]. Mit dem Stabilitätssatz von Lee aus [119] wurde beispielsweise in Kapitel 5 der Stabilitätsnachweis für das hydraulische Positioniersystem erbracht. Mit quadratischen LjapunovFunktionen gelingt dies nicht.

Trotzdem basieren alle bekannten Entwurfsverfahren für lineares modellbasiertes Anti-Windup [55, 80, 173, 174, 191, 208, 211], die globale Stabilität garantieren, auf quadratischen Ljapunov-Funktionen. Die sichere Lösbarkeit des konvexen Optimierungsproblems wird gegenüber einer höheren lokalen Regelgüte priorisiert ${ }^{2}$. Der zu zahlende Preis ist eine in vielen Fällen starke Abweichung des Anti-Windup-Regelkreises von dem unbeschränkten Verhalten, wie auch in [191] erläutert wird.

${ }^{1}$ Die Menge aller $\underline{\boldsymbol{K}}$, die sämtliche Nebenbedingungen des Optimierungsproblems erfüllen, bezeichnet man als zulässige Menge (siehe Anhang B).

${ }^{2}$ Diese Aussage trifft, bis auf eine Ausnahme, auch auf das allgemeine lineare AntiWindup aus Abschnitt 4.3 zu. In [187] wird auf Kosten der Konvexität des Optimierungsproblems ein Entwurfsverfahren vorgestellt, das globale Stabilität sowie eine hohe Regelgüte garantiert und auf der Stabilitätstheorie [99, 100] basiert. 
Um eine höhere Regelgüte zu erzielen und die Konvexität des Optimierungsproblems zu erhalten, wird in [55, 95, 175, 182, 191, 211] die Forderung nach globaler Stabilität fallen gelassen. Mit lokalen Gütekriterien, wie zum Beispiel der $L_{2}$-Kleinsignalverstärkung, kann dann eine hohe lokale Regelgüte erzielt werden. Nachteilig ist, dass die Ruhelage des Regelkreises nur lokal stabil und die Abschätzung eines Einzugsgebietes aufgrund der entkoppelten Struktur des Mismatch-Systems schwierig ist.

Die beschriebenen Vorgehensweisen garantieren somit entweder globale Stabilität auf Kosten der Regelgüte oder eine hohe Regelgüte mit lokaler Stabilitätsgarantie. Das Ziel dieses Kapitels ist die Entwicklung einer Entwurfsmethodik, die eine hohe lokale Regelgüte und globale Stabilität des Anti-Windup-Regelkreises garantiert. Der zu zahlende Preis ist ein Optimierungsproblem, das nicht konvex ist, für dessen lokale Lösung aber ein einfach anzuwendender Algorithmus angegeben wird.

\subsection{Entwurfsbedingungen}

In diesem Abschnitt werden zunächst Bedingungen an die Matrix $\underline{\boldsymbol{K}}$ und den Parameter $\alpha$ hergeleitet, die globale Stabilität und eine hohe lokale Regelgüte des Anti-Windup-Regelkreises garantieren. Anschließend erfolgt die Formulierung des Entwurfs als Optimierungsproblem. Für eine kompaktere Notation wird der in diesem Kapitel verwendete Begriff AntiWindup-Regelkreis in der folgenden Anmerkung detailliert beschrieben.

Anmerkung 7.1 (Anti-Windup-Regelkreis). Der Anti-Windup-Regelkreis besteht aus der Strecke $\mathbf{S}$, dem Regler $\mathbf{R}$ und einem linearen modellbasierten Anti-Windup

$$
\mathbf{A W}:\left\{\begin{array}{l}
\dot{\boldsymbol{x}}_{\mathrm{aw}}=\boldsymbol{A}_{\mathrm{s}} \boldsymbol{x}_{\mathrm{aw}}+\boldsymbol{B}_{\mathrm{s}, u} \mathbf{s a t}_{\alpha \overline{\mathbf{u}}}\left(-\underline{\boldsymbol{K}} \boldsymbol{x}_{\mathrm{aw}}\right)+\boldsymbol{B}_{\mathrm{s}, u} \boldsymbol{\delta}, \\
\boldsymbol{y}_{\mathrm{aw}, \mathrm{u}}=-\boldsymbol{C}_{\mathrm{s}} \boldsymbol{x}_{\mathrm{aw}} \\
\boldsymbol{y}_{\mathrm{aw}, \mathrm{y}}=-\mathbf{s a t}_{\alpha \overline{\mathbf{u}}}\left(-\underline{\boldsymbol{K}} \boldsymbol{x}_{\mathrm{aw}}\right) .
\end{array}\right.
$$

Die Stellbegrenzung des Aktors ist durch $\overline{\mathbf{u}}=\left[\overline{\mathrm{u}}_{1} \ldots \overline{\mathrm{u}}_{m}\right]^{\top}$ charakterisiert. Die Verschaltung der Teilsysteme gemäß Abbildung 6.1 führt mit dem Zustandsvektor $\boldsymbol{x}_{\mathrm{awrk}}^{\top}=\left[\begin{array}{lll}\boldsymbol{x}_{\mathrm{s}}^{\top} & \boldsymbol{x}_{\mathrm{r}}^{\top} & \boldsymbol{x}_{\mathrm{aw}}^{\top}\end{array}\right]$ zu der Systemdynamik

$$
\begin{aligned}
& \dot{\boldsymbol{x}}_{\mathrm{awrk}}=\tilde{\boldsymbol{A}} \boldsymbol{x}_{\mathrm{awrk}}+\tilde{\boldsymbol{B}}_{\mathrm{u}} \mathbf{s a t}_{\overline{\mathbf{u}}}\left(\boldsymbol{u}_{\mathrm{awrk}}\right)+\tilde{\boldsymbol{B}}_{\mathrm{w}} \boldsymbol{z}+\tilde{\boldsymbol{B}}_{\mathrm{z}} \boldsymbol{w}, \\
& \boldsymbol{u}_{\mathrm{awrk}}=\boldsymbol{C}_{\mathrm{r}} \boldsymbol{x}_{\mathrm{r}}+\boldsymbol{D}_{\mathrm{r}, u} \boldsymbol{C}_{\mathrm{s}}\left(\boldsymbol{x}_{\mathrm{s}}+\boldsymbol{x}_{\mathrm{aw}}\right)-\mathbf{s a t}_{\alpha \overline{\mathbf{u}}}\left(-\underline{\boldsymbol{K}} \boldsymbol{x}_{\mathrm{aw}}\right)+\boldsymbol{D}_{\mathrm{r}, w} \boldsymbol{w}
\end{aligned}
$$

des Anti-Windup-Regelkreises. Dabei ist $\boldsymbol{w}$ die Sollgröße und $\boldsymbol{z}$ die auf die Strecke wirkende Störgröße. Die Systemmatrizen $\tilde{\boldsymbol{A}}, \tilde{\boldsymbol{B}}_{\mathrm{u}}, \tilde{\boldsymbol{B}}_{\mathrm{w}}$, und $\tilde{\boldsymbol{B}}_{\mathrm{z}}$ sind in Abschnitt A.3.2 des Anhangs gegeben. 


\subsubsection{Bedingungen für globale Stabilität}

Bedingungen für die Stabilität des Anti-Windup-Regelkreises wurden in Kapitel 6 in einem allgemeineren Rahmen ausführlich diskutiert. Entscheidend dabei war die Darstellung des Regelkreises in Mismatch-Koordinaten und das geschickte Ausnutzen der Kaskadenstruktur.

Übertragen auf den hier betrachteten Spezialfall lautet die wesentliche Stabilitätsbedingung wie folgt. Die globale Stabilität zulässiger Ruhelagen (vgl. Definition 6.2) des Anti-Windup-Regelkreises hängt nach Satz 6.6 davon ab, ob die Ruhelage $\boldsymbol{\xi}_{\mathrm{aw}}^{(\mathrm{R})}=\mathbf{0}$ des autonomen Ersatzsystems

$$
\text { ES : }\left\{\begin{aligned}
\dot{\boldsymbol{\xi}}_{\mathrm{aw}} & =\boldsymbol{A}_{\mathrm{s}} \boldsymbol{\xi}_{\mathrm{aw}}+\boldsymbol{B}_{\mathrm{s}, u} \mathbf{s a t}_{\alpha \overline{\mathbf{u}}}\left(-\underline{\boldsymbol{K}} \boldsymbol{\xi}_{\mathrm{aw}}\right), \\
\boldsymbol{y}_{\mathrm{aw}, \mathrm{u}} & =\boldsymbol{C}_{\mathrm{s}} \boldsymbol{\xi}_{\mathrm{aw}}
\end{aligned}\right.
$$

global asymptotisch stabil ist. Details präzisiert das unmittelbar aus dem Satz 6.6 folgende

Lemma 7.1. Gegeben sei der Anti-Windup-Regelkreis gemäß Anmerkung 7.1. Des Weiteren sei $\boldsymbol{x}_{\mathrm{awrk}}(0)=\boldsymbol{x}_{\mathrm{awrk}}^{(0)} \in \mathbb{R}^{2 n_{\mathrm{s}}+n_{\mathrm{r}}}$ der Anfangszustand und $\boldsymbol{x}_{\mathrm{awrk}}^{(\mathrm{R})}$ eine gemäß Definition 6.2 zulässige Ruhelage des Anti-WindupRegelkreises mit einer zugehörigen stationären Stellgröße $\boldsymbol{y}_{\mathrm{r}}^{(\mathrm{R})}$. Wenn der Anti-Windup-Parameter $\alpha$ aus dem offenen Intervall $(0, \hat{\alpha})$ mit

$$
\hat{\alpha}=1-\max _{i \in\{1, \ldots, m\}}\left\{\left|y_{\mathrm{r}, i}^{(\mathrm{R})}\right| \bar{u}_{i}^{-1}\right\}
$$

gewählt wird und die Ruhelage $\boldsymbol{\xi}_{\mathrm{aw}}^{(\mathrm{R})}=\mathbf{0}$ des autonomen Ersatzsystems

$$
\dot{\boldsymbol{\xi}}_{\mathrm{aw}}=\boldsymbol{A}_{\mathrm{s}} \boldsymbol{\xi}_{\text {aw }}+\boldsymbol{B}_{\mathrm{s}, u} \boldsymbol{s} \boldsymbol{a} \boldsymbol{t}_{\alpha \overline{\boldsymbol{u}}}\left(-\underline{\boldsymbol{K}} \boldsymbol{\xi}_{\mathrm{aw}}\right)
$$

global asymptotisch stabil ist, dann existiert ein c $>0$, so dass für die Trajektorien des Anti-Windup-Regelkreises gilt

$$
\left\|\boldsymbol{x}_{\mathrm{awrk}}(t)-\boldsymbol{x}_{\mathrm{awrk}}^{(\mathrm{R})}\right\|<c \forall t \geq 0, \quad \lim _{t \rightarrow \infty} \boldsymbol{x}_{\mathrm{awrk}}(t)=\boldsymbol{x}_{\mathrm{awrk}}^{(\mathrm{R})} .
$$

Beweis. Das Lemma folgt direkt aus Satz 6.6.

Für den Entwurf ist folglich nur das Anti-Windup-Ersatzsystem (7.3) zu betrachten. Gelingt es, durch eine geeignete Wahl von $\underline{\boldsymbol{K}}$, den Ursprung dieses Systems mit der zur Verfügung stehenden Stellgröße $\alpha \overline{\mathbf{u}}$ global zu stabilisieren, dann ist auch die Stabilität der Ruhelage $\boldsymbol{x}_{\mathrm{awrk}}^{(\mathrm{R})}$ des AntiWindup-Regelkreises gewährleistet. Entscheidend ist deshalb, möglichst alle stabilisierenden Matrizen $\underline{\boldsymbol{K}}$ durch entsprechende Nebenbedingungen zu charakterisieren. Die Auswahl erfolgt danach durch die Wahl eines Gütekriteriums und das Lösen eines Optimierungsproblems. 


\section{Charakterisierung stabilisierender Matrizen}

Wie in Abschnitt 7.1 erwähnt, nutzen viele Entwurfsverfahren quadratische Ljapunov-Funktionen, um eine Menge stabilisierender Matrizen $\underline{\boldsymbol{K}} \mathrm{zu}$ charakterisieren. Das ist jedoch konservativ, denn es werden viele Matrizen $\underline{\boldsymbol{K}}$ ausgeschlossen, obwohl sie zu einem stabilen Regelkreis führen.

Das hier vorgestellte neue Entwurfsverfahren basiert auf dem weniger konservativen Stabilitätssatz von Lee [119], der nichtquadratische Ljapunov-Funktionen verwendet. Für die Charakterisierung der stabilisierenden Matrizen $\underline{\boldsymbol{K}}$ wird erneut die Menge $\mathcal{M}(m)$ aller Diagonalmatrizen benötigt, deren Diagonalelemente entweder null oder eins sind, d. h.

$$
\mathcal{M}(m):=\left\{\operatorname{diag}\left(\nu_{1}, \ldots, \nu_{2 m}\right) \in \mathbb{S}^{2 m} \mid \nu_{i} \in\{0,1\}\right\} .
$$

Um eine kompakte Notation zu erreichen werden im Folgenden vier Matrizen $\boldsymbol{A}_{*} \in \mathbb{R}^{(n+m) \times(n+m)}, \boldsymbol{B}_{*} \in \mathbb{R}^{(n+m) \times m}, \boldsymbol{C}_{*} \in \mathbb{R}^{4 m \times(n+m)}$ und $\boldsymbol{D}_{*} \in \mathbb{R}^{4 m \times m}$ benötigt. Diese sind durch

$$
\begin{array}{lll}
\boldsymbol{A}_{*}=\left[\begin{array}{cc}
\boldsymbol{A}_{\mathrm{s}} & \boldsymbol{B}_{\mathrm{s}, u} \\
\mathbf{0} & \mathbf{0}
\end{array}\right], & \boldsymbol{B}_{*}=\left[\begin{array}{l}
\mathbf{0} \\
\mathbb{I}
\end{array}\right], \\
\boldsymbol{C}_{*}=\left[\begin{array}{cc}
-\underline{\boldsymbol{K}} & \mathbf{0} \\
-\underline{\boldsymbol{K}} \boldsymbol{A}_{\mathrm{s}} & -\underline{\boldsymbol{K}} \boldsymbol{B}_{\mathrm{s}, u} \\
\mathbf{0} & \mathbb{I} \\
\mathbf{0} & \mathbf{0}
\end{array}\right], & \boldsymbol{D}_{*}=\left[\begin{array}{l}
\mathbf{0} \\
\mathbf{0} \\
\mathbf{0} \\
\mathbb{I}
\end{array}\right]
\end{array}
$$

gegeben. Nun lässt sich das nachfolgende Lemma formulieren.

Lemma 7.2 (Globale Stabilität des Anti-Windup-Regelkreises). Gegeben sei der Anti-Windup-Regelkreis gemäß Anmerkung 7.1. Des Weiteren sei $\boldsymbol{x}_{\mathrm{awrk}}(0)=\boldsymbol{x}_{\mathrm{awrk}}^{(0)} \in \mathbb{R}^{2 n_{\mathrm{s}}+n_{\mathrm{r}}}$ der Anfangszustand und $\boldsymbol{x}_{\mathrm{awrk}}^{(\mathrm{R})}$ eine gemäß Definition 6.2 zulässige Ruhelage des Anti-Windup-Regelkreises mit einer zugehörigen stationären Stellgröße $\boldsymbol{y}_{\mathrm{r}}^{(\mathrm{R})}$. Wenn der Anti-WindupParameter $\alpha$ aus dem offenen Intervall $(0, \hat{\alpha})$ mit

$$
\hat{\alpha}=1-\max _{i \in\{1, \ldots, m\}}\left\{\left|y_{\mathrm{r}, i}^{(\mathrm{R})}\right| \bar{u}_{i}^{-1}\right\}
$$

gewählt wird und zwei symmetrische, positiv definite Matrizen $\boldsymbol{R}_{\mathrm{g}} \in \mathbb{S}^{n+m}$, $\Theta \in \mathbb{S}^{4 m}$ existieren, so dass die Matrixungleichungen

$$
\begin{aligned}
\mathrm{He}\left[\begin{array}{cc}
\boldsymbol{A}_{*}^{\top} \boldsymbol{R}_{\mathrm{g}}+\frac{1}{2} \boldsymbol{C}_{*}^{\top} \boldsymbol{\Theta} \boldsymbol{C}_{*} & \mathbf{0} \\
\boldsymbol{D}_{*}^{\top} \boldsymbol{\Theta} \boldsymbol{C}_{*}+\boldsymbol{B}_{*}^{\top} \boldsymbol{R}_{\mathrm{g}} & \frac{1}{2} \boldsymbol{D}_{*}^{\top} \boldsymbol{\Theta} \boldsymbol{D}_{*}
\end{array}\right] & \prec \mathbf{0}, \\
{\left[\begin{array}{ll}
\mathbb{I} & \boldsymbol{M}_{i}
\end{array}\right] \boldsymbol{\Theta}\left[\begin{array}{c}
\mathbb{I} \\
\boldsymbol{M}_{i}
\end{array}\right] } & \succeq \mathbf{0} \forall i=1, \ldots, 4^{m}, \quad \boldsymbol{M}_{i} \in \mathcal{M}(m)
\end{aligned}
$$


mit der Menge $\mathcal{M}(m)$ gemäß (7.4) sowie den Abkürzungen (7.5) erfüllt sind, dann existiert ein $\mathrm{c}>0$, so dass für die Trajektorien des AntiWindup-Regelkreises gilt

$$
\left\|\boldsymbol{x}_{\text {awrk }}(t)-\boldsymbol{x}_{\text {awrk }}^{(\mathrm{R})}\right\|<\mathrm{c} \forall t \geq 0, \quad \lim _{t \rightarrow \infty} \boldsymbol{x}_{\text {awrk }}(t)=\boldsymbol{x}_{\text {awrk }}^{(\mathrm{R})} .
$$

Beweis. Die angegebenen Matrixungleichungen garantieren, dass die Ruhelage $\boldsymbol{\xi}_{\mathrm{aw}}^{(\mathrm{R})}=\mathbf{0}$ des Anti-Windup-Ersatzsystems (7.3) global asymptotisch stabil ist. Der Beweis basiert auf einer Ljapunov-Funktion der Form

$$
V(\boldsymbol{\xi})=\boldsymbol{\xi}^{\top} \boldsymbol{R} \boldsymbol{\xi}, \quad \boldsymbol{R} \succ \mathbf{0},
$$

mit dem erweiterten Zustandsvektor $\boldsymbol{\xi}^{\top}=\left[\boldsymbol{\xi}_{\mathrm{aw}}^{\top} \boldsymbol{s a t}_{\overline{\mathbf{u}}}\left(\boldsymbol{C}_{\mathrm{s}} \boldsymbol{\xi}_{\mathrm{aw}}\right)^{\top}\right]$. Er findet sich in [119] (vgl. Beweis von Lemma 5.1). Aus Lemma 7.1 folgt die Stabilität der Ruhelage des Anti-Windup-Regelkreises.

Nachdem eine Menge an stabilisierenden Matrizen $\underline{\boldsymbol{K}}$ mit einer weniger konservativen nichtquadratischen Ljapunov-Funktion charakterisiert ist, erfolgt im nächsten Abschnitt die Wahl des Gütekriteriums.

\subsubsection{Bedingungen für Regelgüte}

Die Regelgüte für ein gegebenes $\underline{\boldsymbol{K}}$ wird mittels der $L_{2}$-Verstärkung des Anti-Windup-Ersatzsystems

$$
\text { ES: }\left\{\begin{aligned}
\dot{\boldsymbol{\xi}}_{\mathrm{aw}} & =\boldsymbol{A}_{\mathrm{s}} \boldsymbol{\xi}_{\mathrm{aw}}+\boldsymbol{B}_{\mathrm{s}, u} \mathbf{s a t}_{\alpha \overline{\mathbf{u}}}\left(-\underline{\boldsymbol{K}} \boldsymbol{\xi}_{\mathrm{aw}}\right)+\boldsymbol{B}_{\mathrm{s}, u} \boldsymbol{\boldsymbol { y } _ { \mathrm { aw } , \mathrm { u } }}=\boldsymbol{C}_{\mathrm{s}} \boldsymbol{\xi}_{\mathrm{aw}}
\end{aligned}\right.
$$

vom Störeingang $\boldsymbol{\zeta}$ zum Ausgang $\boldsymbol{y}_{\mathrm{aw}, \mathrm{u}}$ bewertet, der die Abweichung vom unbeschränkten Verhalten darstellt.

Wie in Kapitel 5 diskutiert, lässt sich für kleine Störungen eine präzisere Aussage über die Regelgüte treffen, wenn die $L_{2}$-Kleinsignalverstärkung betrachtet wird. In Abschnitt 5.3.3 wurde dazu die lokale Sektorzugehörigkeit der Totzone ausgenutzt. Um diese Ergebnisse auch hier verwenden zu können, ist eine Umformung des Ersatzsystems notwendig. Aufgrund des Zusammenhangs

$$
\operatorname{sat}_{\alpha \overline{\mathbf{u}}}\left(-\underline{\boldsymbol{K}} \boldsymbol{\xi}_{\mathrm{aw}}\right)+\mathrm{dz} \mathrm{z}_{\alpha \overline{\mathbf{u}}}\left(-\underline{\boldsymbol{K}} \boldsymbol{\xi}_{\mathrm{aw}}\right)=-\underline{\boldsymbol{K}} \boldsymbol{\xi}_{\text {aw }}
$$

ergibt sich aus (7.6) das äquivalente Anti-Windup-Ersatzsystem

$$
\begin{aligned}
& \dot{\boldsymbol{\xi}}_{\text {aw }}=\left(\boldsymbol{A}_{\mathrm{s}}-\boldsymbol{B}_{\mathrm{s}, u} \underline{\boldsymbol{K}}\right) \boldsymbol{\xi}_{\mathrm{aw}}+\boldsymbol{B}_{\mathrm{s}, u} \mathrm{dz}_{\alpha \overline{\mathbf{u}}}\left(\underline{\boldsymbol{K}} \xi_{\mathrm{aw}}\right)+\boldsymbol{B}_{\mathrm{s}, u} \boldsymbol{\zeta}, \\
& \boldsymbol{y}_{\mathrm{aw}, u}=\boldsymbol{C}_{\mathrm{s}} \boldsymbol{\xi}_{\mathrm{aw}}
\end{aligned}
$$


mit einer Totzone, die global im Sektor $\mathcal{S}(\mathbf{0}, \mathbb{I})$ liegt.

Für ausreichend kleine Werte des Totzonenarguments $\tilde{\boldsymbol{u}}=\underline{\boldsymbol{K}} \boldsymbol{\xi}_{\text {aw }}$ liegt die Totzone in einem schmaleren Sektor $\mathcal{S}(\mathbf{0}, \boldsymbol{\Phi}) \operatorname{mit} \boldsymbol{\Phi}=\operatorname{diag}\left(\phi_{1}, \ldots, \phi_{m}\right)$ und erfüllt die lokale Sektorbedingung aus Lemma 5.4, wie Abbildung 5.6 illustriert. Für einen Sektor $\mathcal{S}(\mathbf{0}, \boldsymbol{\Phi})$ lassen sich diese Signale $\tilde{\boldsymbol{u}}(t)$ in der Signalklasse

$$
\begin{aligned}
\mathcal{W}(\boldsymbol{\Phi})=\left\{\tilde{\boldsymbol{u}}: \mathbb{R}_{\geq 0} \mapsto \mathbb{R}^{m}|| \tilde{u}_{i} \mid \leq \hat{u}_{i}=\frac{\alpha \overline{\mathrm{u}}_{i}}{1-\phi_{i}}\right. & \\
& \forall i=1, \ldots, m, \forall t \geq 0\}
\end{aligned}
$$

zusammenfassen. Eine Abschätzung der $L_{2}$-Verstärkung des Systems (7.7), die auf der lokalen Sektorbedingung basiert, ist deshalb nur für die Eingangssignale $\boldsymbol{\zeta}$ gültig, die zu Signalen $\tilde{\boldsymbol{u}} \in \mathcal{W}(\boldsymbol{\Phi})$ führen. Kompakt lassen sich diese Eingangssignale in der Signalklasse

$$
\mathcal{Y}(\boldsymbol{\Phi})=\left\{\boldsymbol{\zeta}: \mathbb{R}_{\geq 0} \mapsto \mathbb{R}^{m} \mid \tilde{\boldsymbol{u}}(t) \in \mathcal{W}(\boldsymbol{\Phi}) \quad \forall t \leq 0\right\}
$$

zusammenfassen. Für die Eingangssignalklasse $\mathcal{Y}(\boldsymbol{\Phi})$ kann die $L_{2}$-Kleinsignalverstärkung des Ersatzsystems gemäß Definition 5.3 abgeschätzt werden. Details erläutert

Lemma 7.3 ( $L_{2}$-Kleinsignalverstärkung). Gegeben sei eine positiv definite Diagonalmatrix $\boldsymbol{\Phi}=\operatorname{diag}\left(\phi_{1}, \ldots, \phi_{m}\right)$ und das Anti-WindupErsatzsystem (7.7) mit $\boldsymbol{\xi}_{\text {aw }} \in \mathbb{R}^{n}$ sowie $\boldsymbol{\zeta}$, $\boldsymbol{y}_{\text {aw,u }} \in \mathbb{R}^{m}$ und $\underline{\boldsymbol{K}} \in \mathbb{R}^{m \times n}$. Wenn eine quadratische Matrix $\boldsymbol{Q} \in \mathbb{R}^{n \times n}$ und eine Diagonalmatrix $\boldsymbol{\Psi} \in \mathbb{R}^{m \times m}$ mit den Eigenschaften

$$
\boldsymbol{Q} \succ \mathbf{0}, \quad \boldsymbol{\Psi}=\operatorname{diag}\left(\psi_{1}, \ldots, \psi_{m}\right) \succ \mathbf{0}
$$

sowie ein positiver Skalar $\gamma_{\mathcal{y}}$ existieren, so dass die Matrixungleichung

$$
\operatorname{He}\left[\begin{array}{crcc}
\boldsymbol{A}_{\mathrm{s}} \boldsymbol{Q}-\boldsymbol{B}_{\mathrm{s}, u} \underline{\boldsymbol{K}} \boldsymbol{Q} & \mathbf{0} & \mathbf{0} & \mathbf{0} \\
\boldsymbol{\Psi} \boldsymbol{B}_{\mathrm{s}, u}^{\top}+\boldsymbol{\Phi} \underline{\boldsymbol{K}} \boldsymbol{Q} & -\boldsymbol{\Psi} & \mathbf{0} & \mathbf{0} \\
\boldsymbol{B}_{\mathrm{s}, u}^{\top} & \mathbf{0} & -\frac{1}{2} \gamma_{\mathcal{Y}} \mathbb{I} & \mathbf{0} \\
\boldsymbol{C}_{\mathrm{s}} \boldsymbol{Q} & \mathbf{0} & \mathbf{0} & -\frac{1}{2} \gamma_{\mathcal{\nu}} \mathbb{I}
\end{array}\right] \prec \mathbf{0}
$$

erfüllt ist, dann ist die Ruhelage $\boldsymbol{\xi}_{\mathrm{aw}}^{(0)}=\mathbf{0}$ des Systems (7.7) für $\boldsymbol{\zeta} \equiv \mathbf{0}$ lokal asymptotisch stabil. Des Weiteren ist die $L_{2}$-Kleinsignalverstärkung des Systems vom Eingang $\boldsymbol{\zeta}$ zum Ausgang $\boldsymbol{y}_{\text {aw,u }}$ für Signale mit endlicher $L_{2}$-Norm aus der Signalklasse $\mathcal{Y}(\boldsymbol{\Phi})$ kleiner gleich $\gamma_{\mathcal{y}}$. 
Beweis. Der Beweis findet sich in Abschnitt C.3.11.

Für eine gegebene Eingangssignalklasse $\mathcal{Y}(\boldsymbol{\Phi})$ lässt sich mithilfe des angegebenen Lemmas die $L_{2}$-Kleinsignalverstärkung des Systems (7.7) abschätzen. Ein Sektor $\mathcal{S}(\mathbf{0}, \boldsymbol{\Phi})$ mit $\boldsymbol{\Phi}=\mathbb{I}$ schließt die Totzone komplett ein. Dann garantiert das Lemma globale Stabilität des Systems (7.7) und $\gamma_{\mathcal{y}}$ ist eine obere Schranke für die $L_{2}$-Verstärkung. Eine Kombination des Stabilitätslemmas und Lemma 7.3 führt zu dem folgenden Satz, der die Entwurfsbedingungen für die Anti-Windup-Matrix $\underline{\boldsymbol{K}}$ angibt.

Satz 7.1 (Entwurfsbedingungen). Gegeben sei eine positiv definite Diagonalmatrix $\boldsymbol{\Phi}=\operatorname{diag}\left(\phi_{1}, \ldots, \phi_{m}\right)$ und der Anti-Windup-Regelkreis gemäß Anmerkung 7.1. Des Weiteren sei $\boldsymbol{x}_{\mathrm{awrk}}(0)=\boldsymbol{x}_{\mathrm{awrk}}^{(0)} \in \mathbb{R}^{2 n_{\mathrm{s}}+n_{\mathrm{r}}} \operatorname{der} A n$ fangszustand und $\boldsymbol{x}_{\mathrm{awrk}}^{(\mathrm{R})}$ eine gemäß Definition 6.2 zulässige Ruhelage des Regelkreises mit einer zugehörigen stationären Stellgröße $\boldsymbol{y}_{\mathrm{r}}^{(\mathrm{R})}$. Der AntiWindup-Parameter $\alpha$ sei aus dem offenen Intervall $(0, \hat{\alpha})$ mit

$$
\hat{\alpha}=1-\max _{i \in\{1, \ldots, m\}}\left\{\left|y_{\mathrm{r}, i}^{(\mathrm{R})}\right| \bar{u}_{i}^{-1}\right\}
$$

gewählt. Wenn drei symmetrische, positiv definite Matrizen $\boldsymbol{R}_{\mathrm{g}} \in \mathbb{S}^{n+m}$, $\boldsymbol{\Theta} \in \mathbb{S}^{4 m}, \boldsymbol{Q} \in \mathbb{S}^{n}$, eine Diagonalmatrix

$$
\mathbf{\Psi}=\operatorname{diag}\left(\psi_{1}, \ldots, \psi_{m}\right) \succ \mathbf{0}
$$

und ein positiver Skalar $\gamma_{\mathcal{y}}$ existieren, so dass die Matrixungleichungen

$$
\begin{aligned}
& \mathrm{He}\left[\begin{array}{cc}
\boldsymbol{A}_{*}^{\top} \boldsymbol{R}_{\mathrm{g}}+\frac{1}{2} \boldsymbol{C}_{*}^{\mathrm{\top}} \boldsymbol{\Theta} \boldsymbol{C}_{*} & \mathbf{0} \\
\boldsymbol{D}_{*}^{\top} \boldsymbol{\Theta} \boldsymbol{C}_{*}+\boldsymbol{B}_{*}^{\top} \boldsymbol{R}_{\mathrm{g}} & \frac{1}{2} \boldsymbol{D}_{*}^{\top} \boldsymbol{\Theta} \boldsymbol{D}_{*}
\end{array}\right] \prec \mathbf{0}, \\
& {\left[\begin{array}{ll}
\mathbb{I} & M_{i}
\end{array}\right] \Theta\left[\begin{array}{c}
\mathbb{I} \\
M_{i}
\end{array}\right] \succeq \mathbf{0},} \\
& \mathrm{He}\left[\begin{array}{crcc}
\boldsymbol{A}_{\mathrm{s}} \boldsymbol{Q}-\boldsymbol{B}_{\mathrm{s}, u} \underline{\boldsymbol{K}} \boldsymbol{Q} & \mathbf{0} & \mathbf{0} & \mathbf{0} \\
\boldsymbol{\Psi} \boldsymbol{B}_{\mathrm{s}, u}^{\mathrm{T}}+\boldsymbol{\Phi} \underline{\boldsymbol{K}} \boldsymbol{Q} & -\boldsymbol{\Psi} & \mathbf{0} & \mathbf{0} \\
\boldsymbol{B}_{\mathrm{s}, u}^{\top} & \mathbf{0} & -\frac{1}{2} \gamma_{\mathcal{Y}} \mathbb{I} & \mathbf{0} \\
\boldsymbol{C}_{\mathrm{s}} \boldsymbol{Q} & \mathbf{0} & \mathbf{0} & -\frac{1}{2} \gamma_{\mathcal{Y}} \mathbb{I}
\end{array}\right] \prec \mathbf{0}
\end{aligned}
$$

für alle $\boldsymbol{M}_{i} \in \mathcal{M}_{m}$ mit der Menge $\mathcal{M}$ gemäß (7.4) und den Abkürzungen (7.5) erfüllt sind, dann

1. existiert ein c $>0$, so dass für die Trajektorien des Anti-WindupRegelkreises gilt

$$
\left\|\boldsymbol{x}_{\mathrm{awrk}}(t)-\boldsymbol{x}_{\mathrm{awrk}}^{(\mathrm{R})}\right\|<\mathrm{c} \forall t \geq 0, \quad \lim _{t \rightarrow \infty} \boldsymbol{x}_{\mathrm{awrk}}(t)=\boldsymbol{x}_{\mathrm{awrk}}^{(\mathrm{R})},
$$


2. ist die $L_{2}$-Kleinsignalverstärkung des Systems (7.7) vom Eingang $\boldsymbol{\zeta}$ zum Ausgang $\boldsymbol{y}_{\text {aw,u }}$ für Signale mit endlicher $L_{2}$-Norm aus der Signalklasse $\mathcal{Y}(\boldsymbol{\Phi})$ kleiner gleich $\gamma_{\mathcal{\nu}}$.

Beweis. Der Satz ergibt sich durch eine Kombination von Lemma 7.2 und Lemma 7.3.

Der Satz 7.1 kann genutzt werden, um für eine gegebene Matrix $\underline{\boldsymbol{K}}$ die globale Stabilität des Anti-Windup-Regelkreises zu überprüfen und für eine gegebene Eingangssignalklasse $\mathcal{Y}(\boldsymbol{\Phi})$ eine obere Schranke der $L_{2^{-}}$ Kleinsignalverstärkung abzuschätzen. Dazu ist das folgende konvexe Optimierungsproblem zu lösen.

Optimierungsproblem 7.1. Gegeben seien die Matrizen $\underline{K}$ und

$$
\boldsymbol{\Phi}=\operatorname{diag}\left(\phi_{1}, \ldots, \phi_{m}\right) \succ \mathbf{0}
$$

sowie die Abkürzungen $\boldsymbol{A}_{*}, \boldsymbol{B}_{*}, \boldsymbol{C}_{*}$ und $\boldsymbol{D}_{*}$ gemäß (7.5).

$$
\begin{aligned}
& \underset{\boldsymbol{R}_{\mathrm{g}}, \boldsymbol{\Theta}, \boldsymbol{Q}, \boldsymbol{\Psi}, \gamma_{\mathcal{\nu}}}{\operatorname{Minimiere}} \gamma_{\boldsymbol{\nu}} \quad \text { unter den Nebenbedingungen } \\
& \boldsymbol{R}_{\mathrm{g}} \succ \mathbf{0}, \boldsymbol{\Theta} \succ \mathbf{0}, \boldsymbol{Q} \succ \mathbf{0}, \boldsymbol{\Psi}=\operatorname{diag}\left(\psi_{1}, \ldots, \psi_{m}\right) \succ \mathbf{0}, \gamma_{\mathcal{y}}>0, \\
& \mathrm{He}\left[\begin{array}{cc}
\boldsymbol{A}_{*}^{\top} \boldsymbol{R}_{\mathrm{g}}+\frac{1}{2} \boldsymbol{C}_{*}^{\mathrm{\top}} \boldsymbol{\Theta} \boldsymbol{C}_{*} & \mathbf{0} \\
\boldsymbol{D}_{*}^{\top} \boldsymbol{\Theta} \boldsymbol{C}_{*}+\boldsymbol{B}_{*}^{\top} \boldsymbol{R}_{\mathrm{g}} & \frac{1}{2} \boldsymbol{D}_{*}^{\top} \boldsymbol{\Theta} \boldsymbol{D}_{*}
\end{array}\right] \prec \mathbf{0}, \\
& {\left[\begin{array}{ll}
\mathbb{I} & \boldsymbol{M}_{i}
\end{array}\right] \boldsymbol{\Theta}\left[\begin{array}{c}
\mathbb{I} \\
\boldsymbol{M}_{i}
\end{array}\right] \succeq \mathbf{0} \quad \forall \boldsymbol{M}_{i} \in \mathcal{M}_{m},} \\
& \mathrm{He}\left[\begin{array}{crcc}
\left(\boldsymbol{A}_{\mathrm{s}}-\boldsymbol{B}_{\mathrm{s}, u} \underline{\boldsymbol{K}}\right) \boldsymbol{Q} & \mathbf{0} & \mathbf{0} & \mathbf{0} \\
\boldsymbol{\Psi} \boldsymbol{B}_{\mathrm{s}, u}^{\top}+\boldsymbol{\Phi} \underline{\boldsymbol{K}} \boldsymbol{Q} & -\boldsymbol{\Psi} & \mathbf{0} & \mathbf{0} \\
\boldsymbol{B}_{\mathrm{s}, u}^{\top} & \mathbf{0} & -\frac{1}{2} \gamma_{\mathcal{Y}} \mathbb{I} & \mathbf{0} \\
\boldsymbol{C}_{\mathrm{s}} \boldsymbol{Q} & \mathbf{0} & \mathbf{0} & -\frac{1}{2} \gamma_{\mathcal{Y}} \mathbb{I}
\end{array}\right] \prec \mathbf{0} .
\end{aligned}
$$

Neben der Analyse eines durch $\underline{\boldsymbol{K}}$ gegebenen Anti-Windup interessiert vor allem der Entwurf, d.h. die Bestimmung einer Anti-Windup-Matrix $\underline{\boldsymbol{K}}$ unter Berücksichtigung der Entwurfsbedingungen. Da die Matrix $\underline{\boldsymbol{K}}$ dann unbekannt ist, stellt sie neben $\boldsymbol{R}_{\mathrm{g}}, \boldsymbol{Q}, \boldsymbol{\Theta}, \boldsymbol{\Psi}$ und $\gamma_{\mathcal{\nu}}$ eine weitere Variable für das Optimierungsproblem dar. Hinderlich für die Lösung des Optimierungsproblems ist, dass die Nebenbedingungen (7.12) und (7.14) Produktterme in den Matrixvariablen $\underline{\boldsymbol{K}}$ und $\boldsymbol{Q}$ sowie $\underline{\boldsymbol{K}}$ und $\boldsymbol{\Theta}$ (aufgrund von $\boldsymbol{C}_{*}$ in (7.5)) enthalten und somit nichtlinear in den Entwurfsvariablen sind. Das Optimierungsproblem verliert folglich die Eigenschaft der Konvexität und lässt sich nicht mit den gebräuchlichen Algorithmen [180, 188] 
lösen. Um dennoch eine Matrix $\underline{\boldsymbol{K}}$ bestimmen zu können, wird im nächsten Abschnitt ein Entwurfsverfahren vorgestellt, das auf der Methode des Path-Following basiert [79, 151].

\subsection{Entwurf mit Path-Following}

Der Grundgedanke des Path-Following ist es, nichtlineare Matrixungleichungen, die als Nebenbedingungen eines Optimierungsproblems in Erscheinung treten, um eine zuvor gefundene Lösung zu linearisieren. Die entstehenden linearen Matrixungleichungen lassen sich dann als konvexe Nebenbedingungen in einem konvexen Optimierungsproblem einsetzen, um in einer Umgebung der gültigen Lösung neue Lösungen mit einem höherem Gütemaß zu suchen. Die Größe der Umgebung wird durch zusätzliche Nebenbedingungen beschränkt, damit sich die linearisierten Matrixungleichungen möglichst wenig von den nichtlinearen Matrixungleichungen unterscheiden. Diese Vorgehensweise wird iterativ fortgesetzt, bis eine Abbruchbedingung erfüllt ist.

Um die nichtlinearen Matrixungleichungen (7.12) und (7.14) des Optimierungsproblems 7.1 linearisieren zu können, wird zuerst eine gültige Lösung benötigt, die aus einem Parametersatz

$$
\boldsymbol{R}_{\mathrm{g}, 0}, \quad \boldsymbol{\Theta}_{0}, \quad \boldsymbol{Q}_{0}, \quad \boldsymbol{\Psi}_{0}, \underline{\boldsymbol{K}}_{0}, \gamma_{\mathcal{Y}, 0}
$$

besteht. Eine derartige initiale Lösung lässt sich z. B. durch Ausprobieren finden, was aber umständlich und ineffizient ist. Eine zielführende Vorgehensweise besteht in der Bestimmung der initialen Lösung durch ein konvexes Optimierungsproblem, wie nachfolgend erläutert wird.

\section{Bestimmen einer initialen Lösung durch konvexe Optimierung}

Die Berechnung eines gültigen initialen Parametersatzes (7.15) erfolgt in zwei Schritten. Zuerst wird die initiale Anti-Windup-Matrix $\underline{\boldsymbol{K}}_{0}$ bestimmt. Die Basis hierfür bildet Lemma 7.3 mit der oberen Sektorgrenze $\boldsymbol{\Phi}=\mathbb{I}$. Die Totzone des betrachteten Ersatzsystems liegt dann komplett im Sektor $\mathcal{S}(\mathbf{0}, \mathbb{I})$ und die Bedingung (7.10) mit $\underline{\boldsymbol{K}}=\underline{\boldsymbol{K}}_{0}$ garantiert globale Stabilität des Anti-Windup-Regelkreises.

Um die Anti-Windup-Matrix $\underline{\boldsymbol{K}}=\underline{\boldsymbol{K}}_{0}$ bestimmen zu können, werden die Produkte aus $\underline{\boldsymbol{K}}_{0}$ und $\boldsymbol{Q}$ in der Nebenbedingung (7.10) durch

$$
\boldsymbol{X}=\underline{\boldsymbol{K}}_{0} \boldsymbol{Q}
$$


substituiert. Die Nebenbedingung ist dann eine lineare Matrixungleichung in den Variablen $\boldsymbol{X}, \boldsymbol{Q}, \boldsymbol{\Psi}, \gamma$ und somit konvex. Das Minimieren der $L_{2^{-}}$ Verstärkung $\gamma$ führt direkt zu dem konvexen

\section{Optimierungsproblem 7.2.}

$$
\begin{gathered}
\underset{\boldsymbol{Q}, \boldsymbol{\Psi}, \boldsymbol{X}, \gamma}{\operatorname{Minimiere}} \gamma \text { unter den Nebenbedingungen } \\
\boldsymbol{Q} \succ \mathbf{0}, \boldsymbol{\Psi}=\operatorname{diag}\left(\psi_{1}, \ldots, \psi_{m}\right) \succ \mathbf{0}, \quad \gamma>0, \\
\operatorname{He}\left[\begin{array}{cccc}
\boldsymbol{A}_{\mathrm{s}} \boldsymbol{Q}-\boldsymbol{B}_{\mathrm{s}, u} \boldsymbol{X} & \mathbf{0} & \mathbf{0} & \mathbf{0} \\
\boldsymbol{\Psi} \boldsymbol{B}_{\mathrm{s}, u}^{\top}+\boldsymbol{X} & -\mathbf{\Psi} & \mathbf{0} & \mathbf{0} \\
\boldsymbol{B}_{\mathrm{s}, u}^{\top} & \mathbf{0} & -\frac{1}{2} \gamma \mathbb{I} & \mathbf{0} \\
\boldsymbol{C}_{\mathrm{s}} \boldsymbol{Q} & \mathbf{0} & \mathbf{0} & -\frac{1}{2} \gamma \mathbb{I}
\end{array}\right] \prec \mathbf{0 .}
\end{gathered}
$$

Die gesuchte Matrix $\underline{\boldsymbol{K}}_{0}$ ergibt sich durch Rücksubstitution

$$
\underline{\boldsymbol{K}}_{0}=\boldsymbol{X} \boldsymbol{Q}^{-1} \text {. }
$$

Anmerkung 7.2. Aus numerischen Gründen kann es für die Implementierung des Anti-Windup sinnvoll sein, den Betrag der Eigenwerte von

$$
\boldsymbol{A}_{\mathrm{s}}-\boldsymbol{B}_{\mathrm{s}, u} \underline{\boldsymbol{K}}_{0}
$$

auf einen maximalen Wert $\bar{\lambda}>0$ zu beschränken. Ähnliches wurde auch in [120] für den Entwurf von sättigenden Zustandsreglern vorgeschlagen. Eine derartige Beschränkung kann mit der in [34] angegebenen Bedingung

$$
\mathrm{He}\left[\begin{array}{cc}
-\frac{1}{2} \bar{\lambda} \boldsymbol{Q} & \boldsymbol{A}_{\mathrm{s}} \boldsymbol{Q}-\boldsymbol{B}_{\mathrm{s}, u} \underline{\boldsymbol{K}}_{0} \boldsymbol{Q} \\
\mathbf{0} & -\frac{1}{2} \bar{\lambda} \boldsymbol{Q}
\end{array}\right] \prec \mathbf{0}
$$

berücksichtigt werden. Substituieren von $\underline{\boldsymbol{K}}_{0} \boldsymbol{Q}=\boldsymbol{X}$ führt dann zu der weiteren Nebenbedingung

$$
\mathrm{He}\left[\begin{array}{cc}
-\frac{1}{2} \bar{\lambda} \boldsymbol{Q} & \boldsymbol{A}_{\mathrm{s}} \boldsymbol{Q}-\boldsymbol{B}_{\mathrm{s}, u} \boldsymbol{X} \\
\mathbf{0} & -\frac{1}{2} \bar{\lambda} \boldsymbol{Q}
\end{array}\right] \prec \mathbf{0}
$$

für das Optimierungsproblem 7.2.

Nachdem eine initiale Anti-Windup-Matrix $\underline{\boldsymbol{K}}_{0}$ bestimmt ist, werden in einem zweiten Schritt die initialen Parameter

$$
\boldsymbol{R}_{\mathrm{g}, 0}, \boldsymbol{\Theta}_{0}, \boldsymbol{Q}_{0}, \boldsymbol{\Psi}_{0}, \gamma_{\mathcal{y}, 0}
$$


berechnet. Hierfür kann das Optimierungsproblem 7.1 mit

$$
\boldsymbol{R}_{\mathrm{g}}=\boldsymbol{R}_{\mathrm{g}, 0}, \quad \boldsymbol{\Theta}=\boldsymbol{\Theta}_{0}, \quad \boldsymbol{Q}=\boldsymbol{Q}_{0}, \quad \boldsymbol{\Psi}=\boldsymbol{\Psi}_{0}, \gamma_{\mathcal{\nu}}=\gamma_{\mathcal{y}, 0}
$$

verwendet werden, das aufgrund der bereits bekannten Matrix $\underline{\boldsymbol{K}}=\underline{\boldsymbol{K}}_{0}$ konvex ist. Zuvor muss noch die obere Sektorgrenze $\boldsymbol{\Phi}$ und damit die Eingangssignalklasse $\mathcal{Y}(\boldsymbol{\Phi})$ festgelegt werden, für die später eine Minimierung der $L_{2}$-Kleinsignalverstärkung erfolgen soll.

Der so berechnete initiale Parametersatz (7.15) erfüllt alle Nebenbedingungen des Optimierungsproblems 7.1. Somit garantiert die Anti-WindupMatrix $\underline{\boldsymbol{K}}_{0}$ globale Stabilität und eine $L_{2^{-}}$Kleinsignalverstärkung $\gamma_{\mathcal{y}, 0}$ des Ersatzsystems (7.6) für die Eingangssignalklasse $\mathcal{Y}(\boldsymbol{\Phi})$.

Dabei ist zu beachten, dass die Anti-Windup-Matrix $\underline{\boldsymbol{K}}_{0}$ mit dem Optimierungsproblem 7.2 bestimmt wurde, dessen Nebenbedingung (7.16) auf quadratischen Ljapunov-Funktionen basiert. Des Weiteren wurde bei der Optimierung die $L_{2}$-Verstärkung minimiert. Dieser Entwurf ähnelt stark dem Stand der Technik [191]. Er ist einfach und effizient durchführbar, weist aber die in Abschnitt 7.1 beschriebenen Nachteile auf. Vor allem findet keine Minimierung der $L_{2}$-Kleinsignalverstärkung statt.

Deshalb wird mithilfe der Path-Following-Methodik in einer Umgebung der initialen Lösung nach Anti-Windup-Matrizen $\underline{\boldsymbol{K}}$ gesucht, die eine kleinere obere Schranke $\gamma_{\mathcal{y}}<\gamma_{\mathcal{y}, 0}$ der $L_{2}$-Kleinsignalverstärkung garantieren. Die dazu nötigen Schritte sind nachfolgend beschrieben. In einem ersten Schritt werden die Nebenbedingungen des Optimierungsproblems $7.1 \mathrm{um}$ die gefundene initiale Lösung linearisiert.

\section{Schritt 1: Linearisierung}

In einer kleinen Umgebung der Lösung $\boldsymbol{R}_{\mathrm{g}, 0}$ lässt sich die Variable $\boldsymbol{R}_{\mathrm{g}}$ wie folgt schreiben

$$
\boldsymbol{R}_{\mathrm{g}}=\boldsymbol{R}_{\mathrm{g}, 0}+\boldsymbol{R}_{\mathrm{g}, \Delta}
$$

Dabei kennzeichnet der Index $\Delta$ die Abweichung von der initialen Lösung, die nun die neue Variable darstellt. Für die anderen Variablen des Optimierungsproblems 7.1 gilt analog

$$
\begin{aligned}
\underline{\boldsymbol{K}}=\underline{\boldsymbol{K}}_{0}+\underline{\boldsymbol{K}}_{\Delta}, & \boldsymbol{Q}=\boldsymbol{Q}_{0}+\boldsymbol{Q}_{\Delta}, \\
\boldsymbol{\Psi} & =\underline{\boldsymbol{\Psi}}_{0}+\underline{\boldsymbol{\Psi}}_{\Delta}, \quad \gamma_{\mathcal{Y}}=\gamma_{\mathcal{Y}, 0}+\gamma_{\mathcal{Y}, \Delta}
\end{aligned}
$$


Mittels dieses Ansatzes lässt sich beispielsweise ein Produkt der zwei Variablen $\boldsymbol{Q}$ und $\underline{\boldsymbol{K}}$ wie folgt berechnen

$$
\boldsymbol{Q} \underline{\boldsymbol{K}}=\left(\underline{\boldsymbol{K}}_{0}+\underline{\boldsymbol{K}}_{\Delta}\right)\left(\boldsymbol{Q}_{0}+\boldsymbol{Q}_{\Delta}\right)=\underline{\boldsymbol{K}}_{0} \boldsymbol{Q}_{0}+\underline{\boldsymbol{K}}_{0} \boldsymbol{Q}_{\Delta}+\underline{\boldsymbol{K}}_{\Delta} \boldsymbol{Q}_{0}+\underline{\boldsymbol{K}}_{\Delta} \boldsymbol{Q}_{\Delta} .
$$

Unter der Voraussetzung, dass die Abweichungen $\underline{\boldsymbol{K}}_{\Delta}$ und $\boldsymbol{Q}_{\Delta}$ klein sind, kann der letzte Term $\underline{\boldsymbol{K}}_{\Delta} \boldsymbol{Q}_{\Delta}$ vernachlässigt werden [79]. Auf diese Weise wird das Produkt zweier unbekannter Variablen $\boldsymbol{Q} \underline{\boldsymbol{K}}$ in einer kleinen Umgebung der bekannten Lösungen $\boldsymbol{Q}_{0}, \underline{\boldsymbol{K}}_{0}$ durch einen in den Abweichungen $\boldsymbol{Q}_{\Delta}$ und $\underline{\boldsymbol{K}}_{\Delta}$ linearen Ausdruck

$$
Q \underline{\boldsymbol{K}} \approx \underline{\boldsymbol{K}}_{0} Q_{0}+\underline{\boldsymbol{K}}_{0} Q_{\Delta}+\underline{\boldsymbol{K}}_{\Delta} Q_{0}
$$

approximiert. Dieses Prinzip wird nun auf die Nebenbedingungen (7.12) und (7.14) des Optimierungsproblems 7.1 übertragen.

Für die Nebenbedingung (7.12) ergibt sich die Approximation

$$
\operatorname{He}\left[\begin{array}{cc}
\boldsymbol{X}_{A} & \mathbf{0} \\
\boldsymbol{X}_{B} & \frac{1}{2} \boldsymbol{D}_{L}^{\top} \boldsymbol{\Theta}_{0} \boldsymbol{D}_{L}
\end{array}\right] \prec \mathbf{0},
$$

wobei für eine kompaktere Notation die Abkürzungen

$$
\begin{aligned}
& \boldsymbol{X}_{A}=\boldsymbol{A}_{L}^{\mathrm{\top}}\left(\boldsymbol{R}_{\mathrm{g}, 0}+\boldsymbol{R}_{\mathrm{g}, \Delta}\right)+\frac{1}{2} \boldsymbol{C}_{L, 0}^{\mathrm{\top}} \boldsymbol{\Theta}_{0} \boldsymbol{C}_{L, 0}+\boldsymbol{C}_{L, \Delta}^{\mathrm{T}} \boldsymbol{\Theta}_{0} \boldsymbol{C}_{L, 0}, \\
& \boldsymbol{X}_{B}=\boldsymbol{D}_{L}^{\mathrm{\top}} \boldsymbol{\Theta}_{0}\left(\boldsymbol{C}_{L, 0}+\boldsymbol{C}_{L, \Delta}\right)+\boldsymbol{B}_{L}^{\mathrm{\top}}\left(\boldsymbol{R}_{\mathrm{g}, 0}+\boldsymbol{R}_{\mathrm{g}, \Delta}\right)
\end{aligned}
$$

und die Schreibweisen

$$
\boldsymbol{C}_{L, 0}=\left[\begin{array}{cc}
-\underline{\boldsymbol{K}}_{0} & \mathbf{0} \\
-\underline{\boldsymbol{K}}_{0} \boldsymbol{A}_{\mathrm{s}} & -\underline{\boldsymbol{K}}_{0} \boldsymbol{B}_{\mathrm{s}, u} \\
\mathbf{0} & \mathbb{I} \\
\mathbf{0} & \mathbf{0}
\end{array}\right], C_{L, \Delta}=\left[\begin{array}{cc}
-\underline{\boldsymbol{K}}_{\Delta} & \mathbf{0} \\
-\underline{\boldsymbol{K}}_{\Delta} \boldsymbol{A}_{\mathrm{s}} & -\underline{\boldsymbol{K}}_{\Delta} \boldsymbol{B}_{\mathrm{s}, u} \\
\mathbf{0} & \mathbb{I} \\
\mathbf{0} & \mathbf{0}
\end{array}\right]
$$

verwendet werden. Diese Nebenbedingung ist eine lineare Matrixungleichung in den Variablen $\underline{\boldsymbol{K}}_{\Delta}$ und $\boldsymbol{R}_{\mathrm{g}, \Delta}$. Für die Nebenbedingung (7.14) ergibt sich

$$
\operatorname{He}\left[\begin{array}{cccc}
\boldsymbol{X}_{11} & \mathbf{0} & \mathbf{0} & \mathbf{0} \\
\boldsymbol{X}_{21} & -\boldsymbol{\Psi}_{0}+\boldsymbol{\Psi}_{\Delta} & \mathbf{0} & \mathbf{0} \\
\boldsymbol{B}_{\mathrm{s}, u}^{\top} & \mathbf{0} & \boldsymbol{X}_{33} & \mathbf{0} \\
\boldsymbol{C}_{\mathrm{s}}\left(\boldsymbol{Q}_{0}+\boldsymbol{Q}_{\Delta}\right) & \mathbf{0} & \mathbf{0} & \boldsymbol{X}_{33}
\end{array}\right] \prec \mathbf{0}
$$


mit den abkürzenden Schreibweisen

$$
\begin{aligned}
\boldsymbol{X}_{K Q} & =\underline{\boldsymbol{K}}_{0} \boldsymbol{Q}_{0}+\underline{\boldsymbol{K}}_{0} \boldsymbol{Q}_{\Delta}+\underline{\boldsymbol{K}}_{\Delta} \boldsymbol{Q}_{0} \\
\boldsymbol{X}_{11} & =\boldsymbol{A}_{\mathrm{s}}\left(\boldsymbol{Q}_{0}+\boldsymbol{Q}_{\Delta}\right)-\boldsymbol{B}_{\mathrm{s}, u} \boldsymbol{X}_{K Q} \\
\boldsymbol{X}_{21} & =\left(\boldsymbol{\Psi}_{0}+\boldsymbol{\Psi}_{\Delta}\right) \boldsymbol{B}_{\mathrm{s}, u}^{\top}+\boldsymbol{\Phi} \boldsymbol{X}_{K Q} \\
\boldsymbol{X}_{33} & =-\frac{1}{2}\left(\gamma_{\mathcal{\nu}, 0}+\gamma_{\mathcal{y}, \Delta}\right) \mathbb{I}
\end{aligned}
$$

Dabei handelt es sich ebenfalls um eine lineare Matrixungleichung. Die Variablen sind die Abweichungen $\underline{\boldsymbol{K}}_{\Delta}, \boldsymbol{Q}_{\Delta}, \boldsymbol{\Psi}_{\Delta}$ und $\gamma_{\boldsymbol{\nu}, \Delta}$. Die optionale Nebenbedingung aus Anmerkung 7.2 lässt sich ebenfalls als lineare Matrixungleichung

$$
\operatorname{He}\left[\begin{array}{cc}
-\frac{1}{2} \bar{\lambda}\left(\boldsymbol{Q}_{0}+\boldsymbol{Q}_{\Delta}\right) & \boldsymbol{A}_{\mathrm{s}}\left(\boldsymbol{Q}_{0}+\boldsymbol{Q}_{\Delta}\right)-\boldsymbol{B}_{\mathrm{s}, u} \boldsymbol{X}_{K Q} \\
\mathbf{0} & -\frac{1}{2} \bar{\lambda}\left(\boldsymbol{Q}_{0}+\boldsymbol{Q}_{\Delta}\right)
\end{array}\right] \prec \mathbf{0}
$$

in den Variablen $\underline{\boldsymbol{K}}_{\Delta}$ und $\boldsymbol{Q}_{\Delta}$ approximieren.

Die linearen Matrixungleichungen (7.19) bis (7.21) stellen somit in einer kleinen Umgebung der initialen Lösung gute Approximationen für die nichtlinearen Nebenbedingungen (7.12), (7.14) und der optionalen Nebenbedingung (7.18) des Optimierungsproblems 7.1 dar. Diese Approximation kann genutzt werden, um die initiale Lösung zu verbessern.

\section{Schritt 2: Initiale Lösung verbessern}

Um die initiale Lösung zu verbessern wird ein konvexes Optimierungsproblem basierend auf den approximierten Nebenbedingungen gelöst. Damit die Abweichungen von der initialen Lösung klein bleiben und die Approximation ihre Gültigkeit behält, fordert man, dass die Variablen im Verlauf der Optimierung keine allzu großen Werte annehmen. Das führt zu der Normbegrenzung $\left\|\boldsymbol{Q}_{\Delta}\right\|_{2}<\varkappa\left\|\boldsymbol{Q}_{0}\right\|_{2}$ und $\left\|\boldsymbol{R}_{\mathrm{g}, \Delta}\right\|_{2}<\varkappa\left\|\boldsymbol{R}_{\mathrm{g}, 0}\right\|_{2}$, wobei $\varkappa$ ein positiver Skalar ist, der eine Art Schrittweite für das Optimierungsproblem darstellt. Nach Lemma C.3 im Abschnitt C.2 des Anhangs ergeben sich aus diesen Begrenzungen die zusätzlichen Nebenbedingungen

$$
\left[\begin{array}{cc}
\varkappa \boldsymbol{Q}_{0} & \boldsymbol{Q}_{\Delta} \\
\boldsymbol{Q}_{\Delta} & \varkappa \boldsymbol{Q}_{0}
\end{array}\right] \succ \mathbf{0}, \quad\left[\begin{array}{cc}
\varkappa \boldsymbol{R}_{\mathrm{g}, 0} & \boldsymbol{R}_{\mathrm{g}, \Delta} \\
\boldsymbol{R}_{\mathrm{g}, \Delta} & \varkappa \boldsymbol{R}_{\mathrm{g}, 0}
\end{array}\right] \succ \mathbf{0} .
$$

Das zu lösende konvexe Optimierungsproblem stellt eine Approximation für das Optimierungsproblem 7.1 dar. Es lautet wie folgt. 


\section{Optimierungsproblem 7.3.}

$$
\begin{aligned}
& \underset{\boldsymbol{R}_{\mathrm{g}, \Delta}, \boldsymbol{Q}_{\Delta}, \boldsymbol{\Psi}_{\Delta}, \underline{\boldsymbol{K}}_{\Delta}, \gamma_{\mathcal{Y}, \Delta}}{\text { Minimiere }} \gamma_{\boldsymbol{\mathcal { \nu } , \Delta}} \quad \text { unter den Nebenbedingungen } \\
& \boldsymbol{R}_{\mathrm{g}, 0}+\boldsymbol{R}_{\mathrm{g}, \Delta} \succ \mathbf{0}, \boldsymbol{Q}_{0}+\boldsymbol{Q}_{\Delta} \succ \mathbf{0}, \quad \boldsymbol{\Psi}_{0}+\boldsymbol{\Psi}_{\Delta} \succ \mathbf{0}, \\
& \gamma_{\mathcal{Y}, \Delta} \leq 0, \gamma_{\mathcal{Y}, 0}+\gamma_{\mathcal{\nu}, \Delta}>0 \\
& (7.19),(7.20),(7.21),(7.22) .
\end{aligned}
$$

Dieses Problem ist immer lösbar, da der Parametersatz

$$
\boldsymbol{R}_{\mathrm{g}, \Delta}=\mathbf{0}, \quad \boldsymbol{Q}_{\Delta}=\mathbf{0}, \quad \boldsymbol{\Psi}_{\Delta}=\mathbf{0}, \underline{\boldsymbol{K}}_{\Delta}=\mathbf{0}, \quad \gamma_{\mathcal{\nu}, \Delta}=0
$$

eine gültige Lösung darstellt. Das Ziel ist allerdings, einen Parametersatz mit $\gamma_{\boldsymbol{y}, \Delta}<0 \mathrm{zu}$ finden. Denn dann besitzt die Matrix $\underline{\boldsymbol{K}}_{0}+\underline{\boldsymbol{K}}_{\Delta}$ das Potential, eine im Vergleich zur initialen Lösung niedrigere $L_{2}$-Kleinsignalverstärkung zu garantieren. Ob dies tatsächlich der Fall ist, wird in Schritt 4 überprüft. Zunächst folgt ein Schritt, um die Variablen $\underline{\boldsymbol{K}}$ und $\gamma_{\mathcal{y}} \mathrm{zu}$ aktualisieren.

\section{Schritt 3: Update-Schritt}

Die Variablen $\underline{\boldsymbol{K}}$ und $\gamma_{\boldsymbol{y}}$ werden aktualisiert. Die neuen Werte sind

$$
\underline{\boldsymbol{K}}:=\underline{\boldsymbol{K}}_{0}+\underline{\boldsymbol{K}}_{\Delta}, \quad \gamma_{\mathcal{Y}}:=\gamma_{\mathcal{Y}, 0}+\gamma_{\mathcal{Y}, \Delta}
$$

mit $\underline{\boldsymbol{K}}_{\Delta}$ und $\gamma_{\mathcal{\nu}, \Delta}$ aus Optimierungsproblem 7.3.

\section{Schritt 4: Validierung}

Mit den aktualisierten Werten wird versucht, eine Lösung für das folgende konvexe Validierungsproblem zu finden.

Validierungsproblem 7.1. Für $\underline{\boldsymbol{K}}$ und $\gamma_{\mathcal{y}}$ aus Schritt 3,

$$
\begin{aligned}
& \underset{\boldsymbol{R}_{\mathrm{g}}, \boldsymbol{Q}, \boldsymbol{\Theta}, \boldsymbol{\Psi}}{\text { finde }} \boldsymbol{R}_{\mathrm{g}}, \boldsymbol{Q}, \boldsymbol{\Theta}, \boldsymbol{\Psi} \quad \text { unter den Nebenbedingungen } \\
& \boldsymbol{R}_{\mathrm{g}} \succ \mathbf{0}, \boldsymbol{Q} \succ \mathbf{0}, \boldsymbol{\Theta} \succ \mathbf{0}, \boldsymbol{\Psi}=\operatorname{diag}\left(\psi_{1}, \ldots, \psi_{m}\right) \succ \mathbf{0}, \\
& (7.17),(7.12),(7.13),(7.14) .
\end{aligned}
$$

Falls das Problem lösbar ist, stellen die Matrizen $\boldsymbol{R}_{\mathrm{g}}, \boldsymbol{Q}, \boldsymbol{\Theta}, \Psi, \underline{\boldsymbol{K}}$ und der Skalar $\gamma_{\nu}$ eine neue Lösung dar und garantieren globale Stabilität des Anti-Windup-Regelkreises sowie eine niedrigere $L_{2}$-Kleinsignalverstärkung 


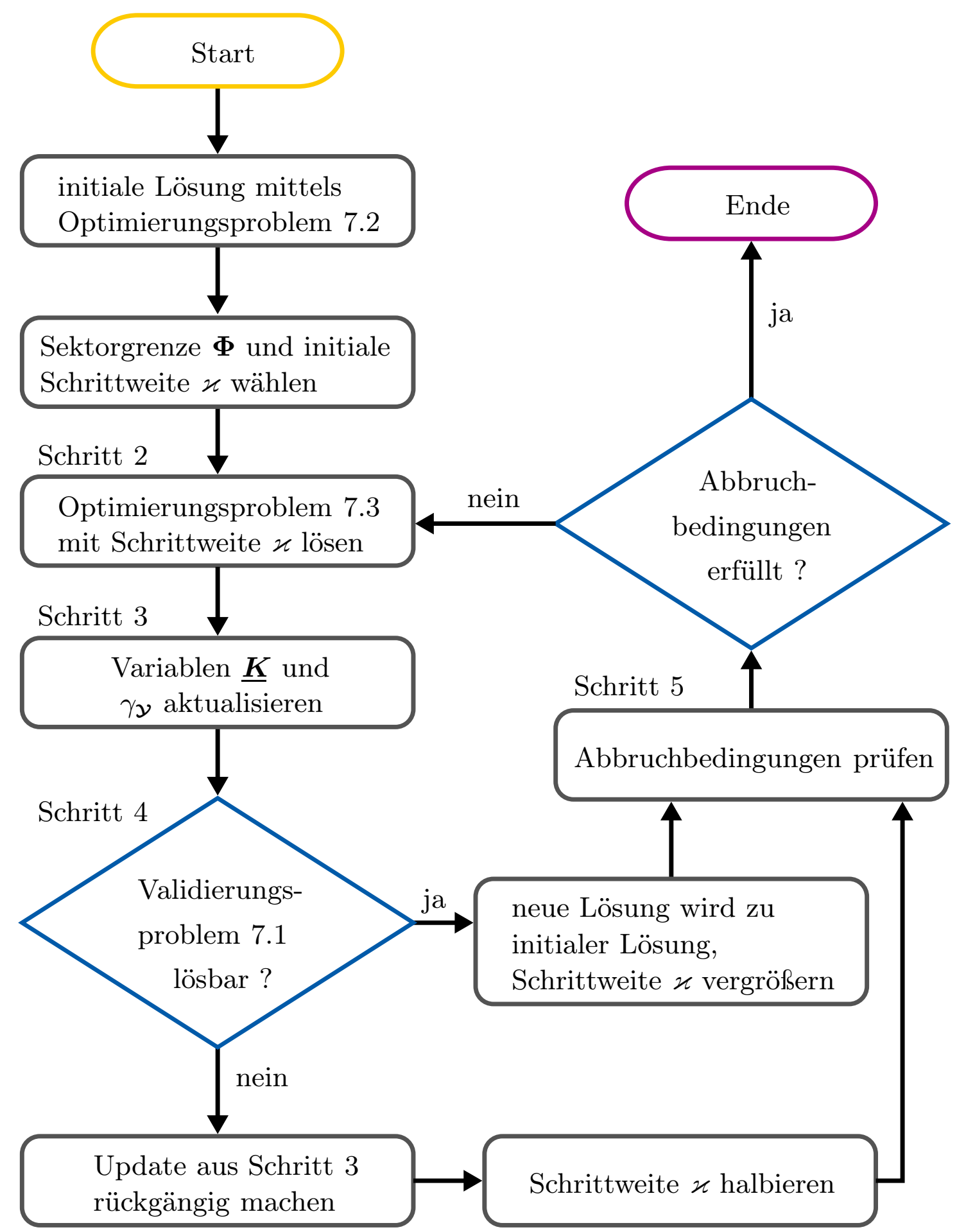

Abbildung 7.2: Path-Following-Algorithmus für den Entwurf des linearen, modellbasierten Anti-Windup. 
als die initiale Lösung. Die initiale Lösung wird deshalb in der nächsten Iteration durch die neue Lösung ersetzt, d. h.

$$
\boldsymbol{R}_{\mathrm{g}, 0}:=\boldsymbol{R}_{\mathrm{g}}, \boldsymbol{Q}_{0}:=\boldsymbol{Q}, \boldsymbol{\Theta}_{0}:=\boldsymbol{\Theta}, \boldsymbol{\Psi}_{0}:=\boldsymbol{\Psi}, \underline{\boldsymbol{K}}_{0}:=\underline{\boldsymbol{K}}, \gamma_{\mathcal{Y}, 0}:=\gamma_{\mathcal{Y}} .
$$

Außerdem wird die Schrittweite $\varkappa$ für die nächste Iteration leicht vergrößert, z. B. $\varkappa:=1,1 \cdot \varkappa$. Falls keine Lösung gefunden wurde, wird das Update der Variablen aus Schritt 3 rückgängig gemacht und die Schrittweite $\varkappa$ halbiert. Danach folgt Schritt 5 .

\section{Schritt 5: Abbruchbedingung prüfen}

Der Algorithmus wird entweder nach einer festgelegten Anzahl von Iterationen beendet oder wenn $\gamma_{\boldsymbol{y}}$ sich im Vergleich zur letzten Iteration kaum verändert hat. Falls die Abbruchbedingungen nicht zutreffen, startet die nächste Iteration des Algorithmus bei Schritt 2.

\section{Zusammenfassung des Entwurfsalgorithmus}

Der Entwurfsalgorithmus für das lineare modellbasierte Anti-Windup besteht aus zwei Stufen. Zunächst wird ein initialer Entwurf auf Basis quadratischer Ljapunov-Funktionen durchgeführt. Die initiale Anti-WindupMatrix $\underline{\boldsymbol{K}}_{0}$ kann dabei durch ein konvexes Optimierungsproblem bestimmt werden. Im Anschluss wird die initiale Lösung durch einen auf PathFollowing basierenden Algorithmus iterativ verbessert. Dabei sind abwechselnd ein konvexes Optimierungsproblem und ein konvexes Validierungsproblem zu lösen. Abbildung 7.2 illustriert den Entwurfsprozess.

Der konvexe initiale Entwurf bzw. die Nebenbedingungen des Optimierungsproblems 7.2 entsprechen dabei dem Stand der Technik [191]. Durch die iterative Optimierung der $L_{2}$-Kleinsignalverstärkung dieser initialen Lösung wird in vielen Fällen eine deutliche Verbesserung erreicht. Das wird in Abschnitt 7.4 anhand eines Beispiels demonstriert.

\subsection{Beispiel: Längsdynamik eines Flugzeugs}

Zur Demonstration des neuen Entwurfsverfahrens wird die Regelung der Längsdynamik des in [70, 102] angegebenen Flugzeugmodells einer F-8 betrachtet. Die F-8 ist ein durch Strahltriebwerke angetriebenes Flugzeug, das in den 1970er Jahren von der NASA zur Erforschung digitaler Fly-ByWire-Konzepte eingesetzt wurde [43]. Abbildung 7.3 zeigt das Flugzeug. In 


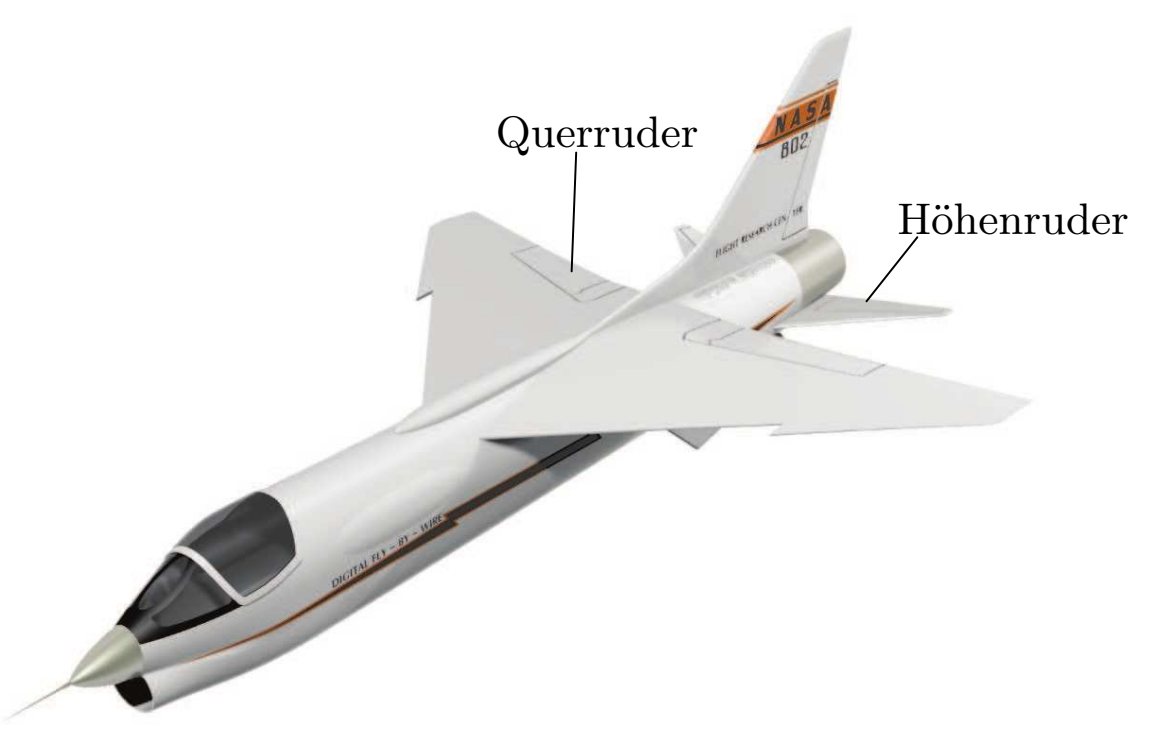

Abbildung 7.3: Experimentalflugzeug F-8 für das in den 1970er Jahren begonnene Fly-By-Wire-Programm der NASA.

insgesamt 211 Testflügen wurde das Fly-By-Wire-System der F-8 erprobt. Es stellt den Vorläufer modernerer Systeme dar, die zum Beispiel Einsatz in den Space Shuttles, militärischen oder zivilen Flugzeugen fanden [142].

Ein lineares Modell der Längsdynamik des Flugzeugs ist durch (6.1) und

$$
\begin{aligned}
\boldsymbol{A}_{\mathrm{s}} & =\left[\begin{array}{cccc}
-0,80 & -6,0 \cdot 10^{-4} & -12,00 & 0 \\
0 & -1,4 \cdot 10^{-2} & -16,64 & -32,20 \\
1,00 & -1,0 \cdot 10^{-4} & -1,50 & 0 \\
1,00 & 0 & 0 & 0
\end{array}\right], \\
\boldsymbol{B}_{\mathrm{s}, u} & =\left[\begin{array}{cc}
-19,00 & -3,00 \\
-0,66 & -0,50 \\
-0,16 & -0,50 \\
0 & 0
\end{array}\right], \boldsymbol{C}_{\mathrm{s}}=\left[\begin{array}{llll}
0 & 0 & 0 & 1 \\
0 & 0 & -1 & 1
\end{array}\right]
\end{aligned}
$$

gegeben [102]. Die Komponenten des Zustandsvektors $\boldsymbol{x}_{\mathrm{s}}$ stellen Abweichungen von einem Arbeitspunkt dar. Dabei ist $x_{\mathrm{s}, 1}$ die Pitchrate in deg/s, $x_{\mathrm{s}, 2}$ die Geschwindigkeit in $\mathrm{ft} / \mathrm{s}, x_{\mathrm{s}, 3}$ der Anstellwinkel in deg und $x_{\mathrm{s} 4}$ der Pitchwinkel in deg. Abbildung 7.4 illustriert den Zusammenhang zwischen Pitch- und Anstellwinkel. Die Stellgrößen sind der Winkel des Höhenruders $u_{\mathrm{s}, 1}$ in deg und der Winkel des Querruders $u_{\mathrm{s}, 2}$ in deg (vgl. Abbildung 7.3). Beide Stellgrößen sind durch den Aktor auf \pm 25 deg begrenzt. Die 


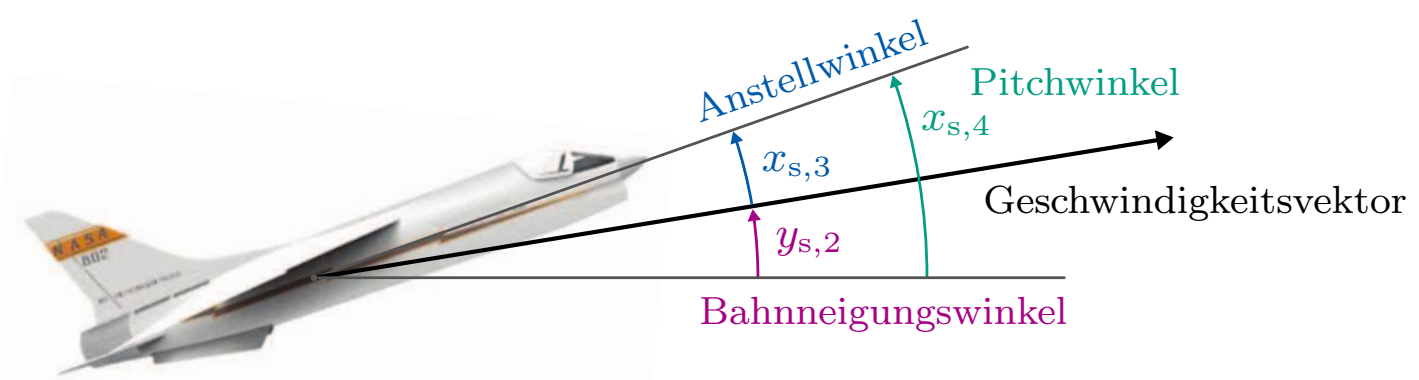

Erdhorizontale

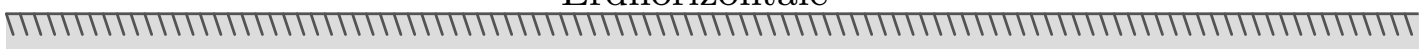

Abbildung 7.4: Definition der Zustände und Ausgänge.

Ausgänge sind der Pitchwinkel $y_{\mathrm{s}, 1}$ in deg und der Bahnneigungswinkel $y_{\mathrm{s}, 2}$ in deg, der ebenfalls in Abbildung 7.4 dargestellt ist.

Die Matrizen des in [102] entworfenen linearen Reglers (6.2) sind im Anhang A.3.3 angegeben. Um die Stellbegrenzungen zu berücksichtigen, kommt ein lineares modellbasiertes Anti-Windup (7.2) zum Einsatz. Die in Abschnitt 6.1 beschriebene Verschaltung von Regler, Strecke und der Anti-Windup-Maßnahme führt zu dem Anti-Windup-Regelkreis.

\subsubsection{Parametrierung des Anti-Windup}

Der Entwurf des Anti-Windup besteht in der Parametrierung der Matrix $\underline{\boldsymbol{K}}$ und der Festlegung des Parameters $\alpha$. Zunächst wird die Stabilisierung der Ruhelage $\boldsymbol{x}_{\text {awrk }}^{(\mathrm{R})}=\mathbf{0}$ betrachtet, d. h. $\boldsymbol{w}=\mathbf{0}$ und $\boldsymbol{z}=\mathbf{0}$, woraus die stationäre Stellgröße $\boldsymbol{y}_{\mathrm{r}}^{(\mathrm{R})}=\mathbf{0}$ folgt. Nach Satz 7.1 kann der Parameter $\alpha$ in diesem Fall beliebig aus dem Intervall $(0,1)$ gewählt werden. Hier wird $\alpha=0,999$ gewählt, damit die Einflussmöglichkeit der Zustandsrückführung $\mathbf{s a t}_{\alpha \overline{\mathbf{u}}}\left(-\underline{\boldsymbol{K}} \boldsymbol{\xi}_{\text {aw }}\right)$ auf die Systemdynamik von ES nicht zu stark eingeschränkt wird.

Die noch zu wählende Anti-Windup-Matrix $\underline{\boldsymbol{K}}$ liefert der neue Entwurfsalgorithmus. Um die initiale Lösung zu berechnen wird das Optimierungsproblem 7.2 mit der optionalen Nebenbedingung (7.18) und $\bar{\lambda}=250$ gelöst. Das Ergebnis ist die Matrix

$$
\underline{\boldsymbol{K}}_{0}=\left[\begin{array}{rrrr}
-5,913 & -0,016 & 47,260 & -51,947 \\
91,727 & 0,325 & -763,830 & 832,884
\end{array}\right]
$$

die eine $L_{2}$-Verstärkung des Ersatzsystems (7.7) von $\gamma=269,832$ garantiert. Für Eingangssignale der Signalklasse $\mathcal{Y}(0,97 \mathbb{I})$ liegt die $L_{2^{-}}$ 


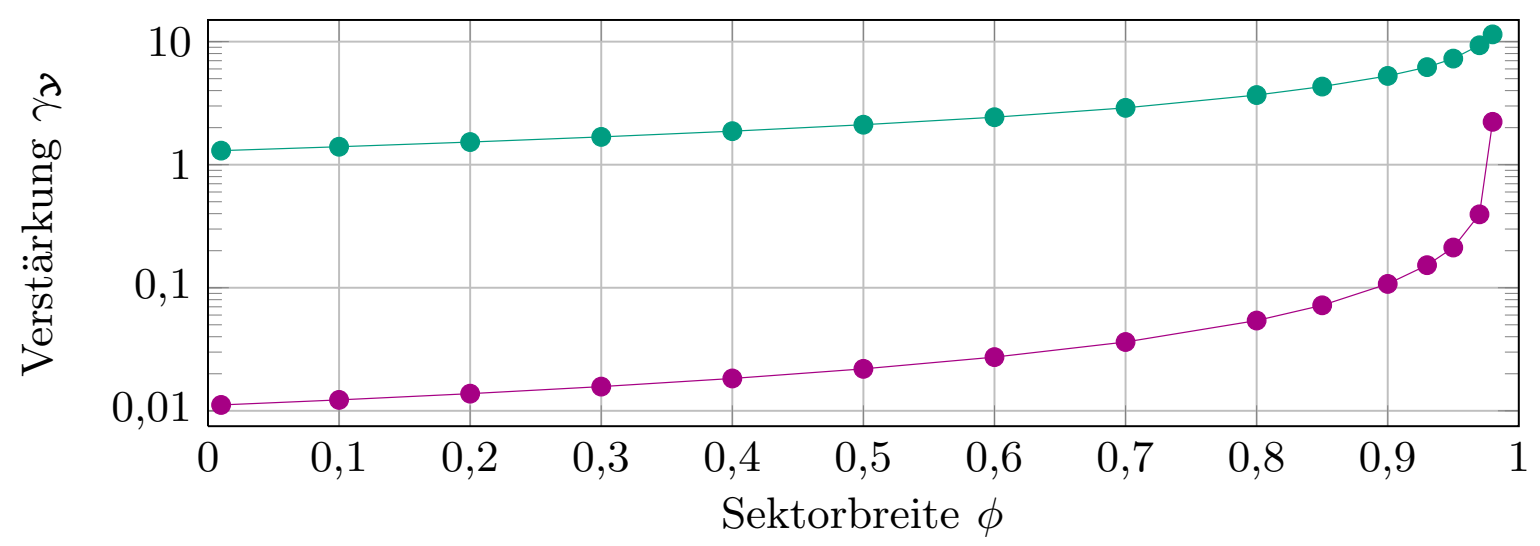

Abbildung 7.5: Logarithmisches Verstärkungsdiagramm. Je kleiner die Verstärkung, desto geringer ist die Abweichung des Anti-Windup-Regelkreises vom unbeschränkten Verhalten. Die initiale Lösung $\underline{\boldsymbol{K}}_{0}$ führt zu großen Verstärkungen $(-\bullet)$. Die optimierte Matrix $\underline{K}$ garantiert eine niedrigere $L_{2^{-}}$ Kleinsignalverstärkung $(-\bullet)$ für die Signalklassen $\mathcal{Y}(0,97$ I $)$, aber auch für alle anderen getesteten Signalklassen $\mathcal{Y}(\phi \mathbb{I})$ bzw. Sektoren $\boldsymbol{\Phi}(\mathbf{0}, \phi \mathbb{I})$.

Kleinsignalverstärkung bei $\gamma_{\mathcal{y}, 0}=9,345$. Mit einer initialen Schrittweite von $\varkappa=0,1$ wird diese Lösung iterativ mit dem in Abbildung 7.2 gezeigten Algorithmus verbessert. Ein Abbruch der Optimierung erfolgt, wenn sich die obere Schranke der $L_{2}$-Kleinsignalverstärkung des Ersatzsystems um weniger als $10^{-5}$ ändert. Das führt zu dem Ergebnis

$$
\underline{\boldsymbol{K}}=\left[\begin{array}{rrrr}
-13,297 & 0,003 & 36,994 & -123,335 \\
-0,813 & 0,037 & -138,817 & 114,507
\end{array}\right] .
$$

Diese Matrix garantiert für die betrachtete Eingangssignalklasse eine rund 23-mal niedrigere $L_{2}$-Kleinsignalverstärkung von $\gamma_{\mathcal{y}}=0,395$. Auch für andere Eingangssignale ergibt sich eine kleinere Verstärkung, wie das aus Kapitel 5 bekannte Verstärkungsdiagramm in Abbildung 7.5 verdeutlicht.

Die höhere Regelgüte der optimierten Anti-Windup-Matrix zeigt sich auch in der Simulation. Betrachtet wird die Ausregelung einer Abweichung des Pitchwinkels $x_{\mathrm{s}, 4}$ von $15 \mathrm{deg}$. Das entspricht dem Anfangszustand

$$
\boldsymbol{x}_{\mathrm{s}}(0)^{\top}=\left[\begin{array}{llll}
0 & 0 & 0 & 15
\end{array}\right], \quad \boldsymbol{x}_{\mathrm{r}}(0)=\mathbf{0}, \quad \boldsymbol{x}_{\mathrm{aw}}(0)=\mathbf{0} .
$$

Die Ausregel- und Stellgrößenverläufe für den unbeschränkten und den beschränkten Regelkreis sowie die Anti-Windup-Regelkreise mit $\underline{\boldsymbol{K}}_{0}$ und der optimierten Matrix $\underline{\boldsymbol{K}}$ finden sich in Abbildung 7.6. Es ist deutlich erkennbar, dass der Anti-Windup-Regelkreis mit $\underline{\boldsymbol{K}}$ die geringste Abwei- 

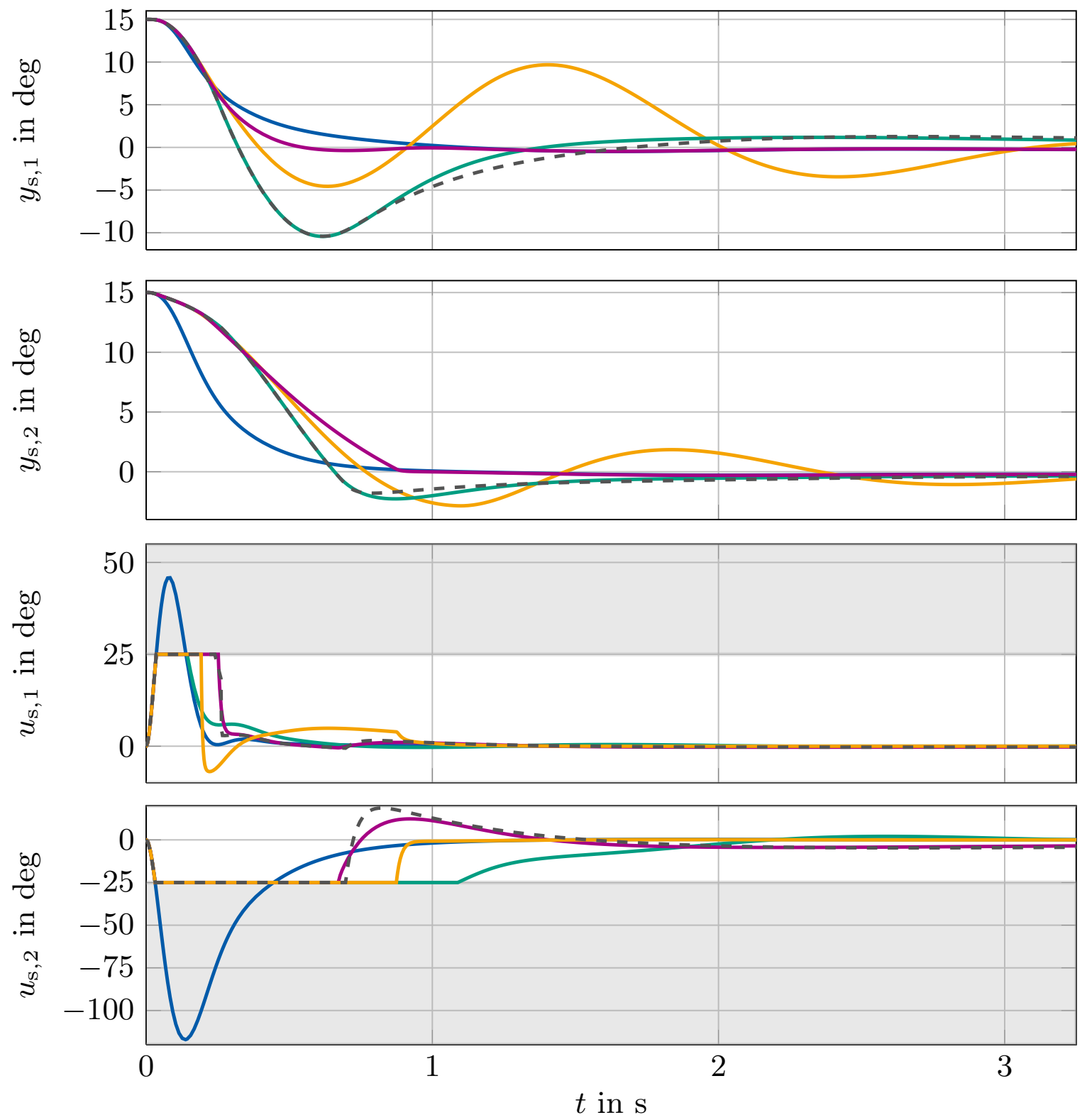

Abbildung 7.6: Verläufe der Ausgänge und Stellgrößen des unbeschränkten Regelkreises (-), des beschränkten Regelkreises ( - ), des Anti-WindupRegelkreises mit $\underline{\boldsymbol{K}}_{0}(-)$ sowie des Anti-Windup-Regelkreises mit der optimierten Matrix $\underline{\boldsymbol{K}}$ (-) für den Anfangszustand (7.23). Zum Vergleich zeigt (- - ) den Ausregelverlauf für ein Anti-Windup nach Turner [191]. 
chung von dem aufgrund der Stellbegrenzungen nicht realisierbaren unbeschränkten Verhalten aufweist. Zum Vergleich wurde auch ein Entwurf mit der Methode von Turner [191, Satz 1] durchgeführt, die auf quadratischen Ljapunov-Funktionen basiert. Die Simulation zeigt, dass dieses Anti-Windup zu ähnlichen Verläufen führt wie das Anti-Windup mit $\underline{\boldsymbol{K}}_{0}$. Somit bleibt festzuhalten, dass durch die Verwendung nichtquadratischer Ljapunov-Funktionen eine deutliche Steigerung der Regelgüte möglich ist.

\subsubsection{Führungsregelung}

Um die Fähigkeiten des Anti-Windup-Regelkreises im Bezug auf die Festwert-Führungsregelung zu demonstrieren wird nun die Aufgabe betrachtet, den Streckenausgang $\boldsymbol{y}_{\mathrm{s}}$ mit dem Sollwert $\boldsymbol{w}^{\top}=\left[\begin{array}{ll}25 & 20\end{array}\right]$ in Übereinstimmung zu bringen. Das entspricht einem Pitchwinkel $y_{\mathrm{s}, 1}$ von $25 \mathrm{deg}$ und einem Bahnneigungswinkel $y_{\mathrm{s}, 2}$ von 20 deg. Der Anfangszustand sei $\boldsymbol{x}_{\text {awrk }}(0)=\mathbf{0}$ und eine Störung tritt nicht auf, d. h. $\boldsymbol{z}=\mathbf{0}$.

Die zu stabilisierende Ruhelage ergibt sich aus (6.13) und ist zulässig, da die benötigte stationäre Stellgröße

$$
\boldsymbol{y}_{\mathrm{r}}^{(\mathrm{R})}=\left[\begin{array}{ll}
-0,832 & -2,068
\end{array}\right]^{\top}
$$

mit der Stellbegrenzung $\overline{\mathrm{u}}_{1}=\overline{\mathrm{u}}_{2}=25$ realisierbar ist. Um die Stabilität der Ruhelage zu garantieren, muss das modellbasierte Anti-Windup angepasst werden. Die Matrix $\underline{\boldsymbol{K}}$ bleibt mit Abschnitt 7.4.1 identisch, nur der Parameter $\alpha$ wird verändert und aus dem Intervall $(0, \hat{\alpha})$ gewählt. Für die obere Schranke (7.11) des Intervalls gilt

$$
\hat{\alpha}=1-\max \left\{\frac{0,832}{25}, \frac{2,068}{25}\right\}=1-\frac{2,068}{25}=0,9173 .
$$

Hier wird $\alpha=0,9$ gewählt, womit nach Satz 7.1 globale Stabilität der Ruhelage garantiert ist.

Die Simulationsergebnisse zeigt Abbildung 7.7. Zum Vergleich ist auch der Verlauf des Anti-Windup-Regelkreises mit $\underline{\boldsymbol{K}}_{0}$ aus dem letzten Abschnitt dargestellt. Es ist deutlich erkennbar, dass sich die gewünschten Sollwerte an den Streckenausgängen einstellen. Die Ruhelage wird erfolgreich stabilisiert. Im Bezug auf die Regelgüte zeigt sich erneut, dass die mit dem neuen Entwurfsalgorithmus parametrierte Matrix $\underline{\boldsymbol{K}}$ dem AntiWindup-Regelkreis durch gute Ausnutzung der Stellgröße zu einem Verhalten verhilft, welches sehr nahe an der unbeschränkten Dynamik liegt. 

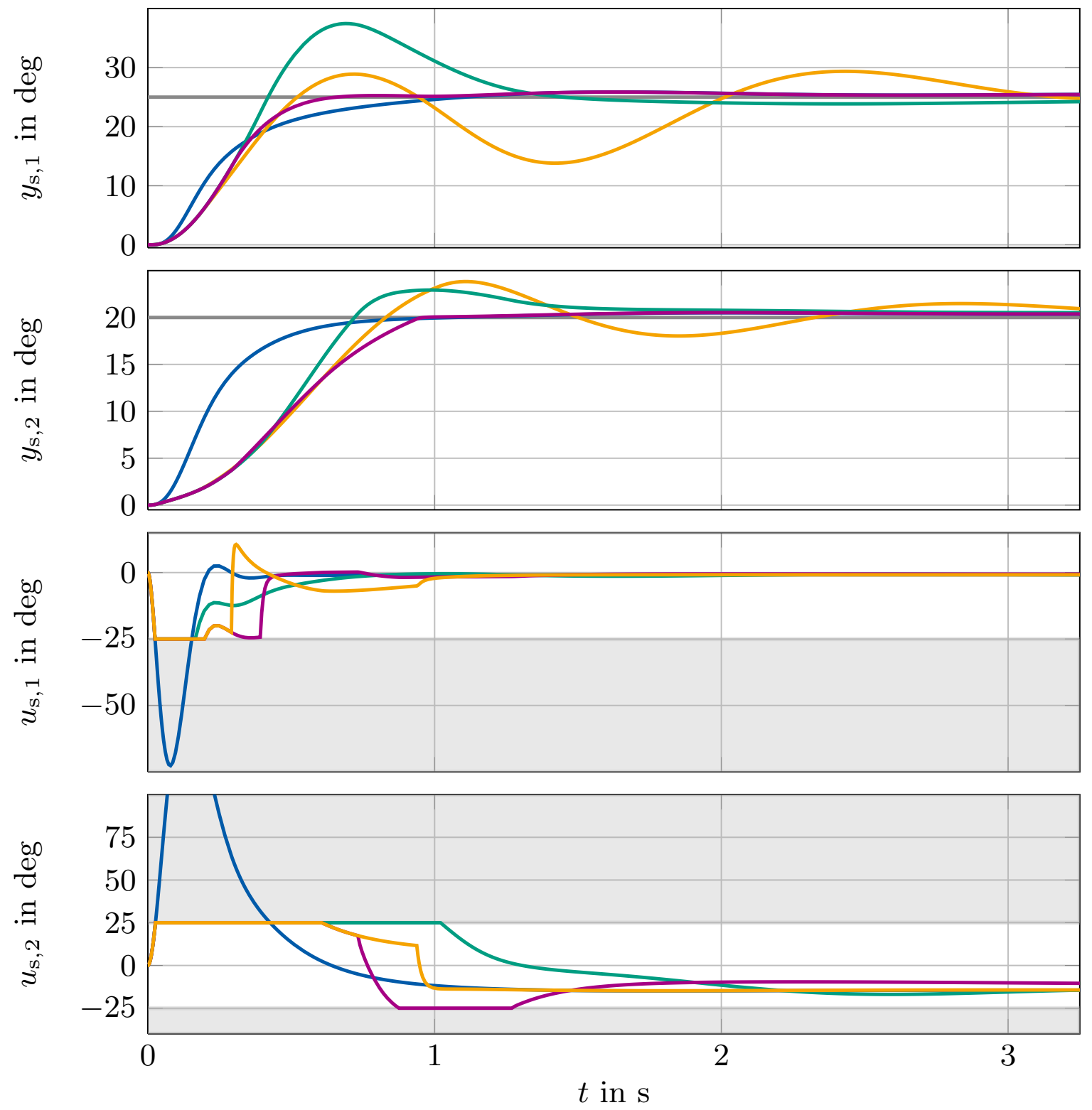

Abbildung 7.7: Verläufe der Ausgänge und Stellgrößen des unbeschränkten Regelkreises (-), des beschränkten Regelkreises ( - ), des Anti-WindupRegelkreises mit $\underline{\boldsymbol{K}}_{0}(-)$ sowie des Anti-Windup-Regelkreises mit der optimierten Matrix $\underline{\boldsymbol{K}}(-)$ für den Anfangszustand $\boldsymbol{x}_{\text {awrk }}(0)=\mathbf{0}$ und die Sollgröße $(-)$ mit dem Wert $\boldsymbol{w}^{\top}=\left[\begin{array}{ll}25 & 20\end{array}\right]$. 


\subsection{Zusammenfassung und Fazit}

In diesem Kapitel wurde ein neues Entwurfsverfahren für lineares modellbasiertes Anti-Windup vorgestellt. Im Vergleich zu den existierenden Entwurfsmethoden garantiert es globale Stabilität und verwendet ein auf der $L_{2}$-Kleinsignalverstärkung des Ersatzsystems ES basierendes Gütekriterium. Dazu ist ein nichtkonvexes Optimierungsproblem lokal zu lösen. Der benötigte initiale Parametersatz kann allerdings durch Lösen eines konvexen Optimierungsproblems sehr einfach bestimmt werden. Ein Beispiel demonstriert, dass mit dem Verfahren eine hohe Regelgüte erzielbar ist.

Wie eingangs erwähnt, wurde die Grundidee der Entwurfsmethodik bereits in [148] veröffentlicht. Die hier vorgestellte Variante stellt allerdings eine bedeutende Vereinfachung dar, weil lediglich der Entwurfsparameter $\boldsymbol{\Phi}$ (optional $\bar{\lambda}$ ) zu wählen ist. In [148] kommen in Abhängigkeit der Streckenordnung $n_{\mathrm{s}}$ noch mehrere Parameter hinzu. 


\section{Anti-Windup mit variabler Struktur für stabile Strecken}

In diesem Kapitel wird ein neues nichtlineares modellbasiertes AntiWindup auf Basis des in [98] beschriebenen sättigenden weich strukturvariablen Zustandsreglers entwickelt. Die Vorteile dieses weich strukturvariablen Anti-Windup bestehen in einem einfachen Entwurf, einer schlanken Systemstruktur und einer hohen Regelgüte. Auszüge dieses Kapitels wurden bereits in [150] veröffentlicht.

\subsection{Problembeschreibung}

Das letzte Kapitel hat gezeigt, dass sich der Entwurf eines linearen modellbasierten Anti-Windup

$$
\mathbf{A W}:\left\{\begin{array}{l}
\dot{\boldsymbol{x}}_{\mathrm{aw}}=\boldsymbol{A}_{\mathrm{s}} \boldsymbol{x}_{\mathrm{aw}}+\boldsymbol{B}_{\mathrm{s}, u} \mathbf{s a t} \mathbf{t}_{\alpha \overline{\mathbf{u}}}\left(-\underline{\boldsymbol{K}} \boldsymbol{x}_{\mathrm{aw}}\right)+\boldsymbol{B}_{\mathrm{s}, u} \boldsymbol{\delta}, \\
\boldsymbol{y}_{\mathrm{aw}, \mathrm{u}}=-\boldsymbol{C}_{\mathrm{s}} \boldsymbol{x}_{\mathrm{aw}}, \\
\boldsymbol{y}_{\mathrm{aw}, \mathrm{y}}=-\mathbf{s a t}_{\alpha \overline{\mathbf{u}}}\left(-\underline{\boldsymbol{K}} \boldsymbol{x}_{\mathrm{aw}}\right)
\end{array}\right.
$$

auf den Entwurf eines sättigenden linearen Zustandsreglers für das in Mismatch-Koordinaten vorliegende Anti-Windup-Ersatzsystem

$$
\begin{aligned}
\dot{\boldsymbol{\xi}}_{\mathrm{aw}} & =\boldsymbol{A}_{\mathrm{s}} \boldsymbol{\xi}_{\mathrm{aw}}+\boldsymbol{B}_{\mathrm{s}, u} \mathbf{s a t}_{\alpha \overline{\mathbf{u}}}\left(-\underline{\boldsymbol{K}} \boldsymbol{\xi}_{\mathrm{aw}}\right)+\boldsymbol{B}_{\mathrm{s}, u} \boldsymbol{\zeta}, \\
\boldsymbol{y}_{\mathrm{aw}, \mathrm{u}} & =\boldsymbol{C}_{\mathrm{s}} \boldsymbol{\xi}_{\mathrm{aw}}
\end{aligned}
$$

reduziert. Da der Ausgang $\boldsymbol{y}_{\mathrm{aw}, \mathrm{u}}=\boldsymbol{C}_{\mathrm{s}} \boldsymbol{\xi}_{\mathrm{aw}}$ die Abweichung von dem idealen unbeschränkten Verhalten darstellt, besteht das Entwurfsziel für die Zustandsrückführung darin, den Zustand $\boldsymbol{\xi}_{\text {aw }}$ in einer möglichst kleinen Umgebung des Ursprungs zu halten bzw. eine möglichst schnelle Ausregelung in den Ursprung zu garantieren. Durch die Verwendung eines linearen $\mathrm{Zu}-$ standsreglers entsteht die einfachst mögliche Anti-Windup-Strategie, die in vielen Fällen gute Ergebnisse liefert.

Der Einfachheit einer linearen Zustandsrückführung steht jedoch eine mäßige Regelgüte in einer Umgebung des Ursprungs gegenüber. Das liegt 
an der durch die Linearität des Regelgesetzes bedingte schlechte Ausnutzung der Stellgröße. Eine strukturvariable Zustandsrückführung löst dieses Problem. Die seit langem bekannte Grundidee ist, immer aggressivere Zustandsregler zu aktivieren je näher die Systemzustände dem Ursprung kommen [109, 110].

Die Arbeiten [53, 210] setzen diese Idee erstmals im Kontext von modellbasiertem Anti-Windup um. Dabei wird in Abhängigkeit des Systemzustandes zwischen einer festen Anzahl an linearen Zustandsrückführungen $\underline{\boldsymbol{K}}_{1}, \ldots, \underline{\boldsymbol{K}}_{N}$ umgeschaltet. Eine weitere Steigerung ist durch unendlich viele Zustandsregler und infinitesimal kleine Umschaltintervalle erreichbar. Die Reglerparameter ändern sich dann kontinuierlich und man spricht von weich strukturvariablen Zustandsregelungen [4, 38, 97].

Die hinsichtlich der Regelgüte am weitesten entwickelte modellbasierte Anti-Windup-Maßnahme ist bislang das weich strukturvariable AntiWindup aus [52, 54]. Dabei werden die Parameter der Zustandsrückführung durch eine konvexe Kombination linearer Zustandsregler kontinuierlich an den aktuellen Systemzustand angepasst. Problematisch ist der hohe Entwurfsaufwand und die Komplexität des entstehenden Anti-Windup. Letztere kann zu Schwierigkeiten bei der Implementierung führen. Wünschenswert ist deshalb ein Anti-Windup mit vergleichbarer hoher Regelgüte, das eine schlankere Systemstruktur aufweist und sich einfach entwerfen und implementieren lässt.

Dieses Kapitel stellt ein neues, auf impliziten Ljapunov-Funktionen basierendes Anti-Windup-Konzept vor, das mit ISOVAW (engl. Implicit SOft Variable-structure Anti-Windup) bezeichnet wird. Es baut auf der in $[38,122,123]$ entwickelten und in [98] vereinfachten impliziten weich strukturvariablen Zustandsregelung auf. Die Vorteile gegenüber [52, 54] sind ein wesentlich geringerer Entwurfsaufwand und eine unkompliziertere Regelstrategie bei vergleichbarer Regelgüte.

\subsection{Weich strukturvariable Regelung}

Im diesem Abschnitt werden die Grundlagen der vereinfachten impliziten weich strukturvariablen Zustandsregelung aus [98] kurz zusammengefasst. Anschließend erfolgt eine Erweiterung des Regelkonzeptes, die es ermöglicht, stabile Strecken global zu stabilisieren. Diese Vorüberlegungen bilden die Basis für Abschnitt 8.3, in dem die Zustandsregelung mithilfe der Ergebnisse aus Kapitel 6 in ein modellbasiertes Anti-Windup integriert wird. 


\subsubsection{Grundlagen}

Ausgangspunkt ist eine lineare, steuer- und beobachtbare Strecke

$$
\dot{\boldsymbol{x}}=\boldsymbol{A} \boldsymbol{x}+\boldsymbol{b} \operatorname{sat}_{\overline{\mathrm{u}}}(u)
$$

mit dem Zustand $\boldsymbol{x} \in \mathbb{R}^{n}$ und dem Eingang $\operatorname{sat}_{\overline{\mathrm{u}}}(u) \in \mathbb{R}$. Diese liege in Steuerungsnormalform vor, d. h., die Systemmatrizen besitzen die Gestalt

$$
\boldsymbol{A}=\left[\begin{array}{ccccc}
0 & 1 & 0 & \cdots & 0 \\
\vdots & \ddots & \ddots & \ddots & \vdots \\
\vdots & & \ddots & \ddots & 0 \\
0 & \cdots & \cdots & 0 & 1 \\
-a_{0} & \cdots & \cdots & \cdots & -a_{n-1}
\end{array}\right], \quad \boldsymbol{b}=\left[\begin{array}{c}
0 \\
\vdots \\
\vdots \\
0 \\
1
\end{array}\right]
$$

Die letzte Zeile der Matrix $\boldsymbol{A}$ enthält die negativen Koeffizienten des charakteristischen Polynoms der Strecke. Da die Polynomkoeffizienten im Weiteren mehrfach benötigt werden, bietet es sich an, diese kompakt zusammenzufassen in dem Vektor

$$
\boldsymbol{a}^{\boldsymbol{\top}}=\left[\begin{array}{lll}
a_{0} & \ldots & a_{n-1}
\end{array}\right] .
$$

Nun lässt sich das weich strukturvariable Regelgesetz angeben. Dessen grundlegende Idee, die schon in $[1,110]$ formuliert wurde, besteht darin, die Reglerparameter einer linearen Zustandsrückführung kontinuierlich zu verändern, so dass „eine gute Ausnutzung der Stellgröße trotz des zunehmend geringer werdenden Abstandes zur Ruhelage gewährleistet ist" [97].

Dies wird mit der Reglerstruktur

$$
\begin{aligned}
u & =-\boldsymbol{k}^{\top}(v) \boldsymbol{x} \\
\boldsymbol{k}(v) & =\boldsymbol{H}^{-1}(v) \boldsymbol{k}_{1}-\boldsymbol{a}, \\
\boldsymbol{H}(v) & =\operatorname{diag}\left(v^{n}, \ldots, v^{2}, v\right)
\end{aligned}
$$

realisiert [1,4], wobei $\boldsymbol{k}_{1} \in \mathbb{R}^{n}$ ein beim Entwurf zu bestimmender Vektor und $v \in(0,1]$ der während des Ausregelverlaufes zu berechnende Selektionsparameter ist. Letzterer bestimmt die Veränderung der Reglerkoeffizienten und wird in Abhängigkeit des Systemzustandes durch ein Auswahlgesetz, die sogenannte Selektionsstrategie, berechnet. Abbildung 8.1 zeigt das Blockschaltbild des Regelkreises.

Der Selektionsparameter ist implizit über das Auswahlgesetz

$$
g(v, \boldsymbol{x})=0
$$




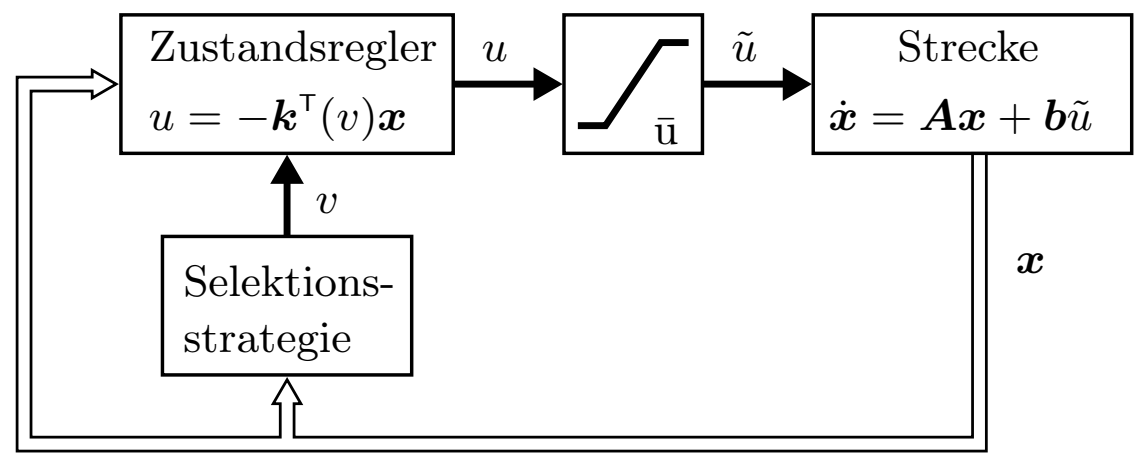

Abbildung 8.1: Regelkreis mit weich strukturvariabler Zustandsregelung.

definiert, wobei die linke Seite der Gleichung durch

$$
\begin{aligned}
g(v, \boldsymbol{x}) & =\boldsymbol{x}^{\top} \boldsymbol{R}(v) \boldsymbol{x}-1 \\
\boldsymbol{R}(v) & =\boldsymbol{H}^{-1}(v) \boldsymbol{R}_{1} \boldsymbol{H}^{-1}(v)
\end{aligned}
$$

mit einer beim Entwurf zu bestimmenden symmetrischen, positiv definiten Matrix $\boldsymbol{R}_{1} \in \mathbb{S}^{n_{\mathrm{s}}}$ gegeben ist. Somit beschreibt die Menge

$$
\mathcal{E}(v)=\left\{\boldsymbol{x} \in \mathbb{R}^{n_{\mathrm{s}}} \mid g(v, \boldsymbol{x})<0\right\}
$$

für alle $v \in(0,1]$ ellipsoide Bereiche im Zustandsraum. Diese Hyperellipsoide sind ineinander geschachtelt, d. h. für $0<v_{1}<v_{2} \leq 1$ gilt

$$
\mathcal{E}\left(v_{1}\right) \subset \mathcal{E}\left(v_{2}\right) .
$$

Im Fall $n=2$ ergeben sich Ellipsen, wie Abbildung 8.2 beispielhaft für drei Werte des Selektionsparameters illustriert. Jeder Ellipse $\mathcal{E}(v)$ ist dabei über die Selektionsstrategie ein Reglervektor $\boldsymbol{k}(v)$ zugeordnet. Die spezielle Wahl des Regelgesetzes (8.3) garantiert, dass die Eigenwerte der Matrix $\boldsymbol{A}-\boldsymbol{b} \boldsymbol{k}^{\top}(v)$ mit sinkendem $v$ in der komplexen Ebene auf Strahlen nach links verschoben werden [4, 122]. Beim Eintritt des Systemzustandes in eine kleinere Ellipse wird demnach ein aggressiverer Regler aktiviert. Das führt zu einer schnellen Ausregelung. Bezüglich der Stabilität des geschlossenen Regelkreises gilt

Satz 8.1 (vgl. Satz 1, Satz 4 in [98]). Betrachtet wird die Strecke (8.1) mit der Zustandsrückführung (8.3) und der Selektionsstrategie (8.4). Wenn eine positiv definite Matrix $\boldsymbol{R}_{1} \in \mathbb{S}^{n}$ und Vektoren $\boldsymbol{k}_{1}, \boldsymbol{k}_{2} \in \mathbb{R}^{n}$ existieren, 


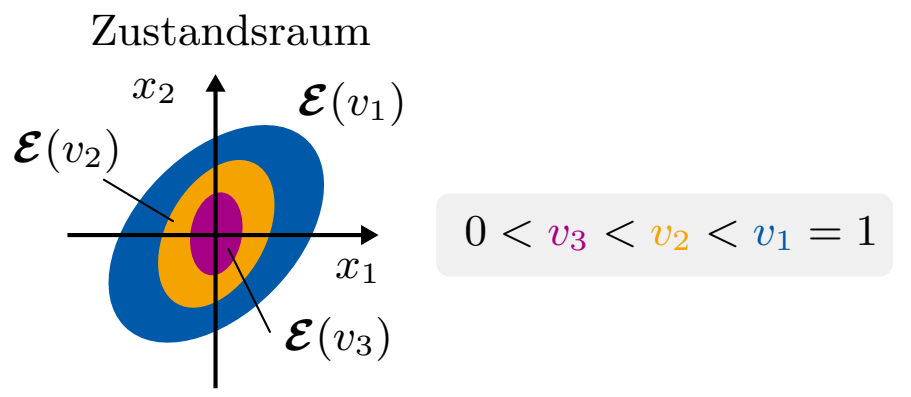

komplexe Ebene

ellipsoide Bereiche $\mathcal{E}(v)$

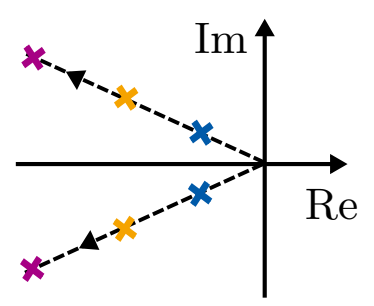

Eigenwerte von $\boldsymbol{A}-\boldsymbol{b}^{\top}(v)$

Abbildung 8.2: Die Selektionsstrategie (8.4) definiert ineinander geschachtelte Hyperellipsoide $\mathcal{E}(v)$ im Zustandsraum. Jedem Hyperellipsoid ist ein Reglervektor $\boldsymbol{k}(v)$ zugeordnet. Beim Eintritt des Systemzustandes in eine kleinere Ellipse wird ein Regler aktiv, der die Eigenwerte der Matrix $\boldsymbol{A}-\boldsymbol{b} \boldsymbol{k}^{\top}(v)$ in der komplexen Ebene weiter nach links verschiebt.

so dass für $\boldsymbol{N}=-\operatorname{diag}(n, n-1, \ldots, 1)$ und alle $v \in(0,1]$ gilt

$$
\begin{aligned}
\left(\boldsymbol{A}_{0}-\boldsymbol{b} \boldsymbol{k}_{1}^{\top}\right)^{\top} \boldsymbol{R}_{1}+\boldsymbol{R}_{1}\left(\boldsymbol{A}_{0}-\boldsymbol{b} \boldsymbol{k}_{1}^{\top}\right) & \prec \mathbf{0}, \\
\left(\boldsymbol{A}_{0}-\boldsymbol{b} \boldsymbol{k}_{2}^{\top}\right)^{\top} \boldsymbol{R}_{1}+\boldsymbol{R}_{1}\left(\boldsymbol{A}_{0}-\boldsymbol{b} \boldsymbol{k}_{2}^{\top}\right) & \prec \mathbf{0}, \\
{\left[\begin{array}{cc}
\bar{u}^{2} & \boldsymbol{k}_{2}^{\top}-\boldsymbol{a}^{\top} \boldsymbol{H}(v) \\
\boldsymbol{k}_{2}-\boldsymbol{H}(v) \boldsymbol{a} & \boldsymbol{R}_{1}
\end{array}\right] } & \succeq \mathbf{0}, \\
\boldsymbol{N} \boldsymbol{R}_{1}+\boldsymbol{R}_{1} \boldsymbol{N} & \prec \mathbf{0},
\end{aligned}
$$

wobei $\boldsymbol{A}_{0}=\boldsymbol{A}+\boldsymbol{b a}^{\top}$, dann

(a) hat die Selektionsstrategie (8.4) eine eindeutige Lösung auf dem Intervall $(0,1]$ für alle $\boldsymbol{x} \in \mathcal{E}(1)=\left\{\boldsymbol{x} \in \mathbb{R}^{n} \mid \boldsymbol{x}^{\top} \boldsymbol{R}_{1} \boldsymbol{x}<1\right\} \backslash\{\mathbf{0}\}$ und ist stetig erweiterbar in $\boldsymbol{x}=\mathbf{0}$,

(b) ist die Ruhelage $\boldsymbol{x}^{(\mathrm{R})}=\mathbf{0}$ des geschlossenen Regelkreises asymptotisch stabil,

(c) ist durch (8.4) in $\mathcal{E}(1) \backslash\{\mathbf{0}\}$ eine Funktion $v(\boldsymbol{x})$ implizit definiert, die in $\boldsymbol{x}=\mathbf{0}$ durch $v(\mathbf{0})=0$ stetig erweiterbar ist. Diese erweiterte Funktion ist eine Ljapunov-Funktion des geschlossenen Regelkreises,

(d) ist für $v \in(0,1]$ jede Ellipse $\mathcal{E}(v)=\left\{\boldsymbol{x} \in \mathbb{R}^{n} \mid g(v, \boldsymbol{x})<0\right\}$ ein Einzugsgebiet der Ruhelage $\boldsymbol{x}^{(\mathrm{R})}=\mathbf{0}$. 
Beweis. Die Punkte (a) bis (c) folgen direkt aus Satz 1 bzw. Satz 4 in [98]. Punkt (d) folgt aus Satz 5 in [2].

Aus numerischen Gründen ist es im Hinblick auf eine praktische Implementierung der Regelung sinnvoll, einen Minimalwert $v_{\min }>0$ für den Selektionsparameter $v$ festzulegen $[4,122]$, zum Beispiel $v_{\min }=0,01$. Nachfolgend wird deshalb $v \in\left[v_{\min }, 1\right]$ angenommen. Für $v \leq v_{\min }, \mathrm{d}$. h. für alle Zustände $\boldsymbol{x}$ in der Hyperellipse

$$
\overline{\mathcal{E}}\left(v_{\text {min }}\right)=\left\{\boldsymbol{x} \in \mathbb{R}^{n_{\mathrm{s}}} \mid \boldsymbol{x}^{\top} \boldsymbol{R}\left(v_{\text {min }}\right) \boldsymbol{x}-1 \leq 0\right\},
$$

wird dann $\boldsymbol{k}(v)=\boldsymbol{k}\left(v_{\min }\right)$ gewählt. Die Selektionsstrategie lautet

$$
v=\left\{\begin{array}{cl}
v_{\min }, & \boldsymbol{x} \in \overline{\mathcal{E}}\left(v_{\min }\right), \\
\text { eindeutige Lösung von } g(v, \boldsymbol{x})=0, & \boldsymbol{x} \in \mathcal{E}(1) \backslash \overline{\mathcal{E}}\left(v_{\min }\right) .
\end{array}\right.
$$

Auch mit dieser Selektionsstrategie bleibt der Ursprung des geschlossenen Regelkreises eine asymptotisch stabile Ruhelage mit dem Einzugsgebiet $\mathcal{E}(1)$. Das garantiert

Lemma 8.1. Betrachtet wird die Strecke (8.1) mit der Zustandsrückführung (8.3) und der Selektionsstrategie (8.6). Wenn alle Bedingungen des Satzes 8.1 erfüllt sind, dann ist die Ruhelage $\boldsymbol{x}^{(\mathrm{R})}=\mathbf{0}$ des geschlossenen Regelkreises asymptotisch stabil und $\mathcal{E}(1)$ ist ein Einzugsgebiet.

Beweis. Der Beweis findet sich in C.3.12

\subsubsection{Entwurf mittels konvexer Optimierung}

Wie in [98] lassen sich die Bedingungen (8.5) aus Satz 8.1 mittels der Substitutionen

$$
\boldsymbol{Q}=\boldsymbol{R}_{1}^{-1}, \boldsymbol{q}_{1}=\boldsymbol{R}_{1}^{-1} \boldsymbol{k}_{1}, \boldsymbol{q}_{2}=\boldsymbol{R}_{1}^{-1} \boldsymbol{k}_{2}
$$

für den Reglerentwurf als lineare Matrixungleichungen formulieren. Es muss dann für alle $v \in\left[v_{\min }, 1\right]$ gelten

$$
\begin{aligned}
& Q \succ \mathbf{0}, \\
& Q N+N Q \prec \mathbf{0}, \\
& \boldsymbol{P}(v)=\left[\begin{array}{cc}
\overline{\mathrm{u}}^{2} & \boldsymbol{q}_{2}^{\top}-\boldsymbol{a}^{\top} \boldsymbol{H}(v) \boldsymbol{Q} \\
\boldsymbol{q}_{2}-\boldsymbol{Q} \boldsymbol{H}(v) \boldsymbol{a} & \boldsymbol{Q}
\end{array}\right] \succeq \mathbf{0}, \\
& \boldsymbol{Q} \boldsymbol{A}_{0}^{\top}+\boldsymbol{A}_{0} \boldsymbol{Q}-\boldsymbol{q}_{2} \boldsymbol{b}^{\top}-\boldsymbol{b} \boldsymbol{q}_{2}^{\top} \prec \mathbf{0}, \\
& \boldsymbol{Q} \boldsymbol{A}_{0}^{\top}+\boldsymbol{A}_{0} \boldsymbol{Q}-\boldsymbol{q}_{1} \boldsymbol{b}^{\top}-\boldsymbol{b} \boldsymbol{q}_{1}^{\top} \prec \mathbf{0} .
\end{aligned}
$$


Die Matrix $\boldsymbol{P}(v)$ in (8.7c) ist aufgrund von $\boldsymbol{H}(v)$ gemäß folgender Definition ein Matrixpolynom.

Definition 8.1 (Matrixpolynom). Eine Matrix $\boldsymbol{P}(v)$, deren Elemente $p_{i, j}(v)$ Polynome in $v$ sind, heißt Matrixpolynom.

Beim Entwurf müssen die Matrix $\boldsymbol{Q}$ und der Vektor $\boldsymbol{q}_{2}$ so gewählt werden, dass die positive Semidefinitheit von $\boldsymbol{P}(v)$ für alle $v \in\left[v_{\min }, 1\right]$ garantiert ist. Im Allgemeinen ist das ein schwieriges Problem. Beschränkt man sich allerdings auf Sum-of-Squares-Matrixpolynome (SOSMatrixpolynome) $\boldsymbol{P}(v)$, so kann die Wahl von $\boldsymbol{Q}$ und $\boldsymbol{q}_{2}$ mittels der Sumof-Squares-Zerlegung sehr recheneffizient durchgeführt werden ${ }^{1}[33,153]$. SOS-Matrixpolynome sind wie folgt definiert.

Definition 8.2 (SOS-Matrixpolynom, [73]). Die Menge der SOS-Matrixpolynome $\Sigma^{r}[v]$ der Dimension $r$ ist die Menge aller symmetrischen Matrixpolynome $\boldsymbol{P}(v) \in \mathbb{S}^{r}$, die sich mit den Matrixpolynomen $\boldsymbol{P}_{i}(v) \in \mathbb{R}^{r \times r}$ darstellen lassen als

$$
\boldsymbol{P}(v)=\sum_{i} \boldsymbol{P}_{i}^{\top}(v) \boldsymbol{P}_{i}(v)
$$

Alle SOS-Matrixpolynome sind positiv semidefinit für alle $v \in \mathbb{R}$. Wenn sich zeigen lässt, dass ein Matrixpolynom zur Menge $\Sigma^{r}[v]$ gehört, dann ist dessen positive Semidefinitheit gezeigt, d.h.

$$
\boldsymbol{P}(v) \in \Sigma^{r}[v] \Rightarrow \boldsymbol{P}(v) \succeq 0 \quad \forall v \in \mathbb{R} .
$$

Um die positive Semidefinitheit eines Matrixpolynoms in einem Intervall zu testen, verwendet man

Lemma 8.2 (S-Prozedur, [96]). Gegeben sei ein Matrixpolynom $\boldsymbol{P}(v) \in \mathbb{S}^{r}$ und ein Polynom

$$
\eta(v)=\mathrm{c}_{n} v^{n}+\ldots+\mathrm{c}_{1} v+\mathrm{c}_{0} .
$$

Wenn ein SOS-Matrixpolynom $\boldsymbol{T}(v) \in \Sigma^{r}[v]$ existiert, so dass

$$
\boldsymbol{P}(v)-\boldsymbol{T}(v) \eta(v) \in \Sigma^{r}[v],
$$

dann gilt $\boldsymbol{P}(v) \succeq 0 \quad \forall v \in \mathcal{H}=\{v \in \mathbb{R} \mid \eta(v) \geq 0\}$.

\footnotetext{
${ }^{1}$ In [98] wird eine alternative Vorgehensweise gewählt.
} 
Eine sinnvolle Wahl für die Festlegung des Intervalls $\mathcal{H}=\left[v_{\min }, 1\right]$ ist das Polynom

$$
\eta(v)=-\left(v-v_{\min }\right)(v-1),
$$

dessen zwei Nullstellen den Intervallgrenzen entsprechen. Um die positive Semidefinitheit von $\boldsymbol{P}(v)$ in $\mathcal{H}$ zu testen, muss nach einem SOSMatrixpolynom $\boldsymbol{T}(v)$ gesucht werden, so dass Bedingung (8.8) erfüllt ist. Die spezielle Struktur von $\boldsymbol{P}(v)$ aus $(8.7 \mathrm{c})$ erlaubt allerdings eine Vereinfachung der Bedingung, so dass sich die Suche auf ein SOS-Polynom beschränken kann.

Mithilfe des Schur-Komplements (Lemma B.1) kann gezeigt werden, dass folgende Bedingungen äquivalent sind

$$
\begin{gathered}
\boldsymbol{P}(v)=\left[\begin{array}{cc}
\overline{\mathrm{u}}^{2} & \boldsymbol{q}_{2}^{\top}-\boldsymbol{a}^{\top} \boldsymbol{H}(v) \boldsymbol{Q} \\
\boldsymbol{q}_{2}-\boldsymbol{Q} \boldsymbol{H}(v) \boldsymbol{a} & \boldsymbol{Q}
\end{array}\right] \succeq \mathbf{0}, \\
\overline{\mathrm{u}}^{2}-\left(\boldsymbol{q}_{2}^{\top}-\boldsymbol{a}^{\top} \boldsymbol{H}(v) \boldsymbol{Q}\right) \boldsymbol{Q}^{-1}
\end{gathered}
$$

Es reicht somit aus, die Nichtnegativität des Polynoms (8.9) für alle $v \in$ $\left[v_{\min }, 1\right]$ sicherzustellen. Nach Lemma 8.2 führt das zu der Forderung

$$
\overline{\mathrm{u}}^{2}-\left(\boldsymbol{q}_{2}^{\top}-\boldsymbol{a}^{\top} \boldsymbol{H}(v) \boldsymbol{Q}\right) \boldsymbol{Q}^{-1}\left(\boldsymbol{q}_{2}^{\top}-\boldsymbol{a}^{\top} \boldsymbol{H}(v) \boldsymbol{Q}\right)^{\top}-\chi(v) \eta(v) \geq 0
$$

mit dem Polynom $\chi(v) \geq 0$ für alle $v \in \mathbb{R}$.

Eine erneute Anwendung des Schur-Komplements liefert

$$
\hat{\boldsymbol{P}}(v)=\left[\begin{array}{cc}
\overline{\mathrm{u}}^{2}-\chi(v) \eta(v) & \boldsymbol{q}_{2}^{\top}-\boldsymbol{a}^{\top} \boldsymbol{H}(v) \boldsymbol{Q} \\
\boldsymbol{q}_{2}-\boldsymbol{Q} \boldsymbol{H}(v) \boldsymbol{a} & \boldsymbol{Q}
\end{array}\right] \succeq \mathbf{0}
$$

mit $\chi(v) \geq 0$ für alle $v \in \mathbb{R}$. Ersetzen von $\chi(v)$ durch ein SOS-Polynom und die Forderung $\hat{\boldsymbol{P}}(v) \in \Sigma^{n_{\mathrm{s}}+1}[v]$ führen zu SOS-Entwurfsbedingungen, welche mittels der frei erhältlichen Matlab-Toolbox YALmiP [134] automatisiert in LMI-Bedingungen umgewandelt werden können.

Möchte man den Vektor $\boldsymbol{k}_{1}$ vorgeben und ausschließlich $\boldsymbol{R}_{1}$ entwerfen, ergeben sich nach der Rücksubstitution

$$
\boldsymbol{q}_{1}=\boldsymbol{R}_{1}^{-1} \boldsymbol{k}_{1}
$$


aus (8.7) die Bedingungen

$$
\begin{aligned}
& \boldsymbol{Q} \succ \mathbf{0}, \\
& Q N+N Q \prec \mathbf{0}, \\
& \boldsymbol{Q} \boldsymbol{A}_{0}^{\top}+\boldsymbol{A}_{0} \boldsymbol{Q}-\boldsymbol{q}_{2} \boldsymbol{b}^{\top}-\boldsymbol{b} \boldsymbol{q}_{2}^{\top} \prec \mathbf{0}, \\
& \boldsymbol{Q} \boldsymbol{A}_{0}^{\top}+\boldsymbol{A}_{0} \boldsymbol{Q}-\boldsymbol{Q} \boldsymbol{k}_{1} \boldsymbol{b}^{\top}-\boldsymbol{b} \boldsymbol{k}_{1}^{\top} \boldsymbol{Q} \prec \mathbf{0}, \\
& {\left[\begin{array}{lc}
\overline{\mathrm{u}}^{2}-\chi(v) \eta(v) & \boldsymbol{q}_{2}^{\top}-\boldsymbol{a}^{\top} \boldsymbol{H}(v) \boldsymbol{Q} \\
\boldsymbol{q}_{2}-\boldsymbol{Q} \boldsymbol{H}(v) \boldsymbol{a} & \boldsymbol{Q}
\end{array}\right] \in \Sigma^{n_{\mathrm{s}}+1}[v],} \\
& \chi(v) \in \Sigma[v],
\end{aligned}
$$

mit dem Polynom $\eta(v)=-\left(v-v_{\min }\right)(v-1)$. Der Grad des Hilfspolynoms $\chi(v)$ kann frei gewählt werden.

Ein Nachteil der in diesem Abschnitt vorgestellten weich strukturvariablen Zustandsregelung ist, dass der Ursprung immer nur lokal stabilisiert werden kann. Ein Einzugsgebiet ist durch die äußerste Ellipse $\mathcal{E}(1)$ gegeben. Durch eine kleine Modifikation der Selektionsstrategie und einen weiteren Entwurfsschritt lässt sich jedoch ein unendlich großes Einzugsgebiet für stabile Strecken erreichen. Details des zugehörigen nichtlinearen Zustandsreglers werden im nächsten Abschnitt erläutert.

\subsubsection{Erweiterung des Regelkonzeptes}

In diesem Abschnitt wird eine stabile SISO-Strecke der Form

$$
\dot{\boldsymbol{x}}=\boldsymbol{A} \boldsymbol{x}+\boldsymbol{b} \operatorname{sat}_{\overline{\mathrm{u}}}(u)
$$

betrachtet, die durch ein modifiziertes weich strukturvariables Regelgesetz global stabilisiert werden soll. Das Ziel der weich strukturvariablen Zustandsregelung besteht darin, den Ausregelvorgang in der Nähe der Ruhelage zu beschleunigen. Dazu wird der Parameter $v$ und damit der Reglervektor $\underline{\boldsymbol{k}}$ in Abhängigkeit des Systemzustandes verändert. In einer ellipsoiden Umgebung der Ruhelage

$$
\mathcal{E}(1)=\left\{\boldsymbol{x} \in \mathbb{R}^{n_{\mathrm{s}}} \mid \boldsymbol{x}^{\top} \boldsymbol{R}(1) \boldsymbol{x}<1\right\}
$$

kommt das in Abschnitt 8.2.1 vorgestellte Regelgesetz

$$
\begin{aligned}
u & =-\boldsymbol{k}^{\top}(v) \boldsymbol{x}, \\
\boldsymbol{k}(v) & =\boldsymbol{H}^{-1}(v) \boldsymbol{k}_{1}-\boldsymbol{a}
\end{aligned}
$$


zum Einsatz, mit dem zu wählenden Vektor $\boldsymbol{k}_{1}$. Der Parameter $v$ wird in Abhängigkeit des Zustandes $\boldsymbol{x}$ wie folgt bestimmt

$$
v=\left\{\begin{array}{cl}
v_{\min }, & \boldsymbol{x} \in \overline{\mathcal{E}}\left(v_{\min }\right), \\
\text { eindeutige Lösung von } g(v, \boldsymbol{x})=0, & \boldsymbol{x} \in \mathcal{E}(1) \backslash \overline{\mathcal{E}}\left(v_{\text {min }}\right),
\end{array}\right.
$$

wobei die Hyperellipse $\overline{\mathcal{E}}\left(v_{\text {min }}\right)$ wie folgt definiert ist

$$
\overline{\mathcal{E}}\left(v_{\text {min }}\right)=\left\{\boldsymbol{x} \in \mathbb{R}^{n_{\mathrm{s}}} \mid \boldsymbol{x}^{\top} \boldsymbol{R}\left(v_{\text {min }}\right) \boldsymbol{x} \leq 1\right\} .
$$

Offensichtlich ist das Regelgesetz bzw. die Selektionsstrategie nur auf einer Teilmenge $\mathcal{E}(1)$ des $\mathbb{R}^{n_{\mathrm{s}}}$ definiert. Um globale Stabilität zu erreichen, muss die Definition auf den gesamten Zustandsraum ausgeweitet werden. Eine naheliegende Wahl ist

$$
v=\left\{\begin{array}{cl}
v_{\text {min }}, & \boldsymbol{x} \in \overline{\mathcal{E}}\left(v_{\min }\right), \\
\text { eindeutige Lösung von } g(v, \boldsymbol{x})=0, & \boldsymbol{x} \in \mathcal{E}(1) \backslash \overline{\mathcal{E}}\left(v_{\min }\right), \\
1, & \boldsymbol{x} \in \mathbb{R}^{n_{\mathrm{s}}} \backslash \mathcal{E}(1) .
\end{array}\right.
$$

Abbildung 8.3 visualisiert die neue Selektionsstrategie beispielhaft im $\mathbb{R}^{2}$ für $v_{\min }=0,3$. Außerhalb der Ellipse $\mathcal{E}(1)$ wird demnach der lineare Zustandsregler $\boldsymbol{k}(1)$ eingesetzt, für den gemäß (8.12) gilt

$$
\boldsymbol{k}(1)=\boldsymbol{H}^{-1}(1) \boldsymbol{k}_{1}-\boldsymbol{a}=\boldsymbol{k}_{1}-\boldsymbol{a} .
$$

Der Vektor $\boldsymbol{a}$ lässt sich durch die spezielle Wahl

$$
\boldsymbol{k}_{1}=\boldsymbol{k}_{g}+\boldsymbol{a}
$$

eliminieren, so dass sich für die Dynamik des geschlossenen Regelkreises außerhalb der Ellipse $\mathcal{E}(1)$ die folgende Differentialgleichung ergibt

$$
\dot{\boldsymbol{x}}=\boldsymbol{A} \boldsymbol{x}+\boldsymbol{b} \operatorname{sat}_{\overline{\mathrm{u}}}\left(-\boldsymbol{k}_{g}^{\top} \boldsymbol{x}\right) .
$$

Innerhalb der Ellipse $\mathcal{E}(1)$ gilt wie in den vorherigen Abschnitten

$$
\dot{\boldsymbol{x}}=\boldsymbol{A} \boldsymbol{x}+\boldsymbol{b} \text { sat }_{\overline{\mathrm{u}}}\left(-\boldsymbol{k}^{\top}(v) \boldsymbol{x}\right),
$$

wobei $v$ über die Selektionsstrategie (8.14) bestimmt wird. Der entscheidende Punkt für die weiteren Überlegungen ist, dass die Ellipse $\mathcal{E}(1)$ durch eine geschickte Wahl des Reglers $\boldsymbol{k}_{g}$ global attraktiv wird. Dann streben alle Trajektorien, die außerhalb von $\mathcal{E}(1)$ starten, in die Ellipse. Ist darüber hinaus die Ellipse ein Einzugsgebiet der Ruhelage $\boldsymbol{x}^{(\mathrm{R})}=\mathbf{0}$, streben die Trajektorien sogar in die Ruhelage. Es gilt 


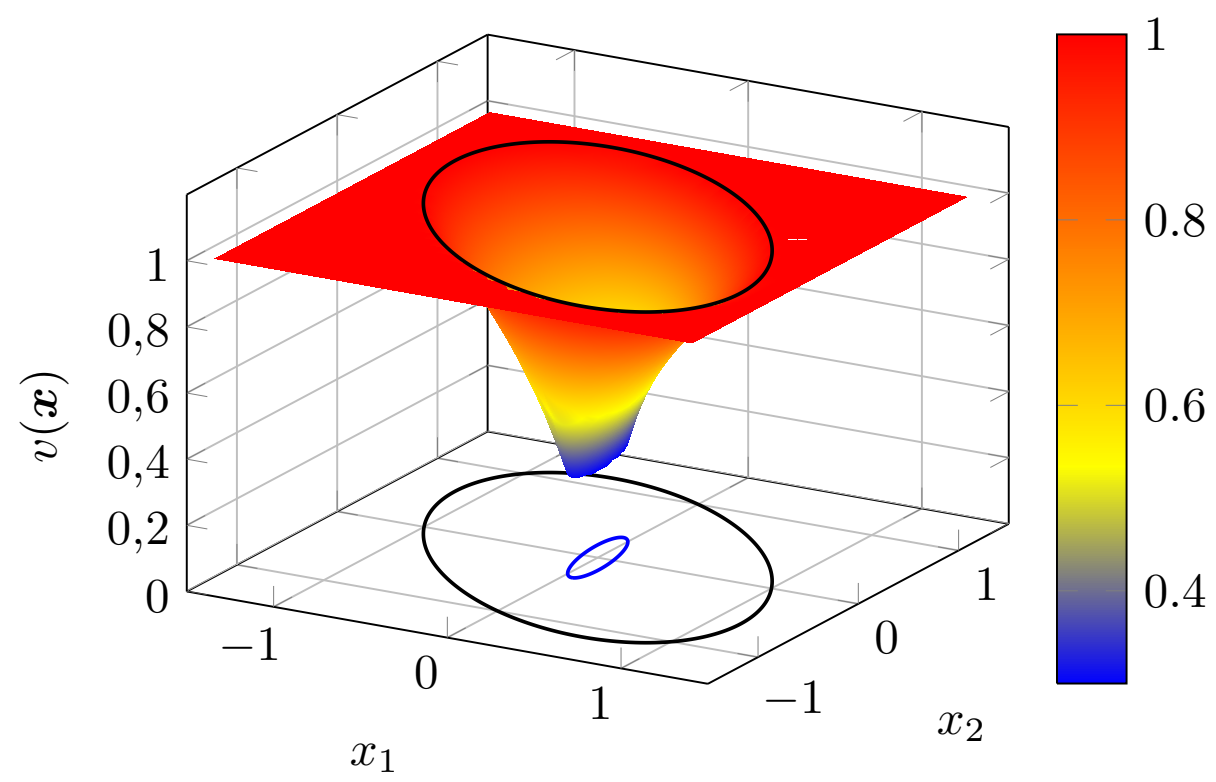

Abbildung 8.3: Visualisierung der Selektionsstrategie $v(\boldsymbol{x})$ im $\mathbb{R}^{2}$. Der Rand von $\mathcal{E}(1)$ ist in $(\longrightarrow)$ dargestellt, der Rand von $\mathcal{E}\left(v_{\min }=0,3\right)$ in $(-)$.

Lemma 8.3. Gegeben sei die stabile Strecke (8.11), das Regelgesetz

$$
\begin{aligned}
u & =-\boldsymbol{k}^{\top}(v) \boldsymbol{x}, \\
\boldsymbol{k}(v) & =\boldsymbol{H}^{-1}(v)\left(\boldsymbol{k}_{g}+\boldsymbol{a}\right)-\boldsymbol{a}
\end{aligned}
$$

und die Selektionsstrategie (8.14) mit

$$
g(v, \boldsymbol{x})=\boldsymbol{x}^{\top} \boldsymbol{H}^{-1}(v) \boldsymbol{R}_{1} \boldsymbol{H}^{-1}(v) \boldsymbol{x}-1 .
$$

Die Matrix $\boldsymbol{R}_{1}$ und der Vektor $\boldsymbol{k}_{1}=\boldsymbol{k}_{g}+\boldsymbol{a}$ erfüllen die Bedingungen (8.10). Wenn die Ruhelage $\boldsymbol{x}^{(\mathrm{R})}=\mathbf{0}$ des Systems

$$
\dot{\boldsymbol{x}}=\boldsymbol{A} \boldsymbol{x}+\boldsymbol{b} \operatorname{sat}_{\bar{u}}\left(-\boldsymbol{k}_{g}^{\top} \boldsymbol{x}\right)
$$

global asymptotisch stabil ist, dann ist auch der Ursprung des geschlossenen Regelkreises

$$
\dot{\boldsymbol{x}}=\boldsymbol{A} \boldsymbol{x}+\boldsymbol{b} \operatorname{sat}_{\bar{u}}\left(-\boldsymbol{k}(v)^{\top} \boldsymbol{x}\right)
$$

eine global asymptotisch stabile Ruhelage.

Beweis. Der Beweis findet sich im Anhang C.3.13.

Im nächsten Abschnitt wird gezeigt, wie die global stabilisierende weich strukturvariable Zustandsregelung in einer modellbasierten Anti-WindupMaßnahme eingesetzt werden kann. 


\subsection{Weich strukturvariables Anti-Windup}

Erneut wird der in Kapitel 6 beschriebene Anti-Windup-Regelkreis mit der Dynamik (6.3) betrachtet, der aus einer in Regelungsnormalform vorliegenden SISO-Strecke

$$
\mathbf{S}:\left\{\begin{array}{l}
\dot{\boldsymbol{x}}_{\mathrm{s}}=\boldsymbol{A}_{\mathrm{s}} \boldsymbol{x}_{\mathrm{s}}+\boldsymbol{b}_{\mathrm{s}, u} u_{\mathrm{s}}+\boldsymbol{b}_{\mathrm{s}, z} z \\
y_{\mathrm{s}}=\boldsymbol{c}_{\mathrm{s}}^{\top} \boldsymbol{x}_{\mathrm{s}}
\end{array}\right.
$$

einem Regler $\mathbf{R}$ und einem modellbasierten Anti-Windup der Form

$$
\mathbf{A W}:\left\{\begin{array}{l}
\dot{\boldsymbol{x}}_{\mathrm{aw}}=\boldsymbol{A}_{\mathrm{s}} \boldsymbol{x}_{\mathrm{aw}}+\boldsymbol{b}_{\mathrm{s}, u} \operatorname{sat}_{\alpha \overline{\mathrm{u}}}\left(-k\left(\boldsymbol{x}_{\mathrm{aw}}\right)\right)+\boldsymbol{b}_{\mathrm{s}, u} \delta \\
y_{\mathrm{aw}, \mathrm{u}}=-\boldsymbol{c}_{\mathrm{s}}^{\top} \boldsymbol{x}_{\mathrm{aw}} \\
y_{\mathrm{aw}, \mathrm{y}}=-\mathrm{sat}_{\alpha \overline{\mathrm{u}}}\left(-k\left(\boldsymbol{x}_{\mathrm{aw}}\right)\right)
\end{array}\right.
$$

besteht. Die Zustandsrückführung $k\left(\boldsymbol{x}_{\mathrm{aw}}\right)$ wird gemäß den Überlegungen aus dem letzten Abschnitt wie folgt festgelegt

$$
\begin{aligned}
k\left(\boldsymbol{x}_{\mathrm{aw}}\right) & =\left(\boldsymbol{H}^{-1}(v)\left(\boldsymbol{k}_{g}+\boldsymbol{a}\right)-\boldsymbol{a}\right)^{\top} \boldsymbol{x}_{\mathrm{aw}}, \\
\boldsymbol{H}^{-1}(v) & =\operatorname{diag}\left(v^{-n_{\mathrm{s}}}, \ldots, v^{-1}\right)
\end{aligned}
$$

mit der Selektionsstrategie

$v\left(\boldsymbol{x}_{\mathrm{aw}}\right)=\left\{\begin{array}{cl}v_{\text {min }}, & \boldsymbol{x}_{\mathrm{aw}} \in \overline{\mathcal{E}}\left(v_{\text {min }}\right), \\ \text { eindeutige Lösung von } g\left(v, \boldsymbol{x}_{\mathrm{aw}}\right)=0, & \boldsymbol{x}_{\mathrm{aw}} \in \mathcal{E}(1) \backslash \overline{\mathcal{E}}\left(v_{\text {min }}\right), \\ 1, & \boldsymbol{x}_{\mathrm{aw}} \in \mathbb{R}^{n_{\mathrm{s}}} \backslash \mathcal{E}(1),\end{array}\right.$

wobei die Funktion $g\left(v, \boldsymbol{x}_{\text {aw }}\right)$ für ein $\boldsymbol{R}_{1} \succ \mathbf{0}$ wie folgt definiert ist

$$
g\left(v, \boldsymbol{x}_{\mathrm{aw}}\right)=\boldsymbol{x}_{\mathrm{aw}}^{\top} \boldsymbol{H}^{-1}(v) \boldsymbol{R}_{1} \boldsymbol{H}^{-1}(v) \boldsymbol{x}_{\mathrm{aw}}-1 .
$$

Das so entstehende weich strukturvariable Anti-Windup wird mit ISOVAW (Implicit SOft Variable-structure Anti-Windup) bezeichnet.

Wenn die Matrix $\boldsymbol{R}_{1}$ und der Vektor $\boldsymbol{k}_{1}=\boldsymbol{k}_{g}+\boldsymbol{a}$ die Bedingungen (8.10) erfüllen, ist sichergestellt, dass die Selektionsstrategie eindeutig ist bzw. die Gleichung $g(v, \boldsymbol{x})=0$ in $\mathcal{E}(1) \backslash \overline{\mathcal{E}}\left(v_{\text {min }}\right)$ eine eindeutige Lösung besitzt (vgl. Satz 8.1). Des Weiteren ist die Funktion (8.18) punktsymmetrisch. Das folgt aus $v\left(\boldsymbol{x}_{\text {aw }}\right)=v\left(-\boldsymbol{x}_{\text {aw }}\right)$, da $\boldsymbol{x}_{\text {aw }}$ und $-\boldsymbol{x}_{\text {aw }}$ auf dem Rand der gleichen Ellipse $\mathcal{E}(v)$ liegen und

$$
\begin{aligned}
k\left(\boldsymbol{x}_{\mathrm{aw}}\right) & =\left(\boldsymbol{H}^{-1}\left(v\left(\boldsymbol{x}_{\mathrm{aw}}\right)\right)\left(\boldsymbol{k}_{g}-\boldsymbol{a}\right)-\boldsymbol{a}\right)^{\top} \boldsymbol{x}_{\mathrm{aw}} \\
& =-\left(\boldsymbol{H}^{-1}\left(v\left(-\boldsymbol{x}_{\mathrm{aw}}\right)\right)\left(\boldsymbol{k}_{g}-\boldsymbol{a}\right)-\boldsymbol{a}\right)^{\top}\left(-\boldsymbol{x}_{\mathrm{aw}}\right)=-k\left(-\boldsymbol{x}_{\mathrm{aw}}\right) .
\end{aligned}
$$


Die lokale Lipschitzstetigkeit des Regelgesetzes (8.18) ist ebenfalls gegeben, denn es gilt

Lemma 8.4. Wenn die Matrix $\boldsymbol{R}_{1}$ und der Vektor $\boldsymbol{k}_{1}=\boldsymbol{k}_{g}+\boldsymbol{a}$ des Regelgesetzes (8.18), (8.19) die Bedingungen (8.10) erfüllen, dann ist das Regelgesetz lokal lipschitzstetig in $\boldsymbol{x}_{\text {aw }}$ gemäß Definition 6.1.

Beweis. Der Beweis findet sich in Abschnitt C.3.14.

Damit sind die in Annahme 6.1 vorausgesetzten Eigenschaften sichergestellt und alle Sätze aus Kapitel 6 besitzen Gültigkeit. Um die globale Stabilität einer zulässigen Ruhelage $\boldsymbol{x}_{\mathrm{awrk}}^{(\mathrm{R})}$ des Anti-Windup-Regelkreises mit zugehöriger stationärer Stellgröße $y_{\mathrm{r}}^{(\mathrm{R})}$ sicherzustellen, kann Satz 6.6 genutzt werden. Dieser Satz stellt die folgenden zwei Forderungen.

1. Der Parameter $\alpha$ muss aus dem offenen Intervall $(0, \hat{\alpha})$ gewählt werden, wobei gilt

$$
\hat{\alpha}=1-\left|y_{\mathrm{r}}^{(\mathrm{R})}\right| \overline{\mathrm{u}}^{-1} \text {. }
$$

2. Die Ruhelage $\boldsymbol{\xi}_{\mathrm{aw}}^{(\mathrm{R})}=\mathbf{0}$ des autonomen Anti-Windup-Ersatzsystems

$$
\dot{\boldsymbol{\xi}}_{\mathrm{aw}}=\boldsymbol{A}_{\mathrm{s}} \boldsymbol{\xi}_{\mathrm{aw}}+\boldsymbol{b}_{\mathrm{s}, u} \operatorname{sat}_{\alpha \overline{\mathrm{u}}}\left(-k\left(\boldsymbol{\xi}_{\mathrm{aw}}\right)\right)
$$

muss global asymptotisch stabil sein.

Die zweite Forderung kann nach Lemma 8.3 durch zwei alternative Bedingungen ersetzt werden. Zum einen müssen die Ungleichungen (8.10) für die reduzierte Stellbegrenzung $\alpha \bar{u}$ erfüllt sein und zum anderen muss die Ruhelage $\boldsymbol{\xi}_{\mathrm{aw}}^{(\mathrm{R})}=\mathbf{0}$ des Systems

$$
\dot{\boldsymbol{\xi}}_{\mathrm{aw}}=\boldsymbol{A}_{\mathrm{s}} \boldsymbol{\xi}_{\mathrm{aw}}+\boldsymbol{b}_{\mathrm{s}, u} \operatorname{sat}_{\alpha \overline{\mathrm{u}}}\left(-\boldsymbol{k}_{g}^{\top} \boldsymbol{\xi}_{\mathrm{aw}}\right)
$$

mit dem linearen sättigenden Zustandsregler $\boldsymbol{k}_{g}$ global asymptotisch stabil sein. Diese Überlegung führt unmittelbar zu

Satz 8.2. Gegeben sei der Anti-Windup-Regelkreis aus Abbildung 6.1 mit der Dynamik (6.3), der aus einer in Regelungsnormalform vorliegenden Strecke (8.16), einem Regler $\mathbf{R}$ und einem modellbasierten Anti-Windup (8.17) besteht. Die Funktion $k: \mathbb{R}^{n_{\mathrm{s}}} \mapsto \mathbb{R}$ des Anti-Windup sei weich strukturvariabel gemäß (8.18) mit der Selektionsstrategie (8.19). Der Anfangszustand des Anti-Windup-Regelkreises sei $\boldsymbol{x}_{\mathrm{awrk}}^{(0)} \in \mathbb{R}^{2 n_{\mathrm{s}}+n_{\mathrm{r}}}$, die $z u$ 
stabilisierende zulässige Ruhelage $\boldsymbol{x}_{\mathrm{awrk}}^{(\mathrm{R})}$ besitze die stationäre Stellgröße $y_{\mathrm{r}}^{(\mathrm{R})}$. Wenn $\alpha$ aus dem Intervall

$$
\left(0,1-\left|y_{\mathrm{r}}^{(\mathrm{R})}\right| \bar{u}^{-1}\right)
$$

gewählt wird, die Matrix $\boldsymbol{R}_{1}$ und der Vektor $\boldsymbol{k}_{1}=\boldsymbol{k}_{g}+\boldsymbol{a}$ die Bedingungen (8.10) für die reduzierte Stellbegrenzung $\alpha \bar{u}$ erfüllen und die Ruhelage $\boldsymbol{\xi}_{\mathrm{aw}}^{(\mathrm{R})}=\mathbf{0}$ des Systems

$$
\dot{\boldsymbol{\xi}}_{\mathrm{aw}}=\boldsymbol{A}_{\mathrm{s}} \boldsymbol{\xi}_{\mathrm{aw}}+\boldsymbol{b}_{\mathrm{s}, u} \boldsymbol{s} \boldsymbol{a} \boldsymbol{t}_{\alpha \overline{\boldsymbol{u}}}\left(-\boldsymbol{k}_{g}^{\top} \boldsymbol{\xi}_{\mathrm{aw}}\right)
$$

global asymptotisch stabil ist, dann existiert ein $\mathrm{c}>0$, so dass für die Trajektorien des Anti-Windup-Regelkreises gilt

$$
\left\|\boldsymbol{x}_{\mathrm{awrk}}(t)-\boldsymbol{x}_{\mathrm{awrk}}^{(\mathrm{R})}\right\|<c \forall t \geq 0, \lim _{t \rightarrow \infty} \boldsymbol{x}_{\mathrm{awrk}}(t)=\boldsymbol{x}_{\mathrm{awrk}}^{(\mathrm{R})} .
$$

Der Entwurf des Anti-Windup reduziert sich damit auf die Parametrierung der Matrix $\boldsymbol{R}_{1}$ und des Vektors $\boldsymbol{k}_{g}$. Es bietet sich ein zweistufiges Entwurfsverfahren an. Zuerst wird durch eine geeignete Wahl des Vektors $\boldsymbol{k}_{g}$ die globale Stabilität der Ruhelage $\boldsymbol{\xi}_{\mathrm{aw}}^{(\mathrm{R})}=\mathbf{0}$ des Ersatzsystems

$$
\begin{aligned}
\dot{\boldsymbol{\xi}}_{\mathrm{aw}} & =\boldsymbol{A}_{\mathrm{s}} \boldsymbol{\xi}_{\mathrm{aw}}+\boldsymbol{b}_{\mathrm{s}, u} \mathbf{s a t}_{\alpha \overline{\mathbf{u}}}\left(-\boldsymbol{k}_{g}^{\top} \boldsymbol{\xi}_{\mathrm{aw}}\right)+\boldsymbol{b}_{\mathrm{s}, u} \zeta, \\
y_{\mathrm{aw}, \mathrm{u}} & =\boldsymbol{c}_{\mathrm{s}}^{\top} \boldsymbol{\xi}_{\mathrm{aw}}
\end{aligned}
$$

für $\zeta=0$ sichergestellt. Um die Regelgüte zu berücksichtigen, kann dabei die $L_{2}$-Verstärkung $\gamma$ des Systems vom Eingang $\zeta$ zum Ausgang $y_{\mathrm{aw}, \mathrm{u}}$ minimiert werden. Ein derartiger Regler $\boldsymbol{k}_{g}$ lässt sich beispielsweise mit Lemma 7.3 entwerfen. Dazu wird die Sektorgrenze $\boldsymbol{\Phi}=\phi_{1}=1$ gewählt und das Optimierungsproblem 7.2 gelöst. Angepasst an den hier vorliegenden SISO-Fall lautet das konvexe Optimierungsproblem wie folgt.

\section{Optimierungsproblem 8.1.}

$\underset{\boldsymbol{Q}_{g}, \Psi, \boldsymbol{m}, \gamma}{\text { Minimiere }} \gamma$ unter den Nebenbedingungen

$$
\boldsymbol{Q}_{g} \succ \mathbf{0}, \Psi>0, \gamma>0,
$$$$
\operatorname{He}\left[\begin{array}{cccc}
\boldsymbol{A}_{\mathrm{s}} \boldsymbol{Q}_{g}-\boldsymbol{b}_{\mathrm{s}, u} \boldsymbol{m}^{\top} & \mathbf{0} & \mathbf{0} & \mathbf{0} \\
\Psi \boldsymbol{b}_{\mathrm{s}, u}^{\top}+\boldsymbol{m}^{\top} & -\Psi & \mathbf{0} & \mathbf{0} \\
\boldsymbol{b}_{\mathrm{s}, u}^{\top} & \mathbf{0} & -\frac{1}{2} \gamma & \mathbf{0} \\
\boldsymbol{c}_{\mathrm{s}}^{\top} \boldsymbol{Q}_{g} & \mathbf{0} & \mathbf{0} & -\frac{1}{2} \gamma
\end{array}\right] \prec \mathbf{0} .
$$ 
Der gesuchte Vektor $\boldsymbol{k}_{g}$ ergibt sich durch den Zusammenhang

$$
\boldsymbol{k}_{g}^{\top}=\boldsymbol{m}^{\top} \boldsymbol{Q}_{g}^{-1} .
$$

In einem zweiten Entwurfsschritt wird eine passende Matrix $\boldsymbol{R}_{1}$ gesucht, so dass der Vektor $\boldsymbol{k}_{1}=\boldsymbol{k}_{g}+\boldsymbol{a}$ und die Matrix $\boldsymbol{R}_{1}$ die Bedingungen (8.10) für die reduzierte Stellbegrenzung $\alpha \overline{\mathrm{u}}$ erfüllen. Um die Regelgüte zu erhöhen, empfiehlt es sich, die Matrix $\boldsymbol{R}_{1}$ so zu wählen, dass die Hyperellipse $\mathcal{E}(1)$ möglichst groß wird. Dann werden aggressivere Zustandsregler zu einem früheren Zeitpunkt aktiviert. Ein sinnvolles Maß für die Größe einer Hyperellipse ist das Volumen. In [120, Anhang B.2] wird gezeigt, dass die Maximierung des Volumens von $\mathcal{E}(1)$ auf die Minimierung der konvexen Funktion

$$
-(\operatorname{det} \boldsymbol{Q})^{1 /\left(2 n_{\mathrm{s}}\right)} \quad \text { mit } \quad \boldsymbol{Q}=\boldsymbol{R}_{1}^{-1} \succ \mathbf{0}
$$

zurückgeführt werden kann. Diese Zielfunktion in Kombination mit den bekannten Nebenbedingungen bildet das konvexe

\section{Optimierungsproblem 8.2.}

$\underset{\boldsymbol{Q}, \boldsymbol{q}_{2}, \chi(v)}{\operatorname{Minimiere}}-(\operatorname{det} \boldsymbol{Q})^{1 /\left(2 n_{\mathrm{s}}\right)} \quad$ unter den Nebenbedingungen

$$
\begin{aligned}
& \boldsymbol{Q} \succ \mathbf{0}, \\
& \boldsymbol{Q N}+\mathbf{N} \boldsymbol{Q} \prec \mathbf{0}, \\
& \boldsymbol{Q} \underbrace{\left(\boldsymbol{A}_{\mathrm{s}}+\boldsymbol{b} \boldsymbol{a}^{\top}\right)^{\top}}_{\boldsymbol{A}_{0}^{\top}}+\boldsymbol{A}_{0} \boldsymbol{Q}-\boldsymbol{q}_{2} \boldsymbol{b}^{\top}-\boldsymbol{b} \boldsymbol{q}_{2}^{\top} \prec \mathbf{0}, \\
& \boldsymbol{Q} \boldsymbol{A}_{\mathrm{s}}^{\top}+\boldsymbol{A}_{\mathrm{s}} \boldsymbol{Q}-\boldsymbol{Q} \boldsymbol{k}_{g} \boldsymbol{b}^{\top}-\boldsymbol{b} \boldsymbol{k}_{g}^{\top} \boldsymbol{Q} \prec \mathbf{0}, \\
& {\left[\begin{array}{cc}
(\alpha \overline{\mathrm{u}})^{2}-\chi(v) \eta(v) & \boldsymbol{q}_{2}^{\top}-\boldsymbol{a}^{\top} \boldsymbol{H}(v) \boldsymbol{Q} \\
\boldsymbol{q}_{2}-\boldsymbol{Q} \boldsymbol{H}(v) \boldsymbol{a} & \boldsymbol{Q}
\end{array}\right] \in \Sigma^{n_{\mathrm{s}}+1}[v],} \\
& \chi(v) \in \Sigma[v] .
\end{aligned}
$$

Anmerkung 8.1. Im Vergleich mit dem existierenden weich strukturvariablen Anti-Windup [52, 54] hat das ISOVAW eine deutlich schlankere Struktur, da weniger Parameter benötigt werden, wie Tabelle 8.1 zeigt. Das liegt daran, dass in $[52,54]$ zwischen $N$ Zustandsreglern interpoliert wird und jeder Zustandsregler gesondert entworfen werden muss. In [150] wird anhand eines Beispiels gezeigt, dass sich eine vergleichbare Regelgüte für $N=5$ ergibt. Ein weiterer Vorteil des ISOVAW ist der einfache Entwurf, da keine Entwurfsparameter zu wählen und lediglich zwei konvexe Optimierungsprobleme zu lösen sind. 
Um $k\left(\boldsymbol{x}_{\mathrm{aw}}\right)$ bestimmen zu können, muss der Selektionsparameter $v$ während des Ausregelvorganges für den aktuellen Zustand $\boldsymbol{x}_{\text {aw }}$ berechnet werden. Dazu ist in dem Gebiet $\mathcal{E}(1) \backslash \overline{\mathcal{E}}\left(v_{\text {min }}\right)$ die Gleichung $g\left(v, \boldsymbol{x}_{\text {aw }}\right)=0$ zu lösen. Das lässt sich wie folgt implementieren (vgl. [98]). Eine Multiplikation der Gleichung mit $v^{2 n_{\mathrm{s}}}$ liefert

$$
g\left(v, \boldsymbol{x}_{\mathrm{aw}}\right) v^{2 n_{\mathrm{s}}}=\boldsymbol{x}_{\mathrm{aw}}^{\top} \hat{\boldsymbol{H}}(v) \boldsymbol{R}_{1} \hat{\boldsymbol{H}}(v) \boldsymbol{x}_{\mathrm{aw}}-v^{2 n_{\mathrm{s}}}=0
$$

mit $\hat{\boldsymbol{H}}(v)=\operatorname{diag}\left(1, v, \ldots, v^{n_{\mathrm{s}}-1}\right)$. Das ist ein Polynom in $v$ vom Grad $2 n_{\mathrm{s}}$. Nach Satz 8.1 besitzt es für alle $\boldsymbol{x}_{\mathrm{aw}} \in \mathcal{E}(1)$ genau eine Nullstelle im Intervall $(0,1]$, die sich durch ein Bisektionsverfahren einfach finden lässt. Anmerkung 8.2. In $[52,54]$ ist die direkte Berechnung des dort benötigten Selektionsparameters wesentlich komplizierter und führt auf ein Optimierungsproblem [211, Seite 206]. Die in [54, 211] vorgeschlagene alternative Methode schätzt deshalb den Selektionsparameter mit einer beobachterähnlichen Struktur. Die Frage nach einem geeigneten Entwurf des Beobachters wird allerdings nicht beantwortet. Auch der Zusammenhang zwischen der Parametrierung des Beobachters und der Regelgüte wird nicht thematisiert. Der Anwender ist auf Ausprobieren angewiesen.

\subsection{Beispiel: elektrisches Netzwerk}

Es wird das elektrische Netzwerk aus [71] betrachtet, das in Abbildung 8.4 dargestellt ist. Die Eingangsspannung $u_{\mathrm{s}}$ ist durch eine Sättigungsfunktion auf $\pm 1 \mathrm{~V}$ begrenzt. Die Ausgangsspannung $y_{\mathrm{s}}$ soll geregelt werden.

Durch Anwendung der Kirchhoff'schen Gesetze ergibt sich ein Zustandsraummodell dritter Ordnung des Systems. Die Zustände beschreiben dabei

Tabelle 8.1: Gegenüberstellung des Aufwandes für den Entwurf eines weich strukturvariablen Anti-Windup gemäß $[52,54]$ und eines ISOVAW. Es bezeichnet $n_{\mathrm{s}}$ die Systemordnung der Strecke, $n^{*}=2^{-1} n_{\mathrm{s}}\left(n_{\mathrm{s}}+1\right)$ und $N$ die Anzahl der Zustandsrückführungen, zwischen denen in $[52,54]$ interpoliert wird. Für eine hohe Regelgüte ist $N>1 \mathrm{zu}$ wählen.

\begin{tabular}{lcc}
\hline Anzahl der ... & ISOVAW & {$[52,54]$} \\
\hline$\ldots$ AW-Parameter & $n^{*}+n_{\mathrm{s}}$ & $(N+1)\left(n^{*}+n_{\mathrm{s}}\right)$ \\
$\ldots$ Entwurfsparameter & 0 & 2 \\
... Optimierungsprobleme & 2 & $N+1$ \\
\hline
\end{tabular}




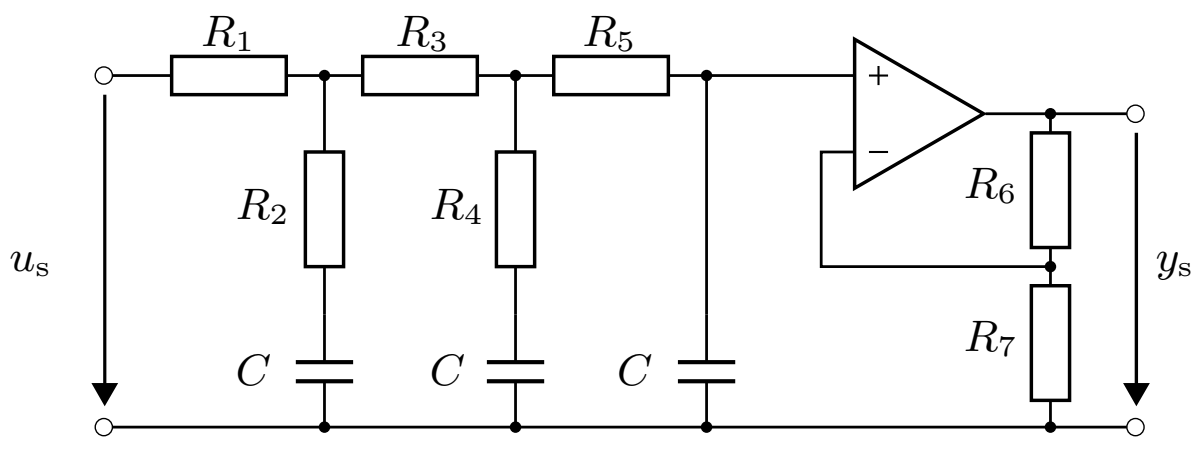

Abbildung 8.4: Elektrisches Netzwerk mit der Eingangsspannung $u_{\mathrm{s}}$ und der Ausgangsspannung $y_{\mathrm{s}}$. Die Widerstände besitzen folgende Werte $R_{1}=313 \Omega$, $R_{2}=20 \Omega, R_{3}=315 \Omega, R_{4}=17 \Omega, R_{5}=10 \Omega, R_{6}=88,9 \Omega, R_{7}=1 \Omega$ und die Kapazitäten $C=0,01$ F. Der Operationsverstärker sei ideal.

die Ladungsmenge auf den Kapazitäten. Eine Transformation in Regelungsnormalform liefert die Systemmatrizen

$$
\begin{aligned}
\boldsymbol{A}_{\mathrm{s}} & =\left[\begin{array}{ccc}
0 & 1 & 0 \\
0 & 0 & 1 \\
-0,33 & -5,29 & -8,12
\end{array}\right], \boldsymbol{b}_{\mathrm{s}}=\left[\begin{array}{l}
0 \\
0 \\
1
\end{array}\right], \\
\boldsymbol{c}_{\mathrm{s}}^{\top} & =\left[\begin{array}{lll}
29,41 & 10,88 & 1
\end{array}\right] .
\end{aligned}
$$

Zur Regelung des Systems wird ein PID-Regler mit der Übertragungsfunktion

$$
y_{\mathrm{r}}(s)=\frac{3,961(s+0,062)(s+0,641)}{s(s+3,658)}\left(w(s)-y_{\mathrm{s}}(s)\right)
$$

verwendet, der die Sollwertfolge sicherstellt und dabei Überschwingen vermeidet. In dem nachfolgend betrachteten Szenario wird ein Ausgangsspannung von $y_{\mathrm{s}}=2 \mathrm{~V}$ gefordert. Die zugehörige stationäre Stellgröße beträgt $y_{\mathrm{r}}^{(\mathrm{R})}=0,0222 \mathrm{~V}$. Zum Zeitpunkt $t=0$ befinden sich alle Zustände im Ursprung. Abbildung 8.5 zeigt das ideale Verhalten des unbeschränkten Regelkreises mit einer Ausregelzeit von 0,9 s (1\%-Band). Ebenfalls dargestellt ist das Verhalten des beschränkten Regelkreises ohne Anti-Windup. Auffällig ist die sehr langsame Konvergenz zu dem Sollwert und das Überschwingen. Ohne Anti-Windup verlängert sich die Ausregelzeit auf etwa $29 \mathrm{~s}$, was aus Gründen der Übersichtlichkeit nicht dargestellt ist.

Um die Windup-Effekte zu minimieren wird zunächst mit Optimierungsproblem 8.1 ein lineares modellbasiertes Anti-Windup entworfen. Der AntiWindup-Parameter $\alpha$ wird aus dem Intervall $\left(0,1-y_{\mathrm{r}}^{(\mathrm{R})}\right)=(0,0,9778) \mathrm{zu}$ 

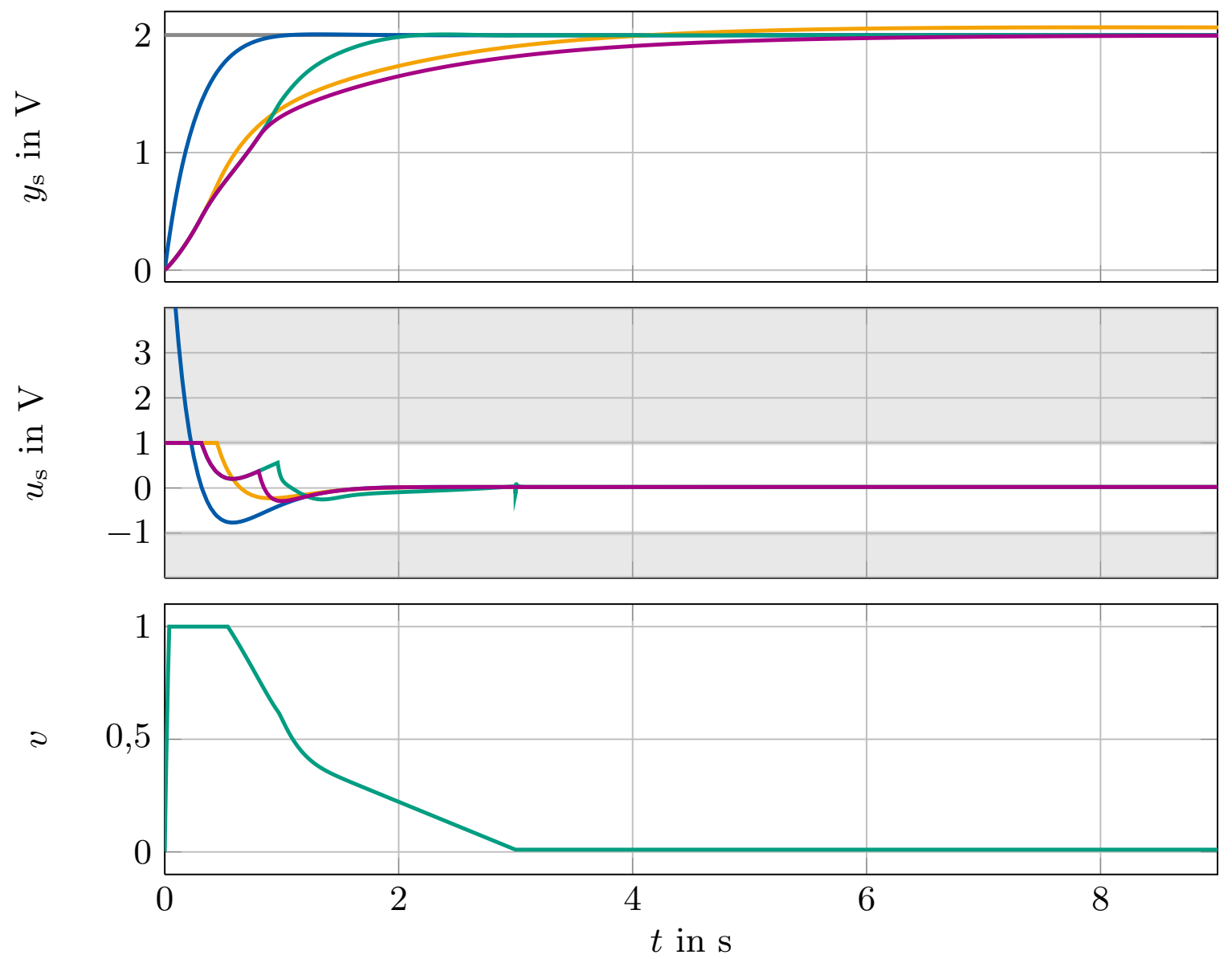

Abbildung 8.5: Verläufe der Ausgänge und Stellgrößen des unbeschränkten Regelkreises (-), des beschränkten Regelkreises ( - ), des Anti-WindupRegelkreises mit einem linearen Anti-Windup (-) und des ISOVAW (-). Ebenfalls dargestellt ist der Verlauf des Selektionsparameters $v$.

$\alpha=0,97$ gewählt. Für den Vektor $\boldsymbol{k}_{g}$ ergibt sich

$$
\boldsymbol{k}_{g}^{\top}=\left[\begin{array}{lll}
-60,570 & -98,668 & -12,200
\end{array}\right] \text {. }
$$

Abbildung 8.5 zeigt die Simulationsergebnisse. Das Überschwingen wird vermieden, die Ausregelzeit von 6,77 s (1\%-Band) ist allerdings noch weit von dem idealen Verhalten entfernt. Deshalb wird durch das Lösen des Optimierungsproblems 8.2 mit $v_{\min }=0,01$, dem Polynom

$$
\eta(v)=-\left(v-v_{\min }\right)(v-1)
$$


und einem Hilfspolynom $\chi(v)$ vierten Grades eine passende Matrix

$$
\boldsymbol{R}_{1}=\left[\begin{array}{rrr}
175,691 & 278,788 & 32,654 \\
278,788 & 463,423 & 63,533 \\
32,654 & 63,533 & 23,417
\end{array}\right]
$$

bestimmt, die zusammen mit dem Vektor $\boldsymbol{k}_{g}$ ein ISOVAW parametriert. Dieses weich strukturvariable Anti-Windup nutzt die Stellgröße besser aus und führt zu einer Ausregelzeit von 1,98 s.

\subsection{Zusammenfassung und Fazit}

In diesem Kapitel wurde ein nichtlineares modellbasiertes Anti-Windup vorgestellt, das im Vergleich zu linearen Anti-Windup-Maßnahmen eine sehr hohe Regelgüte erzielen kann. Die Zustandsrückführung des modellbasierten Anti-Windup basiert auf dem in [98] beschriebenen Konzept der vereinfachten impliziten weich strukturvariablen Zustandsregelung. In Abschnitt 8.2.3 wurde dieses Regelgesetz so modifiziert, dass die globale Stabilisierung von Strecken mit ausschließlich negativen Eigenwerten möglich wird. Der resultierende Zustandsregler ließs sich dann in einem modellbasierten Anti-Windup einsetzen.

Die Vorteile dieses impliziten weich strukturvariablen Anti-Windup (ISOVAW) bestehen im Vergleich zu der existierenden Methode [52, 54] in einem einfacheren Entwurf, da keine Entwurfsparameter zu wählen und lediglich zwei konvexe Optimierungsprobleme zu lösen sind (vgl. Tabelle 8.1). Darüber hinaus reduziert sich die Berechnung des Selektionsparameters auf die Berechnung einer Nullstelle eines Polynoms. In [52, 54] muss zur Berechnung des Selektionsparameters ein Optimierungsproblem gelöst werden, was die Echtzeitfähigkeit des Ansatzes beeinträchtigt [211, Seite 206]. Alternativ kann eine beobachterähnliche Struktur zur Schätzung des Selektionsparameters ausgelegt werden, deren Entwurf aufwendig ist. 


\section{Lineares Anti-Windup für grenz- und instabile Strecken}

Bisher wurden ausschließlich Anti-Windup-Entwurfsverfahren für stabile Strecken vorgestellt. In diesem Kapitel wird gezeigt, wie modellbasiertes Anti-Windup mithilfe des Stabilitätssatzes 6.7 aus Kapitel 6 auch in Regelkreisen mit grenz- und instabilen Strecken eingesetzt werden kann. Die Vorgehensweise beim Entwurf stellt eine Erweiterung der bereits in [149] veröffentlichten Grundidee dar.

\subsection{Problembeschreibung}

Betrachtet wird der in Kapitel 6 detailliert beschriebene Anti-WindupRegelkreis. Dieser besteht aus einer linearen Strecke

$$
\mathbf{S}:\left\{\begin{array}{l}
\dot{x}_{\mathrm{s}}=A_{\mathrm{s}} \boldsymbol{x}_{\mathrm{s}}+\boldsymbol{B}_{\mathrm{s}, u} \boldsymbol{u}_{\mathrm{s}}+\boldsymbol{B}_{\mathrm{s}, z} \boldsymbol{z} \\
\boldsymbol{y}_{\mathrm{s}}=C_{\mathrm{s}} \boldsymbol{x}_{\mathrm{s}}
\end{array}\right.
$$

einem linearen Regler mit der Dynamik

$$
\mathbf{R}:\left\{\begin{array}{l}
\dot{\boldsymbol{x}}_{\mathrm{r}}=\boldsymbol{A}_{\mathrm{r}} \boldsymbol{x}_{\mathrm{r}}+\boldsymbol{B}_{\mathrm{r}, u} \boldsymbol{u}_{\mathrm{r}}+\boldsymbol{B}_{\mathrm{r}, w} \boldsymbol{w} \\
\boldsymbol{y}_{\mathrm{r}}=\boldsymbol{C}_{\mathrm{r}} \boldsymbol{x}_{\mathrm{r}}+\boldsymbol{D}_{\mathrm{r}, u} \boldsymbol{u}_{\mathrm{r}}+\boldsymbol{D}_{\mathrm{r}, w} \boldsymbol{w}
\end{array}\right.
$$

und einem durch $\overline{\mathbf{u}}$ charakterisierten realen Aktor, der den Streckeneingang $\boldsymbol{u}_{\mathrm{s}}$ begrenzt. Auftretende Windup-Probleme lassen sich durch ein lineares modellbasiertes Anti-Windup der Form

$$
\mathbf{A W}:\left\{\begin{array}{l}
\dot{\boldsymbol{x}}_{\mathrm{aw}}=\boldsymbol{A}_{\mathrm{s}} \boldsymbol{x}_{\mathrm{aw}}+\boldsymbol{B}_{\mathrm{s}, u} \mathbf{s a t}_{\alpha \overline{\mathbf{u}}}\left(-\underline{\boldsymbol{K}} \boldsymbol{x}_{\mathrm{aw}}\right)+\boldsymbol{B}_{\mathrm{s}, u} \boldsymbol{\delta}, \\
\boldsymbol{y}_{\mathrm{aw}, \mathrm{u}}=-\boldsymbol{C}_{\mathrm{s}} \boldsymbol{x}_{\mathrm{aw}} \\
\boldsymbol{y}_{\mathrm{aw}, \mathrm{y}}=-\mathbf{s a t}_{\alpha \overline{\mathbf{u}}}\left(-\underline{\boldsymbol{K}} \boldsymbol{x}_{\mathrm{aw}}\right)
\end{array}\right.
$$

beheben. Abbildung 9.1 zeigt den durch die Verschaltung der Teilsysteme entstehenden Anti-Windup-Regelkreis.

Wie in Kapitel 6 detailliert erläutert wurde, reduziert sich der AntiWindup-Entwurf auf die Wahl der Funktion $\boldsymbol{k}_{\text {aw }}(\boldsymbol{x})$, die hier durch 


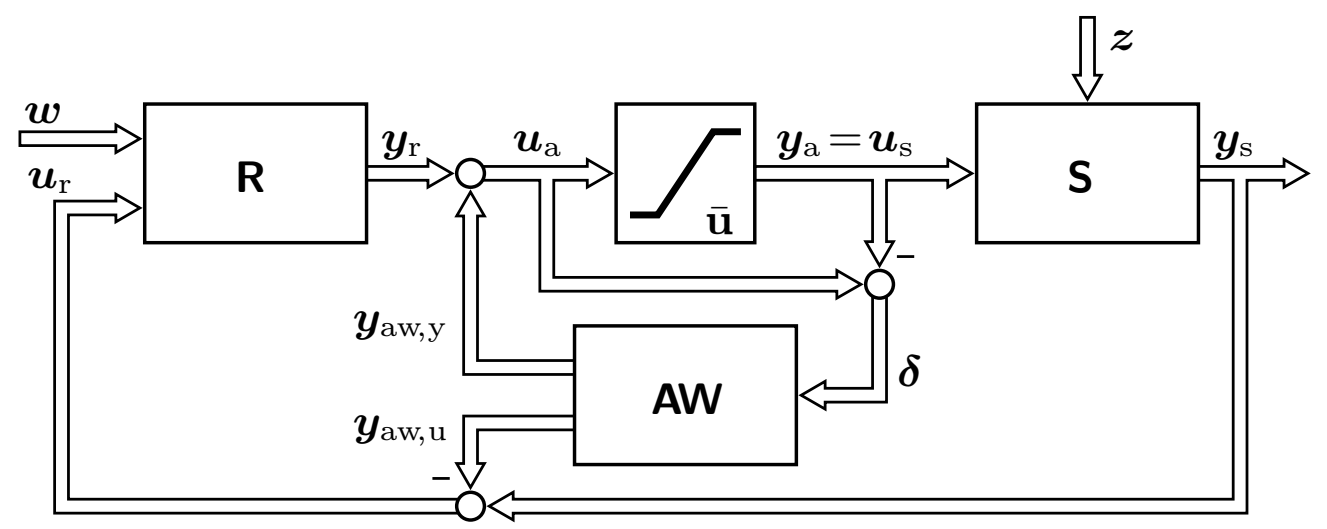

Abbildung 9.1: Anti-Windup-Regelkreis.

$\operatorname{sat}_{\alpha \overline{\mathbf{u}}}\left(-\underline{\boldsymbol{K}} \boldsymbol{x}_{\text {aw }}\right)$ gegeben ist. Folglich sind beim Entwurf die Matrix $\underline{\boldsymbol{K}}$ und der Parameter $\alpha \in(0,1)$ so festzulegen, dass die in Abschnitt 4.2 beschriebenen Ziele erreicht werden. Insbesondere soll das Verhalten des AntiWindup-Regelkreises nahe an dem idealen Verhalten des unbeschränkten Regelkreises liegen. In einer Ruhelage dürfen keine Abweichungen vorhanden sein. Eine Ruhelage des unbeschränkten Regelkreises entspricht deshalb im Anti-Windup-Regelkreis der Ruhelage

$$
\boldsymbol{x}_{\mathrm{s}}^{(\mathrm{R})}=\boldsymbol{x}_{\mathrm{s}, \mathrm{ub}}^{(\mathrm{R})}, \quad \boldsymbol{x}_{\mathrm{r}}^{(\mathrm{R})}=\boldsymbol{x}_{\mathrm{r}, \mathrm{ub}}^{(\mathrm{R})}, \quad \boldsymbol{x}_{\mathrm{aw}}^{(\mathrm{R})}=\mathbf{0} .
$$

Dabei besteht die in Abschnitt 6.4 beschriebene Einschränkung auf zulässige Ruhelagen (Definition 6.2), d. h., die stationär benötigte Stellgröße muss physikalisch mit dem vorhandenen Aktor realisierbar sein.

In Mismatch-Koordinaten $\boldsymbol{\xi}_{\mathrm{ms}}$ lässt sich das Entwurfsproblem sehr übersichtlich darstellen, da der Regelkreis in das Teilsystem

$$
\mathbf{L}:\left\{\begin{array}{l}
\dot{\boldsymbol{\xi}}_{\mathrm{s}}=\boldsymbol{A}_{\mathrm{s}} \boldsymbol{\xi}_{\mathrm{s}}+\boldsymbol{B}_{\mathrm{s}, u} \boldsymbol{y}_{\mathrm{r}}+\boldsymbol{B}_{\mathrm{s}, z} \boldsymbol{z} \\
\dot{\boldsymbol{\xi}}_{\mathrm{r}}=\boldsymbol{A}_{\mathrm{r}} \boldsymbol{\xi}_{\mathrm{r}}+\boldsymbol{B}_{\mathrm{r}, u} \boldsymbol{C}_{\mathrm{s}} \boldsymbol{\xi}_{\mathrm{s}}+\boldsymbol{B}_{\mathrm{r}, w} \boldsymbol{w} \\
\boldsymbol{y}_{\mathrm{r}}=C_{\mathrm{r}} \boldsymbol{\xi}_{\mathrm{r}}+\boldsymbol{D}_{\mathrm{r}, u} \boldsymbol{C}_{\mathrm{s}} \boldsymbol{\xi}_{\mathrm{s}}+\boldsymbol{D}_{\mathrm{r}, w} \boldsymbol{w}
\end{array}\right.
$$

welches dem unbeschränkten Regelkreis entspricht, und das Teilsystem

$$
\text { ES : }\left\{\begin{aligned}
\dot{\boldsymbol{\xi}}_{\mathrm{aw}} & =\boldsymbol{A}_{\mathrm{s}} \boldsymbol{\xi}_{\mathrm{aw}}+\boldsymbol{B}_{\mathrm{s}, u} \mathbf{s a t}_{\alpha \overline{\mathbf{u}}}\left(-\underline{\boldsymbol{K}} \boldsymbol{\xi}_{\mathrm{aw}}\right)+\boldsymbol{B}_{\mathrm{s}, u} \boldsymbol{\zeta} \\
\boldsymbol{y}_{\mathrm{aw}, \mathrm{u}} & =\boldsymbol{C}_{\mathrm{s}} \boldsymbol{\xi}_{\mathrm{aw}}
\end{aligned}\right.
$$

mit dem Zustand $\boldsymbol{\xi}_{\text {aw }}$ zerfällt. Die Ruhelage (9.1) entspricht

$$
\boldsymbol{\xi}_{\mathrm{s}}^{(\mathrm{R})}=\boldsymbol{x}_{\mathrm{s}, \mathrm{ub}}^{(\mathrm{R})}, \quad \boldsymbol{\xi}_{\mathrm{r}}^{(\mathrm{R})}=\boldsymbol{x}_{\mathrm{r}, \mathrm{ub}}^{(\mathrm{R})}, \quad \boldsymbol{\xi}_{\mathrm{aw}}^{(\mathrm{R})}=\mathbf{0}
$$




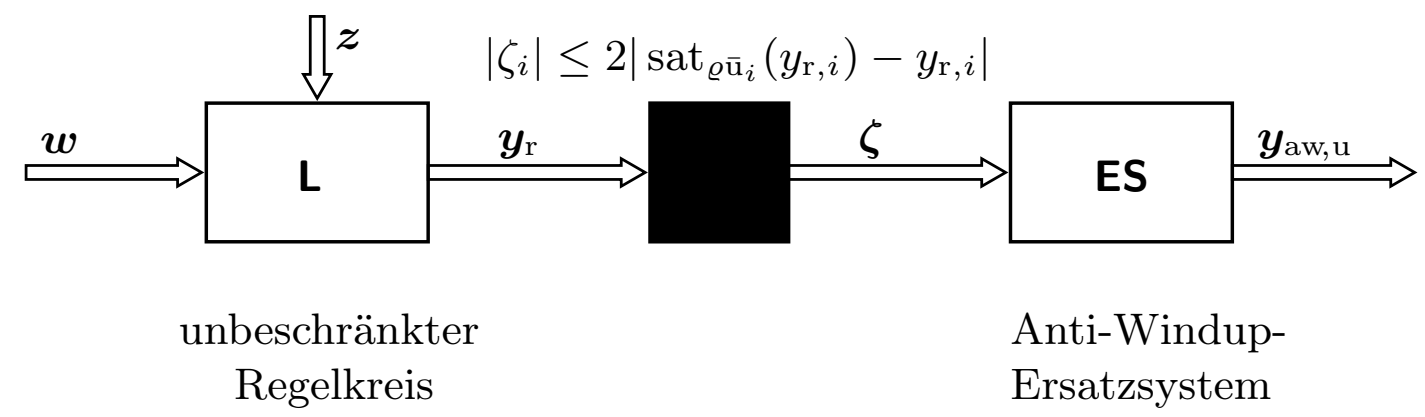

Abbildung 9.2: Reihenschaltung aus dem linearen Teilsystem L, dem Ersatzsystem ES . Das schwarz eingefärbte System erzeugt die stetigen Störsignale $\boldsymbol{\zeta}$, die der komponentenweisen Abschätzung (9.3) genügen.

und der Entwurf reduziert sich auf den Entwurf eines Zustandsreglers für ES. Gelingt es den Ausgang $\boldsymbol{y}_{\mathrm{aw}, \mathrm{u}}$ klein zu halten, bleibt auch die Abweichung des Anti-Windup-Regelkreises vom unbeschränkten Systemverhalten klein. Diese Aufgabe wird durch die Störung $\boldsymbol{\zeta}$ erschwert, für die eine komponentenweise Abschätzung

$$
\left|\zeta_{i}\right| \leq 2\left|\operatorname{sat}_{\varrho \bar{u}_{i}}\left(y_{\mathrm{r}, i}\right)-y_{\mathrm{r}, i}\right|=2\left|\mathrm{dz}_{\varrho \overline{\mathrm{u}}_{i}}\left(y_{\mathrm{r}, i}\right)\right| \leq 2\left|y_{\mathrm{r}, i}\right|
$$

gilt. Dabei ist $\varrho=1-\alpha$ und $y_{\mathrm{r}, i}$ entspricht der $i$-ten Komponente des Ausgangs von L. Abbildung 9.2 zeigt das für den Entwurf in MismatchKoordinaten maßgebliche Blockschaltbild.

Die Wahl des Anti-Windup-Parameters $\alpha$ wurde bereits in Abschnitt 6.4 geklärt. Entscheidend ist dabei die durch $\boldsymbol{w}=\boldsymbol{w}_{0}$ und $\boldsymbol{z}=\boldsymbol{z}_{0}$ vorgegebene Ruhelage und damit die benötigte stationäre Stellgröße $\boldsymbol{y}_{\mathrm{r}}^{(\mathrm{R})}$. Der Beweis des Stabilitätssatzes 6.7 beruht darauf, dass die Festlegung von $\alpha=1-\varrho$ sicherstellt, dass $\mathbf{d} \mathbf{z}_{\varrho \overline{\mathbf{u}}}\left(\boldsymbol{y}_{\mathrm{r}}\right)$ und damit gemäß (9.3) auch $\boldsymbol{\zeta}$ zu einem endlichen Zeitpunkt $0<t^{*}<\infty$ null wird. Nach Lemma 6.3 ist das der Fall, wenn der Anti-Windup-Parameter $\alpha$ aus dem offenen Intervall $(0, \hat{\alpha})$ gewählt wird mit

$$
\hat{\alpha}=1-\max _{i \in\{1, \ldots, m\}}\left\{\left|y_{\mathrm{r}, i}^{(\mathrm{R})}\right| \overline{\mathrm{u}}_{i}^{-1}\right\} .
$$

Für den Anti-Windup-Entwurf ist demnach nur noch die Frage nach der Parametrierung von $\underline{\boldsymbol{K}}$ zu beantworten. Aufgabe des Zustandsreglers ist es, die Stabilität der Ruhelage $\boldsymbol{\xi}_{\text {aw }}^{(\mathrm{R})}=\mathbf{0}$ des Systems ES mit dem Anfangszustand $\boldsymbol{\xi}_{\mathrm{aw}}^{(0)}=\mathbf{0}$ trotz auftretender Störungen $\boldsymbol{\zeta}$ zu garantieren. Dabei steht jedoch lediglich die begrenzte Stellgröße $\alpha \overline{\mathbf{u}}$ zur Verfügung. Dies ist 
problematisch, wenn die Strecke $\mathbf{S}$ grenz- oder instabil ist, wie in Abschnitt 3.1 ausgeführt wurde. Weist die Matrix $\boldsymbol{A}_{\mathrm{s}}$ Eigenwerte mit nichtnegativem Realteil auf, lässt sich für das System ES nur lokale Stabilität der Ruhelage $\boldsymbol{\xi}_{\mathrm{aw}}^{(\mathrm{R})}=\mathbf{0}$ erreichen. Eine große Störung könnte den Systemzustand $\boldsymbol{\xi}_{\text {aw }}$ aus diesem lokalen Stabilitätsgebiet hinaustragen und eine Stabilisierung wäre dann selbst bei einer zum Zeitpunkt $t^{*}$ verschwindenden Störung nicht mehr möglich.

Da Stabilität nicht für beliebig große Störungen erreichbar ist, wird im Weiteren angenommen, dass die Störung $\boldsymbol{\zeta}$ begrenzt ist, was eine Begrenzung der Sollwerte $\boldsymbol{w}$, Störgrößen $\boldsymbol{z}$ und Anfangswerte $\boldsymbol{\xi}_{\mathrm{s}}^{(0)}, \boldsymbol{\xi}_{\mathrm{r}}^{(0)}$ des linearen Systems $\mathbf{L}$ nach sich zieht. Der Anti-Windup-Entwurf zerfällt deshalb bei genauerer Betrachtung in zwei Teilprobleme:

1. Wie lässt sich der Zusammenhang zwischen $\boldsymbol{w}, \boldsymbol{z}, \boldsymbol{\xi}_{\mathrm{s}}^{(0)}, \boldsymbol{\xi}_{\mathrm{r}}^{(0)}$ und der Störung $\boldsymbol{\zeta}$ quantifizieren? Inwieweit ist $\boldsymbol{\zeta}$ begrenzt, wenn $\boldsymbol{w}, \boldsymbol{z}, \boldsymbol{\xi}_{\mathrm{s}}^{(0)}$ und $\boldsymbol{\xi}_{\mathrm{r}}^{(0)}$ begrenzt sind?

2. Wie muss der Zustandsregler $\underline{\boldsymbol{K}}$ parametriert werden, so dass die Stabilität der Ruhelage $\boldsymbol{\xi}_{\text {aw }}^{(\mathrm{R})}=\mathbf{0}$ des Ersatzsystems ES für die begrenzte Störung $\boldsymbol{\zeta}$ garantiert ist?

Die für grenz- und instabile Strecken geeigneten Entwurfsverfahren $[52,173,175,185,191,210]$ für lineares modellbasiertes Anti-Windup lösen nur das zweite Teilproblem. Stabilität des Anti-Windup-Regelkreises ist dann aufgrund der Linearität des Systems L für genügend kleine Anfangszustände, Sollwerte und Störungen garantiert. Eine Quantifizierung von genügend klein erfolgt nicht. Man ist auf Ausprobieren und Simulieren angewiesen. Eine Ausnahme bildet das Entwurfsverfahren [208], das mit einem linearen Zustandsregler globale Stabilität des Anti-WindupRegelkreises für eine spezielle Streckenklasse garantiert. Diese Klasse umfasst alle Strecken mit nichtpositiven Eigenwerten, wobei die Eigenwerte auf der imaginären Achse nur einfach seien dürfen. Der Nachteil des Entwurfs ist, dass die Regelgüte nicht optimiert werden kann [211, S. 182ff].

Eine alternative Entwurfsmethode, die beide Teilprobleme berücksichtigt, beschreiben die nächsten Abschnitte. Das erste Teilproblem wird durch einen Zusammenhang zwischen den Anfangswerten $\boldsymbol{\xi}_{\mathrm{s}}^{(0)}, \boldsymbol{\xi}_{\mathrm{r}}^{(0)}$ und der Energie der Störung $\boldsymbol{\zeta}$ gelöst. Abschnitt 9.3 stellt einen auf dem $\mathrm{Zu}-$ standsreglerentwurf [31] basierendes Anti-Windup vor. Abschließend folgt die Demonstration der Entwurfsmethodik an einem Beispiel. 


\subsection{Abschätzen der Störenergie}

Eine Möglichkeit, das Ausmaß der Störung $\zeta$ zu charakterisieren bietet die $L_{2}$-Norm $\|\boldsymbol{\zeta}\|_{L_{2}}$ (vgl. Abschnitt 4.2), die ein Maß für die Energie des Signals ist. Nach Definition 4.1 gilt

$$
\|\boldsymbol{\zeta}\|_{L_{2}}^{2}=\lim _{t \rightarrow \infty} \int_{0}^{t} \boldsymbol{\zeta}^{\top}(\tau) \boldsymbol{\zeta}(\tau) d \tau .
$$

Um die $L_{2}$-Norm abzuschätzen, erweist sich der Zusammenhang (9.3) als sehr nützlich. Ist jede Komponente von $\boldsymbol{\zeta}$ durch die entsprechende Komponente von $\boldsymbol{y}_{\mathrm{r}}$ beschränkt, so folgt

$$
\|\boldsymbol{\zeta}(t)\|_{2} \leq 2\left\|\mathbf{d} \mathbf{z}_{\varrho \overline{\mathbf{u}}}\left(\boldsymbol{y}_{\mathrm{r}}(t)\right)\right\|_{2} .
$$

Quadrieren dieser Ungleichung und anschließendes Integrieren nach der Zeit führt zu

$$
\int_{0}^{t} \boldsymbol{\zeta}(\tau)^{\top} \boldsymbol{\zeta}(\tau) d \tau \leq 4 \int_{0}^{t} \mathbf{d} \mathbf{z}_{\varrho \overline{\mathbf{u}}}\left(\boldsymbol{y}_{\mathrm{r}}(\tau)\right)^{\top} \mathbf{d} \mathbf{z}_{\varrho \overline{\mathbf{u}}}\left(\boldsymbol{y}_{\mathrm{r}}(\tau)\right) d \tau .
$$

Die Grenzwertbildung $t \rightarrow \infty$ liefert das Endergebnis

$$
\|\boldsymbol{\zeta}\|_{L_{2}}^{2} \leq 4\left\|\mathbf{d} \mathbf{z}_{\varrho \overline{\mathbf{u}}}\left(\boldsymbol{y}_{\mathrm{r}}\right)\right\|_{L_{2}}^{2} .
$$

Um die Energie von $\boldsymbol{\zeta}$ abzuschätzen, wird demnach die Energie von $\mathbf{d z}_{\varrho \overline{\mathbf{u}}}\left(\boldsymbol{y}_{\mathrm{r}}\right)$ benötigt, die offensichtlich von dem Ausgang $\boldsymbol{y}_{\mathrm{r}}$ des linearen Teilsystems L abhängt. Der nächste Abschnitt beschreibt, wie die Abschätzung gelingen kann.

\subsubsection{Zustandstransformation}

Betrachtet wird das lineare Teilsystem L gemäß (9.2), das dem unbeschränkten Regelkreis (2.3) entspricht. Fasst man die Zustände $\boldsymbol{\xi}_{\mathrm{s}}$ und $\boldsymbol{\xi}_{\mathrm{r}}$ in einem Vektor

$$
\boldsymbol{\xi}_{\mathrm{L}}^{\top}=\left[\begin{array}{ll}
\boldsymbol{\xi}_{\mathrm{s}}^{\top} & \boldsymbol{\xi}_{\mathrm{r}}^{\top}
\end{array}\right]
$$

und die Eingänge $\boldsymbol{w}, \boldsymbol{z}$ in dem Vektor

$$
\boldsymbol{r}^{\top}=\left[\begin{array}{ll}
\boldsymbol{w}^{\top} & \boldsymbol{z}^{\top}
\end{array}\right]
$$


zusammen, lässt es sich durch die Dynamik

$$
\begin{aligned}
& \dot{\boldsymbol{\xi}}_{\mathrm{L}}=\boldsymbol{A}_{\mathrm{ub}} \boldsymbol{\xi}_{\mathrm{L}}+\boldsymbol{B}_{\mathrm{ub}} \boldsymbol{r} \\
& \boldsymbol{y}_{\mathrm{r}}=\boldsymbol{C}_{\mathrm{ub}} \boldsymbol{\xi}_{\mathrm{L}}+\boldsymbol{D}_{\mathrm{ub}} \boldsymbol{r}
\end{aligned}
$$

beschreiben, mit $\boldsymbol{A}_{\mathrm{ub}}, \boldsymbol{B}_{\mathrm{ub}}$ gemäß (2.4) und $\boldsymbol{C}_{\mathrm{ub}}, \boldsymbol{D}_{\mathrm{ub}}$ gemäß (6.14). Der Anfangswert ist $\boldsymbol{\xi}_{\mathrm{L}}(0)=\boldsymbol{\xi}_{\mathrm{L}}^{(0)}$. Für $\boldsymbol{r}=\mathbf{0}$ liegt die Ruhelage des Systems im Ursprung. Für $\boldsymbol{r}=\boldsymbol{r}_{0} \neq \mathbf{0}$ ergibt sich die Ruhelage zu

$$
\boldsymbol{\xi}_{\mathrm{L}}^{(\mathrm{R})}=-\boldsymbol{A}_{\mathrm{ub}}^{-1} \boldsymbol{B}_{\mathrm{ub}} \boldsymbol{r}_{0} .
$$

Die zugehörige stationäre Stellgröße $\boldsymbol{y}_{\mathrm{r}}(t \rightarrow \infty)$ lautet

$$
\boldsymbol{y}_{\mathrm{r}}^{(\mathrm{R})}=-\left(\boldsymbol{C}_{\mathrm{ub}} \boldsymbol{A}_{\mathrm{ub}}^{-1} \boldsymbol{B}_{\mathrm{ub}}-\boldsymbol{D}_{\mathrm{ub}}\right) \boldsymbol{r}_{0} .
$$

Um die Energie von $\mathbf{d z}_{\varrho \overline{\mathbf{u}}}\left(\boldsymbol{y}_{\mathrm{r}}\right)$ abzuschätzen, wird es sich als nützlich erweisen, die Ruhelage im Fall $\boldsymbol{r}=\boldsymbol{r}_{0} \neq \mathbf{0}$ in den Ursprung zu transformieren. Dazu wird das System (9.4) mithilfe des neuen Zustandsvektors

$$
\boldsymbol{\xi}_{*}=\boldsymbol{\xi}_{\mathrm{L}}-\boldsymbol{\xi}_{\mathrm{L}}^{(\mathrm{R})} \quad \Leftrightarrow \quad \boldsymbol{\xi}_{\mathrm{L}}=\boldsymbol{\xi}_{*}+\boldsymbol{\xi}_{\mathrm{L}}^{(\mathrm{R})}
$$

dargestellt. Das führt zu dem neuen Anfangswert $\boldsymbol{\xi}_{*}(0)=\boldsymbol{\xi}_{\mathrm{L}}^{(0)}-\boldsymbol{\xi}_{\mathrm{L}}^{(\mathrm{R})}$ und

$$
\begin{aligned}
& \dot{\boldsymbol{\xi}}_{*}=\boldsymbol{A}_{\mathrm{ub}} \boldsymbol{\xi}_{*}, \\
& \boldsymbol{y}_{\mathrm{r}}=\boldsymbol{C}_{\mathrm{ub}} \boldsymbol{\xi}_{*}-\left(\boldsymbol{C}_{\mathrm{ub}} \boldsymbol{A}_{\mathrm{ub}}^{-1} \boldsymbol{B}_{\mathrm{ub}}-\boldsymbol{D}_{\mathrm{ub}}\right) \boldsymbol{r}_{0}=\boldsymbol{C}_{\mathrm{ub}} \boldsymbol{\xi}_{*}+\boldsymbol{y}_{\mathrm{r}}^{(\mathrm{R})} .
\end{aligned}
$$

Der Vorteil dieser Systemdarstellung ist, dass die Differentialgleichung (9.5a) ein autonomes System beschreibt und somit die Abschätzung der Ausgangsenergie mittels der in [21, Abschnitt 6.2.1] beschriebenen Vorgehensweise einfach möglich ist. Störend ist lediglich der konstante Summand $\boldsymbol{y}_{\mathrm{r}}^{(\mathrm{R})}$ der Ausgangsgröße $\boldsymbol{y}_{\mathrm{r}}$. Hier kommt allerdings die Tatsache zu Hilfe, dass nicht die Energie von $\boldsymbol{y}_{\mathrm{r}}$, sondern die von $\mathbf{d z}_{\varrho \overline{\mathbf{u}}}\left(\boldsymbol{y}_{\mathrm{r}}(t)\right)$ interessiert. Anstelle von (9.5) wird deshalb das System

$$
\begin{aligned}
\dot{\boldsymbol{\xi}}_{*} & =\boldsymbol{A}_{\mathrm{ub}} \boldsymbol{\xi}_{*}, \\
\mathrm{~d} \mathbf{z}_{\varrho \overline{\mathbf{u}}}\left(\boldsymbol{y}_{\mathrm{r}}\right) & =\mathrm{dz}_{\varrho \overline{\mathbf{u}}}\left(\boldsymbol{C}_{\mathrm{ub}} \boldsymbol{\xi}_{*}+\boldsymbol{y}_{\mathrm{r}}^{(\mathrm{R})}\right)
\end{aligned}
$$

betrachtet, für das sich folgendes Lemma beweisen lässt.

Lemma 9.1. Gegeben sei das System (9.6). Wenn die Bedingung @ $\bar{u}_{i}>$ $\left|y_{\mathrm{r}, i}^{(\mathrm{R})}\right|$ für alle $i=1, \ldots, m$, positive Skalare $\bar{u}_{i}$ und ein $\varrho \in(0,1)$ erfüllt ist, dann gilt für alle $t \geq 0$ folgende Abschätzung

$$
\left\|\boldsymbol{d} \boldsymbol{z}_{\varrho} \overline{\boldsymbol{u}}\left(\boldsymbol{y}_{\mathrm{r}}(t)\right)\right\|_{2}=\left\|\boldsymbol{d} \boldsymbol{z}_{\varrho} \overline{\boldsymbol{u}}\left(\boldsymbol{C}_{\mathrm{ub}} \boldsymbol{\xi}_{*}(t)+\boldsymbol{y}_{\mathrm{r}}^{(\mathrm{R})}\right)\right\|_{2} \leq\left\|\boldsymbol{C}_{\mathrm{ub}} \boldsymbol{\xi}_{*}(t)\right\|_{2}
$$

mit $\overline{\boldsymbol{u}}=\left[\bar{u}_{1} \ldots \bar{u}_{m}\right]^{\top}$. 
Beweis. Der Beweis basiert auf einer komponentenweisen Betrachtung der Totzone und findet sich in Abschnitt C.3.15.

Die Bedingung $\varrho \overline{\mathrm{u}}_{i}>\left|y_{\mathrm{r}, i}^{(\mathrm{R})}\right|$ ist für alle $i=1, \ldots, m$ erfüllt, da $\varrho=$ $1-\alpha$ gilt und $\alpha$ aus dem Intervall $(0, \hat{\alpha})$ gemäß den Überlegungen aus Abschnitt 9.1 gewählt wird. Die Abschätzung (9.7) gilt deshalb für den hier betrachteten Fall. Quadrieren dieser Ungleichung und Integrieren über die Zeit führt zu

$$
\int_{0}^{t}\left\|\mathbf{d} \mathbf{z}_{\varrho \overline{\mathbf{u}}}\left(\boldsymbol{y}_{\mathrm{r}}(\tau)\right)\right\|_{2}^{2} d \tau \leq \int_{0}^{t}\left\|\boldsymbol{C}_{\mathrm{ub}} \boldsymbol{\xi}_{*}(\tau)\right\|_{2}^{2} d \tau .
$$

Bilden des Grenzwertes für $t \rightarrow \infty$ führt zu

$$
\left\|\mathbf{d} \mathbf{z}_{\varrho \overline{\mathbf{u}}}\left(\boldsymbol{y}_{\mathrm{r}}(\tau)\right)\right\|_{L_{2}}^{2} \leq\left\|\boldsymbol{C}_{\mathrm{ub}} \boldsymbol{\xi}_{*}(\tau)\right\|_{L_{2}}^{2} .
$$

Der entscheidende Punkt ist, dass die Energie von $\boldsymbol{y}_{*}(t)=\boldsymbol{C}_{\mathrm{ub}} \boldsymbol{\xi}_{*}(t)$ immer größer ist als die Energie von $\mathbf{d} \mathbf{z}_{\varrho \overline{\mathbf{u}}}\left(\boldsymbol{y}_{\mathrm{r}}(t)\right)$. Folglich genügt es, für die Energieabschätzung das lineare System

$$
\begin{aligned}
\dot{\boldsymbol{\xi}}_{*} & =\boldsymbol{A}_{\mathrm{ub}} \boldsymbol{\xi}_{*}, \\
\boldsymbol{y}_{*} & =\boldsymbol{C}_{\mathrm{ub}} \boldsymbol{\xi}_{*}
\end{aligned}
$$

mit dem Anfangswert $\boldsymbol{\xi}_{*}(0)$ zu betrachten. Der nächste Abschnitt zeigt, wie eine Abschätzung der Ausgangsenergie durch Lösen eines konvexen Optimierungsproblems möglich ist.

\subsubsection{Ausgangsenergie eines linearen Systems}

Nach einer Idee aus [21] lässt sich die Ausgangsenergie des Systems (9.8) mithilfe der Ljapunov-Funktion

$$
V\left(\boldsymbol{\xi}_{*}\right)=\boldsymbol{\xi}_{*}^{\top} \boldsymbol{R} \boldsymbol{\xi}_{*}, \quad \boldsymbol{R} \succ \mathbf{0}
$$

abschätzen. Ausgangspunkt ist der Ansatz

$$
\dot{V}\left(\boldsymbol{\xi}_{*}\right)+\boldsymbol{\xi}_{*}^{\top} \boldsymbol{C}_{\mathrm{ub}}^{\mathrm{T}} \boldsymbol{C}_{\mathrm{ub}} \boldsymbol{\xi}_{*}<0 \quad \forall \boldsymbol{\xi}_{*} \neq \mathbf{0} .
$$

Integrieren dieser Ungleichung nach der Zeit führt zu

$$
V\left(\boldsymbol{\xi}_{*}(t)\right)-V\left(\boldsymbol{\xi}_{*}(0)\right)+\int_{0}^{t} \boldsymbol{\xi}_{*}^{\mathrm{\top}}(\tau) \boldsymbol{C}_{\mathrm{ub}}^{\mathrm{T}} \boldsymbol{C}_{\mathrm{ub}} \boldsymbol{\xi}_{*}(\tau) d \tau<0 .
$$


Da $V\left(\boldsymbol{\xi}_{*}\right)>0$ für alle $\boldsymbol{\xi}_{*} \neq \mathbf{0}$ gilt, folgt die Abschätzung

$$
\begin{aligned}
\int_{0}^{t} \boldsymbol{\xi}_{*}^{\mathrm{\top}}(\tau) \boldsymbol{C}_{\mathrm{ub}}^{\mathrm{\top}} \boldsymbol{C}_{\mathrm{ub}} \boldsymbol{\xi}_{*}(\tau) d \tau \leq \\
V\left(\boldsymbol{\xi}_{*}(t)\right)+\int_{0}^{t} \boldsymbol{\xi}_{*}^{\mathrm{\top}}(\tau) \boldsymbol{C}_{\mathrm{ub}}^{\mathrm{\top}} \boldsymbol{C}_{\mathrm{ub}} \boldsymbol{\xi}_{*}(\tau) d \tau<V\left(\boldsymbol{\xi}_{*}(0)\right)
\end{aligned}
$$

für alle $t \geq 0$. Bilden des Grenzwertes $t \rightarrow \infty$ liefert

$$
\left\|\boldsymbol{C}_{\mathrm{ub}} \boldsymbol{\xi}_{*}\right\|_{L_{2}}^{2}<V\left(\boldsymbol{\xi}_{*}(0)\right)=\boldsymbol{\xi}_{*}^{\top}(0) \boldsymbol{R} \boldsymbol{\xi}_{*}(0)
$$

Somit ist der Ausdruck $\boldsymbol{\xi}_{*}^{\top}(0) \boldsymbol{R} \boldsymbol{\xi}_{*}(0)$ eine obere Abschätzung für die Energie von $\boldsymbol{C}_{\mathrm{ub}} \boldsymbol{\xi}_{*}$ und damit auch von $\mathbf{d} \mathbf{z}_{\varrho \overline{\mathbf{u}}}\left(\boldsymbol{y}_{\mathrm{r}}\right)$, wobei $\boldsymbol{R}$ eine symmetrische, positiv definite Matrix ist, die (9.9) erfüllen muss. Mit

$$
\dot{V}=\dot{\boldsymbol{\xi}}_{*} \boldsymbol{R} \boldsymbol{\xi}_{*}+\boldsymbol{\xi}_{*} \boldsymbol{R} \dot{\boldsymbol{\xi}}_{*}=\boldsymbol{\xi}_{*}^{\top}\left(\boldsymbol{A}_{\mathrm{ub}}^{\top} \boldsymbol{R}+\boldsymbol{R} \boldsymbol{A}_{\mathrm{ub}}\right) \boldsymbol{\xi}_{*}
$$

lässt sich diese Bedingung auf die lineare Matrixungleichung

$$
\boldsymbol{A}_{\mathrm{ub}}^{\top} \boldsymbol{R}+\boldsymbol{R} \boldsymbol{A}_{\mathrm{ub}}+\boldsymbol{C}_{\mathrm{ub}}^{\mathrm{\top}} \boldsymbol{C}_{\mathrm{ub}} \prec 0
$$

zurückführen. Der folgende Satz fasst die Überlegungen zusammen.

Satz 9.1. Gegeben sei das System (9.4) mit dem Anfangswert $\boldsymbol{\xi}_{\mathrm{L}}^{(0)}$. Die Ruhelage dieses Systems für Eingangsgrößen $\boldsymbol{w}=\boldsymbol{w}_{0}$ und $\boldsymbol{z}=\boldsymbol{z}_{0}$ ist

$$
\boldsymbol{\xi}_{\mathrm{L}}^{(\mathrm{R})}=-\boldsymbol{A}_{\mathrm{ub}}^{-1} \boldsymbol{B}_{\mathrm{ub}} \boldsymbol{r}_{0} \quad \text { mit } \boldsymbol{r}_{0}^{\top}=\left[\begin{array}{ll}
\boldsymbol{w}_{0}^{\top} & \boldsymbol{z}_{0}^{\top}
\end{array}\right] .
$$

Für den stationären Wert der Ausgangsgröße $\boldsymbol{y}_{\mathrm{r}}^{(\mathrm{R})}$ gelte $\varrho \bar{u}_{i}>\left|y_{\mathrm{r}, i}^{(\mathrm{R})}\right|$ für alle $i=1, \ldots, m$, positive Skalare $\bar{u}_{i}$ und ein $\varrho \in(0,1)$. Wenn eine symmetrische, positiv definite Matrix $\boldsymbol{R}$ existiert, so dass die Matrixungleichung

$$
\boldsymbol{A}_{\mathrm{ub}}^{\mathrm{\top}} \boldsymbol{R}+\boldsymbol{R} \boldsymbol{A}_{\mathrm{ub}}+\boldsymbol{C}_{\mathrm{ub}}^{\mathrm{\top}} \boldsymbol{C}_{\mathrm{ub}} \prec \mathbf{0}
$$

erfüllt ist, dann ist $\boldsymbol{\xi}_{*}^{\top}(0) \boldsymbol{R} \boldsymbol{\xi}_{*}(0)$ mit $\boldsymbol{\xi}_{*}(0)=\boldsymbol{\xi}_{\mathrm{L}}(0)-\boldsymbol{\xi}_{\mathrm{L}}^{(\mathrm{R})}$ eine obere Grenze für $\left\|\boldsymbol{d} z_{\varrho} \overline{\boldsymbol{u}}\left(\boldsymbol{y}_{\mathrm{r}}\right)\right\|_{2}^{2}$ mit $\overline{\boldsymbol{u}}=\left[\bar{u}_{1} \ldots \bar{u}_{m}\right]^{\top}$.

Um eine möglichst gute Abschätzung zu erhalten, bietet es sich an, die Funktion $\boldsymbol{\xi}_{*}^{\top}(0) \boldsymbol{R} \boldsymbol{\xi}_{*}(0) \mathrm{zu}$ minimieren. Das führt zu dem konvexen 


\section{Optimierungsproblem 9.1.}

$\underset{\boldsymbol{R}}{\operatorname{Minimiere}}\left(\boldsymbol{\xi}_{\mathrm{L}}^{(0)}-\boldsymbol{\xi}_{\mathrm{L}}^{(\mathrm{R})}\right)^{\top} \boldsymbol{R}\left(\boldsymbol{\xi}_{\mathrm{L}}^{(0)}-\boldsymbol{\xi}_{\mathrm{L}}^{(\mathrm{R})}\right) \quad$ unter den Nebenbedingungen

$$
\begin{aligned}
\boldsymbol{R} & \succ \mathbf{0}, \\
\boldsymbol{A}_{\mathrm{ub}}^{\top} \boldsymbol{R}+\boldsymbol{R} \boldsymbol{A}_{\mathrm{ub}}+C_{\mathrm{ub}}^{\mathrm{T}} C_{\mathrm{ub}} & \prec \mathbf{0} .
\end{aligned}
$$

Bisher wurde nur die Energie von $\mathbf{d} \mathbf{z}_{\varrho \overline{\mathbf{u}}}\left(\boldsymbol{y}_{\mathrm{r}}\right)$ für einen Anfangswert $\boldsymbol{\xi}_{\mathrm{L}}^{(0)}$ abgeschätzt. Oftmals liegen mögliche Anfangszustände in einem Gebiet $\mathcal{X}^{(0)} \in \mathbb{R}^{n_{\mathrm{s}}+n_{\mathrm{r}}}$. Auch in diesem Fall lässt sich eine obere Grenze für die maximale Ausgangsenergie abschätzen, die für alle $\boldsymbol{\xi}_{\mathrm{L}}^{(0)} \in \boldsymbol{\mathcal { X }}^{(0)}$ gilt. Besonders einfach ist dies für Anfangsgebiete, die sich durch ein konvexes Polytop beschreiben lassen. Konvexe Polytope sind vollständig durch ihre Eckpunkte definiert, denn jeder Punkt im Inneren des Polytops lässt sich als konvexe Kombination der Eckpunkte darstellen. Im Weiteren wird eine Menge $\mathcal{P}$ bestehend aus $p \in \mathbb{N} \backslash\{0\}$ Eckpunkten

$$
\mathcal{P}=\left\{\boldsymbol{\xi}_{\mathrm{L} 1}^{(0)}, \ldots, \boldsymbol{\xi}_{\mathrm{L} p}^{(0)}\right\}
$$

betrachtet. Das konvexe Polytop $\boldsymbol{\mathcal { X }}^{(0)}$ ergibt sich dann als konvexe Hülle von $\mathcal{P}[22]$ wie folgt

$$
\begin{aligned}
\boldsymbol{\mathcal { X }}^{(0)} & =\operatorname{co} \mathcal{P}=\operatorname{co}\left\{\boldsymbol{\xi}_{\mathrm{L} 1}^{(0)}, \ldots, \boldsymbol{\xi}_{\mathrm{L} p}^{(0)}\right\} \\
& =\left\{\boldsymbol{\xi}_{\mathrm{L}} \in \mathbb{R}^{n_{\mathrm{s}}+n_{\mathrm{r}}} \mid \boldsymbol{\xi}_{\mathrm{L}}=\sum_{i}^{p} \mathrm{c}_{i} \boldsymbol{\xi}_{\mathrm{L} i}^{(0)}, c_{i} \geq 0, \sum_{i}^{p} \mathrm{c}_{i}=1\right\} .
\end{aligned}
$$

Nach den Überlegungen in [21, Abschnitt 6.2.1] nimmt die Zielfunktion

$$
\left(\boldsymbol{\xi}_{\mathrm{L}}^{(0)}-\boldsymbol{\xi}_{\mathrm{L}}^{(\mathrm{R})}\right)^{\top} \boldsymbol{R}\left(\boldsymbol{\xi}_{\mathrm{L}}^{(0)}-\boldsymbol{\xi}_{\mathrm{L}}^{(\mathrm{R})}\right)
$$

auf der Menge $\boldsymbol{\mathcal { X }}^{(0)}$ an einem der Eckpunkte $\boldsymbol{\xi}_{\mathrm{Li}}^{(0)}$ ihr Maximum an. Somit ist das Optimierungsproblem 9.1 für jedes Element $\boldsymbol{\xi}_{\mathrm{L} i}^{(0)}$ der Menge $\mathcal{P}$ zu lösen, um die entsprechende Ausgangsenergie abzuschätzen. Die größte Ausgangsenergie $\theta^{*}$ stellt die gesuchte Abschätzung dar. Für die auf das Anti-Windup-Ersatzsystem ES wirkende Störung $\boldsymbol{\zeta}$ gilt folglich

$$
\|\boldsymbol{\zeta}\|_{L_{2}}^{2} \leq 4 \theta^{*}=\theta
$$

Damit ist ein Zusammenhang zwischen der Begrenzung der Anfangswerte $\boldsymbol{\xi}_{\mathrm{L}}^{(0)}=\left[\begin{array}{ll}\boldsymbol{\xi}_{\mathrm{s}}^{(0)} & \boldsymbol{\xi}_{\mathrm{r}}^{(0)}\end{array}\right]$ sowie der Begrenzung der Eingangsgrößen $\boldsymbol{w}, \boldsymbol{z}$ und der Größe der Störung $\boldsymbol{\zeta}$ hergestellt. Das in Abschnitt 9.1 beschriebene erste Teilproblem kann als gelöst betrachtet werden. Der nächste Abschnitt betrachtet das zweite Teilproblem. 


\subsection{Anti-Windup-Entwurf}

Das zweite Teilproblem besteht in der Fragestellung, wie die Anti-WindupMatrix $\underline{\boldsymbol{K}}$ parametriert werden muss, so dass die Stabilität von

$$
\begin{aligned}
\dot{\boldsymbol{\xi}}_{\mathrm{aw}} & =\boldsymbol{A}_{\mathrm{s}} \boldsymbol{\xi}_{\mathrm{aw}}+\boldsymbol{B}_{\mathrm{s}, u} \mathbf{s a t}_{\alpha \overline{\mathbf{u}}}\left(-\underline{\boldsymbol{K}} \boldsymbol{\xi}_{\mathrm{aw}}\right)+\boldsymbol{B}_{\mathrm{s}, u} \boldsymbol{\zeta}, \\
\boldsymbol{y}_{\mathrm{aw}, \mathrm{y}} & =\boldsymbol{C}_{\mathrm{s}} \boldsymbol{\xi}_{\mathrm{aw}}
\end{aligned}
$$

mit $\boldsymbol{\xi}_{\text {aw }}^{(0)}=\mathbf{0}$ für die durch (9.11) begrenzte Störung $\boldsymbol{\zeta}$ garantiert ist. Eine allgemeine Antwort gibt Satz 6.7, der die folgenden Bedingungen stellt.

(A) Der Zustand $\boldsymbol{\xi}_{\text {aw }}(t)$ verbleibt für $\boldsymbol{\zeta} \neq \mathbf{0}$ und alle $t \geq 0$ in einer begrenzten Umgebung des Ursprungs $\mathcal{G}_{u} \subset \mathbb{R}^{n_{\mathrm{s}}}$.

(B) Für $\boldsymbol{\zeta}=\mathbf{0}$ soll der Zustand $\boldsymbol{\xi}_{\text {aw }}$ in die im Ursprung gelegene Ruhelage streben. Das ist garantiert, wenn $\mathcal{G}_{u}$ im Einzugsgebiet $\mathcal{G}(\mathbf{0})$ der Ruhelage liegt, d. h. $\mathcal{G}(\mathbf{0}) \supset \mathcal{G}_{u}$.

Um auch das Anti-Windup-Ziel Rückgewinnung des unbeschränkten Verhaltens in den Entwurf mit einzubeziehen, werden die beiden Forderungen um eine dritte Anforderung ergänzt.

(C) Die $L_{2}$-Verstärkung von $\boldsymbol{\zeta}$ nach $\boldsymbol{y}_{\text {aw,y }}$ ist begrenzt und möglichst klein für energiebegrenzte Eingangssignale $\boldsymbol{\zeta}$.

\subsubsection{Parametrierung der Anti-Windup-Matrix}

Der in [31] vorgestellte Zustandsreglerentwurf gibt hinreichende Bedingungen für die Matrix $\underline{\boldsymbol{K}}$ in Form linearer Matrixungleichungen an, die obige Anforderungen sicherstellen. Die Grundidee ist, das Gebiet $\mathcal{G}_{u}$ durch eine Höhenlinie der quadratischen Ljapunov-Funktion

$$
V\left(\boldsymbol{\xi}_{\text {aw }}\right)=\boldsymbol{\xi}_{\text {aw }}^{\top} \boldsymbol{P} \boldsymbol{\xi}_{\text {aw }}, \quad \boldsymbol{P} \succ \mathbf{0}
$$

zu beschreiben. Im Weiteren wird eine Umgebung des Ursprungs $\mathbb{D} \subset \mathbb{R}^{n_{\mathrm{s}}}$ betrachtet. Der Ansatz

$$
\dot{V}\left(\boldsymbol{\xi}_{\mathrm{aw}}\right)+\boldsymbol{y}_{\mathrm{aw}, \mathrm{y}}^{\top} \boldsymbol{y}_{\mathrm{aw}, \mathrm{y}}-\frac{1}{\eta} \boldsymbol{\zeta}^{\top} \boldsymbol{\zeta}<0 \quad \forall \boldsymbol{\xi}_{\mathrm{aw}} \in \mathbb{D}
$$

mit $\eta>0$ und

$$
\dot{V}\left(\boldsymbol{\xi}_{\mathrm{aw}}\right)=\dot{\boldsymbol{\xi}}_{\mathrm{aw}}^{\top} \boldsymbol{P} \boldsymbol{\xi}_{\mathrm{aw}}+\boldsymbol{\xi}_{\mathrm{aw}}^{\top} \boldsymbol{P} \dot{\boldsymbol{\xi}}_{\mathrm{aw}}
$$


führt zu geeigneten Entwurfsbedingungen. Integrieren von (9.12) nach der Zeit liefert unter Beachtung von $V\left(\boldsymbol{\xi}_{\text {aw }}(0)\right)=0$ die Ungleichung

$$
V\left(\boldsymbol{\xi}_{\mathrm{aw}}(t)\right)+\int_{0}^{t} \boldsymbol{y}_{\mathrm{aw}, \mathrm{y}}^{\top}(\tau) \boldsymbol{y}_{\mathrm{aw}, \mathrm{y}}(\tau) d \tau-\frac{1}{\eta} \int_{0}^{t} \boldsymbol{\zeta}^{\top}(\tau) \boldsymbol{\zeta}(\tau) d \tau<0,
$$

welche die gewünschten Eigenschaften garantiert.

(A) Stabilität lässt sich durch Auflösen nach $V\left(\boldsymbol{\xi}_{\text {aw }}(t)\right)$ zeigen, was zu

$$
V\left(\boldsymbol{\xi}_{\text {aw }}(t)\right)<\frac{1}{\eta} \int_{0}^{t} \boldsymbol{\zeta}^{\top}(\tau) \boldsymbol{\zeta}(\tau) d \tau \leq \frac{\theta}{\eta}
$$

führt. Da $V\left(\boldsymbol{\xi}_{\text {aw }}(t)\right)$ für alle $t \geq 0$ durch $\theta / \eta$ begrenzt ist, verbleibt der Zustand $\boldsymbol{\xi}_{\text {aw }}(t)$ für alle $t \geq 0$ in dem Gebiet

$$
\mathcal{G}_{u}=\mathcal{E}(\boldsymbol{P}, \theta / \eta)=\left\{\boldsymbol{\xi}_{\text {aw }} \in \mathbb{R}^{n_{\mathrm{s}}} \mid \boldsymbol{\xi}_{\text {aw }}^{\top} \boldsymbol{P} \boldsymbol{\xi}_{\text {aw }} \leq \theta / \eta\right\}
$$

(B) Des Weiteren gilt für $\boldsymbol{\zeta}=\mathbf{0}$, dass $\dot{V}\left(\boldsymbol{\xi}_{\text {aw }}\right)<0$ für alle $\boldsymbol{\xi}_{\text {aw }} \in \mathbb{D}$. Wenn das Gebiet $\mathcal{G}_{u}$ im Inneren von $\mathbb{D}$ liegt, was durch eine zusätzliche Bedingung überprüft werden kann, gilt $\dot{V}\left(\boldsymbol{\xi}_{\text {aw }}\right)<0$ auch für alle $\boldsymbol{\xi}_{\text {aw }} \in \mathcal{G}_{u}$. Folglich ist $\mathcal{G}_{u}$ ein Einzugsgebiet der Ruhelage $\boldsymbol{\xi}_{\text {aw }}^{(\mathrm{R})}=\mathbf{0}$.

(C) Auch die $L_{2}$-Verstärkung des Systems von $\boldsymbol{\zeta}$ zum Ausgang $\boldsymbol{y}_{\text {aw,y }}$ ist begrenzt, wenn (9.13) gilt. Bilden des Grenzwertes $t \rightarrow \infty$ liefert

$$
\left\|\boldsymbol{y}_{\text {aw }, \mathrm{y}}\right\|_{L_{2}}^{2}<\frac{1}{\eta}\|\boldsymbol{\zeta}\|_{L_{2}}^{2} .
$$

Die konsequente Weiterentwicklung der oben skizzierten Grundidee führt zu einem Satz, der hinreichende Stabilitätsbedingungen angibt.

Satz 9.2 (nach [31]). Gegeben sei das System

$$
\begin{aligned}
\dot{\boldsymbol{\xi}}_{\mathrm{aw}} & =\boldsymbol{A}_{\mathrm{s}} \boldsymbol{\xi}_{\mathrm{aw}}+\boldsymbol{B}_{\mathrm{s}, u} \boldsymbol{s} \boldsymbol{a} \boldsymbol{t}_{\alpha \overline{\boldsymbol{u}}}(\boldsymbol{u})+\boldsymbol{B}_{\mathrm{s}, u} \boldsymbol{\zeta} \\
\boldsymbol{y}_{\mathrm{aw}, \mathrm{y}} & =\boldsymbol{C}_{\mathrm{s}} \boldsymbol{\xi}_{\mathrm{aw}}
\end{aligned}
$$

mit $\boldsymbol{\xi}_{\mathrm{aw}}^{(0)}=\mathbf{0}$. Die Störung $\boldsymbol{\zeta}$ besitze eine begrenzte Energie, d. $h .\|\boldsymbol{\zeta}\|_{L_{2}}^{2} \leq \theta$. Wenn eine symmetrische, positiv definite Matrix $\boldsymbol{W} \in \mathbb{S}^{n_{\mathrm{s}}}$, eine positiv definite Diagonalmatrix $\boldsymbol{N} \in \mathbb{S}^{m}$, Matrizen $\boldsymbol{J} \in \mathbb{R}^{n_{\mathrm{s}} \times n_{\mathrm{s}}}, \boldsymbol{X} \in \mathbb{R}^{m \times n_{\mathrm{s}}}$, 
$\boldsymbol{Y} \in \mathbb{R}^{m \times n_{\mathrm{s}}}$ und positive Skalare $\eta, \beta$ existieren, so dass mit der Abkürzung $\boldsymbol{M}^{*}=\boldsymbol{W}-\boldsymbol{J}^{\top}+\boldsymbol{A}_{\mathrm{s}} \boldsymbol{J}+\boldsymbol{B}_{\mathrm{s}, u} \boldsymbol{X}$ für alle $i=1, \ldots, m$ die Ungleichungen

$$
\begin{array}{r}
\left.\operatorname{He} \begin{array}{ccccc}
\boldsymbol{A}_{\mathrm{s}} \boldsymbol{J}+\boldsymbol{B}_{\mathrm{s}, u} \boldsymbol{X} & \mathbf{0} & \mathbf{0} & \mathbf{0} & \mathbf{0} \\
\boldsymbol{M}^{*} & -\boldsymbol{J} & \mathbf{0} & \mathbf{0} & \mathbf{0} \\
\boldsymbol{Y}-\boldsymbol{N}^{\top} \boldsymbol{B}_{\mathrm{s}, u}^{\top} & -\boldsymbol{N}^{\top} \boldsymbol{B}_{\mathrm{s}, u}^{\top} & -\boldsymbol{N} & \mathbf{0} & \mathbf{0} \\
\eta \boldsymbol{B}_{\mathrm{s}, u}^{\top} & \eta \boldsymbol{B}_{\mathrm{s}, u}^{\top} & \mathbf{0} & -\frac{1}{2} \eta \mathbb{I} & \mathbf{0} \\
\boldsymbol{C}_{\mathrm{s}} \boldsymbol{J} & \mathbf{0} & \mathbf{0} & \mathbf{0} & -\frac{1}{2} \mathbb{I}
\end{array}\right] \\
{\left[\begin{array}{ccc}
\boldsymbol{W} & \left(\boldsymbol{X}_{\langle i\rangle}-\boldsymbol{Y}_{\langle i\rangle}\right)^{\top} & \left(\boldsymbol{X}_{\langle i\rangle}-\boldsymbol{Y}_{\langle i\rangle}\right)^{\top} \\
\boldsymbol{X}_{\langle i\rangle}-\boldsymbol{Y}_{\langle i\rangle} & 1 / \beta \cdot\left(\alpha \bar{u}_{i}\right)^{2} & 0 \\
\boldsymbol{X}_{\langle i\rangle}-\boldsymbol{Y}_{\langle i\rangle} & 0 & \eta / \theta \cdot\left(\alpha \bar{u}_{i}\right)^{2}
\end{array}\right] \succeq \mathbf{0}}
\end{array}
$$

erfüllt sind, wobei $\boldsymbol{X}_{\langle i\rangle}$ bzw. $\boldsymbol{Y}_{\langle i\rangle}$ die i-te Zeile der Matrix $\boldsymbol{X}$ bzw. $\boldsymbol{Y}$ bezeichnet, dann garantiert der Regler $\boldsymbol{u}=-\underline{\boldsymbol{K}} \boldsymbol{\xi}_{\text {aw }}$ mit $\underline{\boldsymbol{K}}=-\boldsymbol{X} \boldsymbol{J}^{-1}$ folgende Eigenschaften des geschlossenen Regelkreises:

(a) Für $\boldsymbol{\zeta} \neq \mathbf{0}$ verbleiben die Trajektorien des geschlossenen Regelkreises für alle $t \geq 0$ in der Hyperellipse

$$
\overline{\mathcal{E}}(\boldsymbol{P}, \mathrm{c})=\left\{\boldsymbol{\xi}_{\text {aw }} \in \mathbb{R}^{n_{\mathrm{s}}} \mid \boldsymbol{\xi}_{\mathrm{aw}}^{\top} \boldsymbol{P} \boldsymbol{\xi}_{\text {aw }} \leq \mathrm{c}\right\}
$$

mit $\boldsymbol{P}=\boldsymbol{J}^{-1} \boldsymbol{W} \boldsymbol{J} \succ \mathbf{0}$ und $\mathbf{c}=\beta+\theta / \eta$.

(b) Für $\boldsymbol{\zeta}=\mathbf{0}$ ist die Ruhelage $\boldsymbol{\xi}_{\mathrm{aw}}^{(\mathrm{R})}=\mathbf{0}$ lokal asymptotisch stabil und $\overline{\mathcal{E}}(\boldsymbol{P}, \mathrm{c})$ ist ein Einzugsgebiet der Ruhelage.

(c) Der geschlossene Regelkreis besitzt eine endliche $L_{2}$-Verstärkung für Störungen $\|\boldsymbol{\zeta}\|_{L_{2}}^{2} \leq \theta$. Es gilt

$$
\left\|\boldsymbol{y}_{\mathrm{aw}, \mathrm{y}}\right\|_{L_{2}} \leq(1 / \sqrt{\eta})\|\boldsymbol{\zeta}\|_{L_{2}}+\sqrt{\beta}
$$

Beweis. Der Satz folgt direkt aus Proposition 2.1 in [31].

Durch Hinzufügen einer weiteren Entwurfsbedingung lässt sich eine gewisse Regelgüte in der Nähe des Ursprungs vorgeben. Für einen gegebenen Skalar $\underline{\lambda}>0$ und ein $\boldsymbol{W}_{\boldsymbol{\lambda}} \succ \mathbf{0}$ garantiert die lineare Matrixungleichung

$$
\operatorname{He}\left[\begin{array}{cc}
\boldsymbol{A}_{\mathrm{s}} \boldsymbol{J}+\boldsymbol{B}_{\mathrm{s}, u} \boldsymbol{X}+\underline{\lambda} \boldsymbol{J} & \mathbf{0} \\
\boldsymbol{W}_{\lambda}+\boldsymbol{A}_{\mathrm{s}} \boldsymbol{J}+\boldsymbol{B}_{\mathrm{s}, u} \boldsymbol{X}-\boldsymbol{J}^{\top}+\underline{\lambda} \boldsymbol{J} & -\boldsymbol{J}^{\top}
\end{array}\right] \prec \mathbf{0},
$$

dass die Realteile der Eigenwerte von $\boldsymbol{A}_{\mathrm{s}}-\boldsymbol{B}_{\mathrm{s}, u} \underline{\boldsymbol{K}}$ kleiner als $-\underline{\boldsymbol{\lambda}}$ sind [31]. Der Zustandsreglerentwurf lässt sich folglich als konvexes Optimierungsproblem formulieren. 
Optimierungsproblem 9.2. Für gegebene Skalare $\theta>0, \underline{\lambda}>0$,

$$
\begin{gathered}
\underset{\boldsymbol{W}, \boldsymbol{W}_{\lambda}, \boldsymbol{J}, \boldsymbol{N}, \boldsymbol{X}, \boldsymbol{Y}, \eta, \beta}{\operatorname{minimiere}}-\eta \quad \text { unter den Nebenbedingungen } \\
\boldsymbol{W} \succ \mathbf{0}, \boldsymbol{W}_{\lambda} \succ \mathbf{0}, \boldsymbol{N} \succ \mathbf{0}, \\
\eta>0, \beta>0, \\
(9.15),(9.16) \text { für alle } i=1, \ldots, m,(9.17) .
\end{gathered}
$$

Mit Optimierungsproblem 9.1 und Optimierungsproblem 9.2 stehen alle nötigen Werkzeuge bereit, um den Anti-Windup-Entwurf durchzuführen. Der nächste Abschnitt fasst die einzelnen Entwurfsschritte zusammen.

\subsubsection{Zusammenfassung der Entwurfsschritte}

Gegeben sei der Anti-Windup-Regelkreis bestehend aus einer linearen Strecke $\mathbf{S}$, einem linearen Regler $\mathbf{R}$ und dem durch $\overline{\mathbf{u}}$ charakterisierten realen Aktor. Der geforderte Sollwert sei $\boldsymbol{w}=\boldsymbol{w}_{0}$ und die auftretende konstante Störung sei $\boldsymbol{z}=\boldsymbol{z}_{0}$. Aus Sollwert und Störung ergibt sich mit $\boldsymbol{r}_{0}^{\top}=\left[\begin{array}{ll}\boldsymbol{w}_{0}^{\top} & \boldsymbol{z}_{0}^{\top}\end{array}\right]$ und $\boldsymbol{A}_{\mathrm{ub}}, \boldsymbol{B}_{\mathrm{ub}}$ gemäß (2.4) die zu stabilisierende Ruhelage des Anti-Windup-Regelkreises wie folgt

$$
\left[\begin{array}{c}
\boldsymbol{x}_{\mathrm{s}}^{(\mathrm{R})} \\
\boldsymbol{x}_{\mathrm{r}}^{(\mathrm{R})}
\end{array}\right]=\boldsymbol{x}_{\mathrm{ub}}^{(\mathrm{R})}=-\boldsymbol{A}_{\mathrm{ub}}^{-1} \boldsymbol{B}_{\mathrm{ub}} \boldsymbol{r}_{0}, \quad \boldsymbol{x}_{\mathrm{aw}}^{(\mathrm{R})}=\mathbf{0} .
$$

Die zugehörige stationäre Stellgröße

$$
\boldsymbol{y}_{\mathrm{r}}^{(\mathrm{R})}=\boldsymbol{C}_{\mathrm{ub}} \boldsymbol{x}_{\mathrm{ub}}^{(\mathrm{R})}+\boldsymbol{D}_{\mathrm{ub}} \boldsymbol{r}_{0}
$$

mit den Matrizen $\boldsymbol{C}_{\mathrm{ub}}, \boldsymbol{D}_{\mathrm{ub}}$ nach (6.14) sei mit der zur Verfügung stehenden Stellgröße realisierbar und damit die Ruhelage gemäß Definition 6.2 zulässig. Aufgrund der Beziehung (4.9) ist die Ruhelage invariant gegenüber einer Transformation in Mismatch-Koordinaten, d. h., es gilt

$$
\boldsymbol{\xi}_{\mathrm{L}}^{(\mathrm{R})}=\boldsymbol{x}_{\mathrm{ub}}^{(\mathrm{R})}, \quad \boldsymbol{\xi}_{\mathrm{aw}}^{(\mathrm{R})}=\boldsymbol{x}_{\mathrm{aw}}^{(\mathrm{R})} .
$$

Die Anfangszustände $\boldsymbol{x}_{\mathrm{s}}^{(0)}, \boldsymbol{x}_{\mathrm{r}}^{(0)}$ von Strecke und Regler liegen in einem konvexen Polytop $\boldsymbol{\mathcal { X }}^{(0)}$, das durch die Menge seiner Eckpunkte

$$
\mathcal{P}=\left\{\left[\begin{array}{l}
\boldsymbol{x}_{\mathrm{s} 1}^{(0)} \\
\boldsymbol{x}_{\mathrm{r} 1}^{(0)}
\end{array}\right], \ldots,\left[\begin{array}{l}
\boldsymbol{x}_{\mathrm{sp}}^{(0)} \\
\boldsymbol{x}_{\mathrm{r} p}^{(0)}
\end{array}\right]\right\}
$$


eindeutig gegeben ist, d. h. $\mathcal{X}^{(0)}=$ co $\mathcal{P}$. Da für den Anfangszustand des linearen modellbasierten Anti-Windup $\boldsymbol{x}_{\mathrm{aw}}^{(0)}=\mathbf{0}$ gilt, ist $\boldsymbol{\mathcal { X }}^{(0)}$ ebenfalls invariant gegenüber der Transformation in Mismatch-Koordinaten und kann somit direkt für den Entwurf verwendet werden. Insbesondere gilt

$$
\boldsymbol{x}_{\mathrm{s}}^{(0)}=\boldsymbol{\xi}_{\mathrm{s}}^{(0)}, \quad \boldsymbol{x}_{\mathrm{r}}^{(0)}=\boldsymbol{\xi}_{\mathrm{r}}^{(0)}, \quad \boldsymbol{x}_{\mathrm{aw}}^{(0)}=-\boldsymbol{\xi}_{\mathrm{aw}}^{(0)}=\mathbf{0} .
$$

Für den Entwurf empfiehlt sich ein Vorgehen in drei Schritten.

Schritt 1: Wahl des Anti-Windup-Parameters $\alpha=1-\varrho$ aus dem offenen Intervall $(0, \hat{\alpha})$ mit

$$
\hat{\alpha}=1-\max _{i \in\{1, \ldots, m\}}\left\{\left|y_{\mathrm{r}, i}^{(\mathrm{R})}\right| \overline{\mathrm{u}}_{i}^{-1}\right\} .
$$

Dabei sollte $\alpha$ möglichst groß gewählt werden, um die Einflussmöglichkeit der Zustandsrückführung $\boldsymbol{u}=\mathbf{s a t}_{\alpha \overline{\mathbf{u}}}\left(-\underline{\boldsymbol{K}} \boldsymbol{\xi}_{\text {aw }}\right)$ auf die Systemdynamik (9.14) nicht zu stark einzuschränken.

Schritt 2: Abschätzen einer oberen Grenze $\theta$ der Energie von $\boldsymbol{\zeta}$ für das konvexe Polytop $\mathcal{X}^{(0)}$ möglicher Anfangszustände. Dazu wird das Optimierungsproblem 9.1 p-mal gelöst, d. h. für die berechnete Ruhelage

$$
\boldsymbol{\xi}_{\mathrm{L}}^{(\mathrm{R})}=\left[\begin{array}{l}
\boldsymbol{\xi}_{\mathrm{s}}^{(\mathrm{R})} \\
\boldsymbol{\xi}_{\mathrm{r}}^{\mathrm{R})}
\end{array}\right]=\left[\begin{array}{l}
\boldsymbol{x}_{\mathrm{s}}^{(\mathrm{R})} \\
\boldsymbol{x}_{\mathrm{r}}^{(\mathrm{R})}
\end{array}\right]
$$

und jeweils einen der $p$ Eckpunkte von $\boldsymbol{\mathcal { X }}^{(0)}$. Bezeichne $\theta^{*}$ das Maximum der $p$ Lösungen, dann gilt

$$
\|\boldsymbol{\zeta}\|_{L_{2}}^{2} \leq 4 \theta^{*}=\theta
$$

Schritt 3. Parametrieren der Anti-Windup-Matrix $\underline{\boldsymbol{K}}$ durch Lösen des Optimierungsproblems 9.2.

Falls das Optimierungsproblem 9.2 lösbar ist, wird die Stabilität der Ruhelage des Anti-Windup-Regelkreises durch Satz 6.7 sichergestellt. Die Existenz einer Lösung ist allerdings nicht garantiert, denn mit einer begrenzten Stellgröße sind die Einflussmöglichkeiten auf den Zustand $\boldsymbol{\xi}_{\text {aw }}$ des Systems ES beschränkt. Zu große Störungen führen, unabhängig von dem verwendeten Zustandsregler, immer zu einem instabilen Verhalten. In diesem Fall ist Stabilität des Anti-Windup-Regelkreises nur für ein kleineres Anfangsgebiet $\boldsymbol{\mathcal { X }}^{(0)}$ oder einen weniger aggressiven linearen Regler $\mathbf{R}$ erreichbar. 


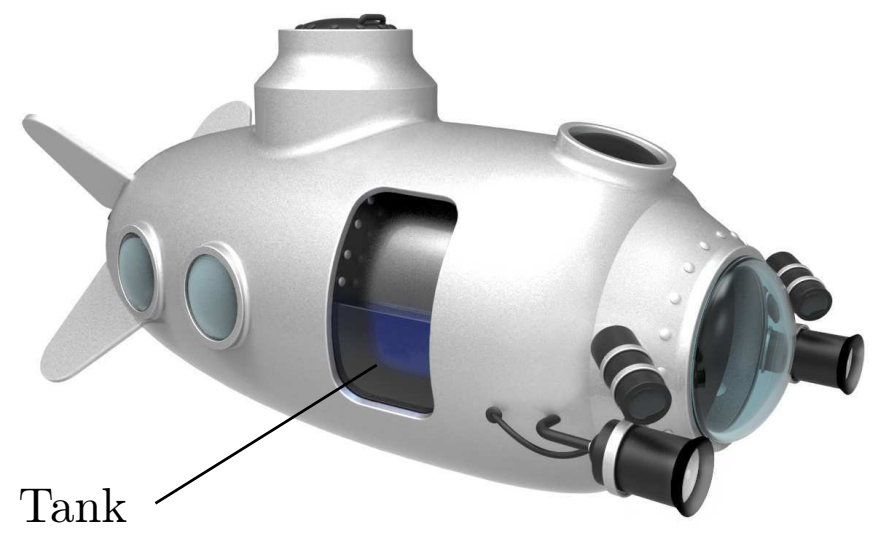

Abbildung 9.3: Forschungsunterseeboot mit Ballasttank.

\subsection{Beispiel: Tauchtiefe eines U-Bootes}

Dieser Abschnitt demonstriert das neue Entwurfsverfahren für lineares modellbasiertes Anti-Windup an einem Beispiel. Betrachtet wird die Tiefenregelung eines U-Bootes für statisches Tauchen. Das sogenannte statische Tauchen basiert auf einer Änderung der Bootsmasse durch Aufnahme oder Abgabe von Meerwasser [3]. Das Wasser kann in speziellen Tanks gespeichert werden, wie Abbildung 9.3 zeigt. Die zusätzliche Masse erzeugt eine vertikale Kraft, die das U-Boot absinken lässt.

Das in $[3,74]$ verwendete lineare Modell eines U-Bootes für statisches Tauchen besitzt drei Zustände und ist durch die Matrizen

$$
\boldsymbol{A}_{\mathrm{s}}=\left[\begin{array}{ccc}
0 & 1 & 0 \\
0 & 0 & 1 \\
0 & 0 & -0,005
\end{array}\right], \quad \boldsymbol{b}_{\mathrm{s}, u}=\left[\begin{array}{l}
0 \\
0 \\
0,005
\end{array}\right]
$$

gegeben. Dabei beschreibt $x_{\mathrm{s}, 1}$ die Abweichung der Tauchtiefe von einem Referenzwert in $\mathrm{m}$. Der Zustand $x_{\mathrm{s}, 2}$ stellt die vertikale Geschwindigkeit in $\mathrm{m} / \mathrm{s}$ dar und $x_{\mathrm{s}, 3}$ die vertikale Beschleunigung des U-Bootes in $\mathrm{m} / \mathrm{s}^{2}$. Der einzig messbare Zustand sei die Tauchtiefe, d. h.

$$
\boldsymbol{c}_{\mathrm{s}}^{\top}=\left[\begin{array}{lll}
1 & 0 & 0
\end{array}\right] \text {. }
$$

Zur Regelung kommt ein Kontrollbeobachter zum Einsatz. Die Reglermatrizen sind folglich durch

$$
\begin{aligned}
& \boldsymbol{A}_{\mathrm{r}}=\boldsymbol{A}_{\mathrm{s}}-\boldsymbol{l}_{\mathrm{r}} \boldsymbol{c}_{\mathrm{S}}^{\top}-\boldsymbol{b}_{\mathrm{s}, u} \boldsymbol{k}_{\mathrm{r}}^{\top}, \quad \boldsymbol{b}_{\mathrm{r}, u}=\boldsymbol{l}_{\mathrm{r}}, \quad \boldsymbol{b}_{\mathrm{s}, w}=\mathbf{0}, \\
& \boldsymbol{c}_{\mathrm{r}}^{\top}=-\boldsymbol{k}_{\mathrm{r}}^{\top}, \quad d_{\mathrm{r}, u}=0, \quad d_{\mathrm{r}, w}=0
\end{aligned}
$$


gegeben und durch die zwei Vektoren

$$
\boldsymbol{k}_{\mathrm{r}}^{\top}=\left[\begin{array}{lll}
10^{-4} & 0,128 & 49,373
\end{array}\right], \quad \boldsymbol{l}_{\mathrm{r}}^{\top}=\left[\begin{array}{lll}
5,569 & 9,770 & 5,438
\end{array}\right]
$$

vollständig parametriert. Die in der Arbeit [74] betrachteten Anfangszustände der Strecke lassen sich durch

$$
\left|x_{\mathrm{s}, 1}\right| \leq 10,\left|x_{\mathrm{s}, 2}\right| \leq 0,05, \quad\left|x_{\mathrm{s}, 3}\right| \leq 0,005
$$

beschreiben. Für den Anfangszustand des Reglers wird $\boldsymbol{x}_{\mathrm{r}}^{(0)}=\mathbf{0}$ angenommen. Mit den Vektoren

$$
\begin{aligned}
& \boldsymbol{x}_{\mathrm{ub}, 1}^{(0)}=\left[\begin{array}{llllll}
10 & 0,05 & 0,005 & 0 & 0 & 0
\end{array}\right]^{\top}, \\
& \boldsymbol{x}_{\mathrm{ub}, 2}^{(0)}=\left[\begin{array}{llllll}
10 & 0,05 & -0,005 & 0 & 0 & 0
\end{array}\right]^{\top}, \\
& \boldsymbol{x}_{\mathrm{ub}, 3}^{(0)}=\left[\begin{array}{llllll}
10 & -0,05 & 0,005 & 0 & 0 & 0
\end{array}\right]^{\top}, \\
& \boldsymbol{x}_{\mathrm{ub}, 4}^{(0)}=\left[\begin{array}{llllll}
10 & -0,05 & -0,005 & 0 & 0 & 0
\end{array}\right]^{\top}
\end{aligned}
$$

führt dies zu dem Anfangsgebiet

$$
\boldsymbol{\mathcal { X }}^{(0)}=\mathrm{co}\left\{\boldsymbol{x}_{\mathrm{ub}, 1}^{(0)}, \boldsymbol{x}_{\mathrm{ub}, 2}^{(0)}, \boldsymbol{x}_{\mathrm{ub}, 3}^{(0)}, \boldsymbol{x}_{\mathrm{ub}, 4}^{(0)},-\boldsymbol{x}_{\mathrm{ub}, 1}^{(0)},-\boldsymbol{x}_{\mathrm{ub}, 2}^{(0)},-\boldsymbol{x}_{\mathrm{ub}, 3}^{(0)},-\boldsymbol{x}_{\mathrm{ub}, 4}^{(0)}\right\} \text {. }
$$

Führungsgrößen und Sollwerte werden in dem betrachteten Szenario zu null angenommen, d. h. $w=0$ und $z=0$. Die zu stabilisierende Ruhelage des Regelkreises ist folglich der Ursprung. Abbildung 9.4 zeigt den Verlauf der Tauchtiefe sowie die zugehörige Stellgröße für den Anfangswert der Strecke $\boldsymbol{x}_{\mathrm{S}}^{\top}(0)=\left[\begin{array}{lll}-10 & 0 & 0\end{array}\right]$. In der Realität lässt sich ein derartiger Verlauf nicht erreichen, da die aufzunehmende bzw. abzugebende Menge des Ballastwassers pro Zeit durch physikalische Gegebenheiten begrenzt ist. In [74] wird dies durch die Stellbegrenzung $\overline{\mathrm{u}}=0,005$ berücksichtigt, die Windup-Effekte nach sich zieht. In Abbildung 9.5 ist zu erkennen, dass die Ausregelung mit Stellbegrenzung viel länger dauert. Die Ausregelzeit beträgt in etwa 83 Minuten. Um die Situation zu verbessern wird ein lineares modellbasiertes Anti-Windup entworfen.

Der Entwurf erfolgt, wie im letzten Abschnitt beschrieben, in drei Schritten. Zunächst wird im ersten Schritt der Parameter $\alpha$ festgelegt. Da die Ruhelage im Ursprung liegt, gilt $\hat{\alpha}=1$ und eine zulässige Wahl für $\alpha$ aus dem Intervall $(0,1)$ ist $\alpha=0,99$. Der zweite Schritt besteht in der Abschätzung der Störenergie $\|\zeta\|_{L_{2}}^{2}$. Dazu wird Optimierungsproblem $9.1 \mathrm{mit}$ 

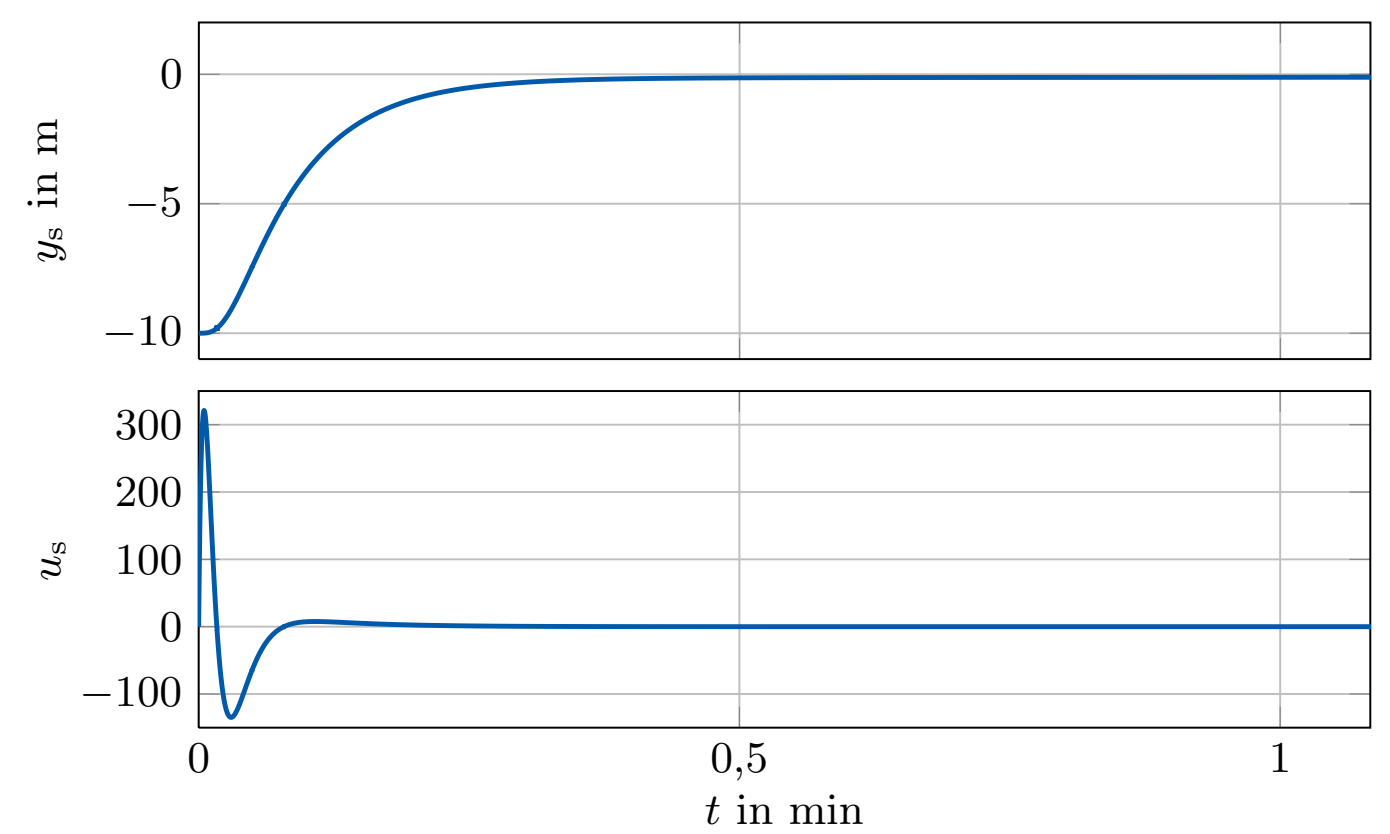

Abbildung 9.4: Verlauf der Tauchtiefe und der Stellgröße für den unbeschränkten Regelkreis mit dem Anfangszustand $\boldsymbol{x}_{\mathrm{s}}^{\top}(0)=\left[\begin{array}{lll}-10 & 0 & 0\end{array}\right]$ der Strecke.

$\boldsymbol{\xi}_{\mathrm{L}}^{(\mathrm{R})}=\mathbf{0}$ für jeden der acht Eckpunkte des Gebietes $\boldsymbol{\mathcal { X }}^{(0)}$ gelöst. Für die quadrierte $L_{2}$-Norm von $\zeta$ ergibt sich

$$
\|\zeta\|_{L_{2}}^{2} \leq 4 \theta^{*}=\theta=2,834 \cdot 10^{5} .
$$

Im dritten Schritt kann der Anti-Windup-Vektor $\underline{\boldsymbol{k}}$ parametriert werden. Mit dem berechneten Wert für $\theta$ und der Wahl $\underline{\lambda}=0,01$ führt das Lösen des Optimierungsproblems $9.2 \mathrm{zu}$

$$
\underline{\boldsymbol{k}}^{\top}=\left[\begin{array}{lll}
0,067 & 11,744 & 567,801
\end{array}\right] .
$$

Abbildung 9.5 zeigt den Verlauf der Tauchtiefe für den Anti-WindupRegelkreis mit dem Anfangszustand

$$
\begin{aligned}
\boldsymbol{x}_{\mathrm{s}}^{\top}(0) & =\left[\begin{array}{lll}
-10 & 0 & 0
\end{array}\right], \\
\boldsymbol{x}_{\mathrm{awrk}}^{\top}(0) & =\left[\begin{array}{lll}
\boldsymbol{x}_{\mathrm{s}}^{\top}(0) & \mathbf{0} & \mathbf{0}
\end{array}\right] .
\end{aligned}
$$

Die Ausregelzeit sinkt erheblich auf etwa 10 min, was einer Reduzierung um $88 \%$ gegenüber dem beschränkten Regelkreis entspricht. 

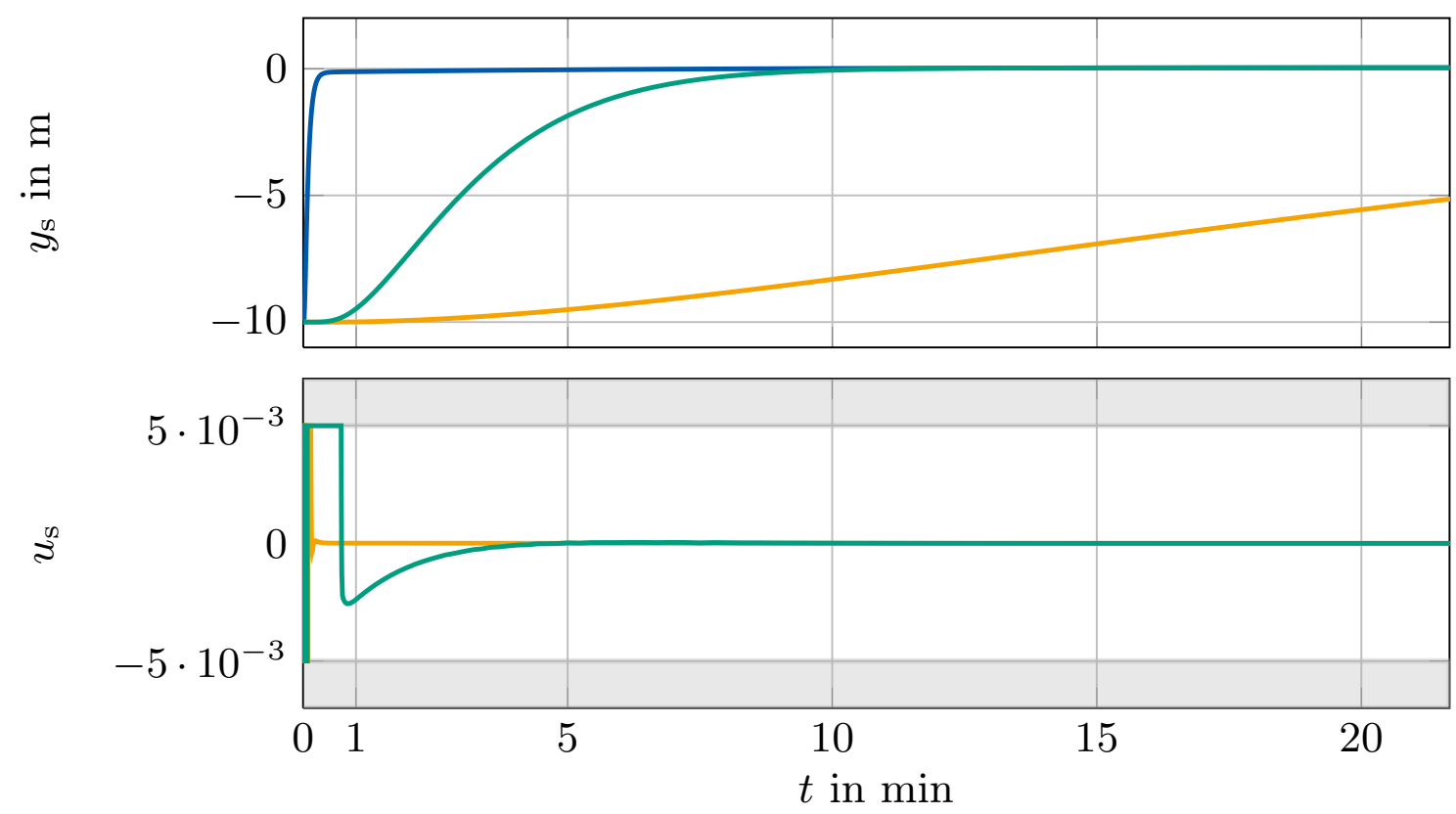

Abbildung 9.5: Verlauf der Tauchtiefe und der Stellgröße für den beschränkten Regelkreis ( - ) und den Anti-Windup-Regelkreis ( - ) mit dem Anfangszustand $\boldsymbol{x}_{\mathrm{s}}^{\top}(0)=\left[\begin{array}{lll}-10 & 0 & 0\end{array}\right]$ der Strecke. Zum Vergleich ist auch der Ausregelverlauf des unbeschränkten Regelkreises (-) dargestellt.

\subsection{Zusammenfassung und Fazit}

In diesem Kapitel wurde ein Entwurfsverfahren für lineares modellbasiertes Anti-Windup vorgestellt, das in Regelkreisen mit grenz- und instabilen Strecken eingesetzt werden kann. Für den benötigten Zustandsreglerentwurf kommen Ergebnisse aus [31] zum Tragen. Im Gegensatz zu $[52,173,175,185,191,210]$ garantiert der Anti-Windup-Entwurf die Stabilität des Anti-Windup-Regelkreises für ein Gebiet möglicher Anfangszustände, wenn das angegebene Optimierungsproblem lösbar ist. Die genaue Beschreibung des Stabilitätsgebietes durch ein konvexes Polytop in Kombination mit einem Entwurf, der ein die Regelgüte betreffendes Gütekriterium minimiert, stellt eine Neuerung gegenüber dem Stand der Technik dar. Anhand der Tauchtiefenregelung eines U-Bootes wird die Effektivität der Methode demonstriert. 


\section{Zusammenfassung}

Ziel dieser Arbeit war die Entwicklung von systematischen Entwurfsmethoden für Anti-Windup-Maßnahmen, die drei wesentliche Anforderungen erfüllen. Neben einer Stabilitätsgarantie des Anti-Windup-Regelkreises für ein möglichst großes, genau spezifiziertes Anfangsgebiet und einer hohen Regelgüte, lag der Fokus auf einem einfach durchführbaren Entwurf mit möglichst wenigen vom Anwender zu wählenden Parametern.

Nach einer detaillierten Charakterisierung des durch Stellbegrenzungen ausgelösten Windup-Problems in Kapitel 2, beschreiben die Kapitel 3 und 4 die historische Entwicklung der Anti-Windup-Strategien bis hin zum aktuellen Stand der Technik. Dabei stellt sich heraus, dass die zunehmende Komplexität der Anti-Windup-Methoden mit einer höheren Leistungsfähigkeit in Bezug auf die Stabilität und Regelgüte einhergeht.

Um das gesteckte Ziel zu erreichen, erweist sich das Prinzip des modellbasierten Anti-Windup-Ansatzes als gut geeignet. Das liegt zum einen daran, dass sich der Entwurf auf die Parametrierung eines Zustandsreglers reduziert, was im Einklang mit der Anforderung nach Einfachheit steht. Zum anderen kann die Regelgüte des Anti-Windup auf eine sehr intuitive Art und Weise bewertet werden, wodurch sich die Forderung nach einer hohen Regelgüte direkt im Entwurfsprozess berücksichtigen lässt.

Die Bewertung der Regelgüte fußt auf einer Transformation des AntiWindup-Regelkreises, der in neuen Koordinaten einer Reihenschaltung aus zwei Teilsystemen entspricht. Das erste Teilsystem ist identisch mit dem unbeschränkten Regelkreis, der Ausgang des zweiten Systems repräsentiert die Abweichung von diesem Verhalten. Die $L_{2}$-Verstärkung des zweiten Systems eignet sich deshalb zur Bewertung der Regelgüte.

Die in Kapitel 5 vorgestellte Umformung macht diese Vorgehensweise auch für die Parametrierung der Beobachtertechnik des modularen AntiWindup nutzbar. Die Beobachtertechnik ist eine statische Anti-WindupMaßnahme für stabile Strecken, deren Entwurf sich auf die Wahl eines Vektors aus einer gegebenen Menge reduziert. Dabei ist zunächst nicht klar, welcher Vektor globale Stabilität garantiert und zu der höchsten Regelgüte führt. Die neu entwickelte Auswahlmethode beantwortet diese Frage und ersetzt das bisher notwendige iterative Ausprobieren und Simulieren. 
Um auf Basis des modellbasierten Anti-Windup-Ansatzes neue AntiWindup-Strategien mit passenden Entwurfsverfahren entwickeln zu können, werden grundlegende Stabilitätssätze benötigt. Kapitel 6 erweitert die bisher in der Literatur verfügbaren Resultate und bildet die Grundlage für die drei folgenden Kapitel 7 bis 9 .

Die Kapitel 7 und 8 behandeln stabile Strecken und zielen auf globale Stabilität des Anti-Windup-Regelkreises ab. Das ist Stand der Technik und steht im Einklang mit der Forderung nach einem möglichst großen Stabilitätsgebiet. Um eine hohe Regelgüte zu erzielen werden zwei unterschiedliche Wege eingeschlagen.

Kapitel 7 betrachtet lineares modellbasiertes Anti-Windup, d. h., der zu entwerfende Zustandsregler besitzt eine lineare Struktur. Im Gegensatz zu existierenden Verfahren basiert dessen Parametrierung allerdings auf einem lokalen Maß für die Regelgüte, der $L_{2}$-Kleinsignalverstärkung des erwähnten zweiten Teilsystems. Dadurch wird lokal eine sehr hohe Regelgüte erreicht, was zu einer geringeren Abweichung vom idealen unbeschränkten Regelkreisverhalten führt. Globale Stabilität lässt sich mit dem Satz von Lee garantieren. Zur Lösung des resultierenden nichtkonvexen Optimierungsproblems wird ein geeigneter Algorithmus vorgeschlagen.

Das in Kapitel 8 vorgestellte nichtlineare modellbasierte Anti-Windup basiert auf der impliziten weich strukturvariablen Zustandsregelung [98]. Durch die variable Struktur lässt sich ein großer Nachteil linearer Zustandsregler umgehen: die schlechte Ausnutzung der Stellgröße in einer Umgebung des Ursprungs. Im Vergleich zu linearen Anti-WindupMethoden kann auf diese Weise eine hohe Regelgüte erreicht werden. Die wesentlichen Vorteile gegenüber dem bislang existierenden strukturvariablen Anti-Windup [52] bestehen in einem einfacheren Entwurf, da keine Entwurfsparameter zu wählen sind, einem weniger komplexen Regelgesetz und somit einer besseren Implementierbarkeit.

Kapitel 9 behandelt lokale Stabilität. Die vorgestellte Entwurfsmethodik für lineares modellbasiertes Anti-Windup ist somit für Regelkreise mit grenz- und instabilen Strecken geeignet. Der Hauptunterschied im Vergleich zu existierenden Entwurfsverfahren besteht in der Stabilitätsgarantie. Falls ein konvexes Optimierungsproblem lösbar ist, garantiert der Entwurf die Stabilität des Anti-Windup-Regelkreises für ein exakt spezifizierbares Gebiet möglicher Anfangszustände.

Zusammenfassend bleibt festzuhalten, dass die neuen Verfahren durch den Einsatz konvexer Optimierung und einer geringen Zahl zu wählender Entwurfsparameter einfach anwendbar sind. Die erzielbare hohe Regelgüte konnte anhand vier verschiedener Beispiele demonstriert werden. 


\section{A Mathematischer Anhang}

\section{A.1 Eigenschaften der Spektralnorm}

Die Spektralnorm $\|\cdot\|_{2}$ einer Matrix $\boldsymbol{Q} \in \mathbb{R}^{n \times n}$, auch induzierte 2-Norm genannt, ist wie folgt definiert

$$
\|\boldsymbol{Q}\|_{2}=\max _{\boldsymbol{x} \in \mathbb{R}^{n} \backslash \mathbf{0}} \frac{\|\boldsymbol{Q} \boldsymbol{x}\|_{2}}{\|\boldsymbol{x}\|_{2}}
$$

wobei $\|\boldsymbol{x}\|_{2}$ die euklidische Norm des Vektors $\boldsymbol{x} \in \mathbb{R}^{n}$ ist.

Diese induzierte Norm besitzt folgende Eigenschaften.

1. Gemäß Theorem 9.4.2 in [16] gilt $\|\mathbb{I}\|_{2}=1$ für die Einheitsmatrix.

2. Die induzierte 2-Norm einer Matrix ist verträglich mit der euklidischen Norm eines Vektors. Somit gilt

$$
\|\boldsymbol{Q} \boldsymbol{x}\|_{2} \leq\|\boldsymbol{Q}\|_{2}\|\boldsymbol{x}\|_{2} .
$$

3. Die induzierte 2-Norm ist nach [16, Proposition 9.3.5] submultiplikativ, d. h., es gilt

$$
\|\boldsymbol{Q B}\|_{2} \leq\|\boldsymbol{Q}\|_{2}\|\boldsymbol{B}\|_{2}
$$

\section{A.2 Sätze über lipschitzstetige Funktionen}

Satz A.1 (Verkettung). Gegeben sei die Funktion $\boldsymbol{g}: \mathbb{D}_{g} \subseteq \mathbb{R}^{m} \mapsto \mathbb{R}^{n}$ und die Funktion $\boldsymbol{f}: \mathbb{D}_{f} \subseteq \mathbb{R}^{n} \mapsto \mathbb{D}_{g}$. Des Weiteren genüge $\boldsymbol{g}$ auf $\mathbb{D}_{g}$ der Lipschitz-Bedingung

$$
\|\boldsymbol{g}(\boldsymbol{x})-\boldsymbol{g}(\tilde{\boldsymbol{x}})\|_{2} \leq L_{g}\|\boldsymbol{x}-\tilde{\boldsymbol{x}}\|_{2} \quad \forall \boldsymbol{x}, \tilde{\boldsymbol{x}} \in \mathbb{D}_{g}
$$

mit $L_{g}>0$ und $\boldsymbol{f}$ genüge auf $\mathbb{D}_{f}$ der Lipschitz-Bedingung

$$
\|\boldsymbol{f}(\boldsymbol{x})-\boldsymbol{f}(\tilde{\boldsymbol{x}})\|_{2} \leq L_{f}\|\boldsymbol{x}-\tilde{\boldsymbol{x}}\|_{2} \quad \forall \boldsymbol{x}, \tilde{\boldsymbol{x}} \in \mathbb{D}_{f}
$$

mit $L_{f}>0$. Unter diesen Voraussetzungen ist die Verkettung $(\boldsymbol{g} \circ \boldsymbol{f})(\boldsymbol{x})$ auf $\mathbb{D}_{f}$ global lipschitzstetig mit der Lipschitz-Konstante $L=L_{g} L_{f}$. 
Beweis. Da die Funktion $\boldsymbol{g}$ auf $\mathbb{D}_{g}$ einer Lipschitz-Bedingung genügt, gilt für die Verkettung von $\boldsymbol{g}$ und $\boldsymbol{f}$ die Abschätzung

$$
\|\boldsymbol{g}(\boldsymbol{f}(\boldsymbol{x}))-\boldsymbol{g}(\boldsymbol{f}(\tilde{\boldsymbol{x}}))\|_{2} \leq L_{g}\|\boldsymbol{f}(\boldsymbol{x})-\boldsymbol{f}(\tilde{\boldsymbol{x}})\|_{2} \quad \forall \boldsymbol{f}(\boldsymbol{x}), \boldsymbol{f}(\tilde{\boldsymbol{x}}) \in \mathbb{D}_{g} .
$$

Da die Funktion $\boldsymbol{f}$ auf $\mathbb{D}_{f}$ ebenfalls einer Lipschitz-Bedingung genügt, lässt sich die rechte Seite nach oben abschätzen. Es gilt

$$
\|\boldsymbol{f}(\boldsymbol{x})-\boldsymbol{f}(\tilde{\boldsymbol{x}})\|_{2} \leq L_{f}\|\boldsymbol{x}-\tilde{\boldsymbol{x}}\|_{2} \quad \forall \boldsymbol{x}, \tilde{\boldsymbol{x}} \in \mathbb{D}_{f} .
$$

Kombinieren der beiden Ergebnisse liefert

$$
\|\boldsymbol{g}(\boldsymbol{f}(\boldsymbol{x}))-\boldsymbol{g}(\boldsymbol{f}(\tilde{\boldsymbol{x}}))\|_{2} \leq L_{g} L_{f}\|\boldsymbol{x}-\tilde{\boldsymbol{x}}\|_{2} \quad \forall \boldsymbol{x}, \tilde{\boldsymbol{x}} \in \mathbb{D}_{f}
$$

womit die globale Lipschitz-Stetigkeit der Verkettung $\boldsymbol{g} \circ \boldsymbol{f}$ auf $\mathbb{D}_{f}$ bewiesen ist.

Satz A.2 (Produkt). Gegeben sei eine offene Menge $\mathbb{D} \subseteq \mathbb{R}^{n}$, die Funktion $\boldsymbol{g}: \mathbb{D} \mapsto \mathbb{R}^{n}$ und die Funktion $\boldsymbol{f}: \mathbb{D} \mapsto \mathbb{R}^{n}$. Beide Funktionen seien stetig und genügen auf $\mathbb{D}$ einer Lipschitz-Bedingung, d. h., für zwei positive Konstanten $L_{f}$ und $L_{g}$ gilt

$$
\begin{aligned}
\|\boldsymbol{g}(\boldsymbol{x})-\boldsymbol{g}(\tilde{\boldsymbol{x}})\|_{2} \leq L_{g}\|\boldsymbol{x}-\tilde{\boldsymbol{x}}\|_{2} & \forall \boldsymbol{x}, \tilde{\boldsymbol{x}} \in \mathbb{D}, \\
\|\boldsymbol{f}(\boldsymbol{x})-\boldsymbol{f}(\tilde{\boldsymbol{x}})\|_{2} \leq L_{f}\|\boldsymbol{x}-\tilde{\boldsymbol{x}}\|_{2} & \forall \boldsymbol{x}, \tilde{\boldsymbol{x}} \in \mathbb{D}
\end{aligned}
$$

Unter diesen Voraussetzungen ist das Produkt $\boldsymbol{f}^{\top}(\boldsymbol{x}) \boldsymbol{g}(\boldsymbol{x})$ lokal lipschitzstetig in $\mathbb{D}$.

Beweis. Der Beweis orientiert sich an [10, Beweis von Satz 2.4.7]. Zu jedem Punkt $\boldsymbol{x}$ in $\mathbb{D}$ gibt es wegen der Offenheit von $\mathbb{D}$ eine kompakte, ganz in $\mathbb{D}$ gelegene Umgebung $\mathbb{K}$. Auf dieser Umgebung sind die stetigen, reellwertigen Funktionen $\|\boldsymbol{f}(\boldsymbol{x})\|_{2}$ und $\|\boldsymbol{g}(\boldsymbol{x})\|_{2}$ beschränkt und neh-

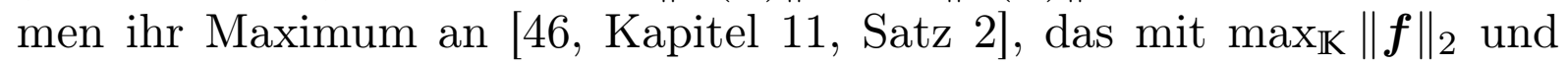
$\max _{\mathbb{K}}\|\boldsymbol{g}\|_{2}$ bezeichnet wird. Für die lokale Lipschitz-Stetigkeit des Produktes $\boldsymbol{f}^{\top}(\boldsymbol{x}) \boldsymbol{g}(\boldsymbol{x})$ auf $\mathbb{D}$ ist zu zeigen, dass mit einem $L>0$ gilt

$$
\left\|\boldsymbol{f}^{\top}(\boldsymbol{x}) \boldsymbol{g}(\boldsymbol{x})-\boldsymbol{f}^{\top}(\tilde{\boldsymbol{x}}) \boldsymbol{g}(\tilde{\boldsymbol{x}})\right\|_{2} \leq L\|\boldsymbol{x}-\tilde{\boldsymbol{x}}\|_{2} \quad \forall \boldsymbol{x}, \tilde{\boldsymbol{x}} \in \mathbb{K} .
$$


Die linke Seite lässt sich wie folgt umschreiben

$$
\begin{aligned}
& \left\|\boldsymbol{f}^{\top}(\boldsymbol{x}) \boldsymbol{g}(\boldsymbol{x})-\boldsymbol{f}^{\top}(\tilde{\boldsymbol{x}}) \boldsymbol{g}(\tilde{\boldsymbol{x}})\right\|_{2} \\
= & \left\|\boldsymbol{f}^{\top}(\boldsymbol{x}) \boldsymbol{g}(\boldsymbol{x})-\boldsymbol{f}^{\top}(\tilde{\boldsymbol{x}}) \boldsymbol{g}(\tilde{\boldsymbol{x}})-\boldsymbol{f}^{\top}(\tilde{\boldsymbol{x}}) \boldsymbol{g}(\boldsymbol{x})+\boldsymbol{f}^{\top}(\tilde{\boldsymbol{x}}) \boldsymbol{g}(\boldsymbol{x})\right\|_{2} \\
\leq & \left\|\boldsymbol{f}^{\top}(\boldsymbol{x}) \boldsymbol{g}(\boldsymbol{x})-\boldsymbol{f}^{\top}(\tilde{\boldsymbol{x}}) \boldsymbol{g}(\boldsymbol{x})\right\|_{2}+\left\|\boldsymbol{f}^{\top}(\tilde{\boldsymbol{x}}) \boldsymbol{g}(\boldsymbol{x})-\boldsymbol{f}^{\top}(\tilde{\boldsymbol{x}}) \boldsymbol{g}(\tilde{\boldsymbol{x}})\right\|_{2} \\
= & \left\|\left[\boldsymbol{f}^{\top}(\boldsymbol{x})-\boldsymbol{f}^{\top}(\tilde{\boldsymbol{x}})\right] \boldsymbol{g}(\boldsymbol{x})\right\|_{2}+\left\|\boldsymbol{f}^{\top}(\tilde{\boldsymbol{x}})[\boldsymbol{g}(\boldsymbol{x})-\boldsymbol{g}(\tilde{\boldsymbol{x}})]\right\|_{2} \\
\leq & \left\|\boldsymbol{f}^{\top}(\boldsymbol{x})-\boldsymbol{f}^{\top}(\tilde{\boldsymbol{x}})\right\|_{2}\|\boldsymbol{g}(\boldsymbol{x})\|_{2}+\left\|\boldsymbol{f}^{\top}(\tilde{\boldsymbol{x}})\right\|_{2}\|\boldsymbol{g}(\boldsymbol{x})-\boldsymbol{g}(\tilde{\boldsymbol{x}})\|_{2} \\
\leq & L_{f}\|\boldsymbol{x}-\tilde{\boldsymbol{x}}\|_{2} \max _{\mathbb{K}}\|\boldsymbol{g}\|_{2}+\max _{\mathbb{K}}\|\boldsymbol{f}\|_{2} L_{g}\|\boldsymbol{x}-\tilde{\boldsymbol{x}}\|_{2} \\
= & \left(L_{f} \max _{\mathbb{K}}\|\boldsymbol{g}\|_{2}+L_{g} \max _{\mathbb{K}}\|\boldsymbol{f}\|_{2}\right)\|\boldsymbol{x}-\tilde{\boldsymbol{x}}\|_{2},
\end{aligned}
$$

womit die Aussage des Satzes gezeigt ist.

\section{A.3 Matrizen und Details zu den Beispielen}

A.3.1 Matrizen des linearen Teilsystems

$$
\begin{gathered}
\boldsymbol{A}_{\ell}=\left[\begin{array}{ccc}
\boldsymbol{A}_{\mathrm{s}} & \mathbf{0} & \mathbf{0} \\
\left.\boldsymbol{B}_{\mathrm{r}, u}+\boldsymbol{D}_{\mathrm{aw}, \mathrm{x}} \boldsymbol{D}_{\mathrm{r}, u}\right) \boldsymbol{C}_{\mathrm{s}} & \boldsymbol{A}_{\mathrm{r}}+\boldsymbol{D}_{\mathrm{aw}, \mathrm{x}} \boldsymbol{C}_{\mathrm{r}} & \boldsymbol{C}_{\mathrm{z}} \\
\boldsymbol{B}_{\mathrm{z}} \boldsymbol{D}_{\mathrm{r}, u} \boldsymbol{C}_{\mathrm{s}} & \boldsymbol{B}_{\mathrm{z}} \boldsymbol{C}_{\mathrm{r}} & \boldsymbol{A}_{\mathrm{z}}+\boldsymbol{B}_{\mathrm{z}} \boldsymbol{C}_{\mathrm{z}}
\end{array}\right] \\
\boldsymbol{B}_{\ell, \mathrm{w}}=\left[\begin{array}{c}
\mathbf{0} \\
\boldsymbol{B}_{\mathrm{r}, w}+\boldsymbol{D}_{\mathrm{aw}, \mathrm{x}} \boldsymbol{D}_{\mathrm{r}, w} \\
\boldsymbol{B}_{\mathrm{z}} \boldsymbol{D}_{\mathrm{r}, w}
\end{array}\right], \quad \boldsymbol{B}_{\ell, \mathrm{u}}=\left[\begin{array}{c}
\boldsymbol{B}_{\mathrm{s}, u} \\
-\boldsymbol{D}_{\mathrm{aw}, \mathrm{x}} \\
-\boldsymbol{B}_{\mathrm{z}}
\end{array}\right] \\
\boldsymbol{B}_{\ell, \mathrm{z}}=\left[\begin{array}{c}
\boldsymbol{B}_{\mathrm{s}, z} \\
\mathbf{0} \\
\mathbf{0}
\end{array}\right]
\end{gathered}
$$

A.3.2 Matrizen des Anti-Windup-Regelkreises

$$
\begin{gathered}
\tilde{\boldsymbol{A}}=\left[\begin{array}{ccc}
\boldsymbol{A}_{\mathrm{s}} & \mathbf{0} & \mathbf{0} \\
\boldsymbol{B}_{\mathrm{r}, u} \boldsymbol{C}_{\mathrm{s}} & \boldsymbol{A}_{\mathrm{r}} & \boldsymbol{B}_{\mathrm{r}, u} \boldsymbol{C}_{\mathrm{s}} \\
\boldsymbol{B}_{\mathrm{s}, u} \boldsymbol{D}_{\mathrm{r}, u} \boldsymbol{C}_{\mathrm{s}} & \boldsymbol{B}_{\mathrm{s}, u} \boldsymbol{C}_{\mathrm{r}} & \boldsymbol{A}_{\mathrm{s}}+\boldsymbol{B}_{\mathrm{s}, u} \boldsymbol{D}_{\mathrm{r}, u} \boldsymbol{C}_{\mathrm{s}}
\end{array}\right] \\
\tilde{\boldsymbol{B}}_{\mathrm{u}}=\left[\begin{array}{c}
\boldsymbol{B}_{\mathrm{s}, u} \\
\mathbf{0} \\
-\boldsymbol{B}_{\mathrm{s}, u}
\end{array}\right], \quad \tilde{\boldsymbol{B}}_{\mathrm{w}}=\left[\begin{array}{c}
\boldsymbol{B}_{\mathrm{s}, z} \\
\mathbf{0} \\
\mathbf{0}
\end{array}\right], \quad \tilde{\boldsymbol{B}}_{\mathrm{z}}=\left[\begin{array}{c}
\mathbf{0} \\
\boldsymbol{B}_{\mathrm{r}, w} \\
\boldsymbol{B}_{\mathrm{s}, u} \boldsymbol{D}_{\mathrm{r}, w}
\end{array}\right]
\end{gathered}
$$




\section{A.3.3 Reglermatrizen aus Abschnitt 7.4}

$$
\begin{aligned}
\boldsymbol{A}_{\mathrm{r}}= & {\left[\begin{array}{rrrrrrrr}
-52,23 & -3,36 & 73,10 & -6 \cdot 10^{-4} & -93,48 & 1072,03 & 0 & 0 \\
-3,36 & -29,70 & -2,19 & -6 \cdot 10^{-3} & 922,30 & -922,93 & 0 & 0 \\
-19,00 & -3,00 & -0,80 & -6 \cdot 10^{-4} & -11,70 & 0,61 & 0 & 0 \\
-0,66 & -0,50 & 0 & -1,4 \cdot 10^{-2} & -1,64 & 0,20 & 0 & 0 \\
-0,16 & -0,50 & 1,00 & -10^{-4} & -6,30 & 0,12 & 0 & 0 \\
0 & 0 & 1,00 & 0 & 0,14 & -4,96 & 0 & 0 \\
-52,23 & -3,36 & 73,10 & -6 \cdot 10^{-4} & -94,30 & 1072,00 & 0 & 0 \\
-3,36 & -29,70 & -2,19 & -6 \cdot 10^{-3} & 908,90 & -921,00 & 0 & 0
\end{array}\right] } \\
\boldsymbol{B}_{\mathrm{r}, u}= & {\left[\begin{array}{cc}
-0,84 & 0,82 \\
-11,54 & 13,47 \\
-0,86 & 0,25 \\
-47,40 & 15,00 \\
4,68 & -4,80 \\
4,82 & 0,14 \\
0 & 0 \\
0 & 0
\end{array}\right]=-\boldsymbol{B}_{\mathrm{r}, w}, \boldsymbol{C}_{\mathrm{r}}^{\boldsymbol{\top}}=\left[\begin{array}{cc}
0 & 0 \\
0 & 0 \\
0 & 0 \\
0 & 0 \\
0 & 0 \\
0 & 0 \\
1 & 0 \\
0 & 1
\end{array}\right], \boldsymbol{D}_{\mathrm{r}, u}=\boldsymbol{D}_{\mathrm{r}, w}=\mathbf{0} . }
\end{aligned}
$$

\section{A.3.4 Gütefunktion aus Abschnitt 3.3.3}
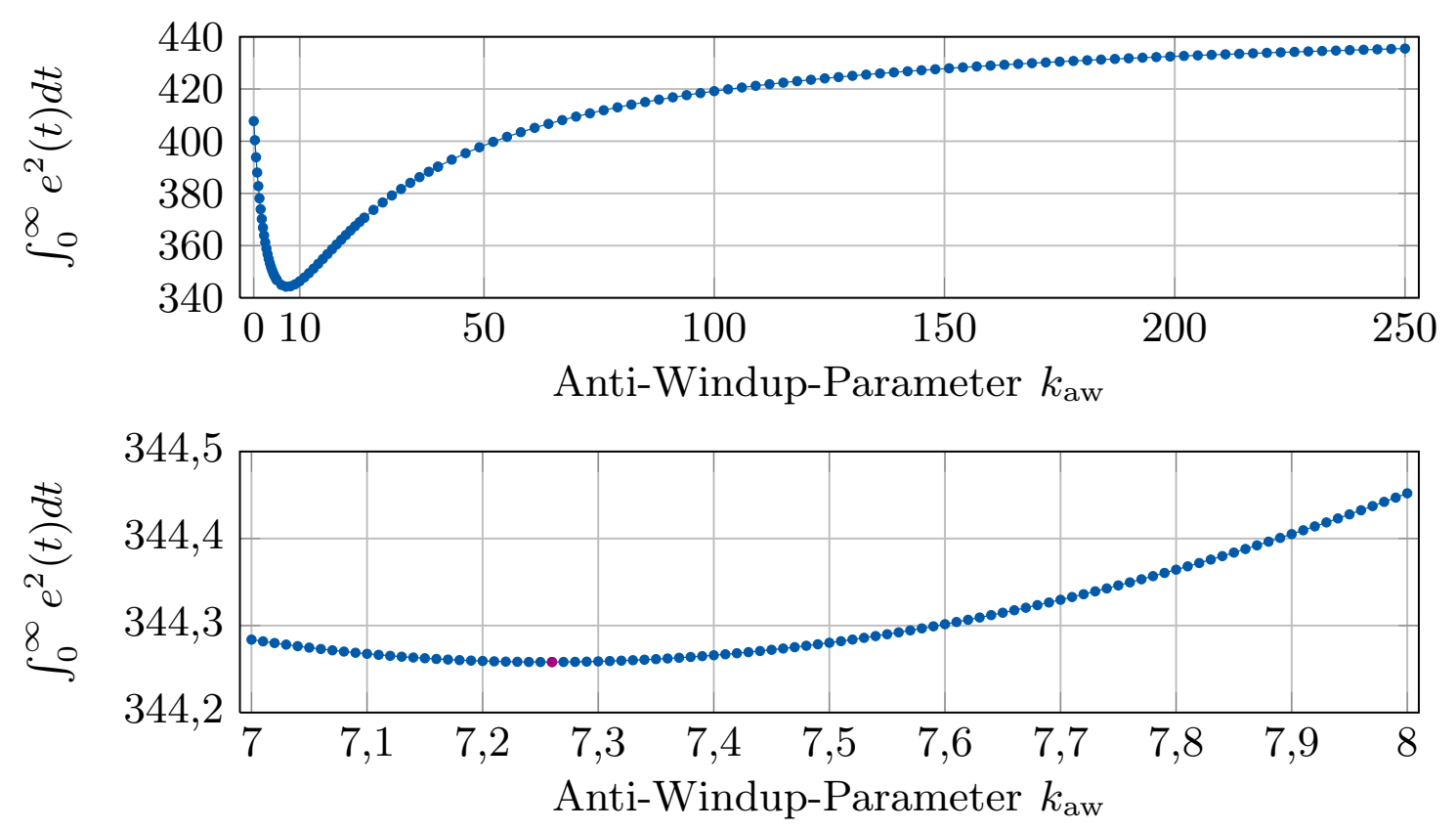

Abbildung A.1: Bestimmung von $k_{\text {aw }}$ für das Beispiel aus Abschnitt 3.3.3. Dargestellt ist das Integral der quadrierten Regelabweichung in Abhängigkeit ausgewählter Anti-Windup-Parameter $k_{\text {aw }}$ für den Anti-Windup-Regelkreis mit Back Calculation und einem Sollwert von $w=20 \mathrm{~cm}$. Der kleinste Wert der Gütefunktion ergibt sich für $k_{\mathrm{aw}}=7,26$. 


\section{B Konvexe Optimierung}

Die in der vorliegenden Arbeit präsentierten Entwurfsmethoden für AntiWindup-Maßnahmen basieren alle auf konvexer Optimierung. Dieses Kapitel definiert deshalb einige Grundbegriffe, die im Zusammenhang mit Optimierungsproblemen und konvexer Optimierung auftreten. Den Vorteil, ein Entwurfsproblem als konvexes Optimierungsproblem zu formulieren, beschreibt Boyd in [22] wie folgt:

„There are great advantages to recognizing or formulating a problem as a convex optimization problem. The most basic advantage is that the problem can then be solved, very reliably and efficiently $[\ldots]^{*}$.

Die folgende Darstellung orientiert sich an [21, 22, 38, 73]. Dabei stellt das Buch [22] ein Standardwerk dar, das konvexe Optimierung ausführlich und mit Blick auf regelungstechnische Probleme behandelt.

\section{B.1 Optimierungsprobleme}

Ein mathematisches Optimierungsproblem besitzt die folgende Form:

$$
\begin{gathered}
\underset{\mathbf{x}}{\operatorname{Minimiere}} f_{0}(\mathbf{x}) \text { unter den Nebenbedingungen } \\
\qquad f_{i}(\mathbf{x}) \leq 0, \quad i=1, \ldots, m .
\end{gathered}
$$

Dabei beinhaltet der Vektor $\mathbf{x}=\left[\mathrm{x}_{1} \ldots \mathrm{x}_{n}\right]^{\top}$ die Variablen des Optimierungsproblems. Die Funktion $f_{0}: \mathbb{R}^{n} \mapsto \mathbb{R}$ ist die Zielfunktion und die Funktionen $f_{i}: \mathbb{R}^{n} \mapsto \mathbb{R}, i=1, \ldots, m$ stellen die Nebenbedingungen des Optimierungsproblems dar. Vektoren $\mathbf{x}$, die sämtliche Nebenbedingungen erfüllen, werden in der zulässigen Menge

$$
\mathbf{X}=\left\{\mathbf{x} \in \mathbb{R}^{n} \mid f_{i}(\mathbf{x}) \leq 0, i=1, \ldots, m\right\}
$$

zusammengefasst. Ein Vektor $\mathbf{x}^{*} \in \mathbf{X}$ wird als optimal oder als Lösung des Optimierungsproblems (B.1) bezeichnet, wenn der ihm über die Zielfunktion $f_{0}$ zugeordnete Wert $f_{0}\left(\mathbf{x}^{*}\right)$ kleiner ist als die entsprechenden Werte $f_{0}(\mathbf{x})$ aller anderen Vektoren $\mathbf{x}$ der zulässigen Menge $\mathbf{X}$. 
Anhand der Eigenschaften und der Gestalt der Funktionen $f_{0}, \ldots, f_{m}$ lassen sich Optimierungsprobleme in verschiedene Klassen einteilen. Sind die Funktionen $f_{i}, i=0, \ldots, m$ beispielsweise linear, d. h., es gilt

$$
f_{i}(\alpha \mathbf{x}+\beta \mathbf{y})=\alpha f_{i}(\mathbf{x})+\beta f_{i}(\mathbf{y})
$$

für alle $\mathbf{x}, \mathbf{y} \in \mathbb{R}^{n}$ und alle $\alpha, \beta \in \mathbb{R}$, wird das Optimierungsproblem als lineares Programm bezeichnet. Eine andere Klasse stellen sogenannte konvexe Optimierungsprobleme dar. Diese zeichnen sich dadurch aus, dass die Funktionen $f_{i}, i=0, \ldots, m$ konvex sind, d. h. die Bedingung

$$
f_{i}(\alpha \mathbf{x}+\beta \mathbf{y}) \leq \alpha f_{i}(\mathbf{x})+\beta f_{i}(\mathbf{y})
$$

für alle $\mathbf{x}, \mathbf{y} \in \mathbb{R}^{n}$ und alle positiven $\alpha, \beta \in \mathbb{R}_{>0}$ mit $\alpha+\beta=1$ erfüllen. Die zulässige Menge $\mathbf{X}$ ist in diesem Fall eine konvexe Menge. Konvexe Mengen besitzen die Eigenschaft, dass jede Verbindungslinie zwischen zwei Punkten $\mathbf{x}, \mathbf{y}$ der Menge $\mathbf{X}$ ebenfalls in der Menge $\mathbf{X}$ liegt.

Der große Vorteil eines konvexen Optimierungsproblems ist, dass schnelle und zuverlässige numerische Lösungsmethoden existieren, wie zum Beispiel Innere-Punkte-Methoden [22]. Die Lösung $\mathbf{x}^{*}$ von (B.1) lässt sich damit auf einem heute üblichen Rechner in sehr kurzer Zeit berechnen. Das ist ein wesentlicher Unterschied zu allgemeinen nichtlinearen Optimierungsproblemen, die - wenn überhaupt - in der Regel nur mit sehr hohem Aufwand gelöst werden können. Wie im nächsten Abschnitt gezeigt wird, lassen sich viele regelungstechnische Probleme durch gewisse Umformungen als spezielles konvexes Optimierungsproblem, als sogenanntes semidefinites Programm formulieren.

\section{B.2 Lineare Matrixungleichungen und semidefinite Programme}

Ein semidefinites Programm ist ein konvexes Optimierungsproblem mit einer linearen Zielfunktion und einer Nebenbedingung in Form einer linearen Matrixungleichung ${ }^{1}$ [22]. Das ist eine Ungleichung der Form

$$
\boldsymbol{F}(\mathbf{x})=\boldsymbol{F}_{0}+\sum_{i=1}^{n} \mathrm{x}_{i} \boldsymbol{F}_{i} \succeq 0
$$

\footnotetext{
${ }^{1}$ engl. linear matrix inequality, Abkürzung $L M I$.
} 
in den Variablen $\mathbf{x}^{\top}=\left[\mathrm{x}_{1} \ldots \mathrm{x}_{n}\right]$ mit gegebenen symmetrischen Matrizen $\boldsymbol{F}_{i} \in \mathbb{S}^{m}, i=0, \ldots, n$. Die Notation $\succeq$ bedeutet hier, dass die Matrix $\boldsymbol{F}(\mathbf{x})$ positiv semidefinit für alle $\mathbf{x} \in \mathbb{R}^{n}$ ist. Semidefinite Programme haben demnach folgende Struktur:

$$
\begin{array}{ll}
\underset{\mathbf{x}}{\operatorname{Minimiere}} & \boldsymbol{c}^{\top} \mathbf{x} \text { unter der Nebenbedingung } \\
& \boldsymbol{F}(\mathbf{x})=\boldsymbol{F}_{0}+\mathrm{x}_{1} \boldsymbol{F}_{1}+\cdots+\mathrm{x}_{n} \boldsymbol{F}_{n} \succeq 0 .
\end{array}
$$

Aus dem letzten Abschnitt ist bekannt, dass die Nebenbedingungen eines konvexen Optimierungsproblems konvexe Funktionen sind. Dies muss folglich auch auf die linearen Matrixungleichungen zutreffen. In der Tat lässt sich mit Bedingung (B.2) die Konvexität zeigen. Für $\beta=1-\alpha$ folgt

$$
\begin{aligned}
\boldsymbol{F}(\alpha \mathbf{x}+\beta \mathbf{y}) & =\boldsymbol{F}_{0}+\sum_{i=1}^{n}\left(\alpha \mathrm{x}_{i}+(1-\alpha) \mathrm{y}_{i}\right) \boldsymbol{F}_{i} \\
& =\boldsymbol{F}_{0}+\alpha \sum_{i=1}^{n} \mathrm{x}_{i} \boldsymbol{F}_{i}+(1-\alpha) \sum_{i=1}^{n} \mathrm{y}_{i} \boldsymbol{F}_{i} \\
& =\boldsymbol{F}_{0}+\alpha \boldsymbol{F}_{0}-\alpha \boldsymbol{F}_{0}+\alpha \sum_{i=1}^{n} \mathrm{x}_{i} \boldsymbol{F}_{i}+(1-\alpha) \sum_{i=1}^{n} \mathrm{y}_{i} \boldsymbol{F}_{i} \\
& =\alpha \boldsymbol{F}_{0}+\alpha \sum_{i=1}^{n} \mathrm{x}_{i} \boldsymbol{F}_{i}+(1-\alpha) \boldsymbol{F}_{0}+(1-\alpha) \sum_{i=1}^{n} \mathrm{y}_{i} \boldsymbol{F}_{i} \\
& =\alpha \boldsymbol{F}(\mathbf{x})+\beta \boldsymbol{F}(\mathbf{y}) .
\end{aligned}
$$

Die bisher verwendete Schreibweise (B.3) einer linearen Matrixungleichung wird als kanonische Form bezeichnet [38]. Im Zusammenhang mit regelungstechnischen Problemstellungen taucht häufig eine andere Form auf, in der die Variablen $\mathbf{x}$ in einer Matrix zusammengefasst sind. Beispielsweise besitzt die Ljapunov-Ungleichung die Form

$$
\boldsymbol{A}^{\top} \boldsymbol{Q}+\boldsymbol{Q} \boldsymbol{A} \prec \mathbf{0}, \quad \boldsymbol{Q} \succ \mathbf{0},
$$

wobei die Matrix $\boldsymbol{A} \in \mathbb{R}^{n}$ gegeben ist und die Variablen den Elementen der symmetrischen Matrix $\boldsymbol{Q} \in \mathbb{S}^{n}$ entsprechen. Diese Form lässt sich durch eine geeignete Wahl von $n(n+1) / 2$ Basismatrizen immer in die kanonische 
Darstellung überführen [21]. Für $n=2$ gilt exemplarisch

$$
\begin{aligned}
\boldsymbol{Q}=\left[\begin{array}{ll}
q_{11} & q_{12} \\
q_{12} & q_{22}
\end{array}\right] & =q_{11} \boldsymbol{E}_{11}+q_{12} \boldsymbol{E}_{12}+q_{22} \boldsymbol{E}_{22} \\
& =q_{11}\left[\begin{array}{ll}
1 & 0 \\
0 & 0
\end{array}\right]+q_{12}\left[\begin{array}{ll}
0 & 1 \\
1 & 0
\end{array}\right]+q_{22}\left[\begin{array}{ll}
0 & 0 \\
0 & 1
\end{array}\right] .
\end{aligned}
$$

Daraus folgt die Ljapunov-Ungleichung in kanonischer Darstellung

$$
q_{11}\left(\boldsymbol{A}^{\top} \boldsymbol{E}_{11}+\boldsymbol{E}_{11} \boldsymbol{A}\right)+q_{12}\left(\boldsymbol{A}^{\top} \boldsymbol{E}_{12}+\boldsymbol{E}_{12} \boldsymbol{A}\right)+q_{22}\left(\boldsymbol{A}^{\top} \boldsymbol{E}_{22}+\boldsymbol{E}_{22} \boldsymbol{A}\right) \prec \mathbf{0} .
$$

Da die Darstellung einer linearen Matrixungleichung mit Matrixvariablen viel übersichtlicher ist, wird in der vorliegenden Arbeit ausschließlich diese Schreibweise verwendet, um konvexe Optimierungsprobleme bzw. semidefinite Programme zu formulieren. Ebenso werden mehrere lineare Matrixungleichungen nicht zu einer Ungleichung zusammengefasst, um die Form (B.4) zu erhalten. Dass dies immer möglich ist, lässt sich erneut an der Ljapunov-Ungleichung veranschaulichen, denn (B.5) ist äquivalent zu

$$
\left[\begin{array}{cc}
-\left(\boldsymbol{A}^{\top} \boldsymbol{Q}+\boldsymbol{Q} \boldsymbol{A}\right) & \mathbf{0} \\
\mathbf{0} & \boldsymbol{Q}
\end{array}\right] \succ \mathbf{0}
$$

Um die formulierten konvexen Optimierungsprobleme zu lösen, ist allerdings eine Umformung der Nebenbedingungen und der Zielfunktion in eine für den Lösungsalgorithmus geeignete Darstellung erforderlich. Dies kann jedoch automatisiert mit der Matlab-Toolbox YALMIP [134] erfolgen, die kompatibel zu den meisten Algorithmen ist. In dieser Arbeit wird ausschließlich der Lösungsalgorithmus SDPT3 [188] verwendet.

\section{B.3 Rechenregeln für LMIs}

Viele regelungstechnische Entwurfsprobleme lassen sich als konvexes Optimierungsproblem mit Nebenbedingungen in Form linearer Matrixungleichungen formulieren. Allerdings sind dazu oft einige Umformungen nötig. Die Wichtigsten werden im Folgenden angegeben.

\section{Kongruenztransformation}

Die beidseitige Multiplikation einer Matrix $\boldsymbol{A}$ mit einer regulären Matrix $\boldsymbol{M}$ bzw. ihrer Transponierten $\boldsymbol{M}^{\top}$ ändert die Definitheit der Matrix $\boldsymbol{A}$ 
nicht [81]. Folglich sind die beiden Forderungen

$$
\boldsymbol{A} \succ \mathbf{0} \quad \Leftrightarrow \quad M^{\top} \boldsymbol{A} M \succ \mathbf{0}
$$

zueinander äquivalent.

\section{Variablensubstitution}

Eine Matrixungleichung, in der Produkte aus zwei oder mehr Matrixvariablen vorkommen, ist nicht mehr linear. Beispielsweise trifft dies auf die Matrixungleichung

$$
\boldsymbol{A}^{\top} \boldsymbol{Q}^{\top}+\boldsymbol{Q A}+\boldsymbol{B F} \boldsymbol{Q}^{\top}+\boldsymbol{Q} \boldsymbol{F}^{\top} \boldsymbol{B}^{\top} \prec \mathbf{0}
$$

mit den Matrixvariablen $\boldsymbol{Q}$ und $\boldsymbol{F}$ zu. Falls $\boldsymbol{Q}$ regulär ist, lässt sich das Produkt jedoch durch eine neue Matrixvariable

$$
\boldsymbol{L}=\boldsymbol{F} \boldsymbol{Q}^{\top}
$$

substituieren. Die so entstehende Matrixungleichung

$$
\boldsymbol{A}^{\top} \boldsymbol{Q}^{\top}+\boldsymbol{Q} \boldsymbol{A}+\boldsymbol{B} \boldsymbol{L}+\boldsymbol{L}^{\top} \boldsymbol{B}^{\top} \prec \mathbf{0}
$$

ist eine LMI in den Variablen $\boldsymbol{Q}, \boldsymbol{L}$ und kann als konvexe Nebenbedingung in einem Optimierungsproblem verwendet werden. Nach der Lösung des Optimierungsproblems ergibt sich $\boldsymbol{F}$ aus

$$
\boldsymbol{F}=\boldsymbol{L}\left(\boldsymbol{Q}^{\top}\right)^{-1}
$$

\section{Schur-Komplement}

Eine andere Möglichkeit, eine nichtlineare Matrixungleichung in eine äquivalente lineare Matrixungleichung zu überführen, bietet das SchurKomplement. Details beschreibt

Lemma B.1 (Schur-Komplement, [21, 190]). Für eine symmetrische Matrix $\boldsymbol{A}_{1} \in \mathbb{S}^{k}$ und eine symmetrische positiv definite Matrix $\boldsymbol{A}_{3} \in \mathbb{S}^{n}$ sowie eine Matrix $\boldsymbol{A}_{2} \in \mathbb{R}^{k \times n}$ gilt

$$
\boldsymbol{A}_{1}-\boldsymbol{A}_{2} \boldsymbol{A}_{3}^{-1} \boldsymbol{A}_{2}^{\top} \succ \mathbf{0} \Leftrightarrow\left[\begin{array}{ll}
\boldsymbol{A}_{1} & \boldsymbol{A}_{2} \\
\boldsymbol{A}_{2}^{\top} & \boldsymbol{A}_{3}
\end{array}\right] \succ \mathbf{0} .
$$

Für eine symmetrische Matrix $\boldsymbol{B}_{1} \in \mathbb{S}^{k}$ und eine symmetrische negativ definite Matrix $\boldsymbol{B}_{3} \in \mathbb{S}^{n}$ sowie eine Matrix $\boldsymbol{B}_{2} \in \mathbb{R}^{k \times n}$ gilt

$$
\boldsymbol{B}_{1}-\boldsymbol{B}_{2} \boldsymbol{B}_{3}^{-1} \boldsymbol{B}_{2}^{\top} \prec \mathbf{0} \Leftrightarrow\left[\begin{array}{cc}
\boldsymbol{B}_{1} & \boldsymbol{B}_{2} \\
\boldsymbol{B}_{2}^{\top} & \boldsymbol{B}_{3}
\end{array}\right] \prec \mathbf{0} .
$$




\section{Herleitungen und Beweise}

\section{C.1 Herleitung des Ersatzsystems}

Zunächst wird gezeigt, dass das Anti-Windup-System

$$
\begin{aligned}
& \dot{\boldsymbol{\xi}}_{\mathrm{aw}}=\boldsymbol{A}_{\mathrm{s}} \boldsymbol{\xi}_{\mathrm{aw}}+\boldsymbol{B}_{\mathrm{s}, u}\left(\operatorname{sat}_{\overline{\mathbf{u}}}\left(\boldsymbol{y}_{\mathrm{r}}+\boldsymbol{y}_{\mathrm{aw}, \mathrm{y}}\right)-\boldsymbol{y}_{\mathrm{r}}\right), \\
& \boldsymbol{y}_{\mathrm{aw}, \mathrm{y}}=\boldsymbol{k}_{\mathrm{aw}}\left(\boldsymbol{\xi}_{\mathrm{aw}}\right), \\
& \boldsymbol{y}_{\mathrm{aw}, \mathrm{u}}=\boldsymbol{C}_{\mathrm{s}} \boldsymbol{\xi}_{\mathrm{aw}}
\end{aligned}
$$

und das Ersatzsystem

$$
\begin{aligned}
& \dot{\boldsymbol{\xi}}_{\mathrm{aw}}=\boldsymbol{A}_{\mathrm{s}} \boldsymbol{\xi}_{\mathrm{aw}}+\boldsymbol{B}_{\mathrm{s}, u} \mathbf{s a t}_{[-\overline{\mathbf{u}}+\boldsymbol{p}(t), \overline{\mathbf{u}}+\boldsymbol{p}(t)]}\left(\boldsymbol{y}_{\mathrm{aw}, \mathrm{y}}\right)+\boldsymbol{B}_{\mathrm{s}, u} \boldsymbol{\zeta}, \\
& \boldsymbol{y}_{\mathrm{aw}, \mathrm{y}}=\boldsymbol{k}_{\text {aw }}\left(\boldsymbol{\xi}_{\mathrm{aw}}\right), \\
& \boldsymbol{y}_{\mathrm{aw}, \mathrm{u}}=\boldsymbol{C}_{\mathrm{s}} \boldsymbol{\xi}_{\mathrm{aw}}
\end{aligned}
$$

für die spezielle Wahl

$$
\begin{aligned}
& \boldsymbol{p}=\operatorname{sat}_{\varrho \overline{\mathbf{u}}}\left(\boldsymbol{y}_{\mathrm{r}}\right) \\
& \boldsymbol{\zeta}=\boldsymbol{p}+\mathbf{s a t}_{\overline{\mathbf{u}}}\left(\boldsymbol{y}_{\mathrm{r}}+\boldsymbol{y}_{\mathrm{aw}, \mathrm{y}}\right)-\mathbf{s a t}_{\overline{\mathbf{u}}}\left(\boldsymbol{p}+\boldsymbol{y}_{\mathrm{aw}, \mathrm{y}}\right)-\boldsymbol{y}_{\mathrm{r}}
\end{aligned}
$$

äquivalent sind. Danach werden die Größen $\boldsymbol{p}$ und $\boldsymbol{\zeta}$ geeignet abgeschätzt.

\section{C.1.1 Äquivalenz}

Um die Äquivalenz zu zeigen, wird die in (2.7) definierte Sättigungsfunktion $\mathbf{s a t}_{\varrho \overline{\mathbf{u}}}: \mathbb{R}^{m} \mapsto \mathbb{R}^{m}$ mit den Sättigungsgrenzen

$$
\varrho \overline{\mathbf{u}}^{\top}=\left[\varrho \overline{\mathbf{u}}_{1} \quad \cdots \quad \varrho \overline{\mathrm{u}}_{m}\right]
$$

benötigt, wobei $\varrho \in(0,1)$ gilt. Nun wird der Eingang

$$
\tilde{\boldsymbol{u}}=\operatorname{sat}_{\overline{\mathbf{u}}}\left(\boldsymbol{y}_{\mathrm{r}}+\boldsymbol{y}_{\mathrm{aw}, \mathrm{y}}\right)-\boldsymbol{y}_{\mathrm{r}}
$$

des Anti-Windup-Systems betrachtet. Durch geschickte Addition von null lässt sich dieser nach einer Idee aus $[52,53]$ wie folgt schreiben

$$
\begin{aligned}
\tilde{\boldsymbol{u}} & =\operatorname{sat}_{\overline{\mathbf{u}}}\left(\boldsymbol{y}_{\mathrm{r}}+\boldsymbol{y}_{\mathrm{aw}, \mathrm{y}}\right)-\boldsymbol{y}_{\mathrm{r}}+\underbrace{\mathbf{s a t}_{\overline{\mathbf{u}}}(\boldsymbol{\eta})-\mathbf{s a t}_{\overline{\mathbf{u}}}(\boldsymbol{\eta})}_{0} \\
& =\operatorname{sat}_{\overline{\mathbf{u}}}(\boldsymbol{\eta})-\boldsymbol{y}_{\mathrm{r}}+\boldsymbol{\theta},
\end{aligned}
$$


wobei zwecks einer kompakteren Notation die Abkürzungen

$$
\begin{aligned}
& \boldsymbol{\eta}=\operatorname{sat}_{\varrho \overline{\mathbf{u}}}\left(\boldsymbol{y}_{\mathrm{r}}\right)+\boldsymbol{y}_{\mathrm{aw}, \mathrm{y}}, \\
& \boldsymbol{\theta}=\operatorname{sat}_{\overline{\mathbf{u}}}\left(\boldsymbol{y}_{\mathrm{r}}+\boldsymbol{y}_{\mathrm{aw}, \mathrm{y}}\right)-\operatorname{sat}_{\overline{\mathbf{u}}}(\boldsymbol{\eta})
\end{aligned}
$$

verwendet werden. Eine erneute Addition von null führt zu

$$
\tilde{\boldsymbol{u}}=\operatorname{sat}_{\overline{\mathbf{u}}}(\boldsymbol{\eta})-\boldsymbol{y}_{\mathrm{r}}+\boldsymbol{\theta}+\underbrace{\mathbf{s a t}_{\varrho \overline{\mathbf{u}}}\left(\boldsymbol{y}_{\mathrm{r}}\right)-\mathbf{s a t}_{\varrho \overline{\mathbf{u}}}\left(\boldsymbol{y}_{\mathrm{r}}\right)}_{0}
$$

und schließlich zu dem gewünschten Endergebnis

$$
\begin{aligned}
\tilde{\boldsymbol{u}} & =\operatorname{sat}_{\overline{\mathbf{u}}}\left(\operatorname{sat}_{\varrho \overline{\mathbf{u}}}\left(\boldsymbol{y}_{\mathrm{r}}\right)+\boldsymbol{y}_{\mathrm{aw}, \mathrm{y}}\right)-\mathbf{s a t}_{\varrho \overline{\mathbf{u}}}\left(\boldsymbol{y}_{\mathrm{r}}\right)+\boldsymbol{\zeta} \\
& =\left[\operatorname{sat}_{\overline{\mathbf{u}}}\left(\boldsymbol{p}+\boldsymbol{y}_{\mathrm{aw}, \mathrm{y}}\right)-\boldsymbol{p}\right]+\boldsymbol{\zeta}
\end{aligned}
$$

mit den abermals abkürzenden Schreibweisen

$$
\begin{aligned}
& \boldsymbol{p}=\operatorname{sat}_{\varrho \overline{\mathbf{u}}}\left(\boldsymbol{y}_{\mathrm{r}}\right) \\
& \boldsymbol{\zeta}=\boldsymbol{p}+\operatorname{sat}_{\overline{\mathbf{u}}}\left(\boldsymbol{y}_{\mathrm{r}}+\boldsymbol{y}_{\mathrm{aw}, \mathrm{y}}\right)-\mathbf{s a t}_{\overline{\mathbf{u}}}\left(\boldsymbol{p}+\boldsymbol{y}_{\mathrm{aw}, \mathrm{y}}\right)-\boldsymbol{y}_{\mathrm{r}}
\end{aligned}
$$

Einsetzen der umgeformten Eingangsgröße (C.4) in die Systemdynamik (C.1) liefert das äquivalente Anti-Windup-System

$$
\begin{aligned}
& \dot{\boldsymbol{\xi}}_{\mathrm{aw}}=\boldsymbol{A}_{\mathrm{s}} \boldsymbol{\xi}_{\mathrm{aw}}+\boldsymbol{B}_{\mathrm{s}, u}\left[\mathbf{s a t}_{\overline{\mathbf{u}}}\left(\boldsymbol{p}+\boldsymbol{y}_{\mathrm{aw}, \mathrm{y}}\right)-\boldsymbol{p}\right]+\boldsymbol{B}_{\mathrm{s}, u} \boldsymbol{\zeta} \\
& \boldsymbol{y}_{\mathrm{aw}, \mathrm{y}}=\boldsymbol{k}_{\mathrm{aw}}\left(\boldsymbol{\xi}_{\mathrm{aw}}\right) \\
& \boldsymbol{y}_{\mathrm{aw}, \mathrm{u}}=\boldsymbol{C}_{\mathrm{s}} \boldsymbol{\xi}_{\mathrm{aw}} .
\end{aligned}
$$

Dabei kann der Ausdruck

$$
\operatorname{sat}_{\overline{\mathbf{u}}}\left(\boldsymbol{p}+\boldsymbol{y}_{\mathrm{aw}, \mathrm{y}}\right)-\boldsymbol{p}
$$

als zeitvariante Sättigungsfunktion interpretiert werden, denn die einzelnen Komponenten

$$
\operatorname{sat}_{\overline{\mathrm{u}}_{\mathrm{i}}}\left(p_{i}+y_{\mathrm{aw}, \mathrm{y}, \mathrm{i}}\right)-p_{i}= \begin{cases}\left(p_{i}-1\right) \overline{\mathrm{u}}_{i}, & y_{\mathrm{aw}, \mathrm{y}, \mathrm{i}}<\left(p_{i}-1\right) \overline{\mathrm{u}}_{i}, \\ \left(1+p_{i}\right) \overline{\mathrm{u}}_{i}, & y_{\mathrm{aw}, \mathrm{y}, \mathrm{i}}>\left(1+p_{i}\right) \overline{\mathrm{u}}_{i}, \\ y_{\mathrm{aw}, \mathrm{y}, \mathrm{i}}, & \text { sonst }\end{cases}
$$

mit $p_{i}=\operatorname{sat}_{\varrho \overline{\mathrm{u}}_{i}}\left(y_{\mathrm{r}, i}\right)$ sind entlang der ersten Winkelhalbierenden verschobene Sättigungsfunktionen. Die maximale Verschiebung ist durch $p_{i}=\varrho \overline{\mathrm{u}}_{i}$ bzw. $p_{i}=-\varrho \overline{\mathrm{u}}_{i}$ gegeben. Für diese Werte von $p_{i}$ ist die Funktion (C.6) 
in Abbildung 4.9 des Abschnitts 4.4.2 (Seite 57) dargestellt. Aufgrund der Zeitabhängigkeit von $p_{i}$ ist (C.6) eine zeitvariante Sättigungsfunktion, deren Grenzen variieren. Deshalb lässt sich für (C.5) schreiben

$$
\operatorname{sat}_{\overline{\mathbf{u}}}\left(\boldsymbol{p}+\boldsymbol{y}_{\mathrm{aw}, \mathrm{y}}\right)-\boldsymbol{p}=\mathbf{s a t}_{[-\overline{\mathbf{u}}+\boldsymbol{p}(t), \overline{\mathbf{u}}+\boldsymbol{p}(t)]}\left(\boldsymbol{y}_{\mathrm{aw}, \mathrm{y}}\right),
$$

woraus die Äquivalenz von (C.1) und (C.2) für $\boldsymbol{p}$ und $\zeta$ gemäß (C.3) folgt.

\section{C.1.2 Abschätzung}

Die Größen $\boldsymbol{p}$ und $\boldsymbol{\zeta}$ lassen sich geeignet abschätzen. Für $\boldsymbol{p}$ gilt nach (C.3) offensichtlich

$$
-\varrho \overline{\mathrm{u}}_{i} \leq p_{i} \leq \varrho \overline{\mathrm{u}}_{i}
$$

Eine Abschätzung von $\boldsymbol{\zeta}$ ist komplizierter und erfordert eine längere Vorbereitung. Zunächst wird das folgende Lemma benötigt.

Lemma C.1. Gegeben sei die entlang der Ursprungsgeraden mit Steigung eins verschobene Sättigungsfunktion

$$
\operatorname{sat}_{\bar{u}}(y+\mathrm{c})-\mathrm{c}=\left\{\begin{array}{lr}
-\bar{u}-\mathrm{c}, & y<-\bar{u}-\mathrm{c}, \\
y, & -\bar{u}-\mathrm{c} \leq y \leq \bar{u}-\mathrm{c}, \\
\bar{u}-\mathrm{c}, & y>\bar{u}-\mathrm{c}
\end{array}\right.
$$

mit der Konstanten $c \in \mathbb{R}$ und dem Sättigungslevel $\bar{u} \in \mathbb{R}_{>0}$. Für Konstanten $\mathrm{c}_{1}, \mathrm{c}_{2} \in \mathbb{R}$ mit $\mathrm{c}_{1}>\mathrm{c}_{2}$ gilt

$$
\operatorname{sat}_{\bar{u}}\left(y+\mathrm{c}_{1}\right)-\mathrm{c}_{1} \leq \operatorname{sat}_{\bar{u}}\left(y+\mathrm{c}_{2}\right)-\mathrm{c}_{2} \quad \forall y \in \mathbb{R} .
$$

Beweis. $\mathrm{Zu}$ beweisen ist, dass (C.7) für beliebige Konstanten $\mathrm{c}_{1}, \mathrm{c}_{2} \in \mathbb{R}$, $c_{1}>c_{2}$ gilt. Bei der Funktion auf der linken Seite handelt es sich um eine Sättigungsfunktion mit Sättigungslevel $\bar{u}$, die entlang der Ursprungsgeraden mit Steigung eins verschoben ist. Gleiches gilt für die Funktion auf der rechten Seite. Das Ausmaß der Verschiebung wird durch die Konstanten $c_{1}$ und $c_{2}$ bestimmt. Abbildung C.1 stellt die Sättigungsfunktionen

$$
\operatorname{sat}_{\overline{\mathrm{u}}}\left(y+\mathrm{c}_{1}\right)-\mathrm{c}_{1}, \quad \operatorname{sat}_{\overline{\mathrm{u}}}\left(y+\mathrm{c}_{2}\right)-\mathrm{c}_{2}, \quad \mathrm{c}_{1}>\mathrm{c}_{2}
$$

für den Fall (a): $\overline{\mathrm{u}}-\mathrm{c}_{1}>-\overline{\mathrm{u}}-\mathrm{c}_{2}$ und den Fall (b): $\overline{\mathrm{u}}-\mathrm{c}_{1}<-\overline{\mathrm{u}}-\mathrm{c}_{2}$ dar. Im Fall (a) überlappen beide Funktionen, im Fall (b) gibt es keine gemeinsamen Punkte. Offensichtlich gilt für jeden der fünf Bereiche I-V in beiden Fällen die Ungleichung (C.7). 
(a) $\overline{\mathrm{u}}-\mathrm{c}_{1}>-\overline{\mathrm{u}}-\mathrm{c}_{2}$

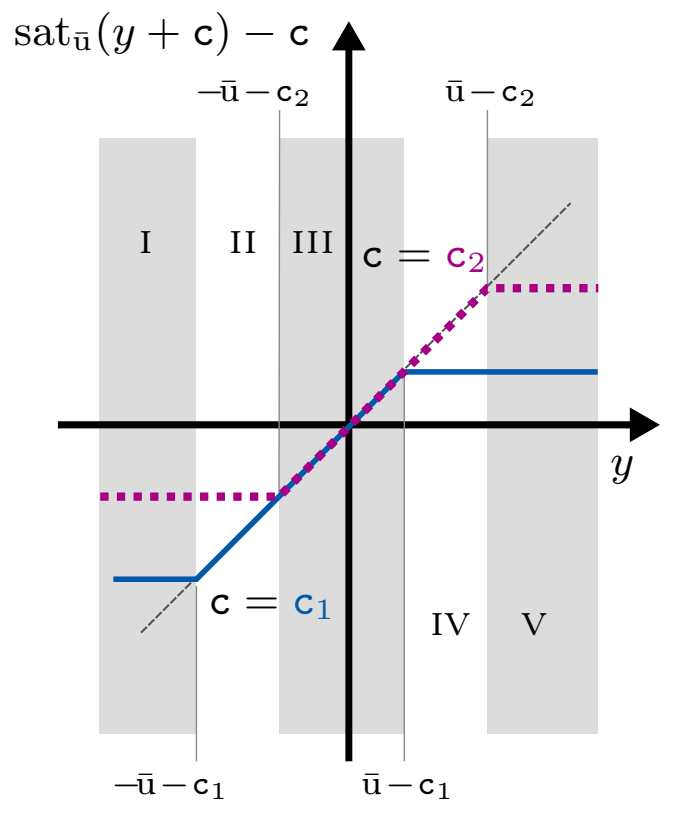

(b) $\overline{\mathrm{u}}-\mathrm{c}_{1}<-\overline{\mathrm{u}}-\mathrm{c}_{2}$

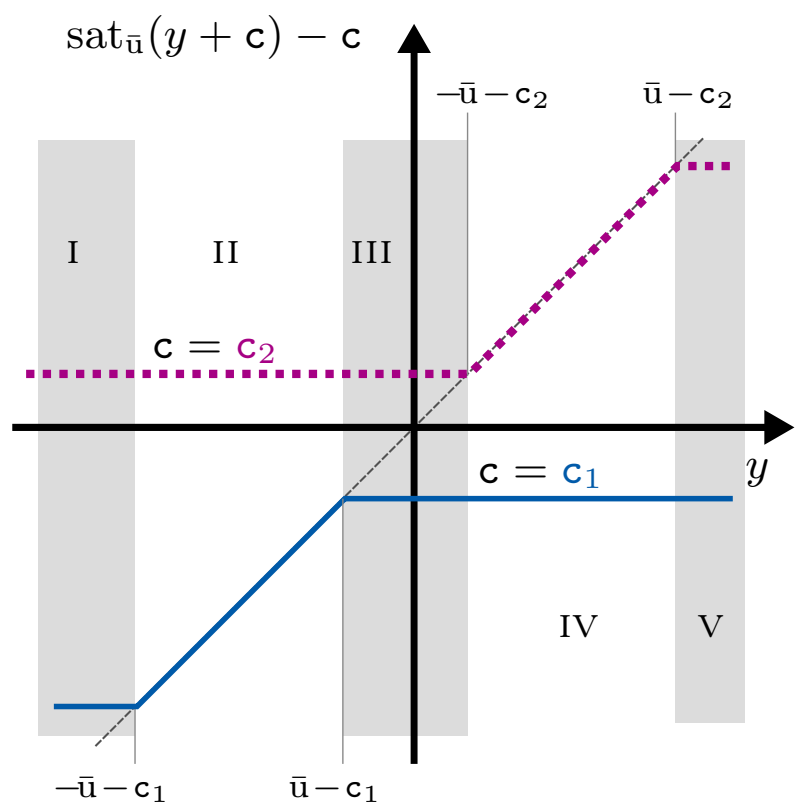

Abbildung C.1: Verschobene Sättigungsfunktionen.

Mithilfe des obigen Lemmas lässt sich dann die Abschätzung von $\boldsymbol{\zeta}$ vornehmen. Es gilt

Lemma C.2. Die von $\boldsymbol{y}_{\mathrm{r}}$ und $\boldsymbol{y}_{\mathrm{aw}, \mathrm{y}}$ abhängende Größe

$$
\boldsymbol{\zeta}=\boldsymbol{s a t} \boldsymbol{t}_{\varrho \bar{u}}\left(\boldsymbol{y}_{\mathrm{r}}\right)+\boldsymbol{s a t} \bar{t}_{\overline{\boldsymbol{u}}}\left(\boldsymbol{y}_{\mathrm{r}}+\boldsymbol{y}_{\mathrm{aw}, \mathrm{y}}\right)-\boldsymbol{s} \boldsymbol{a} \bar{t}_{\overline{\boldsymbol{u}}}\left(\boldsymbol{s a t} \boldsymbol{t}_{\varrho \bar{u}}\left(\boldsymbol{y}_{\mathrm{r}}\right)+\boldsymbol{y}_{\mathrm{aw}, \mathrm{y}}\right)-\boldsymbol{y}_{\mathrm{r}}
$$

mit $\varrho \in(0,1)$ besitzt die folgenden Eigenschaften:

(I) Der Betrag jeder Komponente kann abgeschätzt werden zu

$$
\left|\zeta_{i}\right| \leq 2\left|\operatorname{sat}_{\varrho} \bar{u}_{i}\left(y_{\mathrm{r}, i}\right)-y_{\mathrm{r}, i}\right| \leq 2\left|y_{\mathrm{r}, i}\right| .
$$

(II) Für den Sonderfall $\boldsymbol{s a t}_{\overline{\boldsymbol{u}}}\left(\boldsymbol{y}_{\mathrm{r}}\right)=\boldsymbol{y}_{\mathrm{r}}$ und $\boldsymbol{y}_{\mathrm{aw}, \mathrm{y}}=\mathbf{0}$ gilt $\boldsymbol{\zeta}=\mathbf{0}$.

Beweis. Zum Beweis von (I) wird der Betrag von (C.8) gebildet. Es gilt

$$
|\zeta|=\left|\operatorname{sat}_{\varrho \overline{\mathbf{u}}}\left(\boldsymbol{y}_{\mathrm{r}}\right)+\operatorname{sat}_{\overline{\mathbf{u}}}\left(\boldsymbol{y}_{\mathrm{r}}+\boldsymbol{y}_{\mathrm{aw}, \mathrm{y}}\right)-\operatorname{sat}_{\overline{\mathbf{u}}}\left(\operatorname{sat}_{\varrho \overline{\mathbf{u}}}\left(\boldsymbol{y}_{\mathrm{r}}\right)+\boldsymbol{y}_{\mathrm{aw}, \mathrm{y}}\right)-\boldsymbol{y}_{\mathrm{r}}\right| .
$$

Eine Abschätzung des Betrags liefert

$$
|\boldsymbol{\zeta}| \leq\left|\operatorname{sat}_{\overline{\mathbf{u}}}\left(\boldsymbol{y}_{\mathrm{r}}+\boldsymbol{y}_{\mathrm{aw}, \mathrm{y}}\right)-\operatorname{sat}_{\overline{\mathbf{u}}}\left(\operatorname{sat}_{\varrho \overline{\mathbf{u}}}\left(\boldsymbol{y}_{\mathrm{r}}\right)+\boldsymbol{y}_{\mathrm{aw}, \mathrm{y}}\right)\right|+\left|\mathbf{s a t}_{\varrho \overline{\mathbf{u}}}\left(\boldsymbol{y}_{\mathrm{r}}\right)-\boldsymbol{y}_{\mathrm{r}}\right| .
$$


Um zu zeigen, dass $\left|\zeta_{i}\right| \leq 2\left|\operatorname{sat}_{\varrho \overline{\mathrm{u}}_{i}}\left(y_{\mathrm{r}, i}\right)-y_{\mathrm{r}, i}\right|$ gilt, muss bewiesen werden, dass folgende Ungleichung erfüllt ist

$$
\begin{aligned}
\left|\operatorname{sat}_{\overline{\mathrm{u}}_{\mathrm{i}}}\left(y_{\mathrm{r}, i}+y_{\mathrm{aw}, \mathrm{y}, \mathrm{i}}\right)-\operatorname{sat}_{\overline{\mathrm{u}}_{\mathrm{i}}}\left(\operatorname{sat}_{\varrho \overline{\mathrm{u}}_{i}}\left(y_{\mathrm{r}, i}\right)+y_{\mathrm{aw}, \mathrm{y}, \mathrm{i}}\right)\right| \\
\quad \leq\left|\operatorname{sat}_{\varrho \overline{\mathrm{u}}_{i}}\left(y_{\mathrm{r}, i}\right)-y_{\mathrm{r}, i}\right| .
\end{aligned}
$$

Ungleichung (C.9) muss für drei Fälle untersucht werden. Im ersten Fall gilt $-\varrho \overline{\mathrm{u}}_{i} \leq y_{\mathrm{r}, i} \leq \varrho \overline{\mathrm{u}}_{i}$. Der zweite Fall betrachtet $0<\varrho \overline{\mathrm{u}}_{i}<y_{\mathrm{r}, i}$ und der dritte Fall $y_{\mathrm{r}, i}<-\varrho \overline{\mathrm{u}}_{i}<0$. Nachfolgend werden die Fälle behandelt.

Fall 1. Es gilt $\operatorname{sat}_{\varrho \overline{\mathrm{u}}_{i}}\left(y_{\mathrm{r}, i}\right)=y_{\mathrm{r}, i}$. Somit vereinfacht sich (C.9) zu

$$
\left|\operatorname{sat}_{\overline{\mathrm{u}}_{\mathrm{i}}}\left(y_{\mathrm{r}, i}+y_{\mathrm{aw}, \mathrm{y}, \mathrm{i}}\right)-\operatorname{sat}_{\overline{\mathrm{u}}_{\mathrm{i}}}\left(y_{\mathrm{r}, i}+y_{\mathrm{aw}, \mathrm{y}, \mathrm{i}}\right)\right| \leq\left|y_{\mathrm{r}, i}-y_{\mathrm{r}, i}\right| .
$$

Das ist immer erfüllt.

Fall 2. Es gilt $\operatorname{sat}_{\varrho \overline{\mathrm{u}}_{i}}\left(y_{\mathrm{r}, i}\right)=\varrho \overline{\mathrm{u}}_{i}$. Somit vereinfacht sich (C.9) zu

$$
\left|\operatorname{sat}_{\varrho \overline{\mathrm{u}}_{i}}\left(y_{\mathrm{r}, i}+y_{\mathrm{aw}, \mathrm{y}, \mathrm{i}}\right)-\operatorname{sat}_{\varrho \overline{\mathrm{u}}_{i}}\left(\varrho \overline{\mathrm{u}}_{i}+y_{\mathrm{aw}, \mathrm{y}, \mathrm{i}}\right)\right| \leq\left|\varrho \overline{\mathrm{u}}_{i}-y_{\mathrm{r}, i}\right| .
$$

Der nächste Schritt besteht im Auflösen der Beträge. Auf der rechten Seite ergibt sich

$$
\left|\varrho \overline{\mathrm{u}}_{i}-y_{\mathrm{r}, i}\right|=-\left(\varrho \overline{\mathrm{u}}_{i}-y_{\mathrm{r}, i}\right)=y_{\mathrm{r}, i}-\varrho \overline{\mathrm{u}}_{i},
$$

da nach Voraussetzung $\varrho \overline{\mathrm{u}}_{i}<y_{\mathrm{r}, i}$ gilt. Ebenso gilt

$$
\varrho \overline{\mathrm{u}}_{i}+y_{\mathrm{aw}, \mathrm{y}, \mathrm{i}}<y_{\mathrm{r}, i}+y_{\mathrm{aw}, \mathrm{y}, \mathrm{i}}
$$

und deshalb aufgrund der Monotonie der Sättigungsfunktion

$$
\operatorname{sat}_{\varrho \overline{\mathrm{u}}_{i}}\left(\varrho \overline{\mathrm{u}}_{i}+y_{\mathrm{aw}, \mathrm{y}, \mathrm{i}}\right) \leq \operatorname{sat}_{\varrho \overline{\mathrm{u}}_{i}}\left(y_{\mathrm{r}, i}+y_{\mathrm{aw}, \mathrm{y}, \mathrm{i}}\right) .
$$

Folglich kann man den linken Betrag in (C.10) durch den Ausdruck

$$
\operatorname{sat}_{\varrho \overline{\mathrm{u}}_{i}}\left(y_{\mathrm{r}, i}+y_{\mathrm{aw}, \mathrm{y}, \mathrm{i}}\right)-\operatorname{sat}_{\varrho \overline{\mathrm{u}}_{i}}\left(\varrho \overline{\mathrm{u}}_{i}+y_{\mathrm{aw}, \mathrm{y}, \mathrm{i}}\right)
$$

ersetzen und mit (C.11) resultiert die Ungleichung

$$
\operatorname{sat}_{\varrho \overline{\mathrm{u}}_{i}}\left(y_{\mathrm{r}, i}+y_{\mathrm{aw}, \mathrm{y}, \mathrm{i}}\right)-y_{\mathrm{r}, i} \leq \operatorname{sat}_{\varrho \overline{\mathrm{u}}_{i}}\left(\varrho \overline{\mathrm{u}}_{i}+y_{\mathrm{aw}, \mathrm{y}, \mathrm{i}}\right)-\varrho \overline{\mathrm{u}}_{i} .
$$

Da $y_{\mathrm{r}, i}>\varrho \overline{\mathrm{u}}_{i}$ ist, gilt die Ungleichung nach Lemma C.1. 
Fall 3. Es gilt $\operatorname{sat}_{\varrho \overline{\mathrm{u}}_{i}}\left(y_{\mathrm{r}, i}\right)=-\varrho \overline{\mathrm{u}}_{i}$. Somit vereinfacht sich (C.9) zu

$$
\left|\operatorname{sat}_{\varrho \overline{\mathrm{u}}_{i}}\left(y_{\mathrm{r}, i}+y_{\mathrm{aw}, \mathrm{y}, \mathrm{i}}\right)-\operatorname{sat}_{\varrho \overline{\mathrm{u}}_{i}}\left(-\varrho \overline{\mathrm{u}}_{i}+y_{\mathrm{aw}, \mathrm{y}, \mathrm{i}}\right)\right| \leq\left|-\varrho \overline{\mathrm{u}}_{i}-y_{\mathrm{r}, i}\right| .
$$

Analog zum zweiten Fall werden die Beträge aufgelöst. Unter Beachtung der für Fall 3 geltenden Ungleichung

$$
y_{\mathrm{r}, i}<-\varrho \overline{\mathrm{u}}_{i}<0 \Leftrightarrow 0<-y_{\mathrm{r}, i}-\varrho \overline{\mathrm{u}}_{i}<-y_{\mathrm{r}, i}
$$

folgt für den Betrag auf der rechten Seite

$$
\left|-\varrho \overline{\mathrm{u}}_{i}-y_{\mathrm{r}, i}\right|=-y_{\mathrm{r}, i}-\varrho \overline{\mathrm{u}}_{i} .
$$

Ebenso gilt für ein $y_{\mathrm{aw}, \mathrm{y}, \mathrm{i}} \in \mathbb{R}$ die Ungleichung

$$
y_{\mathrm{r}, i}+y_{\mathrm{aw}, \mathrm{y}, \mathrm{i}}<-\varrho \overline{\mathrm{u}}_{i}+y_{\mathrm{aw}, \mathrm{y}, \mathrm{i}}
$$

und deshalb aufgrund der Monotonie der Sättigungsfunktion

$$
\operatorname{sat}_{\varrho \overline{\mathrm{u}}_{i}}\left(y_{\mathrm{r}, i}+y_{\mathrm{aw}, \mathrm{y}, \mathrm{i}}\right) \leq \operatorname{sat}_{\varrho \overline{\mathrm{u}}_{i}}\left(-\varrho \overline{\mathrm{u}}_{i}+y_{\mathrm{aw}, \mathrm{y}, \mathrm{i}}\right) .
$$

Mit diesem Ergebnis folgt für den Betrag auf der linken Seite

$$
\operatorname{sat}_{\varrho \overline{\mathrm{u}}_{i}}\left(-\varrho \overline{\mathrm{u}}_{i}+y_{\mathrm{aw}, \mathrm{y}, \mathrm{i}}\right)-\operatorname{sat}_{\varrho \overline{\mathrm{u}}_{i}}\left(y_{\mathrm{r}, i}+y_{\mathrm{aw}, \mathrm{y}, \mathrm{i}}\right)
$$

und man erhält für (C.12) nach Einsetzen der bisherigen Ergebnisse

$$
\operatorname{sat}_{\varrho \overline{\mathrm{u}}_{i}}\left(-\varrho \overline{\mathrm{u}}_{i}+y_{\mathrm{aw}, \mathrm{y}, \mathrm{i}}\right)-\operatorname{sat}_{\varrho \overline{\mathrm{u}}_{i}}\left(y_{\mathrm{r}, i}+y_{\mathrm{aw}, \mathrm{y}, \mathrm{i}}\right) \leq-y_{\mathrm{r}, i}-\varrho \overline{\mathrm{u}}_{i} .
$$

Substituieren von $\mathrm{c}=-\varrho \overline{\mathrm{u}}_{i}$ und Umstellen der Ungleichung liefert

$$
\operatorname{sat}_{\varrho \bar{u}_{i}}\left(\mathrm{c}+y_{\mathrm{aw}, \mathrm{y}, \mathrm{i}}\right)-\mathrm{c} \leq \operatorname{sat}_{\varrho \overline{\mathrm{u}}_{i}}\left(y_{\mathrm{r}, i}+y_{\mathrm{aw}, \mathrm{y}, \mathrm{i}}\right)-y_{\mathrm{r}, i} .
$$

Diese Ungleichung gilt nach Lemma C.1, da $y_{\mathrm{r}, i}<\mathrm{c}=-\varrho \overline{\mathrm{u}}_{i}$ erfüllt ist.

Eigenschaft (II) ergibt sich durch Einsetzen der Bedingungen $\boldsymbol{y}_{\mathrm{aw}, \mathrm{y}}=\mathbf{0}$ sowie $\operatorname{sat}_{\overline{\mathbf{u}}}\left(\boldsymbol{y}_{\mathrm{r}}\right)=\boldsymbol{y}_{\mathrm{r}}$ in (C.8) und Ausnutzen von

$$
\operatorname{sat}_{\overline{\mathbf{u}}}\left(\operatorname{sat}_{\varrho \overline{\mathbf{u}}}\left(\boldsymbol{y}_{\mathrm{r}}\right)\right)=\operatorname{sat}_{\varrho \overline{\mathbf{u}}}\left(\boldsymbol{y}_{\mathrm{r}}\right) .
$$

Damit ist das Lemma bewiesen. 


\section{C.2 Schrittweite beim Path-Following}

In [151] wird folgendes Resultat verwendet, aber nicht bewiesen.

Lemma C.3. Gegeben sei eine symmetrische, positiv definite Matrix $\boldsymbol{Q} \in \mathbb{R}^{n \times n}$, eine symmetrische Matrix $\boldsymbol{Q}_{\Delta}$ und ein positiver Skalar $\alpha$. Des Weiteren sei $\|\boldsymbol{Q}\|_{2}$ die induzierte 2 -Norm von $\boldsymbol{Q}$. Wenn die Matrixungleichung

$$
\left[\begin{array}{ll}
\alpha \boldsymbol{Q} & \boldsymbol{Q}_{\Delta} \\
\boldsymbol{Q}_{\Delta} & \alpha \boldsymbol{Q}
\end{array}\right] \succ \mathbf{0}
$$

erfüllt ist, dann gilt

$$
\left\|\boldsymbol{Q}_{\Delta}\right\|_{2}<\alpha\|\boldsymbol{Q}\|_{2}
$$

Für den Beweis werden Eigenschaften der induzierten 2-Norm ausgenutzt, die in Abschnitt A.1 angegeben sind. Details finden sich in [16].

Beweis. Ausgangspunkt der Überlegungen ist (C.13). Nach einer Umformung mit Lemma B.1, ergibt sich die äquivalente Matrixungleichung

$$
\alpha \boldsymbol{Q}-\boldsymbol{Q}_{\Delta} \alpha^{-1} \boldsymbol{Q}^{-1} \boldsymbol{Q}_{\Delta} \succ \mathbf{0},
$$

die wie folgt umformbar ist

$$
\boldsymbol{Q}_{\Delta} \boldsymbol{Q}^{-1} \boldsymbol{Q}_{\Delta} \boldsymbol{Q}^{-1}-\alpha^{2} \mathbb{I} \prec \mathbf{0} .
$$

Offensichtlich muss die Matrix auf der linken Seite negativ definit sein, was äquivalent $\mathrm{zu}$

$$
\boldsymbol{x}^{\top}\left(\boldsymbol{Q}_{\Delta} \boldsymbol{Q}^{-1} \boldsymbol{Q}_{\Delta} \boldsymbol{Q}^{-1}-\alpha^{2} \mathbb{I}\right) \boldsymbol{x}<0 \quad \forall \boldsymbol{x} \in \mathbb{R}^{n} \backslash \mathbf{0}
$$

ist. Umsortieren führt zu

$$
\boldsymbol{x}^{\top}\left(\boldsymbol{Q}_{\Delta} \boldsymbol{Q}^{-1} \boldsymbol{Q}_{\Delta} \boldsymbol{Q}^{-1}\right) \boldsymbol{x}<\boldsymbol{x}^{\top} \alpha^{2} \boldsymbol{x}=\alpha^{2}\|\boldsymbol{x}\|_{2}^{2} .
$$

Die Substitution $\boldsymbol{y}=\left(\boldsymbol{Q}_{\Delta} \boldsymbol{Q}^{-1} \boldsymbol{Q}_{\Delta} \boldsymbol{Q}^{-1}\right) \boldsymbol{x} \in \mathbb{R}^{n}$ und die Verträglichkeit der induzierten 2-Norm mit der euklidischen Norm (A.1) führen zusammen mit der Eigenschaft $\left\|\boldsymbol{x}^{\top} \boldsymbol{y}\right\|_{2} \leq\|\boldsymbol{x}\|_{2}\|\boldsymbol{y}\|_{2}$ der euklidischen Norm [16, Proposition 9.1.6] zu einer Abschätzung der linken Seite der Ungleichung. Es gilt

$$
\begin{aligned}
\left\|\boldsymbol{x}^{\top}\left(\boldsymbol{Q}_{\Delta} \boldsymbol{Q}^{-1} \boldsymbol{Q}_{\Delta} \boldsymbol{Q}^{-1}\right) \boldsymbol{x}\right\|_{2}=\left\|\boldsymbol{x}^{\top} \boldsymbol{y}\right\|_{2} & \leq\|\boldsymbol{x}\|_{2}\|\boldsymbol{y}\|_{2} \\
& \leq\|\boldsymbol{x}\|_{2}\left\|\boldsymbol{Q}_{\Delta} \boldsymbol{Q}^{-1} \boldsymbol{Q}_{\Delta} \boldsymbol{Q}^{-1}\right\|_{2}\|\boldsymbol{x}\|_{2} \\
& \leq\left\|\boldsymbol{Q}_{\Delta} \boldsymbol{Q}^{-1} \boldsymbol{Q}_{\Delta} \boldsymbol{Q}^{-1}\right\|_{2}\|\boldsymbol{x}\|_{2}^{2}
\end{aligned}
$$


Kombiniert man (C.14) und die letzte Ungleichung ergibt sich

$$
\left\|\boldsymbol{x}^{\top}\left(\boldsymbol{Q}_{\Delta} \boldsymbol{Q}^{-1} \boldsymbol{Q}_{\Delta} \boldsymbol{Q}^{-1}\right) \boldsymbol{x}\right\|_{2} \leq\left\|\boldsymbol{Q}_{\Delta} \boldsymbol{Q}^{-1} \boldsymbol{Q}_{\Delta} \boldsymbol{Q}^{-1}\right\|_{2}\|\boldsymbol{x}\|_{2}^{2}<\alpha^{2}\|\boldsymbol{x}\|_{2}^{2},
$$

was für alle $\boldsymbol{x} \in \mathbb{R}^{n} \backslash \mathbf{0}$ erfüllt ist, wenn

$$
\left\|\boldsymbol{Q}_{\Delta} \boldsymbol{Q}^{-1} \boldsymbol{Q}_{\Delta} \boldsymbol{Q}^{-1}\right\|_{2}<\alpha^{2}
$$

gilt. Aufgrund der Submultiplikativität (A.2) der 2-Norm folgt

$$
\left\|\boldsymbol{Q}_{\Delta} \boldsymbol{Q}^{-1} \boldsymbol{Q}_{\Delta} \boldsymbol{Q}^{-1}\right\|_{2} \leq\left\|\boldsymbol{Q}_{\Delta}\right\|_{2}\left\|\boldsymbol{Q}^{-1}\right\|_{2}\left\|\boldsymbol{Q}_{\Delta}\right\|_{2}\left\|\boldsymbol{Q}^{-1}\right\|_{2}=\left\|\boldsymbol{Q}_{\Delta}\right\|_{2}^{2}\left\|\boldsymbol{Q}^{-1}\right\|_{2}^{2}
$$

Offensichtlich impliziert die Ungleichung

$$
\left\|\boldsymbol{Q}_{\Delta}\right\|_{2}^{2}\left\|\boldsymbol{Q}^{-1}\right\|_{2}^{2}<\alpha^{2}
$$

die Forderung (C.15), die wiederum (C.14) garantiert. Für den letzten Schritt wird folgendes Resultat für nichtsinguläre Matrizen benötigt

$$
\left\|\boldsymbol{Q}^{-1}\right\|_{2} \geq \frac{\|\mathbb{I}\|_{2}}{\|\boldsymbol{Q}\|_{2}}=\frac{1}{\|\boldsymbol{Q}\|_{2}}
$$

das in [16, Fact 9.8.5] angegeben ist. Das führt zu

$$
\frac{\left\|\boldsymbol{Q}_{\Delta}\right\|_{2}^{2}}{\|\boldsymbol{Q}\|_{2}^{2}} \leq\left\|\boldsymbol{Q}_{\Delta}\right\|_{2}^{2}\left\|\boldsymbol{Q}^{-1}\right\|_{2}^{2}<\alpha^{2}
$$

und nach einer Umformung ergibt sich

$$
\left\|\boldsymbol{Q}_{\Delta}\right\|_{2}<\alpha\|\boldsymbol{Q}\|_{2}
$$

womit das Lemma bewiesen ist.

\section{C.3 Beweise}

\section{C.3.1 Beweis von Lemma 5.2}

Beweis. Zuerst werden die Übertragungsfunktionen (5.11) betrachtet. Die Übertragungsfunktion (5.11a) lässt sich durch Ausnutzen der Äquivalenz (5.16) wie folgt schreiben

$$
G_{1}(s)=\frac{-\operatorname{det}\left(\tilde{\boldsymbol{Z}}_{\mathrm{r}, w}(s)\right)}{\operatorname{det}\left(s \mathbb{I}-\boldsymbol{A}_{\mathrm{r}}-\boldsymbol{l}_{\mathrm{aw}, \mathrm{x}} \boldsymbol{c}_{\mathrm{r}}^{\top}\right)}
$$


mit

$$
\tilde{\boldsymbol{Z}}_{\mathrm{r}, w}(s)=\left[\begin{array}{cc}
s \mathbb{I}-\boldsymbol{A}_{\mathrm{r}}-\boldsymbol{l}_{\mathrm{aw}, \mathrm{x}} \boldsymbol{c}_{\mathrm{r}}^{\top} & \boldsymbol{b}_{\mathrm{r}, w}+\boldsymbol{l}_{\mathrm{aw}, \mathrm{x}} d_{\mathrm{r}, w} \\
\boldsymbol{c}_{\mathrm{r}}^{\top} & -d_{\mathrm{r}, w}
\end{array}\right] .
$$

Bekanntlich wird durch elementare Zeilenumformungen die Determinante einer Matrix nicht verändert [16, Proposition 2.7.2], [194]. Dazu zählt z. B. die Addition eines Vielfachen einer Zeile zu einer anderen Zeile. Multipliziert man die zweite Zeile von links mit $\boldsymbol{l}_{\mathrm{aw}, \mathrm{x}}$ und addiert diese zu der ersten Zeile, ergibt sich die Matrix

$$
\boldsymbol{Z}_{\mathrm{r}, w}(s)=\left[\begin{array}{cc}
s \mathbb{I}-\boldsymbol{A}_{\mathrm{r}} & \boldsymbol{b}_{\mathrm{r}, w} \\
\boldsymbol{c}_{\mathrm{r}}^{\top} & -d_{\mathrm{r}, w}
\end{array}\right]
$$

und folglich die Übertragungsfunktion (5.17a). Die Übertragungsfunktion (5.17b) lässt sich auf analoge Weise aus (5.11b) und der Äquivalenz (5.16) herleiten. Für die Übertragungsfunktion $(5.17 \mathrm{c})$ wird $(5.11 \mathrm{c})$ zunächst geschickt umgeformt. Eine Addition von null liefert

$$
G_{3}(s)=G_{3}(s)-1+1=\tilde{G}_{3}(s)+1 .
$$

Jetzt wird die Äquivalenz (5.16) ausgenutzt. Für $\tilde{G}_{3}(s)$ lässt sich schreiben

$$
\tilde{G}_{3}(s)=G_{3}(s)-1=\frac{-\operatorname{det}\left(\boldsymbol{Z}_{3}(s)\right)}{\operatorname{det}\left(s \mathbb{I}-\boldsymbol{A}_{\mathrm{r}}-\boldsymbol{l}_{\mathrm{aw}, \mathrm{x}} \boldsymbol{c}_{\mathrm{r}}^{\top}\right)}
$$

mit

$$
\boldsymbol{Z}_{3}(s)=\left[\begin{array}{cc}
s \mathbb{I}-\boldsymbol{A}_{\mathrm{r}}-\boldsymbol{l}_{\mathrm{aw}, \mathrm{x}} \boldsymbol{c}_{\mathrm{r}}^{\top} & -\boldsymbol{l}_{\mathrm{aw}, \mathrm{x}} \\
\boldsymbol{c}_{\mathrm{r}}^{\top} & 1
\end{array}\right] .
$$

Eine Multiplikation der dritten Zeile mit $\boldsymbol{l}_{\mathrm{aw}, \mathrm{x}}$ und eine Addition zu der ersten Zeile ändert die Determinante der Matrix nicht und führt zu

$$
\operatorname{det}\left(\boldsymbol{Z}_{3}(s)\right)=\operatorname{det}\left[\begin{array}{cc}
s \mathbb{I}-\boldsymbol{A}_{\mathrm{r}} & \mathbf{0} \\
\boldsymbol{c}_{\mathrm{r}}^{\top} & 1
\end{array}\right] .
$$

Die Determinante einer unteren Blockdreiecksmatrix ist das Produkt der Determinanten der Diagonalblöcke [16, Proposition 2.8.1]. Daraus folgt

$$
\operatorname{det}\left(\boldsymbol{Z}_{3}(s)\right)=\operatorname{det}\left(s \mathbb{I}-\boldsymbol{A}_{\mathrm{r}}\right) \operatorname{det}(1)=\operatorname{det}\left(s \mathbb{I}-\boldsymbol{A}_{\mathrm{r}}\right) .
$$

Einsetzen dieses Ergebnisses in (C.16) liefert (5.17c). 
Mit den Übertragungsfunktionen (5.14) wird ähnlich verfahren. Aus (5.16) folgt, dass sich (5.14a) auch schreiben lässt als

$$
H_{1}(s)=\frac{-\operatorname{det}\left(\tilde{\boldsymbol{Z}}_{\mathrm{r}, w}(s)\right)}{\operatorname{det}\left(s \mathbb{I}-\boldsymbol{A}_{\mathrm{ub}}\right)},
$$

wobei für die im Zähler auftretende Matrix gilt

$$
\tilde{\boldsymbol{Z}}_{\mathrm{r}, w}(s)=\left[\begin{array}{ccc}
s \mathbb{I}-\boldsymbol{A}_{\mathrm{s}}-\boldsymbol{b}_{\mathrm{s}, u} d_{\mathrm{r}, u} \boldsymbol{c}_{\mathrm{s}}^{\top} & -\boldsymbol{b}_{\mathrm{s}, u} \boldsymbol{c}_{\mathrm{r}}^{\top} & \boldsymbol{b}_{\mathrm{s}, u} d_{\mathrm{r}, w} \\
-\boldsymbol{b}_{\mathrm{r}, u} \boldsymbol{c}_{\mathrm{s}}^{\top} & s \mathbb{I}-\boldsymbol{A}_{\mathrm{r}} & \boldsymbol{b}_{\mathrm{r}, w} \\
d_{\mathrm{r}, u} \boldsymbol{c}_{\mathrm{S}}^{\top}+\underline{\boldsymbol{k}}^{\top} & \boldsymbol{c}_{\mathrm{r}}^{\top} & -d_{\mathrm{r}, w}
\end{array}\right] .
$$

Eine Multiplikation der dritten Zeile von links mit $\boldsymbol{b}_{\mathrm{s}, u}$ und eine Addition des Ergebnisses zu der ersten Zeile führt zu

$$
\operatorname{det}\left(\tilde{\boldsymbol{Z}}_{\mathrm{r}, w}(s)\right)=\operatorname{det}\left[\begin{array}{ccc}
s \mathbb{I}-\boldsymbol{A}_{\mathrm{s}}+\boldsymbol{b}_{\mathrm{s}, u} \underline{\boldsymbol{k}}^{\top} & \mathbf{0} & \mathbf{0} \\
-\boldsymbol{b}_{\mathrm{r}, u} \boldsymbol{c}_{\mathrm{s}}^{\top} & s \mathbb{I}-\boldsymbol{A}_{\mathrm{r}} & \boldsymbol{b}_{\mathrm{r}, w} \\
d_{\mathrm{r}, u} \boldsymbol{c}_{\mathrm{s}}^{\top}+\underline{\boldsymbol{k}}^{\top} & \boldsymbol{c}_{\mathrm{r}}^{\top} & -d_{\mathrm{r}, w}
\end{array}\right] .
$$

Ausnutzen der Struktur dieser unteren Blockdreiecksmatrix liefert

$$
\begin{aligned}
& \operatorname{det}\left(\tilde{\boldsymbol{Z}}_{\mathrm{r}, w}(s)\right)=\operatorname{det}\left(s \mathbb{I}-\boldsymbol{A}_{\mathrm{s}}+\boldsymbol{b}_{\mathrm{s}, u} \underline{\boldsymbol{k}}^{\top}\right) \operatorname{det}\left[\begin{array}{cc}
s \mathbb{I}-\boldsymbol{A}_{\mathrm{r}} & \boldsymbol{b}_{\mathrm{r}, w} \\
\boldsymbol{c}_{\mathrm{r}}^{\top} & -d_{\mathrm{r}, w}
\end{array}\right] \\
& =\operatorname{det}\left(s \mathbb{I}-\boldsymbol{A}_{\mathrm{s}}+\boldsymbol{b}_{\mathrm{s}, u} \underline{\boldsymbol{k}}^{\top}\right) \operatorname{det}\left(\boldsymbol{Z}_{\mathrm{r}, w}(s)\right)
\end{aligned}
$$

und somit (5.18a). Die Übertragungsfunktion (5.18b) ergibt sich nach dem gleichen Prinzip aus (5.14b). Die Übertragungsfunktion (5.14c) wird zunächst geschickt umgeformt. Die Addition von null führt zu

$$
H_{3}(s)=H_{3}(s)-1+1=\tilde{H}_{3}(s)+1,
$$

wobei aus (5.16) für $\tilde{H}_{3}(s)$ folgt

$$
\tilde{H}_{3}(s)=H_{3}(s)-1=\frac{-\operatorname{det}(\tilde{\boldsymbol{Z}}(s))}{\operatorname{det}\left(s \mathbb{I}-\boldsymbol{A}_{\mathrm{ub}}\right)}
$$

mit

$$
\tilde{\boldsymbol{Z}}(s)=\left[\begin{array}{ccc}
s \mathbb{I}-\boldsymbol{A}_{\mathrm{s}}-\boldsymbol{b}_{\mathrm{s}, u} d_{\mathrm{r}, u} \boldsymbol{c}_{\mathrm{s}}^{\top} & -\boldsymbol{b}_{\mathrm{s}, u} \boldsymbol{c}_{\mathrm{r}}^{\top} & -\boldsymbol{b}_{\mathrm{s}, u} \\
-\boldsymbol{b}_{\mathrm{r}, u} \boldsymbol{c}_{\mathrm{s}}^{\top} & s \mathbb{I}-\boldsymbol{A}_{\mathrm{r}} & \mathbf{0} \\
d_{\mathrm{r}, u} \boldsymbol{c}_{\mathrm{s}}^{\top}+\underline{\boldsymbol{k}}^{\top} & \boldsymbol{c}_{\mathrm{r}}^{\top} & 1
\end{array}\right]
$$


Eine Multiplikation der dritten Zeile von links mit $\boldsymbol{b}_{\mathrm{s}, u}$ und eine Addition des Ergebnisses zu der ersten Zeile führt zu

$$
\begin{aligned}
\tilde{\boldsymbol{Z}}(s) & =\operatorname{det}\left[\begin{array}{ccc}
s \mathbb{I}-\boldsymbol{A}_{\mathrm{s}}+\boldsymbol{b}_{\mathrm{s}, u} \underline{\boldsymbol{k}}^{\top} & \mathbf{0} & \mathbf{0} \\
-\boldsymbol{b}_{\mathrm{r}, u} \boldsymbol{c}_{\mathrm{s}}^{\top} & s \mathbb{I}-\boldsymbol{A}_{\mathrm{r}} & \mathbf{0} \\
d_{\mathrm{r}, u} \boldsymbol{c}_{\mathrm{s}}^{\top}+\underline{\boldsymbol{k}}^{\top} & \boldsymbol{c}_{\mathrm{r}}^{\top} & 1
\end{array}\right] \\
& =\operatorname{det}\left(s \mathbb{I}-\boldsymbol{A}_{\mathrm{s}}+\boldsymbol{b}_{\mathrm{s}, u} \underline{\boldsymbol{k}}^{\top}\right) \operatorname{det}\left(s \mathbb{I}-\boldsymbol{A}_{\mathrm{r}}\right) .
\end{aligned}
$$

Einsetzen dieses Ergebnisses in (C.17) liefert (5.18c).

\section{C.3.2 Beweis von Lemma 5.3}

Beweis. Die Sektorbedingung folgt aus (5.6a) und (5.6b). Zuerst wird der Fall $u_{i} \in \mathbb{R}_{\geq 0}$ betrachtet. Für einen positiven Skalar $\hat{\psi}_{i}$ gilt $\hat{\psi}_{i} y_{i}=$ $\hat{\psi} \mu_{i}\left(u_{i}, t\right) \geq 0$. Multiplizieren von (5.6a) mit $\hat{\psi}_{i} y_{i} \geq 0$ liefert

$$
0 \leq \hat{\psi}_{i} y_{i}^{2} \leq \hat{\psi}_{i} y_{i} \phi_{i} u_{i} \quad \Leftrightarrow \quad 0 \leq \hat{\psi}_{i} y_{i}\left(\phi_{i} u_{i}-y_{i}\right) .
$$

Für den Fall $u_{i} \in \mathbb{R}_{<0}$ wird analog vorgegangen. Für $\hat{\psi}_{i}>0$ gilt $\hat{\psi}_{i} y_{i}=$ $\hat{\psi} \mu_{i}\left(u_{i}, t\right) \leq 0$. Multiplizieren von $(5.6 \mathrm{~b})$ mit $\hat{\psi}_{i} y_{i} \leq 0$ liefert

$$
\hat{\psi}_{i} y_{i} \phi_{i} u_{i} \geq \hat{\psi}_{i} y_{i}^{2} \geq 0 \quad \Leftrightarrow \quad 0 \leq \hat{\psi}_{i} y_{i}\left(\phi_{i} u_{i}-y_{i}\right) .
$$

Fasst man die erhaltenen Bedingungen für alle Komponenten der Nichtlinearität zusammen ergibt sich (5.24).

\section{C.3.3 Beweis von Satz 5.2}

Beweis. Zum Beweis wird eine quadratische Ljapunov-Funktion

$$
V\left(\boldsymbol{\xi}_{\text {aw }}\right)=\boldsymbol{\xi}_{\text {aw }}^{\top} \boldsymbol{R} \boldsymbol{\xi}_{\text {aw }}
$$

mit einer symmetrischen, positiv definiten Matrix $\boldsymbol{R} \in \mathbb{S}^{n}$ benötigt. Der Ansatz

$$
\dot{V}\left(\boldsymbol{\xi}_{\mathrm{aw}}\right)=\frac{\partial V\left(\boldsymbol{\xi}_{\mathrm{aw}}\right)}{\partial t}<\gamma^{2} \boldsymbol{y}_{\mathrm{r}}^{\top} \boldsymbol{y}_{\mathrm{r}}-\boldsymbol{y}_{\mathrm{aw}, \mathrm{u}}^{\top} \boldsymbol{y}_{\mathrm{aw}, \mathrm{u}}
$$

garantiert für $\boldsymbol{y}_{\mathrm{r}}=\mathbf{0}$ ein global stabiles System, da für alle $\boldsymbol{\xi}_{\text {aw }} \in \mathbb{R}^{n}$

$$
\dot{V}\left(\boldsymbol{\xi}_{\mathrm{aw}}\right)<-\boldsymbol{y}_{\mathrm{aw}, \mathrm{u}}^{\top} \boldsymbol{y}_{\mathrm{aw}, \mathrm{u}} \leq 0
$$


gilt und die Ljapunov-Funktion radial unbeschränkt ist. Für energiebeschränkte Störungen, d. h. $\left\|\boldsymbol{y}_{\mathrm{r}}\right\|_{L_{2}}<\infty$, führt die Integration von (C.18) nach der Zeit im Intervall $\left[0, T_{e}\right] \mathrm{zu}$

$$
V\left(\boldsymbol{\xi}_{\text {aw }}\left(T_{e}\right)\right)-V\left(\boldsymbol{\xi}_{\text {aw }}(0)\right)<\gamma^{2} \int_{0}^{T_{e}} \boldsymbol{y}_{\mathrm{r}}^{\top} \boldsymbol{y}_{\mathrm{r}} d t-\int_{0}^{T_{e}} \boldsymbol{y}_{\mathrm{aw}, \mathrm{u}}^{\top} \boldsymbol{y}_{\mathrm{aw}, \mathrm{u}} d t .
$$

Aufgrund von $\boldsymbol{\xi}_{\text {aw }}(0)=\mathbf{0}$ folgt mit $V\left(\boldsymbol{\xi}_{\text {aw }}(0)\right)=0$ sofort

$$
\int_{0}^{T_{e}} \boldsymbol{y}_{\mathrm{aw}, \mathrm{u}}^{\top} \boldsymbol{y}_{\mathrm{aw}, \mathrm{u}} d t<\gamma^{2} \int_{0}^{T_{e}} \boldsymbol{y}_{\mathrm{r}}^{\top} \boldsymbol{y}_{\mathrm{r}} d t-V\left(\boldsymbol{\xi}_{\mathrm{aw}}\left(T_{e}\right)\right) .
$$

Für $T_{e} \rightarrow \infty$ ergibt sich gemäß Definition 4.2 der gesuchte Zusammenhang $\left\|\boldsymbol{y}_{\text {aw }, \mathrm{u}}\right\|_{L_{2}}<\gamma\left\|\boldsymbol{y}_{\mathrm{r}}\right\|_{L_{2}}$, da $V\left(\boldsymbol{\xi}_{\text {aw }}\left(T_{e}\right)\right) \geq 0$. Der Ansatz sichert demnach die gewünschten Eigenschaften des Systems.

Es bleibt noch zu zeigen, dass der Ansatz erfüllt ist, wenn die Matrixungleichung (5.26) gilt. Mit

$$
\dot{V}=\dot{\boldsymbol{\xi}}_{\mathrm{aw}}^{\top} \boldsymbol{R} \boldsymbol{\xi}_{\mathrm{aw}}+\boldsymbol{\xi}_{\mathrm{aw}}^{\top} \boldsymbol{R} \dot{\boldsymbol{\xi}}_{\mathrm{aw}}
$$

und der Systemdynamik (5.25) ergibt sich aus (C.18) sowie den Abkürzungen

$$
\begin{aligned}
\boldsymbol{A} & =\boldsymbol{A}_{\mathrm{s}}-\boldsymbol{B}_{\mathrm{s}, u} \underline{\boldsymbol{K}}, \\
\tilde{\boldsymbol{y}} & =\mathbf{d z}_{\overline{\mathbf{u}}}\left(\underline{\boldsymbol{K}} \boldsymbol{\xi}_{\mathrm{aw}}-\boldsymbol{y}_{\mathrm{r}}\right)
\end{aligned}
$$

die Ungleichung

$$
\boldsymbol{\xi}_{\mathrm{aw}}^{\top}\left(\boldsymbol{A}^{\top} \boldsymbol{R}+\boldsymbol{R} \boldsymbol{A}+\boldsymbol{C}_{\mathrm{s}}^{\top} \boldsymbol{C}_{\mathrm{s}}\right) \boldsymbol{\xi}_{\mathrm{aw}}+2 \tilde{\boldsymbol{y}}^{\top} \boldsymbol{B}_{\mathrm{s}, u}^{\top} \boldsymbol{R} \boldsymbol{\xi}_{\mathrm{aw}}-\gamma^{2} \boldsymbol{y}_{\mathrm{r}}^{\top} \boldsymbol{y}_{\mathrm{r}}<0 .
$$

Die Totzone (C.19) erfüllt die Sektorbedingung

$$
\tilde{\boldsymbol{y}}^{\top} 2 \hat{\boldsymbol{\Psi}}\left(\left(\underline{\boldsymbol{K}} \boldsymbol{\xi}_{\mathrm{aw}}-\boldsymbol{y}_{\mathrm{r}}\right)-\tilde{\boldsymbol{y}}\right) \geq 0
$$

aus Definition 5.1 für jede positiv definite Diagonalmatrix $\hat{\mathbf{\Psi}}$. Folglich gilt (C.20), wenn die restriktivere Ungleichung

$$
\begin{aligned}
\boldsymbol{\xi}_{\mathrm{aw}}^{\top}\left(\boldsymbol{A}^{\top} \boldsymbol{R}+\boldsymbol{R} \boldsymbol{A}+\boldsymbol{C}_{\mathrm{s}}^{\top} \boldsymbol{C}_{\mathrm{s}}\right) \boldsymbol{\xi}_{\mathrm{aw}}+2 \tilde{\boldsymbol{y}}^{\top} \boldsymbol{B}_{\mathrm{s}, u}^{\top} \boldsymbol{R}_{\mathrm{aw}} & \\
& -\gamma^{2} \boldsymbol{y}_{\mathrm{r}}^{\top} \boldsymbol{y}_{\mathrm{r}}+\tilde{\boldsymbol{y}}^{\top} 2 \hat{\boldsymbol{\Psi}}\left(\left(\underline{\boldsymbol{K}} \boldsymbol{\xi}_{\mathrm{aw}}-\boldsymbol{y}_{\mathrm{r}}\right)-\tilde{\boldsymbol{y}}\right)<0
\end{aligned}
$$


erfüllt ist. Letztere kann in der kompakteren Schreibweise

$$
\left[\begin{array}{lll}
\boldsymbol{\xi}_{\mathrm{aw}}^{\top} & \tilde{\boldsymbol{y}}^{\top} & \boldsymbol{y}_{\mathrm{r}}^{\top}
\end{array}\right]\left[\begin{array}{ccc}
\boldsymbol{A}^{\top} \boldsymbol{R}+\boldsymbol{R} \boldsymbol{A}+\boldsymbol{C}_{\mathrm{s}}^{\top} \boldsymbol{C}_{\mathrm{s}} & \star & \star \\
\boldsymbol{B}_{\mathrm{s}, u}^{\top} \boldsymbol{R}+\hat{\boldsymbol{\Psi}} \underline{\boldsymbol{K}} & -2 \hat{\boldsymbol{\Psi}} & \star \\
\mathbf{0} & \hat{\boldsymbol{\Psi}} & -\gamma^{2} \mathrm{II}
\end{array}\right]\left[\begin{array}{c}
\boldsymbol{\xi}_{\mathrm{aw}} \\
\tilde{\boldsymbol{y}} \\
\boldsymbol{y}_{\mathrm{r}}
\end{array}\right]<0
$$

dargestellt werden ${ }^{1}$. Offensichtlich ist diese Ungleichung immer erfüllt, wenn die Matrix negativ definit ist. Eine Umformung der Matrix mit Lemma B.1 (Schur-Komplement) liefert

$$
\left[\begin{array}{crcc}
\boldsymbol{A}^{\top} \boldsymbol{R}+\boldsymbol{R} \boldsymbol{A} & \star & \star & \star \\
\boldsymbol{B}_{\mathrm{s}, u}^{\top} \boldsymbol{R}+\hat{\boldsymbol{\Psi}} \underline{\boldsymbol{K}} & -2 \hat{\mathbf{\Psi}} & \star & \star \\
\mathbf{0} & \hat{\boldsymbol{\Psi}} & -\gamma^{2} \mathbb{I} & \star \\
\boldsymbol{C}_{\mathrm{s}} & \mathbf{0} & \mathbf{0} & -\mathbb{I}
\end{array}\right] \prec \mathbf{0}
$$

Um die Bedingung (5.26) aus dem Satz zu erhalten, sind einige weitere Schritte notwendig. Zuerst erfolgt eine Kongruenztransformation, d. h., die Ungleichung (C.21) wird von links und rechts mit der Matrix

$$
\gamma^{-\frac{1}{2}} \operatorname{diag}(\mathbb{I}, \mathbb{I}, \mathbb{I}, \gamma \mathbb{I})
$$

multipliziert. Das führt zu dem Zwischenergebnis

$$
\left[\begin{array}{cccc}
\boldsymbol{A}^{\top} \boldsymbol{R} \gamma^{-1}+\gamma^{-1} \boldsymbol{R} \boldsymbol{A} & \star & \star & \star \\
\boldsymbol{B}_{\mathrm{s}, u}^{\top} \boldsymbol{R} \gamma^{-1}+\gamma^{-1} \hat{\mathbf{\Psi}} \underline{\boldsymbol{K}} & -2 \hat{\mathbf{\Psi}} \gamma^{-1} & \star & \star \\
\mathbf{0} & \hat{\mathbf{\Psi}} \gamma^{-1} & -\gamma \mathbb{I} & \star \\
\boldsymbol{C}_{\mathrm{s}} & \mathbf{0} & \mathbf{0} & -\gamma \mathbb{I}
\end{array}\right] \prec \mathbf{0 .}
$$

Eine anschließende Substitution

$$
\boldsymbol{Q}^{-1}=\boldsymbol{R} \gamma^{-1}, \Psi^{-1}=\hat{\mathbf{\Psi}} \gamma^{-1}
$$

und eine erneute Kongruenztransformation mit

$$
\operatorname{diag}(\boldsymbol{Q}, \boldsymbol{\Psi}, \mathbb{I}, \mathbb{I})
$$

führt zu der Bedingung (5.26).

\footnotetext{
${ }^{1}$ Dabei bezeichnet $\star$ den Term, der benötigt wird, um eine symmetrische Matrix zu erhalten.
} 


\section{C.3.4 Beweis von Satz 5.3}

Beweis. Der Beweis erfolgt in zwei Schritten. Zuerst wird in Schritt 1 gezeigt, dass die Matrixungleichung (5.30) aus dem Satz äquivalent ist zu

$$
\begin{aligned}
& \frac{d}{d t} \boldsymbol{\xi}_{\mathrm{aw}}^{\top} \boldsymbol{R} \boldsymbol{\xi}_{\mathrm{aw}}-\gamma_{\mathcal{y}}^{2} \boldsymbol{y}_{\mathrm{r}}^{\top} \boldsymbol{y}_{\mathrm{r}}+\boldsymbol{y}_{\mathrm{aw}, \mathrm{u}}^{\top} \boldsymbol{y}_{\mathrm{aw}, \mathrm{u}}+ \\
& \tilde{\boldsymbol{y}}^{\top} 2 \hat{\boldsymbol{\Psi}}\left(\boldsymbol{\Phi}\left(\underline{\boldsymbol{K}} \boldsymbol{\xi}_{\mathrm{aw}}-\boldsymbol{y}_{\mathrm{r}}\right)-\tilde{\boldsymbol{y}}\right)<0
\end{aligned}
$$

für einen Skalar $\gamma_{\mathcal{y}}>0$, eine symmetrische, positiv definite Matrix $\boldsymbol{R}=\boldsymbol{Q}^{-1} \gamma_{\mathcal{Y}}$, eine positiv definite Diagonalmatrix $\hat{\boldsymbol{\Psi}}=\boldsymbol{\Psi}^{-1} \gamma_{\mathcal{\nu}}$ und alle $\boldsymbol{\xi}_{\text {aw }}, \boldsymbol{y}_{\mathrm{r}}, \boldsymbol{y}_{\mathrm{aw}, \mathrm{u}}$. Danach wird in Schritt 2 gezeigt, dass diese Ungleichung die im Satz angegebenen Systemeigenschaften garantiert, d. h. lokale asymptotische Stabilität der Ruhelage $\boldsymbol{\xi}_{\mathrm{aw}}^{(0)}=\mathbf{0}$ für $\boldsymbol{y}_{\mathrm{r}} \equiv \mathbf{0}$ und eine obere Schranke $\gamma_{\nu}$ für die $L_{2}$-Kleinsignalverstärkung.

Schritt 1. Analog zu dem Beweis von Satz 5.2 ergibt sich mit

$$
\frac{d}{d t} \boldsymbol{\xi}_{\mathrm{aw}}^{\top} \boldsymbol{R} \boldsymbol{\xi}_{\mathrm{aw}}=\dot{\boldsymbol{\xi}}_{\mathrm{aw}}^{\top} \boldsymbol{R} \boldsymbol{\xi}_{\mathrm{aw}}+\boldsymbol{\xi}_{\mathrm{aw}}^{\top} \boldsymbol{R} \dot{\boldsymbol{\xi}}_{\mathrm{aw}}
$$

und der Systemdynamik (5.29) sowie den Abkürzungen

$$
\begin{aligned}
\boldsymbol{A} & =\boldsymbol{A}_{\mathrm{s}}-\boldsymbol{B}_{\mathrm{s}, u} \underline{\boldsymbol{K}}, \\
\tilde{\boldsymbol{y}} & =\mathrm{dz}_{\overline{\mathbf{u}}}\left(\underline{\boldsymbol{K}} \boldsymbol{\xi}_{\mathrm{aw}}-\boldsymbol{y}_{\mathrm{r}}\right)
\end{aligned}
$$

aus (C.22) die Ungleichung ${ }^{2}$

$$
\left[\begin{array}{lll}
\boldsymbol{\xi}_{\mathrm{aw}}^{\top} & \tilde{\boldsymbol{y}}^{\top} & \boldsymbol{y}_{\mathrm{r}}^{\top}
\end{array}\right]\left[\begin{array}{ccc}
\boldsymbol{A}^{\top} \boldsymbol{R}+\boldsymbol{R} \boldsymbol{A}+\boldsymbol{C}_{\mathrm{s}}^{\top} \boldsymbol{C}_{\mathrm{s}} & \star & \star \\
\boldsymbol{B}_{\mathrm{s}, u}^{\top} \boldsymbol{R}+\hat{\boldsymbol{\Psi}} \boldsymbol{\Phi} \underline{\boldsymbol{K}} & -2 \hat{\boldsymbol{\Psi}} & \star \\
\mathbf{0} & \boldsymbol{\Phi} \hat{\boldsymbol{\Psi}} & -\gamma^{2} \mathbb{I}
\end{array}\right]\left[\begin{array}{c}
\boldsymbol{\xi}_{\mathrm{aw}} \\
\tilde{\boldsymbol{y}} \\
\boldsymbol{y}_{\mathrm{r}}
\end{array}\right]<0 .
$$

Offensichtlich ist diese Ungleichung für alle $\boldsymbol{\xi}_{\text {aw }}, \boldsymbol{y}_{\mathrm{r}}$ und $\boldsymbol{y}_{\mathrm{aw}, \mathrm{u}}$ erfüllt, wenn die Matrix negativ definit ist. Eine Umformung der Matrix mit Lemma B.1 (Schur-Komplement) liefert

$$
\left[\begin{array}{cccc}
\boldsymbol{A}^{\top} \boldsymbol{R}+\boldsymbol{R} \boldsymbol{A} & \star & \star & \star \\
\boldsymbol{B}_{\mathrm{s}, u}^{\top} \boldsymbol{R}+\hat{\boldsymbol{\Psi}} \boldsymbol{\Phi} \underline{\boldsymbol{K}} & -2 \hat{\mathbf{\Psi}} & \star & \star \\
\mathbf{0} & \mathbf{\Phi} \hat{\mathbf{\Psi}} & -\gamma^{2} \mathbb{I} & \star \\
\boldsymbol{C}_{\mathrm{s}} & \mathbf{0} & \mathbf{0} & -\mathbb{I}
\end{array}\right] \prec \mathbf{0} .
$$

${ }^{2}$ Dabei bezeichnet $\star$ den Term, der benötigt wird, um eine symmetrische Matrix zu erhalten. 
Die Matrixungleichung (5.30) aus dem Satz ergibt sich nach drei weiteren Umformungen. Zuerst erfolgt eine Kongruenztransformation, d. h., die Ungleichung (C.23) wird von links und rechts mit der regulären Matrix

$$
\gamma_{\mathcal{Y}}^{-\frac{1}{2}} \operatorname{diag}\left(\mathbb{I}, \mathbb{I}, \mathbb{I}, \gamma_{\mathcal{Y}} \mathbb{I}\right)
$$

multipliziert. Die Substitution $\boldsymbol{Q}^{-1}=\boldsymbol{R} \gamma_{\mathcal{y}}^{-1}, \boldsymbol{\Psi}^{-1}=\hat{\mathbf{\Psi}} \gamma_{\mathcal{y}}^{-1}$ und eine erneute Kongruenztransformation mit der Diagonalmatrix $\operatorname{diag}(\boldsymbol{Q}, \boldsymbol{\Psi}, \mathbb{I}, \mathbb{I})$ führt zu (5.30).

Schritt 2. Betrachtet wird die positiv definite Funktion

$$
V\left(\boldsymbol{\xi}_{\text {aw }}\right)=\boldsymbol{\xi}_{\text {aw }}^{\top} \boldsymbol{R} \boldsymbol{\xi}_{\text {aw }}, \quad \boldsymbol{R} \succ \mathbf{0}
$$

und die folgenden zwei Fälle.

Fall 1. Sei $\boldsymbol{y}_{\mathrm{r}} \equiv \mathbf{0}$ und $\boldsymbol{\xi}_{\text {aw }} \in \mathcal{E}(\boldsymbol{R}, \mathrm{c})$, wobei

$$
\mathcal{E}(\boldsymbol{R}, \mathrm{c})=\left\{\boldsymbol{\xi}_{\mathrm{aw}} \in \mathbb{R}^{n_{\mathrm{s}}} \mid \boldsymbol{\xi}_{\mathrm{aw}}^{\mathrm{T}} \boldsymbol{R} \boldsymbol{\xi}_{\mathrm{aw}} \leq \mathrm{c}\right\},
$$

mit einem $c>0$. Des Weiteren sei c ausreichend klein, so dass der Eingang $\tilde{\boldsymbol{u}}=\underline{\boldsymbol{K}} \boldsymbol{\xi}_{\text {aw }}$ der multivariablen Totzone

$$
\tilde{\boldsymbol{y}}=\mathbf{d} \mathbf{z}_{\overline{\mathbf{u}}}(\tilde{\boldsymbol{u}})
$$

zur Signalklasse $\mathcal{W}(\boldsymbol{\Phi})$ gehört. Folglich gilt die lokale Sektorbedingung

$$
\tilde{\boldsymbol{y}}^{\top} 2 \hat{\boldsymbol{\Psi}}(\boldsymbol{\Phi} \tilde{\boldsymbol{u}}-\tilde{\boldsymbol{y}}) \geq 0
$$

aus Lemma 5.4 für die Totzone. Ungleichung (C.22) impliziert dann

$$
\dot{V}<0 \quad \forall \boldsymbol{\xi}_{\text {aw }} \in \mathcal{E}(\boldsymbol{R}, \mathrm{c}) .
$$

Somit ist (C.24) eine Ljapunov-Funktion für das System (5.29). Asymptotische Stabilität der Ruhelage ist sichergestellt und $\mathcal{E}(\boldsymbol{R}, \mathrm{c})$ ist ein Einzugsgebiet der Ruhelage, da es durch die Höhenlinie c der Ljapunov-Funktion begrenzt wird.

Fall 2. Sei $\boldsymbol{\xi}_{\mathrm{aw}}^{(0)}=\mathbf{0}$ und $\boldsymbol{y}_{\mathrm{r}} \in \mathcal{Y}(\boldsymbol{\Phi})$. Dann gilt ebenfalls die lokale Sektorbedingung für die Totzone und (C.22) impliziert

$$
\dot{V}<\gamma_{\mathcal{y}}^{2} \boldsymbol{y}_{\mathrm{r}}^{\top} \boldsymbol{y}_{\mathrm{r}}-\boldsymbol{y}_{\mathrm{aw}, \mathrm{u}}^{\top} \boldsymbol{y}_{\mathrm{aw}, \mathrm{u}} .
$$


Die Integration der Ungleichung nach der Zeit im Intervall $\left[0, T_{e}\right]$ führt zu

$$
V\left(T_{e}\right)-V(0)<\gamma_{\mathcal{Y}}^{2} \int_{0}^{T_{e}} \boldsymbol{y}_{\mathrm{r}}^{\top} \boldsymbol{y}_{\mathrm{r}} d t-\int_{0}^{T_{e}} \boldsymbol{y}_{\mathrm{aw}, \mathrm{u}}^{\top} \boldsymbol{y}_{\mathrm{aw}, \mathrm{u}} d t
$$

Aufgrund von $\boldsymbol{\xi}_{\text {aw }}(0)=\mathbf{0}$ folgt mit $V\left(\boldsymbol{\xi}_{\text {aw }}(0)\right)=0$ sofort

$$
\int_{0}^{T_{e}} \boldsymbol{y}_{\mathrm{aw}, \mathrm{u}}^{\top} \boldsymbol{y}_{\mathrm{aw}, \mathrm{u}} d t<\gamma_{\mathcal{y}}^{2} \int_{0}^{T_{e}} \boldsymbol{y}_{\mathrm{r}}^{\top} \boldsymbol{y}_{\mathrm{r}} d t-V\left(\boldsymbol{\xi}_{\mathrm{aw}}\left(T_{e}\right)\right) .
$$

Für $T_{e} \rightarrow \infty$ ergibt sich gemäß Definition 4.2 der gesuchte Zusammenhang $\left\|\boldsymbol{y}_{\text {aw }, \mathrm{u}}\right\|_{L_{2}}<\gamma_{\mathcal{y}}\left\|\boldsymbol{y}_{\mathrm{r}}\right\|_{L_{2}}$, da $V\left(\boldsymbol{\xi}_{\text {aw }}\left(T_{e}\right)\right) \geq 0$. Damit ist gezeigt, dass $\gamma_{\mathcal{y}}$ eine obere Schranke für die $L_{2}$-Kleinsignalverstärkung des Systems ist.

\section{C.3.5 Beweis von Satz 6.1}

Beweis. Der Satz folgt aus dem globalen Existenz- und Eindeutigkeitssatz für Differentialgleichungen mit beschränkter rechter Seite [10, Satz 2.5.6, Seite 87]. Dieser lautet wie folgt.

Satz C.1. Hat die stetige und bezüglich $\boldsymbol{x}$ lokal lipschitzstetige rechte Seite $\boldsymbol{f}: \mathbb{D} \mapsto \mathbb{R}^{n}$ einer Differentialgleichung $\dot{\boldsymbol{x}}=\boldsymbol{f}(t, \boldsymbol{x})$ einen Definitionsbereich der Form

$$
\mathbb{D}:=(a, b) \times \mathbb{R}^{n} \quad \text { mit }-\infty \leq a<b \leq \infty,
$$

und ist $\boldsymbol{f}(t, \boldsymbol{x})$ linear beschränkt, d.h., es gilt

$$
\|\boldsymbol{f}(t, \boldsymbol{x})\| \leq f_{1}(t)\|\boldsymbol{x}\|+f_{2}(t)
$$

für alle $t \in(a, b)$ und $\boldsymbol{x} \in \mathbb{R}^{n}$ mit stetigen Funktionen $f_{1}, f_{2}:(a, b) \mapsto$ $\mathbb{R}_{\geq 0}$, so existiert die maximale Lösung jedes der Anfangswertprobleme

$$
\dot{\boldsymbol{x}}=\boldsymbol{f}(t, \boldsymbol{x}), \quad \boldsymbol{x}\left(t_{0}\right)=\boldsymbol{x}^{(0)},\left(t_{0}, \boldsymbol{x}^{(0)}\right) \in(a, b) \times \mathbb{R}^{n}
$$

auf dem ganzen Intervall $(a, b)$. 
Die hier betrachtete rechte Seite der Differentialgleichung (6.3) ist für alle $t$ und alle $\boldsymbol{x}_{\text {awrk }}$ definiert, d.h., der Definitionsbereich lautet

$$
\mathbb{D}:=(-\infty, \infty) \times \mathbb{R}^{2 n_{\mathrm{s}}+n_{\mathrm{r}}} .
$$

Des Weiteren ist die rechte Seite lokal lipschitzstetig bezüglich $\boldsymbol{x}_{\text {awrk }}$, da $\boldsymbol{k}_{\text {aw }}\left(\boldsymbol{x}_{\text {aw }}\right)$ nach Annahme 6.1 lokal lipschitzstetig ist. Das folgt aus der Tatsache, dass Summen, Produkte und Verkettungen lokal lipschitzstetiger Funktionen wieder lokal lipschitzstetig sind (vgl. Abschnitt A.2).

Abschließend ist noch zu zeigen, dass die rechte Seite linear beschränkt ist. Es gilt mit den nach Annahme 2.1 konstanten Sollwerten $\boldsymbol{w}=\boldsymbol{w}_{0}$ und Störgrößen $\boldsymbol{z}=\boldsymbol{z}_{0}$ die Abschätzung

$$
\begin{aligned}
& \left\|\tilde{\boldsymbol{A}} \boldsymbol{x}_{\mathrm{awrk}}+\tilde{\boldsymbol{B}}_{\mathrm{u}} \operatorname{sat}_{\overline{\mathbf{u}}}\left(\boldsymbol{u}_{\mathrm{awrk}}\right)+\tilde{\boldsymbol{B}}_{\mathrm{w}} \boldsymbol{z}_{0}+\tilde{\boldsymbol{B}}_{\mathrm{z}} \boldsymbol{w}_{0}\right\|_{2} \\
& \leq\left\|\tilde{\boldsymbol{A}} \boldsymbol{x}_{\mathrm{awrk}}\right\|_{2}+\left\|\tilde{\boldsymbol{B}}_{\mathrm{u}} \mathbf{s a t}_{\overline{\mathbf{u}}}\left(\boldsymbol{u}_{\mathrm{awrk}}\right)\right\|_{2}+\left\|\tilde{\boldsymbol{B}}_{\mathrm{w}} \boldsymbol{z}_{0}\right\|_{2}+\left\|\tilde{\boldsymbol{B}}_{\mathrm{z}} \boldsymbol{w}_{0}\right\|_{2} \\
& \leq\|\tilde{\boldsymbol{A}}\|_{2}\left\|\boldsymbol{x}_{\mathrm{awrk}}\right\|_{2}+\left\|\tilde{\boldsymbol{B}}_{\mathrm{u}}\right\|_{2}\left\|\mathbf{s a t}_{\overline{\mathbf{u}}}\left(\boldsymbol{u}_{\mathrm{awrk}}\right)\right\|_{2}+\left\|\tilde{\boldsymbol{B}}_{\mathrm{w}}\right\|_{2}\left\|\boldsymbol{z}_{0}\right\|_{2}+\left\|\tilde{\boldsymbol{B}}_{\mathrm{z}}\right\|_{2}\left\|\boldsymbol{w}_{0}\right\|_{2} \\
& \leq\|\tilde{\boldsymbol{A}}\|_{2}\left\|\boldsymbol{x}_{\mathrm{awrk}}\right\|_{2}+\underbrace{\left\|\tilde{\boldsymbol{B}}_{\mathrm{u}}\right\|_{2}\|\overline{\mathbf{u}}\|_{2}+\left\|\tilde{\boldsymbol{B}}_{\mathrm{w}}\right\|_{2}\left\|\boldsymbol{z}_{0}\right\|_{2}+\left\|\tilde{\boldsymbol{B}}_{\mathrm{z}}\right\|_{2}\left\|\boldsymbol{w}_{0}\right\|_{2}}_{\mathrm{c} \geq 0} \\
& =\|\tilde{\boldsymbol{A}}\|_{2}\left\|\boldsymbol{x}_{\mathrm{awrk}}\right\|_{2}+\mathrm{c} .
\end{aligned}
$$

Das entspricht (C.25) mit den Funktionen $f_{1}=\|\tilde{\boldsymbol{A}}\|_{2}$ und $f_{2}=$ c.

\section{C.3.6 Beweis von Lemma 6.1}

Beweis. Die Dynamik des linearen unbeschränkten Regelkreises $\mathbf{L}$ wird durch (6.4) bestimmt. Die Lösung dieses Differentialgleichungssystems ist zum Beispiel in [132] angegeben. Für $\boldsymbol{w}=\mathbf{0}$ und $\boldsymbol{z}=\mathbf{0}$ ergibt sich

$$
\boldsymbol{\xi}_{\mathrm{L}}(t)=\mathrm{e}^{\boldsymbol{A}_{\mathrm{ub}} t} \boldsymbol{\xi}_{\mathrm{L}}(0)
$$

mit $\boldsymbol{A}_{\text {ub }}$ gemäß (2.4). Aufgrund von Annahme 2.2 besitzt $\boldsymbol{A}_{\text {ub }}$ ausschließlich Eigenwerte mit negativem Realteil. Eine Abschätzung mit der euklidischen Norm liefert

$$
\left\|\boldsymbol{\xi}_{\mathrm{L}}(t)\right\|_{2}=\left\|\mathrm{e}^{\boldsymbol{A}_{\mathrm{ub}} t} \boldsymbol{\xi}_{\mathrm{L}}(0)\right\|_{2} \leq\left\|\mathrm{e}^{\boldsymbol{A}_{\mathrm{ub}} t}\right\|_{2}\left\|\boldsymbol{\xi}_{\mathrm{L}}(0)\right\|_{2},
$$

wobei $\left\|\mathrm{e}^{\boldsymbol{A}_{\mathrm{ub}} t}\right\|_{2}$ die Spektralnorm der Transitionsmatrix ist. Aus dem Ergebnis [16, Fakt 11.18.8] folgt die Existenz von positiven Konstanten $C_{L}$ und $\lambda$, so dass die Abschätzung

$$
\left\|\mathrm{e}^{\boldsymbol{A}_{\mathrm{ub}} t}\right\|_{2} \leq \mathrm{c}_{\mathrm{L}} \mathrm{e}^{-\lambda t}
$$


gilt. Einsetzen in (C.26) liefert

$$
\left\|\boldsymbol{\xi}_{\mathrm{L}}(t)\right\|_{2} \leq\left\|\boldsymbol{\xi}_{\mathrm{L}}(0)\right\|_{2} \mathrm{c}_{\mathrm{L}} \mathrm{e}^{-\lambda t}
$$

und komplettiert den Beweis von Aussage (I).

Für die euklidische Norm des Vektors $\boldsymbol{y}_{\mathrm{r}}$ gilt

$$
\left\|\boldsymbol{y}_{\mathrm{r}}(t)\right\|_{2}=\left\|\left[\begin{array}{ll}
\boldsymbol{D}_{\mathrm{r}, u} \boldsymbol{C}_{\mathrm{s}} & \boldsymbol{C}_{\mathrm{r}}
\end{array}\right]\left[\begin{array}{l}
\boldsymbol{\xi}_{\mathrm{s}}(t) \\
\boldsymbol{\xi}_{\mathrm{r}}(t)
\end{array}\right]\right\|_{2}=\left\|\boldsymbol{M} \boldsymbol{\xi}_{\mathrm{L}}(t)\right\|_{2} \leq\|\boldsymbol{M}\|_{2}\left\|\boldsymbol{\xi}_{\mathrm{L}}(t)\right\|_{2} .
$$

Ausnutzen der Abschätzung (C.27) liefert

$$
\left\|\boldsymbol{y}_{\mathrm{r}}(t)\right\|_{2} \leq\left\|\boldsymbol{\xi}_{\mathrm{L}}(0)\right\|_{2}\|\boldsymbol{M}\|_{2} \mathrm{c}_{\mathrm{L}} \mathrm{e}^{-\lambda t}
$$

und somit den Beweis für Aussage (II) mit $c_{r}=\|M\|_{2} c_{L}$.

\section{C.3.7 Beweis von Satz 6.2}

Beweis. Nach Satz 6.1 besitzt das Anfangswertproblem, das durch die Differentialgleichung des Anti-Windup-Regelkreises (6.3) und einen Anfangswert $\boldsymbol{x}_{\text {awrk }}(0)$ gegeben ist, für alle $t \geq 0$ eine eindeutige Lösung. Das gilt auch für den in Mismatch-Koordinaten transformierten Regelkreis und folglich auch für das Anfangswertproblem

$$
\begin{aligned}
\dot{\boldsymbol{\xi}}_{\mathrm{aw}} & =\boldsymbol{A}_{\mathrm{s}} \boldsymbol{\xi}_{\mathrm{aw}}+\boldsymbol{B}_{\mathrm{s}, u} \mathbf{s a t}_{\alpha \overline{\mathbf{u}}}\left(-\boldsymbol{k}\left(\boldsymbol{\xi}_{\mathrm{aw}}\right)\right)+\boldsymbol{B}_{\mathrm{s}, u} \boldsymbol{\zeta}(t), \\
\boldsymbol{\xi}_{\mathrm{aw}}(0) & =\boldsymbol{\xi}_{\mathrm{aw}}^{(0)} .
\end{aligned}
$$

Die Trajektorie $\boldsymbol{\xi}_{\text {aw }}(t)$ kann deshalb nicht in endlicher Zeit gegen einen unendlichen Wert laufen. Eine endliche Entweichzeit ist ausgeschlossen $[10,200]$. Folglich existiert ein $\delta>0$, das eine obere Schranke für die Norm des Zustandsvektors zum Zeitpunkt $t=t^{*}<\infty$ darstellt, d.h., es gilt die Abschätzung

$$
\left\|\boldsymbol{\xi}_{\text {aw }}\left(t^{*}\right)\right\|<\delta<\infty .
$$

Da zum Zeitpunkt $t=t^{*}$ die Störung $\boldsymbol{\zeta}(t)$ verschwindet, reduziert sich die Systemdynamik auf das autonome System

$$
\dot{\boldsymbol{\xi}}_{\text {aw }}=\boldsymbol{A}_{\mathrm{s}} \boldsymbol{\xi}_{\text {aw }}+\boldsymbol{B}_{\mathrm{s}, u} \mathbf{s a t}_{\alpha \overline{\mathbf{u}}}\left(-\boldsymbol{k}\left(\boldsymbol{\xi}_{\mathrm{aw}}\right)\right)
$$

mit dem Anfangszustand $\boldsymbol{\xi}_{\text {aw }}\left(t^{*}\right)$. Dieser liegt in einer Umgebung der Ruhelage $\boldsymbol{\xi}_{\mathrm{aw}}^{(\mathrm{R})}=\mathbf{0}$, die durch

$$
\mathcal{G}_{\delta}=\left\{\boldsymbol{\xi}_{\text {aw }} \in \mathbb{R}^{n} \mid\left\|\boldsymbol{\xi}_{\text {aw }}\right\|<\delta\right\}
$$


beschrieben werden kann. Da die Ruhelage nach Voraussetzung global asymptotisch stabil ist, existiert nach Definition 2.2 ein $\varepsilon>0$, so dass

$$
\left\|\boldsymbol{\xi}_{\text {aw }}\left(t^{*}\right)\right\|<\delta \Rightarrow\left\|\boldsymbol{\xi}_{\text {aw }}(t)\right\|<\varepsilon \forall t \geq t^{*} .
$$

Daraus folgt $\left\|\boldsymbol{\xi}_{\text {aw }}(t)\right\|<\varepsilon=\mathrm{c}$ für alle $t \geq 0$. Die Konvergenz der Trajektorie $\boldsymbol{\xi}_{\text {aw }}(t)$ zum Ursprung folgt aus der globalen Attraktivität einer global asymptotisch stabilen Ruhelage.

\section{C.3.8 Beweis von Satz 6.3}

Beweis. Aufgrund der einleitenden Überlegungen des Abschnitts 6.3 lässt sich der Beweis in Mismatch-Koordinaten führen. Somit ist zu zeigen, dass für ein $\tilde{c}>0$ und die Trajektorien des Anti-Windup-Regelkreises gilt

$$
\left\|\boldsymbol{\xi}_{\mathrm{ms}}(t)\right\|<\tilde{\mathrm{c}} \forall t \geq 0, \quad \lim _{t \rightarrow \infty} \boldsymbol{\xi}_{\mathrm{ms}}(t)=0
$$

mit dem Zustandsvektor $\boldsymbol{\xi}_{\mathrm{ms}}^{\top}=\left[\begin{array}{lll}\boldsymbol{\xi}_{\mathrm{s}}^{\top} & \boldsymbol{\xi}_{\mathrm{r}}^{\top} & \boldsymbol{\xi}_{\mathrm{aw}}^{\top}\end{array}\right]$.

Zunächst wird das stabile lineare System $\mathbf{L}$ mit der Dynamik (6.4) betrachtet. Offensichtlich sind die Trajektorien $\boldsymbol{\xi}_{\mathrm{s}}(t)$ und $\boldsymbol{\xi}_{\mathrm{r}}(t)$ begrenzt und konvergieren nach Lemma 6.1 für beliebige Anfangswerte gegen null, da der Ursprung eine global exponentiell stabile Ruhelage des Systems ist.

Die Norm der Trajektorien $\boldsymbol{\xi}_{\text {aw }}(t)$ des Teilsystems ES ist nach Satz 6.2 begrenzt, wenn die in Satz 6.3 angegebenen Bedingungen erfüllt sind. Die Konvergenz von $\boldsymbol{\xi}_{\text {aw }}(t)$ gegen die Ruhelage im Ursprung ist ebenfalls durch die Bedingungen und Satz 6.2 sichergestellt.

\section{C.3.9 Beweis von Satz 6.5}

Beweis. Der Beweis erfolgt analog zu dem Beweis von Satz 6.3. Aufgrund der Überlegungen zu Beginn des Abschnitts 6.3 lässt sich der Beweis in Mismatch-Koordinaten führen. Somit ist zu zeigen, dass für ein $\tilde{c}>0$ gilt

$$
\left\|\boldsymbol{\xi}_{\mathrm{ms}}(t)\right\|<\tilde{\mathrm{c}} \forall t \geq 0, \quad \lim _{t \rightarrow \infty} \boldsymbol{\xi}_{\mathrm{ms}}(t)=0
$$

mit dem Zustandsvektor $\boldsymbol{\xi}_{\mathrm{ms}}^{\top}=\left[\begin{array}{lll}\boldsymbol{\xi}_{\mathrm{s}}^{\top} & \boldsymbol{\xi}_{\mathrm{r}}^{\top} & \boldsymbol{\xi}_{\mathrm{aw}}^{\top}\end{array}\right]$.

Zunächst wird das stabile lineare System $\mathbf{L}$ mit der Dynamik (6.4) betrachtet. Offensichtlich sind die Trajektorien $\boldsymbol{\xi}_{\mathrm{s}}(t)$ und $\boldsymbol{\xi}_{\mathrm{r}}(t)$ begrenzt und konvergieren nach Lemma 6.1 für beliebige Anfangswerte gegen null, da der Ursprung eine global exponentiell stabile Ruhelage des Systems ist. 
Die Norm der Trajektorien $\boldsymbol{\xi}_{\text {aw }}(t)$ des Teilsystems ES ist nach Satz 6.4 begrenzt, wenn die in Satz 6.5 angegebenen Bedingungen erfüllt sind. Die Konvergenz von $\boldsymbol{\xi}_{\text {aw }}(t)$ gegen die Ruhelage im Ursprung ist ebenfalls durch die Bedingungen und Satz 6.4 sichergestellt.

\section{C.3.10 Beweis von Lemma 6.2}

Beweis. Die Lösung des Anfangswertproblems (6.17) ist zum Beispiel in [133, Abschnitt 2.4.1] angegeben. Es gilt

$$
\boldsymbol{\xi}_{\mathrm{L}}(t)=\mathrm{e}^{\boldsymbol{A}_{\mathrm{ub}} t} \boldsymbol{\xi}_{\mathrm{L}}^{(0)}+\int_{0}^{t} \mathrm{e}^{\boldsymbol{A}_{\mathrm{ub}}(t-\tau)} \boldsymbol{B}_{\mathrm{ub}} \boldsymbol{r}_{0} d \tau .
$$

Für den Ausgang $\boldsymbol{y}_{\mathrm{r}}$ folgt

$$
\begin{aligned}
\boldsymbol{y}_{\mathrm{r}}(t) & =\boldsymbol{C}_{\mathrm{ub}} \boldsymbol{\xi}_{\mathrm{L}}(t)+\boldsymbol{D}_{\mathrm{ub}} \boldsymbol{r}_{0} \\
& =\boldsymbol{C}_{\mathrm{ub}} \mathrm{e}^{\boldsymbol{A}_{\mathrm{ub} t}} \boldsymbol{\xi}_{\mathrm{L}}^{(0)}+\boldsymbol{C}_{\mathrm{ub}}\left(\int_{0}^{t} \mathrm{e}^{\boldsymbol{A}_{\mathrm{ub}}(t-\tau)} d \tau\right) \boldsymbol{B}_{\mathrm{ub}} \boldsymbol{r}_{0}+\boldsymbol{D}_{\mathrm{ub}} \boldsymbol{r}_{0} .
\end{aligned}
$$

Da $\operatorname{det}\left(\boldsymbol{A}_{\mathrm{ub}}\right) \neq 0$ ist, lässt sich das Integral geschlossen lösen, wie zum Beispiel in [132, Abschnitt 5.5.1] erläutert wird. Das führt zu

$$
\begin{aligned}
\boldsymbol{y}_{\mathrm{r}}(t) & =\boldsymbol{C}_{\mathrm{ub}} \mathrm{e}^{\boldsymbol{A}_{\mathrm{ub} t}} \boldsymbol{\xi}_{\mathrm{L}}^{(0)}+\boldsymbol{C}_{\mathrm{ub}}\left(\boldsymbol{A}_{\mathrm{ub}}^{-1} \mathrm{e}^{\boldsymbol{A}_{\mathrm{ub}} t}-\boldsymbol{A}_{\mathrm{ub}}^{-1}\right) \boldsymbol{B}_{\mathrm{ub}} \boldsymbol{r}_{0}+\boldsymbol{D}_{\mathrm{ub}} \boldsymbol{r}_{0} \\
& =\boldsymbol{C}_{\mathrm{ub}} \mathrm{e}^{\boldsymbol{A}_{\mathrm{ub}} t} \boldsymbol{\xi}_{\mathrm{L}}^{(0)}+\boldsymbol{C}_{\mathrm{ub}} \boldsymbol{A}_{\mathrm{ub}}^{-1} \mathrm{e}^{\boldsymbol{A}_{\mathrm{ub}} t} \boldsymbol{B}_{\mathrm{ub}} \boldsymbol{r}_{0}+\left(\boldsymbol{D}_{\mathrm{ub}}-\boldsymbol{C}_{\mathrm{ub}} \boldsymbol{A}_{\mathrm{ub}}^{-1} \boldsymbol{B}_{\mathrm{ub}}\right) \boldsymbol{r}_{0} \\
& =\boldsymbol{C}_{\mathrm{ub}} \mathrm{e}^{\boldsymbol{A}_{\mathrm{ub} t}} \boldsymbol{\xi}_{\mathrm{L}}^{(0)}+\boldsymbol{C}_{\mathrm{ub}} \boldsymbol{A}_{\mathrm{ub}}^{-1} \mathrm{e}^{\boldsymbol{A}_{\mathrm{ub}} t} \boldsymbol{B}_{\mathrm{ub}} \boldsymbol{r}_{0}+\boldsymbol{y}_{\mathrm{r}}^{(\mathrm{R})} .
\end{aligned}
$$

Umstellen und Berechnen der euklidischen Norm ergibt

$$
\left\|\boldsymbol{y}_{\mathrm{r}}(t)-\boldsymbol{y}_{\mathrm{r}}^{(\mathrm{R})}\right\|_{2}=\left\|\boldsymbol{C}_{\mathrm{ub}} \mathrm{e}^{\boldsymbol{A}_{\mathrm{ub}} t} \boldsymbol{\xi}_{\mathrm{L}}^{(0)}+\boldsymbol{C}_{\mathrm{ub}} \boldsymbol{A}_{\mathrm{ub}}^{-1} \mathrm{e}^{\boldsymbol{A}_{\mathrm{ub}} t} \boldsymbol{B}_{\mathrm{ub}} \boldsymbol{r}_{0}\right\|_{2} .
$$

Dabei lässt sich die rechte Seite wie folgt abschätzen

$$
\begin{aligned}
& \left\|\boldsymbol{C}_{\mathrm{ub}} \mathrm{e}^{\boldsymbol{A}_{\mathrm{ub}} t} \boldsymbol{\xi}_{\mathrm{L}}^{(0)}\right\|_{2}+\left\|\boldsymbol{C}_{\mathrm{ub}} \boldsymbol{A}_{\mathrm{ub}}^{-1} \mathrm{e}^{\boldsymbol{A}_{\mathrm{ub}} t} \boldsymbol{B}_{\mathrm{ub}} \boldsymbol{r}_{0}\right\|_{2} \\
& \leq\left\|\boldsymbol{C}_{\mathrm{ub}}\right\|_{2}\left\|\mathrm{e}^{\boldsymbol{A}_{\mathrm{ub}} t}\right\|_{2}\left\|\boldsymbol{\xi}_{\mathrm{L}}^{(0)}\right\|_{2}+\left\|\boldsymbol{C}_{\mathrm{ub}} \boldsymbol{A}_{\mathrm{ub}}^{-1}\right\|_{2}\left\|\mathrm{e}^{\boldsymbol{A}_{\mathrm{ub}} t}\right\|_{2}\left\|\boldsymbol{B}_{\mathrm{ub}} \boldsymbol{r}_{0}\right\|_{2} .
\end{aligned}
$$

Ausklammern der Matrixexponentialfunktion liefert

$$
\left\|\boldsymbol{y}_{\mathrm{r}}(t)-\boldsymbol{y}_{\mathrm{r}}^{(\mathrm{R})}\right\|_{2} \leq\left(\left\|\boldsymbol{C}_{\mathrm{ub}}\right\|_{2}\left\|\boldsymbol{\xi}_{\mathrm{L}}^{(0)}\right\|_{2}+\left\|\boldsymbol{C}_{\mathrm{ub}} \boldsymbol{A}_{\mathrm{ub}}^{-1}\right\|_{2}\left\|\boldsymbol{B}_{\mathrm{ub}} \boldsymbol{r}_{0}\right\|_{2}\right)\left\|\mathrm{e}^{\boldsymbol{A}_{\mathrm{ub}} t}\right\|_{2} .
$$


Mit [16, Fakt 11.18.8] lässt sich die Norm der Matrixexponentialfunktion abschätzen und es folgt die Existenz von $c_{1}>0, \lambda>0$, so dass gilt

$$
\begin{aligned}
\left\|\boldsymbol{y}_{\mathrm{r}}(t)-\boldsymbol{y}_{\mathrm{r}}^{(\mathrm{R})}\right\|_{2} & \leq\left(\left\|\boldsymbol{C}_{\mathrm{ub}}\right\|_{2}\left\|\boldsymbol{\xi}_{\mathrm{L}}^{(0)}\right\|_{2}+\left\|\boldsymbol{C}_{\mathrm{ub}} \boldsymbol{A}_{\mathrm{ub}}^{-1}\right\|_{2}\left\|\boldsymbol{B}_{\mathrm{ub}} \boldsymbol{r}_{0}\right\|_{2}\right) \mathrm{c}_{1} \mathrm{e}^{-\lambda t} \\
& \leq \mathrm{ce}^{-\lambda t}
\end{aligned}
$$

Damit ist das Lemma bewiesen.

\section{C.3.11 Beweis von Lemma 7.3}

Beweis. Der Beweis erfolgt analog zu dem Beweis von Satz 5.3 in zwei Schritten. Zuerst wird in Schritt 1 gezeigt, dass die Matrixungleichung (7.10) des Lemmas äquivalent ist zu der Bedingung

$$
\frac{d}{d t} \boldsymbol{\xi}_{\mathrm{aw}}^{\top} \boldsymbol{R} \boldsymbol{\xi}_{\mathrm{aw}}-\gamma_{\mathcal{y}}^{2} \boldsymbol{\zeta}_{\mathrm{r}}^{\top} \boldsymbol{\zeta}_{\mathrm{r}}+\boldsymbol{y}_{\mathrm{aw}, \mathrm{u}}^{\top} \boldsymbol{y}_{\mathrm{aw}, \mathrm{u}}+\tilde{\boldsymbol{y}}^{\top} 2 \hat{\boldsymbol{\Psi}}\left(\boldsymbol{\Phi} \underline{\boldsymbol{K}} \boldsymbol{\xi}_{\mathrm{aw}}-\tilde{\boldsymbol{y}}\right)<0
$$

für alle $\boldsymbol{\xi}_{\mathrm{aw}}, \boldsymbol{\zeta}_{\mathrm{r}}, \boldsymbol{y}_{\mathrm{aw}, \mathrm{u}}$. Dabei ist $\gamma_{\mathcal{y}}$ ein positiver Skalar, $\boldsymbol{R}=\boldsymbol{Q}^{-1} \gamma_{\mathcal{y}}$ eine symmetrische, positiv definite Matrix und $\hat{\boldsymbol{\Psi}}=\boldsymbol{\Psi}^{-1} \gamma_{\mathcal{\nu}}$ eine positiv definite Diagonalmatrix. Danach wird in Schritt 2 gezeigt, dass diese Ungleichung die im Lemma angegebenen Eigenschaften des Ersatzsystems (7.7) garantiert, d. h. lokale asymptotische Stabilität der Ruhelage $\boldsymbol{\xi}_{\mathrm{aw}}^{(0)}=\mathbf{0}$ für $\boldsymbol{y}_{\mathrm{r}} \equiv \mathbf{0}$ und eine obere Schranke $\gamma_{\mathcal{y}}$ für die $L_{2}$-Kleinsignalverstärkung.

Schritt 1. Analog zu dem Beweis von Satz 5.2 ergibt sich mit

$$
\frac{d}{d t} \boldsymbol{\xi}_{\mathrm{aw}}^{\top} \boldsymbol{R} \boldsymbol{\xi}_{\mathrm{aw}}=\dot{\boldsymbol{\xi}}_{\mathrm{aw}}^{\top} \boldsymbol{R} \boldsymbol{\xi}_{\mathrm{aw}}+\boldsymbol{\xi}_{\mathrm{aw}}^{\top} \boldsymbol{R} \dot{\boldsymbol{\xi}}_{\mathrm{aw}}
$$

und der Systemdynamik (7.7) sowie den Abkürzungen

$$
\begin{aligned}
\boldsymbol{A} & =\boldsymbol{A}_{\mathrm{s}}-\boldsymbol{B}_{\mathrm{s}, u} \underline{\boldsymbol{K}}, \\
\tilde{\boldsymbol{y}} & =\mathbf{d z}_{\alpha \overline{\mathbf{u}}}\left(\underline{\boldsymbol{K}} \boldsymbol{\xi}_{\mathrm{aw}}\right)
\end{aligned}
$$

aus (C.28) die Ungleichung ${ }^{3}$

$$
\left[\begin{array}{lll}
\boldsymbol{\xi}_{\mathrm{aw}}^{\top} & \tilde{\boldsymbol{y}}^{\top} & \boldsymbol{\zeta}^{\top}
\end{array}\right]\left[\begin{array}{ccc}
\boldsymbol{A}^{\top} \boldsymbol{R}+\boldsymbol{R} \boldsymbol{A}+\boldsymbol{C}_{\mathrm{s}}^{\top} \boldsymbol{C}_{\mathrm{s}} & \star & \star \\
\boldsymbol{B}_{\mathrm{s}, u}^{\top} \boldsymbol{R}+\hat{\boldsymbol{\Psi}} \boldsymbol{\Phi} \underline{\boldsymbol{K}} & -2 \hat{\boldsymbol{\Psi}} & \star \\
\boldsymbol{B}_{\mathrm{s}, u} \boldsymbol{R} & \mathbf{0} & -\gamma^{2} \mathbb{I}
\end{array}\right]\left[\begin{array}{c}
\boldsymbol{\xi}_{\mathrm{aw}} \\
\tilde{\boldsymbol{y}} \\
\boldsymbol{\zeta}
\end{array}\right]<0 .
$$

${ }^{3}$ Dabei bezeichnet $\star$ den Term, der benötigt wird, um eine symmetrische Matrix zu erhalten. 
Offensichtlich ist diese Ungleichung für alle $\boldsymbol{\xi}_{\text {aw }}, \boldsymbol{\zeta}$ und $\tilde{\boldsymbol{y}}$ erfüllt, wenn die Matrix negativ definit ist. Mit dem Schur-Komplement (Lemma B.1) ergibt sich die identische Bedingung

$$
\left[\begin{array}{crcc}
\boldsymbol{A}^{\top} \boldsymbol{R}+\boldsymbol{R} \boldsymbol{A} & \star & \star & \star \\
\boldsymbol{B}_{\mathrm{s}, u}^{\top} \boldsymbol{R}+\hat{\boldsymbol{\Psi}} \boldsymbol{\Phi} \underline{\boldsymbol{K}} & -2 \hat{\boldsymbol{\Psi}} & \star & \star \\
\boldsymbol{B}_{\mathrm{s}, u}^{\top} \boldsymbol{R} & \mathbf{0} & -\gamma^{2} \mathbb{I} & \star \\
\boldsymbol{C}_{\mathrm{s}} & \mathbf{0} & \mathbf{0} & -\mathbb{I}
\end{array}\right] \prec \mathbf{0}
$$

Die Matrixungleichung (7.10) des Lemmas ergibt sich nach drei weiteren Umformungen. Zuerst erfolgt eine Kongruenztransformation, d. h., die Ungleichung (C.29) wird von links und rechts mit der regulären Matrix

$$
\gamma_{\mathcal{Y}}^{-\frac{1}{2}} \operatorname{diag}\left(\mathbb{I}, \mathbb{I}, \mathbb{I}, \gamma_{\mathcal{Y}} \mathbb{I}\right)
$$

multipliziert. Die Substitution $\boldsymbol{Q}^{-1}=\boldsymbol{R} \gamma_{\mathcal{Y}}^{-1}, \Psi^{-1}=\hat{\mathbf{\Psi}} \gamma_{\mathcal{Y}}^{-1}$ und eine Kongruenztransformation mit $\operatorname{diag}(\boldsymbol{Q}, \Psi, \mathbb{I}, \mathbb{I})$ führt zu (7.10).

Schritt 2. Betrachtet wird die positiv definite Funktion

$$
V\left(\boldsymbol{\xi}_{\text {aw }}\right)=\boldsymbol{\xi}_{\text {aw }}^{\top} \boldsymbol{R} \boldsymbol{\xi}_{\text {aw }}, \quad \boldsymbol{R} \succ \mathbf{0}
$$

und das System

$$
\begin{aligned}
\dot{\boldsymbol{\xi}}_{\mathrm{aw}} & =\left(\boldsymbol{A}_{\mathrm{s}}-\boldsymbol{B}_{\mathrm{s}, u} \underline{\boldsymbol{K}}\right) \boldsymbol{\xi}_{\mathrm{aw}}+\boldsymbol{B}_{\mathrm{s}, u} \mathbf{d} \mathbf{z}_{\alpha \overline{\mathbf{u}}}\left(\underline{\boldsymbol{K}} \boldsymbol{\xi}_{\mathrm{aw}}\right)+\boldsymbol{B}_{\mathrm{s}, u} \boldsymbol{\zeta} \\
\boldsymbol{y}_{\mathrm{aw}, \mathrm{u}} & =\boldsymbol{C}_{\mathrm{s}} \boldsymbol{\xi}_{\mathrm{aw}}
\end{aligned}
$$

für die folgenden zwei Fälle.

Fall 1. Sei $\boldsymbol{\zeta} \equiv \mathbf{0}$ und der Anfangszustand $\boldsymbol{\xi}_{\mathrm{aw}}^{(0)} \in \mathcal{E}(\boldsymbol{R}, \mathrm{c})$. Dabei gelte

$$
\mathcal{E}(\boldsymbol{R}, \mathrm{c})=\left\{\boldsymbol{\xi}_{\text {aw }} \in \mathbb{R}^{n_{\mathrm{s}}} \mid \boldsymbol{\xi}_{\mathrm{aw}}^{\top} \boldsymbol{R} \boldsymbol{\xi}_{\mathrm{aw}} \leq \mathrm{c}\right\}
$$

mit einem $c>0$. Des Weiteren sei c ausreichend klein, so dass der Eingang $\tilde{\boldsymbol{u}}=\underline{\boldsymbol{K}} \boldsymbol{\xi}_{\text {aw }}$ der multivariablen Totzone

$$
\tilde{\boldsymbol{y}}=\mathbf{d z}_{\overline{\mathbf{u}}}(\tilde{\boldsymbol{u}})
$$

für alle $\boldsymbol{\xi}_{\text {aw }} \in \mathcal{E}(\boldsymbol{R}, \mathrm{c})$ zur Signalklasse $\mathcal{W}(\boldsymbol{\Phi})$ gemäß (7.8) gehört. Folglich gilt die lokale Sektorbedingung

$$
\tilde{\boldsymbol{y}}^{\top} 2 \hat{\boldsymbol{\Psi}}(\boldsymbol{\Phi} \tilde{\boldsymbol{u}}-\tilde{\boldsymbol{y}}) \geq 0
$$


aus Lemma 5.4 für die Totzone. Ungleichung (C.28) impliziert dann

$$
\dot{V}<0 \quad \forall \boldsymbol{\xi}_{\text {aw }} \in \mathcal{E}(\boldsymbol{R}, \mathrm{c}) .
$$

Somit ist (C.30) eine Ljapunov-Funktion für das System (7.7). Asymptotische Stabilität der Ruhelage ist sichergestellt und $\mathcal{E}(\boldsymbol{R}, \boldsymbol{c})$ ist ein Einzugsgebiet der Ruhelage, da es durch die Höhenlinie c der Ljapunov-Funktion begrenzt wird.

Fall 2. Sei $\boldsymbol{\xi}_{\mathrm{aw}}^{(0)}=\mathbf{0}$ und $\boldsymbol{\zeta} \in \mathcal{Y}(\boldsymbol{\Phi})$ ein Eingangssignal der Signalklasse $\mathcal{Y}(\boldsymbol{\Phi})$ gemäß (7.9). Dann gilt ebenfalls die lokale Sektorbedingung für die Totzone und (C.28) impliziert

$$
\dot{V}<\gamma_{\mathcal{y}}^{2} \boldsymbol{y}_{\mathrm{r}}^{\top} \boldsymbol{y}_{\mathrm{r}}-\boldsymbol{y}_{\mathrm{aw}, \mathrm{u}}^{\top} \boldsymbol{y}_{\mathrm{aw}, \mathrm{u}} .
$$

Die Integration der Ungleichung nach der Zeit im Intervall $\left[0, T_{e}\right]$ führt zu

$$
V\left(T_{e}\right)-V(0)<\gamma_{\mathcal{Y}}^{2} \int_{0}^{T_{e}} \boldsymbol{y}_{\mathrm{r}}^{\top} \boldsymbol{y}_{\mathrm{r}} d t-\int_{0}^{T_{e}} \boldsymbol{y}_{\mathrm{aw}, \mathrm{u}}^{\top} \boldsymbol{y}_{\mathrm{aw}, \mathrm{u}} d t .
$$

Aufgrund von $\boldsymbol{\xi}_{\text {aw }}(0)=\mathbf{0}$ folgt mit $V\left(\boldsymbol{\xi}_{\text {aw }}(0)\right)=0$ sofort

$$
\int_{0}^{T_{e}} \boldsymbol{y}_{\mathrm{aw}, \mathrm{u}}^{\top} \boldsymbol{y}_{\mathrm{aw}, \mathrm{u}} d t<\gamma_{\mathcal{\nu}}^{2} \int_{0}^{T_{e}} \boldsymbol{y}_{\mathrm{r}}^{\top} \boldsymbol{y}_{\mathrm{r}} d t-V\left(\boldsymbol{\xi}_{\mathrm{aw}}\left(T_{e}\right)\right) .
$$

Für $T_{e} \rightarrow \infty$ ergibt sich gemäß Definition 4.2 der gesuchte Zusammenhang $\left\|\boldsymbol{y}_{\text {aw, }}\right\|_{L_{2}}<\gamma_{\mathcal{Y}}\left\|\boldsymbol{y}_{\mathrm{r}}\right\|_{L_{2}}$, da $V\left(\boldsymbol{\xi}_{\text {aw }}\left(T_{e}\right)\right) \geq 0$. Damit ist gezeigt, dass $\gamma_{\mathcal{y}}$ eine obere Schranke für die $L_{2}$-Kleinsignalverstärkung des Systems ist.

\section{C.3.12 Beweis von Lemma 8.1}

Beweis. Da die Selektionsstrategie (8.6) in dem Gebiet $\mathcal{G}_{1}=\mathcal{E}(1) \backslash \overline{\mathcal{E}}\left(v_{\text {min }}\right)$ identisch mit der Selektionsstrategie (8.4) ist, besitzt sie dort gemäß Punkt (a) des Satzes 8.1 eine eindeutige Lösung. Des Weiteren garantiert der Satz 8.1, dass alle Trajektorien, die in $\mathcal{G}_{1}$ starten in Richtung der Ruhelage streben und dabei in immer kleinere Ellipsen eintreten, die nicht mehr verlassen werden können. In $\mathcal{G}_{1}$ erfüllt die implizit definierte Funktion $v(\boldsymbol{x})$ nämlich gemäß Punkt (c) des Satzes für alle $\boldsymbol{x}$ die Bedingungen

$$
v(\boldsymbol{x})>0, \quad \dot{v}(\boldsymbol{x})<0 .
$$


Entlang der Systemtrajektorien ist $\dot{v}$ negativ. Deshalb nimmt $v(\boldsymbol{x})$ entlang aller Trajektorien $\boldsymbol{x}(t)$ ab und der Zustand $\boldsymbol{x}$ läuft aufgrund der Schachtelung der Gebiete $\mathcal{E}(v)$ in Richtung des Ursprungs. Zu einem Zeitpunkt $0<t_{1}<\infty$ tritt er dann in das Gebiet $\overline{\mathcal{E}}\left(v_{\text {min }}\right)$ ein. Der Rand dieser Ellipse entspricht exakt der Höhenlinie eins der Funktion

$$
V(\boldsymbol{x})=\boldsymbol{x}^{\top} \boldsymbol{R}\left(v_{\min }\right) \boldsymbol{x},
$$

die eine Ljapunov-Funktion für die in $\overline{\mathcal{E}}\left(v_{\text {min }}\right)$ aktive Systemdynamik

$$
\dot{\boldsymbol{x}}=\boldsymbol{A} \boldsymbol{x}+\boldsymbol{b} \operatorname{sat}_{\overline{\mathrm{u}}}\left(-\boldsymbol{k}^{\top}\left(v_{\min }\right) \boldsymbol{x}\right)
$$

darstellt. Aus Satz 4.1 folgt die asymptotische Stabilität der Ruhelage.

Abschließend wird gezeigt, dass $V(\boldsymbol{x})$ eine Ljapunov-Funktion ist. Offensichtlich gilt $V(\mathbf{0})=\mathbf{0}$ und $V(\boldsymbol{x})>0$ für alle $\boldsymbol{x} \neq \mathbf{0}$, da $\boldsymbol{R}_{1}$ gemäß den Voraussetzungen von Satz 8.1 positiv definit ist und die Kongruenztransformation

$$
\boldsymbol{R}\left(v_{\min }\right)=\boldsymbol{H}^{-1}\left(v_{\min }\right) \boldsymbol{R}_{1} \boldsymbol{H}^{-1}\left(v_{\min }\right) \succ \mathbf{0}
$$

die Definitheit einer Matrix nicht ändert. Somit bleibt noch zu zeigen, dass für alle $\boldsymbol{x} \in \overline{\mathcal{E}}\left(v_{\text {min }}\right) \backslash\{\boldsymbol{0}\}$ gilt

$$
\dot{V}(\boldsymbol{x})=\frac{\partial V(\boldsymbol{x})}{\partial \boldsymbol{x}}\left(\boldsymbol{A} \boldsymbol{x}+\boldsymbol{b} \operatorname{sat}_{\overline{\mathrm{u}}}\left(-\boldsymbol{k}^{\top}\left(v_{\min }\right) \boldsymbol{x}\right)\right)<0 .
$$

Nach Satz 1 in [122] ist dies erfüllt, wenn für alle $\boldsymbol{x} \in \overline{\mathcal{E}}\left(v_{\min }\right) \backslash\{\mathbf{0}\}$ gilt

$$
\begin{aligned}
& \frac{\partial V(\boldsymbol{x})}{\partial \boldsymbol{x}}\left(\boldsymbol{A}-\boldsymbol{b}^{\top}\left(v_{\min }\right)\right) \boldsymbol{x}<0, \\
& \frac{\partial V(\boldsymbol{x})}{\partial \boldsymbol{x}}\left(\boldsymbol{A}-\boldsymbol{b} \boldsymbol{k}_{*}^{\top}\left(v_{\min }\right)\right) \boldsymbol{x}<0, \\
& \left|\boldsymbol{k}_{*}^{\top}\left(v_{\min }\right) \boldsymbol{x}\right| \leq \overline{\mathrm{u}}
\end{aligned}
$$

mit $\boldsymbol{k}_{*}\left(v_{\min }\right)=\boldsymbol{H}^{-1}\left(v_{\min }\right) \boldsymbol{k}_{2}-\boldsymbol{a}$. Für die erste Bedingung (C.31a) ergibt sich

$$
2 \boldsymbol{x}^{\top} \boldsymbol{R}\left(v_{\min }\right)\left(\boldsymbol{A}-\boldsymbol{b k}^{\top}\left(v_{\min }\right)\right) \boldsymbol{x}<0 .
$$

Einsetzen von $\boldsymbol{R}\left(v_{\min }\right)$ und $\boldsymbol{k}\left(v_{\min }\right)$ führt zu

$$
2 \boldsymbol{x}^{\top} \boldsymbol{H}^{-1}\left(v_{\min }\right) \boldsymbol{R}_{1} \boldsymbol{H}^{-1}\left(v_{\min }\right)\left(\boldsymbol{A}-\boldsymbol{b}\left(\boldsymbol{H}^{-1}\left(v_{\min }\right) \boldsymbol{k}_{1}^{\top}-\boldsymbol{a}^{\top}\right)\right) \boldsymbol{x}<0 .
$$


Da die Strecke in Regelungsnormalform vorliegt, gilt nach [98]

$$
\begin{aligned}
\boldsymbol{A}-\boldsymbol{b}\left(\boldsymbol{H}^{-1}\left(v_{\min }\right) \boldsymbol{k}_{1}^{\top}-\boldsymbol{a}^{\top}\right) & = \\
& \frac{1}{v_{\min }} \boldsymbol{H}\left(v_{\min }\right)\left[\boldsymbol{A}-\boldsymbol{b}\left(\boldsymbol{k}_{1}-\boldsymbol{a}\right)^{\top}\right] \boldsymbol{H}^{-1}\left(v_{\min }\right) .
\end{aligned}
$$

Einsetzen in die obige Bedingung führt zu

$$
2 \frac{1}{v_{\min }} \boldsymbol{x}^{\top} \boldsymbol{H}^{-1}\left(v_{\min }\right) \boldsymbol{R}_{1}\left[\boldsymbol{A}-\boldsymbol{b}\left(\boldsymbol{k}_{1}-\boldsymbol{a}\right)^{\top}\right] \boldsymbol{H}^{-1}\left(v_{\min }\right) \boldsymbol{x}<0 .
$$

Diese Bedingung ist für alle $v_{\min }>0$ und alle $\boldsymbol{x} \neq \mathbf{0}$ erfüllt, wenn

$$
\boldsymbol{R}_{1}\left[\boldsymbol{A}-\boldsymbol{b}\left(\boldsymbol{k}_{1}-\boldsymbol{a}\right)^{\top}\right]+\left[\boldsymbol{A}-\boldsymbol{b}\left(\boldsymbol{k}_{1}-\boldsymbol{a}\right)^{\top}\right]^{\top} \boldsymbol{R}_{1} \prec \mathbf{0}
$$

gilt, was Bedingung (8.5a) des Satzes 8.1 entspricht. Die zweite Bedingung (C.31b) lässt sich analog in Bedingung (8.5b) des Satzes 8.1 umformen. Die dritte Bedingung (C.31c) führt nach Lemma B.1 aus [120, Anhang B.1, Seite 165] zu der Matrixungleichung (8.5c).

\section{C.3.13 Beweis von Lemma 8.3}

Beweis. Um die globale asymptotische Stabilität des Ursprungs nachzuweisen ist nach Definition $2.2 \mathrm{zu}$ zeigen, dass die Ruhelage $\boldsymbol{x}^{(\mathrm{R})}=\mathbf{0}$ stabil im Sinne von Ljapunov sowie global attraktiv ist.

Da gemäß Voraussetzung die Bedingungen (8.10) erfüllt sind, folgt nach Lemma 8.1 die lokale asymptotische Stabilität der Ruhelage $\boldsymbol{x}^{(\mathrm{R})}=\mathbf{0}$ mit dem Einzugsgebiet $\mathcal{E}(1)$. Diese Eigenschaft impliziert nach Definition 2.2 die Stabilität im Sinne von Ljapunov.

Die globale Attraktivität der Ruhelage ist ebenfalls gegeben. Da $\mathcal{E}(1)$ nach Lemma 8.1 ein Einzugsgebiet der Ruhelage ist, folgt für alle Trajektorien mit Anfangswerten $\boldsymbol{x}^{(0)} \in \mathcal{E}(1)$ die Konvergenz in den Ursprung, d. h. $\boldsymbol{x}(t) \rightarrow \mathbf{0}$ für $t \rightarrow \infty$. Alle Trajektorien, die außerhalb der Ellipse $\mathcal{E}(1)$ starten, d. h. $\boldsymbol{x}^{(0)} \notin \mathcal{E}(1)$, streben in endlicher Zeit in die Ellipse und damit gemäß den obigen Ausführungen für $t \rightarrow \infty$ in den Ursprung.

Das lässt sich wie folgt begründen. Außerhalb der Ellipse weist der Regelkreis die Systemdynamik (8.15) auf. Die Ruhelage $\boldsymbol{x}^{(\mathrm{R})}=\mathbf{0}$ dieses Systems wird von dem Regler $\boldsymbol{k}_{g}$ global asymptotisch stabilisiert. Insbesondere ist diese Ruhelage dann global attraktiv. Das bedeutet, alle Trajektorien streben für $t \rightarrow \infty$ in den Ursprung. Da die Ellipse $\mathcal{E}(1)$ eine Umgebung des Ursprungs ist, müssen die Trajektorien diese Umgebung in endlicher Zeit erreichen. 


\section{C.3.14 Beweis von Lemma 8.4}

Beweis. Der Beweis erfolgt in drei Schritten. Im ersten Schritt wird gezeigt, dass das Regelgesetz

$$
\begin{aligned}
k\left(\boldsymbol{x}_{\mathrm{aw}}\right) & =\left(\boldsymbol{H}^{-1}\left(v\left(\boldsymbol{x}_{\mathrm{aw}}\right)\right)\left(\boldsymbol{k}_{g}+\boldsymbol{a}\right)-\boldsymbol{a}\right)^{\top} \boldsymbol{x}_{\mathrm{aw}}, \\
\boldsymbol{H}^{-1}(v) & =\operatorname{diag}\left(v^{-n_{\mathrm{s}}}\left(\boldsymbol{x}_{\mathrm{aw}}\right), \ldots, v^{-1}\left(\boldsymbol{x}_{\mathrm{aw}}\right)\right)
\end{aligned}
$$

lokal lipschitzstetig ist, wenn die Selektionsstrategie

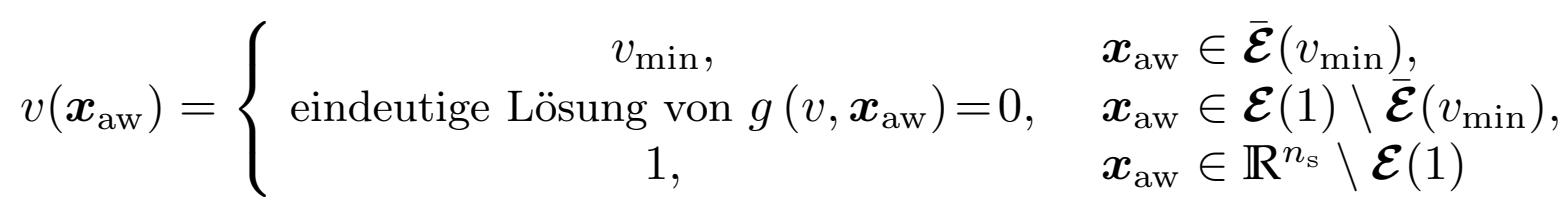

global lipschitzstetig ist. Im zweiten Schritt wird gezeigt, dass $g\left(v, \boldsymbol{x}_{\mathrm{aw}}\right)$ eine streng monoton fallende Funktion bezüglich $v$ ist, wenn die Bedingungen (8.10) erfüllt sind. Dieses Resultat hilft dann im dritten Schritt beim Nachweis der globalen Lipschitz-Stetigkeit der Selektionsstrategie.

Schritt 1. Das Regelgesetz kann als Verkettung der Funktion $\boldsymbol{f}_{1}(u)=$ $\boldsymbol{H}^{-1}(u)\left(\boldsymbol{k}_{g}+\boldsymbol{a}\right)-\boldsymbol{a}$ mit der Funktion $v\left(\boldsymbol{x}_{\text {aw }}\right)$ sowie einer anschließenden Multiplikation mit der Funktion $\boldsymbol{f}_{2}\left(\boldsymbol{x}_{\mathrm{aw}}\right)=\boldsymbol{x}_{\mathrm{aw}}$ aufgefasst werden, d. h.

$$
k\left(\boldsymbol{x}_{\mathrm{aw}}\right)=\left[\left(\boldsymbol{f}_{1} \circ v\right)\left(\boldsymbol{x}_{\mathrm{aw}}\right)\right]^{\top} \boldsymbol{f}_{2}\left(\boldsymbol{x}_{\mathrm{aw}}\right) .
$$

Mit den Sätzen A.1 und A.2 über die Verkettung und das Produkt lipschitzstetiger Funktionen folgt die gewünschte Aussage. Die Funktion $\boldsymbol{f}_{2}\left(\boldsymbol{x}_{\text {aw }}\right)=\boldsymbol{x}_{\text {aw }}$ erfüllt eine Lipschitz-Bedingung auf ganz $\mathbb{R}^{n_{\mathrm{s}}}$, da sie stetig partiell differenzierbar ist und die Komponenten der partiellen Ableitungen beschränkt sind [10, Satz 2.4.6]. Folglich ist sie global lipschitzstetig auf ihrem Definitionsbereich $\mathbb{R}^{n}$. Gleiches gilt für die Funktion $\boldsymbol{f}_{1}(u)$ und den Definitionsbereich $\left[v_{\min }, 1\right]$. Wenn die Selektionsstrategie global lipschitzstetig auf $\mathbb{R}^{n_{\mathrm{s}}}$ ist, folgt aus Satz A.1 die globale Lipschitz-Stetigkeit der Verkettung $\left(\boldsymbol{f}_{1} \circ v\right)\left(\boldsymbol{x}_{\mathrm{aw}}\right)$ auf ganz $\mathbb{R}^{n_{\mathrm{s}}}$ und aus Satz A.2 die lokale Lipschitz-Stetigkeit des Regelgesetzes.

Schritt 2. Da die Bedingungen (8.10) erfüllt sind, gilt $\boldsymbol{R}_{1} \succ \mathbf{0}$ und

$$
\boldsymbol{N} \boldsymbol{R}_{1}+\boldsymbol{R}_{1} \boldsymbol{N} \prec \mathbf{0} \quad \text { mit } \quad \boldsymbol{N}=\operatorname{diag}\left(-n_{\mathrm{s}}, \ldots,-1\right) .
$$

Die Ungleichungen garantierten, dass die Funktion

$$
g\left(v, \boldsymbol{x}_{\mathrm{aw}}\right)=\boldsymbol{x}_{\mathrm{aw}}^{\top} \boldsymbol{R}(v) \boldsymbol{x}_{\mathrm{aw}}-1
$$


streng monoton fallend bezüglich $v$ ist, denn die partielle Ableitung

$$
\frac{\partial g\left(v, \boldsymbol{x}_{\mathrm{aw}}\right)}{\partial v}=\frac{1}{v} \boldsymbol{x}_{\mathrm{aw}}^{\top} \boldsymbol{H}^{-1}(v)\left[\boldsymbol{N} \boldsymbol{R}_{1}+\boldsymbol{R}_{1} \boldsymbol{N}\right] \boldsymbol{H}^{-1}(v) \boldsymbol{x}_{\mathrm{aw}}
$$

ist negativ für alle $\boldsymbol{x}_{\text {aw }}$ und alle $v \in\left[v_{\min }, 1\right]$. Daraus folgt die sogenannte Schachtelungseigenschaft. Es gilt für zwei Konstanten $v_{\min } \leq v_{1}<v_{2} \leq 1$

$$
\boldsymbol{g}\left(v_{1}, \boldsymbol{x}_{\mathrm{aw}}\right)>\boldsymbol{g}\left(v_{2}, \boldsymbol{x}_{\mathrm{aw}}\right) \quad \Leftrightarrow \quad \boldsymbol{x}_{\mathrm{aw}}^{\top} \boldsymbol{R}\left(v_{1}\right) \boldsymbol{x}_{\mathrm{aw}}-1>\boldsymbol{x}_{\mathrm{aw}}^{\top} \boldsymbol{R}\left(v_{2}\right) \boldsymbol{x}_{\mathrm{aw}}-1 .
$$

Fordert man $\boldsymbol{x}_{\mathrm{aw}}^{\top} \boldsymbol{R}\left(v_{1}\right) \boldsymbol{x}_{\mathrm{aw}}-1 \leq 0$ folgt $0 \geq \boldsymbol{g}\left(v_{1}, \boldsymbol{x}_{\mathrm{aw}}\right)>\boldsymbol{g}\left(v_{2}, \boldsymbol{x}_{\mathrm{aw}}\right)$ und daraus

$$
\overline{\mathcal{E}}\left(v_{1}\right) \subset \mathcal{E}\left(v_{2}\right)
$$

mit

$$
\begin{aligned}
& \overline{\mathcal{E}}\left(v_{1}\right)=\left\{\boldsymbol{x}_{\mathrm{aw}} \in \mathbb{R}^{n_{\mathrm{s}}} \mid \boldsymbol{x}_{\mathrm{aw}}^{\top} \boldsymbol{R}\left(v_{1}\right) \boldsymbol{x}_{\mathrm{aw}} \leq 1\right\} \\
& \mathcal{E}\left(v_{2}\right)=\left\{\boldsymbol{x}_{\mathrm{aw}} \in \mathbb{R}^{n_{\mathrm{s}}} \mid \boldsymbol{x}_{\mathrm{aw}}^{\top} \boldsymbol{R}\left(v_{2}\right) \boldsymbol{x}_{\mathrm{aw}}<1\right\}
\end{aligned}
$$

Die Menge $\overline{\mathcal{E}}\left(v_{1}\right)$ liegt im Inneren von $\mathcal{E}\left(v_{2}\right)$. Das gilt auch, wenn $v_{2}$ nur minimal größer ist als $v_{1}$, d. h. $v_{2}=v_{1}+\epsilon$ mit einer beliebig kleinen positiven Konstante $\epsilon$. Das führt zu der Schlussfolgerung: Zwischen den Punkten des Randes $\partial \overline{\mathcal{E}}\left(v_{1}\right)$ und allen Punkten der Ellipse $\mathcal{E}\left(v_{2}\right)$ muss ein Mindestabstand $\delta>0$ existieren, also

$$
\left\|\boldsymbol{x}_{\mathrm{aw}}-\tilde{\boldsymbol{x}}_{\mathrm{aw}}\right\|_{2} \geq \delta>0 \quad \forall \boldsymbol{x}_{\mathrm{aw}} \in \partial \overline{\mathcal{E}}\left(v_{1}\right), \tilde{\boldsymbol{x}}_{\mathrm{aw}} \in \mathcal{E}\left(v_{2}\right)
$$

Schritt 3. Dass die Funktion $v\left(\boldsymbol{x}_{\mathrm{aw}}\right)$ die Lipschitz-Bedingung

$$
\left\|v\left(\boldsymbol{x}_{\mathrm{aw}}\right)-v\left(\tilde{\boldsymbol{x}}_{\mathrm{aw}}\right)\right\|_{2} \leq L\left\|\boldsymbol{x}_{\mathrm{aw}}-\tilde{\boldsymbol{x}}_{\mathrm{aw}}\right\|_{2}
$$

für ein $L>0$ und alle $\boldsymbol{x}_{\mathrm{aw}}, \tilde{\boldsymbol{x}}_{\mathrm{aw}} \in \mathbb{R}^{n_{\mathrm{s}}}$ erfüllt, wird mittels einer Fallunterscheidung gezeigt. Für eine kompaktere Notation bietet sich die Verwendung folgender Abkürzungen an

$$
\begin{aligned}
\overline{\mathcal{G}}_{1} & =\overline{\mathcal{E}}\left(v_{\min }\right), \\
\mathcal{G}_{2} & =\mathcal{E}(1) \backslash \overline{\mathcal{E}}\left(v_{\min }\right), \\
\overline{\mathcal{G}}_{2} & =\overline{\mathcal{E}}(1) \backslash \mathcal{E}\left(v_{\min }\right), \\
\mathcal{G}_{3} & =\mathbb{R}^{n_{\mathrm{s}}} \backslash \mathcal{E}(1) .
\end{aligned}
$$

Dabei beinhaltet die geschlossene Menge $\overline{\mathcal{G}}_{2}$ alle Punkte der offenen Menge $\mathcal{G}_{2}$. Abbildung C.2 stellt die Gebiete beispielhaft für den $\mathbb{R}^{2}$ dar. 


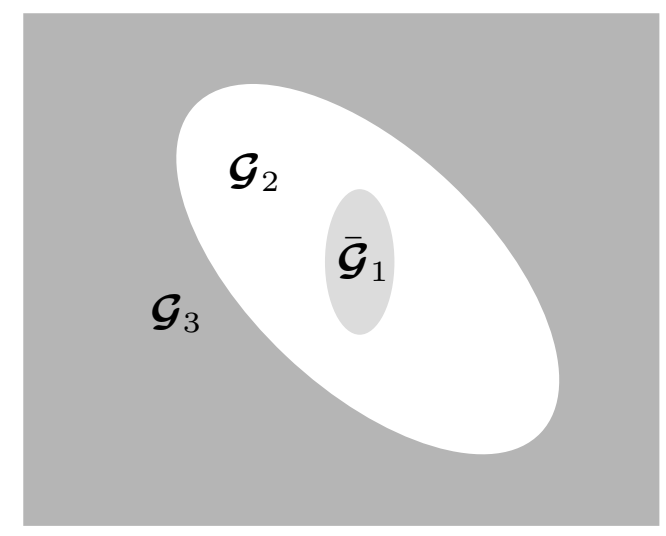

Abbildung C.2: Visualisierung der Gebiete im $\mathbb{R}^{2}$.

Fall 1: $\boldsymbol{x}_{\text {aw }}, \tilde{\boldsymbol{x}}_{\text {aw }} \in \overline{\mathcal{G}}_{1}$. Hier gilt $v\left(\boldsymbol{x}_{\text {aw }}\right)-v\left(\tilde{\boldsymbol{x}}_{\text {aw }}\right)=0$. Die LipschitzBedingung ist erfüllt für ein beliebig kleines $L>0$.

Fall 2: $\boldsymbol{x}_{\mathrm{aw}}, \tilde{\boldsymbol{x}}_{\mathrm{aw}} \in \overline{\mathcal{G}}_{2}$. Für diesen Fall wird zunächst eine Hilfsfunktion $\tilde{v}:[0,1] \mapsto\left[v_{\min }, 1\right]$ definiert, die folgende Gestalt besitzt

$$
\tilde{v}(\tau)=v\left(\boldsymbol{x}_{\mathrm{aw}}+\tau\left(\tilde{\boldsymbol{x}}_{\mathrm{aw}}-\boldsymbol{x}_{\mathrm{aw}}\right)\right) .
$$

Offensichtlich gilt $\tilde{v}(0)=v\left(\boldsymbol{x}_{\text {aw }}\right)$ und $\tilde{v}(1)=v\left(\tilde{\boldsymbol{x}}_{\text {aw }}\right)$. Mit der Kettenregel ergibt sich für die Ableitung

$$
\frac{d}{d \tau} \tilde{v}(\tau)=\tilde{v}^{\prime}=\operatorname{grad}\left(v\left(\boldsymbol{x}_{\mathrm{aw}}+\tau\left(\tilde{\boldsymbol{x}}_{\mathrm{aw}}-\boldsymbol{x}_{\mathrm{aw}}\right)\right)\right) \cdot\left(\tilde{\boldsymbol{x}}_{\mathrm{aw}}-\boldsymbol{x}_{\mathrm{aw}}\right) .
$$

Der Gradient von $v(\boldsymbol{x})$ mit $\boldsymbol{x}=\boldsymbol{x}_{\mathrm{aw}}+\tau\left(\tilde{\boldsymbol{x}}_{\mathrm{aw}}-\boldsymbol{x}_{\text {aw }}\right)$ existiert für alle $\boldsymbol{x} \in \mathcal{G}_{2}$ und alle $v \in\left[v_{\min }, 1\right]$. Er ist stetig, beschränkt und lässt sich berechnen zu

$$
\frac{\partial v}{\partial \boldsymbol{x}}=-\left(\frac{1}{v} \boldsymbol{x}^{\top} \boldsymbol{D}^{-1}(v)\left[\boldsymbol{N} \boldsymbol{R}_{1}+\boldsymbol{R}_{1} \boldsymbol{N}\right] \boldsymbol{D}^{-1}(v) \boldsymbol{x}\right)^{-1} \cdot 2 \boldsymbol{x}^{\top} \boldsymbol{R}(v) .
$$

Da der Gradient von $v$ nur für alle $\boldsymbol{x}=\tilde{\boldsymbol{x}}_{\text {aw }}+\tau\left(\tilde{\boldsymbol{x}}_{\text {aw }}-\boldsymbol{x}_{\text {aw }}\right)$ in der offenen Menge $\mathcal{G}_{2}$ existiert, $\boldsymbol{x}_{\text {aw }}$ und $\tilde{\boldsymbol{x}}_{\text {aw }}$ allerdings Randpunkte von $\overline{\mathcal{G}}_{2}$ sein dürfen, existiert die Ableitung $\tilde{v}^{\prime}(\tau)$ in diesen Fällen nur für $\tau \in(0,1)$. Mehr wird im Weiteren allerdings auch nicht benötigt.

Für die auf dem abgeschlossenen Intervall $[0,1]$ definierte Funktion $\tilde{v}(\tau)$ existiert nach dem Mittelwertsatz der Differentialrechnung ein $\tau \in(0,1)$, so dass gilt

$$
\tilde{v}(0)-\tilde{v}(1)=\tilde{v}^{\prime}(\tau)(0-1)=-\tilde{v}^{\prime}(\tau)
$$


Das lässt sich für die folgende Abschätzung ausnutzen. Es gilt für ein $\tau \in(0,1)$

$$
\begin{aligned}
& \left\|v\left(\boldsymbol{x}_{\mathrm{aw}}\right)-v\left(\tilde{\boldsymbol{x}}_{\mathrm{aw}}\right)\right\|_{2} \\
& =\|\tilde{v}(0)-\tilde{v}(1)\|_{2}=\left\|\tilde{v}^{\prime}(\tau)\right\|_{2} \\
& =\left\|\operatorname{grad}\left(v\left(\boldsymbol{x}_{\mathrm{aw}}+\tau\left(\tilde{\boldsymbol{x}}_{\mathrm{aw}}-\boldsymbol{x}_{\mathrm{aw}}\right)\right)\right) \cdot\left(\tilde{\boldsymbol{x}}_{\mathrm{aw}}-\boldsymbol{x}_{\mathrm{aw}}\right)\right\|_{2} .
\end{aligned}
$$

Mit der Cauchy-Schwartz'schen Ungleichung folgt

$$
\left\|v\left(\boldsymbol{x}_{\mathrm{aw}}\right)-v\left(\tilde{\boldsymbol{x}}_{\mathrm{aw}}\right)\right\|_{2} \leq\left\|\operatorname{grad}\left(v\left(\boldsymbol{x}_{\mathrm{aw}}+\tau\left(\tilde{\boldsymbol{x}}_{\mathrm{aw}}-\boldsymbol{x}_{\mathrm{aw}}\right)\right)\right)\right\|_{2}\left\|\tilde{\boldsymbol{x}}_{\mathrm{aw}}-\boldsymbol{x}_{\mathrm{aw}}\right\|_{2}
$$

und somit

$$
\frac{\left\|v\left(\boldsymbol{x}_{\mathrm{aw}}\right)-v\left(\tilde{\boldsymbol{x}}_{\mathrm{aw}}\right)\right\|_{2}}{\left\|\tilde{\boldsymbol{x}}_{\mathrm{aw}}-\boldsymbol{x}_{\mathrm{aw}}\right\|_{2}} \leq L=\left\|\operatorname{grad}\left(v\left(\boldsymbol{x}_{\mathrm{aw}}+\tau\left(\tilde{\boldsymbol{x}}_{\mathrm{aw}}-\boldsymbol{x}_{\mathrm{aw}}\right)\right)\right)\right\|_{2} .
$$

Dabei ist $L$ begrenzt, weil der Gradient für alle $\tau \in(0,1)$ existiert und dessen Betrag endliche Werte annimmt.

Fall 3: $\boldsymbol{x}_{\text {aw }}, \tilde{\boldsymbol{x}}_{\text {aw }} \in \mathcal{G}_{3}$. Analog zu Fall 1.

Fall 4: $\boldsymbol{x}_{\text {aw }} \in \overline{\mathcal{G}}_{1}, \tilde{\boldsymbol{x}}_{\text {aw }} \in \mathcal{G}_{2}$. Dieser Fall lässt sich auf bereits bekannte Fälle zurückführen.

Es lässt sich immer ein $\boldsymbol{x} \in \partial \overline{\mathcal{G}}_{1}$ finden, dass auf der Verbindungslinie von $\boldsymbol{x}_{\text {aw }}$ und $\tilde{\boldsymbol{x}}_{\text {aw }}$ liegt. Somit gilt

$$
\left\|\boldsymbol{x}_{\mathrm{aw}}-\tilde{\boldsymbol{x}}_{\mathrm{aw}}\right\|_{2}=\left\|\boldsymbol{x}_{\mathrm{aw}}-\boldsymbol{x}\right\|_{2}+\left\|\boldsymbol{x}-\tilde{\boldsymbol{x}}_{\mathrm{aw}}\right\|_{2} .
$$

Weiter gilt

$$
\begin{aligned}
\left\|v\left(\boldsymbol{x}_{\mathrm{aw}}\right)-v\left(\tilde{\boldsymbol{x}}_{\mathrm{aw}}\right)\right\|_{2} & =\left\|v\left(\boldsymbol{x}_{\mathrm{aw}}\right)-v\left(\tilde{\boldsymbol{x}}_{\mathrm{aw}}\right)+v(\boldsymbol{x})-v(\boldsymbol{x})\right\|_{2} \\
& \leq\left\|v\left(\boldsymbol{x}_{\mathrm{aw}}\right)-v(\boldsymbol{x})\right\|_{2}+\left\|v(\boldsymbol{x})-v\left(\tilde{\boldsymbol{x}}_{\mathrm{aw}}\right)\right\|_{2} .
\end{aligned}
$$

Der erste Term ist null, da $\boldsymbol{x}_{\text {aw }}, \boldsymbol{x} \in \overline{\mathcal{G}}_{1}$ und dort ist $v\left(\boldsymbol{x}_{\text {aw }}\right)=v(\boldsymbol{x})=v_{\text {min }}$. Das entspricht Fall 1 und folglich gilt

$$
\left\|v\left(\boldsymbol{x}_{\mathrm{aw}}\right)-v\left(\tilde{\boldsymbol{x}}_{\mathrm{aw}}\right)\right\|_{2} \leq L_{1}\left\|\boldsymbol{x}_{\mathrm{aw}}-\boldsymbol{x}\right\|_{2}
$$

für ein beliebig kleines $L_{1}>0$. Der zweite Term lässt sich nach den Überlegungen zu Fall 2 wie folgt abschätzen

$$
\left\|v(\boldsymbol{x})-v\left(\tilde{\boldsymbol{x}}_{\mathrm{aw}}\right)\right\|_{2} \leq\left\|\operatorname{grad}\left(v\left(\boldsymbol{x}+\tau\left(\tilde{\boldsymbol{x}}_{\mathrm{aw}}-\boldsymbol{x}\right)\right)\right)\right\|_{2}\left\|\tilde{\boldsymbol{x}}_{\mathrm{aw}}-\boldsymbol{x}\right\|_{2}
$$


mit $\tau \in(0,1)$. Somit gilt analog zu Fall 2:

$$
\left\|v(\boldsymbol{x})-v\left(\tilde{\boldsymbol{x}}_{\mathrm{aw}}\right)\right\|_{2} \leq L_{2}=\left\|\operatorname{grad}\left(v\left(\boldsymbol{x}+\tau\left(\tilde{\boldsymbol{x}}_{\mathrm{aw}}-\boldsymbol{x}\right)\right)\right)\right\|_{2}\left\|\tilde{\boldsymbol{x}}_{\mathrm{aw}}-\boldsymbol{x}\right\|_{2} .
$$

Daraus und aus der Tatsache, dass $L_{1}$ beliebig groß gewählt werden kann, ergibt sich mit $L_{1}=L_{2}=L$

$$
\begin{aligned}
\left\|v\left(\boldsymbol{x}_{\mathrm{aw}}\right)-v\left(\tilde{\boldsymbol{x}}_{\mathrm{aw}}\right)\right\|_{2} & \leq\left\|v\left(\boldsymbol{x}_{\mathrm{aw}}\right)-v(\boldsymbol{x})\right\|_{2}+\left\|v(\boldsymbol{x})-v\left(\tilde{\boldsymbol{x}}_{\mathrm{aw}}\right)\right\|_{2} \\
& \leq L\left\|\boldsymbol{x}_{\mathrm{aw}}-\boldsymbol{x}\right\|_{2}+L\left\|\tilde{\boldsymbol{x}}_{\mathrm{aw}}-\boldsymbol{x}\right\|_{2} \\
& \leq L\left(\left\|\boldsymbol{x}_{\mathrm{aw}}-\boldsymbol{x}\right\|_{2}+\left\|\tilde{\boldsymbol{x}}_{\mathrm{aw}}-\boldsymbol{x}\right\|_{2}\right) \\
& \leq L\left\|\boldsymbol{x}_{\mathrm{aw}}-\tilde{\boldsymbol{x}}_{\mathrm{aw}}\right\|_{2} .
\end{aligned}
$$

Aufösen nach $L$ liefert

$$
\frac{\left\|v\left(\boldsymbol{x}_{\mathrm{aw}}\right)-v\left(\tilde{\boldsymbol{x}}_{\mathrm{aw}}\right)\right\|_{2}}{\left\|\boldsymbol{x}_{\mathrm{aw}}-\tilde{\boldsymbol{x}}_{\mathrm{aw}}\right\|_{2}} \leq L<\infty .
$$

Fall 5: $\boldsymbol{x}_{\text {aw }} \in \mathcal{G}_{2}, \tilde{\boldsymbol{x}}_{\mathrm{aw}} \in \mathcal{G}_{3}$. Analog zu Fall 4.

Fall 6: $\boldsymbol{x}_{\text {aw }} \in \overline{\mathcal{G}}_{1}, \tilde{\boldsymbol{x}}_{\text {aw }} \in \mathcal{G}_{3}$. Es gilt $v\left(\boldsymbol{x}_{\text {aw }}\right)=v_{\text {min }}$ und $v\left(\tilde{\boldsymbol{x}}_{\text {aw }}\right)=1$. Daraus folgt

$$
\left\|v\left(\boldsymbol{x}_{\mathrm{aw}}\right)-v\left(\tilde{\boldsymbol{x}}_{\mathrm{aw}}\right)\right\|_{2}=1-v_{\mathrm{min}} .
$$

Die Schachtelungseigenschaft (siehe Schritt 2) garantiert $\overline{\mathcal{G}}_{1} \subset \mathcal{G}_{3}$. Daraus folgt ein Mindestabstand zwischen $\tilde{\boldsymbol{x}}_{\text {aw }} \in \mathcal{G}_{3}$ und $\boldsymbol{x}_{\text {aw }} \in \overline{\mathcal{G}}_{1}$, also

$$
\left\|\boldsymbol{x}_{\mathrm{aw}}-\tilde{\boldsymbol{x}}_{\mathrm{aw}}\right\|_{2} \geq \delta>0 \quad \forall \boldsymbol{x}_{\mathrm{aw}} \in \overline{\mathcal{G}}_{1}, \tilde{\boldsymbol{x}}_{\mathrm{aw}} \in \mathcal{G}_{3}
$$

Aus der Lipschitz-Bedingung

$$
\left\|v\left(\boldsymbol{x}_{\mathrm{aw}}\right)-v\left(\tilde{\boldsymbol{x}}_{\mathrm{aw}}\right)\right\|_{2}=1-v_{\mathrm{min}} \leq L\left\|\boldsymbol{x}_{\mathrm{aw}}-\tilde{\boldsymbol{x}}_{\mathrm{aw}}\right\|_{2}
$$

folgt

$$
L \geq \frac{1-v_{\min }}{\delta}
$$

Ein $L>0$, das in allen sechs Fällen den aufgestellten Bedingungen genügt, garantiert die globale Lipschitz-Stetigkeit von $v\left(\boldsymbol{x}_{\mathrm{aw}}\right)$. 


\section{C.3.15 Beweis von Lemma 9.1}

Beweis. Für jede Komponente von $\mathbf{d} \mathbf{z}_{\varrho \overline{\mathbf{u}}}\left(\boldsymbol{y}_{\mathrm{r}}\right)$ gilt mit $\boldsymbol{y}_{\mathrm{r}}=\boldsymbol{C}_{\mathrm{ub}} \boldsymbol{\xi}_{*}(t)+\boldsymbol{y}^{(\mathrm{R})}$ gemäß der Definition der Totzone

$$
\mathrm{dz}_{\varrho \overline{\mathrm{u}}_{i}}\left(y_{\mathrm{r}, i}\right)=\left\{\begin{array}{lr}
\boldsymbol{C}_{\mathrm{ub}\langle i\rangle} \boldsymbol{\xi}_{*}+y_{\mathrm{r}, i}^{(\mathrm{R})}+\varrho \overline{\mathrm{u}}_{i}, & -\varrho \overline{\mathrm{u}}_{i}>\boldsymbol{C}_{\mathrm{ub}\langle i\rangle} \boldsymbol{\xi}_{*}+y_{\mathrm{r}, i}^{(\mathrm{R})}, \\
0, & -\varrho \overline{\mathrm{u}}_{i} \leq \boldsymbol{C}_{\mathrm{ub}\langle i\rangle} \boldsymbol{\xi}_{*}+y_{\mathrm{r}, i}^{(\mathrm{R})} \leq \varrho \overline{\mathrm{u}}_{i}, \\
\boldsymbol{C}_{\mathrm{ub}\langle i\rangle} \boldsymbol{\xi}_{*}+y_{\mathrm{r}, i}^{(\mathrm{R})}-\varrho \overline{\mathrm{u}}_{i}, & \boldsymbol{C}_{\mathrm{ub}\langle i\rangle} \boldsymbol{\xi}_{*}+y_{\mathrm{r}, i}^{(\mathrm{R})}>\varrho \overline{\mathrm{u}}_{i},
\end{array}\right.
$$

wobei $\boldsymbol{C}_{\mathrm{ub}\langle i\rangle}$ die $i$-te Zeile der Matrix $\boldsymbol{C}_{\mathrm{ub}}$ bezeichnet. Für jeden der drei Bereiche lässt sich der Betrag der Totzone nach oben abschätzen, wie nachfolgend gezeigt wird. Zu beachten ist, dass $\left|y_{\mathrm{r}, i}^{(\mathrm{R})}\right|<\varrho \overline{\mathrm{u}}_{i}$ gilt.

Bereich 1: $-\varrho \overline{\mathrm{u}}_{i}>\boldsymbol{C}_{\mathrm{ub}\langle i\rangle} \boldsymbol{\xi}_{*}+y_{\mathrm{r}, i}^{(\mathrm{R})}$. Aus dieser Ungleichung folgt

$$
\boldsymbol{C}_{\mathrm{ub}\langle i\rangle} \boldsymbol{\xi}_{*}+\underbrace{y_{\mathrm{r}, i}^{(\mathrm{R})}+\varrho \overline{\mathrm{u}}_{i}}_{>0}<0 \Rightarrow \boldsymbol{C}_{\mathrm{ub}\langle i\rangle} \boldsymbol{\xi}_{*}<\boldsymbol{C}_{\mathrm{ub}\langle i\rangle} \boldsymbol{\xi}_{*}+y_{\mathrm{r}, i}^{(\mathrm{R})}+\varrho \overline{\mathrm{u}}_{i}<0 .
$$

Bilden des Betrags liefert die Abschätzung

$$
\left|\boldsymbol{C}_{\mathrm{ub}\langle i\rangle} \boldsymbol{\xi}_{*}\right|>\left|\boldsymbol{C}_{\mathrm{ub}\langle i\rangle} \boldsymbol{\xi}_{*}+y_{\mathrm{r}, i}^{(\mathrm{R})}+\varrho \overline{\mathrm{u}}_{i}\right|>0 .
$$

Bereich 2: $-\varrho \overline{\mathrm{u}}_{i} \leq \boldsymbol{C}_{\mathrm{ub}\langle i\rangle} \boldsymbol{\xi}_{*}+y_{\mathrm{r}, i}^{(\mathrm{R})} \leq \varrho \overline{\mathrm{u}}_{i}$. Hier gilt trivialerweise

$$
\left|\mathrm{dz}_{\varrho \overline{\mathrm{u}}_{i}}\left(y_{\mathrm{r}, i}\right)\right|=0<\left|\boldsymbol{C}_{\mathrm{ub}\langle i\rangle} \boldsymbol{\xi}_{*}\right| .
$$

Bereich 3: $\boldsymbol{C}_{\mathrm{ub}\langle i\rangle} \boldsymbol{\xi}_{*}+y_{\mathrm{r}, i}^{(\mathrm{R})}>\varrho \overline{\mathrm{u}}_{i}$. Aus der Ungleichung folgt direkt

$$
\boldsymbol{C}_{\mathrm{ub}\langle i\rangle} \boldsymbol{\xi}_{*}+\underbrace{y_{\mathrm{r}, i}^{(\mathrm{R})}-\varrho \overline{\mathrm{u}}_{i}}_{<0}>0 \Rightarrow \boldsymbol{C}_{\mathrm{ub}\langle i\rangle} \boldsymbol{\xi}_{*}>\boldsymbol{C}_{\mathrm{ub}\langle i\rangle} \boldsymbol{\xi}_{*}+y_{\mathrm{r}, i}^{(\mathrm{R})}-\varrho \overline{\mathrm{u}}_{i}>0 .
$$

Bilden des Betrags führt zu der Abschätzung

$$
\left|\boldsymbol{C}_{\mathrm{ub}\langle i\rangle} \boldsymbol{\xi}_{*}\right|>\left|\boldsymbol{C}_{\mathrm{ub}\langle i\rangle} \boldsymbol{\xi}_{*}+y_{\mathrm{r}, i}^{(\mathrm{R})}-\varrho \overline{\mathrm{u}}_{i}\right|>0 .
$$

Die Abschätzungen aus den drei Bereichen liefern zusammen die Abschätzung für die $i$-te Komponente der Totzone. Es gilt

$$
\left|\mathrm{dz}_{\varrho \overline{\mathrm{u}}_{i}}\left(y_{\mathrm{r}, i}\right)\right| \leq\left|\boldsymbol{C}_{\mathrm{ub}\langle i\rangle} \boldsymbol{\xi}_{*}\right| .
$$

Daraus folgt die Abschätzung für die multivariable Totzone (9.7). 


\section{Literaturverzeichnis}

[1] Adamy, J.: Strukturvariable Regelungen mittels impliziter Ljapunov-Funktionen, Universität Dortmund, Diss., 1991

[2] Adamy, J.: Implicit Lyapunov functions and isochrones of linear systems. In: IEEE Transactions on Automatic Control 50 (2005), Nr. 6, S. 874-879

[3] Adamy, J.: Nichtlineare Regelungen. Springer, 2009

[4] Adamy, J. ; Flemming, A.: Soft variable-structure controls: a survey. In: Automatica 40 (2004), Nr. 11, S. 1821-1844

[5] Albers, K.: Syntheseverfahren für nichtlineare parameter- und strukturvariable Zustandsregler unter Berücksichtigung von Begrenzungen, Universität Dortmund, Diss., 1983

[6] Antsaklis, P. J. ; Michel, A. N.: A Linear Systems Primer. Birkhäuser, 2007

[7] Aström, K. J. ; Rundqwist, L.: Integrator windup and how to avoid it. In: Proceedings of the American Control Conference. Pittsburg (PA), USA, 1989, S. 1693-1698

[8] Aström, K. J. ; Wittenmark, B.: Computer controlled systems. Prentice-Hall, 1984

[9] Athans, M. ; Falb, P. L.: Optimal Control. McGraw-Hill, 1966

[10] Aulbach, B.: Gewöhnliche Differentialgleichungen. Spektrum, 2004

[11] Bacciotti, A. ; Rosier, L.: Liapunov Functions and Stability in Control Theory. Springer, 2005

[12] Barbu, C. ; Reginatto, R. ; Teel, A. R. ; Zaccarian, L.: Anti-windup for exponentially unstable linear systems with rate and magnitude limits. In: Kapila, V. (Hrsg.) ; Grigoriadis, K. (Hrsg.): Actuator saturation control. Marcel Dekker, 2001

[13] Bemporad, A. ; Casavola, A. ; Mosca, E.: Nonlinear control of constrained linear systems via predictive reference management. In: IEEE Transactions on Automatic Control 42 (1997), Nr. 3, S. 340-349 
[14] Bemporad, A. ; Morari, M. ; Dua, V. ; Pistikopoulos, E. N.: The explicit linear quadratic regulator for constrained systems. In: Automatica 38 (2002), Nr. 1, S. 3-20

[15] Bemporad, A ; Teel, A. R. ; Zaccarian, L.: Anti-windup synthesis via sampled-data piecewise affine optimal control. In: Automatica 40 (2004), S. 549-562

[16] Bernstein, D. S.: Matrix Mathematics. Princeton University Press, 2005

[17] Bernstein, D. S. ; Michel, A. N.: A chronological bibliography on saturating actuators. In: International Journal of Robust and Nonlinear Control 5 (1995), Nr. 5, S. 375-380

[18] Biannic, J-M. ; Roos, C. ; Tarbouriech, S.: A practical method for fixed-order anti-windup design. In: Proceedings of the 7th IFAC Symposium on Nonlinear Control Systems. Pretoria, Südafrika, 2007

[19] Blanchini, F. ; Miani, S.: Any domain of attraction for a linear constrained system ist a tracking domain of attraction. In: SIAM Journal of Optimal Control 38 (2000), Nr. 3, S. 971-994

[20] Bohn, C. ; Atherton, D. P.: An analysis package comparing PID antiwindup strategies. In: IEEE Control Systems Magazine 15 (1995), Nr. 2, S. $34-40$

[21] Boyd, S. ; Ghaoui, L. E. ; Feron, E. ; Balakrishnan, V.: Linear matrix inequalities in system and control theory. SIAM, 1994

[22] Boyd, S. ; Vandenberghe, L.: Convex Optimization. Cambridge University Press, 2004

[23] Brammer, R. F.: Controllability in linear autonomous systems with positive controllers. In: International Journal of Control 10 (1972), Nr. 2, S. 339-353

[24] Buhl, M. ; Joos, P. ; Lohmann, B.: Sättigende weiche strukturvariable Regelung. In: at - Automatisierungstechnik 56 (2008), Nr. 6, S. 316-323

[25] Buhl, Michael: Sättigende strukturvariable Regelungen, Technische Universität München, Diss., 2008

[26] BüHLER, H.: Regelkreise mit Begrenzungen. VDI Verlag, 2000 (FortschrittBerichte VDI, Reihe 8)

[27] Camacho, E. F. ; Bordons, C.: Model Predictive Control. Springer, 2007 
[28] Campo, P. J. ; Morari, M. ; Nett, C. N.: Multivariable anti-windup and bumpless transfer: a general theory. In: Proceedings of the American Control Conference. Pittsburgh (PA), USA, 1989, S. 1706-1711

[29] CAO, Y.-Y.; Lin, Z. ; WARD, D. G.: An antiwindup approach to enlarging domain of attraction for linear systems subject to actuator saturation. In: IEEE Transactions on Automatic Control 47 (2002), Nr. 1, S. 140-145

[30] Casavola, A. ; Mosca, E. ; Angeli, D.: Robust command governors for constrained linear systems. In: IEEE Transactions on Automatic Control 45 (2000), Nr. 11, S. 2071-2077

[31] Castelan, E. B. ; Tarbouriech, S. ; Gomes da Silva, J. M. Jr. ; Queinnec, I.: $L_{2}$-Stabilization of continuous-time linear systems with saturating actuators. In: International Journal of Robust Nonlinear Control 16 (2006), Nr. 18, S. 935-944

[32] Chen, C.-Y. ; Perng, M.-H.: An optimal anti-windup scheme for saturating MIMO systems. In: Proceedings of the Conference on Decision and Control. Kobe, Japan, 1996, S. 2832-2837

[33] Chesi, G.: LMI techniques for optimization over polynomials in control: a survey. In: IEEE Transactions on Automatic Control 55 (2010), Nr. 11, S. $2500-2510$

[34] Chilali, M. ; Gahinet, P.: $H_{\infty}$ design with pole placement constraints: an LMI approach. In: IEEE Transactions on Automatic Control 41 (1996), Nr. 3, S. 358-367

[35] Clarridge, R. E.: An improved pneumatic control system. In: Transactions ASME 73 (1951), S. 297-305

[36] Crawshaw, S. ; Vinnicombe, G.: Anti-windup synthesis for guaranteed $\mathrm{L}_{2}$ performance. In: Proceedings of the Conference on Decision and Control. Sydney (NSW), Australien, 2000, S. 1063-1068

[37] Dai, D. ; Hu, T. ; Teel, A. R. ; Zaccarian, L.: Piecewise-quadratic Lyapunov functions for systems with deadzones or saturations. In: Systems \&3 Control Letters 58 (2009), S. 365-371

[38] Domont-Yankulova, D.: Entwurf strukturvariabler Regelungen mittels linearer Matrixungleichungen, TU Darmstadt, Diss., 2010

[39] Dorf, R. C. ; Bishop, R. H.: Modern Control Systems. Prentice Hall, 2004 
[40] Doyle, J. C. ; Smith, R. S. ; Enns, D. F.: Control of Plants with Input Saturations Nonlinearities. In: Proceedings of American Control Conference. Minneapolis (MN), USA, 1987, S. 1034-1039

[41] Edwards, C. ; Postlethwaite, I.: Anti-windup and bumpless transfer schemes. In: Automatica 34 (1998), Nr. 2, S. 199-210

[42] Edwards, C. ; Postlethwaite, I.: An anti-windup scheme with closedloop stability considerations. In: Automatica 35 (1999), Nr. 4, S. 761-765

[43] Elliott, J.: NASA's advanced control law program for the F-8 digital flyby-wire aircraft. In: IEEE Transactions on Automatic Control 22 (1977), Nr. 5, S. 753-757

[44] Ferreau, H. J. ; Bock, H. G. ; Diehl, M.: An online active set strategy to overcome the limitations of explicit MPC. In: International Journal of Robust and Nonlinear Control 18 (2008), Nr. 8, S. 816-830

[45] Fertik, H. A. ; Ross, C. W.: Direct digital control algorithm with antiwindup feature. In: ISA Transactions 6 (1967), Nr. 4, S. 317-328

[46] Forster, O.: Analysis 1. Vieweg+Teubner, 2004

[47] Franke, D.: Ausschöpfung von Stellgrößenbeschränkungen mittels weicher strukturvariabler Regelung. In: Regelungstechnik 30 (1982), Nr. 10, S. 348-355

[48] Fuller, A. T.: In-the-large stability of relay and saturating control systems with linear controllers. In: International Journal of Control 10 (1969), Nr. 4, S. 457-480

[49] Föllinger, O.: Optimale Regelung und Steuerung. Oldenbourg, 1994

[50] Föllinger, O.: Regelungstechnik. Hüthig, 2008

[51] Galeani, S. ; Massimetti, M. ; Teel, A. R. ; Zaccarian, L.: Reduced order linear anti-windup augmentation for stable linear systems. In: International Journal of Systems Science 37 (2006), Nr. 2, S. 115-127

[52] Galeani, S. ; Onori, S. ; Teel, A. R. ; Zaccarian, L.: Nonlinear $L_{2}$ anti-windup for enlarged stability regions and regional performance. In: 7th IFAC Symposium on Nonlinear Control Systems. Pretoria, Südafrika, 2007

[53] Galeani, S. ; Onori, S. ; Teel, A. R. ; Zaccarian, L.: Regional, semiglobal, global nonlinear anti-windup via switching design. In: Proceedings of the European Control Conference. Kos, Giechenland, 2007, S. 5403-5410 
[54] Galeani, S. ; Onori, S. ; Zaccarian, L.: Nonlinear scheduled control for linear systems subject to saturation with application to anti-windup control. In: Proceedings of the Conference on Decision and Control. New Orleans (LA), USA, 2007

[55] Galeani, S. ; Tarbouriech, S. ; Turner, M. C. ; Zaccarian, L.: A tutorial on modern anti-windup design. In: European Journal of Control 15 (2009), Nr. 3-4, S. 418-440

[56] Galeani, S. ; Teel, A. R. ; Zaccarian, L.: Constructive nonlinear antiwindup design for exponentially unstable linear plants. In: Systems $\&$ Control Letters 56 (2007), Nr. 5, S. 357-365

[57] Gest, C. H.: Balanced and variable-linked automatic reset. In: Instruments 25 (1951), S. 1292-1294

[58] Gilbert, E. G. ; Kolmanovsky, I.: Fast reference governors for systems with state and control constraints and disturbance inputs. In: International Journal of Robust and Nonlinear Control 9 (1999), Nr. 15, S. 1117-1141

[59] Gilbert, E. G. ; Kolmanovsky, I.: Nonlinear tracking control in the presence of state and control constraints: a generalized reference governor. In: Automatica 38 (2002), Nr. 12, S. 2063-2073

[60] Gilbert, E. G. ; Kolmanovsky, I. ; Tan, K. T.: Discrete-time reference governors and the nonlinear control of systems with state and control constraints. In: International Journal of Robust Nonlinear Control 5 (1995), Nr. 5, S. 487-504

[61] Gilbert, E. G. ; Ong, C.-J.: Constrained linear systems with hard constraints and disturbances: an extended command governor with large domain of attraction. In: Automatica 47 (2011), Nr. 2, S. 334-340

[62] Gilbert, E. G. ; Tan, K. T.: Linear systems with state and control constraints: the theory and application of maximal output admissible sets. In: IEEE Transactions on Automatic Control 36 (1991), Nr. 9, S. 10081020

[63] Glattfelder, A. H. ; Schaufelberger, W.: Stability analysis of single loop control systems with saturation and antireset-windup circuits. In: IEEE Transactions on Automatic Control 28 (1983), Nr. 12, S. 1074-1081

[64] Glattfelder, A. H. ; Schaufelberger, W.: Start-up performance of different proportional-integral-anti-wind-up regulators. In: International Journal of Control 44 (1986), Nr. 2, S. 493-505 
[65] Glattfelder, A. H. ; Schaufelberger, W.: Stability of discrete override and cascade-limiter single-loop control systems. In: IEEE Transactions on Automatic Control 33 (1988), Nr. 6, S. 532-540

[66] Glattfelder, A. H. ; Schaufelberger, W.: Control Systems with Input and Output Constraints. Springer, 2003

[67] Glattfelder, A. H. ; Schaufelberger, W. ; Fässler, HP.: Stability of override control systems. In: International Journal of Control 37 (1983), Nr. 5, S. 1023-1037

[68] Gomes da Silva, J. M. Jr. ; Tarbouriech, S.: Antiwindup design with guaranteed regions of stability: an LMI-based approach. In: IEEE Transactions on Automatic Control 50 (2005), Nr. 1, S. 106-111

[69] Gomes da Silva, J. M. Jr. ; Tarbouriech, S.: Anti-windup design with guaranteed regions of stability for discrete-time linear systems. In: Systems \& Control Letters 55 (2006), Nr. 3, S. 184-192

[70] Grimm, G. ; Hatfield, J. ; Postlethwaite, I. ; Teel, A. R. ; Turner, M. C. ; ZacCARIAn, L.: Antiwindup for stable linear systems with input saturation: an LMI-based synthesis. In: IEEE Transactions on Automatic Control 48 (2003), Nr. 9, S. 1509-1525

[71] Grimm, G. ; Postlethwaite, I. ; Teel, A. R. ; Turner, M. C. ; ZaCCARIAN, L.: Case studies using linear matrix inequalities for optimal antiwindup synthesis. In: European Journal of Control 9 (2003), Nr. 5, S. $463-473$

[72] Grimm, G. ; Teel, A. R. ; Zaccarian, L.: Linear LMI-based external anti-windup augmentation for stable linear systems. In: Automatica 40 (2004), Nr. 11, S. 1987-1996

[73] Gussner, T.: Reglerentwurf für nichtlineare Systeme mit Stellgrößenbeschränkungen, TU Darmstadt, Diss., 2011

[74] Gutman, P. ; Hagander, P.: A new design of constrained controllers for linear systems. In: IEEE Transactions on Automatic Control 30 (1985), Nr. 1, S. 22-33

[75] Haddad, W. M. ; Kapila, V.: Absolute stability criteria for multiple slope-restricted monotonic nonlinearities. In: IEEE Transactions on Automatic Control 40 (1995), Nr. 2, S. 361-365

[76] Hanus, R.: A new technique for preventing control windup. In: Journal A 21 (1980), Nr. 1, S. 15-20 
[77] Hanus, R. ; Kinnaert, M.: Control of constrained multivariable systems using the conditioning technique. In: Proceedings of the American Control Conference. Pittsburgh (PA), USA, 1989, S. 1712-1718

[78] Hanus, R. ; Kinnaert, M. ; Henrotte, J. L.: Conditioning technique, a general anti-windup and bumpless transfer method. In: Automatica 23 (1987), Nr. 6, S. 729-739

[79] Hassibi, A. ; How, J. ; Boyd, S.: A path-following method for solving BMI problems in control. In: Proceedings of the American Control Conference Bd. 2. San Diego (CA), USA, 1999, S. 1385-1389

[80] Herrmann, G. ; Turner, M. C. ; Postlethwaite, I.: Discrete-time and sampled-data anti-windup synthesis: stability and performance. In: International Journal of Systems Science 37 (2006), Nr. 2, S. 91-113

[81] Herrmann, G. ; Turner, M. C. ; Postlethwaite, I.: Linear Matrix Inequalities in Control. In: Turner, M. C. (Hrsg.) ; BAtes, D. G. (Hrsg.): Mathematical methods for robust and nonlinear control. Springer, 2007, S. $132-142$

[82] Hippe, P.: Zustandsregelung. Springer, 1985

[83] Hippe, P.: Windup prevention for unstable systems. In: Automatica 39 (2003), Nr. 11, S. 1967-1973

[84] Hippe, P.: Eine neue Methode zur Regelung von Strecken mit Stellbegrenzung. In: Automatisierungstechnik 52 (2004), Nr. 9, S. 421-431

[85] Hippe, P.: Windup in Control. Springer, 2006

[86] Hippe, P.: Windup prevention for stable and unstable MIMO systems. In: International Journal of Systems Science 37 (2006), Nr. 2, S. 67-78

[87] Hippe, P.: Eine systematische Vermeidung der durch Stellbegrenzungen ausgelösten Probleme. In: Automatisierungstechnik 55 (2007), Nr. 8, S. $377-393$

[88] Hippe, P. ; Wurmthaler, C.: Controller and plant windup prevention in MIMO loops with input saturation. In: Proceedings of the European Control Conference. Brüssel, Belgien, 1997

[89] Hippe, P. ; Wurmthaler, C.: Systematic closed-loop design in the presence of input saturations. In: Automatica 35 (1999), S. 689-695

[90] Hippe, P. ; Wurmthaler, C. ; Glattfelder, A. H. ; Schaufelberger, W.: Regelung von Strecken mit Stellbegrenzung. In: Engell, S. (Hrsg.): Entwurf nichtlinearer Regelungen. Oldenbourg, 1995, S. 239-264 
[91] Hu, T. ; Lin, Z.: Control systems with actuator saturation. Birkhäuser, 2001

[92] Hu, T. ; Lin, Z.: Output regulation of linear systems with bounded continuous feedback. In: IEEE Transactions on Automatic Control 49 (2004), Nr. 11, S. 1941-1953

[93] Hu, T. ; Lin, Z. ; Chen, B. M.: An analysis and design method for linear systems subject to actuator saturation and disturbance. In: Automatica 38 (2002), Nr. 2, S. 351-359

[94] Hu, T. ; Teel, A. R. ; Zaccarian, L.: Stability and performance for saturated systems via quadratic and nonquadratic Lyapunov functions. In: IEEE Transactions on Automatic Control 51 (2006), Nr. 11, S. 17701786

[95] Hu, T. ; Teel, A. R. ; Zaccarian, L.: Anti-windup synthesis for linear control systems with input saturation: achieving regional, nonlinear performance. In: Automatica 44 (2008), Nr. 2, S. 512-519

[96] Jarvis-Wloszek, Z. W.: Lyapunov Based Analysis and Controller Synthesis for Polynomial Systems using Sum-of-Squares Optimization, University of California, Berkley, Diss., 2003

[97] Jasniewicz, B.: Über weiche strukturvariable Regelung mittels impliziter Lyapunov-Funktionen - von der impliziten zur expliziten Regelung, TU Darmstadt, Diss., 2009

[98] Jasniewicz, B. ; Adamy, J. ; Domont-Yankulova, D.: Vereinfachte schnelle Regelung von linearen Systemen mit Stellgrößenbeschränkungen. In: at - Automatisierungstechnik 59 (2011), Nr. 2, S. 84-93

[99] Johansson, M.: Piecewise Linear Control Systems, Lund University, Diss., 1999

[100] Johansson, M. ; Rantzer, A.: Computation of piecewise quadratic Lyapunov functions for hybrid systems. In: IEEE Transactions on Automatic Control 43 (1998), Nr. 4, S. 555-559

[101] Kapasouris, P. ; Athans, M.: Multivariable control systems with saturating actuators antireset windup strategies. In: Proceedings of the American Control Conference. Boston (MA), USA, 1985, S. 1579-1584

[102] Kapasouris, P. ; Athans, M. ; Stein, G.: Design of feedback control systems for stable plants with saturating actuators. In: Proceedings of the 27th IEEE Conference on Decision and Control. Austin, Texas, USA, 1988, S. 469-479 vol.1 
[103] Kapila, V. (Hrsg.) ; Grigoriadis, K. M. (Hrsg.): Actuator saturation control. Marcel Dekker, 2002

[104] Kefferpütz, K. ; Adamy, J.: Saturating set point tracking controller for linear systems subject to input constraints. In: Proceedings of the Conference on Decision and Control. Atlanta (GA), USA, 2010, S. 51745179

[105] Kefferpütz, K. ; Adamy, J.: Schnelle Regelung linearer Systeme mit Stellgrößen- und Stellratenbegrenzungen. In: at - Automatisierungstechnik 58 (2010), Nr. 7, S. 361-369

[106] Kefferpütz, K. ; Adamy, J.: A Tracking Controller for Linear Systems subject to Input Amplitude and Rate Constraints. In: Proceedings of the American Control Conference. San Francisco (CA), USA, 2011, S. 37903795

[107] Khalil, H. K.: Nonlinear Systems. Prentice Hall, 2001

[108] Khandheria, J. ; Luyben, W. L.: Experimental evaluation of digital algorithms for antireset windup. In: Industrial \& Engineering Chemistry Process Design and Development 15 (1976), Nr. 2, S. 278-285

[109] KiendL, H.: Suboptimale Regler mit abschnittsweise linearer Struktur. Springer, 1972

[110] Kiendl, H. ; Schneider, G.: Synthese nichtlinearer Regler für die Regelstrecke const $/ s^{2}$ aufgrund ineinandergeschachtelter abgeschlossener Gebiete beschränkter Stellgröße. In: Regelungstechnik und Prozeßdatenverarbeitung 20 (1972), Nr. 7, S. 289-296

[111] Kollmann, E.: Maßnahmen zur Verbesserung des Anfahrens einschleifiger Regelkreise. In: Regelungstechnische Praxis 13 (1971), Nr. 1 und 2, S. 6772 und $96-102$

[112] Kothare, M. V. ; Campo, P. J. ; Morari, M. ; Nett, C. N.: A unified framework for the study of anti-windup designs. In: Automatica 30 (1994), Nr. 12, S. 1869-1883

[113] Kothare, M. V. ; Morari, M.: Multivariable anti-windup controller synthesis using multi-objective optimization. In: Proceedings of the American Control Conference. Albuquerque (NM), USA, 1997, S. 3093-3097

[114] Kothare, M. V. ; Morari, M.: Multiplier theory for stability analysis of anti-windup control systems. In: Automatica 35 (1999), S. 917-928 
[115] Kramer, L. C. ; Jenkins, K. W.: A new technique for preventing direct digital control windup. In: Proceedings of the Joint Automatic Control Conference. St. Louis (MO), USA, 1971, S. 571-577

[116] Krikelis, N. J.: State feedback integral control with ïntelligentïntegrators. In: International Journal of Control 32 (1980), Nr. 3, S. 465-473

[117] Krikelis, N. J. ; Barkas, S. K.: Design of tracking systems subject to actuator saturation and integrator windup. In: International Journal of Control 39 (1984), Nr. 4, S. 667-682

[118] Kroll, A. ; Abel, D.: Modellbasierte Prädiktive Regelung. In: at Automatisierungstechnik 54 (2006), Nr. 12, S. 587-589

[119] Lee, S. M. ; PARK, J. H. ; Kwon, O. M.: Improved asymptotic stability analysis for Lur'e systems with sector and slope restricted nonlinearities. In: Physics Letters A 362 (2007), Nr. 5-6, S. 348-351

[120] Lens, H.: Schnelle Regelung mit Ausgangsrückführung für Systeme mit Stellgrößenbeschränkungen, TU Darmstadt, Diss., 2009

[121] Lens, H. ; AdAmy, J.: Weiche strukturvariable Regelungen mit Beobachtern für einen hydraulischen Aktor. In: Automatisierungstechnik 55 (2007), Nr. 8, S. 411-417

[122] Lens, H. ; Adamy, J.: Schnelle Regelung von linearen Systemen mit Stellgrößenbeschränkungen. In: at - Automatisierungstechnik 57 (2009), Nr. 2, S. 70-79

[123] Lens, H. ; Adamy, J. ; Domont-Yankulova, D.: A fast nonlinear control method for linear systems with input saturation. In: Automatica 47 (2011), Nr. 4, S. 857-860

[124] Lin, Z.: Global control of linear systems with saturating actuators. In: Automatica 34 (1998), Nr. 7, S. 897-905

[125] Lin, Z.: Low gain feedback. Springer, 1998

[126] Lin, Z.: Low gain and low-and-high gain feedback: A review and some recent results. In: Proceedings of the Chinese Control and Decision Conference. Guilin, China, 2009, S. lii-lxi

[127] Lin, Z. ; SABERI, A.: Semi-global exponential stabilization of linear systems subject to input saturation via linear feedbacks. In: Systems and Control Letters 21 (1993), Nr. 3, S. 225-239 
[128] Lin, Z. ; SABERI, A.: A semi-global low-and-high gain design technique for linear systems with input saturation - stabilization and disturbance rejection. In: International Journal of Robust and Nonlinear Control 5 (1995), Nr. 5, S. 381-398

[129] Lin, Z. ; SABERI, A.: Low-and-high gain design technique for linear systems subject to input saturation - a direct method. In: International Journal of Robust and Nonlinear Control 7 (1997), Nr. 12, S. 1071-1101

[130] Lin, Z. ; Stoorvogel, A. A. ; Saberi, A.: Output regulation for linear systems subject to input saturation. In: Automatica 32 (1996), Nr. 1, S. $29-47$

[131] LozIER, J. C.: A steady state approach to the theory of saturable servo systems. In: IRE Transactions on Automatic Control 1 (1956), Nr. 1, S. 19-39

[132] Lunze, J.: Regelungstechnik 1. Springer, 2004

[133] Lunze, J.: Regelungstechnik 2. Springer, 2008

[134] LÖFBERG, J.: YALMIP : a toolbox for modeling and optimization in MATLAB. In: International Symposium on Computer Aided Control System Design. Taipeh, Taiwan, 2004, S. 284-289

[135] Marcopoli, V. R. ; Phillips, S. M.: Analysis and synthesis tools for a class of actuator-limited multivariable control systems: a linear matrix inequality approach. In: International Journal of Robust and Nonlinear Control 6 (1996), Nr. 9-10, S. 1045-1063

[136] Mayne, D. Q. ; Rawlings, J. B. ; Rao, C. V. ; Scokaert, P. O. M.: Constrained model predictive control: Stability and optimality. In: Automatica 36 (2000), Nr. 6, S. 789-814

[137] Megretski, A.: $L_{2}$ BIBO Output feedback stabilization with saturated control. In: Proceedings of the IFAC World Congress Bd. D. San Francisco (CA), USA, 1996, S. 435-440

[138] Miyamoto, S. ; Vinnicombe, G.: Robust Control of plants with saturation nonlinearity based on coprime factor representation. In: Proceedings of the Conference on Decision and Control. Kobe, Japan, 1997, S. 2838-2840

[139] Mulder, E. F. ; Kothare, M. V. ; Morari, M.: Multivariable antiwindup controller synthesis using linear matrix inequalities. In: Automatica 37 (2001), Nr. 9, S. 1407-1416 
[140] Mulder, E. F. ; Tiwari, P. Y. ; Kothare, M. V.: Simultaneous linear and anti-windup controller synthesis using multiobjective convex optimization. In: Automatica 45 (2009), S. 805-811

[141] Narendra, K. S. ; Taylor, J. H.: Frequency Domain Criteria for Absolute Stability. Academic Press, 1973

[142] NASA: F-8 Digital Fly-By-Wire Aircraft. Quelle: NASA-Webseite, URL: http://www.nasa.gov/centers/dryden/news/FactSheets/FS-024DFRC.html, Version vom 2.12.2009, abgerufen im Mai 2012

[143] Nguyen, T. ; JABbARI, F.: Output feedback controllers for disturbance attenuation with actuator amplitude and rate saturation. In: Automatica 36 (2000), Nr. 9, S. 1339-1346

[144] Noisser, R.: Anti-Reset-Windup-Maßnahmen für Eingrößenregelungen mit digitalen Reglern. In: at - Automatisierungstechnik 35 (1987), Nr. 12, S. 499-504

[145] Ohlson, G. F.: Reset inhibiting improves control. In: Instruments \& Control Systems 38 (1965), Nr. 5, S. 143-147

[146] Oppelt, W.: Kleines Handbuch Technischer Regelvorgänge. Verlag Chemie $\mathrm{GmbH}, 1960$

[147] Ortseifen, A. ; Adamy, J.: Eine $L_{2}$-optimale Beobachtertechnik zur Vermeidung von Regler-Windup. In: at - Automatisierungstechnik 59 (2011), Nr. 2, S. 84-93

[148] Ortseifen, A. ; AdAmy, J.: A new design method for mismatch-based anti-windup compensators: achieving local performance and global stability in the SISO case. In: Proceedings of the American Control Conference. San Francisco (CA), USA, 2011, S. 3796-3801

[149] Ortseifen, A. ; AdAmy, J.: A performance-oriented, non-iterative, local design method for mismatch-based anti-windup compensators. In: Proceedings of the IEEE Multiconference on Systems and Control. Denver (CO), USA, 2011, S. 1056-1061

[150] Ortseifen, A. ; Adamy, J.: Ein einfaches weich strukturvariables AntiWindup. In: at - Automatisierungstechnik 60 (2012), Nr. 3, S. 123-133

[151] Ostertag, E.: An improved path-following method for mixed $H_{2} / H_{\infty}$ controller design. In: IEEE Transactions on Automatic Control 53 (2008), Nr. 8, S. 1967-1971 
[152] Pannocchia, G. ; Rawlings, J. B. ; Wright, S. J.: Fast, large-scale model predictive control by partial enumeration. In: Automatica 43 (2007), Nr. 5, S. $852-860$

[153] Parillo, P.: Structured Semidefinite Programs and Semialgebraic Geometry Methods in Robustness and Optimization, California Institute of Technology, Diss., 2000

[154] PARK, P.: Stability criteria of sector- and slope-restricted Lur'e systems. In: IEEE Transactions on Automatic Control 47 (2002), Nr. 2, S. 308-313

[155] Peng, Y. ; Vrancic, D. ; Hanus, R.: Anti-windup, bumpless, and conditioned transfer techniques for PID controllers. In: IEEE Control Systems Magazine 16 (1996), Nr. 4, S. 48-57

[156] Peng, Y. ; Vrancic, D. ; Hanus, R. ; Weller, S. S. R.: Anti-windup designs for multivariable controllers. In: Automatica 34 (1998), Nr. 12, S. $1559-1565$

[157] Piwinger, F.: Automatisches Anfahren von Chargenprozessen. In: Regelungstechnik 10 (1962), Nr. 1, S. 12-16

[158] Rawlings, J. B.: Tutorial overview of model predictive control. In: IEEE Control Systems Magazine 20 (2000), Nr. 3, S. 38-52

[159] Rossiter, J. A.: Model-based predictive control - a practical approach. CRC Press, 2003

[160] Rönnbäck, S. ; Walgama, K. S. ; Sternby, J.: An extension to the generalized anti-windup compensator. In: Proceedings of the IMACS World Congress on Computation and Applied Mathematics. Dublin, Irland, 1991, S. $1192-1196$

[161] Saberi, A. ; Hou, P. ; Stoorvogel, A. A.: On simultaneous global external and global internal stabilization of critically unstable linear systems with saturating actuators. In: IEEE Transactions on Automatic Control 45 (2000), Nr. 6, S. 1042-1052

[162] Saberi, A. ; Lin, Z. ; Teel, A. R.: Control of linear systems with saturating actuators. In: IEEE Transactions on Automatic Control 41 (1996), Nr. 3, S. 368-378

[163] Saberi, A.; Stoorvogel, A. A. ; Sannuti, P.: Control of linear systems with regulation and input constraints. Springer, 1999

[164] Safonov, M. ; Athans, M.: A multiloop generalization of the circle criterion for stability margin analysis. In: IEEE Transactions on Automatic Control 26 (1981), Nr. 2, S. 415-422 
[165] Schmitendorf, W. E. ; Barmish, B. R.: Null controllability of linear systems with constrained controls. In: Journal on Control and Optimization 8 (1980), Nr. 4, S. 327-345

[166] Schmitt, G.: Frequency domain evaluation of circle criterion, Popov criterion and off-axis circle criterion in the MIMO case. In: International Journal of Control 72 (1999), Nr. 14, S. 1299-1309

[167] Schmitт, G.: Stabilitätsanalyse nichtlinearer Mehrgrößen-Regelkreise mit Hilfe des Kriteriums der nicht-axialen Kreise. In: at - Automatisierungstechnik 47 (1999), Nr. 6, S. 270-276

[168] Shamma, J. S.: Anti-windup via constrained regulation with observers. In: Systems $\& 3$ Control Letters 40 (2000), Nr. 4, S. 261-268

[169] Shewchun, J. M. ; Feron, E.: High performance control with position and rate limited actuators. In: International Journal of Robust and Nonlinear Control 9 (1999), Nr. 10, S. 617-630

[170] Shi, G. ; Saberi, A. ; Stoorvogel, A. A.: On the $L_{p}\left(l_{p}\right)$ stabilization of open-loop neutrally stable linear plants with input subject to amplitude saturation. In: International Journal of Robust and Nonlinear Control 13 (2003), Nr. 8, S. 735-754

[171] Shi, G. ; Saberi, A. ; Stoorvogel, A. A. ; Sannuti, P.: Semi-global stabilization and output regulation of constrained linear plants via measurement feedback. In: International Journal of Control 75 (2002), Nr. 18, S. $1525-1538$

[172] Shinskey, F. G.: Process-control systems. McGraw-Hill, 1967

[173] Sofrony, J.: Anti-windup Compensation of Input Constrained Systems. VDM Verlag Dr. Müller, 2009

[174] Sofrony, J. ; Turner, M. C. ; Postlethwaite, I.: Anti-windup synthesis using Riccati equations. In: International Journal of Control 80 (2007), Nr. 1, S. $112-128$

[175] Sofrony, J. ; Turner, M. C. ; Postlethwite, I.: Anti-windup synthesis for systems with rate-limits using Riccati equations. In: International Journal of Control 83 (2010), Nr. 2, S. 233-245

[176] Solyom, S.: A synthesis method for static anti-windup compensators. In: Proceedings of the European Control Conference. Cambridge, Vereinigtes Königreich Großbritannien und Nordirland, 2003, S. 551-554 
[177] SontaG, E. D.: An algebraic approach to bounded controllability of linear systems. In: International Journal of Control 39 (1984), Nr. 1, S. 181-188

[178] Stein, G.: Respect the unstable. In: IEEE Control Systems Magazine 23 (2003), Nr. 4, S. 12-25

[179] Stoorvogel, A. A. ; SABeri, A.: Output regulation of linear plants with actuators subject to amplitude and rate constraints. In: International Journal of Robust and Nonlinear Control 9 (1999), Nr. 10, S. 631-657

[180] Sturm, J. F.: Using SeDuMi 1.02, a Matlab toolbox for optimization over symmetric cones. In: Optimization Methods and Software 11 (1999), Nr. 1-4, S. 625-653

[181] Sussmann, H. J. ; Sontag, E. D. ; YAng, Y.: A general result on the stabilization of linear systems using bounded controls. In: IEEE Transactions on Automatic Control 39 (1994), Nr. 12, S. 2411-2425

[182] Tarbouriech, S. ; Garcia, G. ; Gomes da Silva, J. M. Jr. ; Queinnec, I.: Stability and Stabilization of Linear Systems with Saturating Actuators. Springer, 2011

[183] Tarbouriech, S. ; Turner, M. C.: Anti-windup design: an overview of some recent advances and open problems. In: IET Control Theory and Applications 3 (2009), Nr. 1, S. 1-19

[184] TeEL, A. R.: Semi-global stabilizability of linear null controllable systems with input nonlinearities. In: IEEE Transactions on Automatic Control 40 (1995), Nr. 1, S. 96-100

[185] Teel, A. R. ; Kapoor, N.: The L2 anti-windup problem: its definition and solution. In: Proceedings of the European Control Conference. Brüssel, Belgien, 1997

[186] Teel, A. R. ; Praly, L.: On assigning the derivative of a disturbance attenuation control Lyapunov function. In: Control Signals Systems 13 (2000), S. 95-124

[187] Tiwari, P. Y.; Mulder, E. F. ; Kothare, M. V.: Synthesis of stabilizing antiwindup controllers using piecewise quadratic Lyapunov functions. In: IEEE Transactions on Automatic Control 52 (2007), Nr. 12, S. 2341-2345

[188] Toh, K.-C. ; Todd, M. J. ; Tutuncu, R. H.: SDPT3 - A Matlab software package for semidefinite programming. In: Optimization Methods and Software 11 (1999), Nr. 1-4, S. 545-581 
[189] Toop, G. H.: A technique for minimizing overshoots in discontinuous processes. In: Instrument Practice 6 (1952), S. 369-379

[190] Turner, M. C. (Hrsg.) ; Bates, D. G. (Hrsg.): Mathematical Methods for Robust and Nonlinear Control. Springer, 2007

[191] Turner, M. C. ; Herrmann, G. ; Postlethwaite, I.: Anti-windup compensation using a decoupling architecture. In: TARBOURIECH, S. (Hrsg.): Advanced Strategies in Control Systems with Input and Output constraints. Springer, 2007, S. 121-171

[192] Turner, M. C. ; Kerr, M. ; Postlethwaite, I.: On the existence of multipliers for MIMO systems with repeated slope-restricted nonlinearities. In: ICCAS-SICE, 2009. Fukuoka, Japan, 2009, S. 1052-1057

[193] Turner, M. C. ; Kerr, M. ; Postlethwaite, I.: On the existence of stable, causal multipliers for systems with slope-restricted nonlinearities. In: IEEE Transactions on Automatic Control 54 (2009), Nr. 11, S. 26972702

[194] Voigt, C. ; Adamy, J.: Formelsammlung der Matrizenrechnung. Oldenbourg, 2007

[195] Walgama, K. S. ; Rönnbëck, S. ; Sternby, J.: Generalisation of conditioning technique for anti-windup compensators. In: IEEE Proceedings on Control Theory and Applications Bd. 139, 1992, S. 109-118

[196] Walgama, K. S. ; Sternby, J.: Inherent observer property in a class of anti-windup compensators. In: International Journal of Control 52 (1990), Nr. 3, S. 705-724

[197] Walgama, K. S. ; Sternby, J.: Conditioning technique for multiinputmultioutput processes with input saturation. In: IEE Proceedings - Control Theory and Applications 140 (1993), Nr. 4, S. 231-242

[198] Wang, Y. ; Boyd, S.: Fast model predictive control using online optimization. In: IEEE Transactions on Control Systems Technology 18 (2010), Nr. 2, S. 267-278

[199] Weston, F. ; Postlethwaite, I.: Linear conditioning for systems containing saturating actuators. In: Automatica 36 (2000), Nr. 9, S. 1347-1354

[200] Wirsching, G. J.: Gewöhnliche Differentialgleichungen. Vieweg+Teubner, 2006

[201] Wittenmark, B.: Integrators, Nonlinearities, and Anti-reset Windup for Different Control Structures. In: Proceedings of the American Control Conference. Pittsburgh (PA), USA, 1989, S. 1679-1683 
[202] Wredenhagen, G. F. ; Bélanger, P. R.: Piecewise-linear LQ control for systems with input constraints. In: Automatica 30 (1994), Nr. 3, S. 403-416

[203] Wu, F. ; Lin, Z. ; Zheng, Q.: Output feedback stabilization of linear systems with actuator saturation. In: IEEE Transactions on Automatic Control 52 (2007), Nr. 1, S. 122-128

[204] Wu, F. ; Lu, B.: Anti-windup control design for exponentially unstable LTI systems with actuator saturation. In: Systems \& Control Letters 52 (2004), Nr. 3-4, S. 305-322

[205] Wurmthaler, C.: Einsatz von Zustandsregelungen bei begrenzter Stellgröße und in Ablöseregelungen. In: at - Automatisierungstechnik 25 (1977), Nr. 3, S. 91-92

[206] Wurmthaler, C. ; Hippe, P.: Verbesserung des Stör- und Führungsverhaltens bei stabilen und instabilen Regelstrecken mit Stellbegrenzung. In: at - Automatisierungstechnik 42 (1994), Nr. 7, S. 299-307

[207] Wurmthaler, C. ; Sauerwein, R.: PID-Reglerstruktur zur Berücksichtigung von Stellsignalbegrenzungen. In: at - Automatisierungstechnik 34 (1986), Nr. 9, S. 372-375

[208] Zaccarian, L. ; Li, Y. ; Weyer, E. ; Cantoni, M. ; Teel, A. R.: Antiwindup for marginally stable plants and its application to open water channel control systems. In: Control Engineering Practice 15 (2007), Nr. 2, S. $261-272$

[209] Zaccarian, L. ; Teel, A. R.: A common framework for anti-windup, bumpless transfer and reliable designs. In: Automatica 38 (2002), Nr. 10, S. $1735-1744$

[210] Zaccarian, L. ; Teel, A. R.: Nonlinear scheduled anti-windup design for linear systems. In: IEEE Transactions on Automatic Control 49 (2004), Nr. 11, S. 2055-2061

[211] Zaccarian, L. ; Teel, A. R.: Modern Anti-windup Synthesis. Princeton University Press, 2011

[212] Zames, G.: On the input-output stability of time-varying nonlinear feedback systems - part 1: conditions derived using concepts of loop gain, conicity and positivity. In: IEEE Transactions on Automatic Control AC-11 (1966), Nr. 2, S. 228-238

[213] Zheng, A. ; Kothare, M. V. ; Morari, M.: Anti-windup design for internal model control. In: International Journal of Control 60 (1994), Nr. 5, S. 1015-1024 


\section{Lebenslauf}

Persönliche Daten

Name Andreas Ortseifen

Geburtsdaten 08.10.1983 in Karlsruhe

Schulbildung

1994 - $2003 \quad$ Main-Taunus-Schule in Hofheim

Abschluss: Abitur

Studium und Praktika

\begin{tabular}{|c|c|}
\hline $2003-2009$ & $\begin{array}{l}\text { Studium: Elektrotechnik und Informationstechnik } \\
\text { Technische Universität Darmstadt } \\
\text { Vertiefungsrichtung: Automatisierungstechnik } \\
\text { Abschluss: Dipl.-Ing. }\end{array}$ \\
\hline $\begin{array}{l}2003 \\
2007-2008\end{array}$ & $\begin{array}{l}\text { Grundpraktikum: Braun GmbH, Kronberg } \\
\text { Fachpraktikum: Robert Bosch Corporation, } \\
\text { Anderson (SC), USA }\end{array}$ \\
\hline \multicolumn{2}{|c|}{ Berufstätigkeit } \\
\hline $2009-2012$ & $\begin{array}{l}\text { Wissenschaftlicher Mitarbeiter am Fachgebiet } \\
\text { Regelungstheorie und Robotik des Instituts } \\
\text { für Automatisierungstechnik und Mechatronik } \\
\text { der Technischen Universität Darmstadt }\end{array}$ \\
\hline 2012 - z. Z & $\begin{array}{l}\text { Forschungsingenieur Regelungstechnik, } \\
\text { Robert Bosch GmbH, Schwieberdingen }\end{array}$ \\
\hline
\end{tabular}

